\title{
STOCHASTIC MODELING OF A FRACTURE NETWORK IN A HYDRAULICALLY \\ FRACTURED SHALE-GAS RESERVOIR
}

\author{
A Thesis \\ by \\ ADNENE MHIRI \\ Submitted to the Office of Graduate and Professional Studies of \\ Texas A\&M University \\ in partial fulfillment of the requirements for the degree of \\ MASTER OF SCIENCE \\ Thomas A. Blasingame \\ Walter B. Ayers \\ Maria A. Barrufet \\ A. Daniel Hill
}

Chair of Committee,

Committee Members,

Head of Department,

August 2014

Major Subject: Petroleum Engineering

Copyright 2014 Adnene Mhiri 


\begin{abstract}
The fundamental behavior of fluid production from shale/ultra-low permeability reservoirs that are produced under a constant wellbore pressure remains difficult to quantify, which is believed to be (at least in part) due to the complexity of the hydraulic fracture patterns created during the well stimulation process. This work introduces a novel approach to model the hydraulic fractures in a shale reservoir using a stochastic method called random-walk. We see this approach as a beginning step that could be used to capture a part of the "complexity" of a fracture that has been generated by a hydraulic fracturing treatment and that such "complex" fracture processes may be observed in the Microseismic measurements.

To assess the random-walk fracture concept, we performed numerical simulation of the patterns generated using a given random-walk fracture pattern. Using a total of 83 pattern cases, sensitivity analyses were performed on these fracture patterns; where the tortuosity, the extent (length), the tendency to split, and the number of branching stages were the factors considered. The rate performance of the "random-walk" fracture cases were compared to the standard model of a (single) planar hydraulic fracture. In addition to the mass rate performance, the created pressure distribution was analyzed in "time slices" (or "snapshots) to qualitatively assess each complex-pattern during early production times (before the onset of the pseudosteady-state flow regime).

Our results were used to create a correlation between fracture performance, in terms of cumulative recovery, and the fracture volume and "complexity." In addition, an empirical correlation between the number of stages of bifurcation (splitting) of the fracture pattern and the value of the mass rate $\beta$ derivative for early production times was then established. Finally, as we were limited to a small-scale case due to the intensive gridding required, the feasibility and the advantages of a full-scale reservoir and well model are discussed.
\end{abstract}




\section{DEDICATION}

I dedicate this work to:

The love of my life Hana; for all the encouragement that inspired me to complete this work. My parents, Nabil and Fatma; who never stopped believing in me.

My sisters; Wafa and Hela. 


\section{ACKNOWLEDGEMENTS}

I would like to express my gratitude to the following people that contributed to this work: Dr. Thomas A. Blasingame for his supervision, his patience and his demand for perfection; Dr. Walter B. Ayers for the quality of his lessons and his contribution in this work; Dr. Maria A. Barrufet for her kindness and precious advice; Dr. George J. Moridis for providing the base numerical simulator, and his demand for perfection; Dr. Peter P. Valkó for his assistance and guidance and Dr. Spiros Vellas (Texas A\&M supercomputing facility) who provided supercomputer access. 


\section{TABLE OF CONTENTS}

\section{Page}

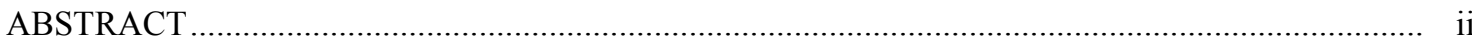

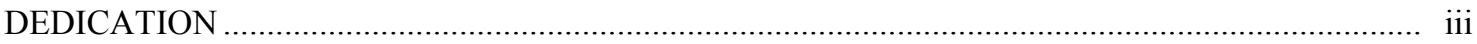

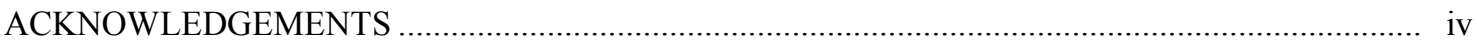

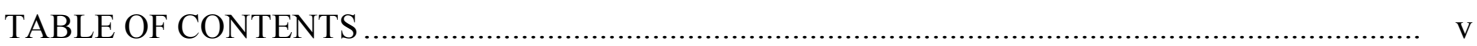

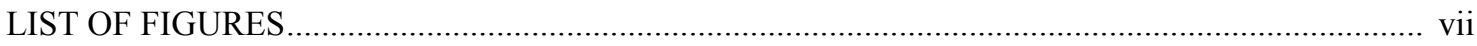

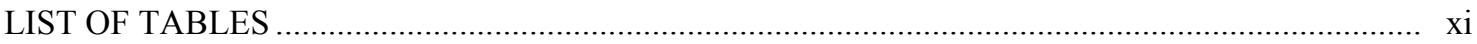

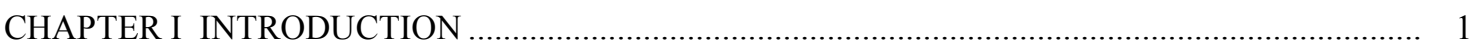

I.1 Statement of the Problem .................................................................................... 1

I.2 Objectives of the Research ................................................................................... 2

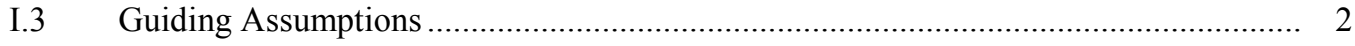

I.4 Parameters Used in Modeling Study ……….......................................................... 4

I.5 Numerical Simulation Model ..................................................................................... 4

I.6 Comparison of Results: Planar Fracture Model...................................................... 5

I.7 Validation: Comparison to a Commercial Modeling Software ……........................... 6

I.8 Challenges in Modeling the Complex Fracture Patterns ............................................. 8

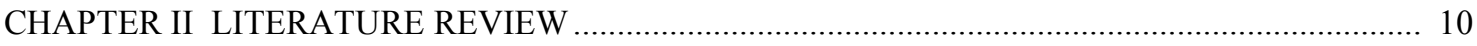

II.1 Analytical and Numerical Models for Fractured Well Performance ............................. 10

II.2 Present Status — Modeling of Complex Hydraulic Fracture Patterns ........................... 11

II.3 Research Hypothesis ......................................................................................... 12

II.4 A Novel Approach to Model 2-D Fractures: "Random-Walk" Fractures....................... 13

II.5 Grid Refinement Issues: Random-Walk Fracture Patterns........................................... 14

CHAPTER III DEVELOPMENT OF A SIMPLESTOCHASTIC FRACTURE PROPAGATION

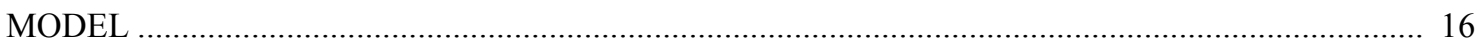

III.1 Introduction to the Stochastic Fracture Propagation Model ..................................... 16

III.2 Mathematical Description of the Random-Walk Fracture Problem .............................. 17

III.3 Constraining the Fracture Network to Preferential Growth Directions ......................... 19

III.4 Bifurcation (Splitting) of a Given Fracture .............................................................. 20

III.5 Generation of the Fracture Network and Reservoir Simulation .................................... 23

III.6 Stochastic Fracture Generation Algorithm ........................................................ 25

III.6.1 Non-Branched Fracture Pattern ................................................................. 25

III.6.2 Mono-Branched Fracture Pattern .................................................................. 27

III.6.3 Dual-Branched Fracture Pattern .................................................................. 30

III.6.4 Tri-Branched Fracture Pattern ...................................................................... 32

III.6.5 Quad-Branched Fracture Pattern .................................................................. 33

III.7 Reservoir Model and Simulation....................................................................... 35 
III.7.1 Consideration of the Multiply-Fractured Horizontal Well Model ................. 35

III.7.2 Optimization of Gridding (Minimum Number of Grid Blocks) .................... 36

III.7.3 Orientation - Modeling Fracture Patterns ..................................................... 36

CHAPTER IV INFLUENCE OF THE STOCHASTIC FRACTURE CHARACTERISTICS ............... 38

IV.1 Methodology to Assess the Performance of the Stochastic Fracture Patterns ............... 38

IV.1.1 Map the Pressure Change to Identify the Flow Regimes.............................. 38

IV.1.2 Reservoir Performance (Mass Production Rate and $\beta$-Derivative)............... 39

IV.1.3 Cumulative Mass Recovery .................................................................... 39

IV.2 Single-Planar Fracture Case (Benchmark/Comparative Standard)................................ 40

IV.2.1 Single Planar Fracture Case: Reservoir Pressure Profiles ............................ 42

IV.2.2 Single Planar Fracture Case: Mass Production Rate and $\beta$-Derivative.......... 42

IV.2.3 Single Planar Fracture Case: Cumulative Mass Recovery ……………….... 44

IV.3 Stochastic Fracture Networks - Non-Branched Fracture Patterns ………................... 45

IV.4 Stochastic Fracture Networks - Mono-Branched Fracture Patterns ............................ 48

IV.5 Stochastic Fracture Networks — Dual-Branched Fracture Patterns................................ 52

IV.6 Stochastic Fracture Networks — Tri-Branched Fracture Patterns ............................... 56

IV.7 Stochastic Fracture Networks — Quad-Branched Fracture Patterns.............................. 60

IV.8 Stochastic Fracture Networks — Impact on Reservoir Performance ............................. 64

IV.9 Stochastic Fracture Networks — Summary of Additional Modeling Cases ................. 65

IV.10 Stochastic Fracture Networks - Correlation of the $\beta$-derivative Performance (all

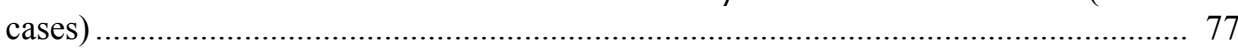

IV.11 Stochastic Fracture Networks — Assumptions, Limitations, and Discussion............... 78

\section{CHAPTER V SUMMARY, CONCLUSIONS AND RECOMMENDATIONS FOR FUTURE}

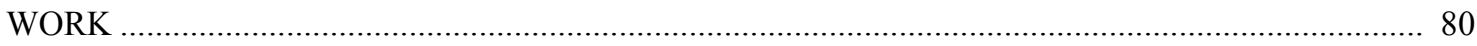

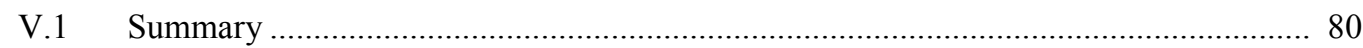

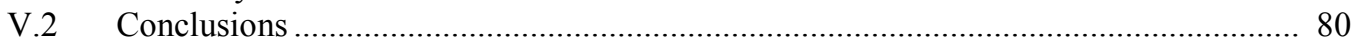

V.3 Recommendations for Future Work ………………................................................. 81

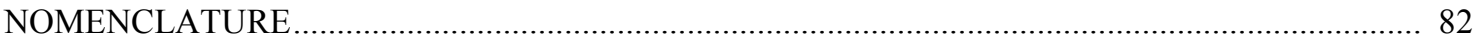

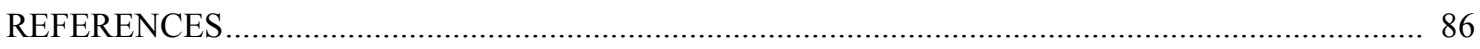

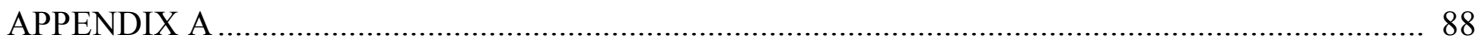

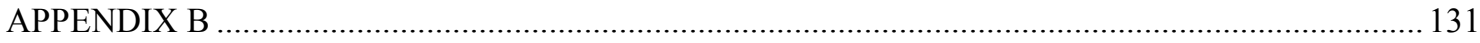

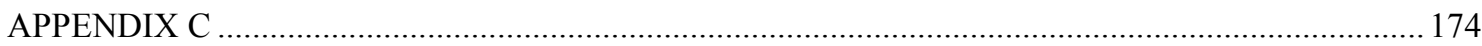

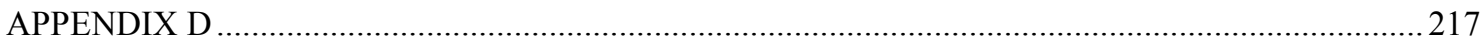




\section{LIST OF FIGURES}

Figure 1 U.S. dry natural gas production (Reproduced with data from EIA (2014))................ 1

Figure $2-$ Base case reservoir model (planar fracture). The right-side shows the complete grid and the left-side is an expanded view of the refined grid fracture region................... 5

Figure $3-$ Synthetic example - producing well has a single vertical (planar) fracture.............. 6

Figure $4-$ Comparison of mass production rates (FTSim and Rubis simulation codes)............. 7

Figure $5-$ Comparison of cumulative produced gas as a percentage of the initial volume of gas for a synthetic case modeled using the FTSim and Ecrin simulators. ........................ 8

Figure 6 - Olorode et al. (2013) imagined variations of the geometry of the main planar

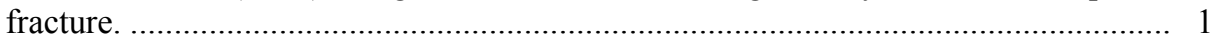

Figure $7-3$ single-branch fracture cases generated using the MESHMODIFIER code. We observe that the tortuosity of the fracture path progressively increases due to the ratio of probabilities $x$ - and $y$-directions [i.e., the $P_{x+1} /\left(P_{y+1}+P_{y-1}\right)$ ratio]....................... 14

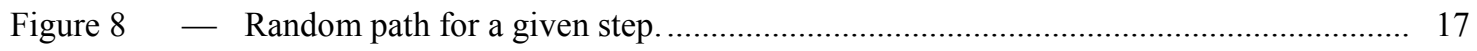

Figure $9-$ Various random-walk paths with uniform probabilities for 1000 iterations................ 18

Figure $10-$ Preferential growth of a fracture direction after 1000 iterations................................. 19

Figure 11 - Random-walk with uniform probabilities for 1000 iterations ( 2 branches)................. 21

Figure $12-$ Random-walk with uniform probabilities for 1000 iterations (4 branches)................ 22

Figure 13 - Random-walk fracture network projected on a 2-D grid structure............................. 23

Figure $14-$ Schematic of a progressively coarsening grid-size near a fracture.............................. 24

Figure $15-2$ 2-D view of the fracture grid in an unfractured reservoir.......................................... 24

Figure 16 - In the upper 3 figures the realizations for a non-branched pattern with an $x_{\max }=2.25 \mathrm{~m}$ constraint are shown. In the lower 3 figures the realizations of a nonbranched pattern with 225 of iterations $(N=225)$ are shown. .................................... 26

Figure $17-5$ Different realizations of the "mono-branch" fracture pattern: the upper left figure shows a fracture for which $F_{01}$ and $F_{02}$ definition verifies $P_{y+1}=P_{y-1}$, the upper right figure shows a fracture which bifurcation occurs late whereas the middle left figure shows a fracture which bifurcation occurs early, the middle right and bottom figures shows fractures for which $F_{01}$ and $F_{02}$ have a ratio $P_{y+1} / P_{y-1}$ higher and lower than 1,

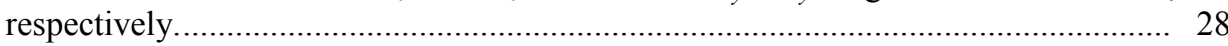

Figure $18-5$ different realizations of the "dual-branch" fracture pattern. In each case the extent of the branch has not been defined (used a random number of iterations). The subfractures have a ratio $P_{y+l} / P_{y-l}$ higher and lower than 1, respectively.

Figure $19-5$ different realizations of the "tri-branch" fracture pattern. In each case the extent of the branch has not been defined (used a random number of iterations). Additionally, the sub-fractures have a ratio $P_{y+1} / P_{y-1}$ higher and lower than 1 , respectively..... 
Figure $20-5$ different realizations of the "quad-branch" fracture pattern. In each case the extent of the branch has not been defined (used a random number of iterations). Additionally, the sub-fractures have a ratio $P_{y+1} / P_{y-1}$ higher and lower than 1 , respectively.

Figure $21-$ Schematic 2-D representation of a full-scale horizontal well producing from a shale reservoir. 10 fracture stages are represented and the stencil shows the minimum repeatable feature in the reservoir.

Figure $22-3-\mathrm{D}$ view of a stochastic pattern network of fractures............................................ 36

Figure $23-$ Schematic of the single-planar hydraulic fracture in the synthetic shale reservoir...... 41

Figure $24-2$-dimensional pressure profile change around a single-planar fracture. ..................... 41

Figure $25-$ Mass rate for a synthetic shale gas reservoir produced from a single-planar fracture. 43

Figure $26-\beta$-derivative of the mass rate for a synthetic shale gas reservoir produced from a single-planar fracture.......................................................................................... 43

Figure 27 - Cumulative mass production as a fraction of the initial mass in place, synthetic shale gas reservoir produced from a single-planar fracture........................................ 44

Figure $28-3$ non-branched fracture patterns (cases 1 to 3 ) ........................................................ 45

Figure 29 - Mass rate for a synthetic shale gas reservoir produced from 3 non-branched fracture patterns and compared to the planar fracture case.................................................... 46

Figure $30-\beta$-derivative of the mass rate for a synthetic shale gas reservoir produced from 3 non-branched fracture patterns and compared to the planar fracture case................... 47

Figure 31 - Cumulative mass production recovery for a shale gas reservoir produced from 3 non-branched fracture patterns and compared to the planar fracture case.................. 47

Figure $32-5$ mono-branched fracture patterns (cases 1 to 5 )................................................... 49

Figure 33 - Mass rate for a synthetic shale gas reservoir produced from 5 mono-branched fracture patterns and compared to the planar fracture case. ....................................... 50

Figure $34-\beta$-derivative of the mass rate for a synthetic shale gas reservoir produced from 5 mono-branched fracture patterns and compared to the planar fracture case............... 51

Figure $35-$ Cumulative mass production recovery for a shale gas reservoir produced from 5 mono-branched fracture patterns and compared to the planar fracture case................ 51

Figure $36-5$ dual-branched fracture patterns (cases 1 to 5)..................................................... 53

Figure 37 - Mass rate for a synthetic shale gas reservoir produced from 5 dual-branched fracture patterns and compared to the planar fracture case. ....................................... 54

Figure $38-\beta$-derivative of the mass rate for a synthetic shale gas reservoir produced from 5 dual-branched fracture patterns and compared to the planar fracture case................. 55

Figure $39-$ Cumulative mass production recovery for a shale gas reservoir produced from 5 dual-branched fracture patterns and compared to the planar fracture case................ 56

Figure $40-5$ tri-branched fracture patterns (cases 1 to 5 ). ......................................................... 57

Figure 41 - Mass rate for a synthetic shale gas reservoir produced from 5 tri-branched fracture patterns and compared to the planar fracture case...................................................... 58 
Figure $42-\beta$-derivative of the mass rate for a synthetic shale gas reservoir produced from 5 tribranched fracture patterns and compared to the planar fracture case........................ 58

Figure $43-$ Cumulative mass production recovery for a shale gas reservoir produced from 5 tribranched fracture patterns and compared to the planar fracture case........................ 59

Figure $44-5$ quad-branched fracture patterns (cases 1 to 5) .................................................. 61

Figure 45 - Mass rate for a synthetic shale gas reservoir produced from 5 quad-branched fracture patterns and compared to the planar fracture case. ....................................... 62

Figure $46-\beta$-derivative of the mass rate for a synthetic shale gas reservoir produced from 5 tribranched fracture patterns and compared to the planar fracture case. ....................... 62

Figure $47-$ Cumulative mass production recovery for a shale gas reservoir produced from 5 quad-branched fracture patterns and compared to the planar fracture case. ................ 63

Figure $48-$ Cumulative mass recovery at 100 days correlated with fracture volume for different stochastic fracture network patterns. ................................................................... 64

Figure $49-5$ mono-branched fracture patterns (cases 6 to 10) .................................................... 66

Figure $50-5$ mono-branched fracture patterns (cases 11 to 15$)$................................................... 67

Figure $51-5$ mono-branched fracture patterns (cases 16 to 20) …........................................... 67

Figure $52-5$ dual-branched fracture patterns (cases 6 to 10)................................................... 68

Figure $53-5$ dual-branched fracture patterns (cases 11 to 15$)$................................................ 68

Figure $54-5$ dual-branched fracture patterns (cases 16 to 20)................................................... 69

Figure $55-5$ tri-branched fracture patterns (cases 6 to 10)...................................................... 69

Figure $56-5$ tri-branched fracture patterns (cases 11 to 15$)$. ................................................... 70

Figure $57-5$ tri-branched fracture patterns (cases 16 to 20). .................................................. 70

Figure $58-5$ quad-branched fracture patterns (cases 6 to 10).................................................. 71

Figure $59-5$ quad-branched fracture patterns (cases 11 to 15$)$.................................................. 71

Figure $60-5$ quad-branched fracture patterns (cases 16 to 20) …............................................. 72

Figure 61 - Mass rate for a synthetic shale gas reservoir produced from 20 mono-branched fracture patterns and compared to the planar fracture case. ..................................... 73

Figure $62-\beta$-derivative of the mass rate for a synthetic shale gas reservoir produced from 20 mono-branched fracture patterns and compared to the planar fracture case................ 73

Figure 63 - Mass rate for a synthetic shale gas reservoir produced from 20 dual-branched fracture patterns and compared to the planar fracture case. ..................................... 74

Figure $64-\beta$-derivative of the mass rate for a synthetic shale gas reservoir produced from 20 dual-branched fracture patterns and compared to the planar fracture case................. 74

Figure $65-$ Mass rate for a synthetic shale gas reservoir produced from 20 tri-branched fracture patterns and compared to the planar fracture case. .................................................... 75

Figure $66-\beta$-derivative of the mass rate for a synthetic shale gas reservoir produced from 20 tri-branched fracture patterns and compared to the planar fracture case. ................... 75 
Figure 67 - Mass rate for a synthetic shale gas reservoir produced from 20 quad-branched fracture patterns and compared to the planar fracture case.

Figure $68-\beta$-derivative of the mass rate for a synthetic shale gas reservoir produced from 20 quad-branched fracture patterns and compared to the planar fracture case................. 76

Figure $69-$ Average value of the $\beta$-derivative and proposed empirical model. ............................ 77 


\section{LIST OF TABLES}

Table 1 Parameters for the Synthetic Shale Reservoir Considered in this Study..................... 4

Table 2 Fracture Parameters for the Synthetic Shale Gas Reservoir Case. .............................. 40

Table 3 - Reservoir Parameters for the Synthetic Shale Gas Reservoir Case. ........................... 40

Table 4 - Statistical Parameters for the Non-Branched Fracture Pattern Case............................. 45

Table 5 Statistical Parameters for the Mono-Branched Fracture Pattern Case......................... 48

Table 6 - Statistical Parameters for the Dual-Branched Fracture Pattern Case............................ 52

Table $7 \quad$ Statistical Parameters for the Tri-Branched Fracture Pattern Case. ............................. 56

Table $8 \quad$ Statistical Parameters for the Quad-Branched Fracture Pattern Case.......................... 60

Table $9-$ Statistical Parameters for the Additional Mono-Branched Fracture Pattern Cases. .... 65

Table 10 - Statistical Parameters for the Additional Dual-Branched Fracture Pattern Cases. ...... 65

Table 11 - Statistical Parameters for the Additional Tri-Branched Fracture Pattern Cases.......... 65

Table 12 - Statistical Parameters for the Additional Quad-Branched Fracture Pattern Cases. .... 66

Table $13-$ Average $\beta$-Derivative Values Derived from Modeling Results $(80$ cases $)$................. 77 


\section{CHAPTER I}

\section{INTRODUCTION}

\section{I.1 Statement of the Problem}

According to data from the U.S Energy information administration (EIA 2014), natural gas production from shale gas reservoirs increased from less than 1 percent in 1990 to 34 percent in 2011, and is projected to reach more than 50 percent in 2040. The portion of the natural gas produced from shale and tight gas of the U.S. total dry gas production was around 60 percent in 2011 and is expected to reach more than 72 percent. Unconventional resources are the main contributors to the domestic dry gas production (Figure 1), and this contribution is projected to increase steadily until 2040. Understanding the performance potential and nature of reserves for unconventional reservoirs remains a significant challenge, and the economic impact of inaccurate reserves estimation may be significant.

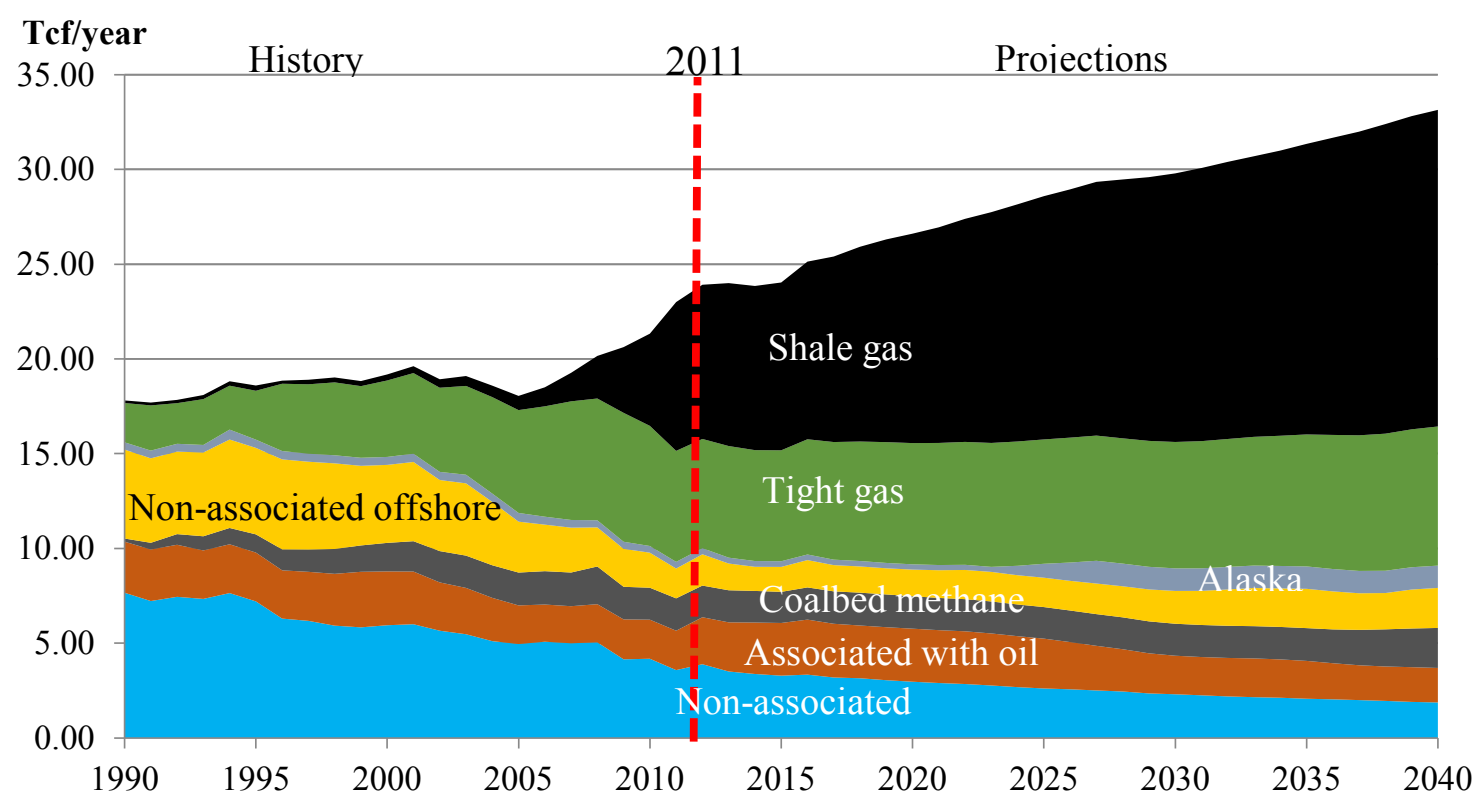

Figure 1 - U.S. dry natural gas production (Reproduced with data from EIA (2014)).

In the context of unconventional reservoirs, we are faced an increasing complexity of reservoir geometries which do not necessarily have analytical solutions - however; the ever-increasing capacity of computational resources has encouraged the use of numerical simulators to solve ever more complex problems in flow geometry, phase behavior, as well as fundamental flow behavior at the nano-scale. 
In conventional and tight gas reservoirs the hydraulic fractures created by fluid injection are modeled by a single plane propagating from the point of fluid entry. This has been proven to be reasonably accurate in small-scale and so-called "mine-back" tests. However, with development of ultra-low permeability reservoirs and in particular, shales, the guiding concept has changed from "planar" fractures to much more "complex fractures," often thought (or assumed) to follow the natural planes of weakness in shales. There are also other concepts whereas shale reservoirs are often over-pressured and require very high fracturing pressures, we may also be creating primarily vertical fractures (as theory suggests), but also fractures with a horizontal fracture component.

In addition, with the wide use of microseismic monitoring of hydraulic fracture treatments, we tend to observe quite complex patterns of the microseismic events, suggesting that we are creating quite complex hydraulic fractures geometries. It is not the purpose of this thesis to pursue a specific concept or geometry. Perhaps on the contrary, our hypothesis is that a somewhat random fracture pattern is created by hydraulic fracturing in shales, and we recall that our goal is to attempt to quantify the behavior of these conceptual fracture patterns for comparison with base models (i.e., the planar fracture) as well as actual field performance (discussed, but not pursued in this work).

\section{I.2 Objectives of the Research}

The overall objectives of this work are to:

- Construct a model that simulates probabilistic random-walk, mono and multi-branched hydraulic fracture networks that propagate in a shale-gas reservoir;

- Analyze the structure of stochastic fracture networks and to determine whether such random networks can render the performance behavior observed in ultra-low permeability/shale reservoirs. A sensitivity analysis on the fracture shape, tendency to bifurcate (split) and the number of bifurcations (splits) is performed to study the impact of the modeled fracture network on the production performance (mass rates and reservoir pressure distributions);

- Study the effect of these "random-walk" fractures on the pressure propagation to determine the timeframe at which fracture interference will occur to better understand and comprehend the effect of each pattern of fractures;

- Use the results of the approach to better explain the flow path from the reservoir to the wellbore; and

- Understand the use (and limitations) of the single-plane fracture compared to a complex fracture network.

\section{I.3 Guiding Assumptions}

The two-dimensional fracture propagation models proposed by Daguier et al. 1996; Mastorakos et al. 2003 use a probabilistic approach for a tensile (mode I) fracture growth. This approach assumes that a fracture 
grows from an initial point (hydraulic fluid injection for example) into a homogeneous media or in a media where heterogeneities are uniformly-spatially distributed. In simple terms, we assume that the $x y$ plane contains a series of discrete integer points $(x, y) \in \mathbb{Z} \times \mathbb{Z}$, and a fracture can grow from a point to the next following the minimum critical fracturing stress $\sigma_{\mathrm{c}}$. Considering that the mechanical properties are distributed in the two-dimensional porous media according to the given probabilistic laws, the fracture growth direction could be randomly directed according to those distributed laws. As a first approach, we suppose that those properties (critical stress) are uniformly distributed, and therefore, the probability for the fracture to move in each direction is constant. The process is mathematically known as a "randomwalk" path.

To summarize, the hypothesis of this work are:

- Uniform distribution of heterogeneities that cause a variation of geomechanical properties such as:

- In-situ stress

- Fracture initiation pressure

- Elastic moduli (Shear modulus and Poisson's ratio)

- No interaction with natural fractures:

- Natural fractures are not modeled

- The pattern shape does not depend on the arrangement of natural fractures

- Fractures are modeled in a 2-D plane (invariance of the structure vertically along the z-axis)

Our approach is to use the "random-walk" stochastic process to generate complex fracture patterns that will conceptually capture the spatial variability of the induced hydraulic fractures. Generation of the "random-walk" path is separate from the numerical modeling — however; to capture the "random-walk" path of the fracture requires the use of extremely refined Cartesian coordinate grid systems (as fine as $1 \mathrm{~cm}$ per grid block dimension). In order to reduce the complexity of this case, we limit ourselves to an $x-y$ fracture pattern, holding the fracture height constant. Reduction of this problem to only 2 dimensions leads to very reasonable computation times for the numerical reservoir simulator deployed in this work.

The complex fracture geometries that will be generated will help us to quantify and assess the effect of each geometric feature (tortuosity, splitting, orientation of branches, and number of branches) on the flow behavior. As validation, we compare the pressure and rate profiles generated using the random-walk fracture patterns with the base case of a planar fracture. Our goal is to assess the hypothesis that a single plane fracture will overestimate the production performance in shale gas reservoir — and to understand the circumstances where the planar fracture case may have value as well as limitations. 


\section{I.4 Parameters Used in Modeling Study}

Table 1 - Parameters for the Synthetic Shale Reservoir Considered in this Study.

\begin{tabular}{lcc} 
Parameters & SI Units & Field Units \\
\hline Fracture width, $w_{f}$ & $1 \mathrm{~cm}$ & $0.0328 \mathrm{ft}$ \\
Reservoir thickness, $h$ & $20 \mathrm{~m}$ & $65.62 \mathrm{ft}$ \\
Matrix permeability, $k$ & $5 \times 10^{-20} \mathrm{~m}^{2}$ & $5 \times 10^{-5} \mathrm{mD}$ \\
Fracture permeability, $k_{f}$ & $5 \times 10^{-11} \mathrm{~m}^{2}$ & $5 \times 10^{4} \mathrm{mD}$ \\
Matrix porosity, $\phi$ & 0.04 & 4 percent \\
Fracture porosity, $\phi_{f}$ & 0.50 & 50 percent \\
Initial reservoir pressure, $p_{i}$ & $2.5 \times 10^{7} \mathrm{~Pa}$ & $3626 \mathrm{psi}$ \\
Wellbore pressure, $p_{f}$ & $1.25 \times 10^{7} \mathrm{~Pa}$ & $1813 \mathrm{psi}$ \\
Fractured region length, $x_{F}$ & $2.5 \mathrm{~m}$ & $8.2 \mathrm{ft}$ \\
Fractured region width, $y_{F}$ & $2.5 \mathrm{~m}$ & $8.2 \mathrm{ft}$ \\
Reservoir length, $x_{R}$ & $20 \mathrm{~m}$ & $65.62 \mathrm{ft}$ \\
Reservoir width, $y_{R}$ & $20 \mathrm{~m}$ & $65.62 \mathrm{ft}$
\end{tabular}

The geometry of this synthetic shale reservoir is a small-scale, square-shaped rectangular. Although this is a synthetic case, parameters such as fracture permeability, matrix porosity and fracture porosity are thought to be representative of the Barnett Shale (Texas) (see Houzé et al. 2010; Shelley et al. 2010). The data used are summarized in Table 1. A dry gas (methane) is used, with densities computed using the Peng-Robinson equation of state (EOS) and viscosity computed using the Chung (1988) correlations.

\section{I.5 Numerical Simulation Model}

A modified version of the single-phase, non-isothermal numerical simulator "Flow Transport Simulator" (or FTSim) has been used for the numerical modeling required in this work. This simulator is based on the TOUGH+ code and is written in standard FORTRAN 95/2003 and was developed (Moridis 2008) at the Lawrence Berkeley National Laboratory (LBNL). This modified version of the FTSim code was developed as a part of a graduate course project at Texas A\&M University (taught by Dr. Moridis). The modifications include the following modules:

- Simplified inputs reading using the "Namelist" object.

- Gas viscosity and density computations using different correlations.

- Gas heat capacity and enthalpy computation.

- Use of save file function (allows the simulation to stop and to resume using saved data).

- Modification of the output format to provide the require rate and pressure data. 
- A more efficient matrix solver was been incorporated (bi-conjugate gradient method (Lanczos type).

Our modified FTSim simulator uses a fully-implicit numerical method for stability and accuracy, which requires significantly more computational time than other simulators. The code is set to generate outputs such as pressure, density, viscosity, rates and volumes at each time-step of the simulation (or at any given number of time-steps). The output data are then read with a small code that extracts the relevant data and puts the data in the form of tables ready to use in plotting software (Igor Pro, Matlab and Mathematica).

The FTSim code also includes a separate Meshmaker code also written in FORTRAN95/2003 and used for space discretization (gridding) where this code provides for the generation of 2-D radially symmetric meshes in cylindrical coordinates as well as 3-D Cartesian grids (which is what we use in this work). This code generates an output file that contains information about the grid (name of the cell, name of the media, volume and coordinates) as well as the connections between the cells (name of the connection elements, directional indicator of the permeability tensor, distance from the centers to the interfaces, interface area and angle between the vertical and the connection line).

The output from the Meshmaker code is used as an input to the MESHMODIFIER code that reads all of the elements of the mesh and stores them in arrays. The MESHMODIFIER code is used to incorporate the random-walk fracture patterns and once created, are exported to the FTSim code to model a given configuration.

\section{I.6 Comparison of Results: Planar Fracture Model}

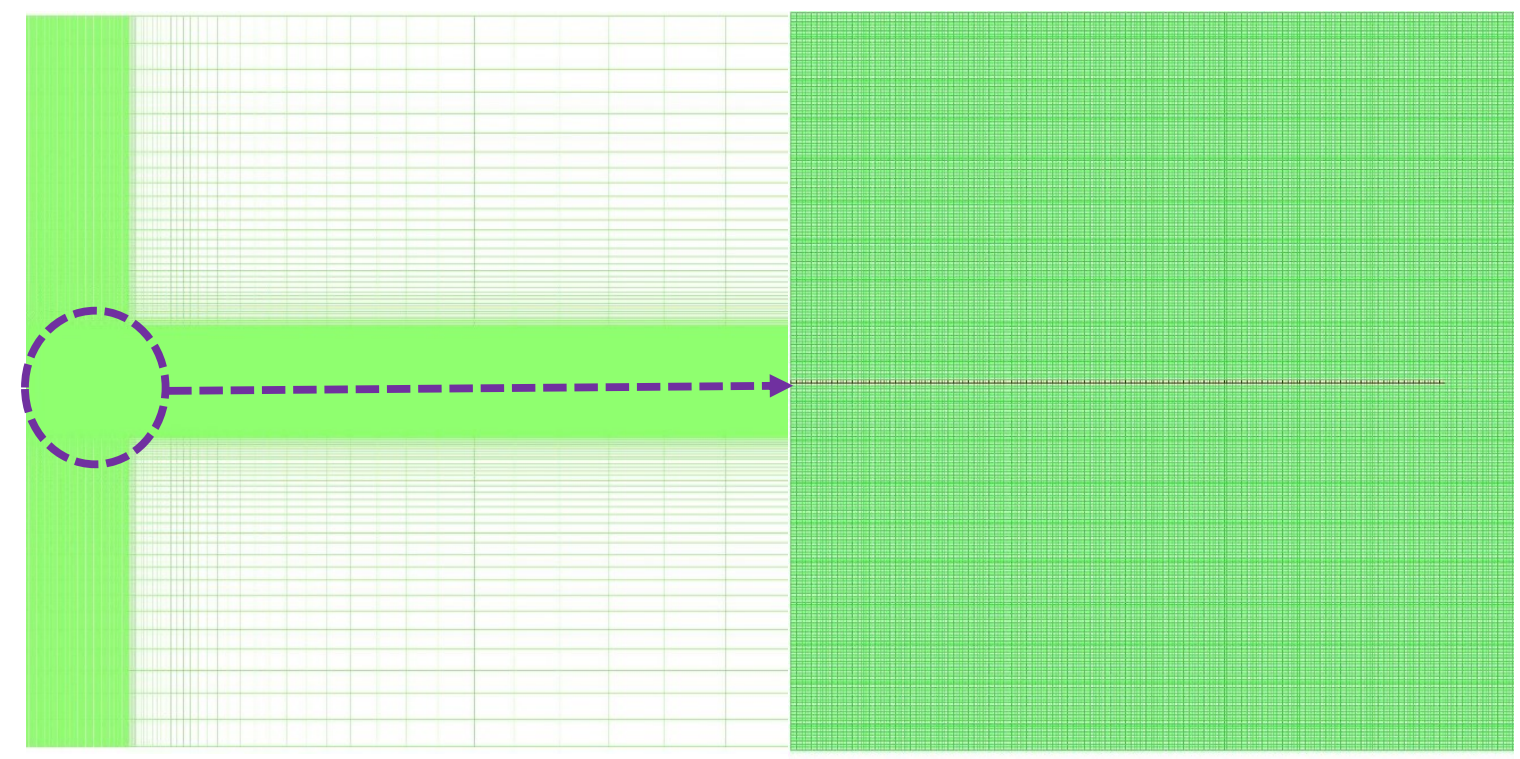

Figure 2 - Base case reservoir model (planar fracture). The right-side shows the complete grid and the left-side is an expanded view of the refined grid fracture region. 
To establish a preliminary validation of our FTSim code we used a conventional planar fracture model (see Figure 2) that assumes that the hydraulic fracture is a limited plane that propagates orthogonally to the wellbore (standard modeling assumption). One objective of this research is to evaluate whether there are differences in the flow behavior between the proposed complex fracture patterns and the standard planar fracture case. As such, this simulation case serves as a base case that will help us identify the effect of different fractures geometries.

\section{I.7 Validation: Comparison to a Commercial Modeling Software}

In addition to the base case of a plane fracture given in the previous section, we also want to validate the Rubis numerical simulation module (Kappa Engineering (2014)). The Rubis module was used to generate production curves for the same reservoir configuration we considered for FTSim code.

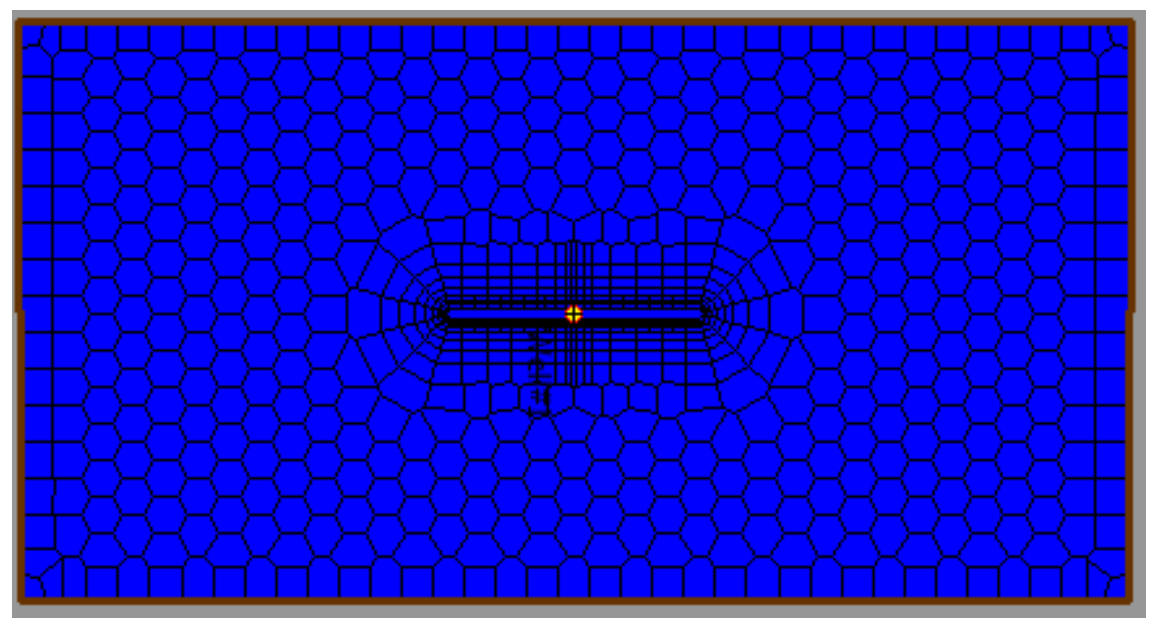

Figure 3 - Synthetic example - producing well has a single vertical (planar) fracture.

The reservoir configuration used in this comparison is shown in Figure 3. As noted, the reservoir is produced from a planar finite-conductivity hydraulic fracture. Although the simulator FTSim has been compared extensively to analytical solutions for radial and cylindrical geometries, we had not made the specific test of a vertically-fractured well during the construction of the simulator. The same geometry and input data that were used in FTSim are now used in the commercial simulator in the Ecrin suite (Kappa Engineering (2014)).

As shown in Figure 4, the computed mass rates for these case compare quite well, extremely well if we consider the fact that the only deviations occur where the "fracture flow" subsides and the "reservoir flow" commences. This phenomena is very difficult to model in general, and given the differences in gridding, model solution schemes, as well as slight differences in PVT properties, this is an excellent match. 


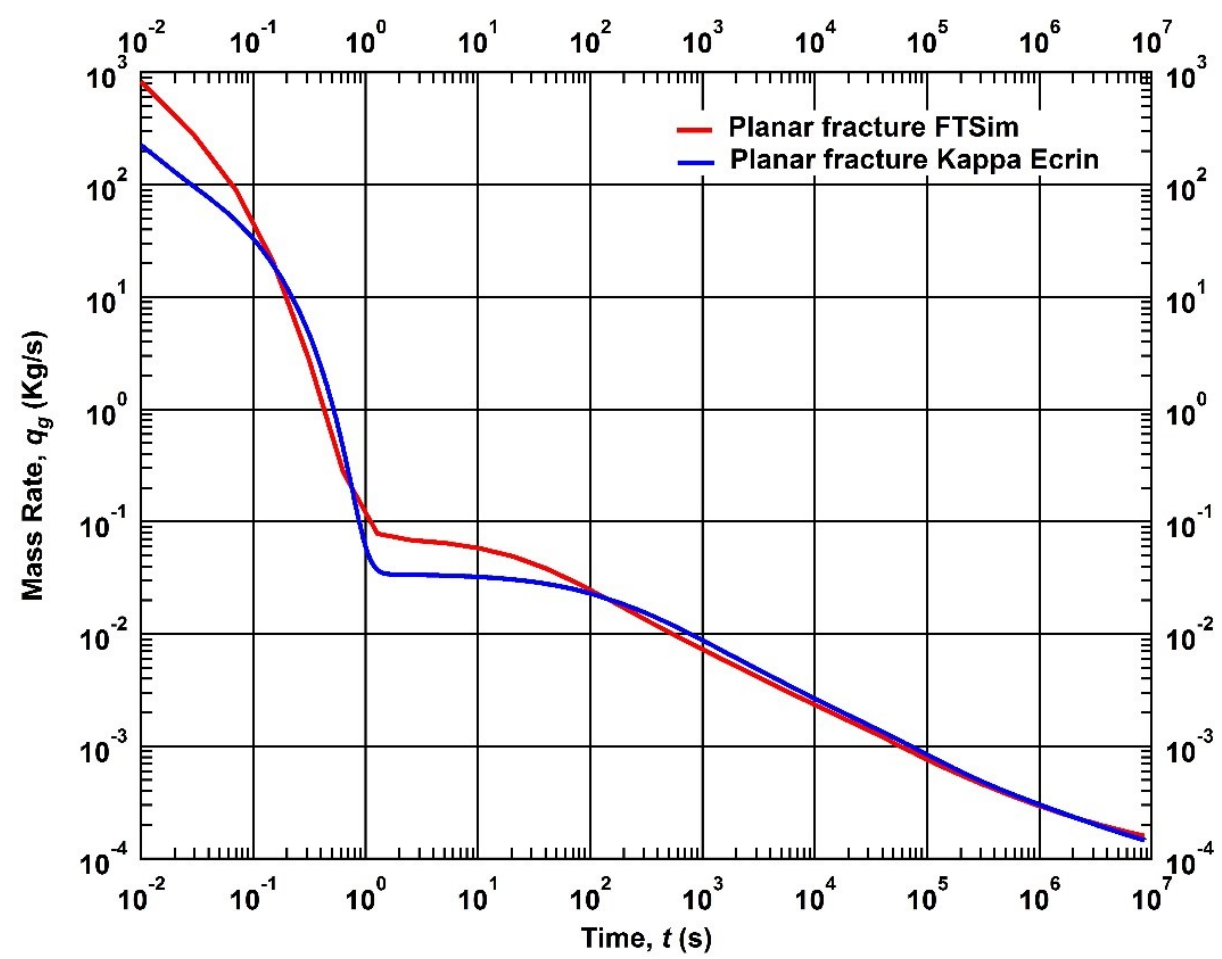

Figure 4 - Comparison of mass production rates (FTSim and Rubis simulation codes).

The small differences in the computed production rate profiles could be attributed to the following:

- Different PVT correlations are deployed in these simulations.

- The wellbore and fracture storage components are slightly different in both cases due to slight differences in the geometries of the well and the fracture.

- Some minor computational differences may be attributed to the finite-difference schemes used in these models.

In addition to the comparison of mass production rates for this study, we also compared the cumulative mass production, where this is expressed as a percentage of the total mass in the reservoir system. The comparison of cumulative mass recovery is shown in Figure 5 (Cartesian format plot) and we note an outstanding correlation of performance based on these variables.

As FTSim has been demonstrated to be comparable to a commercially available product, we now proceed to modeling the "random-walk" fracture cases using FTSim, with the expectation that similar rigor will exist in the computed solutions. Our rationale for using FTSim in this study is that it is our belief that no commercial product currently exists which can incorporate the random-walk fracture patterns (generated in this work) and provide the level of accuracy and stability that we require in this study. 


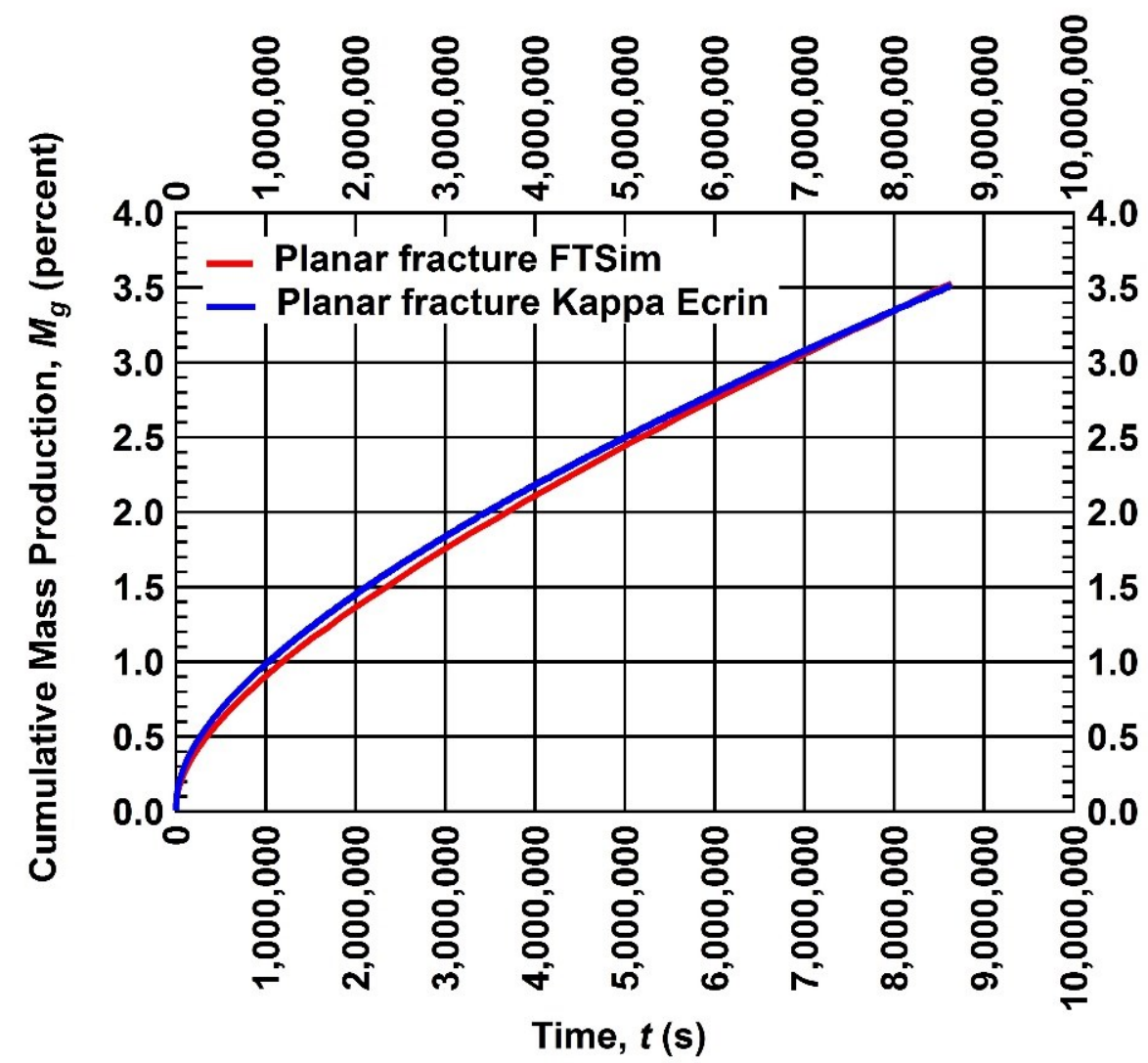

Figure 5 - Comparison of cumulative produced gas as a percentage of the initial volume of gas for a synthetic case modeled using the FTSim and Ecrin simulators.

\section{I.8 Challenges in Modeling the Complex Fracture Patterns}

The majority of the "labor" in this work was dedicated to the development of MESHMODIFIER code which is the code that generates the gridding mesh for the input complex fracture patterns - specifically, this code provides a new mesh file for the FTSim reservoir simulator. Another major challenge in this work was the computational complexity of the problem due to the very highly refined grid-blocks in the region of the complex (random-walk) fractures.

The size and nature of the fracture gridding scheme lead to the reduction of this problem to the centimeter scale for the fracture modeling, which in turn required us to reduce the size of the overall reservoir model to $\Delta x=\Delta y=\Delta z=20 \mathrm{~m}$. This reduced reservoir model including the complex fractures and the extremely low shale matrix permeability (50 nd) was suitable for modeling all of the significant flow behavior and allowed us to generate simulations on the order of 100 days in a practical computational time. To 
facilitate computations, we utilized the Supercomputing Facility at Texas A\&M University, where we will note that if had we not had such computational access, the study could not have been completed. 


\section{CHAPTER II}

\section{LITERATURE REVIEW}

This chapter is dedicated to a review of the present status of reservoir modeling/simulation for unconventional reservoirs. For our work we will focus on the performance of reservoir model which consider induced (hydraulic) fractures and/or naturally-fractured reservoirs. The purpose of this review is to assess and understand the cases of single orthogonal or non-orthogonal planes, patterns of planes (e.g., "zig-zag" patterns), and random assortments of planar fractures. We remind the reader that our hypothesis is that a stochastic "random-walk" fracture (and by extension, patterns of random-walk fractures) will effectively model the production performance of ultra-low permeability/shale reservoir systems.

\section{II.1 Analytical and Numerical Models for Fractured Well Performance}

Early solutions for planar fractures include the analytical and semi-analytical flow solutions for a singlefractured reservoir were developed by (Gringarten et al. 1974) and then by (Blasingame and Poe 1993), respectively. For a multiple-fractured horizontal well (MFHW), several solutions were proposed, but a series of "proxy" semi-analytical solutions (e.g., Medeiros et al. 2006) were used to represent the well/reservoir condition so that diagnostic regimes could be interpreted. Other analytical/semi-analytical models evolved to improve the existing forecasting methods (Anderson et al. 2010; Bello and Wattenbarger 2008; Mattar 2008). Unfortunately, these types of simplified models generalize the complexity of the physical problem (i.e., the fracture system is reduced to a lumped variable and/or a single "super fracture"). In contrast, numerical solutions provide approximate solutions to highlycomplex, non-linear problems using finite-difference and/or finite-element methods and are much better suited to "fracture network" types of modeling cases.

Induced hydraulic fractures in shale-gas and tight reservoirs are commonly seen as $2 \mathrm{D}$ structures that propagate in the plane of maximum principal stress direction $\sigma_{\max }$ (Geertsma and De Klerk 1969; Hossain et al. 2000; Perkins and Kern 1961). Weng et al. (2011) describe the uncertainty/complexity of a fractured reservoir as a complex interaction between the natural fracture network and (any) hydraulically induced fractures. Microseismic data suggest a significant complexity of hydraulic fractures (Li et al. 2012). As such, any approach used to model natural fracture patterns and any interactions with hydraulic fractures must consider a wide variety of configurations of the natural and/or hydraulic fractures. We note that two sets of orthogonal fractures are traditionally used in numerical simulations (Meyer and Bazan 2011; Olorode et al. 2012; Xu et al. 2010). In addition, Olson (2008) developed a more sophisticated fracture network using empirical crack propagation laws. 
Maxwell et al. (2006) show that microseismic events could indicate fracture density; this observation suggests a complex interaction between natural fractures and hydraulic fractures. One of the challenges of numerical modeling of shale-gas reservoirs is to be able to use a given set of microseismic data to construct a fracture pattern that, once modeled, provides an accurate match of the production curves. The ultimate goal is to be able to predict the rate and pressure performance in these reservoirs. While conceptual hydraulic fractures are still modeled in reservoir simulation as extended planes, one may wonder whether the hypothesis of planar fractures accurately describes the "actual" hydraulic fracture path and if this description has a significant impact on the production and on reserve estimation.

\section{II.2 Present Status — Modeling of Complex Hydraulic Fracture Patterns}

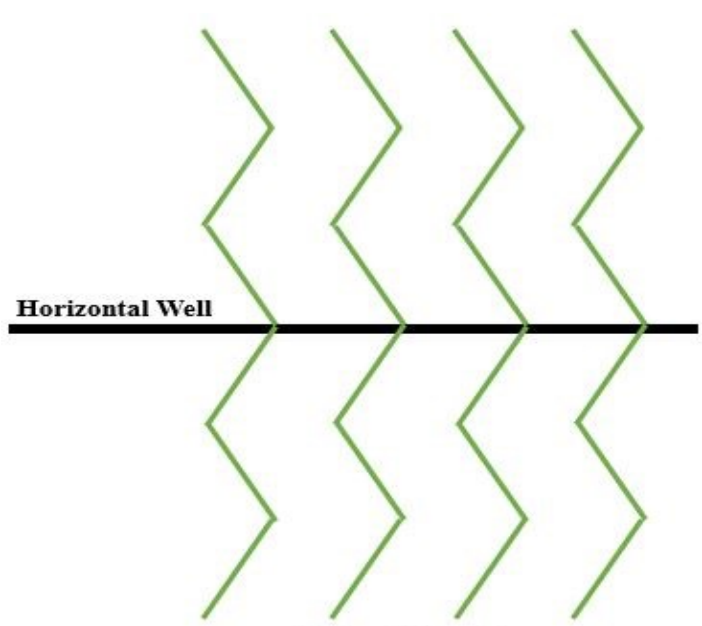

Hydraulic Fractures

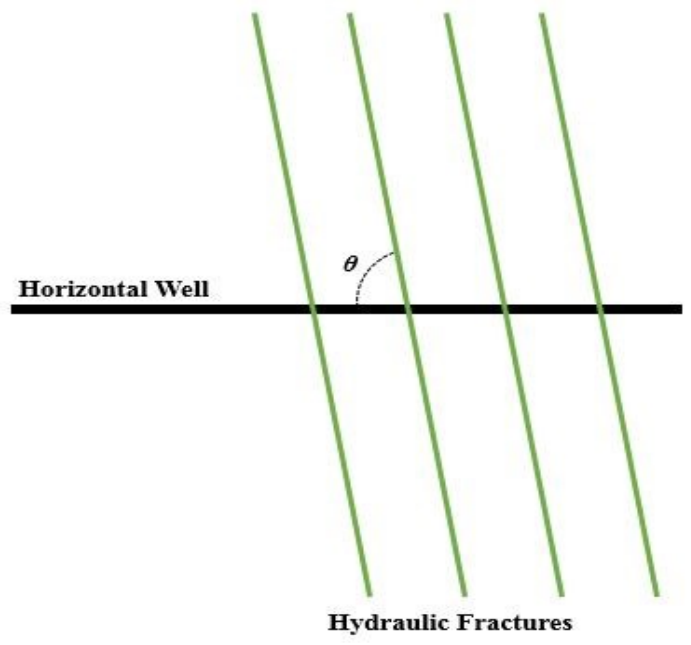

Figure 6 - Olorode et al. (2013) imagined variations of the geometry of the main planar fracture.

Olorode et al. (2013) use geometrically-irregular patterns as shown in Figure 6 (inclined and a triangular wave type) and generated production and pressure histories as means of comparing the performance of these fracture patterns. A major conclusion of the work by Olorode et al. (2013) was that significant differences can exist given the configuration of the fracture systems, in terms of both performance and recovery. In another study, Roussel et al. (2013) also used non parallel orientations of hydraulic fractures to model infill wells and noticed that the stress field could be (and likely is) altered by prior production.

Cipolla et al. (2011) provide a novel workflow that could be used to generate a complex hydraulic fracture network based on microseismic data. In this workflow, Cipolla et al. (2011) generated 2-D structures which are highly complex and accurately model the rate and pressure history as well as provide a 
reasonable production forecast. However, this method appears to be time-consuming as the workflow of pattern detection using microseismic data must be performed for each completion stage.

Zhou et al. (2012) developed a semi-analytical model to simulate the production from a complex fracture network based on a fracture discretization concept. This model considers the behavior of the primary hydraulic fracture connected to a network of secondary fractures. The question remains as to the relevance (and reliability) of these simplified models which attempt to match the complexity of the created and enhanced fracture networks inferred from microseismic data.

Olorode et al. (2013) observe that the shape of the fracture and/or its geometrical orientation can be directly related to the rate and pressure performance (and hence, the estimation of reserves) for "low permeability" (typically "tight gas" cases where the permeability is on the order of $0.001 \mathrm{md}$ ). This observation by Olorode et al. (2013), combined with the belief that the geometry of the created/enhanced hydraulic fracture is quite complex (Cipolla et al. 2011; Olorode et al. 2013; Roussel et al. 2013; Zhou et al. 2012) motivated this research to consider the "random-walk" fracture network. In our work we consider a highly complex, randomly generated 2-D hydraulic fracture network as our mechanism to assess the performance of the created/enhanced fracture network generated by hydraulic fracturing.

Specific to our work, we consider the character of the "random-walk" fracture networks as follows:

- The fracture tortuosity (controlled by the ratio of the probabilities $\frac{P_{x+1}}{P_{y+1}+P_{y-1}}$ ).

- The occurrence of "branching."

- The orientation of the branches (controlled by the ratio of probabilities in the $y$-direction $\frac{P_{y+1}}{P_{y-1}}$ ).

- The total number of branches.

\section{II.3 Research Hypothesis}

Moridis et al. (2010) identified the following fracture systems which influence flow in reservoirs:

- The original fractures present in the reservoir which have been present before any intervention during the production operations. They are called native (or natural) fractures.

- The fracture networks that are created during reservoir stimulation (hydraulic fracturing); usually referred to as primary fractures.

- The fractures that are dynamically created due to the change in the geomechanical properties in the vicinity of the primary fracture these are referred to as secondary fractures and are thought to be orthogonal to primary fractures.

- The fractures that originate due to the change of the geomechanical status of the rock in the vicinity of the well because of drilling, they are called radial fractures. 
In this research we assume that only the primary fracture network is present in the reservoir. This assumption may seem unrealistic as the flow in hydraulically fractured shale or tight reservoir must be modeled as a complex interaction between all of the fracture types given by Moridis et al. (2010). However; the purpose of this research is to assess the relevance of the planar hydraulic fracture model as a "standard" and compare this model to other fracture/fracture network models (in our work, we will limit our study to stochastically generated multi-branched fracture networks).

The primary goal of this research is to assess the performance of "random-walk" fracture networks - in comparison the "standard" planar fracture (i.e., to evaluate the relative performance), but also to establish performance metrics/features - in our case, the $\beta$-derivative, where we note that more complex fracture networks have higher $\beta$-derivative values. We believe that the "random-walk" fracture network model may one approach to integrate/represent the combined effects of all of the fracture types proposed by Moridis et al. (2010).

For in a given shale reservoir, we assume that the geomechanical properties that control the propagation of the hydraulic fracture(s) are homogeneously distributed so that we can justify the use of random-walk fracture networks (i.e., in this work we do not consider the influence of stress field). As a recommendation for future work, we do suggest that some sort of geomechanics be considered in the random-walk fracture workflow (perhaps explicitly, or as some sort of proxy-type penalty function which would guide direction and length of propagation of a given fracture).

Although the random-walk fracture network concept can be extended to 3 dimensions (and we do suggest that other researchers consider such an extension), for this introductory work we believe that the 2dimensional case should be sufficient to prove our research hypothesis. In addition, extension to 3 dimensions will require a significantly higher computational load (our estimates are at least a factor of 10 , perhaps a factor of 100 in computational requirements). At this point our goal is to demonstrate the 2-D random-walk fracture network model, extensions to 3-D models should be relatively straightforward.

\section{II.4 A Novel Approach to Model 2-D Fractures: "Random-Walk" Fractures}

(Daguier et al. 1996; Mastorakos et al. 2003) used a probabilistic approach for the growth of a tensile (mode I) type fracture. The rationale for using such approaches is that created/enhanced hydraulic fracture patterns are inferred from observed microseismic data. In simple terms, we know that we can find many fracture patterns which will "connect the dots" shown by microseismic data. Given the extreme levels of uncertainty in the physical subsurface, we believe that the best starting point for generating fracture patterns is to use a stochastic approach (i.e., where the growth/propagation of the fractures is "controlled" or constrained by probabilities). 
By imposing limits on the stochastic process, the created fracture network is not as "random" as one might think. In Figure 7 we present a very simple case (single fracture) where our algorithm produces constrained fractures by controlling the $x$ - and $y$-probability ratios (as mentioned above). This "control process" yields a relatively straight fracture, while relaxation of the control on the $x$ - and $y$-probability ratios yields as more "tortuous" path.
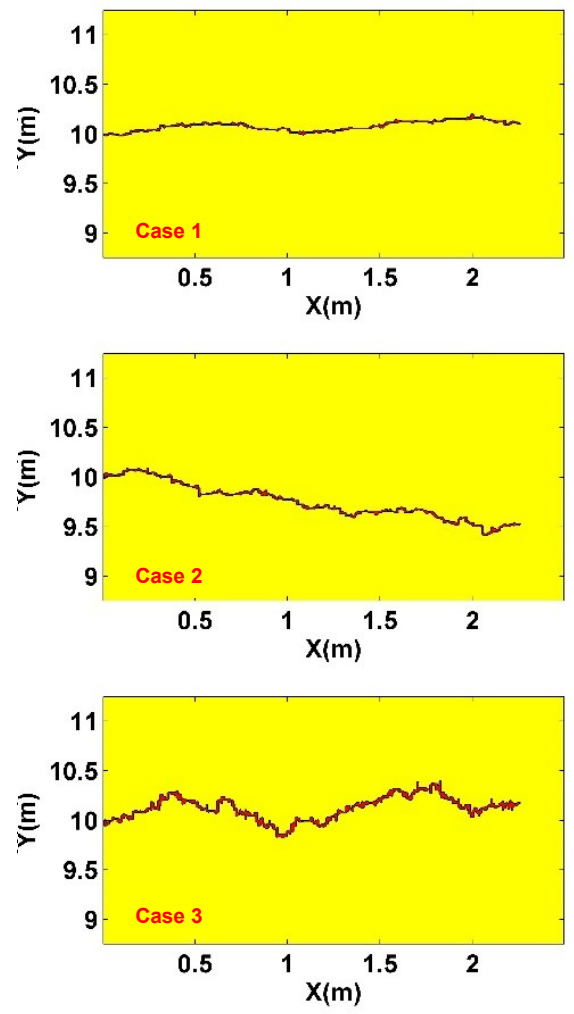

Figure $7-3$ single-branch fracture cases generated using the MESHMODIFIER code. We observe that the tortuosity of the fracture path progressively increases due to the ratio of probabilities $x$ - and $y$-directions [i.e., the $P_{x+1} /\left(P_{y+1}+P_{y-1}\right)$ ratio].

\section{II.5 Grid Refinement Issues: Random-Walk Fracture Patterns}

As comment, the random-walk fracture networks produced in this work require extremely small grid systems for representative and accurate numerical simulation of these fracture networks. In fact, this particular case may be one of the most difficult gridding challenges in fracture modeling. The typical approach is to use complex grid refinement algorithms as the Voronoi gridding method (Olorode et al. 2013) or the logarithmic grid coarsening approach which is typically used in modeling fractured reservoirs. However, for both of these cases the fractures are relatively planar and/or the fracture networks 
are essentially orthogonal — and the length scale is 10 's of meters, certainly not varying on the centimeter scale as we have chosen to consider.

For reference, we could have elected to use a Voronoi-type gridding, but this would have been extremely challenging in terms of our model scale (as mentioned above), but also in terms of the nature of the grids. We elected to model the system as the scale of our smallest change in fracture properties, which is 1 centimeter. As this is an orthogonal system, we elected to use a rectangular Cartesian grid. Given that we permitted fracture network variation on the centimeter-scale, we also had to re-size our "reservoir" to a much smaller than physically normal size - in our case we chose a cube of $20 \mathrm{~m} \times 20 \mathrm{~m} \times 20 \mathrm{~m}$, with the fracture network located in the "core" of the reservoir (a $5 \mathrm{~m} \times 5 \mathrm{~m}$ region). The nature and orientation of our model scale will be discussed in detail in later sections. 


\section{CHAPTER III}

\section{DEVELOPMENT OF A SIMPLESTOCHASTIC FRACTURE PROPAGATION MODEL}

\section{III.1 Introduction to the Stochastic Fracture Propagation Model}

This chapter provides the mathematical definition of the fractures using a 2 dimensional density function. The definition of the function is iterative and the process is called "random-walk." This process is mathematically defined as a succession of random steps, it was introduced for the first time by (Pearson 1905) and the method is assumed to be a Markov chain in the sense where the current time step depends only on the previous one and not on the whole temporal definition of the process. Since the introduction of the random-walk concept, several applications of this mathematical probabilistic concept have been attempted to model different natural phenomenon. Among these attempts are Daguier et al. 1996 and Mastorakos et al. 2003 who used this concept to represent the propagation of macro-cracks in several different media. In this research, we will use the "random-walk" approach to model the propagation of a hydraulic fracture network.

After defining the process that describes the fracture as a mathematical density function, we used the fact that the function is discrete (integer-based) to apply the approach on a rectangular Cartesian coordinate system where we iteratively modify the nature of grid-blocks from matrix to fracture in order to build the discrete structure of the fracture network on a 3-D grid. This process is performed by the fit-for-purpose MESHMODIFIER code that produces a gridding of the fracture pattern for the FTSim numerical simulation code. The MESHMODIFIER code is used to generate a wide range of fracture structures which depend on the characteristic probabilities of the prescribed density function. The various fracture networks will then be used in the FTSim numerical simulation code to predict rate and pressure profiles for comparison and to establish specific features in the performance. In our case we have selected the " $\beta$ derivative" of the gas mass flowrate as our comparator variable.

Further description of the MESHMODIFIER algorithm is provided in this chapter. For simplicity (at least for this initial study), the number of bifurcation (or splitting) stages for a given fracture network has been limited to 4 "branches." Extension to more than 4 branches is straightforward, but additional branches will require significantly more computational work in our present work structure. In short, we believe that we have captured the majority of the likely behavior of a multi-branch fracture network with 4 branches. Finally, it should be noted that as the number of branches increases (and hence complexity of the created fracture network), the higher the "equivalent" permeability of the reservoir system, extension to a very high number of fracture branches essentially renders a reservoir system at permeability near that of the fracture system. 


\section{III.2 Mathematical Description of the Random-Walk Fracture Problem}

The fracture trajectory is a 2-dimensional discrete density function, $F(x, y)$, which is defined as:

$$
\left\{\begin{array}{l}
F(x, y)=1 \text { If }(x, y) \in \text { Fracture } \\
F(x, y)=1 \text { If }(x, y) \in \text { Matrix }
\end{array}\right.
$$

The function is assumed to start from the origin:

$$
F\left(x_{0}, y_{0}\right)=F(0,0)=1
$$

Then, we iteratively define the function for a certain step $n \in \mathbb{N}$ :

$$
\left\{\begin{array}{l}
F\left(x_{n+1}, y_{n+1}\right)=F\left(x_{n}+1, y_{n}\right) \text { with a probability } P_{x+1} \\
F\left(x_{n+1}, y_{n+1}\right)=F\left(x_{n}, y_{n}+1\right) \text { with a probability } P_{y+1} \\
F\left(x_{n+1}, y_{n+1}\right)=F\left(x_{n}, y_{n}-1\right) \text { with a probability } P_{y-1}
\end{array}\right.
$$

The probabilities to progress (grow) in each of the specific directions verify:

$$
P_{x+1}+P_{y+1}+P_{y-1}=1
$$

The following distributions are used to generate a random fracture path:

$$
\left\{\begin{array}{l}
P_{x+1}=\frac{1}{3} \\
P_{y+1}=\frac{1}{3} \\
P_{y-1}=\frac{1}{3}
\end{array}\right.
$$

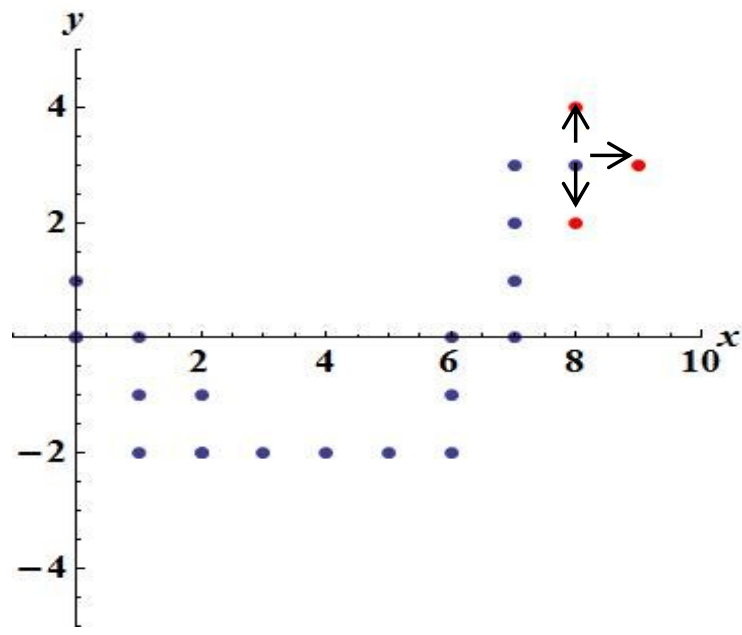

Figure 8 - Random path for a given step.

Figure 8 shows the possible progression paths starting from a certain point $(2,-2)$. Figure 9 shows different "possible" realizations using the same growth probabilities and the same number of 
iterations $N=1000$. One of the main advantages of a probabilistic method is the ability to generate a wide range of possible realizations. The observed patterns of fractures usually include branches which intersect with the networks of natural fractures. These fractures are assumed to be responsible for "linking" the natural fractures (primary and secondary) and are therefore an important aspect of flow.
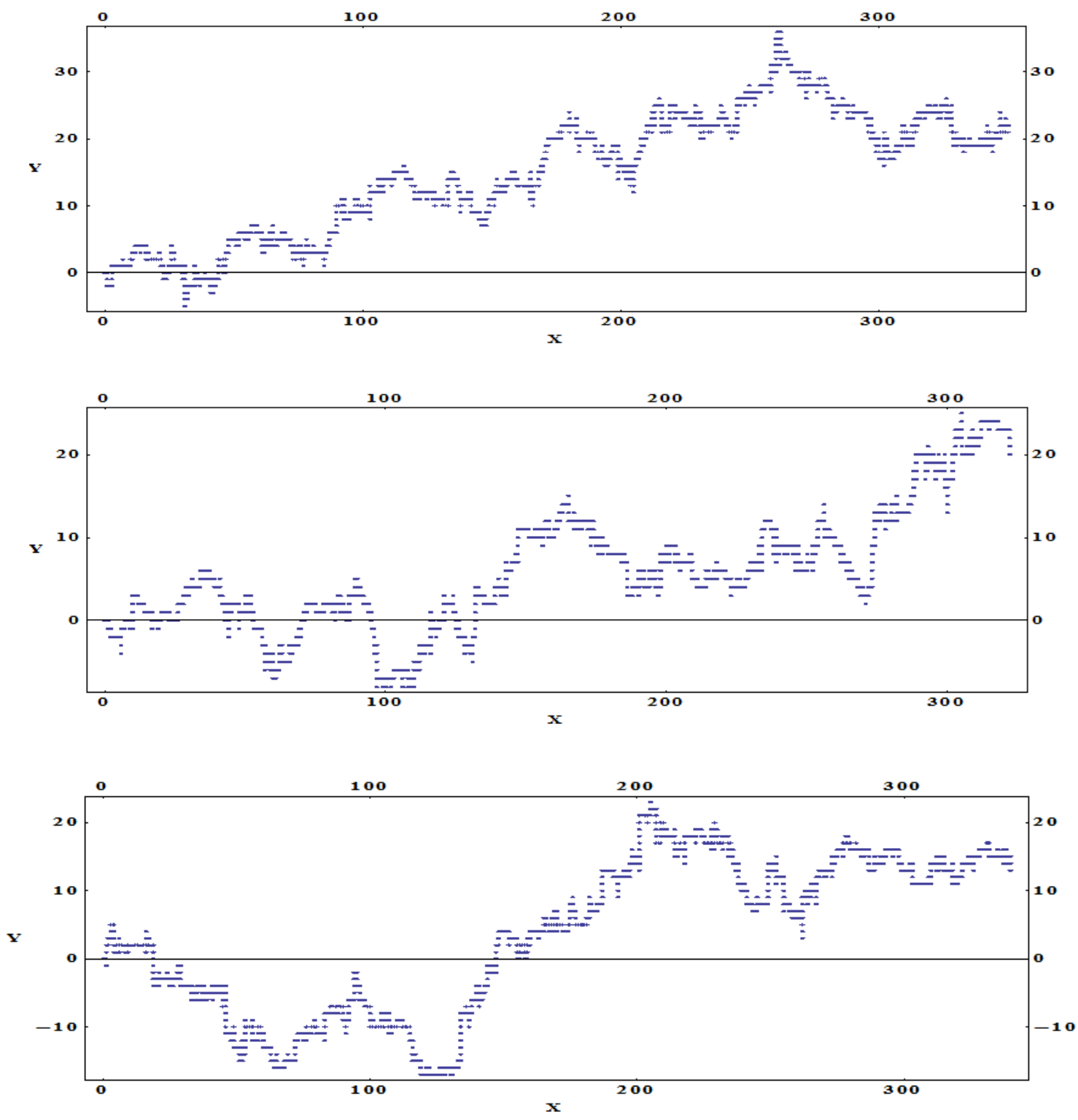

Figure 9 - Various random-walk paths with uniform probabilities for 1000 iterations. 


\section{III.3 Constraining the Fracture Network to Preferential Growth Directions}
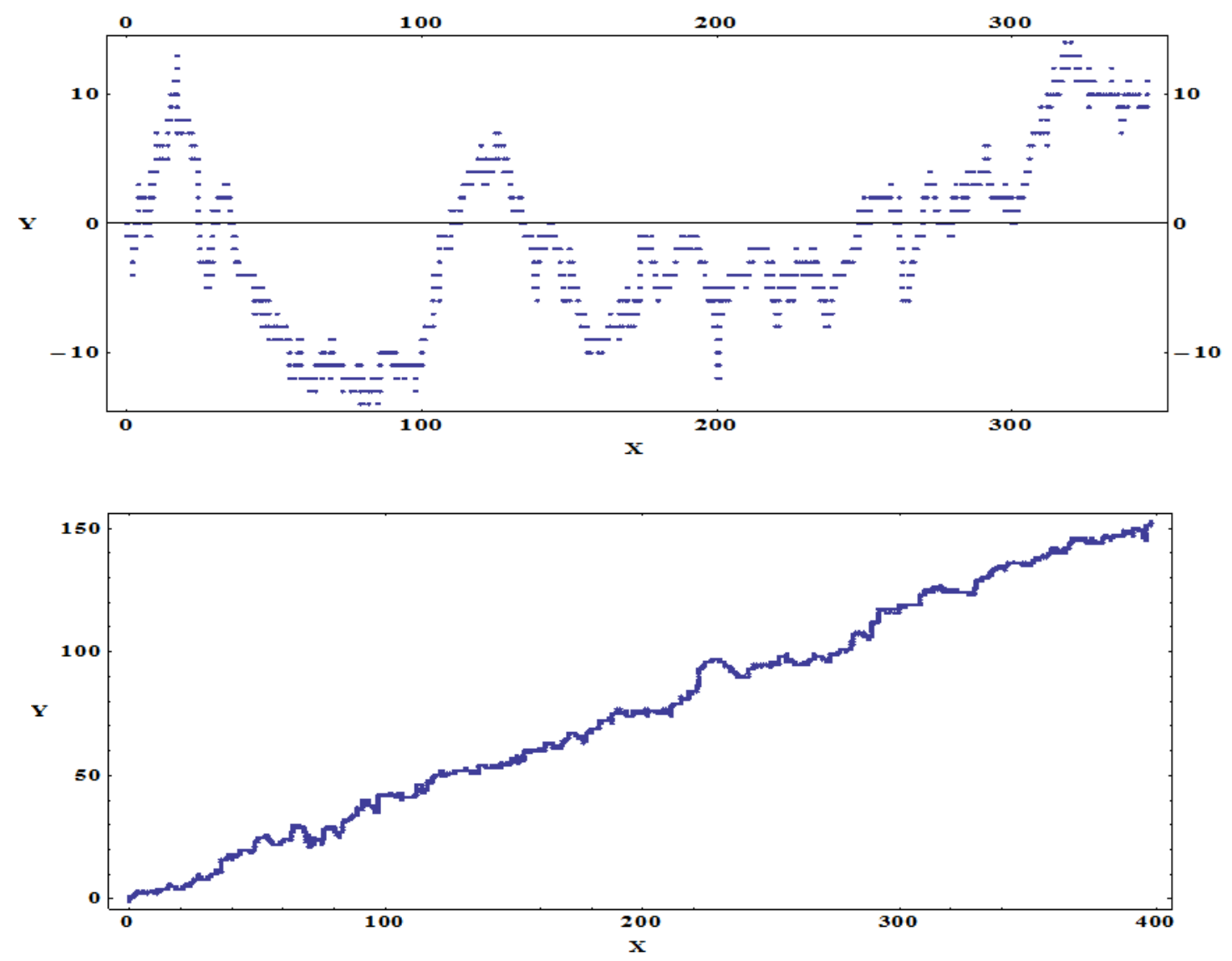

Figure 10 - Preferential growth of a fracture direction after 1000 iterations.

The defined probabilities govern the direction of the fracture. For example, if the probabilities to progress along the $y$-axis are equal:

$$
P_{y+1}=P_{y-1}
$$

Then the fracture is expected to oscillate around the x-axis and the expected value of the $y_{n}$ coordinates of the points where the density function is a fracture is zero:

$$
E\left(y_{n}\right)=1 \times P_{y+1}+(-1) \times P_{y+1}=0
$$

However, when the fracture is chosen to have a preferential progression toward a positive value of $y>0$, then $P_{y+1}$ is slightly higher than $P_{y-1}$

$$
P_{y+1}>P_{y-1}
$$


In this case, the expected value of $E\left(y_{n}\right)$ is positive, and the fracture will have the tendency to grow with a positive slope. For the same number of iterations $N=1000$, the upper plot of Figure 10 shows that the first fracture tends to remain parallel to the $\mathrm{x}$-axis (between $\mathrm{y}=-15$ and $\mathrm{y}=15$ ) when the probabilities to grow in the positive and negative y are equal $P_{y+1}=P_{y-1}$. In the lower plot of Figure 10, the probability

for the fracture to go in the positive y-direction is slightly higher $\left(P_{x+1}=P_{y+1}=\frac{2}{5}, P_{y-1}=\frac{1}{5}\right)$, and there is a steady increase (positive slope) of the fracture.

\section{III.4 Bifurcation (Splitting) of a Given Fracture}

The observation of fracture patterns in shale (or in other materials) show that the fracture does not only necessarily propagate in a linear fashion. While this process could presumed to be described using a fully coupled geomechanical and flow simulation, we will instead investigate the possibility of having what we refer to as "branched fractures" or fractures that have one or more bifurcation (splitting) stages.

This branching character can be captured by the random-walk process. At some randomly generated step, the fracture has the tendency to split between "upper" and "lower" branches. Starting from that point, the propagation continues as if we had to separate fractures whose propagation is governed by the same probability laws. In Figure $\mathbf{1 1}$ we show different realizations generated using the same growth probabilities and the same number of iterations $(N=1000)$ and for which a bifurcation occurs after a randomly generated step $(1<k<N)$.

For $1<i<k$ :

$$
\left\{\begin{array}{l}
P_{x+1}=\frac{1}{2} \\
P_{y+1}=\frac{1}{4} \\
P_{y-1}=\frac{1}{4}
\end{array}\right.
$$

For $k<i<N$ :

$$
\left\{\begin{array}{c}
P_{x+1}=\frac{2}{5} \\
P_{y+1}=\frac{2}{5} \text { for the upper branch and } P_{y+1}=\frac{1}{5} \text { for the lower branch. } \\
P_{y-1}=\frac{1}{5} \text { for the upper branch and } P_{y-1}=\frac{2}{5} \text { for the lower branch }
\end{array}\right.
$$

A multi-branching process is prescribed to generate our most complex fracture patterns (limited to 4 in this study for computational purposes). Figure 12 illustrates different "possible" realizations using the same growth probabilities and the same number of iterations $(N=1000)$ with 4 random bifurcation stages for a randomly generated number of steps for each branch. After each bifurcation, the upper and lower branches progress according the probability law defined previously (for a single branch). 

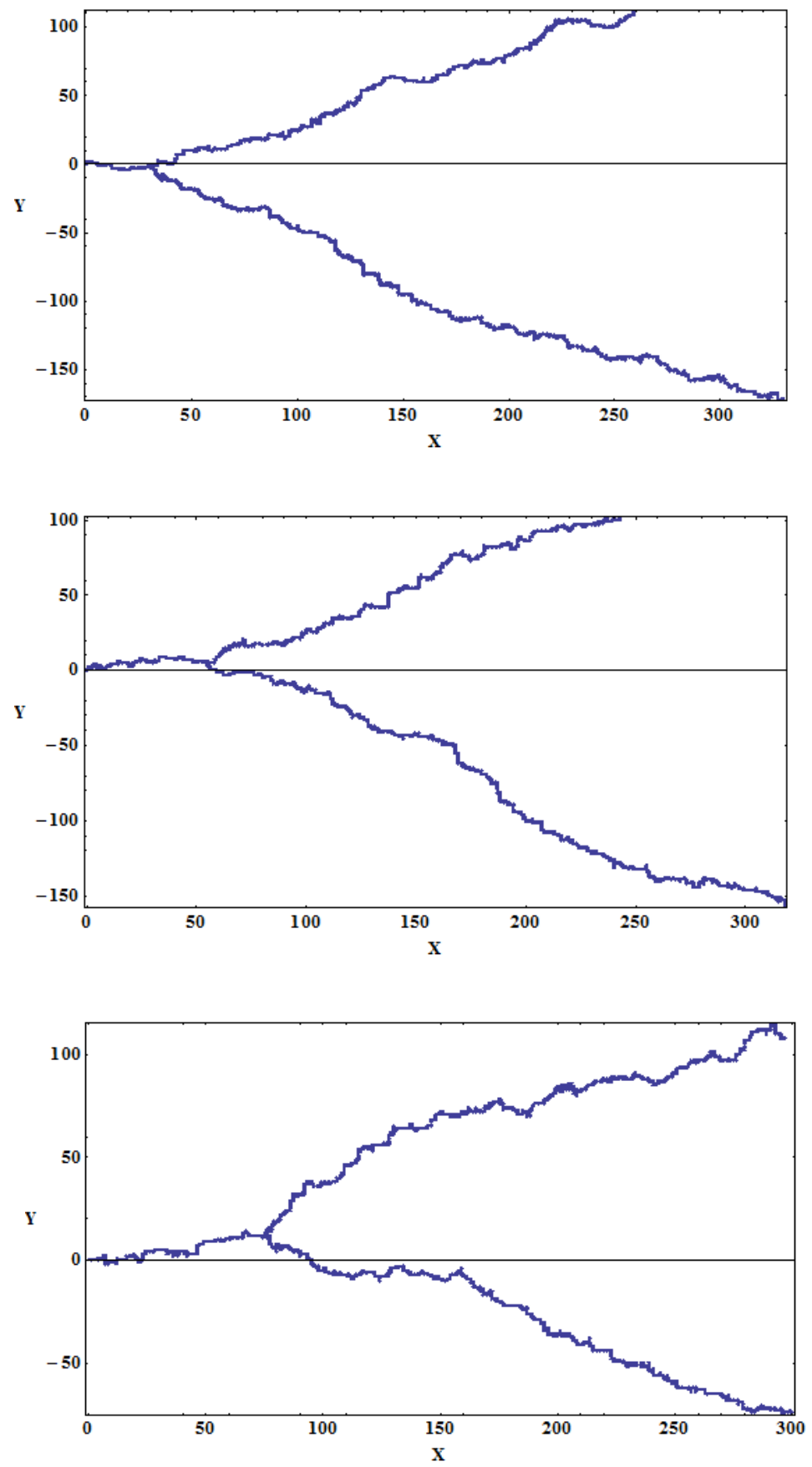

Figure 11 - Random-walk with uniform probabilities for 1000 iterations (2 branches). 

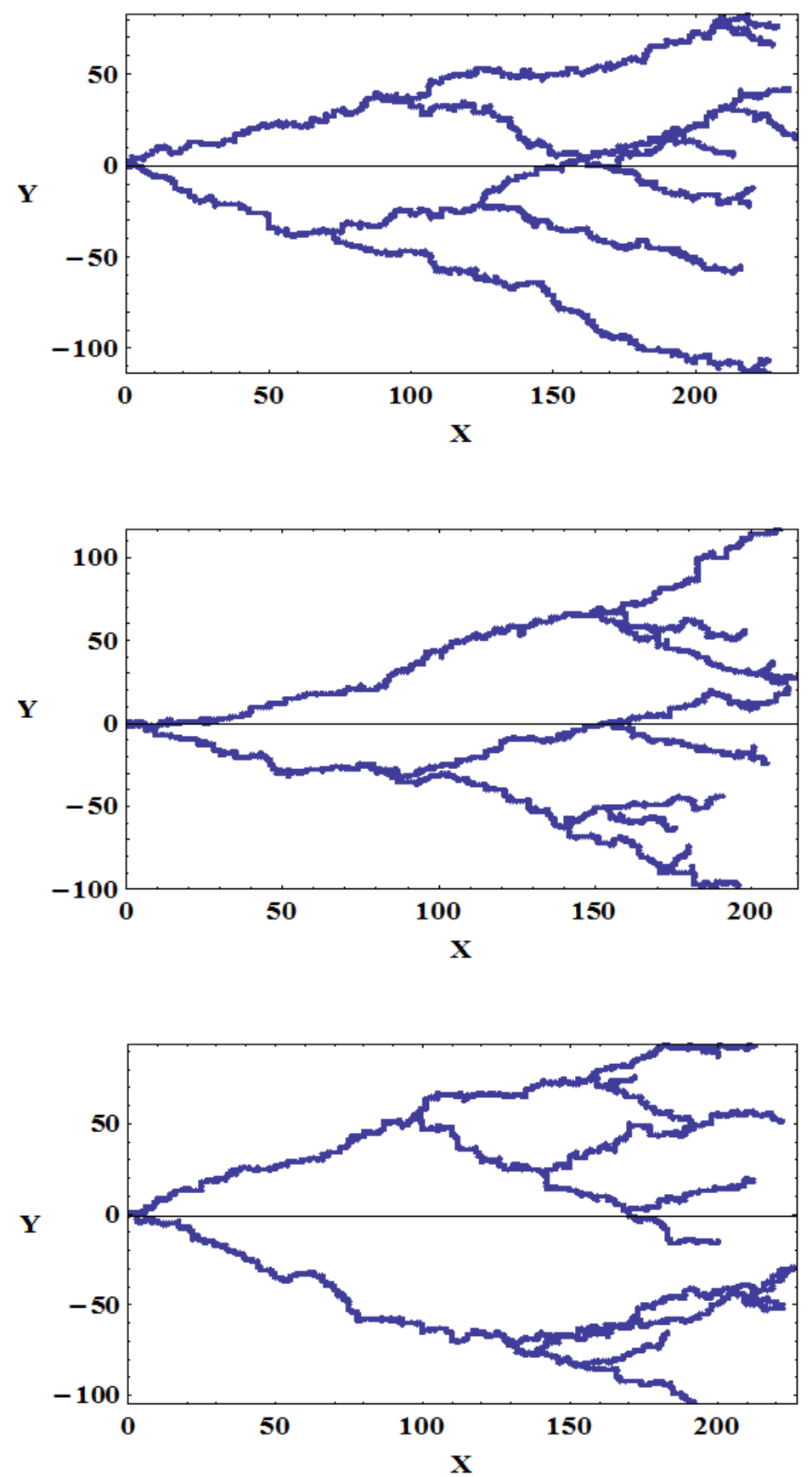

Figure 12 - Random-walk with uniform probabilities for 1000 iterations (4 branches). 


\section{III.5 Generation of the Fracture Network and Reservoir Simulation}

The computed fracture patterns which essentially just a series of points in 2-D space are input into a numerical simulator by transforming these points into a "grid mesh" (a segmentation of the reservoir into prescribed 3-dimensional blocks (or grids). In most cases (and this work) the mesh is generated by considering that each point to the center of a grid-cell.

Based on a rectangular grid we use the MESHMODIFIER code to create the grid matrix grid. In our case we have chosen to use the centimeter scale as our largest grid size to ensure proper representation of the fracture network. To scale our problem we assume that the fracture network will reside in a sub-region of the reservoir ( $\Delta x=\Delta y=2.5 m, \Delta z=20 \mathrm{~m}$ ), beyond the "fracture network" region we use geometrically increasing grid-sizes to achieve better computation efficiencies.

For reference, the dimensions of our synthetic reservoir are:

$$
\Delta x=\Delta y=\Delta z=20 m
$$

Figure 13 shows a gridded fracture network on a 2-D grid $(N x=N y=250, N z=1)$ for a 3-branched fracture network. Figure 14 shows the entire reservoir 2-D grid $(N x=300, N y=350, N z=1)$, the region where the fractures are assumed to grow is highlighted.

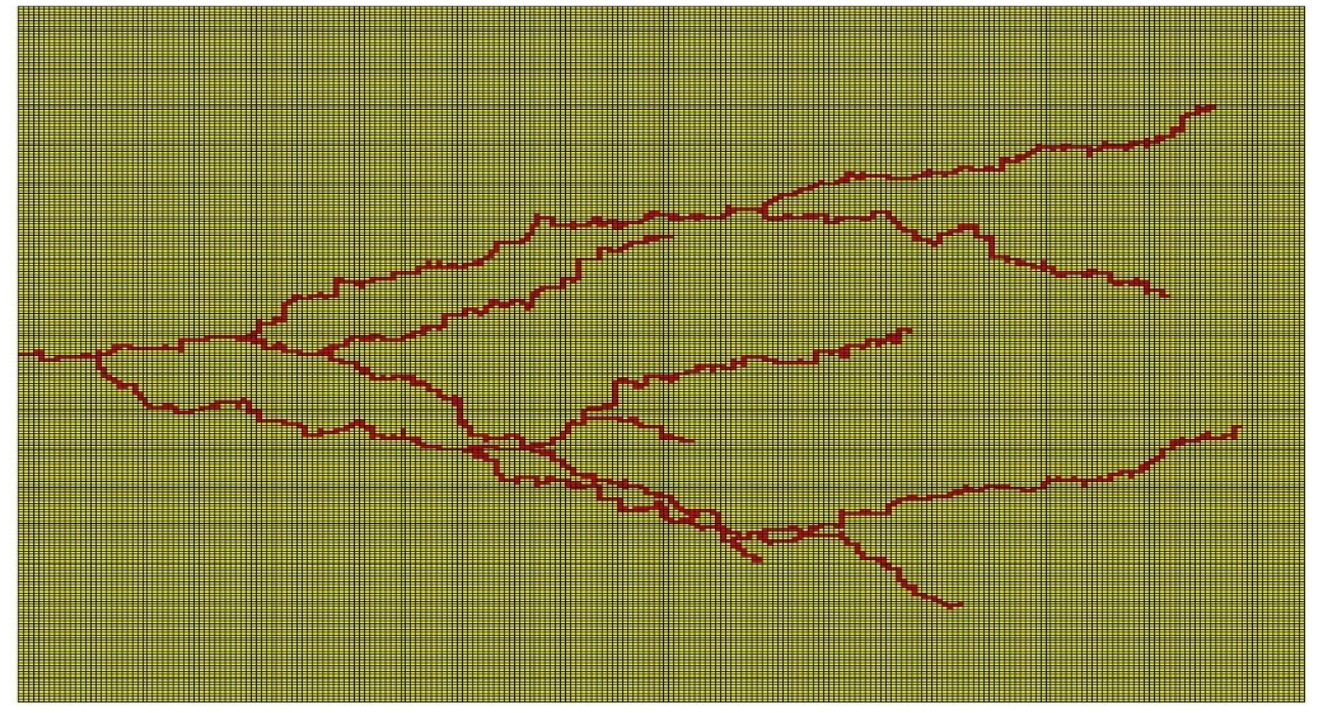

Figure 13 - Random-walk fracture network projected on a 2-D grid structure. 


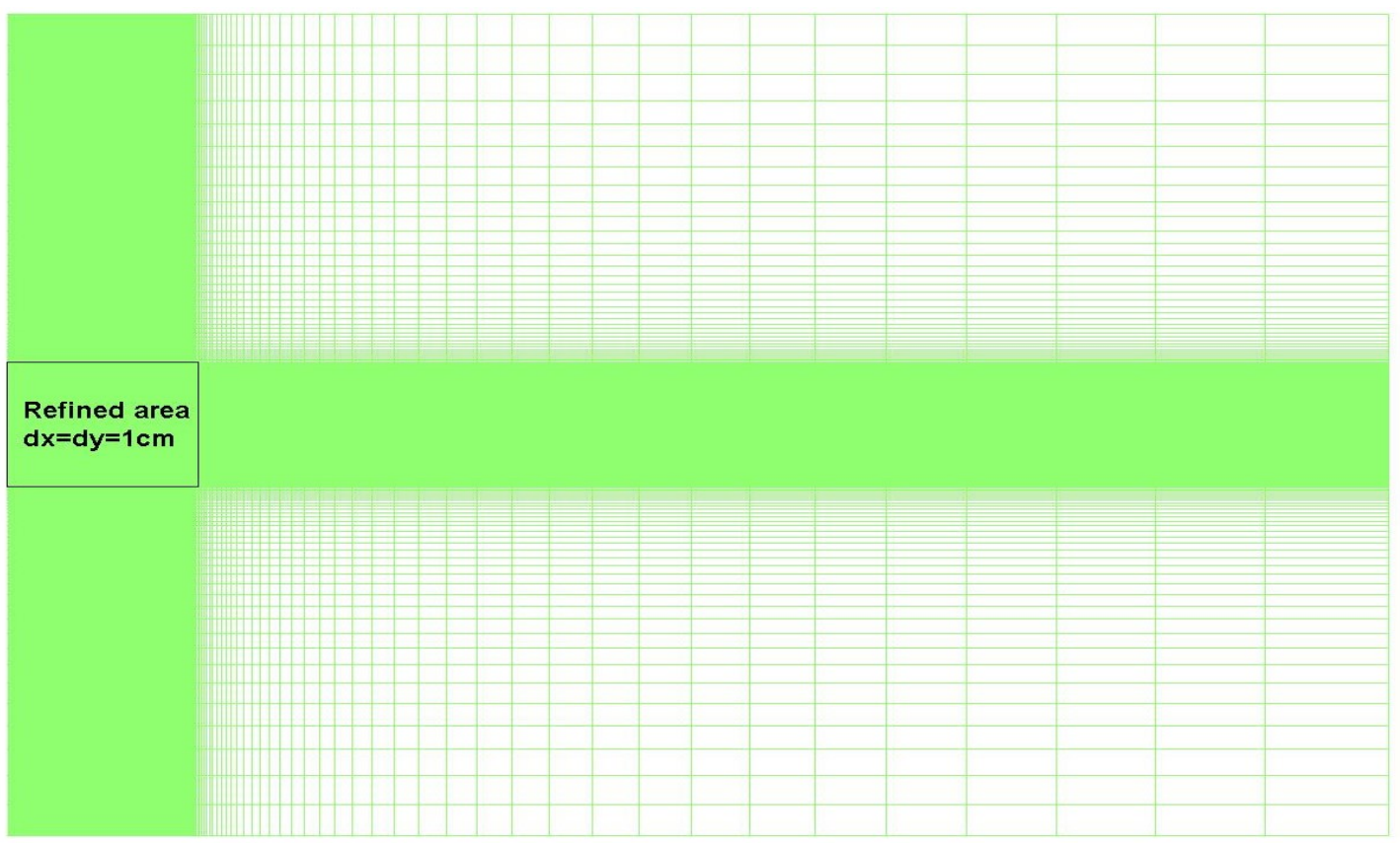

Figure 14 - Schematic of a progressively coarsening grid-size near a fracture.

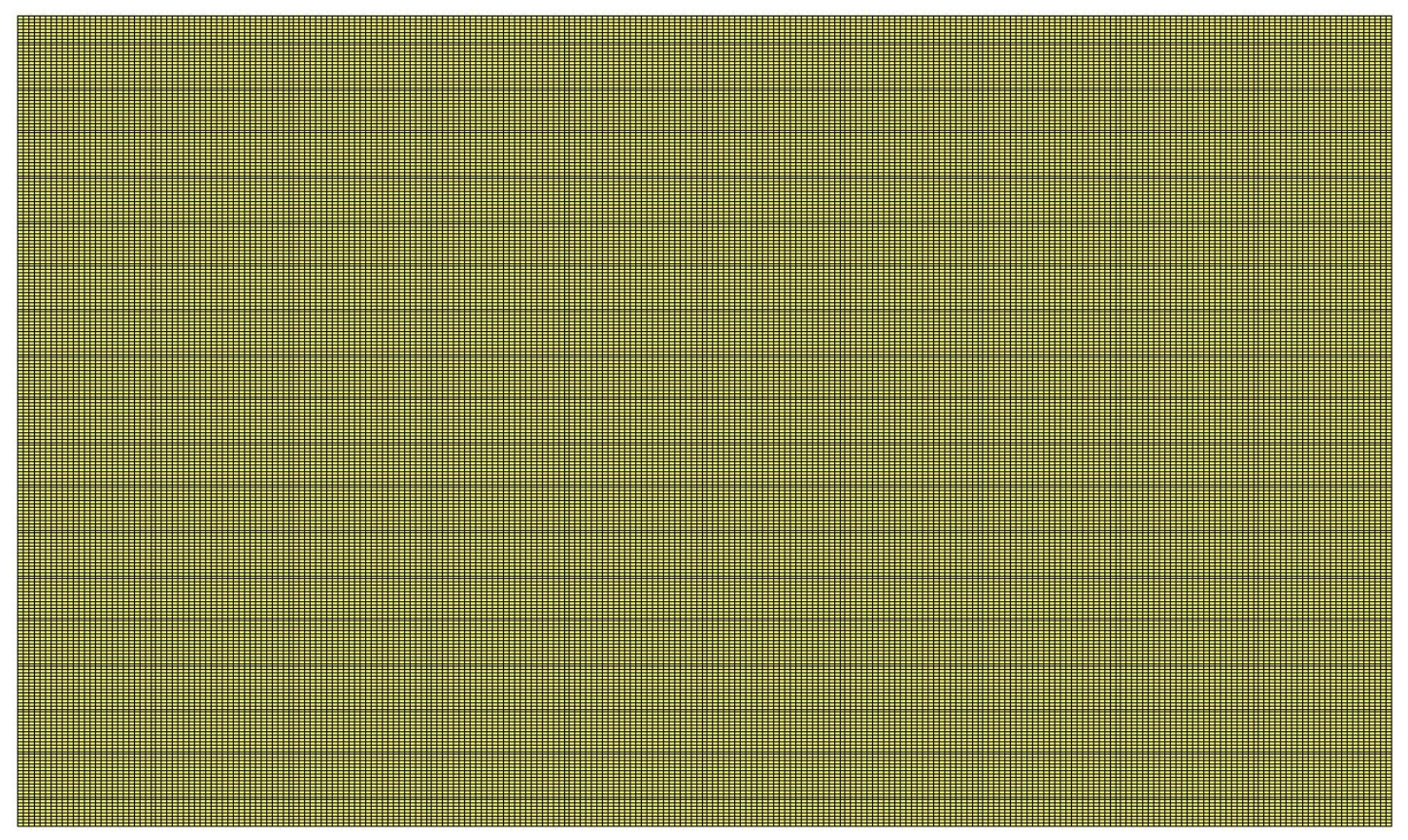

Figure $15-2$-D view of the fracture grid in an unfractured reservoir. 


\section{III.6 Stochastic Fracture Generation Algorithm}

As we previously explained, only a restricted area in our synthetic reservoir contains the stochastic fracture network patterns $\left(x_{F}=y_{F}=2.5 m, z_{F}=20 m\right)$ with a refined grid cell size $\left(d_{x}=d_{y}=\right.$ $\left.0.01 m, d_{z}=20 m\right)$. Consequently, the total number of grid blocks in that area is

$$
N_{T}=N x \times N y \times N z=250 \times 250 \times 1=62500
$$

The initial reservoir simulation grid (without an imposed fracture network) is shown for reference in

Figure 15. As we noted, the reservoir is segregated into fractured and unfractured regions as shown in

Figure 14. For reference, the dimensions of the synthetic reservoir are:

$$
\Delta x=\Delta y=\Delta z=20 m
$$

As mentioned in previous sections, we elected to use this "scaled model" to evaluate the behavior of the stochastic fracture networks. We recognize that our "scaled model" has limitations, but we believe that this model accurately represents all of the expected behaviors in the fracture/reservoir system. As illustrated in Figure 14, the grid blocks size is chosen to be progressively (geometric increase) coarsening out of the fractured region in order to reduce the computational requirements of the simulator. For this configuration, our number of grid-blocks is:

$$
N_{T}=N x \times N y \times N z=300 \times 350 \times 1=105000
$$

Using our MESHMODIFIER code, only the "fractured reservoir" region is modified, the "unfractured reservoir" region is maintained at a constant grid configuration. All the generated fractures that are used in this research are given in Appendix A.

\section{III.6.1 Non-Branched Fracture Pattern}

A non-branched fracture pattern is an unbroken fracture path that contains no bifurcation. The nonbranched fracture is geometrically similar to the planar hydraulic fracture, except that the non-branched fracture can have a zig-zag pattern (but no splits). This fracture uses the density function representation characterized by the probabilities of growth in each direction $\left(P_{x+1}, P_{y+1}\right.$ and $\left.P_{y-1}\right)$. There are two ways to define the dimensions of this structure:

- The total number of iterations $N$.

- The maximum reach of the fracture in the $\mathrm{x}$-direction which we shall call $x_{\max }$ (limiting distance).

Each definition yield a different fracture extent. We note that the maximum extent for $N$ iterations is:

$$
x_{\max 2}=N \times d_{x}
$$


Where $\mathrm{x}_{\max 2}$ is generally different than a fixed maximum extent of the fracture $\left(x_{\max }\right)$. To illustrate this scenario, we performed 3 simulations for a fixed number of steps $N=225$ so that $x_{\max 2}=x_{\max }=$ $2.25 \mathrm{~m}$ and 3 other simulations of pattern with a fixed fracture extent $x_{\max }$.
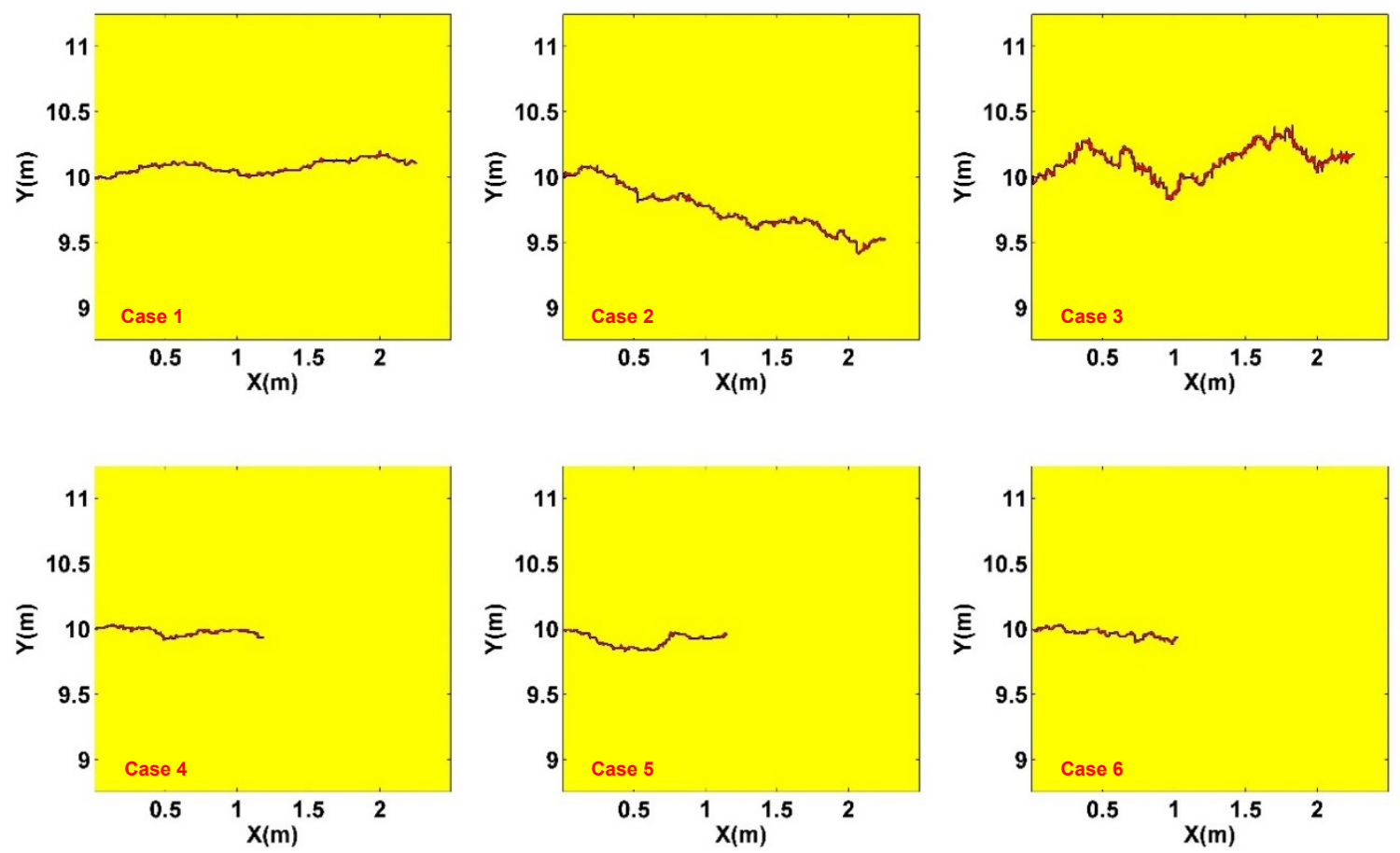

Figure 16 - In the upper 3 figures the realizations for a non-branched pattern with an $x_{\max }=2.25 \mathrm{~m}$ constraint are shown. In the lower 3 figures the realizations of a non-branched pattern with 225 of iterations $(N=225)$ are shown.

Figure 16 shows that the type of constraint in the algorithm that can modify a given the pattern. For example, with a fixed lateral extent $x_{\max }=2.25 \mathrm{~m}$ the fracture always stops at this extent provided that we use a sufficient number of iterations (a very large value is chosen, say $N=5000$, and an exit condition is added). However, with a fixed number of iterations, we know that the maximum extent cannot be more than $x_{\max 2}=2.25 \mathrm{~m}$ - however; this extent changes for each simulation. The steps performed for the non-branched fracture pattern are as follows:

- Reads in the mesh data.

- The origin of the fracture is selected in the basis of the grid $(x=0 \mathrm{~m}, y=10 \mathrm{~m})$.

- The number of iterations (or steps) is selected or randomly generated (for a given range).

- A loop from 1 to the number of iteration is created:

- A random real number is generated (between 0 and 1). 
- If this number is lower than $P_{x+1}$ the current cell flag moves to the adjacent cell along the positive $\mathrm{x}$ direction and modifies its media name from "Shale" to "Fracture."

- If this number is lower than $P_{x+1}+P_{y+1}$ the current cell flag moves to the adjacent cell along the positive y direction and modifies its media name from "Shale" to "Fracture."

- Else if this number is lower than $P_{x+1}+P_{y+1}+P_{y-1}=1$ the current cell flag moves to the adjacent cell along the negative y direction and modifies its media name from "Shale" to "Fracture".

- The code prints all the new mesh information in the output file.

\section{III.6.2 Mono-Branched Fracture Pattern}

A Mono-branched fracture pattern is a 2-dimensional fracture path that contains only one bifurcation. We can subdivide this pattern in 3 scenarios:

- A random non-branched pattern that extends until an $x_{\max 0}$ or that is defined by a number of iterations $k_{0}$. It is constructed by the same algorithm defined in the previous paragraph. We label this portion $F_{0}$.

- An upper sub-fracture that occurs after the bifurcation stage, it may be modeled to reach a certain maximum extent $x_{\max 01}$ or a prescribed number of iterations $k_{01}$. We label this portion $F_{01}$.

- A lower sub-fracture that occurs after the bifurcation stage, it may be modeled to reach a certain maximum extent $x_{\max 02}$ or a prescribed number of iterations $k_{02}$. We label this portion $F_{02}$.

Each of the defined units may be controlled separately:

- Each unit has its own constraints: maximum length $x_{\max }$ or maximum number of iterations $k$.

- Each unit has its own density function and probabilities of propagation $\left(P_{x+1}, P_{y+1}\right.$ and $\left.P_{y-1}\right)$.

As a consequence of these specifications, the propagation of the mono-branched fracture may be controlled and we are able to choose the following propagation characteristics:

- The length of the sub-unit it $F_{0}$ that extends until the fracture "splits", in other terms this controls the moment when the fracture bifurcates into two sub-fractures $F_{01}$ and $F_{02}$.

- The preferential growth direction of each sub-unit: which is controlled by the probabilities of growth or their ratio $\frac{P_{x+1}}{P_{y+1}+P_{y-1}}$ and $\frac{P_{y+1}}{P_{y-1}}$. 

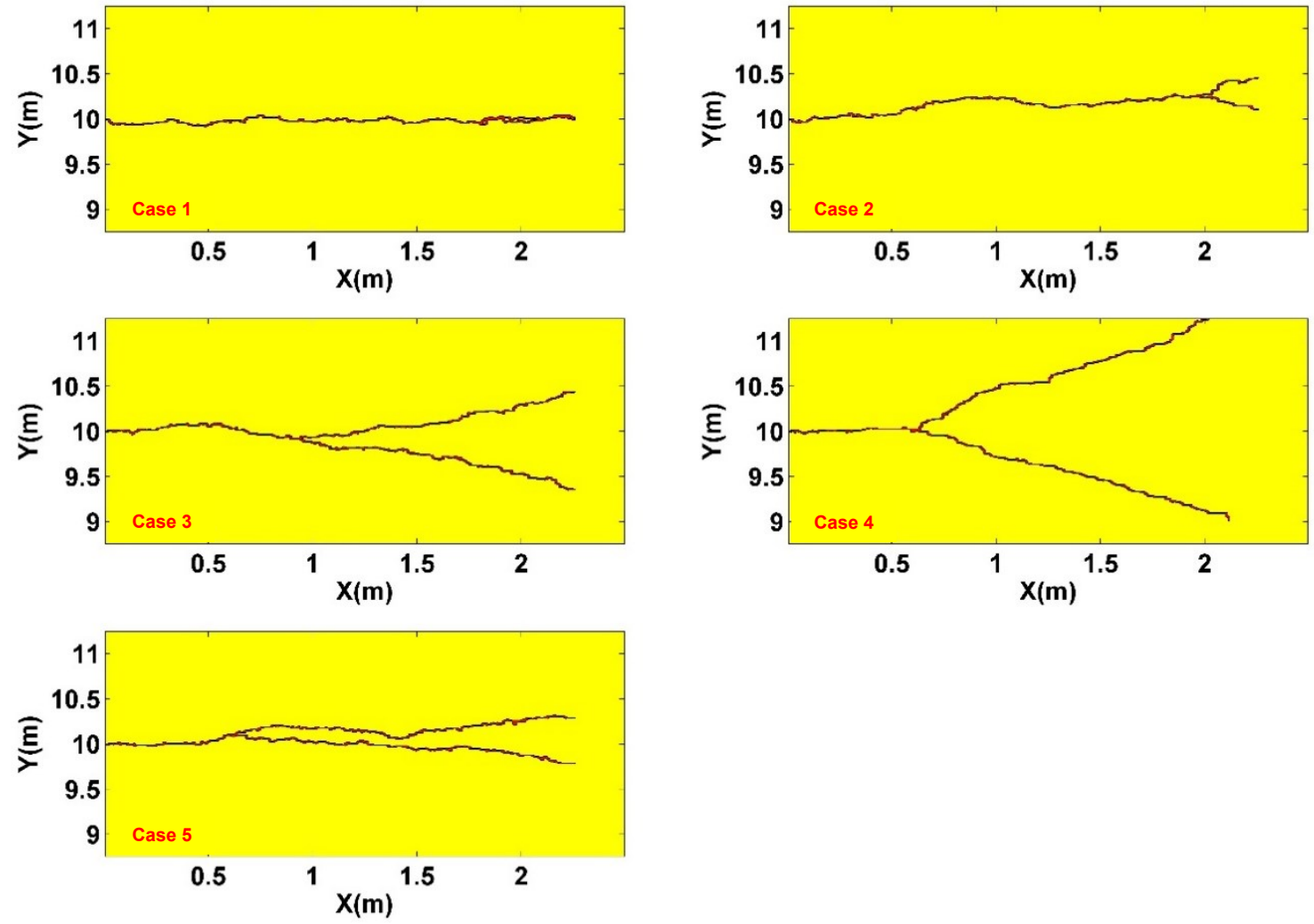

Figure 17 - 5 Different realizations of the "mono-branch" fracture pattern: the upper left figure shows a fracture for which $F_{01}$ and $F_{02}$ definition verifies $P_{y+1}=P_{y-1}$, the upper right figure shows a fracture which bifurcation occurs late whereas the middle left figure shows a fracture which bifurcation occurs early, the middle right and bottom figures shows fractures for which $F_{01}$ and $F_{02}$ have a ratio $P_{y+1} / P_{y-1}$ higher and lower than 1 , respectively.

Figure 17 shows how the mono-branched pattern characteristics are controlled by varying different input parameters in the code (number of iterations and probabilities of growth). The steps used to create a mono-branched fracture pattern are as follows:

- Reads in the mesh data.

- The origin of the fracture is selected in the basis of the grid $(x=0 \mathrm{~m}, y=10 \mathrm{~m})$ and its media name is modified from "Shale" to "Fracture".

- The number of iterations is selected or randomly generated (for a given range): $k_{0}, k_{01}$ and $k_{02}$.

- (primary, non-branched pattern) A loop from 1 to the number of iteration $k_{0}$ is created: 
- A random real number is generated (between 0 and 1).

- If this number is lower than $P_{x+1}$ the current cell flag moves to the adjacent cell along the positive $\mathrm{x}$ direction and modifies its media name from "Shale" to "Fracture".

- If this number is lower than $P_{x+1}+P_{y+1}$ the current cell flag moves to the adjacent cell along the positive y direction and modifies its media name from "Shale" to "Fracture".

- Else, if this number is lower than $P_{x+1}+P_{y+1}+P_{y-1}=1$ the current cell flag moves to the adjacent cell along the negative y direction and modifies its media name from "Shale" to "Fracture".

- (upper sub-fracture) A loop from 1 to the number of iteration $k_{01}$ is created:

- A random real number is generated (between 0 and 1).

- If this number is lower than $P_{x+1}$ the current cell flag moves to the adjacent cell along the positive $\mathrm{x}$ direction and modifies its media name from "Shale" to "Fracture".

- If this number is lower than $P_{x+1}+P_{y+1}$ the current cell flag moves to the adjacent cell along the positive y direction and modifies its media name from "Shale" to "Fracture".

- Else, if this number is lower than $P_{x+1}+P_{y+1}+P_{y-1}=1$ the current cell flag moves to the adjacent cell along the negative y direction and modifies its media name from "Shale" to "Fracture".

- (lower sub-fracture) A loop from 1 to the number of iteration $k_{01}$ is created:

- A random real number is generated (between 0 and 1).

- If this number is lower than $P_{x+1}$ the current cell flag moves to the adjacent cell along the positive $\mathrm{x}$ direction and modifies its media name from "Shale" to "Fracture".

- If this number is lower than $P_{x+1}+P_{y+1}$ the current cell flag moves to the adjacent cell along the positive y direction and modifies its media name from "Shale" to "Fracture".

- Else, if this number is lower than $P_{x+1}+P_{y+1}+P_{y-1}=1$ the current cell flag moves to the adjacent cell along the negative y direction and modifies its media name from "Shale" to "Fracture".

- The code prints all the new mesh information in the output file. 


\section{III.6.3 Dual-Branched Fracture Pattern}
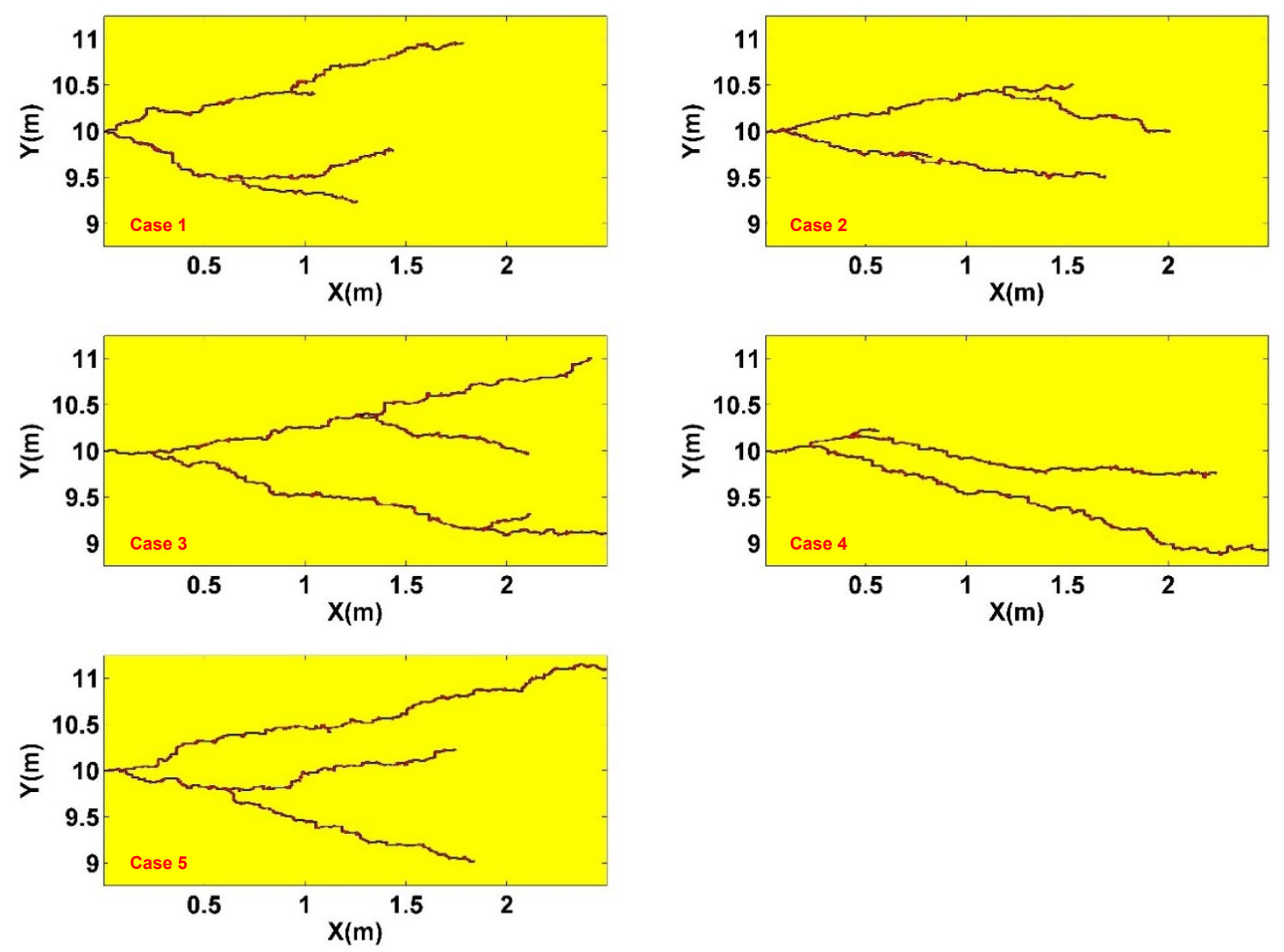

Figure $18-5$ different realizations of the "dual-branch" fracture pattern. In each case the extent of the branch has not been defined (used a random number of iterations). The subfractures have a ratio $P_{y+1} / P_{y-1}$ higher and lower than 1, respectively.

A dual-branch fracture pattern is a 2-dimensional fracture path that contains two bifurcation stages. It contains 7 non-branched fractures that are linked as in Figure 18. We may subdivide this pattern in 7 subunits:

- $F_{0}, F_{01}$ and $F_{02}$ as defined previously.

- An upper sub-fracture that occurs after the second upper bifurcation stage, it may be modeled to reach a certain maximum extent $x_{\max 011}$ or a certain number of iterations $k_{011}$. We label this portion $F_{011}$.

- A lower sub-fracture that occurs after the second upper bifurcation stage, it may be modeled to reach a certain maximum extent $x_{\max 012}$ or a certain number of iterations $k_{012}$. We label this portion $F_{012}$.

- An upper sub-fracture that occurs after the second lower bifurcation stage, it may be modeled to reach a certain maximum extent $x_{\max 021}$ or a certain number of iterations $k_{021}$. We label this portion $F_{011}$. 
- A lower sub-fracture that occurs after the second lower bifurcation stage, it may be modeled to reach a certain maximum extent $x_{\max 022}$ or a certain number of iterations $k_{022}$. We label this portion $F_{012}$.

Each of the defined units may be controlled separately:

- It has its own constraints: maximum length $x_{\max }$ or maximum number of iterations $k$.

- Its own density function and probabilities of propagation $\left(P_{x+1}, P_{y+1}\right.$ and $\left.P_{y-1}\right)$.

As a consequence of these specifications, the propagation of the dual-branched fracture may be controlled and we are able to choose the following propagation characteristics:

- The length of the sub-unit it $F_{0}$ that extends until the fracture "splits", in other terms this controls the moment when the fracture bifurcates into two sub-fractures $F_{01}$ and $F_{02}$.

- The length of the sub-unit it $F_{01}$ that extends until the fracture "splits", in other terms this controls the moment when the fracture bifurcates into two sub-fractures $F_{011}$ and $F_{012}$.

- The length of the sub-unit it $F_{02}$ that extends until the fracture "splits", in other terms this controls the moment when the fracture bifurcates into two sub-fractures $F_{021}$ and $F_{022}$.

- The preferential growth direction of each sub-unit: which is controlled by the probabilities of growth or their ratio $\frac{P_{x+1}}{P_{y+1}+P_{y-1}}$ and $\frac{P_{y+1}}{P_{y-1}}$.

Figure 18 shows how the same characteristics of the dual-branch pattern can yield different fracture patterns. As a preliminary observation we note that the area covered by the different patterns generated using the exact same input parameters is different, therefore we can predict a variety of flow performance behaviors for those 5 different patterns. We will consider the influence of a given dual-branch fracture pattern later in this work. The steps performed by the dual-branch fracture pattern generation code are similar to the code that generates mono-branched pattern:

- Reads in the mesh data.

- The origin of the fracture is selected in the basis of the grid $(x=0 m, y=10 m)$ and its media name is modified from "Shale" to "Fracture".

- The number of iterations (or steps) is selected or randomly generated (in a certain range): $k_{0}, k_{01}$, $k_{02}, k_{011}, k_{012}, k_{021}$ and $k_{022}$.

- Generate $F_{0}, F_{01}$ and $F_{02}$ using the same code as for the mono-branched fracture pattern.

- Similarly generate $F_{011}, F_{012}, F_{021}$ and $F_{022}$.

- The code prints all the new mesh information in the output file. 


\section{III.6.4 Tri-Branched Fracture Pattern}
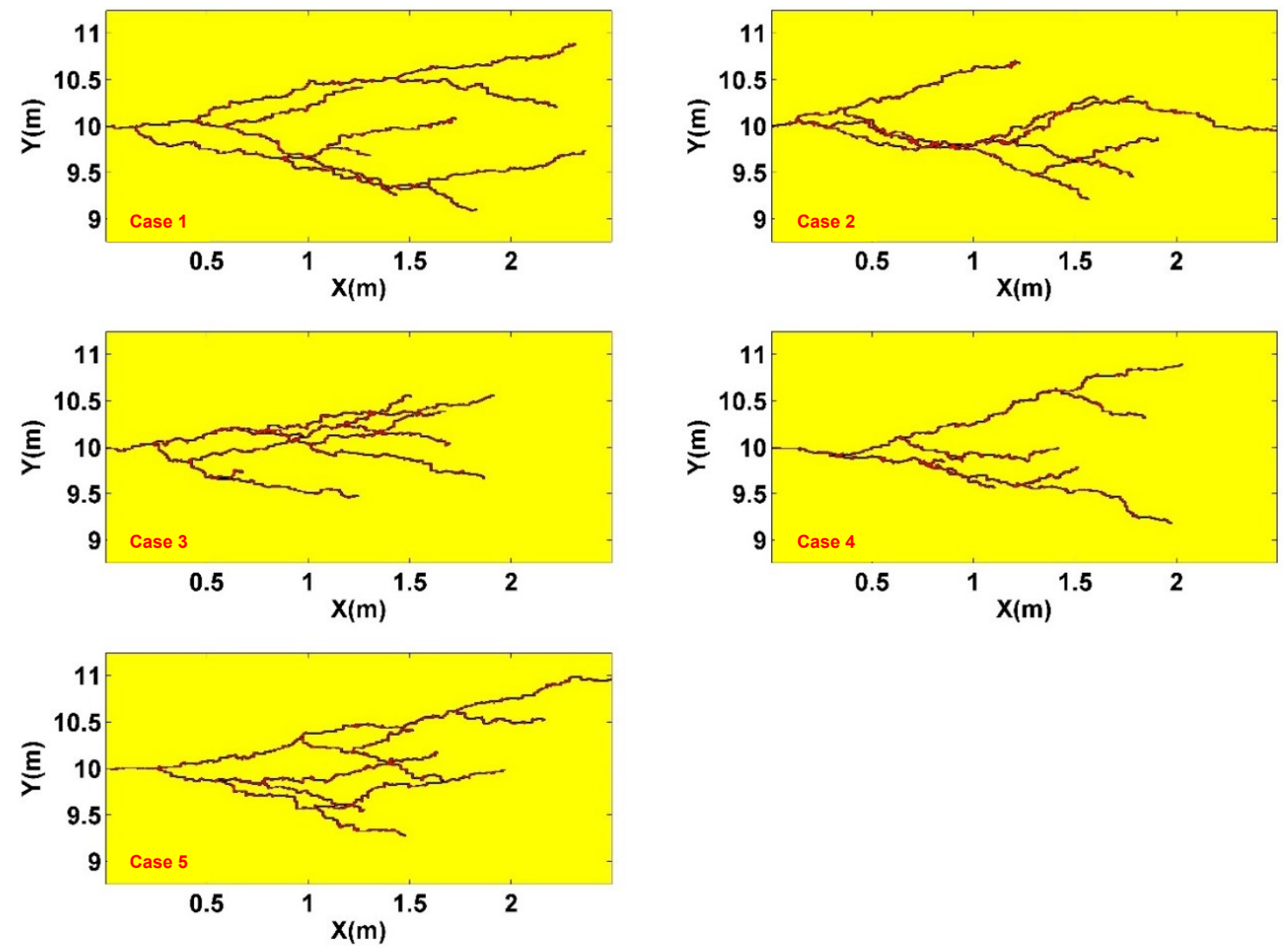

Figure $19-5$ different realizations of the "tri-branch" fracture pattern. In each case the extent of the branch has not been defined (used a random number of iterations). Additionally, the sub-fractures have a ratio $P_{y+1} / P_{y-1}$ higher and lower than 1 , respectively.

A Tri-branch fracture pattern is a 2-dimensional fracture path that contains 3 bifurcation stages as shown in Figure 19. We may subdivide this pattern in 15 sub-units:

$\bullet F_{0}, F_{01}, F_{02}, F_{011}, F_{012}, F_{021}$ and $F_{022}$ as they have been defined previously.

- Two sub-fractures of $F_{011}: F_{0111}$ and $F_{0112}$

- Two sub-fractures of $F_{012}: F_{0121}$ and $F_{0122}$

- Two sub-fractures of $F_{021}: F_{0211}$ and $F_{0212}$

- Two sub-fractures of $F_{022}: F_{2121}$ and $F_{0222}$ 
All the properties of this fracture pattern are similarly defined as for the dual-branch pattern. The direction of propagation, the extent and the occurrence of the branching may be controlled. 5 variations of a randomly generated tri-branch pattern are shown in Figure 19.

The steps performed by the tri-branch fracture pattern generation code are similar to the code that generates the dual-branch pattern:

- Reads in the mesh data.

- The origin of the fracture is selected in the basis of the grid $(x=0 \mathrm{~m}, y=10 \mathrm{~m})$ and its media name is modified from "Shale" to "Fracture"

- The number of iterations (or steps) is selected or randomly generated (in a certain range): $k_{0}, k_{01}$, $k_{02}, k_{011}, k_{012}, k_{021}, k_{022}, k_{0111}, k_{0112}, k_{0121}, k_{0122}, k_{0211}, k_{0212}, k_{0221}$ and $k_{0222}$

- Generate $F_{0}, F_{01}, F_{02}, F_{011}, F_{012}, F_{021}, F_{022}$ using the same code as for the dual-branched fracture pattern.

- Similarly generate $F_{0111}, F_{0112}, F_{0121}, F_{0122}, F_{0211}, F_{0212}, F_{0221}$ and $F_{0222}$

- The code prints all the new mesh information in a the output file

\section{III.6.5 Quad-Branched Fracture Pattern}

A quad-branch fracture pattern is a 2-dimensional fracture path that contains 4 bifurcation stages. The quad-branch fracture pattern contains 31 possible non-branched fractures that are linked as shown in

Figure 20. As suggested, we may subdivide this pattern in 31 sub-units:

$\bullet F_{0}, F_{01}, F_{02}, F_{011}, F_{012}, F_{021}, F_{022}, F_{0111}, F_{0112}, F_{0121}, F_{0122}, F_{0211}, F_{0212}, F_{2121}$ and $F_{0222}$ as defined previously.

- Two sub-fractures of $F_{0111}: F_{01111}$ and $F_{01112}$.

- Two sub-fractures of $F_{0112}: F_{01121}$ and $F_{01122}$.

- Two sub-fractures of $F_{0121}: F_{01211}$ and $F_{01212}$.

- Two sub-fractures of $F_{0122}: F_{01221}$ and $F_{01222}$.

- Two sub-fractures of $F_{0211}: F_{02111}$ and $F_{02112}$.

- Two sub-fractures of $F_{0212}: F_{02121}$ and $F_{02122}$.

- Two sub-fractures of $F_{0221}: F_{02211}$ and $F_{02212}$.

- Two sub-fractures of $F_{0222}: F_{02221}$ and $F_{02222}$. 
All the properties of this fracture pattern are similarly defined as for the tri-branch pattern. The direction of propagation, the extent and the occurrence of the branching may be controlled. 5 variations of a randomly generated quad-branch pattern are shown in Figure 20.
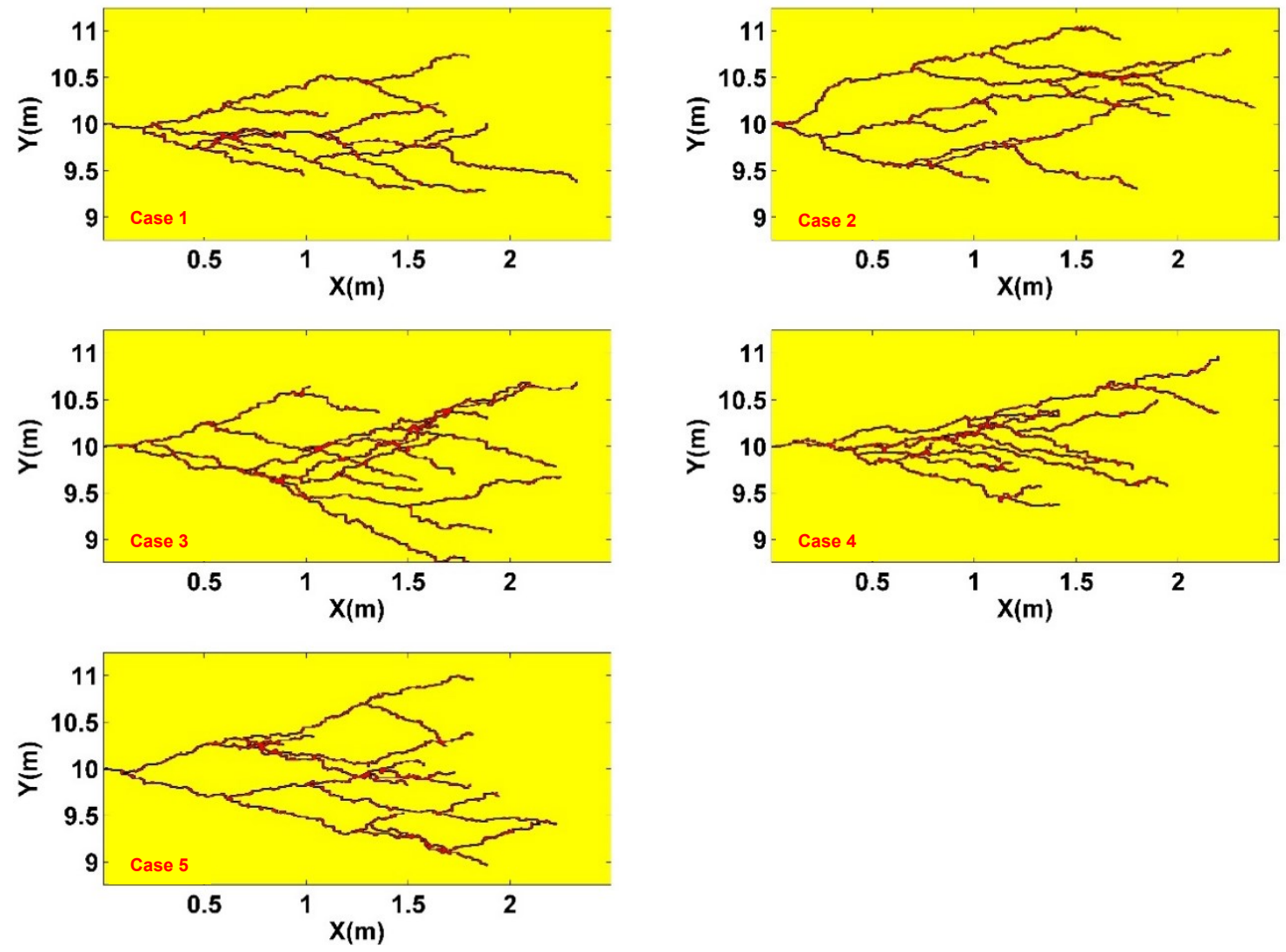

Figure 20 - 5 different realizations of the "quad-branch" fracture pattern. In each case the extent of the branch has not been defined (used a random number of iterations). Additionally, the sub-fractures have a ratio $P_{y+1} / P_{y-1}$ higher and lower than 1 , respectively.

The steps performed by the quad-branch fracture pattern generation code are similar to the code that generates tri-branch pattern:

- Reads in the mesh data.

- The origin of the fracture is selected in the basis of the grid $(x=0 \mathrm{~m}, y=10 \mathrm{~m})$ and its media name is modified from "Shale" to "Fracture".

- The number of iterations (or steps) is selected or randomly generated (in a certain range): $k_{0}, k_{01}$, $k_{02}, k_{011}, k_{012}, k_{021}, k_{022}, k_{0111}, k_{0112}, k_{0121}, k_{0122}, k_{0211}, k_{0212}, k_{0221}, k_{0222}, k_{01111}, k_{01112}$, 
$k_{01121}, k_{01122}, k_{01211}, k_{01212}, F_{01221}, F_{01222}, F_{02111}, F_{02112}, F_{02121}, F_{02122}, F_{02211}, F_{02212}, F_{02221}$ and $F_{02222}$.

- Generate $F_{0}, F_{01}, F_{02}, F_{011}, F_{012}, F_{021}, F_{022}, F_{0111}, F_{0112}, F_{0121}, F_{0122}, F_{0211}, F_{0212}, F_{2121}$ and $F_{0222}$ using the same code as for the tri-branched fracture pattern.

- Similarly generate $F_{01111}, F_{01112}, F_{01121}, F_{01122}, F_{01211}, F_{01212}, F_{01221}, F_{01222}, F_{02111}, F_{02112}, F_{02121}$, $F_{02122}, F_{02211}, F_{02212}, F_{02221}$ and $F_{02222}$.

- The code prints all the new mesh information in the output file.

\section{III.7 Reservoir Model and Simulation}

\section{III.7.1 Consideration of the Multiply-Fractured Horizontal Well Model}

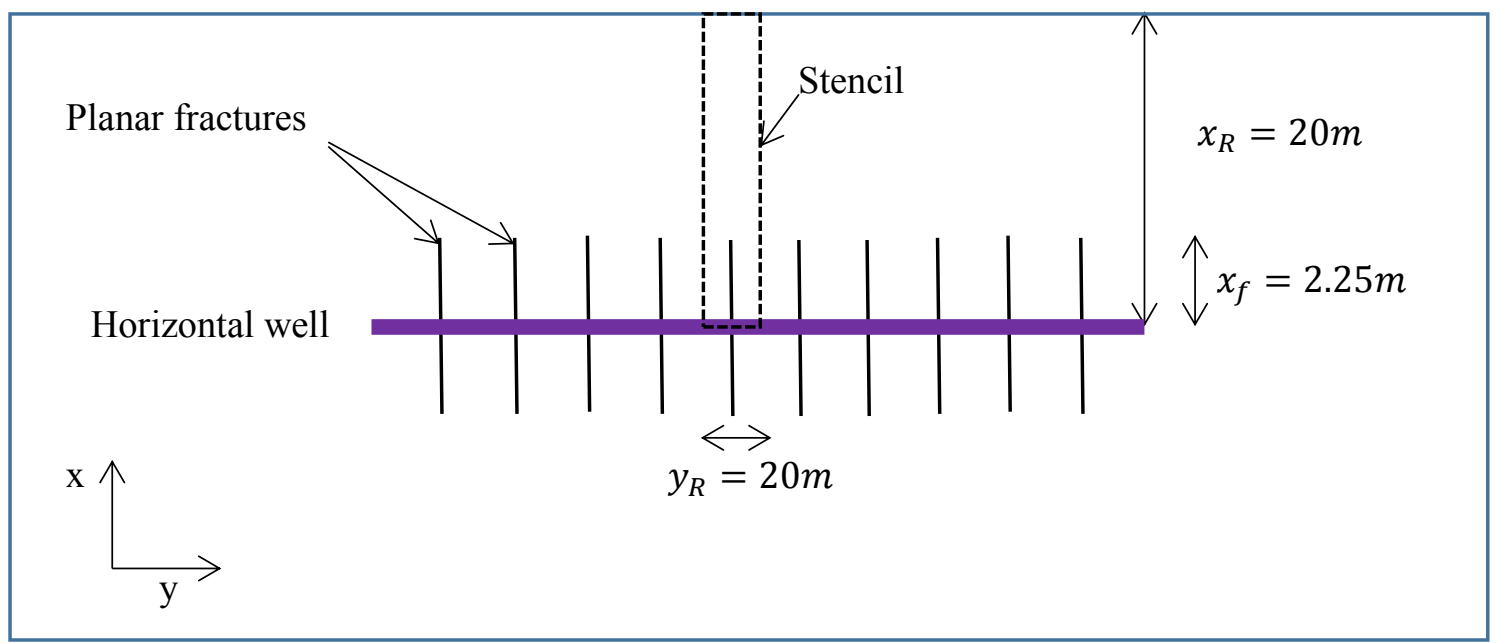

Figure 21 - Schematic 2-D representation of a full-scale horizontal well producing from a shale reservoir. 10 fracture stages are represented and the stencil shows the minimum repeatable feature in the reservoir.

Rather than to study a full-scale horizontal well with multiple fracture stages, our approach is to consider a representative volume of investigation also referred to as "stencil," where the stencil is chosen to be the minimal repetitive element for a multiply fractured horizontal well (Figure 21). Eventually the stencil will fail as fracture interference regimes evolve (Olorode et al. 2013).

As we are only interested in the early time flow performance this effect can be neglected. Our first inclination was to use the multiply-fractured horizontal well model as the basis for comparison of the stochastic fracture network cases considered in this work. However, we felt that, at this stage of the 
investigation, the better comparator would be the simple (single) planar fracture case. That is, our comparator is the single planar fracture case as opposed the multiply-fractured horizontal well.

\section{III.7.2 Optimization of Gridding (Minimum Number of Grid Blocks)}

Our guiding constraint for this work is that we are only considering the 2-D fracture network case (constant fracture height). The "wellbore" is taken as the base cell of the fracture network (defined as the origin point) as shown by the orange line in Figure 22. Later investigators are encouraged to consider 3dimensional fracture distributions, but as noted earlier, our goal is to identify the fundamental nature of flow for a random-walk fracture pattern and we believe that 2-dimensional flow behavior is sufficient for our needs. Extensions to the 3-dimensional case would (likely) be more useful for understanding geomechanical behavior as well as potentially addressing layering issues.

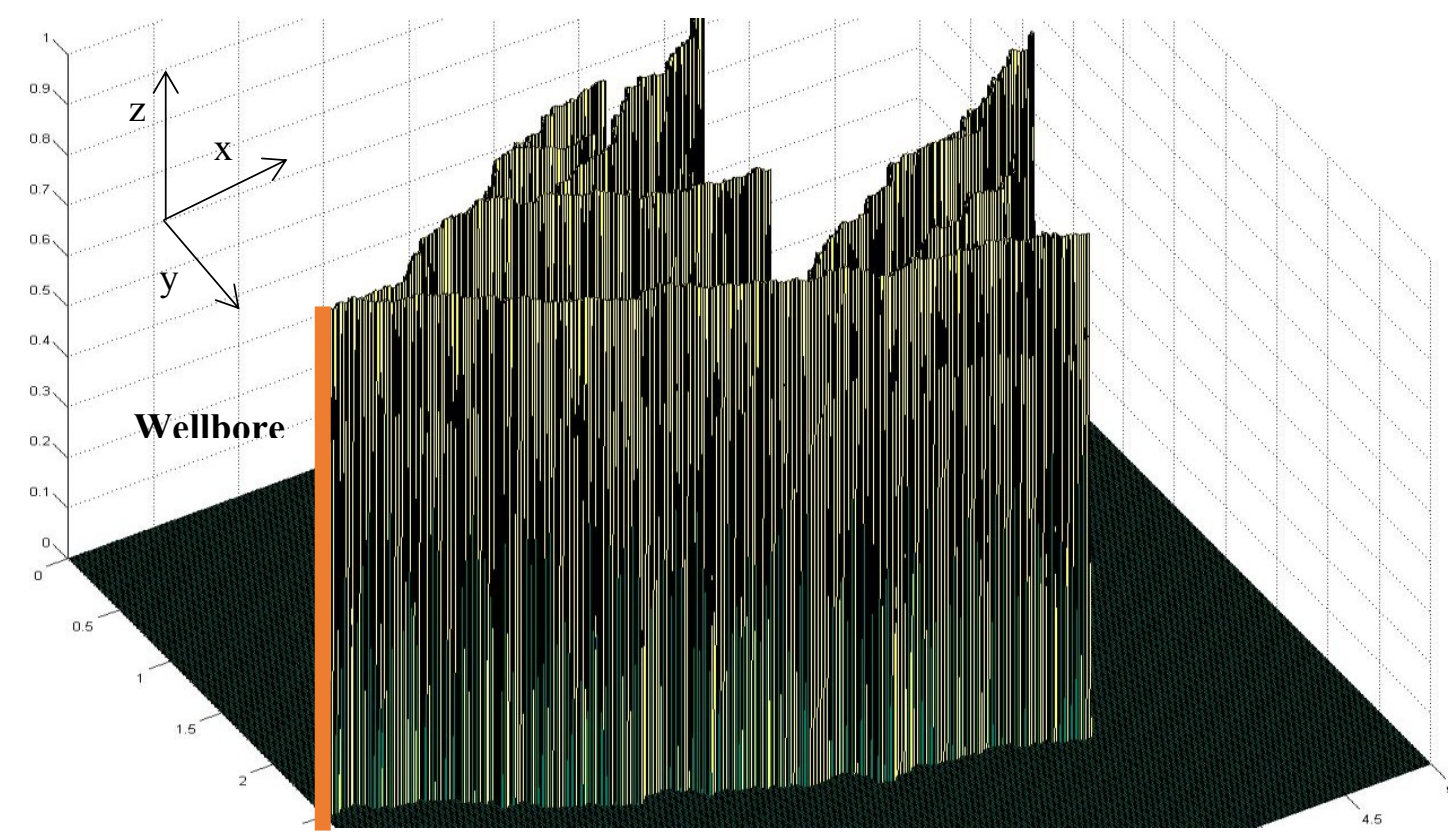

Figure $22-3-D$ view of a stochastic pattern network of fractures.

\section{III.7.3 Orientation - Modeling Fracture Patterns}

The MESHMODIFIER code provides the construction of rectangular Cartesian grids that describe the flow models that we investigate in the next chapter. The shape and the geometric features of the fracture patterns are thought to have an influence on the flow behavior (rate and pressure behavior). The code is used to generate specific sets of fracture patterns (83 patterns are investigated and compared to the planar fracture model). The pattern properties that will be varied are the following: 
- The fracture tortuosity (controlled by the ratio of the probabilities $\frac{P_{x+1}}{P_{y+1}+P_{y-1}}$ ).

- The occurrence of "branching."

- The orientation of the branches (controlled by the ratio of probabilities in the $y$-direction $\frac{P_{y+1}}{P_{y-1}}$ ).

- The total number of branches.

Then, the following workflow is applied to each of the generated fracture network:

- Monitor the pressure change around the fracture using a 2-D pressure profile.

- Use the pressure profile to identify the range of time that is impacted by the shape of the fracture.

- Compare the mass production rate curves of the stochastic-patterns to the planar fracture model.

- Assess the performance (cumulative mass production) of the stochastic patterns.

As noted, the rate and pressure performance of the stochastic fracture networks are compared with those for the (single) planar fracture case (our "standard" case). Based on observations of the pressure distributions, we concluded that the effect of the pattern would "disappear" after a relatively short production period (less than 4 days in this case); primarily due to the reservoir size, recall that this is a "scaled" experiment.

Most simulations were targeted for 100 days (which is an extremely long time for this configuration), and as we noted that most features are observed on the order of 4 days (circa 100 hours) many of the simulations were terminated in this timeframe to reduce computational expense.

The investigation of the different stochastic fracture network patterns allow us to assess the effect of each fracture property on the performance of the reservoir - specifically, tortuosity, occurrence of branching, orientation of branches and number of branches. One of the practical goals of this work is observe the behavior of complex fracture networks on production rate performance - and as a simplistic conclusion, we can state that the fracture networks we have generated in this work provide analogous production signatures to those observed in the field. This observation could have a significant impact on the analysis and interpretation of production performance as well as the evaluation of reserves. 


\section{CHAPTER IV}

\section{INFLUENCE OF THE STOCHASTIC FRACTURE CHARACTERISTICS}

\section{IV.1 Methodology to Assess the Performance of the Stochastic Fracture Patterns}

The following chapter provides an in-depth analysis of stochastic "random-walk" fracture patterns created in this work using numerical simulation (FTSim) via comparison of computed mass rate and pressure functions. Each fracture network is modeled and then the results from each model are compared in groups (by number of fracture branches) as well as to the single-planar fracture case (which is our standard for comparison).

\section{IV.1.1 Map the Pressure Change to Identify the Flow Regimes}

In the work we extract the "pressure maps" at different time values for each fracture network. Each simulation case is generated for at least 100 days of production at a constant wellbore pressure $p_{w f}=$

$1.25 \times 10^{7} \mathrm{~Pa}=\frac{p_{i}}{2}$. The 100-day production period was shown (via modeling) to be sufficient for this synthetic shale matrix permeability $(50 \mathrm{nD})$ where we would definitely observe transient flow features (as we desire) and for some cases we can observe severe interference (in a pressure sense) of the created fracture networks. Given the reservoir size, the matrix permeability, and the various fracture networks, we established that we should begin to see interference/depletion effects at times on the order of 100 days. In short, our approach is based on the observation of transient flow behavior of the fracture network, where we evaluate the mass rate and $\beta$-derivative of the mass rate trends versus production time on a "log-log" style plot.

Specific to the generation and review of the pressure maps for this work, we note that we do indeed observe fracture interference effects, and we note that these interference effects are somewhat unique to a given stochastic fracture network patterns. For reference regarding all of our pressure maps, the displayed variable $(f)$ is the $p_{f} / p_{i}$ ratio. For a given time, the reservoir pressure, $p_{t}$, is given as:

$$
p_{f}=\frac{p_{i}}{2}<p_{t}<p_{i}
$$

Therefore we expect the function $f$ to be:

$$
0.5<f<1
$$

The study of the pressure distributions for a given fracture network provides more insight than the mass production rate in isolation, but obviously we cannot measure pressure maps in the field. As with most tasks in reservoir engineering we use what we can measure as a proxy for what we cannot - so we will 
have to infer fracture interference, reservoir depletion, etc. from the production rate profiles. This is discussed in the next section of this chapter.

\section{IV.1.2 Reservoir Performance (Mass Production Rate and $\beta$-Derivative)}

In this section we present the introduction of the analysis/interpretation using the mass production rate and $\beta$-derivative functions. We present different groups of stochastic fracture networks, focusing (primarily) on the constant portion of the mass production rate $\beta$-derivative function as our diagnostic. Recall that for a single-planar fracture our expectation is "linear flow" (which has a constant $\beta$-derivative value of 0.5 (or $1 / 2)$ ). For the various fracture network cases we would expect a $\beta$-derivative value greater than 0.5 (as a given fracture network should be more productive than the single planar fracture case). As such, we group cases according to the number of bifurcations (i.e., splits or branches), and we find that most cases for a given number of branches do "cluster" around a distinct (constant) value of the $\beta$-derivative.

The $\beta$-derivative of the rate function is defined as

$$
\beta=d_{\beta} q(t)=\left|\frac{d(\log (q))}{d(\log (t))}\right|
$$

Where for all cases in this work $q$ is the mass rate in $\mathrm{Kg} / \mathrm{s}$ and $t$ is the time in $\mathrm{s}$. All the mass production decline rate plots and $\beta$-derivative plots are given in Appendices A and $\mathrm{B}$ respectively.

\section{IV.1.3 Cumulative Mass Recovery}

In this work we also monitor the cumulative mass recovery as a mechanism to assess the performance and recovery of gas (mass) at a given time. The cumulative recovery $C R_{t}$ (mass) at a time $t$ is defined as

$$
C R_{t}=\frac{\text { Produced gas mass (or volume) at } t}{\text { Total initial gas mass (or volume) }}
$$

The ultimate or final value of the cumulative recovery $C R_{\infty}$ is the asymptotic maximum value of recovered gas fraction and this value provides an estimation of reserves for a given reservoir/fracture pattern configuration. As we do not produce the cases in this work to complete depletion due to computational issues (this would require extremely large computing times), we use a "common point" to which all cases are produced. In this work we chose 100 days as our reference point for comparison of recovery. For each stochastic fracture network case we present a graphical correlation of cumulative recovery at 100 days versus total fracture volume (as proxy for stimulation "effectiveness"). We have observed a base trend, but also a trend for each fracture network, grouped according to the number of fracture branches. 


\section{IV.2 Single-Planar Fracture Case (Benchmark/Comparative Standard)}

As explained earlier, the single-planar fracture case is used to compare the performance of each of the stochastic fracture networks. As this work is based on a synthetic shale gas reservoir model, we must define the basic hydraulic fracture characteristics (summarized in Table 2) as well as the properties of the synthetic shale gas reservoir (i.e., the reservoir matrix), which are summarized in Table 3.

Table 2 Fracture Parameters for the Synthetic Shale Gas Reservoir Case.

\begin{tabular}{lcc} 
Parameters & SI Units & Field Units \\
\hline Fracture half-length, $x_{f}$ & $2.25 \mathrm{~m}$ & $7.38 \mathrm{ft}$ \\
Fracture width, $w_{f}$ & $1 \mathrm{~cm}$ & $0.0328 \mathrm{ft}$ \\
Fracture thickness, $h$ & $20 \mathrm{~m}$ & $65.62 \mathrm{ft}$ \\
Fracture permeability, $k_{f}$ & $5 \times 10^{-11} \mathrm{~m}^{2}$ & $5 \times 10^{4} \mathrm{mD}$ \\
Fracture porosity, $\phi_{f}$ & 0.50 & 50 percent
\end{tabular}

Table 3 - Reservoir Parameters for the Synthetic Shale Gas Reservoir Case.

$\begin{array}{lll}\text { Parameters } & \text { SI Units } & \text { Field Units }\end{array}$

\begin{tabular}{lcc}
\hline Reservoir length, $x_{R}$ & $20 \mathrm{~m}$ & $65.62 \mathrm{ft}$ \\
Reservoir width, $y_{R}$ & $20 \mathrm{~m}$ & $65.62 \mathrm{ft}$ \\
Reservoir thickness, $h$ & $20 \mathrm{~m}$ & $65.62 \mathrm{ft}$ \\
Shale permeability, $k$ & $5 \times 10^{-20} \mathrm{~m}^{2}$ & $5 \times 10^{-5} \mathrm{mD}$ \\
Shale porosity, $\phi$ & 0.04 & 4 percent \\
Shale compressiblity, $c_{f}$ & $10^{-9} \mathrm{~Pa}^{-1}$ & $6.9 \times 10^{-6} \mathrm{psi}^{-1}$
\end{tabular}

In Figure 23 we present the schematic of the single-planar hydraulic fracture in the reduced-scale reservoir. As we limit our study to 2 dimensions (constant reservoir and fracture thickness), all of our presentations are made relative to a 2-dimensional view of the reservoir. The wellbore is at the base of the hydraulic fracture and is held at a constant flowing bottomhole pressure $\left(p_{w f}=2.5 \times 10^{7} \mathrm{~Pa}=2 p_{i}\right)$.

As mentioned in previous sections, all cases are produced to at least 100 days, so this is a point of reference for assessing cumulative mass recovery as. 


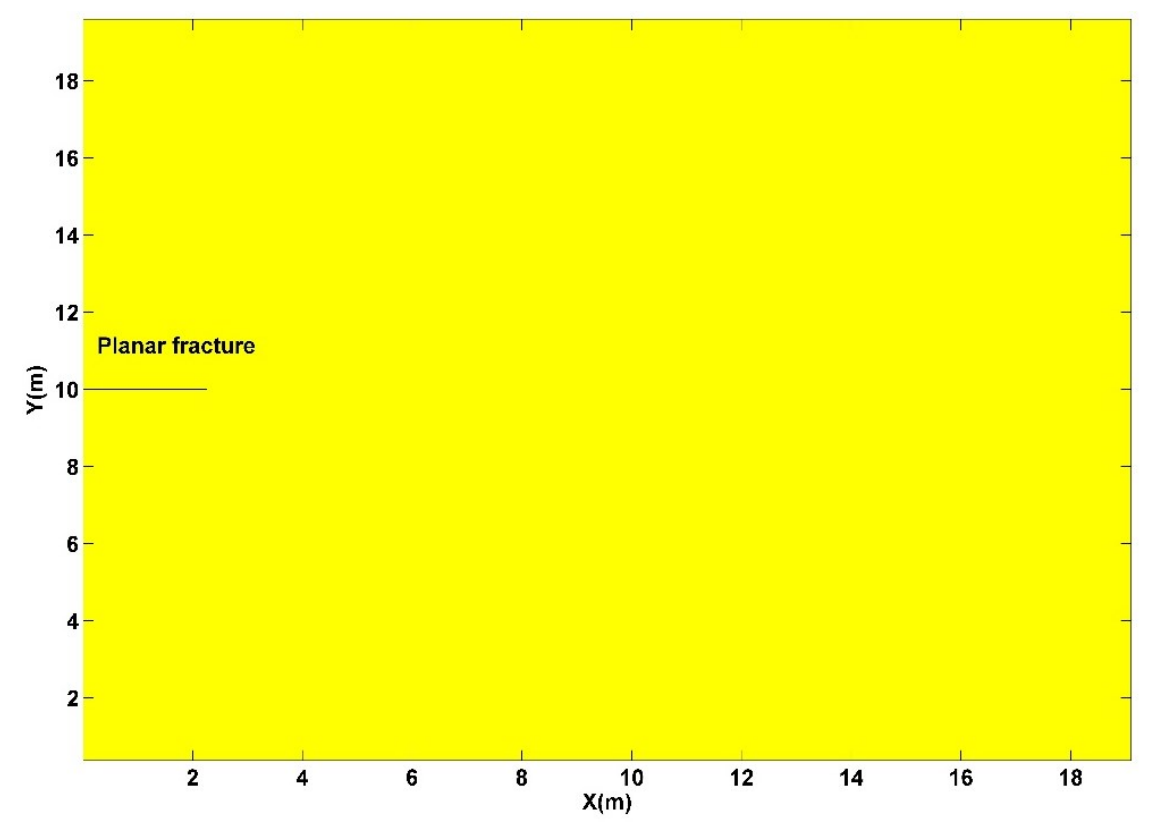

Figure 23 - Schematic of the single-planar hydraulic fracture in the synthetic shale reservoir.
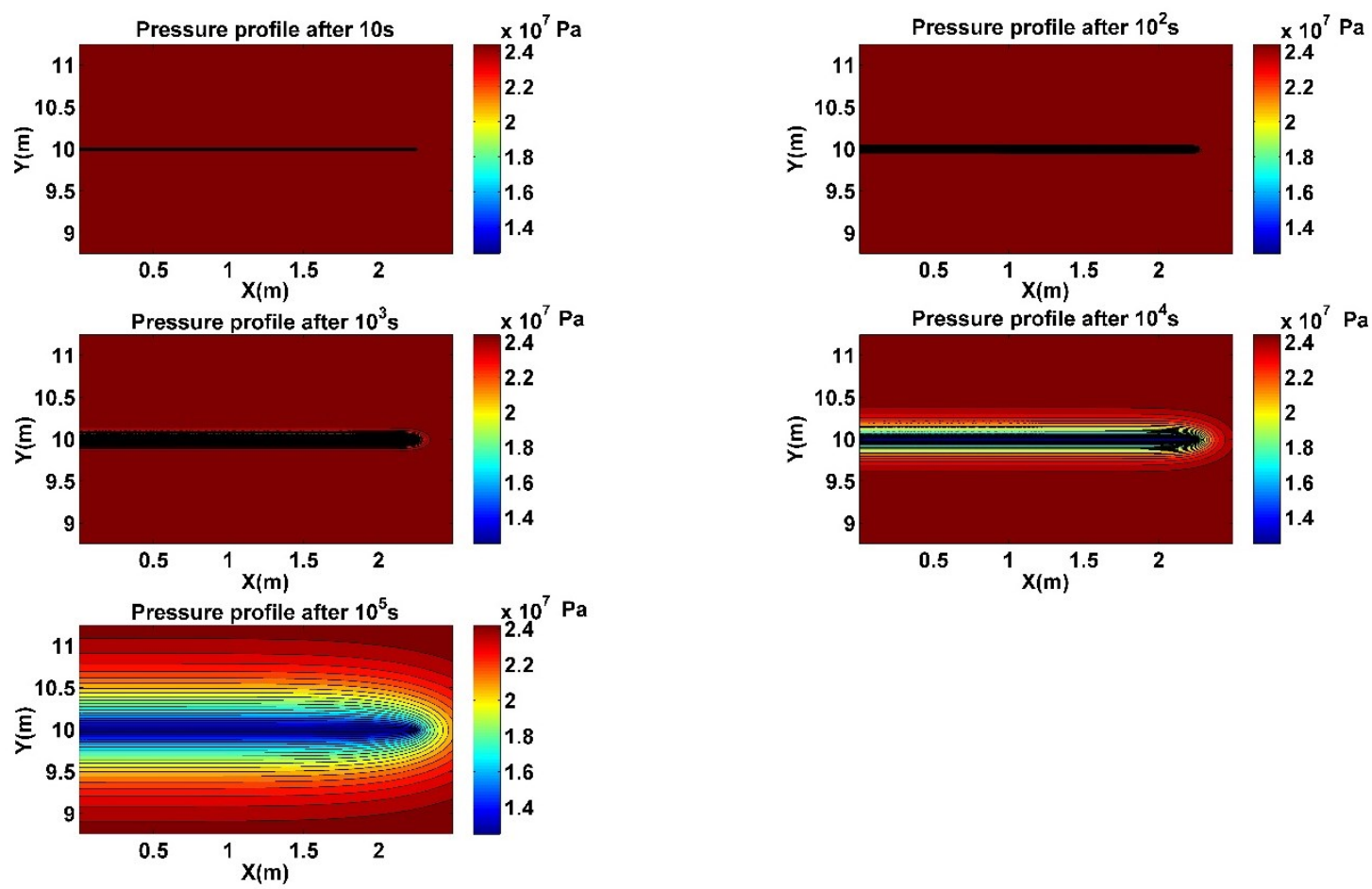

Figure $24-2$-dimensional pressure profile change around a single-planar fracture. 


\section{IV.2.1 Single Planar Fracture Case: Reservoir Pressure Profiles}

The use of pressure maps is a somewhat qualitative exercise, but this effort provides significant insight into the change of the pressure distribution in the reservoir. Our primary goal is to use pressure maps to identify/understand different flow regimes that occur during production. For the case of a single-planar fracture, a series of time-dependent pressure maps are shown in Figure 24. As the single-planar fracture case is well-established in the literature, the flow regimes for this case are well understood. For example, in Figure 24 we note:

- Initial linear flow from the fracture to the wellbore, duration is only a few seconds.

- A transition period that occurs fracture to matrix linear flow as the fracture depletes.

- Linear flow in the matrix commences until fracture interference and/or boundary-dominated flow.

For the convenience of the reader, the pressure maps for all of the stochastic fracture network cases are provided in Appendix D.

\section{IV.2.2 Single Planar Fracture Case: Mass Production Rate and $\beta$-Derivative}

The mass production rate profile for the case of a single-planar fracture is shown in Figure 25. We note that the linear portion of the data on this plot (roughly $50 \mathrm{~s}$ to $100,000 \mathrm{~s}$ ) align to a half-slope straight line $(-1 / 2$ slope on the logarithm of mass production rate versus logarithm of production time). The primary utility of the $\beta$-derivative is that this function "automatically" identifies power-law flow regimes such as linear flow. As shown in Figure 26 the $\beta$-derivative profile confirms the half slope $(\beta$-derivative $=0.5$ ) during the linear flow regime (roughly $50 \mathrm{~s}$ to $100,000 \mathrm{~s}$ ).

As comment, the behavior between $10^{-2} \mathrm{sec}<t<10^{0} \mathrm{sec}$ is an "artifact" of the fracture (or fracture system) unloading (a sort of depletion), and we note the strong influence of this behavior in both Figure 25 (mass production rate profile) and Figure 26 ( $\beta$-derivative profile). This feature is also seen for the stochastic fracture network cases, and (again) is simply seen as an "artifact" of fracture depletion. For reference, other investigations also show this behavior (e.g., Olorode et al. (2013)). 


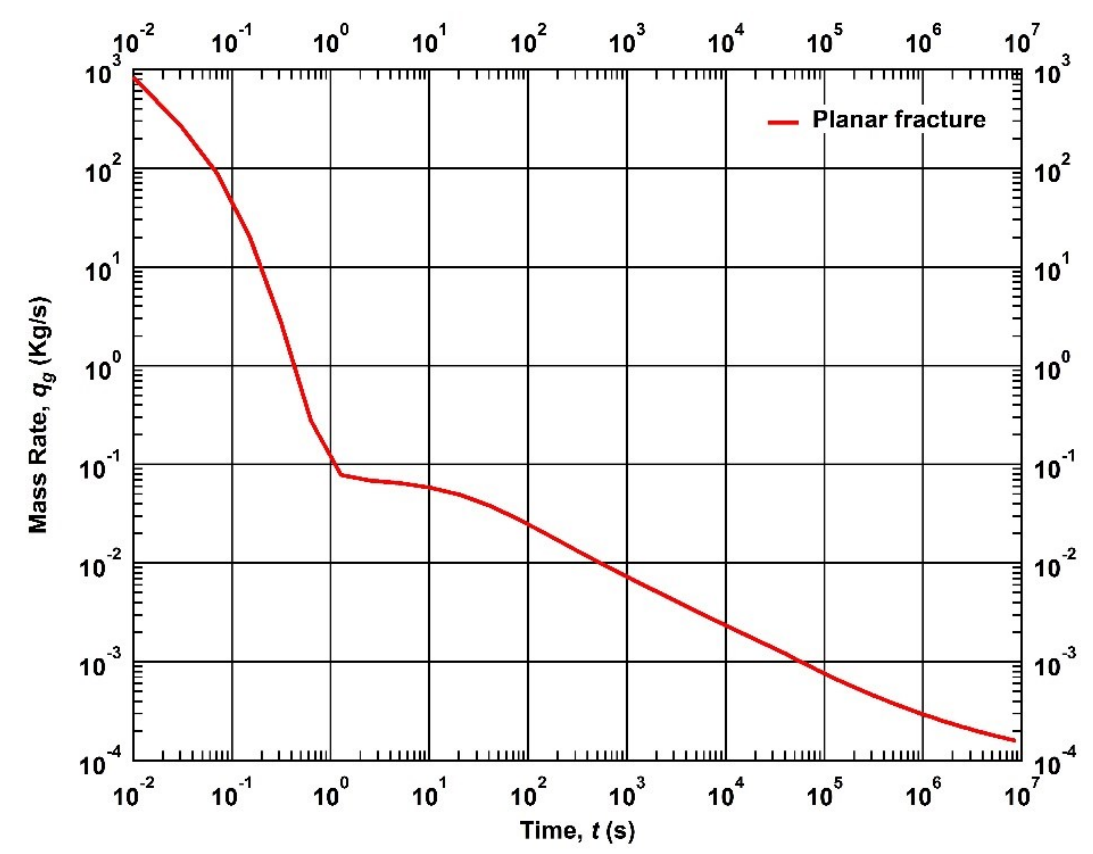

Figure 25 - Mass rate for a synthetic shale gas reservoir produced from a single-planar fracture.

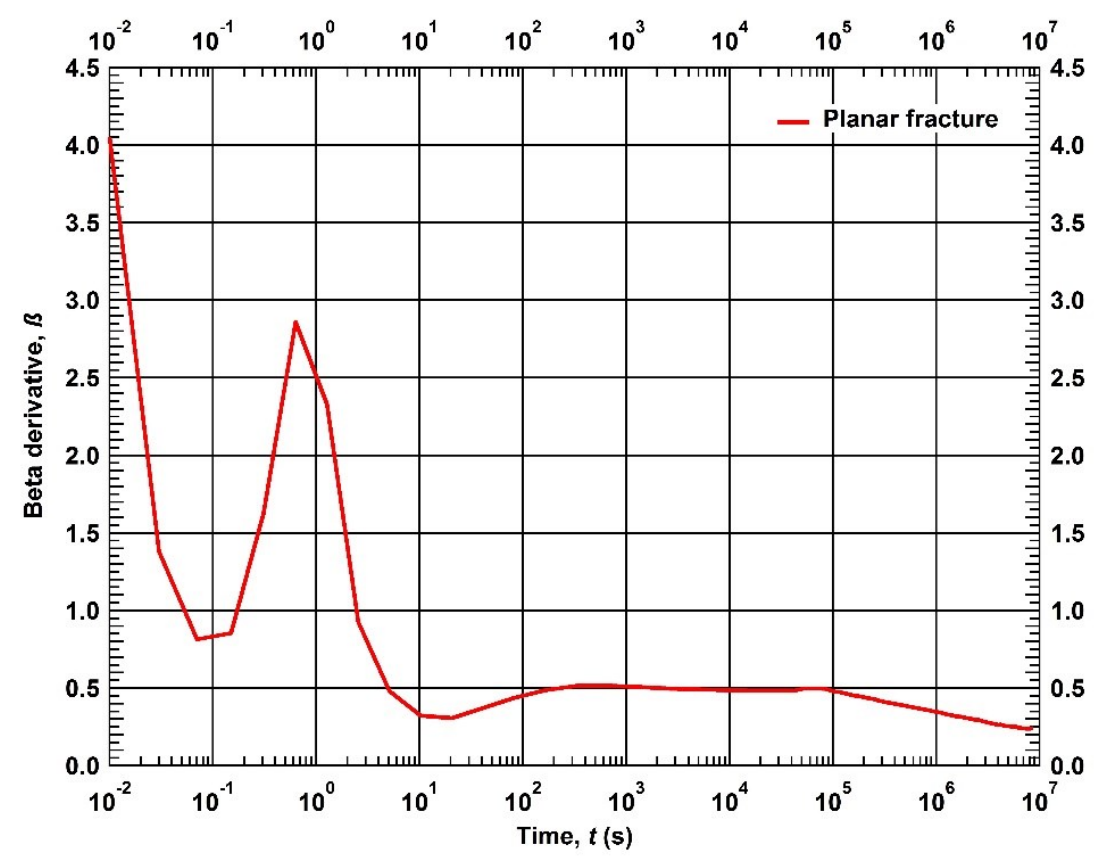

Figure $26-\beta$-derivative of the mass rate for a synthetic shale gas reservoir produced from a single-planar fracture. 


\section{IV.2.3 Single Planar Fracture Case: Cumulative Mass Recovery}

The ratio of cumulative mass production divided by the initial mass of gas in the reservoir is an indicator of the performance of the fracture through time and serves as our "basis" variable for assessing recovery. For the case of the single-planar fracture we note that the fracture only penetrates a portion of the reservoir and that at 100 days the mass recovery is 3.5 percent. As shown in Figure 27, the cumulative mass recovery steadily increases as the reservoir is produced.

Our expectation is that the cumulative mass recovery curve (percent) will vary for different stochastic fracture networks. As mentioned earlier, we do provide a correlation of cumulative mass recovery at 100 days for all cases versus fracture volume - this correlation confirms the expectation of differential recovery for different fracture networks.

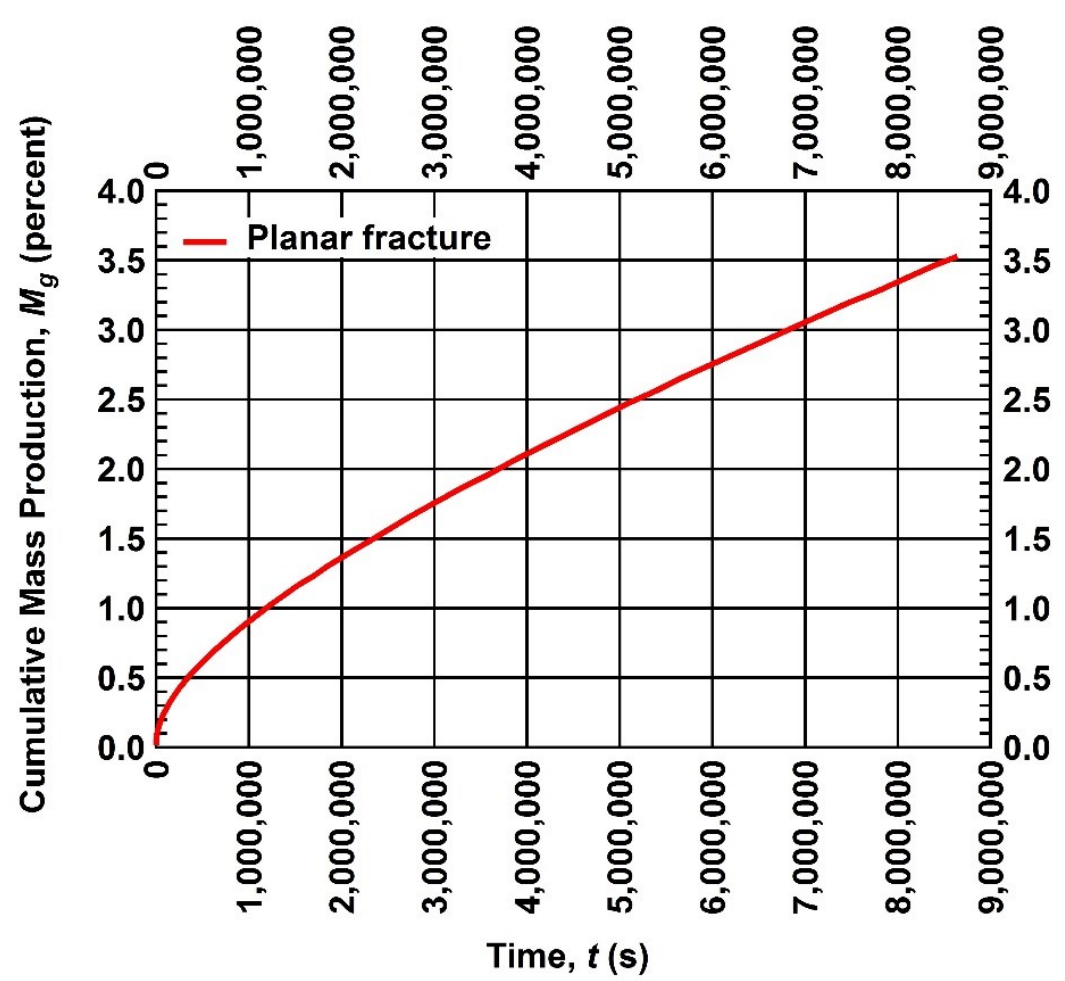

Figure 27 - Cumulative mass production as a fraction of the initial mass in place, synthetic shale gas reservoir produced from a single-planar fracture. 


\section{IV.3 Stochastic Fracture Networks - Non-Branched Fracture Patterns}

A set of 3 different Mono-branched fracture cases are created according to the probabilities provided in

Table 4. These probabilities were selected so that we can observe the effect of the fracture tortuosity ("crookedness") on the flow behavior.

Table 4 - Statistical Parameters for the Non-Branched Fracture Pattern Case.

\begin{tabular}{lccc} 
Parameters & $\boldsymbol{P}_{\boldsymbol{x}+\mathbf{1}}$ & $\boldsymbol{P}_{\boldsymbol{y + 1}}$ & $\boldsymbol{P}_{\boldsymbol{y - 1}}$ \\
\hline Non branched fracture 1 & 0.5 & 0.25 & 0.25 \\
Non branched fracture 2 & 0.3 & 0.35 & 0.35 \\
Non branched fracture 3 & 0.1 & 0.45 & 0.45
\end{tabular}
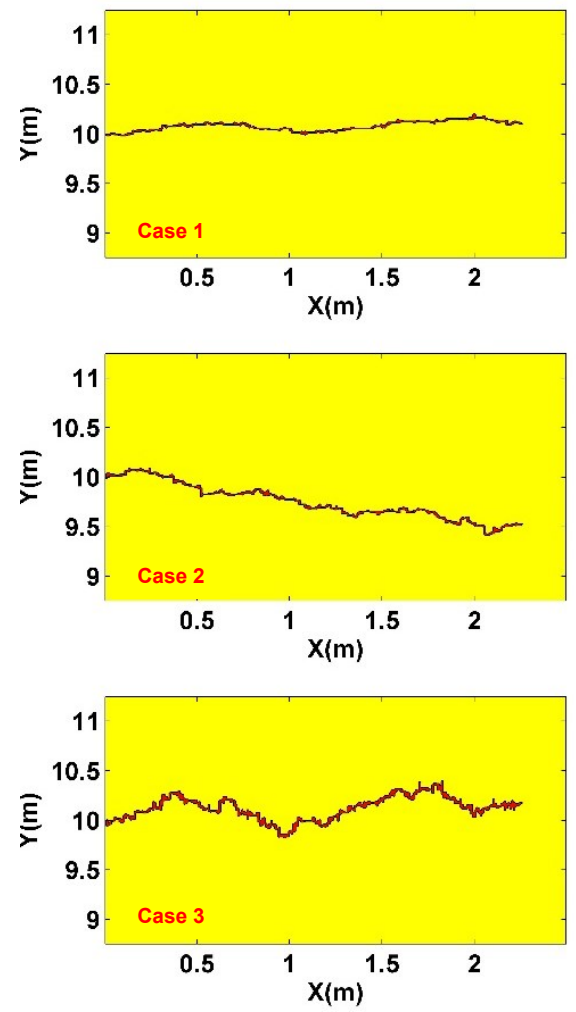

Figure $28-3$ non-branched fracture patterns (cases 1 to 3 ).

These cases presented in Table $\mathbf{4}$ are shown in Figure $\mathbf{2 8}$ and we clearly note the tortuous nature of these fractures compared to the single-planar fracture case. This tortuosity is controlled by the value of $\frac{P_{x+1}}{P_{y+1}+P_{y-1}}$, the higher this ratio is the more likely the fracture will have the tendency to oscillate in the y- 
direction and increase the fracture tortuosity. The length of the fractures (along the $x$-direction) has been constrained to be the same as for the plane fracture case $x_{f}=2.25 \mathrm{~m}$.

The performance of these patterns is analyzed and compared to the plane fracture case. Figure 29 shows that the tortuous and more voluminous fractures exhibit an early time linear fracture flow $\left(t<10^{-2} \mathrm{~s}\right)$. After the "fracture depletion" transition zone the trends converge toward the linear flow (half-slope) trend typically observed for the single-planar fracture case. In Figure 30 we confirm that the $\beta$-derivative function exhibits a near-constant value (approximately 0.5 ) during the apparent linear flow period.

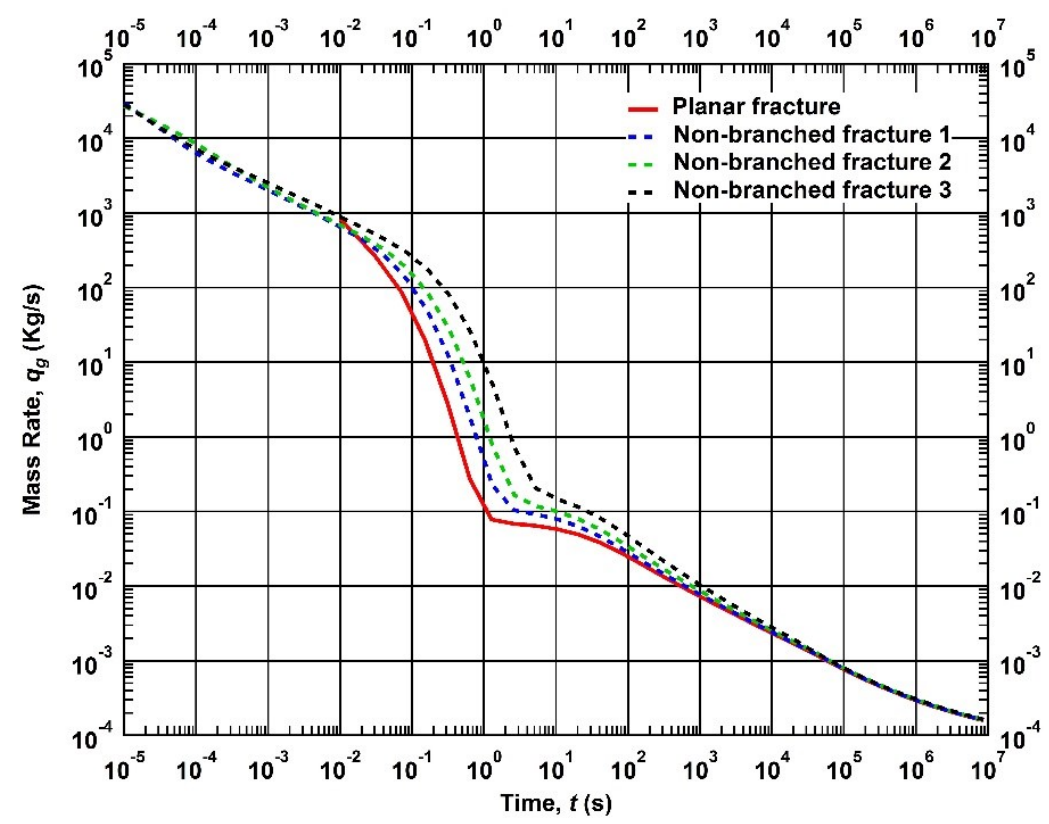

Figure 29 - Mass rate for a synthetic shale gas reservoir produced from 3 non-branched fracture patterns and compared to the planar fracture case.

The cumulative mass recovery comparison of the different non-branched fracture patterns and the singleplanar fracture pattern is shown in Figure 31, and (as expected) the most tortuous fracture pattern is the most productive case. A differential increase of mass recovery of approximately 0.3 percent is observed at the end of the production period (i.e., 100 days). As an observation, we note that the behavior of the nonbranched stochastic fracture cases essentially converge to the single-planar fracture case after one day of production (approximately 86,400 s). 


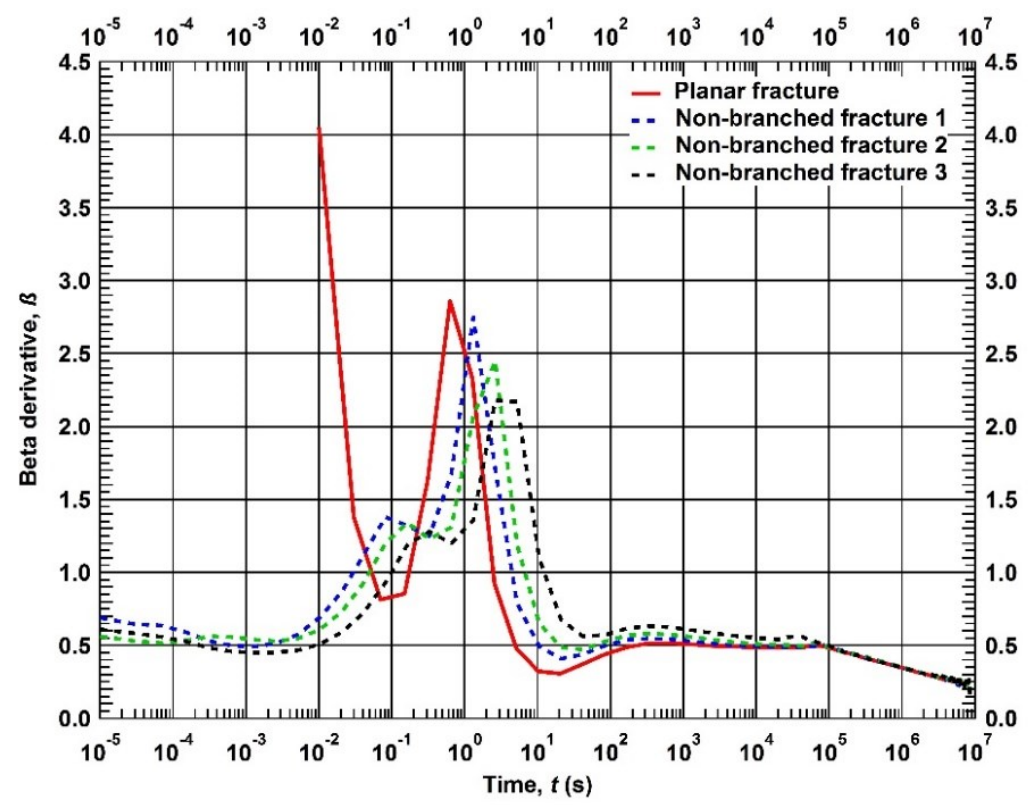

Figure $30-\beta$-derivative of the mass rate for a synthetic shale gas reservoir produced from 3 nonbranched fracture patterns and compared to the planar fracture case.

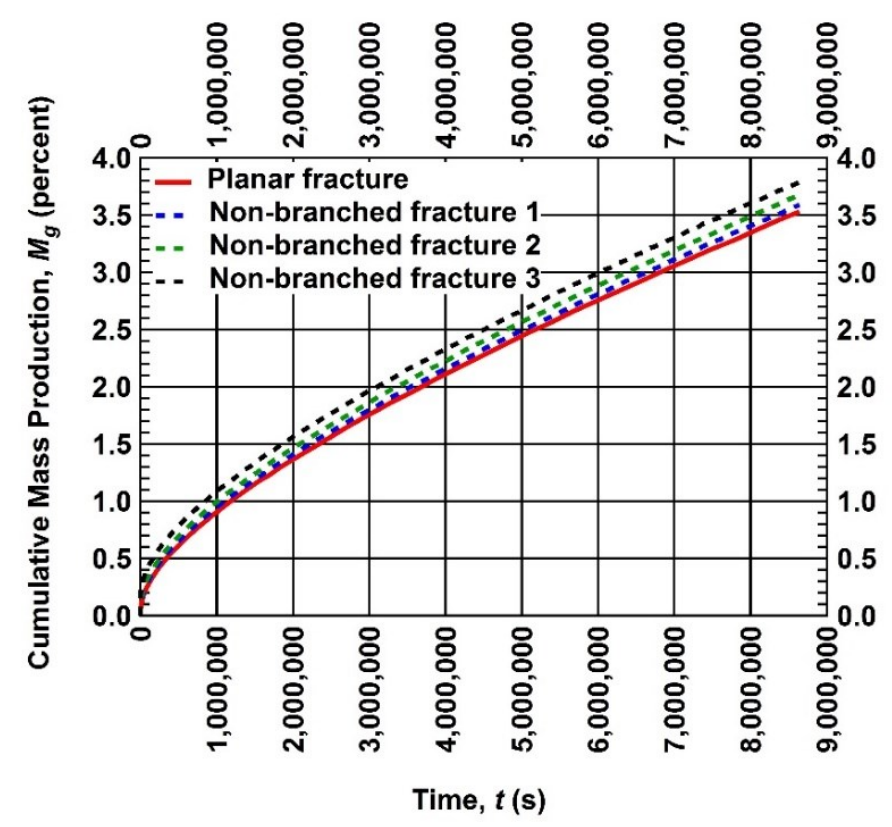

Figure 31 - Cumulative mass production recovery for a shale gas reservoir produced from 3 nonbranched fracture patterns and compared to the planar fracture case. 


\section{IV.4 Stochastic Fracture Networks - Mono-Branched Fracture Patterns}

Table 5 - Statistical Parameters for the Mono-Branched Fracture Pattern Case.

\begin{tabular}{|c|c|c|c|c|c|c|c|c|c|}
\hline \multirow{2}{*}{ Parameters } & \multicolumn{3}{|c|}{$F_{0}$} & \multicolumn{3}{|c|}{$F_{01}$} & \multicolumn{3}{|c|}{$F_{02}$} \\
\hline & $P_{x+1}$ & $P_{y+1}$ & $P_{y-1}$ & $P_{x+1}$ & $P_{y+1}$ & $P_{y-1}$ & $\boldsymbol{P}_{x+1}$ & $P_{y+1}$ & $P_{y-1}$ \\
\hline Mono-branched fracture 1 & 0.5 & 0.25 & 0.25 & 0.5 & 0.25 & 0.25 & 0.5 & 0.25 & 0.25 \\
\hline Mono-branched fracture 2 & 0.5 & 0.25 & 0.25 & 0.5 & 0.35 & 0.15 & 0.5 & 0.15 & 0.35 \\
\hline Mono-branched fracture 3 & 0.5 & 0.25 & 0.25 & 0.5 & 0.35 & 0.15 & 0.5 & 0.15 & 0.35 \\
\hline Mono-branched fracture 4 & 0.5 & 0.25 & 0.25 & 0.5 & 0.45 & 0.05 & 0.5 & 0.05 & 0.45 \\
\hline Mono-branched fracture 5 & 0.5 & 0.25 & 0.25 & 0.4 & 0.3 & 0.3 & 0.4 & 0.3 & 0.3 \\
\hline
\end{tabular}

A set of 5 different Mono-branched fractures is created following the probabilities described in Table 5. The probabilities were selected so that we can analyze the effect the bifurcation occurrence (extent of $F_{0}$ ); and also the distance between the two sub-fractures $F_{01}$ and $F_{02}$ which is controlled by the ratio $\frac{P_{y+1}}{P_{y-1}}$.

The generated mono-branched patterns are shown in Figure 32, we can notice that the first case has a very low opening between the two sub-fractures $F_{01}$ and $F_{02}$; this opening is progressively increased in the other patterns (case 2, case 3 and case 4). Additionally, the occurrence of the bifurcation on the case 2 pattern is delayed compared to the other patterns. 

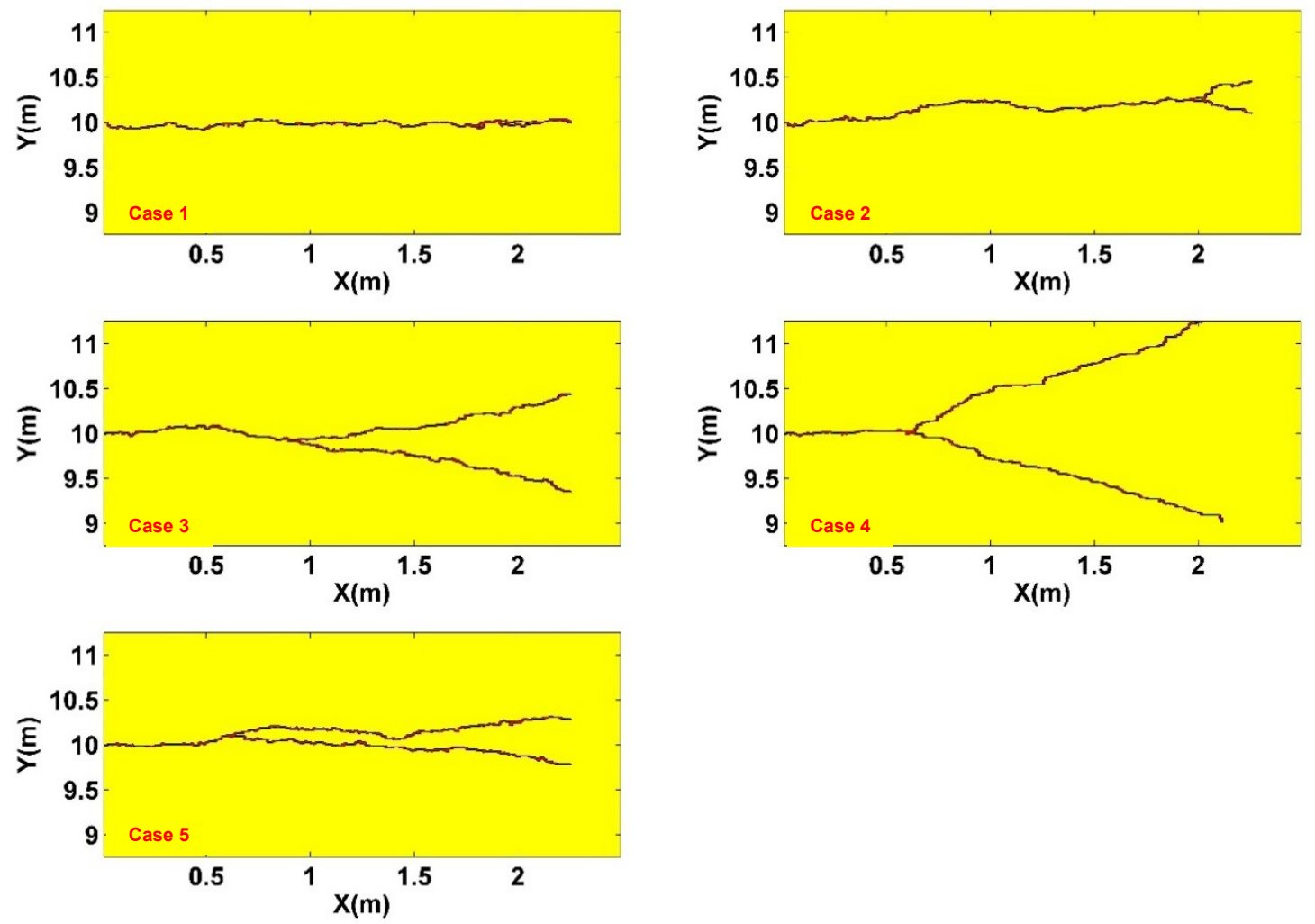

Figure 32 - 5 mono-branched fracture patterns (cases 1 to 5 ).

The shapes variation between the mono-branched fracture aims to capture the effect of different geometric settings of the fractures. The pressure distributions for each of the simulated patterns are joined in the Appendix $\mathrm{D}$ of this thesis. They allow us to identify the period of flow where the two sub-fractures $F_{01}$ and $F_{02}$ start to exhibit an effect on the mass decline rate. The analysis of the pressure distribution shows that the interferences between different sub-fractures occur before $10^{5} \mathrm{~s}$ of production $\left(t<10^{5} \mathrm{~s}\right)$. The diagnosis of the mass production curve during that particular period will be critical for the branchdependent characterization of the mass decline rate curves. From Figure 33 we can notice that between $50 s$ and $10^{5} s$ (approximately) the mass production rate curve exhibits a linear behavior with a slope slightly superior to the planar fracture case; Figure 34 confirms this observation as the $\beta$-derivative has a constant value higher on average than the 0.5 value of the planar fracture. 


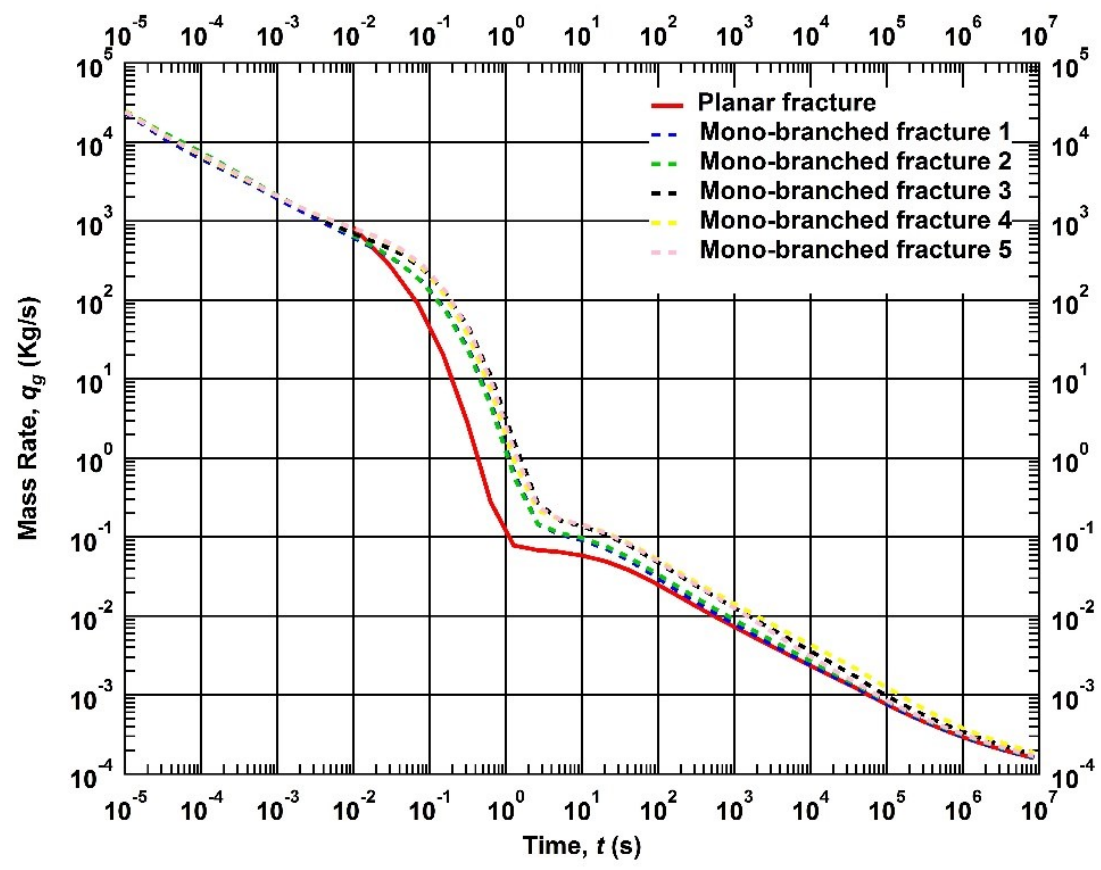

Figure 33 - Mass rate for a synthetic shale gas reservoir produced from 5 mono-branched fracture patterns and compared to the planar fracture case.

The mono-branched fracture of case 1 and 2 seems to have a similar performance as the plane fracture after a relatively short time of production of around $50 \mathrm{~s}$. This effect is due to the fact that the first case pattern has its two sub-fractures $F_{01}$ and $F_{02}$ very close so that they almost behave as a single fracture; it can be somehow "seen" as a non-branched fracture pattern. The mono-branched fracture of case 2 has very limited two sub-fractures, their effect is barely noticed and their interferences occur rather quickly. However, the mono-branched fracture of case 3 has an earlier occurrence of the fracture split and the interferences effect seem to take place for a longer time according to the mass production rate curve of Figure 33 . 


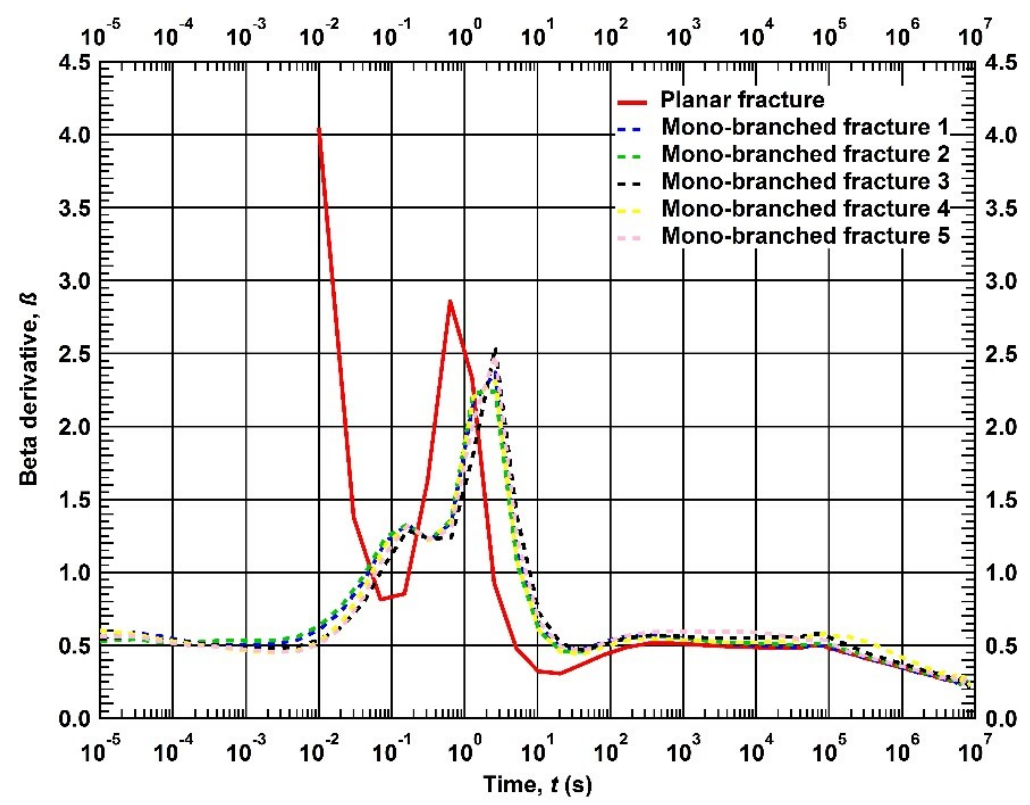

Figure $34-\beta$-derivative of the mass rate for a synthetic shale gas reservoir produced from 5 mono-branched fracture patterns and compared to the planar fracture case.

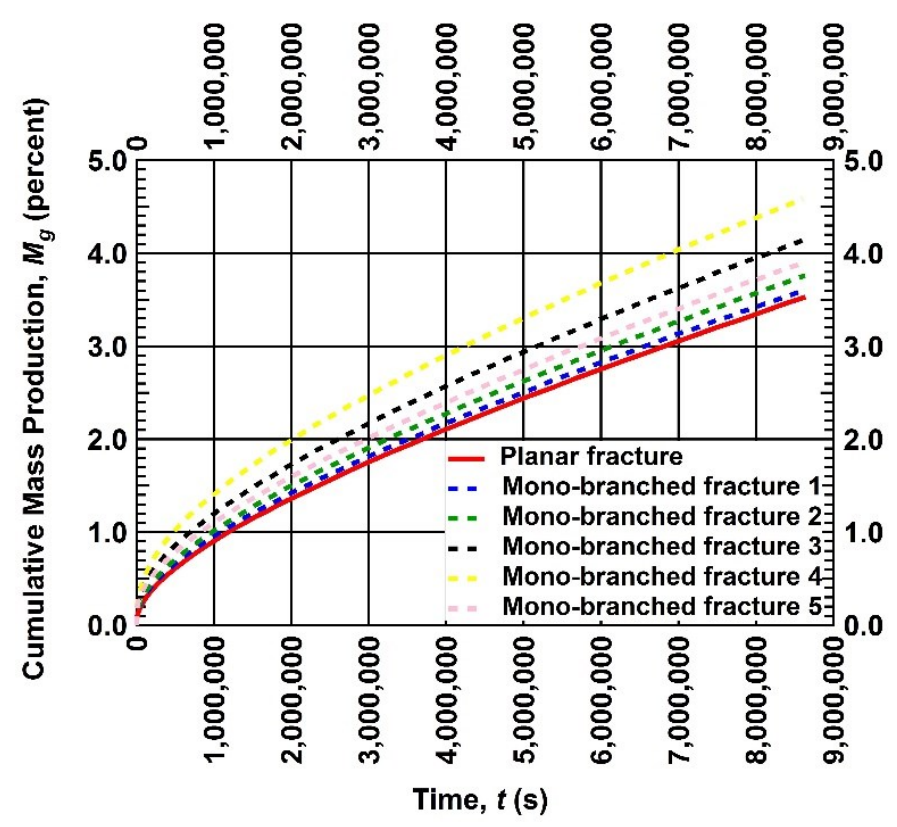

Figure 35 - Cumulative mass production recovery for a shale gas reservoir produced from 5 mono-branched fracture patterns and compared to the planar fracture case. 
Figure 34 gives a more detailed insights on the variations of the mass production rate curve for the monobranched fracture patterns. Apart from the patterns 1 and 2 that converge rather quickly toward a linear half-slope behavior; the mono-branched fracture patterns have a $\beta$-derivative slightly higher on average than 0.5 during the production period where the interferences take place (approximately between $10^{2} s$ and $10^{5} \mathrm{~s}$ ). The interferences are confirmed through the analysis of the pressure change maps which are provided in Appendix D.

The cumulative recovery plots of Figure 35 shows a significant increase in the performance of the different patterns compared to the planar hydraulic fracture. The $4^{\text {th }}$ case pattern has 1 percent more produced gas by the end of the production period which is around 30 percent more than the planar fracture recovery. This $4^{\text {th }}$ pattern covers more area than all the simulated mono-branched patterns because of the opening of its sub-fractures according to Figure 32.

We can also observe that the first mono-branched pattern is very close to the planar fracture case as its two sub-fractures remain very close; it somehow behaves like a non-branched pattern. The second pattern is also "poorly" performing because of the limited extent of its sub-fractures. This might partly explain the fact that the $1^{\text {st }}$ and the $2^{\text {nd }}$ mono-branched patterns have mass production rate curves that are close to the single-plane fracture mass production rate curve. In both cases, the interference effect is too small to be observed macroscopically on the production indicator plots.

\section{IV.5 Stochastic Fracture Networks - Dual-Branched Fracture Patterns}

Table 6 - Statistical Parameters for the Dual-Branched Fracture Pattern Case.

\begin{tabular}{lccccccccc} 
Parameter & \multicolumn{3}{c}{$\boldsymbol{F}_{\mathbf{0}}$} & \multicolumn{4}{c}{$\boldsymbol{F}_{\mathbf{0 1}} \boldsymbol{F}_{\mathbf{0 1 1}}, \boldsymbol{F}_{\mathbf{0 2 1}}$} & \multicolumn{3}{c}{$\boldsymbol{F}_{\mathbf{0 2}}, \boldsymbol{F}_{\mathbf{0 1 2}}, \boldsymbol{F}_{\mathbf{0 2 2}}$} \\
& $\boldsymbol{P}_{\boldsymbol{x}+\mathbf{1}}$ & $\boldsymbol{P}_{\boldsymbol{y + 1}}$ & $\boldsymbol{P}_{\boldsymbol{y - 1}}$ & $\boldsymbol{P}_{\boldsymbol{x + 1}}$ & $\boldsymbol{P}_{\boldsymbol{y}+\mathbf{1}}$ & $\boldsymbol{P}_{\boldsymbol{y - 1}}$ & $\boldsymbol{P}_{\boldsymbol{x + 1}}$ & $\boldsymbol{P}_{\boldsymbol{y + 1}}$ & $\boldsymbol{P}_{\boldsymbol{y}-\mathbf{1}}$ \\
\hline Dual-branched fracture 1 & 0.5 & 0.25 & 0.25 & 0.4 & 0.4 & 0.2 & 0.4 & 0.2 & 0.4 \\
Dual -branched fracture 2 & 0.5 & 0.25 & 0.25 & 0.4 & 0.4 & 0.2 & 0.4 & 0.2 & 0.4 \\
Dual -branched fracture 3 & 0.5 & 0.25 & 0.25 & 0.4 & 0.4 & 0.2 & 0.4 & 0.2 & 0.4 \\
Dual -branched fracture 4 & 0.5 & 0.25 & 0.25 & 0.4 & 0.4 & 0.2 & 0.4 & 0.2 & 0.4 \\
Dual -branched fracture 5 & 0.5 & 0.25 & 0.25 & 0.4 & 0.4 & 0.2 & 0.4 & 0.2 & 0.4
\end{tabular}

Similarly to the mono-fractured pattern cases, we generated 5 different patterns of fractures that have two stages of bifurcations. Table 6 summarizes the parameters that are used to constrain those fractures. Basically, there are no variations between the different patterns; the effects that will be observed are due to the differences between different realizations of the same constrained structure. Additionally to the analysis of the effect of tortuosity, the branches spacing and the placement of the bifurcation we want to analyze the effect that the number of bifurcations stages or branches has on the production. 
Figure 36 shows the different patterns that are investigated in this paragraph. We can notice that the length of each sub-fracture is totally random; however the fracture is constrained to remain in the gridrefined reservoir area. The pressure profile change of different patterns in the Appendix D show that the effect of fracture interferences seems to be occurring between $10 \mathrm{~s}$ and $10^{5} \mathrm{~s}$. That particular period is analyzed on the mass production rate and $\beta$-derivative curves. A particular signature starts to appear in the mass production rate curve of Figure 37. In fact, the half-slope linear behavior tends to become a higher degree linear slope. The $\beta$-derivative curve is slightly higher than 0.5 , and it seems to be considerably higher than the derivatives for the mono-branched fracture patterns. This effect shall be studied later in further depth with more simulated patterns.
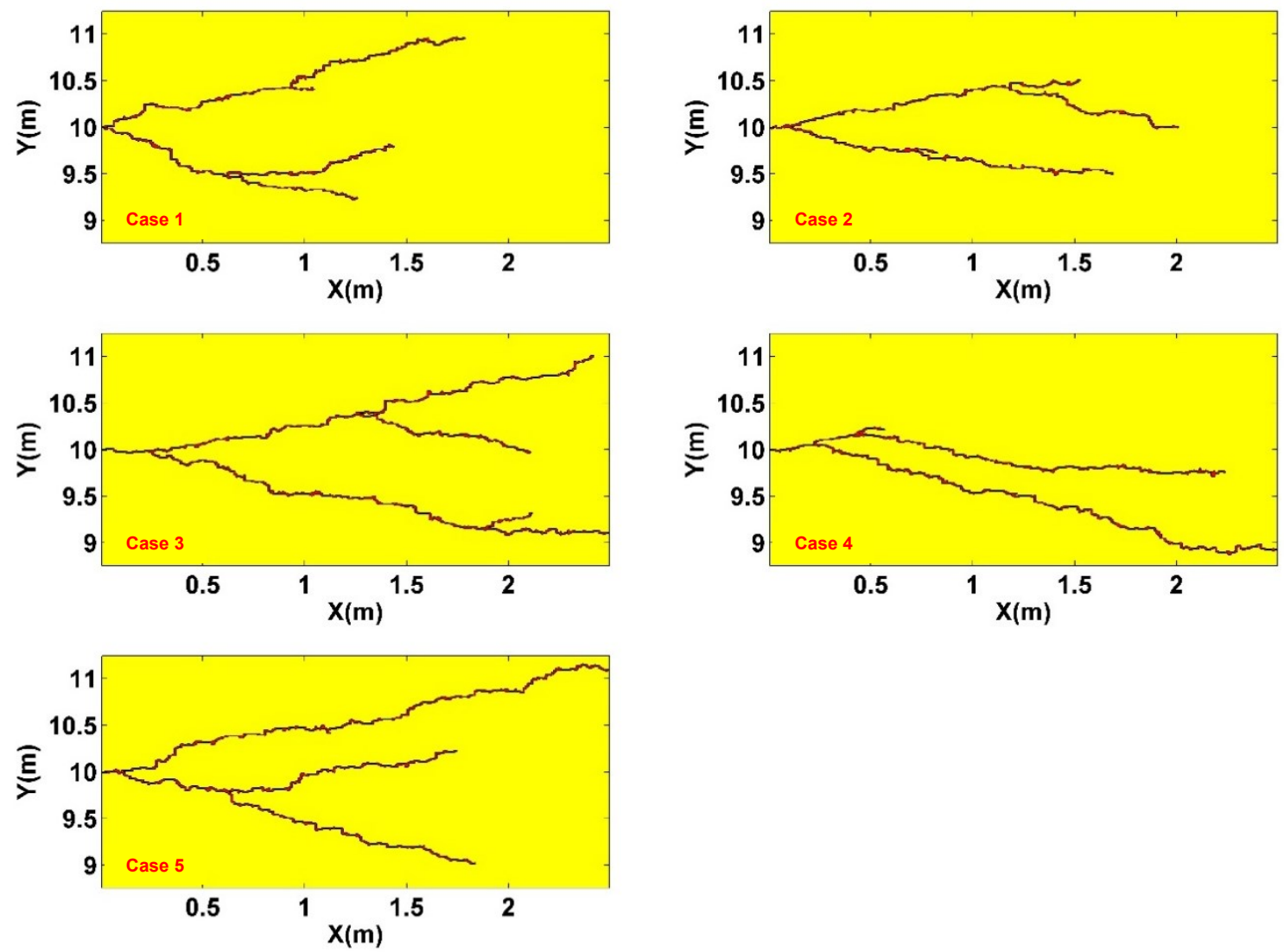

Figure $36-5$ dual-branched fracture patterns (cases 1 to 5 ). 


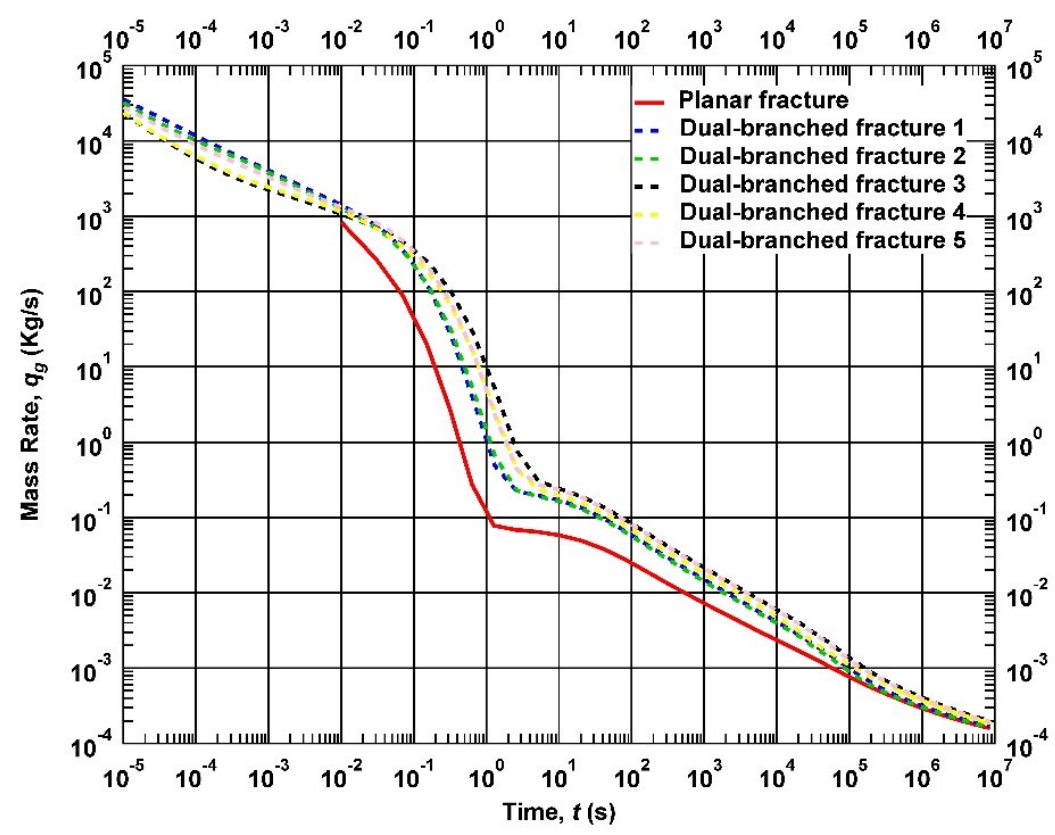

Figure 37 - Mass rate for a synthetic shale gas reservoir produced from 5 dual-branched fracture patterns and compared to the planar fracture case.

The dual-branched pattern mass production rate curves of Figure 37 exhibit a behavior that is similar to the plane fracture after $10^{5} \mathrm{~s}$ of production. According to the pressure change maps of Appendix $\mathrm{D}$, the interferences between the branches seem to extend until $10^{5} \mathrm{~s}$. This observation means that the mass production rate behavior characterized by a higher slope between $10^{2} \mathrm{~s}$ and $10^{5} \mathrm{~s}$ than for the planar fracture case is due to the interferences between the branches. To characterize the mass production rate, the $\beta$ derivative plot of Figure 38 gives a value of the derivative that is higher on average for the dual-branched patterns than the planar fracture and also the mono-branched patterns. As the dual-branched fracture pattern, extends over a larger area the cumulative production from those fracture is significantly higher than the one of a planar fracture. The recovery at the end of the production period is up to 40 percent higher for the $3^{\text {rd }}$ dual-branched pattern. This tendency can somehow be understood as the $3^{\text {rd }}$ pattern seems to extend over more area in the fractured volume Figure 36. Similarly to the mono-branched fractures, the best performer between the simulated patterns is the fracture that is spread over the most volume of the reservoir. The dual-branched pattern 1 and 2 are the worst performers because they cover a significantly lower area than the other dual-branched patterns. While comparing the performance of the mono-branched patterns and the dual branched patterns in Figure 35 and Figure 39 we can already notice that a superior number of branched is not necessarily a guarantee of a better performance. The 
performance of the fractures seems rather to be controlled by the efficient distribution of the pattern over the fractured area.

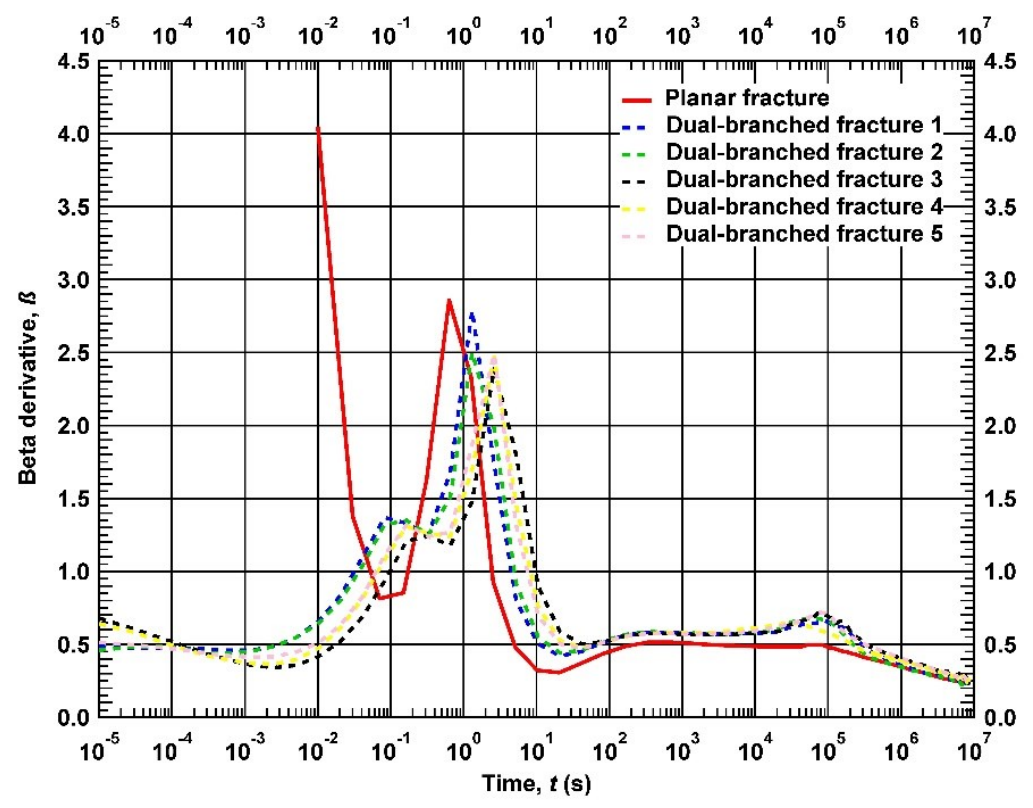

Figure $38-\beta$-derivative of the mass rate for a synthetic shale gas reservoir produced from 5 dualbranched fracture patterns and compared to the planar fracture case. 


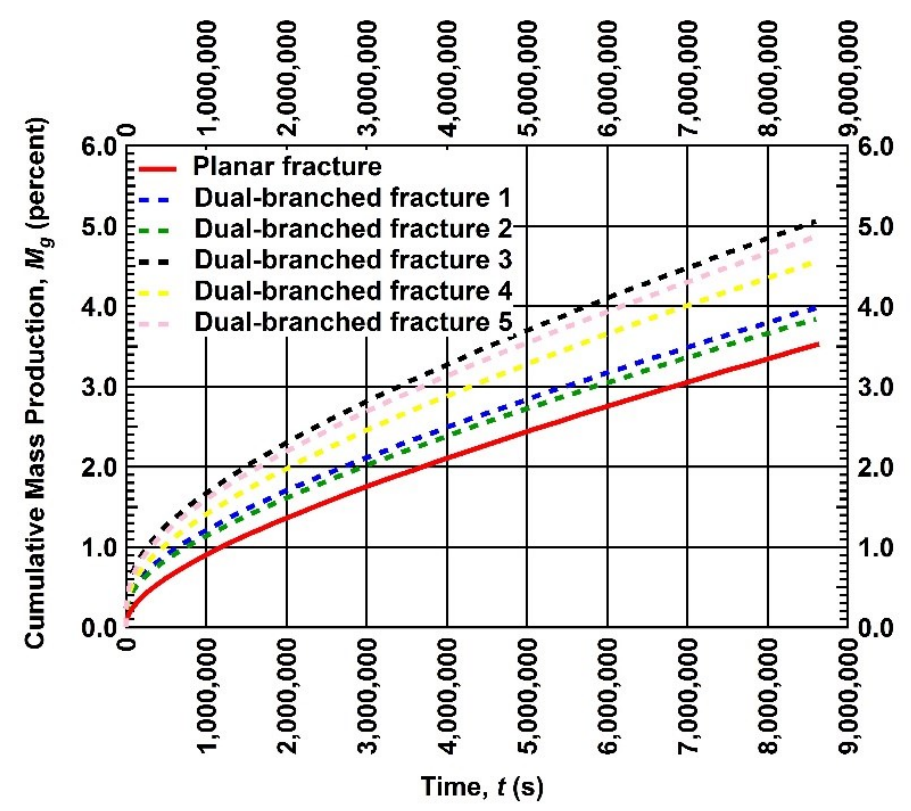

Figure 39 - Cumulative mass production recovery for a shale gas reservoir produced from 5 dualbranched fracture patterns and compared to the planar fracture case.

\section{IV.6 Stochastic Fracture Networks - Tri-Branched Fracture Patterns}

Table 7 - Statistical Parameters for the Tri-Branched Fracture Pattern Case.

\begin{tabular}{|c|c|c|c|c|c|c|c|c|c|}
\hline \multirow[t]{2}{*}{ Parameters } & \multicolumn{3}{|c|}{$F_{0}$} & \multicolumn{3}{|c|}{$\begin{array}{c}F_{01}, F_{011}, F_{021} \\
F_{0111}, F_{0121}, F_{0221}, F_{0211}\end{array}$} & \multicolumn{3}{|c|}{$\begin{array}{c}F_{02}, F_{012}, F_{022} \\
F_{0112}, F_{0122}, F_{0212}, F_{0222}\end{array}$} \\
\hline & $P_{x+1}$ & $P_{y+1}$ & $P_{y-1}$ & $P_{x+1}$ & $P_{y+1}$ & $P_{y-1}$ & $P_{x+1}$ & $P_{y+1}$ & $P_{y-1}$ \\
\hline Tri-branched fracture 1 & 0.5 & 0.25 & 0.25 & 0.4 & 0.4 & 0.2 & 0.4 & 0.2 & 0.4 \\
\hline Tri -branched fracture 2 & 0.5 & 0.25 & 0.25 & 0.4 & 0.4 & 0.2 & 0.4 & 0.2 & 0.4 \\
\hline Tri -branched fracture 3 & 0.5 & 0.25 & 0.25 & 0.4 & 0.4 & 0.2 & 0.4 & 0.2 & 0.4 \\
\hline Tri -branched fracture 4 & 0.5 & 0.25 & 0.25 & 0.4 & 0.4 & 0.2 & 0.4 & 0.2 & 0.4 \\
\hline Tri -branched fracture 5 & 0.5 & 0.25 & 0.25 & 0.4 & 0.4 & 0.2 & 0.4 & 0.2 & 0.4 \\
\hline
\end{tabular}

5 different tri-branched fracture patterns are generated in this section. The obtained 5 structures are displayed in Figure 40. The reader can notice that the tri-branched pattern have a high enough number of branches that makes it more probable to have an intersection between different branches in a limited area.

The same generation probabilities were used to generate those different patterns Table 7. The impact of the presence of 3 stages of branching starts is confirming the observation that we saw in the previous paragraph. 

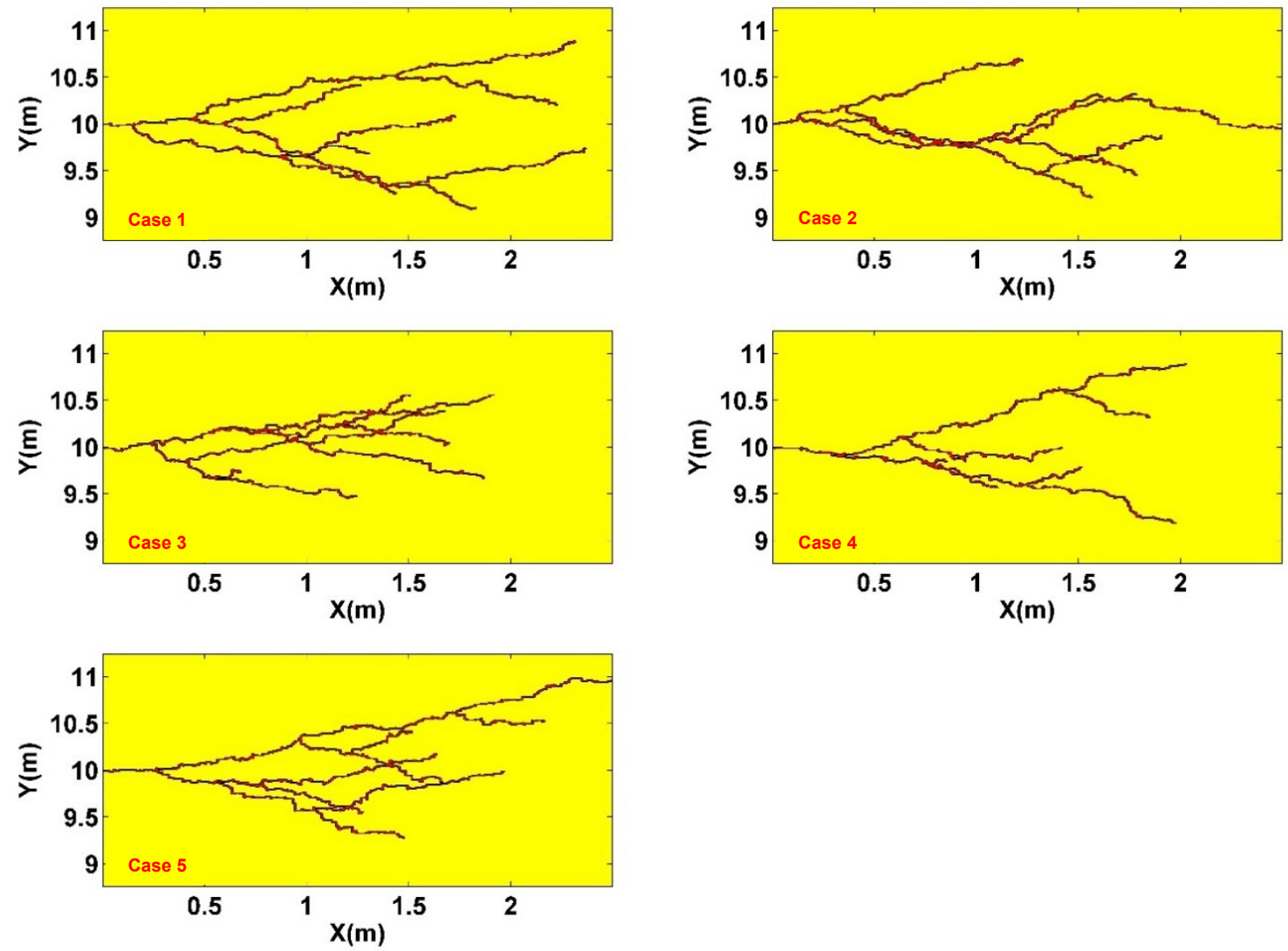

Figure 40 - 5 tri-branched fracture patterns (cases 1 to 5 ).

The investigation of the production curves of Figure 41 and Figure 42 shows that between $10^{2} s$ and $10^{5} \mathrm{~s}$ the flow exhibits a linear behavior with a similar slope for different tri-branched patterns. This period of flow occurs when fractures are interfering (according to pressure profiles of Appendix D). The fractures are somehow defining "channels" which edges produce at a constant pressure, the $\beta$-derivative is therefore higher than for a planar fracture and for lower degree branched fracture that contains less "channels". 


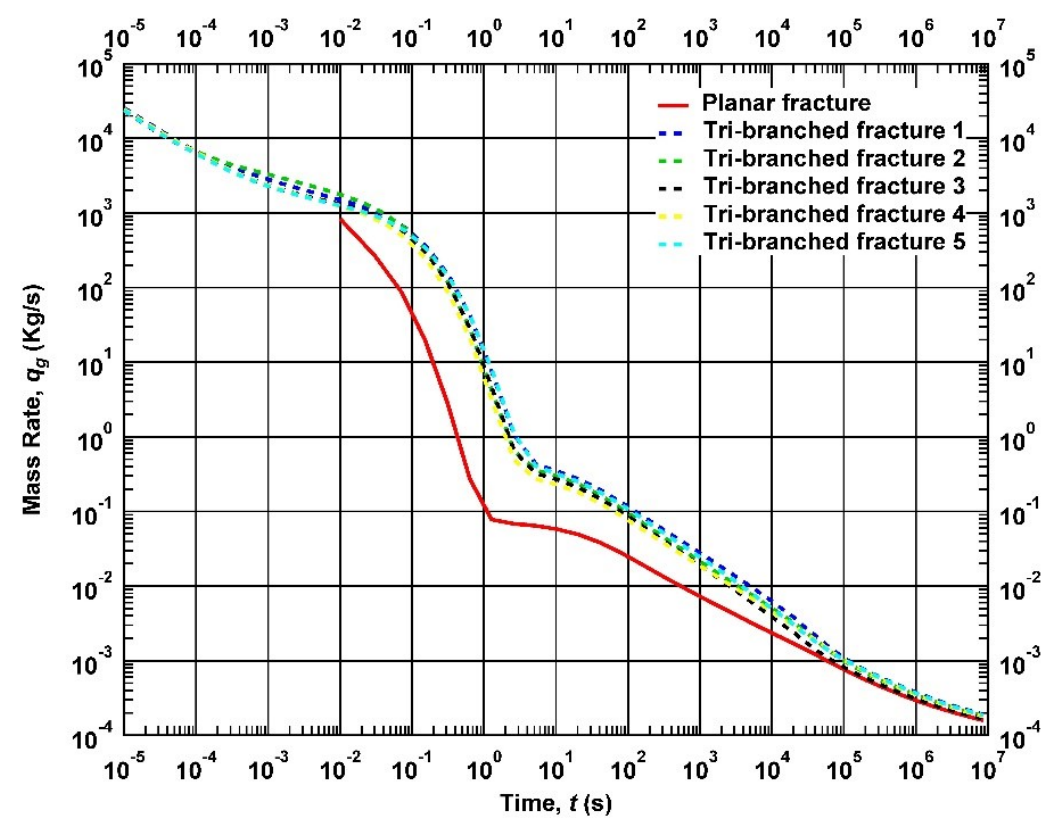

Figure 41 - Mass rate for a synthetic shale gas reservoir produced from 5 tri-branched fracture patterns and compared to the planar fracture case.

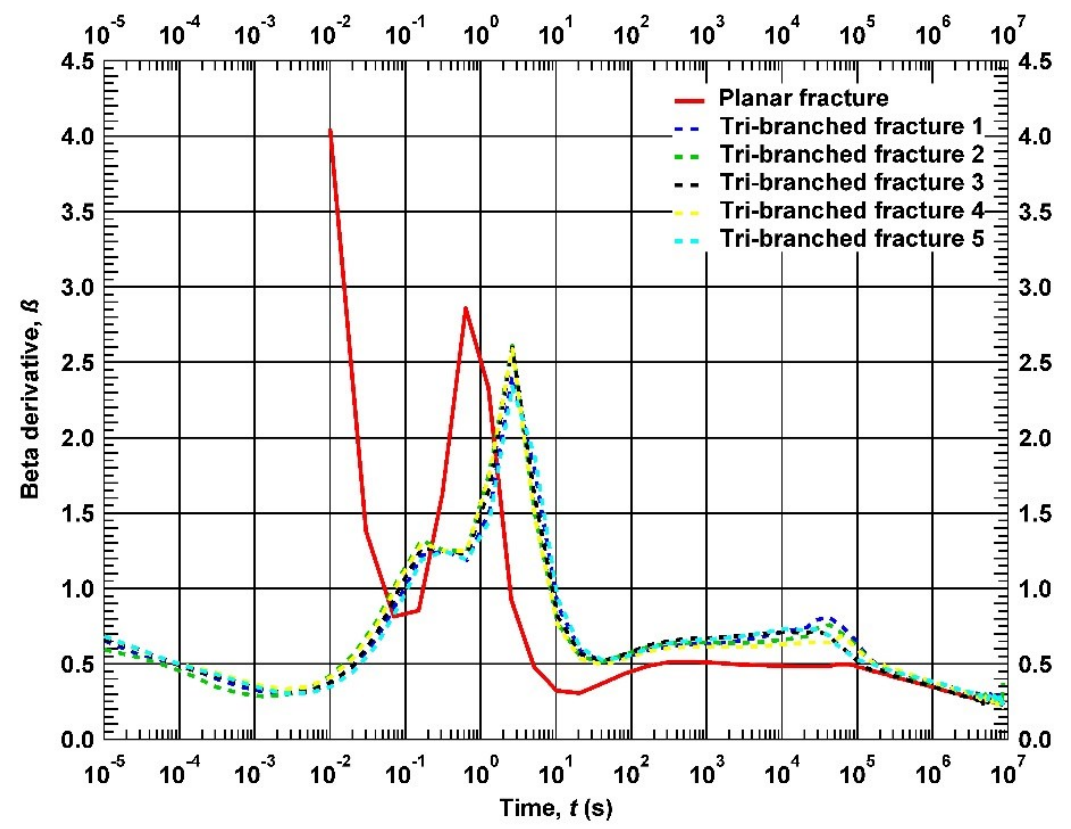

Figure $42-\beta$-derivative of the mass rate for a synthetic shale gas reservoir produced from 5 tribranched fracture patterns and compared to the planar fracture case. 
Even though the simulated tri-branched patterns are quite geometrically different from each other, the mass production rate curves of Figure 41 display a similar behavior between $10^{2} \mathrm{~s}$ and $10^{5} \mathrm{~s}$. Those interferences effect which have also been observed for a lower number of branches could be characterized by the slope of the mass production rate curve between $10^{2} \mathrm{~s}$ and $10^{5} \mathrm{~s}$, this slope value, being 0.5 for the planar fracture, seems to be significantly higher for the simulated set of tri-branched fractures. On Figure 42, the $\beta$-derivative for the tri-branched pattern is 0.65 on average.

Although they are performing better than the plane fracture case, the tri-branched fracture patterns are not notably better than the dual-branched patterns Figure 43; which is possibly due to the distribution of the patterns in the fractured area. The best performer between the 5 simulated tri-branched patterns is the first pattern which is more efficiently distributed over the fractured area of the shale reservoir. Eventually, with a higher number of simulations the tri-branched patterns are expected to behave better on average than the lower-branched fractures. However, the effect of the number of branches is clearly impacting the mass production rate curve as we observe a $\beta$-derivative value of 0.65 that is notably higher than the dualbranched pattern cases.

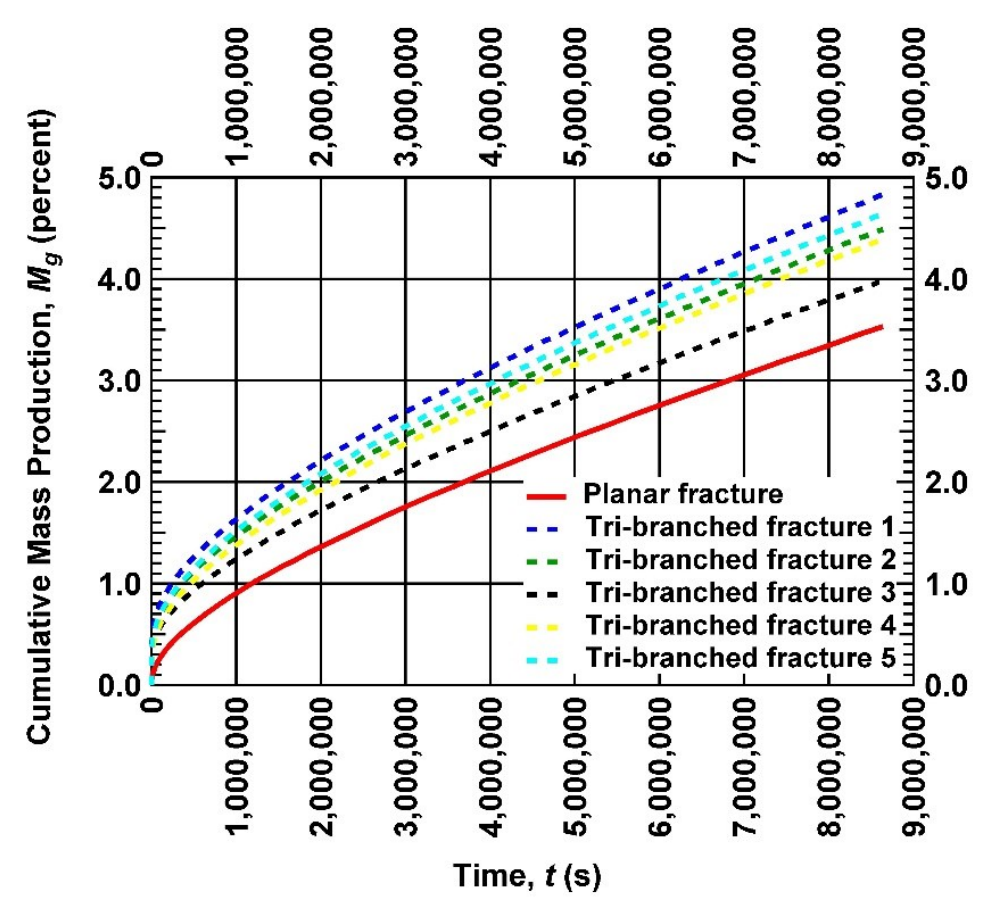

Figure 43 - Cumulative mass production recovery for a shale gas reservoir produced from 5 tribranched fracture patterns and compared to the planar fracture case. 


\section{IV.7 Stochastic Fracture Networks — Quad-Branched Fracture Patterns}

Table 8 - Statistical Parameters for the Quad-Branched Fracture Pattern Case.

\begin{tabular}{|c|c|c|c|c|c|c|c|c|c|}
\hline \multirow[t]{2}{*}{ Parameter } & \multicolumn{3}{|c|}{$F_{0}$} & \multicolumn{3}{|c|}{$\begin{array}{c}F_{01}, F_{011}, F_{021}, \\
F_{0111}, F_{0121}, F_{0221}, F_{0211} \\
F_{01111}, F_{01211}, F_{02211}, F_{02111} \\
F_{01121}, F_{01221}, F_{02121}, F_{02221}\end{array}$} & \multicolumn{3}{|c|}{$\begin{array}{c}F_{02}, F_{012}, F_{022} \\
F_{0112}, F_{0122}, F_{0212}, F_{0222} \\
F_{01112}, F_{01212}, F_{02212}, F_{02112} \\
F_{01122}, F_{01222}, F_{02122}, F_{02222}\end{array}$} \\
\hline & $P_{x+1}$ & $P_{y+1}$ & $P_{y-1}$ & $P_{x+1}$ & $P_{y+1}$ & $P_{y-1}$ & $P_{x+1}$ & $P_{y+1}$ & $P_{y-1}$ \\
\hline Quad-branched fracture 1 & 0.5 & 0.25 & 0.25 & 0.4 & 0.4 & 0.2 & 0.4 & 0.2 & 0.4 \\
\hline Quad -branched fracture 2 & 0.5 & 0.25 & 0.25 & 0.4 & 0.4 & 0.2 & 0.4 & 0.2 & 0.4 \\
\hline Quad -branched fracture 3 & 0.5 & 0.25 & 0.25 & 0.4 & 0.4 & 0.2 & 0.4 & 0.2 & 0.4 \\
\hline Quad -branched fracture 4 & 0.5 & 0.25 & 0.25 & 0.4 & 0.4 & 0.2 & 0.4 & 0.2 & 0.4 \\
\hline Quad -branched fracture 5 & 0.5 & 0.25 & 0.25 & 0.4 & 0.4 & 0.2 & 0.4 & 0.2 & 0.4 \\
\hline
\end{tabular}

Similarly to the Tri-branched fracture pattern cases, 5 different quad-branched fracture patterns are generated in this section. The obtained 5 structures are displayed in Figure 44, as we observed for the tribranched fractures, the quad-branched patterns are dense enough for the branches to intersect between each other. The same generation probabilities were used to generate those different patterns Table 8 . The impact of the presence of 4 stages of branching starts is confirming the observation that we saw in two previous sections. 

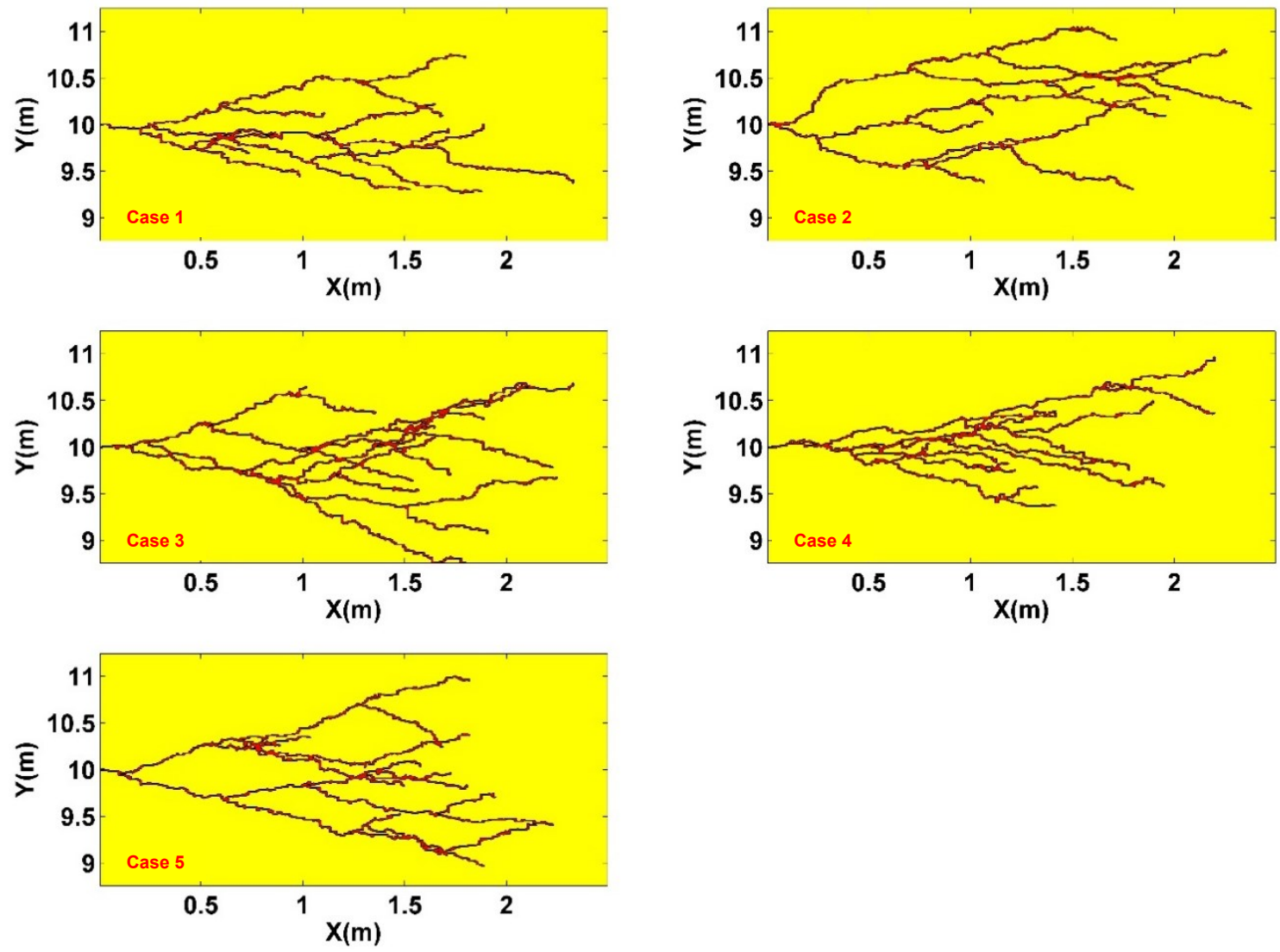

Figure $44-5$ quad-branched fracture patterns (cases 1 to 5 ). 


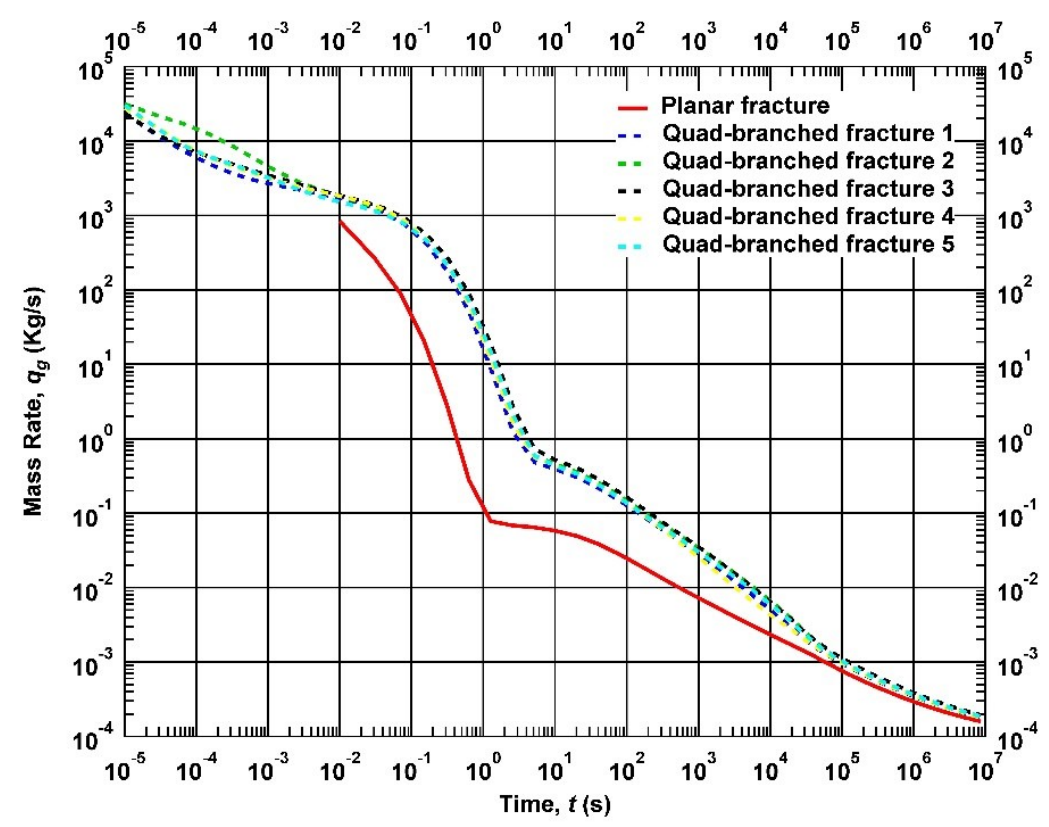

Figure 45 - Mass rate for a synthetic shale gas reservoir produced from 5 quad-branched fracture patterns and compared to the planar fracture case.

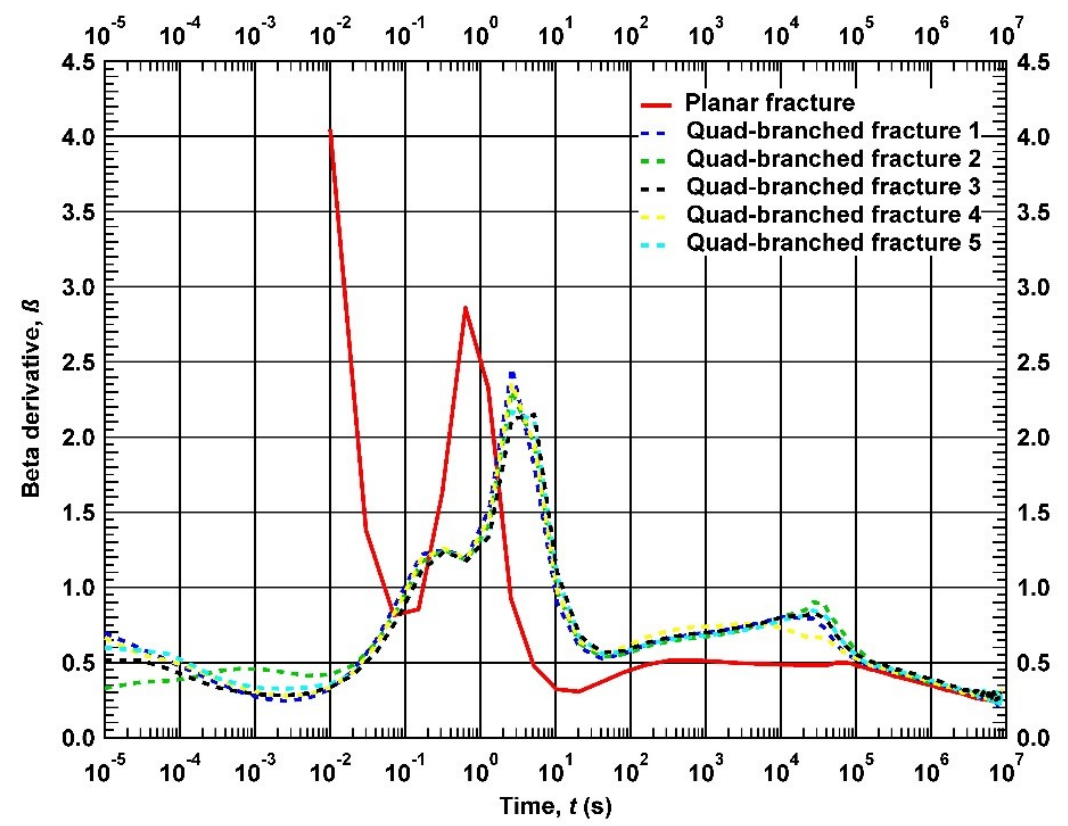

Figure $46-\beta$-derivative of the mass rate for a synthetic shale gas reservoir produced from 5 tribranched fracture patterns and compared to the planar fracture case. 
In Figure 45 and Figure 46 we can observe an early behavior of the mass production rate curve that exhibits a linear flow behavior with a characteristic slope clearly superior to 0.5 . Furthermore, the identified approached value of 0.7 for the derivative between $10^{2} s$ and $10^{5} s$ confirms the increasing tendency of the slope in the mass production rate curve. In other words, the early time behavior of a mass production rate curve is impacted by the number of branches for a given set of stochastically generated fractures as the number of "channels" inside those fractures increase.

Similarly to the mono-branched, dual-branched and tri-branched fracture patterns mass rate change, we observe a similar behavior for all of the quad-branched pattern during the phase where the fractures are interfering. Those interferences are confirmed by the observation of the pressure change maps of Appendix D. They generally occur between $10^{2} \mathrm{~s}$ and $10^{5} \mathrm{~s}$. During that same period, we can identify a signature slope in Figure 45 that characterizes all the quad-branched patterns. The slope value is better read on the $\beta$-derivative plots of Figure 46.

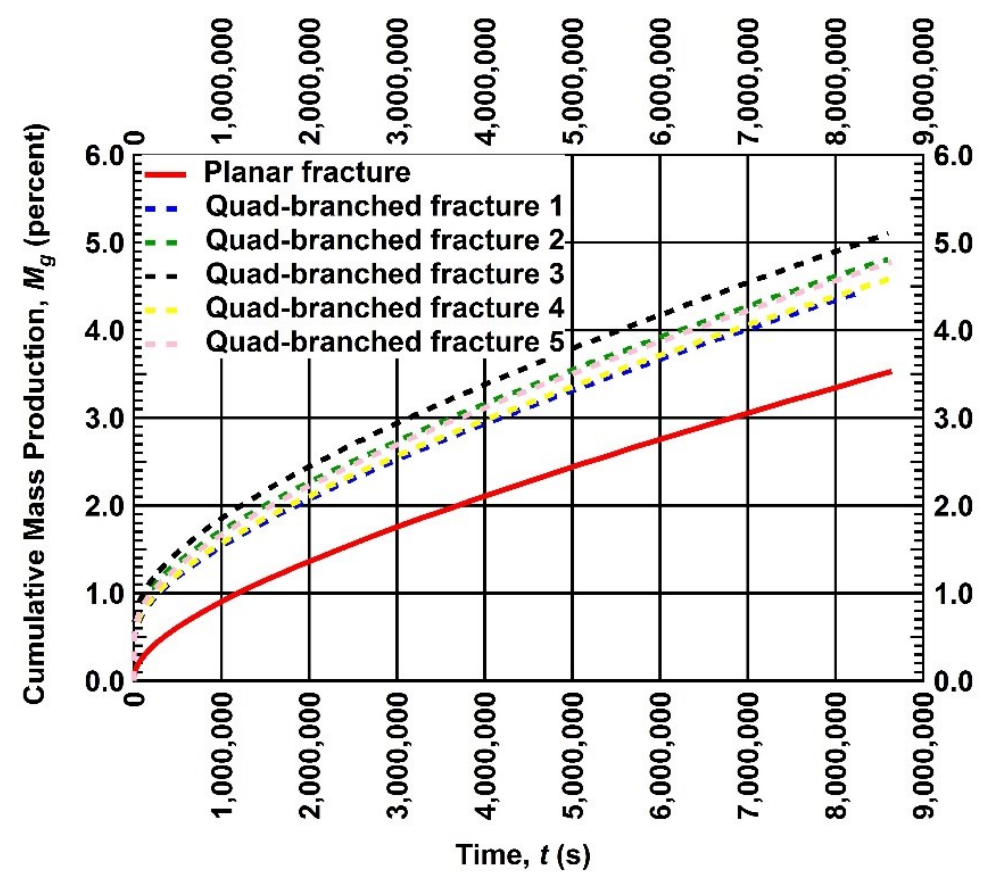

Figure 47 - Cumulative mass production recovery for a shale gas reservoir produced from 5 quadbranched fracture patterns and compared to the planar fracture case.

Figure 47 illustrates that the different quad-branched patterns yield a better recovery after 100 days of production than the single-planar fracture case. Case 3 of the quad-branched cases has the best 
performance in terms of recovery, and Cases 2 and 5 do not yield a significantly different recovery. As an observation, Case 2, 3, and 5 appear to be the most complex fracture networks (see Figure 44).

\section{IV.8 Stochastic Fracture Networks - Impact on Reservoir Performance}

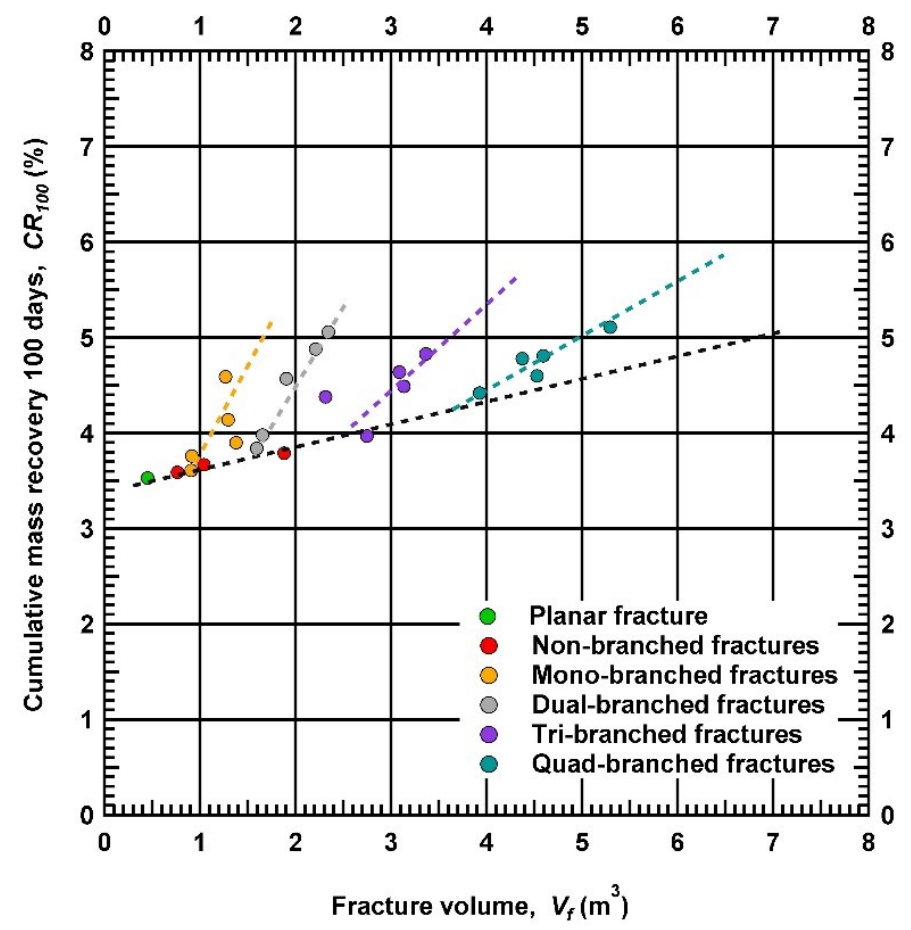

Figure 48 - Cumulative mass recovery at 100 days correlated with fracture volume for different stochastic fracture network patterns.

In Figure 48 we present the cumulative mass recovery at 100 days as a function of fracture volume for the stochastic fracture network patterns created in this work. We clearly note a correlation of the number of branching stages on cumulative mass recovery. While we should not generalize these observations, we should expect such correlations based on the number of branching stages and the total fracture volume per case. The dashed-black line is imposed as a "baseline" for each given fracture network case (i.e., this would be the base correlation of recovery and fracture volume. The individual trend for each stochastic fracture network case suggests a correlation with fracture network "complexity" (i.e., complexity would be a function of the number of branches and the fracture volume). 


\section{IV.9 Stochastic Fracture Networks - Summary of Additional Modeling Cases}

In order to confirm the results of the previous section in terms of the effect of the number of branching stages on the value of the $\beta$-derivative or the slope of the mass production rate curves, we created additional fractures for each case:

- 15 mono-branched patterns of Figure 32, Figure 49, Figure 50 and Figure 51 which generation parameters are given in Table 9.

- 15 dual-branched patterns of Figure 36, Figure 52, Figure 53 and Figure 54 which generation parameters are given in Table $\mathbf{1 0 .}$

- 15 tri-branched patterns of Figure 40, Figure 55, Figure 56 and Figure 57 which generation parameters are given in Table 11.

- 15 quad-branched patterns of Figure 44, Figure 58, Figure 59 and Figure 60 which generation parameters are given in Table 12.

As the effect of the fracture interference is observed after $10^{5} \mathrm{~s}$ of production for all cases, we generated these additional simulation 60 cases only for 100 hours $\left(3.6 \times 10^{5} \mathrm{~s}\right)$ of production to reduce the computational workload.

Table 9 - Statistical Parameters for the Additional Mono-Branched Fracture Pattern Cases.

\begin{tabular}{lccccccccc} 
Parameters & \multicolumn{3}{c}{$\boldsymbol{F}_{\mathbf{0}}$} & \multicolumn{3}{c}{$\boldsymbol{F}_{\mathbf{0 1}}$} & \multicolumn{3}{c}{$\boldsymbol{F}_{\mathbf{0 2}}$} \\
& $\boldsymbol{P}_{\boldsymbol{x}+\mathbf{1}}$ & $\boldsymbol{P}_{\boldsymbol{y}+\mathbf{1}}$ & $\boldsymbol{P}_{\boldsymbol{y}-\mathbf{1}}$ & $\boldsymbol{P}_{\boldsymbol{x}+\mathbf{1}}$ & $\boldsymbol{P}_{\boldsymbol{y}+\mathbf{1}}$ & $\boldsymbol{P}_{\boldsymbol{y}-\mathbf{1}}$ & $\boldsymbol{P}_{\boldsymbol{x}+\mathbf{1}}$ & $\boldsymbol{P}_{\boldsymbol{y}+\mathbf{1}}$ & $\boldsymbol{P}_{\boldsymbol{y}-\mathbf{1}}$ \\
\hline Mono-branched fracture 6 to 20 & 0.5 & 0.25 & 0.25 & 0.4 & 0.3 & 0.3 & 0.4 & 0.3 & 0.3
\end{tabular}

Table 10 - Statistical Parameters for the Additional Dual-Branched Fracture Pattern Cases.

\begin{tabular}{lccccccccc}
\multirow{2}{*}{ Parameters } & \multicolumn{3}{c}{$\boldsymbol{F}_{\mathbf{0}}$} & \multicolumn{4}{c}{$\boldsymbol{F}_{\mathbf{0 1}}, \boldsymbol{F}_{\mathbf{0 1 1}}, \boldsymbol{F}_{\mathbf{0 2 1}}$} & \multicolumn{4}{c}{$\boldsymbol{F}_{\mathbf{0 2}}, \boldsymbol{F}_{\mathbf{0 1 2}}, \boldsymbol{F}_{\mathbf{0 2 2}}$} \\
& $\boldsymbol{P}_{x+1}$ & $\boldsymbol{P}_{\boldsymbol{y}+\mathbf{1}}$ & $\boldsymbol{P}_{\boldsymbol{y}-\mathbf{1}}$ & $\boldsymbol{P}_{x+1}$ & $\boldsymbol{P}_{\boldsymbol{y + 1}}$ & $\boldsymbol{P}_{\boldsymbol{y}-\mathbf{1}}$ & $\boldsymbol{P}_{x+1}$ & $\boldsymbol{P}_{\boldsymbol{y + 1}}$ & $\boldsymbol{P}_{\boldsymbol{y - 1}}$ \\
\hline Dual -branched fracture 6 to 20 & 0.5 & 0.25 & 0.25 & 0.4 & 0.4 & 0.2 & 0.4 & 0.2 & 0.4
\end{tabular}

Table 11 - Statistical Parameters for the Additional Tri-Branched Fracture Pattern Cases.

\begin{tabular}{|c|c|c|c|c|c|c|c|c|c|}
\hline \multirow[t]{2}{*}{ Parameters } & \multicolumn{3}{|c|}{$F_{0}$} & \multicolumn{3}{|c|}{$\begin{array}{c}F_{01}, F_{011}, F_{021} \\
F_{0111}, F_{0121}, F_{0221}, F_{0211}\end{array}$} & \multicolumn{3}{|c|}{$\begin{array}{c}F_{02}, F_{012}, F_{022} \\
F_{0112}, F_{0122}, F_{0212}, F_{022}\end{array}$} \\
\hline & $P_{x+1}$ & $P_{y+1}$ & $P_{y_{-}}$ & $P_{x+1}$ & $P_{y+1}$ & $P_{y-1}$ & $P_{x+1}$ & $P_{y+1}$ & $P_{y-1}$ \\
\hline hed fracture 6 to 2 & 0.5 & 0.25 & $0.2 !$ & 0.4 & 0.4 & 0.2 & 0.4 & 0.2 & 0.4 \\
\hline
\end{tabular}


Table 12 - Statistical Parameters for the Additional Quad-Branched Fracture Pattern Cases.

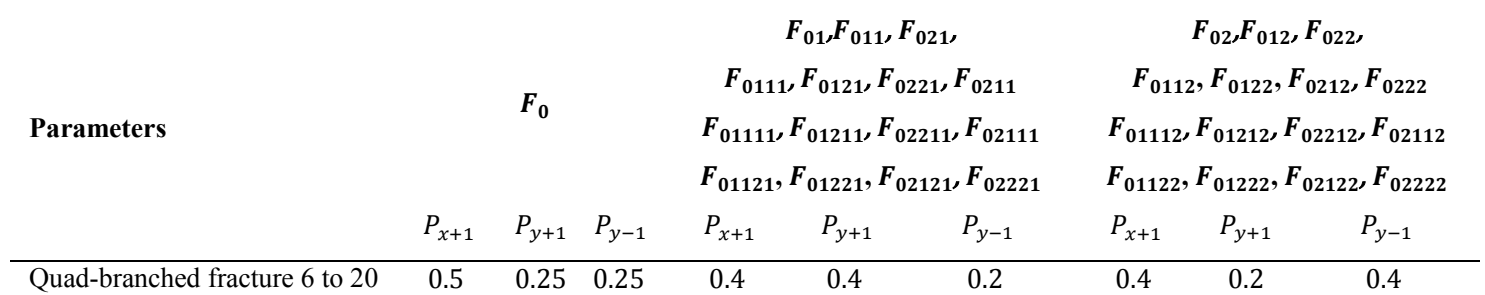
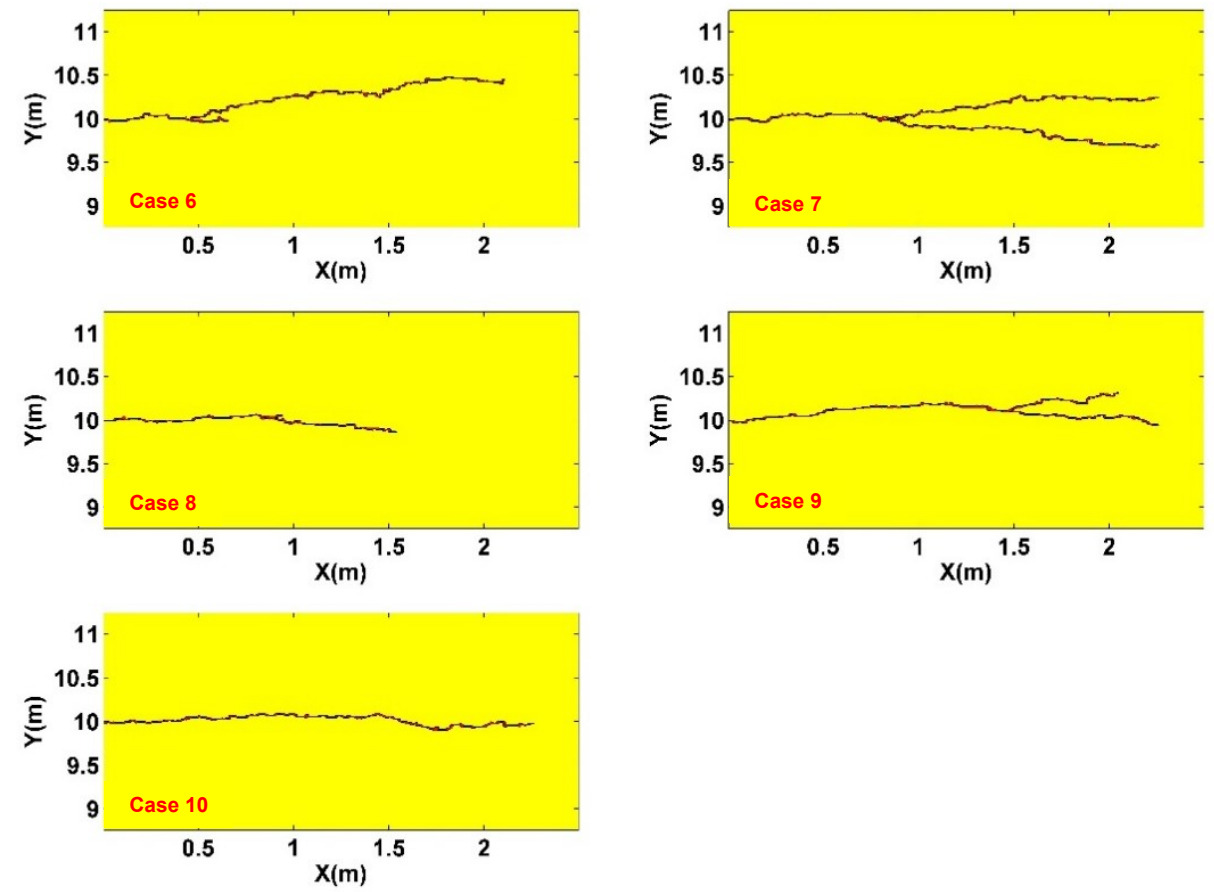

Figure 49 - 5 mono-branched fracture patterns (cases 6 to 10). 

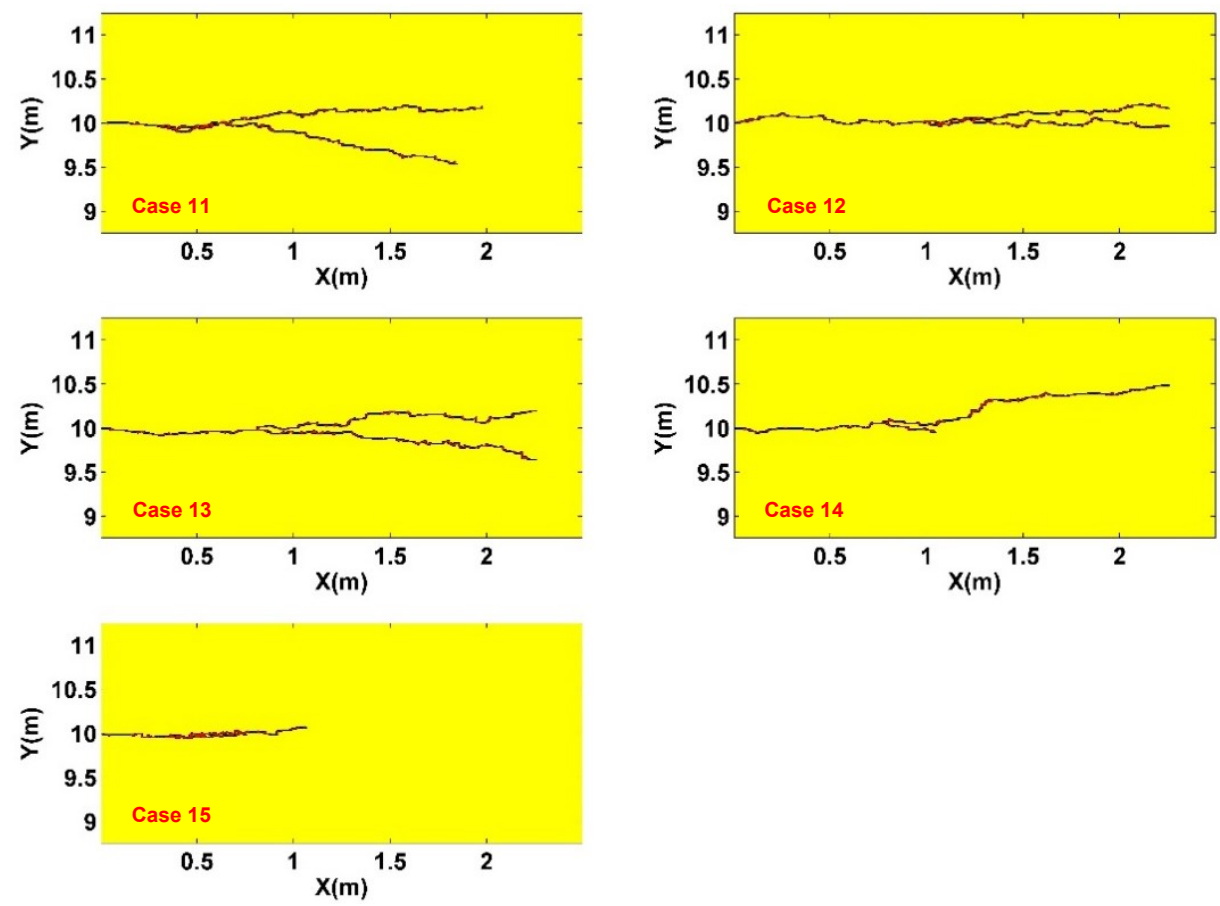

Figure $50-5$ mono-branched fracture patterns (cases 11 to 15).
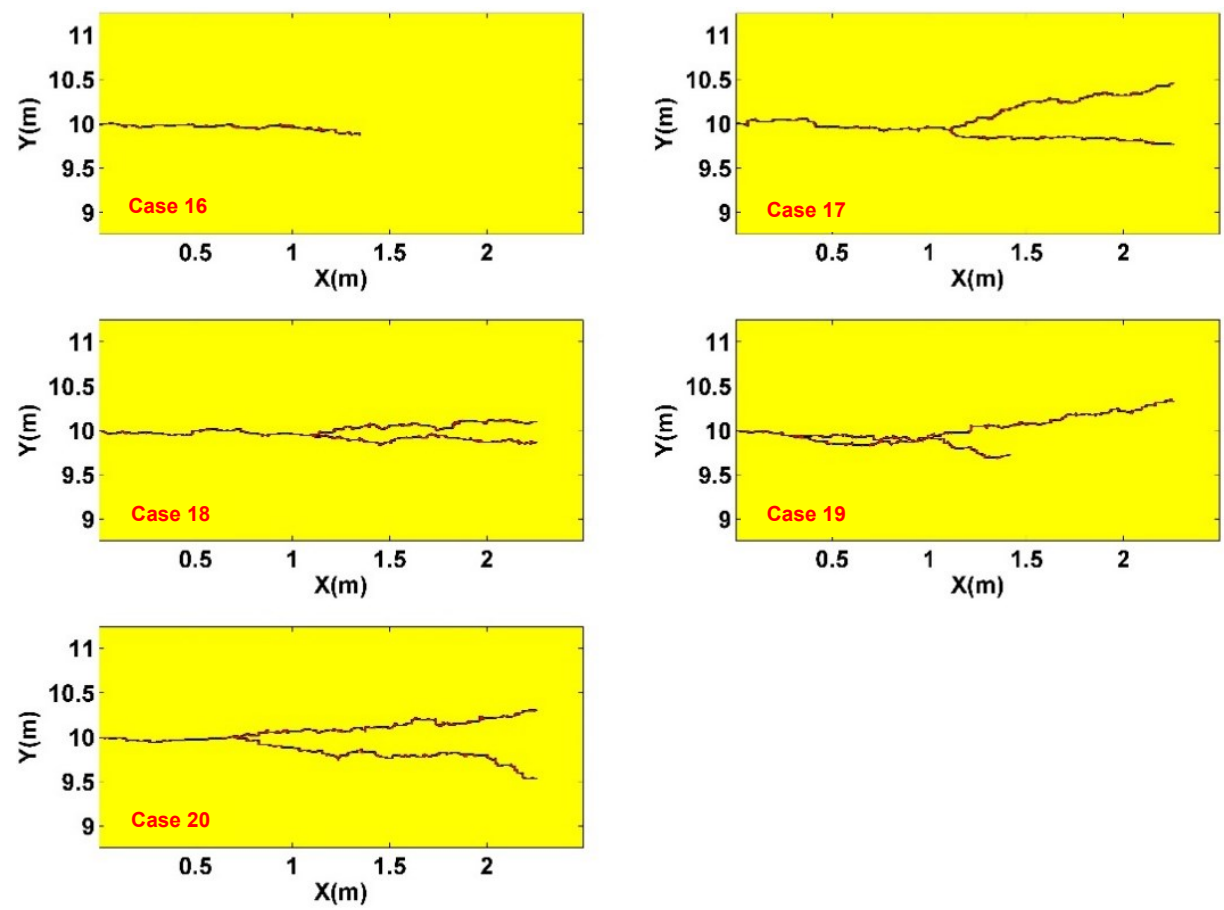

Figure $51-5$ mono-branched fracture patterns (cases 16 to 20). 

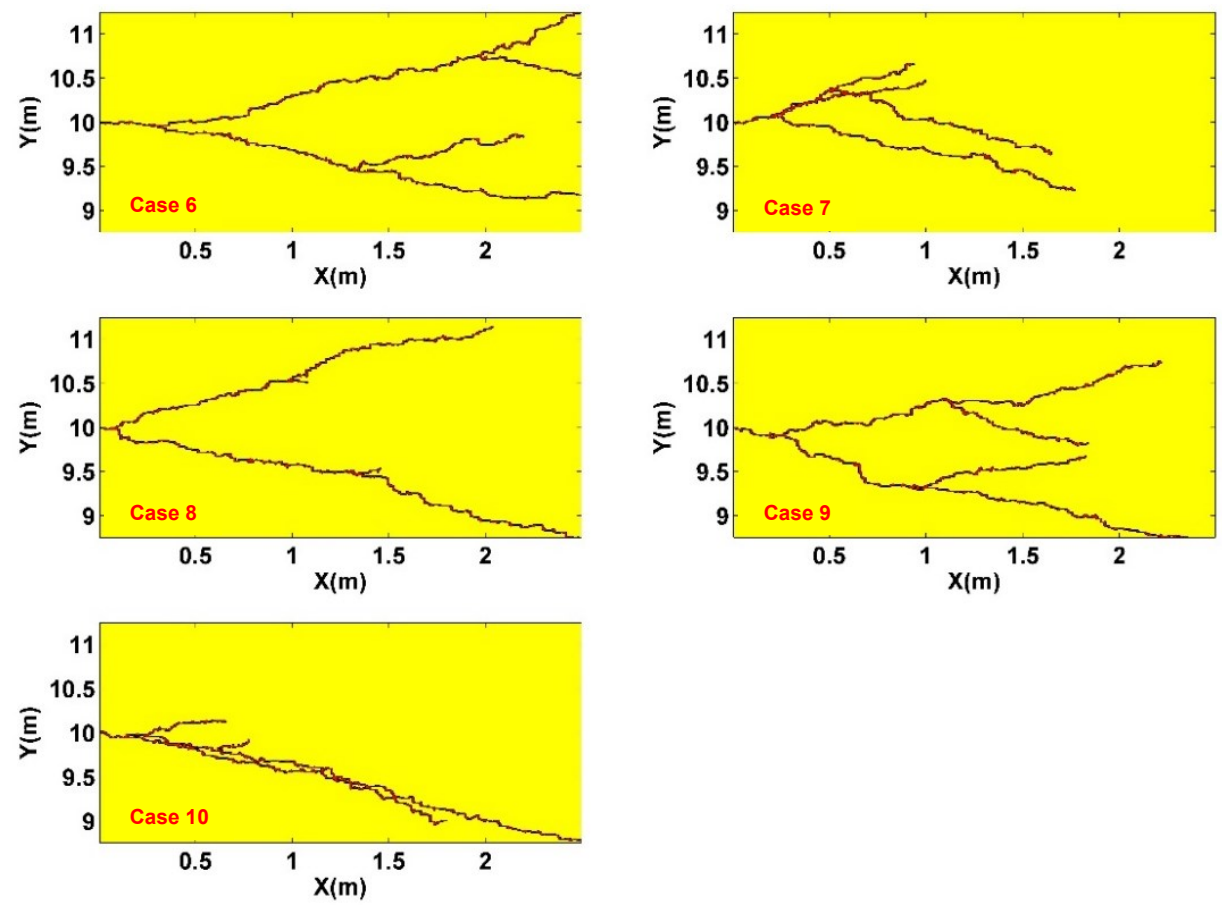

Figure $52-5$ dual-branched fracture patterns (cases 6 to 10).
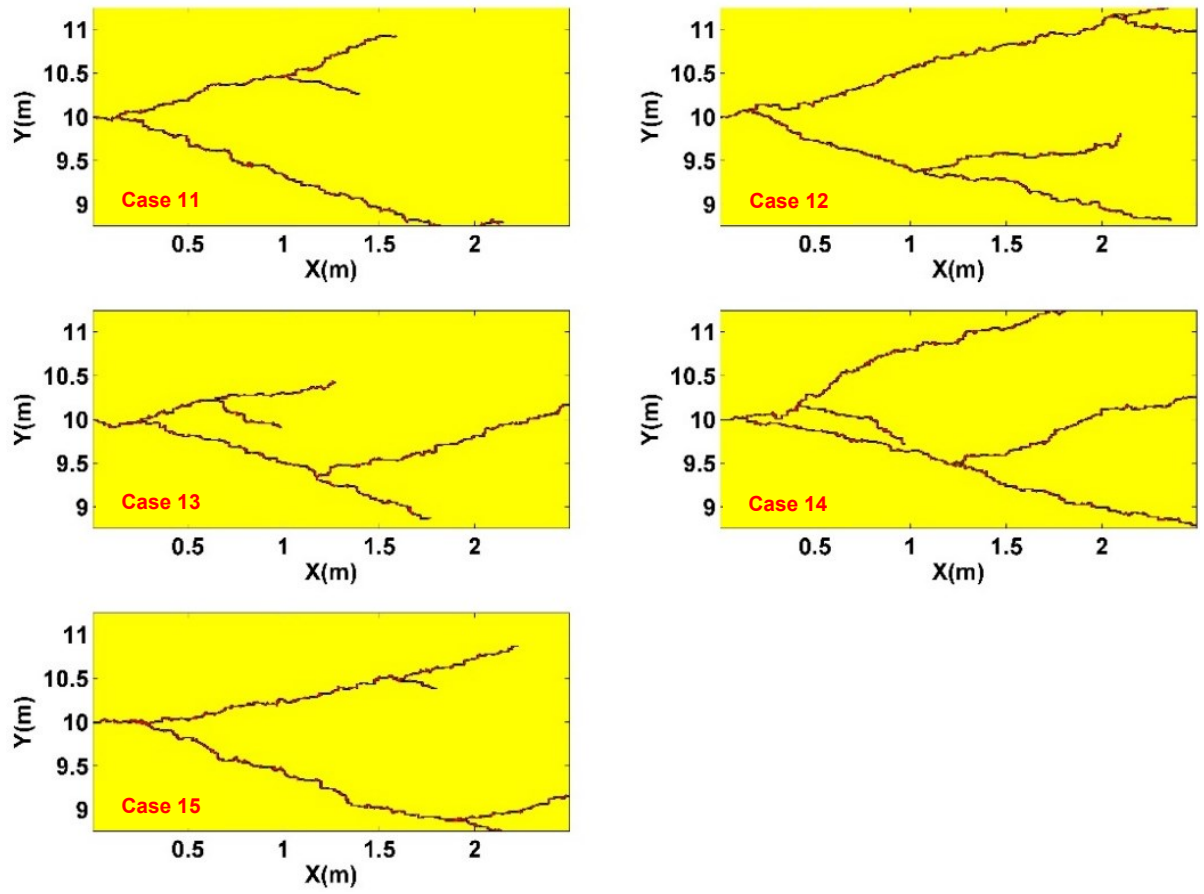

Figure $53-5$ dual-branched fracture patterns (cases 11 to 15). 

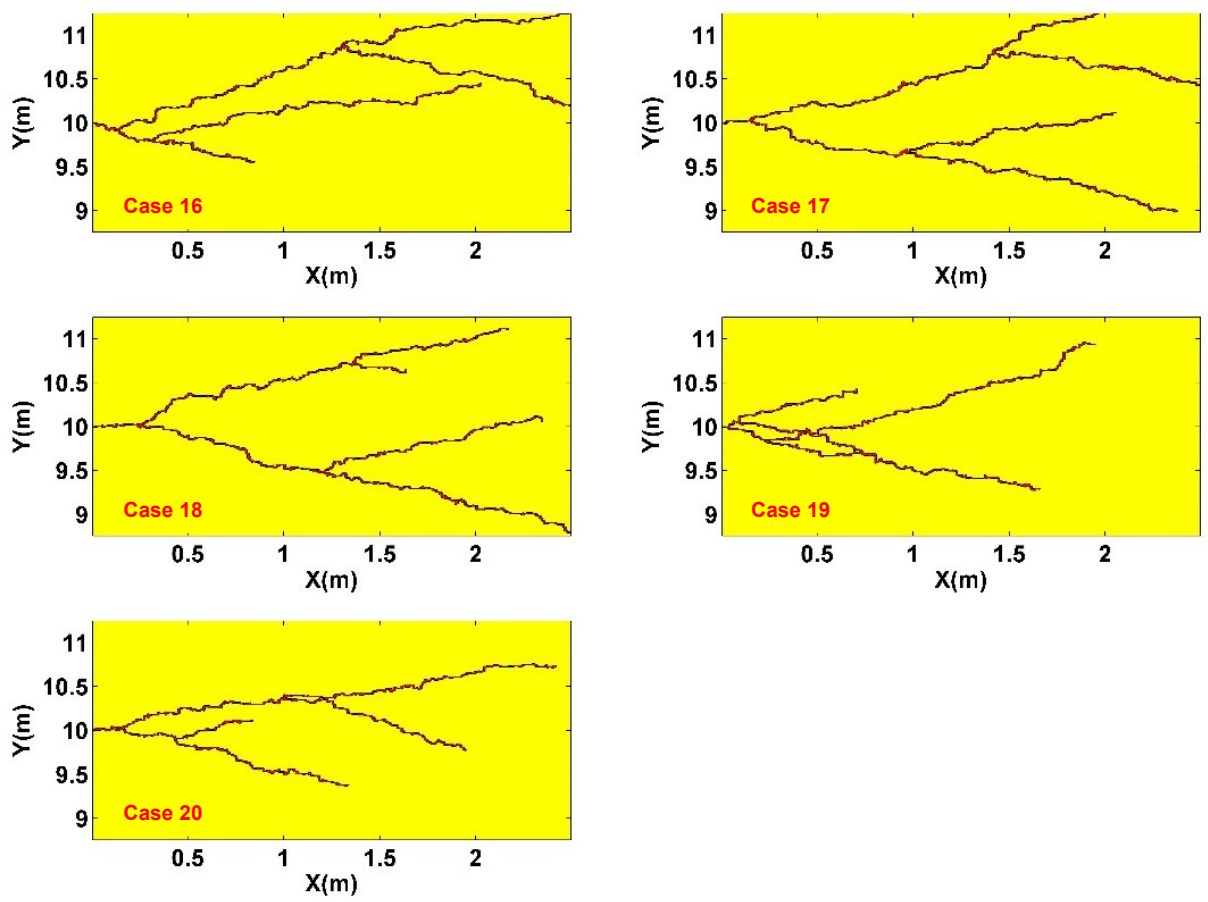

Figure $54-5$ dual-branched fracture patterns (cases 16 to 20).
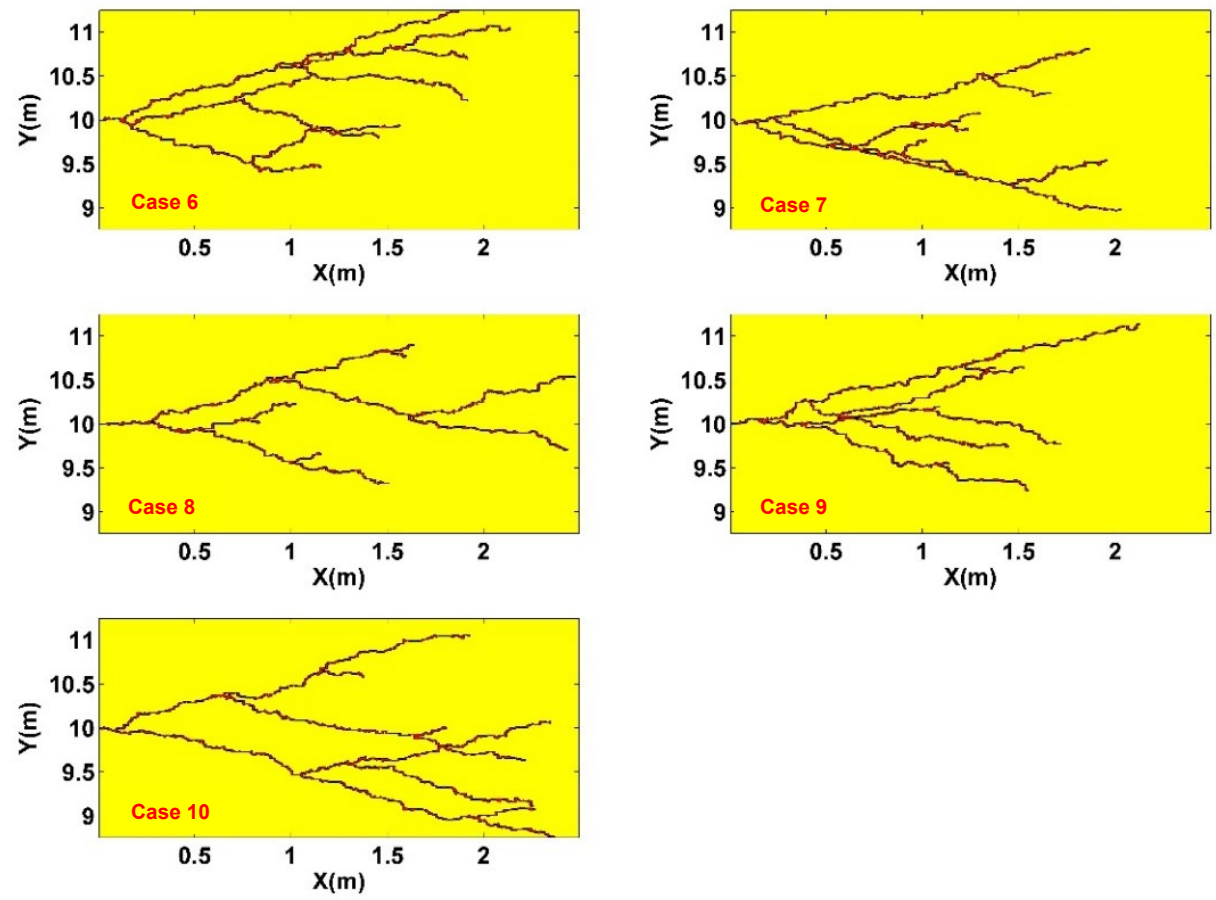

Figure 55 - 5 tri-branched fracture patterns (cases 6 to 10). 

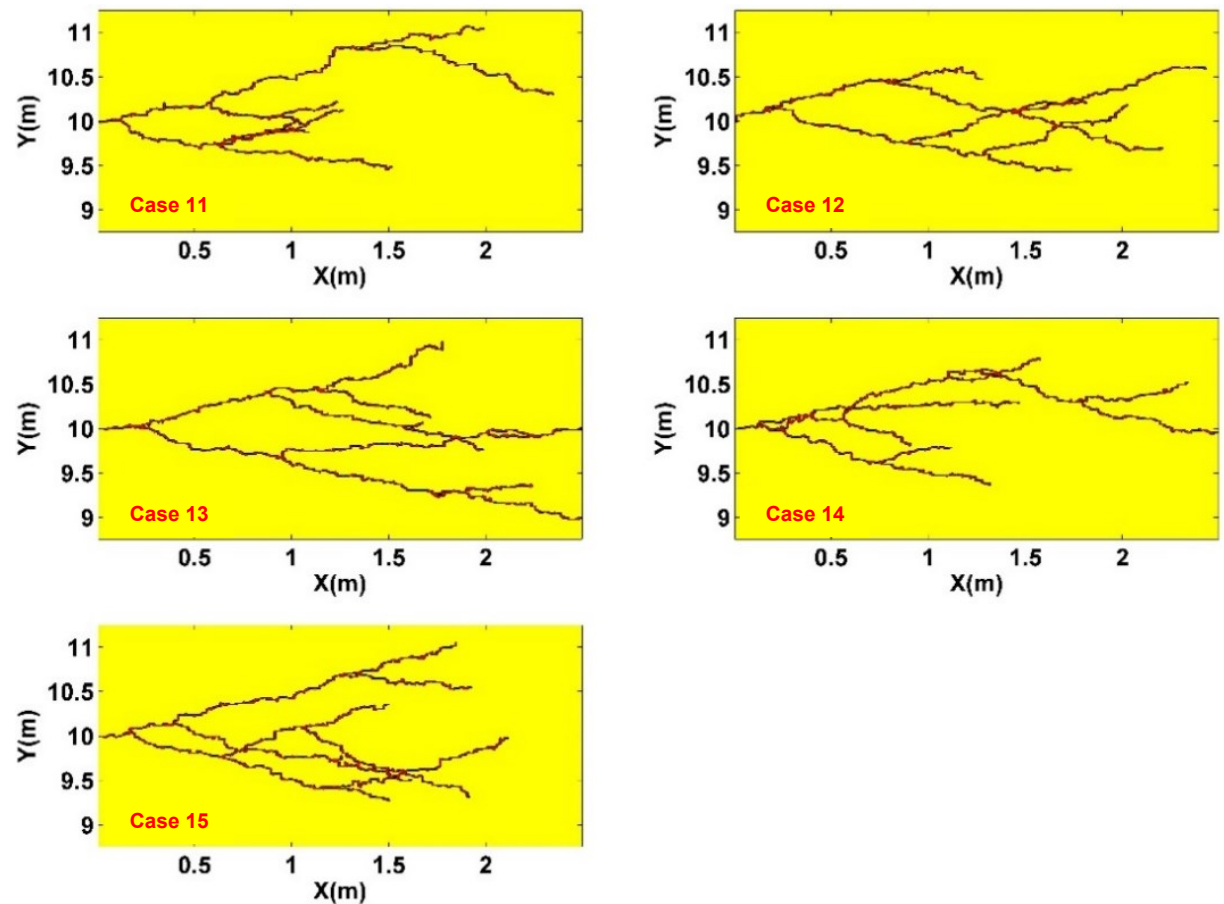

Figure $56-5$ tri-branched fracture patterns (cases 11 to 15).
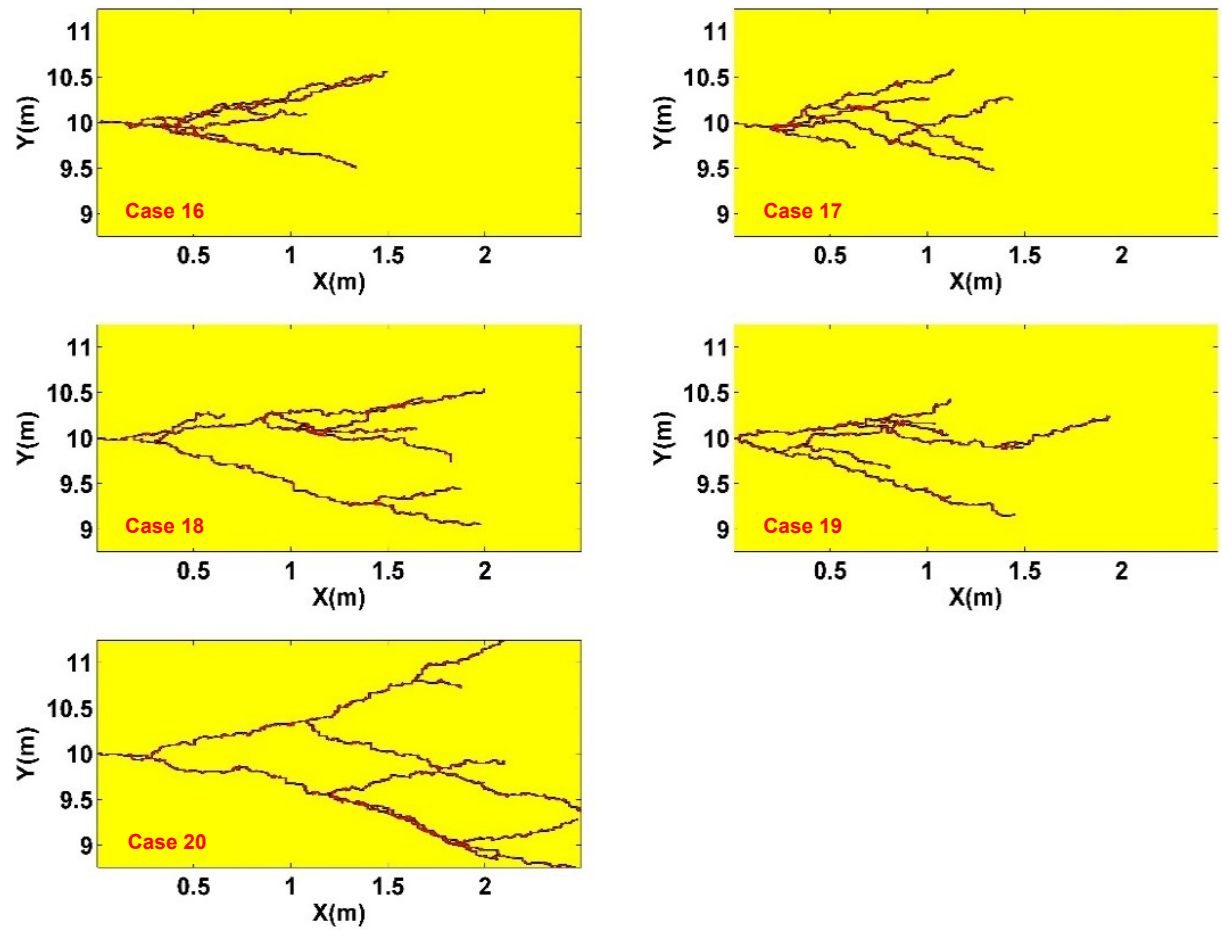

Figure $57-5$ tri-branched fracture patterns (cases 16 to 20). 

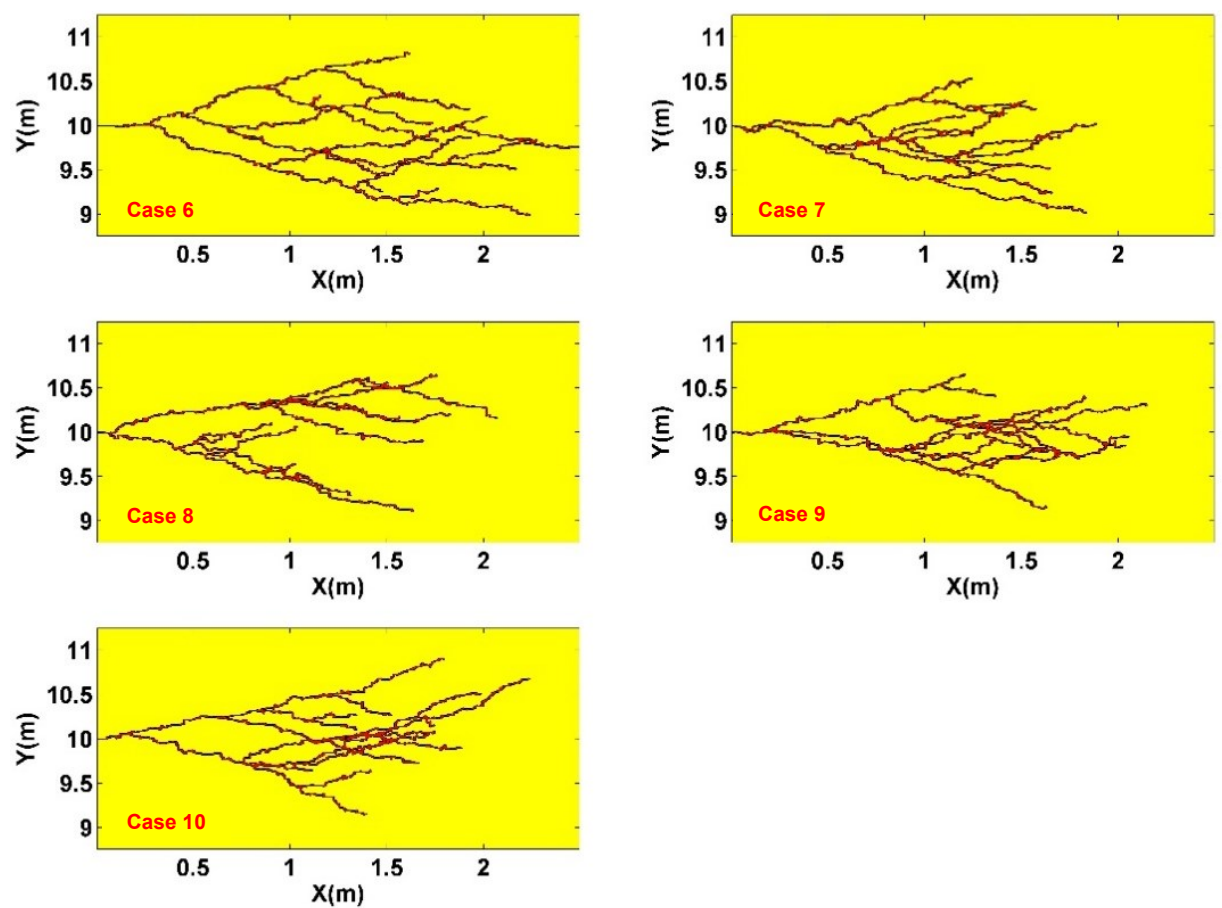

Figure $58-5$ quad-branched fracture patterns (cases 6 to 10).
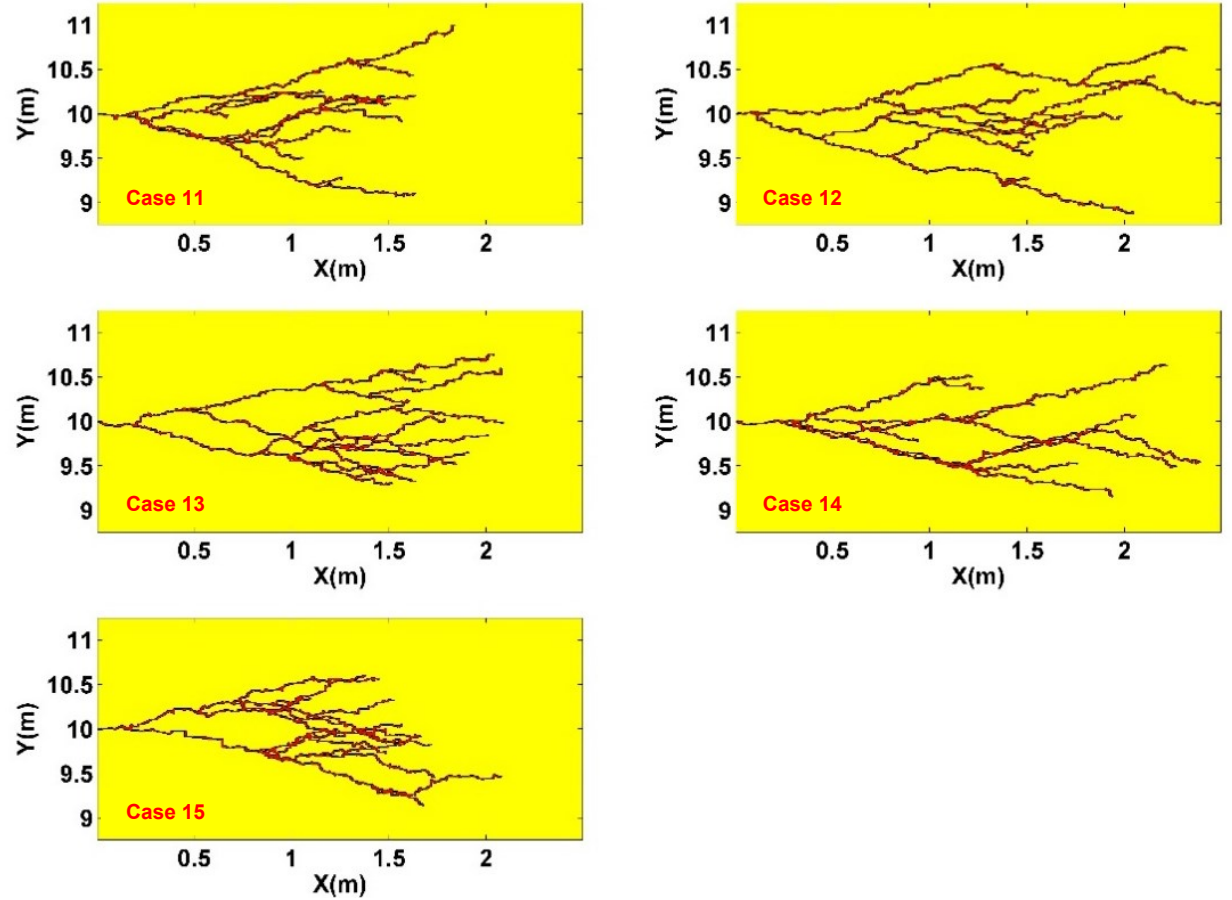

Figure $59-5$ quad-branched fracture patterns (cases 11 to 15). 

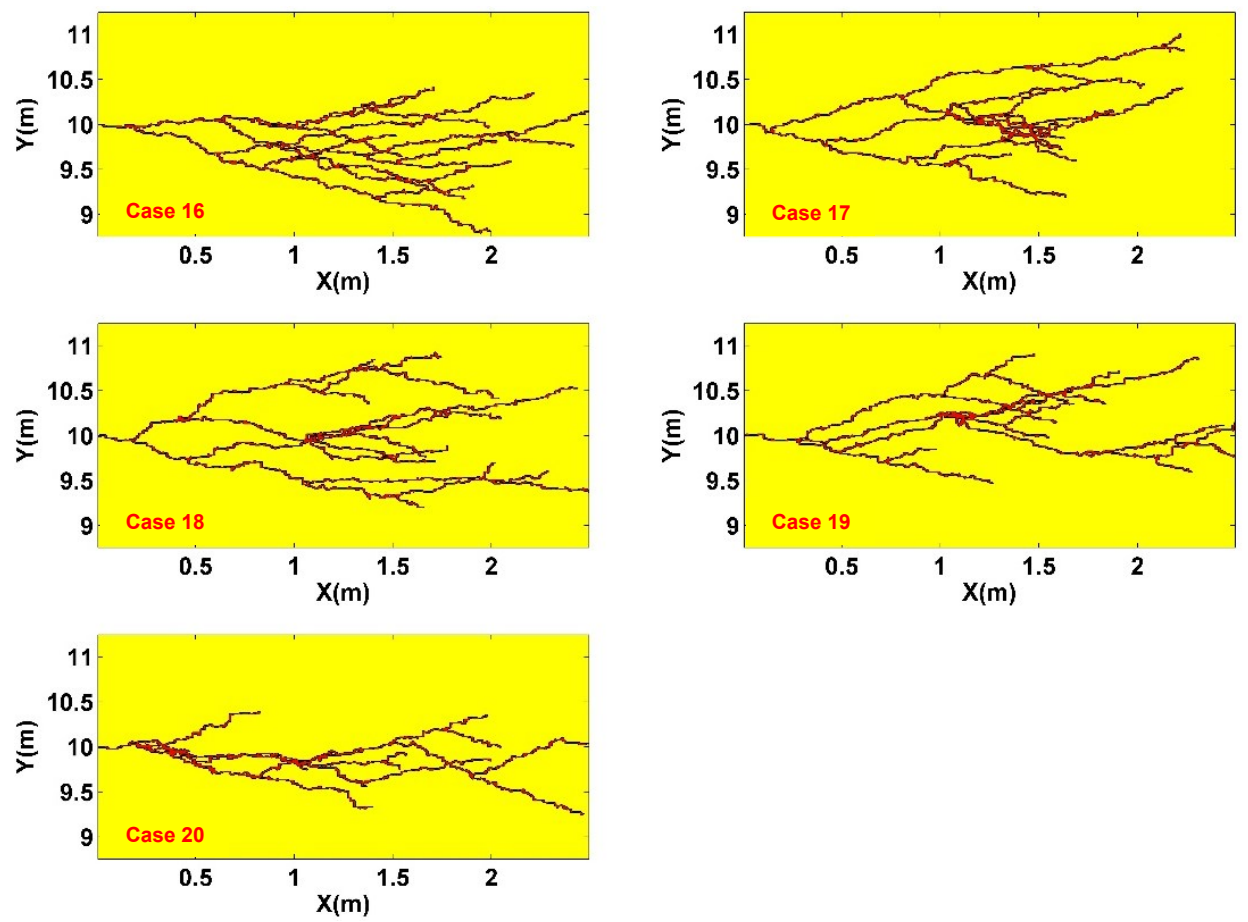

Figure $60-5$ quad-branched fracture patterns (cases 16 to 20).

For each set of patterns, the mass production rate and $\beta$-derivative plot are shows in Figure 61, Figure 62,

Figure 63, Figure 64, Figure 65, Figure 66, Figure 67 and Figure 68. 


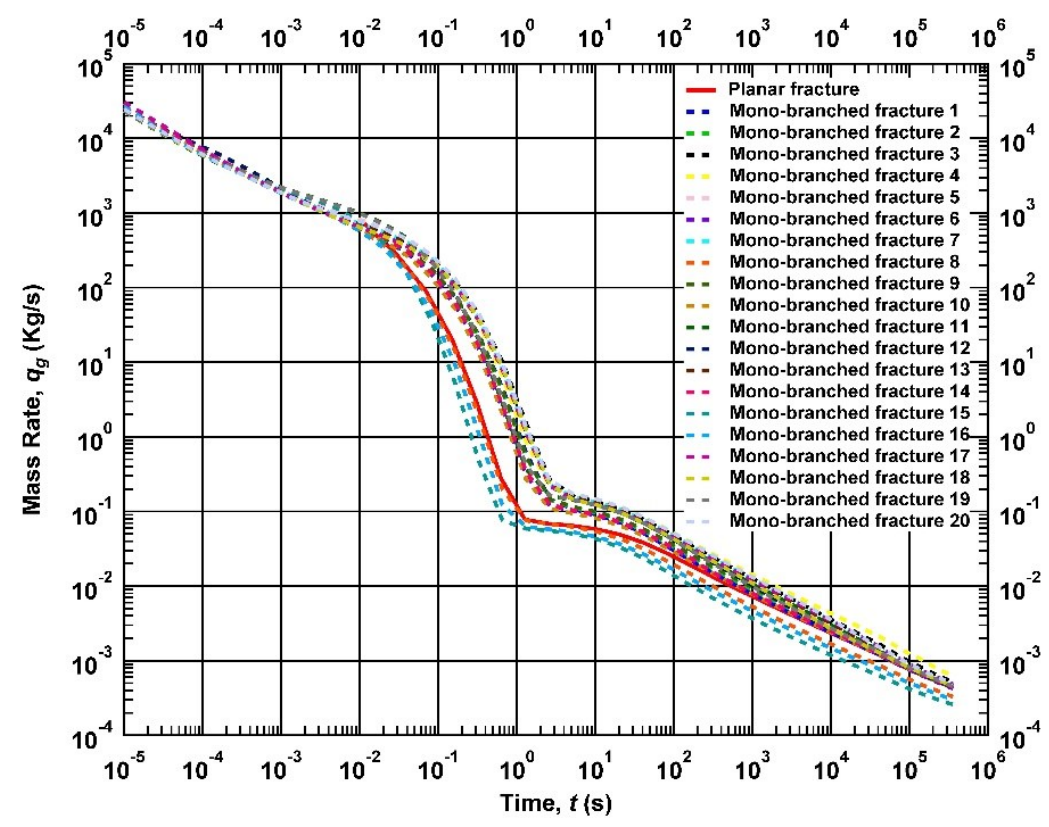

Figure 61 - Mass rate for a synthetic shale gas reservoir produced from 20 mono-branched fracture patterns and compared to the planar fracture case.

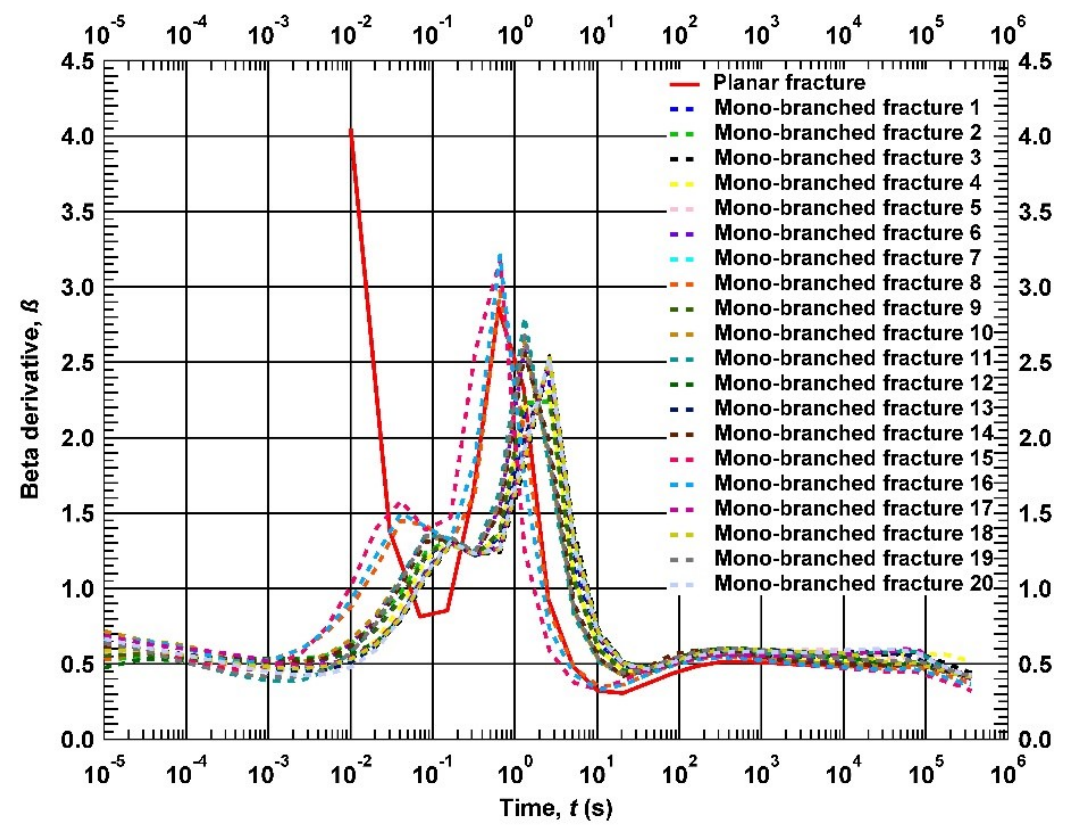

Figure $62-\beta$-derivative of the mass rate for a synthetic shale gas reservoir produced from 20 mono-branched fracture patterns and compared to the planar fracture case. 


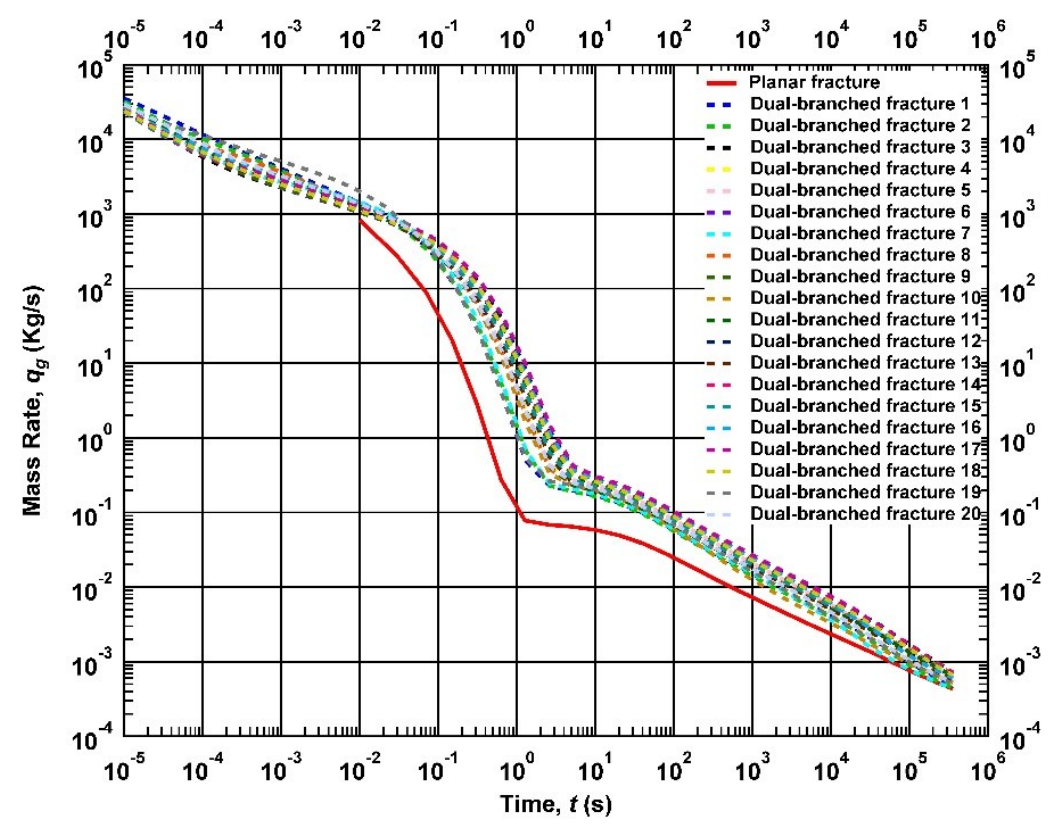

Figure 63 - Mass rate for a synthetic shale gas reservoir produced from 20 dual-branched fracture patterns and compared to the planar fracture case.

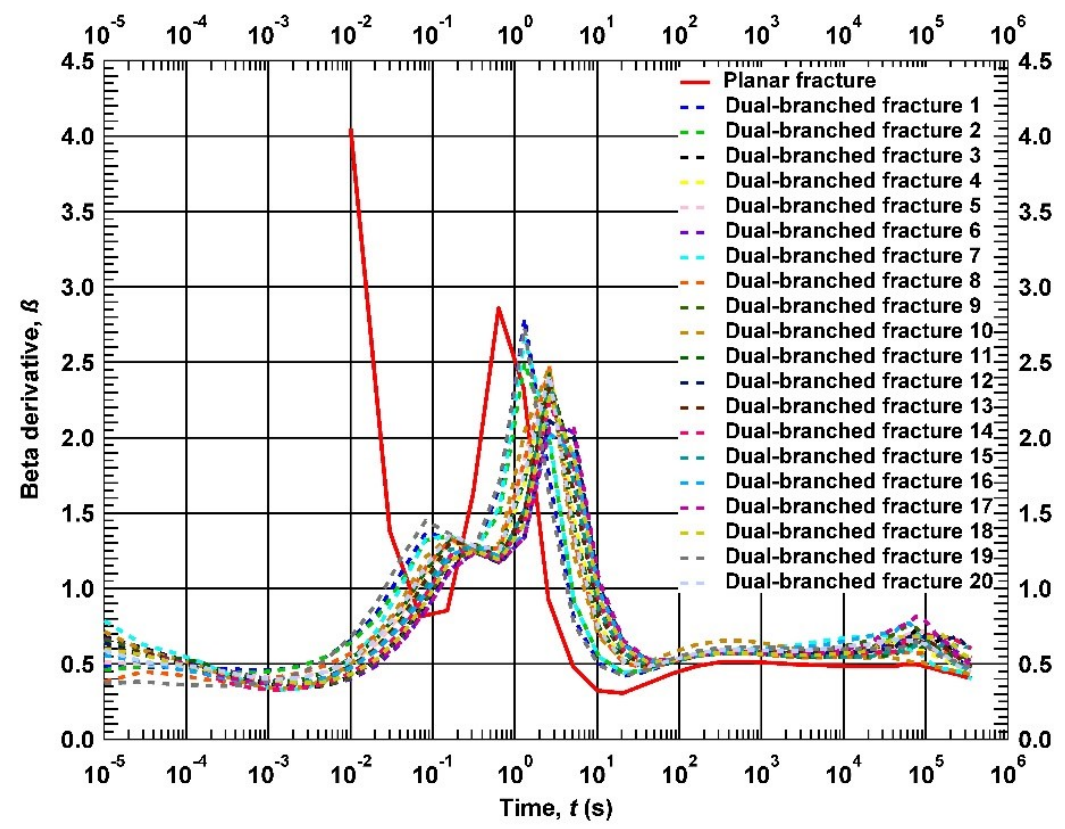

Figure $64-\beta$-derivative of the mass rate for a synthetic shale gas reservoir produced from 20 dual-branched fracture patterns and compared to the planar fracture case. 


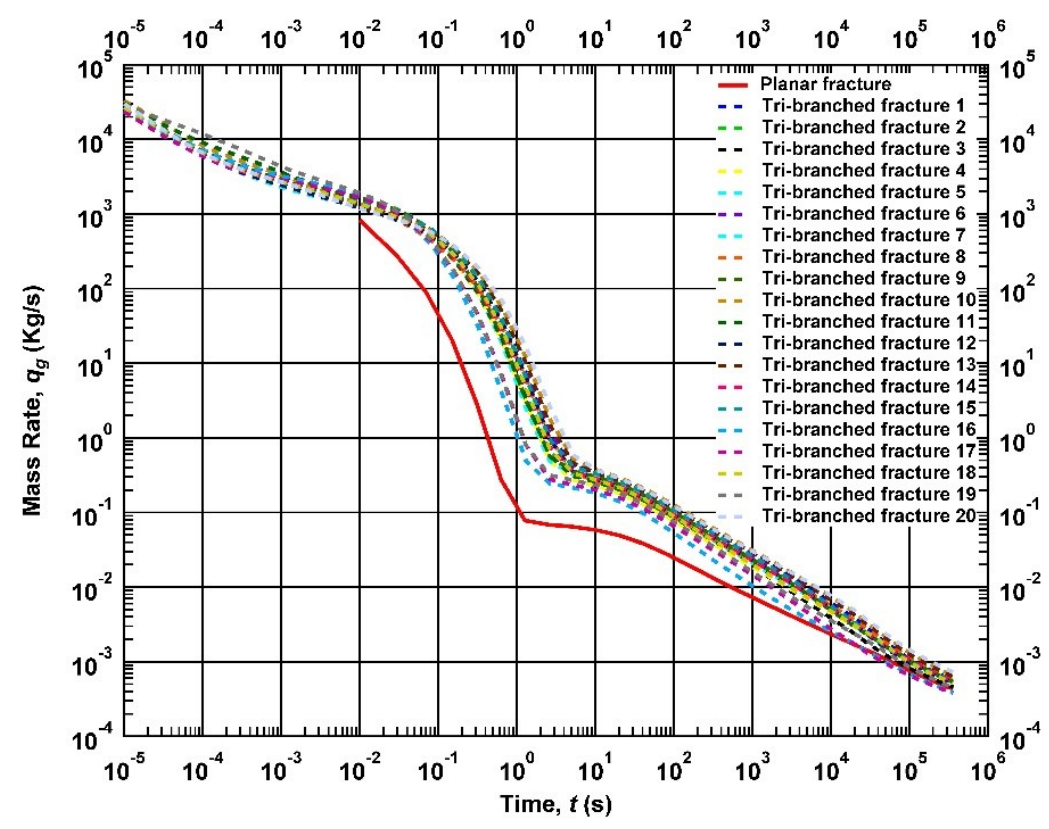

Figure 65 - Mass rate for a synthetic shale gas reservoir produced from 20 tri-branched fracture patterns and compared to the planar fracture case.

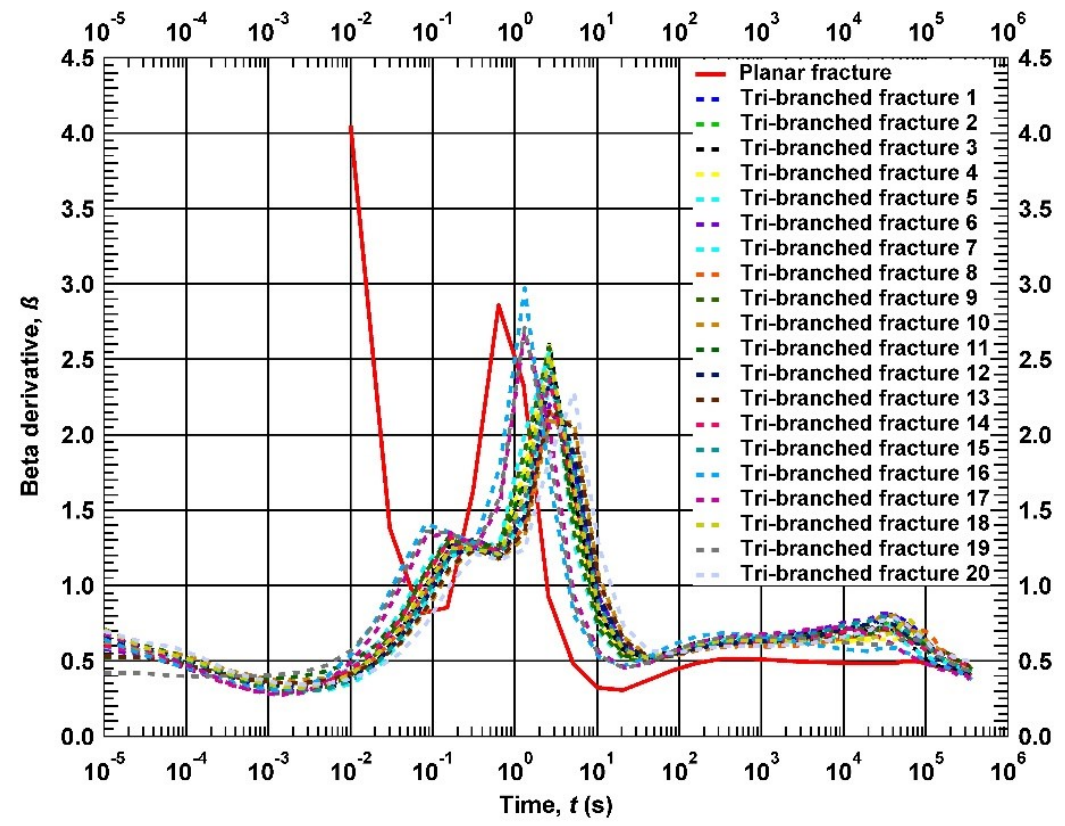

Figure $66-\beta$-derivative of the mass rate for a synthetic shale gas reservoir produced from 20 tribranched fracture patterns and compared to the planar fracture case. 


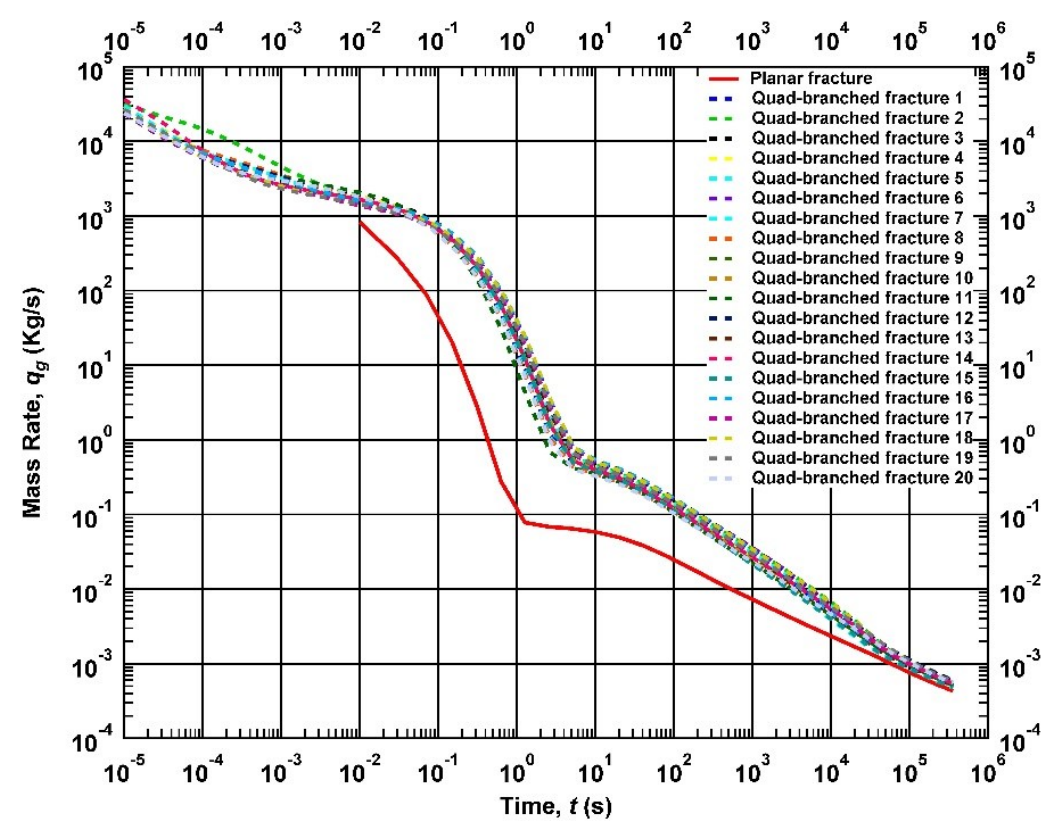

Figure 67 - Mass rate for a synthetic shale gas reservoir produced from 20 quad-branched fracture patterns and compared to the planar fracture case.

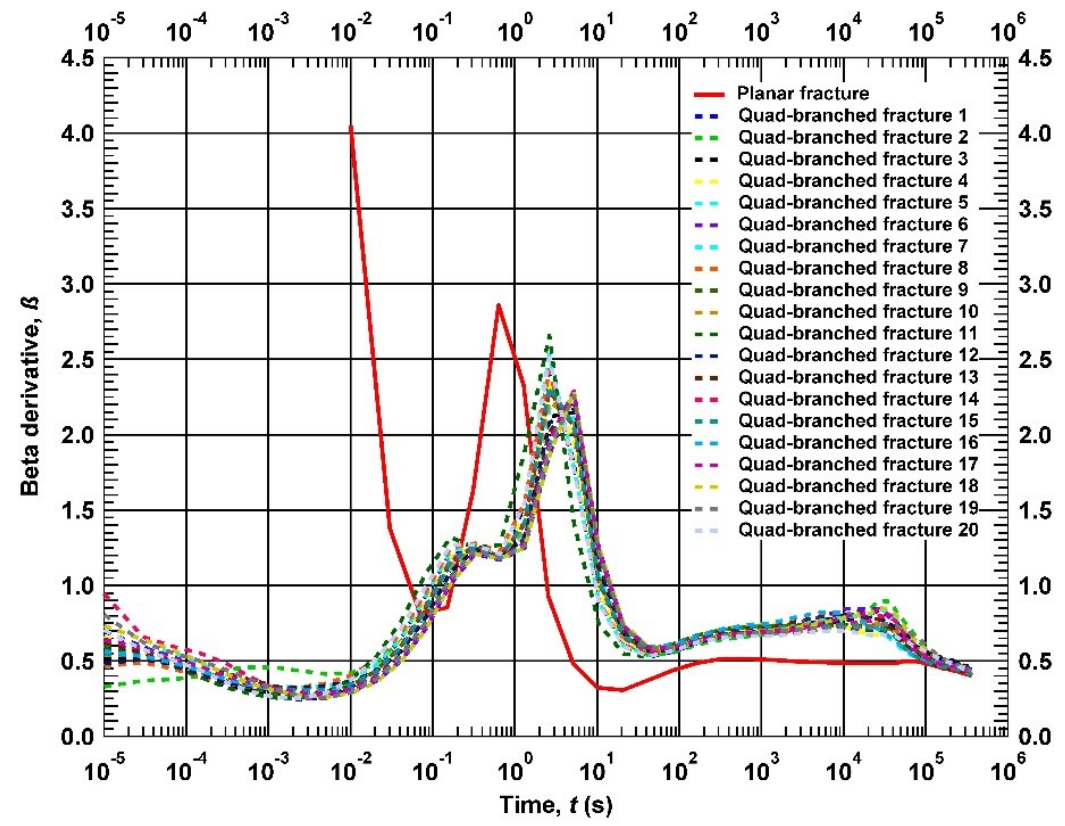

Figure 68 - $\beta$-derivative of the mass rate for a synthetic shale gas reservoir produced from 20 quad-branched fracture patterns and compared to the planar fracture case. 


\section{IV.10 Stochastic Fracture Networks - Correlation of the $\beta$-derivative Performance (all cases)}

The average value of the $\beta$-derivative based on the mass production rate for all of the modeling cases are summarized in Table 13.

Table 13 - Average $\beta$-Derivative Values Derived from Modeling Results (80 cases).

\begin{tabular}{cc}
$\begin{array}{c}\text { Number of } \\
\text { branching stages }\end{array}$ & $\begin{array}{c}\text { Average } \boldsymbol{\beta} \text {-derivative } \\
\text { between } \mathbf{1 0}^{\mathbf{2}} \text { s and } \mathbf{1 0}^{\mathbf{5}} \mathbf{s} .\end{array}$ \\
\hline 1 & 0.56 \\
2 & 0.59 \\
3 & 0.64 \\
4 & 0.70
\end{tabular}

In addition, we can assume that the minimum $\beta$-derivative (0.5) exists for cases where there are no branches. The maximum value of the $\beta$-derivative would be 1 (i.e., unity), which corresponds to "depletion behavior" where the entire volume is fractured and we achieve a (very) high equivalent permeability value for the fractured reservoir volume. Physically, when the number of branches is infinitely high, the reservoir volume is essentially filled with fractures and the reservoir effectively becomes a tank (pseudosteady-state condition would exist).

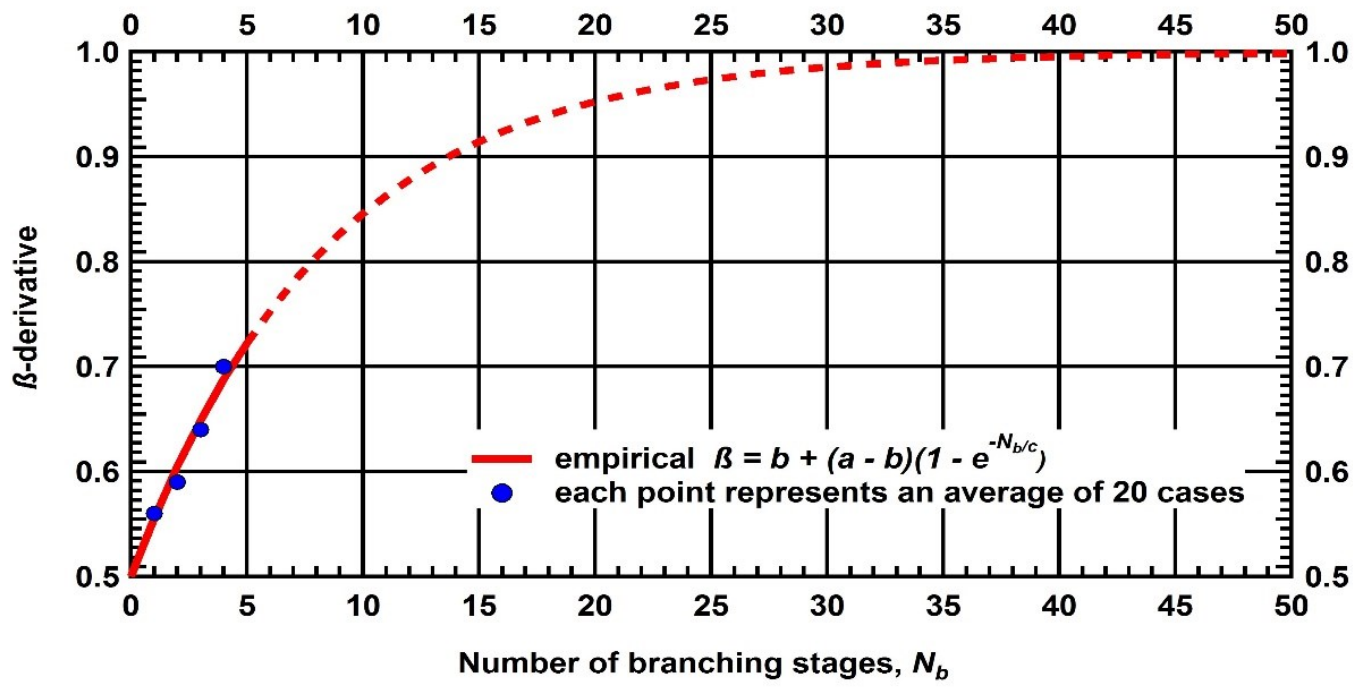

Figure 69 - Average value of the $\beta$-derivative and proposed empirical model.

In Figure 69 we present the average value of the $\beta$-derivative derived from the cases for a given number of fracture branches as a function of the number of branches present in the created stochastic fracture 
network. In order to have a functional representation (for possible use as a design tool), we developed the following model of the $\beta$-derivative as a function of the number of stochastic fracture branches:

$$
\beta\left(N_{b}\right)=b+(a-b)\left(1-e^{-\frac{N_{b}}{c}}\right)
$$

Where the $a, b$, and $c$-coefficients are defined for our work using regression analysis. The specific coefficient values for this case are:

$$
\begin{aligned}
& a=1.00 . \\
& b=0.50 . \\
& c=8.50 .
\end{aligned}
$$

One possible consequence of the trend shown in Figure 69 is that this result could be used to interpret the geometry of an in-situ created/enhanced fracture network using the performance signature of the mass production rate curve (specifically, the $\beta$-derivative). Another conclusion that can be drawn from the trend shown in Figure 69 is that when we are investigating the behavior of $\beta$-derivative in practice (i.e., using field data) we could be observing the combined effect of natural and hydraulically created/enhanced fractures. This hypothesis implies that we should utilize microseismic monitoring as a means of trying to characterize the behavior of the created/enhanced fracture system and the pre-existing natural fracture network.

\section{IV.11 Stochastic Fracture Networks - Assumptions, Limitations, and Discussion}

The understanding of in-situ fracture geometries is essential to the accurate prediction of rate and pressure performance as well as reserves estimations for shale gas reservoirs. Therefore, we must consider that each geometrical feature which has an influence on the performance of the reservoir should be properly assessed and accounted for in a numerical simulation. This research proves that the following factors govern reservoir performance for created/enhanced fracture networks:

- Fracture tortuosity;

- The placement of the branching or bifurcation in the fracture;

- The orientation of the branches; and

- The number of branching stages

Each of these factors has an influence on the rate and pressure performance for a stimulated reservoir system. Although not an objective of this study, it is clear that we must establish how hydraulic fractures intersect/connect with the different types of fractures which were not been modeled in this study (i.e., natural, secondary and radial fractures). The connected fracture network results from the interaction 
between hydraulic fracture and the activation of other fracture networks present in the reservoir. (Cipolla et al. 2011) attempted to quantify this complexity via reservoir modeling. However, one could (and probably should) argue that there is no unique work-path to construct a fracture network model that accurately matches the microseismic data (particularly in light of the fact that we are not certain what physical processes the recorded microseismic events actually represent).

The stochastically created fracture networks used in this research are all based on the same fundamental "random walk" algorithm, and this could be construed as a limitation. Although our investigation was not "exhaustive" due to the tedious nature of the simulations (and their associated computational costs), we are very confident that the results of this research are both representative and reproducible. As we have successfully deployed the $\beta$-derivative function as our diagnostic for interpreting the nature of a given fracture network, we must acknowledge that this is an "aggregate" tool because there are an infinite number of stochastic fracture patterns that could generate a given production signature. 


\section{CHAPTER V}

\section{SUMMARY, CONCLUSIONS AND RECOMMENDATIONS FOR FUTURE WORK}

\section{V.1 Summary}

In this work we considered 83 different stochastic ("random-walk") fracture patterns generated to represent distinct hypothetical complex fracture networks which are created during hydraulic fracture stimulation. The (mass) production rate behavior and the pressure distributions for these fracture patterns were assessed using the FTSim code, a single-phase, single-component numerical reservoir simulator that was developed as a part of a course project taken in the Department of Petroleum Engineering at Texas A\&M University.

Using these stochastic ("random-walk") fracture pattern cases, we identified the effect of different geometric properties of the fractures on the flow behavior using the " $\beta$-derivative" of the mass flowrate generated by the simulator (we identified a specific, characteristic value of the " $\beta$-derivative" function for each set of "fracture branches"). We also utilized the pressure distribution maps created for each case to identify the periods when the "branches" for given fracture network were "interfering" (i.e., the pressure distributions intersect and aggregate).

As noted, the (mass) rate-time and (mass) rate-time $\beta$-derivative curves generated using the FTSim code were used to identify the signatures of different fracture patterns that were investigated. The cumulative gas recovery curves (percent recovery) were also used to assess the "efficiency" of the created stochastic fracture patterns. Using the $\beta$-derivative curves, an empirical model was created to predict the (constant) value of the $\beta$-derivative function for a given number of branching stages in a particular fracture pattern. We believe that this is a diagnostic feature that may be useful for assessing "stimulation efficiency" from field performance data. In a practical sense, assuming the observation of a particular (constant) value of the $\beta$-derivative obtained from production rate data, we can infer the number of fracture branching stages for a given well case.

\section{V.2 Conclusions}

- Early-time rate performance is an indicator of the geometry of the hydraulic fracture pattern.

- The $\beta$-derivative (mass rate) is observed to be directly related to the number of branching stages.

- The $\beta$-derivative (mass rate) for the single-plane hydraulic fracture is $1 / 2$ (one-half), which confirms the assumed theoretical performance for this case (i.e., non-interfering, linear flow).

- In practice, we can infer that for cases where the $\beta$-derivative is approximately equal to $1 / 2$, then linear flow essentially exists for all simple (i.e., near-planar) fracture patterns. 
- In terms of cumulative gas recovery, the more complex stochastic fracture patterns yield higher recoveries than the planar fracture case.

- While we observe a unique performance of the $\beta$-derivative function for a given set of fracture branch patterns, there are an infinite number of patterns which could yield such a signature.

\section{V.3 Recommendations for Future Work}

We suggest the following tasks for future work in the analysis/modeling of well performance behavior for wells with hydraulic fractures in ultra-low permeability/shale reservoirs:

- Create a 3-D "random-walk" fracture scheme to model fracture propagation in 3-dimensions (xyz). Our suggestion is that in this rendering, all fractures could first be in orthogonal planes (i.e., no slant or angled fractures).

- Extend the proposed approach from the case of a single fracture propagating from a single point of entry to the case of a multiply-fractured horizontal well in order to model/analyze the effect of fracture interference behavior (between fracture networks).

- Compare and contrast the proposed "random-walk" fracture patterns with fracture patterns observed in nature (outcrops, core, and in-situ studies if possible). The objective of such comparisons would be to understand the nature of induced (hydraulic) fractures in a field of natural fractures.

- As a major task, we recommend that the proposed "random-walk" fracture model be combined with some type(s) of stress field (which could simply be a penalty function). The ultimate objective of such a task would be to combine the random-walk (multi-branched fracture model) with a coupled flow/rock mechanics simulator.

- Develop workflows that help to estimate the extent and nature of random fracture patterns (the fracture extent, growth direction(s), etc.) in order to constrain a given stochastic (random-walk) fracture network.

- Continue/extend the current investigation to large production times (pseudosteady-state) to verify that a given fracture pattern dominates the early to mid-time performance, not late-time (pseudosteady-state/boundary-dominated flow). As part of such an investigation we recommend a "full-scale" reservoir model, as opposed to the "small-scale" reservoir model deployed in this study (recall that we employed a small-scale model to represent fractures on a centimeter scale to ensure resolution of the various flow features). 


\section{NOMENCLATURE}

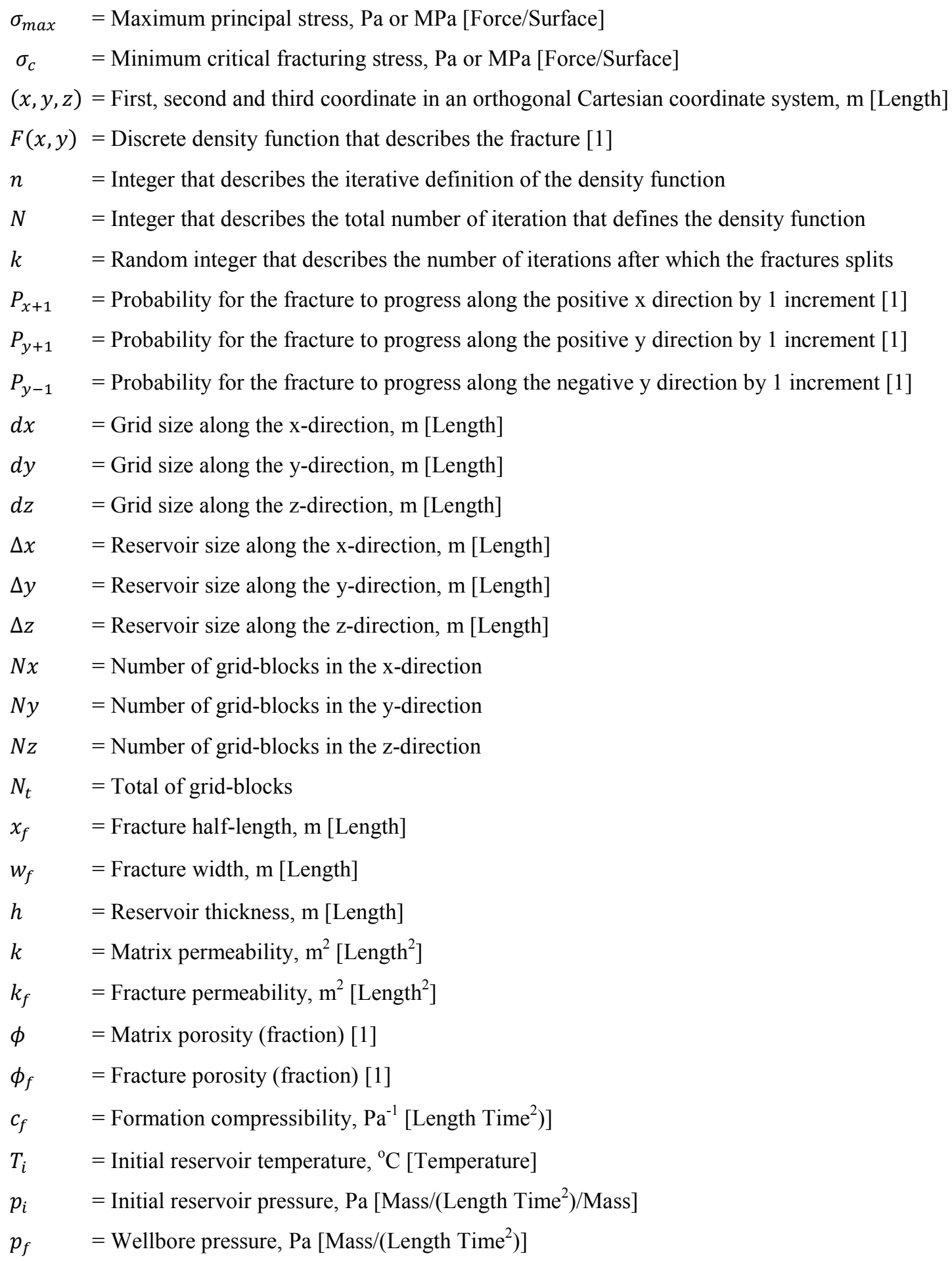




$$
\begin{aligned}
& r_{w e} \quad=\text { Equivalent well radius, } \mathrm{m} \text { [Length] } \\
& x_{F} \quad=\text { Fractured region length, } \mathrm{m} \text { [Length] } \\
& y_{F} \quad=\text { Fractured region width, } \mathrm{m} \text { [Length] } \\
& Z_{F} \quad=\text { Fractured region thickness, } \mathrm{m} \text { [Length] } \\
& x_{R} \quad=\text { Reservoir length, } \mathrm{m} \text { [Length] } \\
& y_{R} \quad=\text { Reservoir width, } \mathrm{m} \text { [Length] } \\
& \left(x_{n}, y_{n}\right)=\text { First and seconds coordinate of an orthogonal Cartesian system at the } \mathrm{n}^{\text {th }} \text { iteration, } \mathrm{m} \text { [Length] } \\
& E\left(y_{n}\right)=\text { Expected value of } y_{n}, \mathrm{~m} \text { [Length] } \\
& x_{\max }=\text { Maximum extent in the } \mathrm{x} \text {-direction of a stochastic fracture, } \mathrm{m} \text { [Length] } \\
& x_{\max 1}=\text { Maximum extent in the x-direction of a stochastic fracture after } N \text { iterations, } \mathrm{m} \text { [Length] } \\
& F_{0} \quad=\text { Stochastic fracture branch before any bifurcation stage } \\
& F_{01} \quad=\text { Upper Branch of the bifurcation of } F_{0}\left(1^{\text {st }} \text { bifurcation stage }\right) \\
& F_{02} . \quad=\text { Lower Branch of the bifurcation of } F_{0}\left(1^{\text {st }} \text { bifurcation stage }\right) \\
& F_{011}=\text { Upper Branch of the bifurcation of } F_{01}\left(2^{\text {nd }} \text { bifurcation stage }\right) \\
& \left.F_{012} \text {. } \quad=\text { Lower Branch of the bifurcation of } F_{01} \text { ( } 2^{\text {nd }} \text { bifurcation stage }\right) \\
& F_{021}=\text { Upper Branch of the bifurcation of } F_{02}\left(2^{\text {nd }} \text { bifurcation stage }\right) \\
& \left.F_{022} \text {. } \quad=\text { Lower Branch of the bifurcation of } F_{02} \text { ( } 2^{\text {nd }} \text { bifurcation stage }\right) \\
& F_{0111}=\text { Upper Branch of the bifurcation of } F_{011}\left(3^{\text {rd }} \text { bifurcation stage }\right) \\
& F_{0112} \text {. }=\text { Lower Branch of the bifurcation of } F_{011} \text { ( }{ }^{\text {rd }} \text { bifurcation stage) } \\
& F_{0121}=\text { Upper Branch of the bifurcation of } F_{012}\left(3^{\text {rd }} \text { bifurcation stage }\right) \\
& F_{0122} \text {. }=\text { Lower Branch of the bifurcation of } F_{012} \text { ( } 3^{\text {rd }} \text { bifurcation stage) } \\
& F_{0211}=\text { Upper Branch of the bifurcation of } F_{021}\left(3^{\text {rd }}\right. \text { bifurcation stage) } \\
& F_{0212} \text {. }=\text { Lower Branch of the bifurcation of } F_{021} \text { ( } 3^{\text {rd }} \text { bifurcation stage) } \\
& F_{0221}=\text { Upper Branch of the bifurcation of } F_{022}\left(3^{\text {rd }} \text { bifurcation stage }\right) \\
& F_{0222} . \quad=\text { Lower Branch of the bifurcation of } F_{022}\left(3^{\text {rd }} \text { bifurcation stage }\right) \\
& F_{01111}=\text { Upper Branch of the bifurcation of } F_{0111} \text { ( } 4^{\text {th }} \text { bifurcation stage) } \\
& F_{01112}=\text { Lower Branch of the bifurcation of } F_{0111}\left(4^{\text {th }}\right. \text { bifurcation stage) } \\
& F_{01121} \text {. }=\text { Upper Branch of the bifurcation of } F_{0112} \text { ( } 4^{\text {th }} \text { bifurcation stage) } \\
& F_{01122} \text {. }=\text { Lower Branch of the bifurcation of } F_{0112}\left(4^{\text {th }} \text { bifurcation stage }\right) \\
& F_{01211}=\text { Upper Branch of the bifurcation of } F_{0121}\left(4^{\text {th }} \text { bifurcation stage }\right) \\
& F_{01212}=\text { Lower Branch of the bifurcation of } F_{0121}\left(4^{\text {th }}\right. \text { bifurcation stage) } \\
& F_{01221} \text {. }=\text { Upper Branch of the bifurcation of } F_{0122} \text { ( } 4^{\text {th }} \text { bifurcation stage) } \\
& F_{01222} \text {. }=\text { Lower Branch of the bifurcation of } F_{0122}\left(4^{\text {th }} \text { bifurcation stage }\right) \\
& F_{02111}=\text { Upper Branch of the bifurcation of } F_{0211}\left(4^{\text {th }} \text { bifurcation stage }\right) \\
& F_{02112}=\text { Lower Branch of the bifurcation of } F_{0211}\left(4^{\text {th }} \text { bifurcation stage }\right)
\end{aligned}
$$


$F_{02121}$. $=$ Upper Branch of the bifurcation of $F_{0212}\left(4^{\text {th }}\right.$ bifurcation stage $)$

$F_{02122}$. $=$ Lower Branch of the bifurcation of $F_{0212}\left(4^{\text {th }}\right.$ bifurcation stage $)$

$F_{02221}$. $=$ Upper Branch of the bifurcation of $F_{0222}\left(4^{\text {th }}\right.$ bifurcation stage $)$

$F_{02222}$. $=$ Lower Branch of the bifurcation of $F_{0222}\left(4^{\text {th }}\right.$ bifurcation stage $)$

$x_{\max 0}=$ Maximum extent in the $\mathrm{x}$-direction of $F_{0}, \mathrm{~m}$ [Length]

$x_{\max 01}=$ Maximum extent in the $\mathrm{x}$-direction of $F_{01}, \mathrm{~m}$ [Length]

$x_{\max 02}=$ Maximum extent in the x-direction of $F_{02}, \mathrm{~m}$ [Length]

$k_{0} \quad=$ Number of iterations for $F_{0}$

$k_{01} \quad=$ Number of iterations for $F_{01}$

$k_{02} . \quad=$ Number of iterations for $F_{02}$

$k_{011}=$ Number of iterations for $F_{011}$

$k_{012}$. $=$ Number of iterations for $F_{012}$

$k_{021}=$ Number of iterations for $F_{021}$

$k_{022}$. $=$ Number of iterations for $F_{022}$

$k_{0111}=$ Number of iterations for $F_{0111}$

$k_{0112} . \quad=$ Number of iterations for $F_{0112}$

$k_{0121}=$ Number of iterations for $F_{0121}$

$k_{0122} . \quad=$ Number of iterations for $F_{0122}$

$k_{0211}=$ Number of iterations for $F_{0211}$

$k_{0212}$. $=$ Number of iterations for $F_{0212}$

$k_{0221}=$ Number of iterations for $F_{0221}$

$k_{0222} . \quad=$ Number of iterations for $F_{0222}$

$k_{01111}=$ Number of iterations for $F_{01111}$

$k_{01112}=$ Number of iterations for $F_{01112}$

$k_{01121}$. $=$ Number of iterations for $F_{01121}$

$k_{01122}$. $=$ Number of iterations for $F_{01122}$

$k_{01211}=$ Number of iterations for $F_{01211}$

$k_{01212}=$ Number of iterations for $F_{01212}$

$k_{01221}$. $=$ Number of iterations for $F_{01221}$

$k_{01222} .=$ Number of iterations for $F_{01222}$

$k_{02111}=$ Number of iterations for $F_{02111}$

$k_{02112}=$ Number of iterations for $F_{02112}$

$k_{02121}$. $=$ Number of iterations for $F_{02121}$

$k_{02122}$. $=$ Number of iterations for $F_{02122}$

$k_{02221}$. $=$ Number of iterations for $F_{02221}$ 


$$
\begin{array}{ll}
k_{02222} . & =\text { Number of iterations for } F_{02222} \\
f & =\text { Fraction of current pressure } p \text { over the initial pressure }\left(p_{i}\right)[1] \\
t & =\text { Time, } \mathrm{s}[\text { Time }] \\
q(t) & =\text { Mass rate at a time } t, \mathrm{Kg}^{-1} \mathrm{~s}^{-1}[\text { Mass/Time] } \\
d_{\beta} q(t) & =\beta \text { - derivative of } q(t)[1] \\
\beta & =\beta \text { - derivative of } q(t)[1] \\
p_{t} & \left.=\text { Pressure at time } t, \mathrm{~Pa}\left[\text { Mass/(Length Time }{ }^{2}\right)\right] \\
C R_{t} & =\text { Cumulative gas recovery (fraction or percent) at a time } t[1] \\
a & =\text { First calibration constant for the } \beta \text {-derivative empirical match [1] } \\
b & =\text { Second calibration constant for the } \beta \text {-derivative empirical match [1] } \\
c & =\text { Third calibration constant for the } \beta \text {-derivative empirical match [1] } \\
N_{b} & =\text { Number of branching stages in the stochastic fractures [1] }
\end{array}
$$




\section{REFERENCES}

Anderson, D.M., Nobakht, M., Moghadam, S. et al. 2010. Analysis of Production Data from Fractured Shale Gas Wells. Society of Petroleum Engineers. DOI: 10.2118/131787-MS.

Bello, R.O. and Wattenbarger, R.A. 2008. Rate Transient Analysis in Naturally Fractured Shale Gas Reservoirs. Society of Petroleum Engineers. DOI: 10.2118/114591-MS.

Blasingame, T.A. and Poe, B.D., Jr. 1993. Semianalytic Solutions for a Well with a Single FiniteConductivity Vertical Fracture. Society of Petroleum Engineers. DOI: 10.2118/26424-MS.

Cipolla, C.L., Fitzpatrick, T., Williams, M.J. et al. 2011. Seismic-to-Simulation for Unconventional Reservoir Development. Society of Petroleum Engineers. DOI: 10.2118/146876-MS.

Daguier, P., Bouchaud, E., and Lapasset, G. 1996. Model of Crack Propagation in Heterogeneous Materials Local Approach. Le Journal de Physique IV 6 (C6): C6-483-C486-490.

EIA. 2014. Annual Energy Outlook 2014. U.S. Energy Information Administration.

Geertsma, J. and De Klerk, F. 1969. A Rapid Method of Predicting Width and Extent of Hydraulically Induced Fractures. DOI: 10.2118/2458-PA

Gringarten, A.C., Ramey, H.J., Jr., and Raghavan, R. 1974. Unsteady-State Pressure Distributions Created by a Well with a Single Infinite-Conductivity Vertical Fracture. DOI: 10.2118/4051-PA

Hossain, M., Rahman, M., and Rahman, S. 2000. Hydraulic Fracture Initiation and Propagation: Roles of Wellbore Trajectory, Perforation and Stress Regimes. Journal of petroleum science and engineering 27 (3): 129-149.

Houzé, O., Tauzin, E., Artus, V. et al. 2010. The Analysis of Dynamic Data in Shale Gas Reservoirs-Part 1. Company report, Kappa Engineering, Houston, Texas, USA.

Li, Y., Wei, C., Qin, G. et al. 2012. Optimizing Hydraulic Fracturing Design for Shale Gas Production through Numerical Simulations. Society of Petroleum Engineers. DOI: 10.2118/157411-MS.

Mastorakos, I., Gallos, L.K., and Aifantis, E.C. 2003. Computer Simulation of Discrete Crack Propagation. Journal of the Mechanical Behavior of Materials 14 (1): 9-22.

Mattar, L. 2008. Production Analysis and Forecasting of Shale Gas Reservoirs: Case History-Based Approach. Society of Petroleum Engineers. DOI: 10.2118/119897-MS.

Maxwell, S.C., Waltman, C., Warpinski, N.R. et al. 2006. Imaging Seismic Deformation Induced by Hydraulic Fracture Complexity. Society of Petroleum Engineers. DOI: 10.2118/102801-MS.

Medeiros, F., Ozkan, E., and Kazemi, H. 2006. A Semianalytical, Pressure-Transient Model for Horizontal and Multilateral Wells in Composite, Layered, and Compartmentalized Reservoirs. Society of Petroleum Engineers. DOI: 10.2118/102834-MS.

Meyer, B.R. and Bazan, L.W. 2011. A Discrete Fracture Network Model for Hydraulically Induced Fractures - Theory, Parametric and Case Studies. Society of Petroleum Engineers. DOI: 10.2118/140514-MS.

Moridis, G. 2008. Tough+ Hydrate V1. 0 User's Manual: A Code for the Simulation of System Behavior in Hydrate-Bearing Geologic Media. 
Moridis, G.J., Blasingame, T.A., and Freeman, C.M. 2010. Analysis of Mechanisms of Flow in Fractured Tight-Gas and Shale-Gas Reservoirs. Society of Petroleum Engineers. DOI: 10.2118/139250-MS.

Olorode, O., Freeman, C.M., Moridis, G. et al. 2013. High-Resolution Numerical Modeling of Complex and Irregular Fracture Patterns in Shale-Gas Reservoirs and Tight Gas Reservoirs. DOI: $10.2118 / 152482-\mathrm{PA}$

Olorode, O.M., Freeman, C.M., Moridis, G.J. et al. 2012. High-Resolution Numerical Modeling of Complex and Irregular Fracture Patterns in Shale Gas and Tight Gas Reservoirs. Society of Petroleum Engineers. DOI: 10.2118/152482-MS.

Olson, J. 2008. Multi-Fracture Propagation Modeling: Applications to Hydraulic Fracturing in Shales and Tight Gas Sands. In The 42nd US rock mechanics symposium (USRMS): American Rock Mechanics Association.

Pearson, K. 1905. The Problem of the Random-walk. Nature 72 (1865): 294.

Perkins, T.K. and Kern, L.R. 1961. Widths of Hydraulic Fractures. DOI: 10.2118/89-PA

Roussel, N.P., Florez, H., and Rodriguez, A.A. 2013. Hydraulic Fracture Propagation from Infill Horizontal Wells. Society of Petroleum Engineers. DOI: 10.2118/166503-MS.

Shelley, R.F., Lolon, E., Dzubin, B. et al. 2010. Quantifying the Effects of Well Type and Hydraulic Fracture Selection on Recovery for Various Reservoir Permeabilities Using a Numerical Reservoir Simulator. Society of Petroleum Engineers. DOI: 10.2118/133985-MS.

Weng, X., Kresse, O., Cohen, C.E. et al. 2011. Modeling of Hydraulic Fracture Network Propagation in a Naturally Fractured Formation. Society of Petroleum Engineers. DOI: 10.2118/140253-MS.

Xu, W., Thiercelin, M.J., Ganguly, U. et al. 2010. Wiremesh: A Novel Shale Fracturing Simulator. Society of Petroleum Engineers. DOI: 10.2118/132218-MS.

Zhou, W., Banerjee, R., Poe, B.D. et al. 2012. Semi-Analytical Production Simulation of Complex Hydraulic Fracture Network. Society of Petroleum Engineers. DOI: 10.2118/157367-MS. 
APPENDIX A

SIMULATED STOCHASTIC FRACTURE PATTERNS

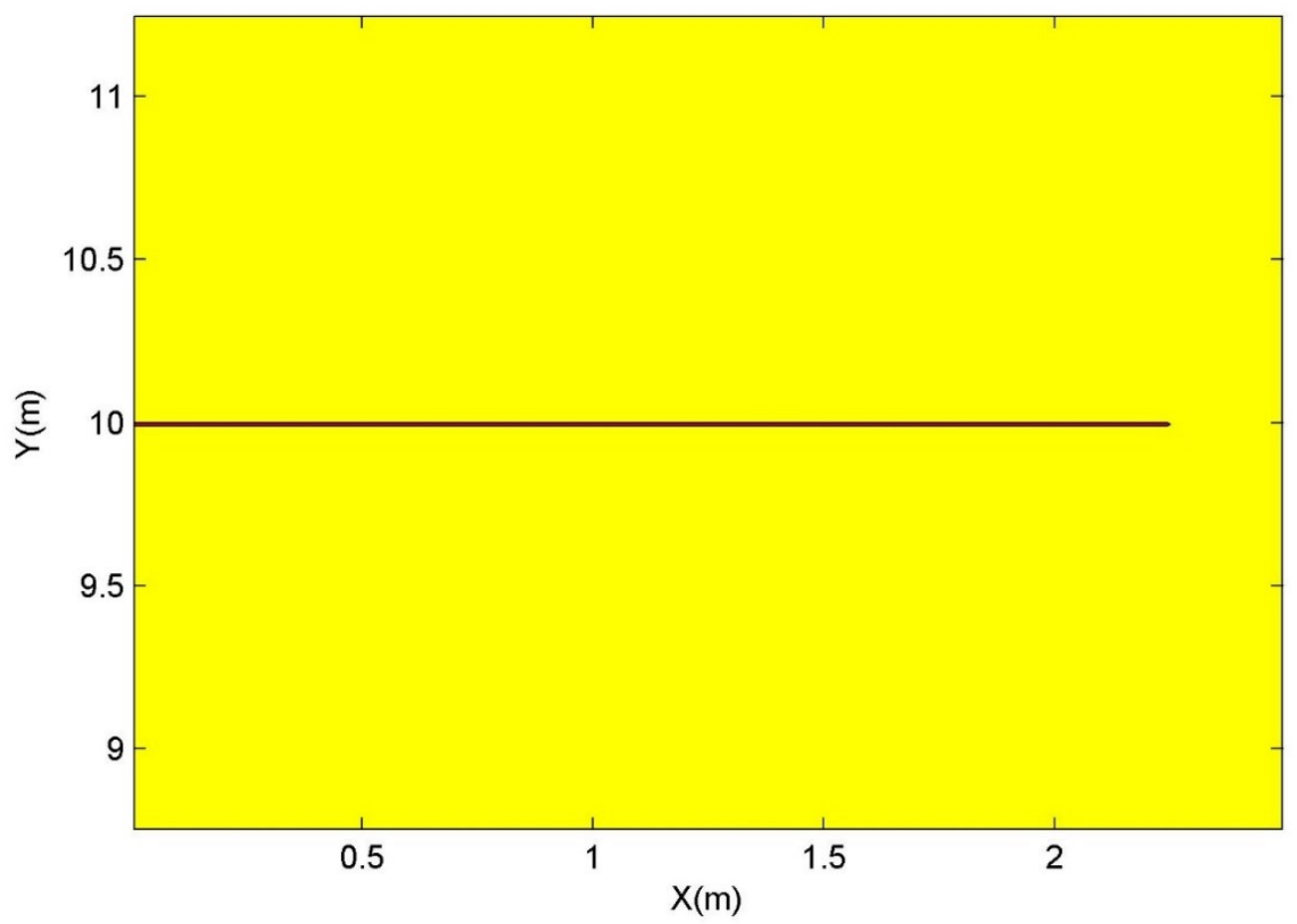

Figure A. $1 \quad$ Planar fracture. 


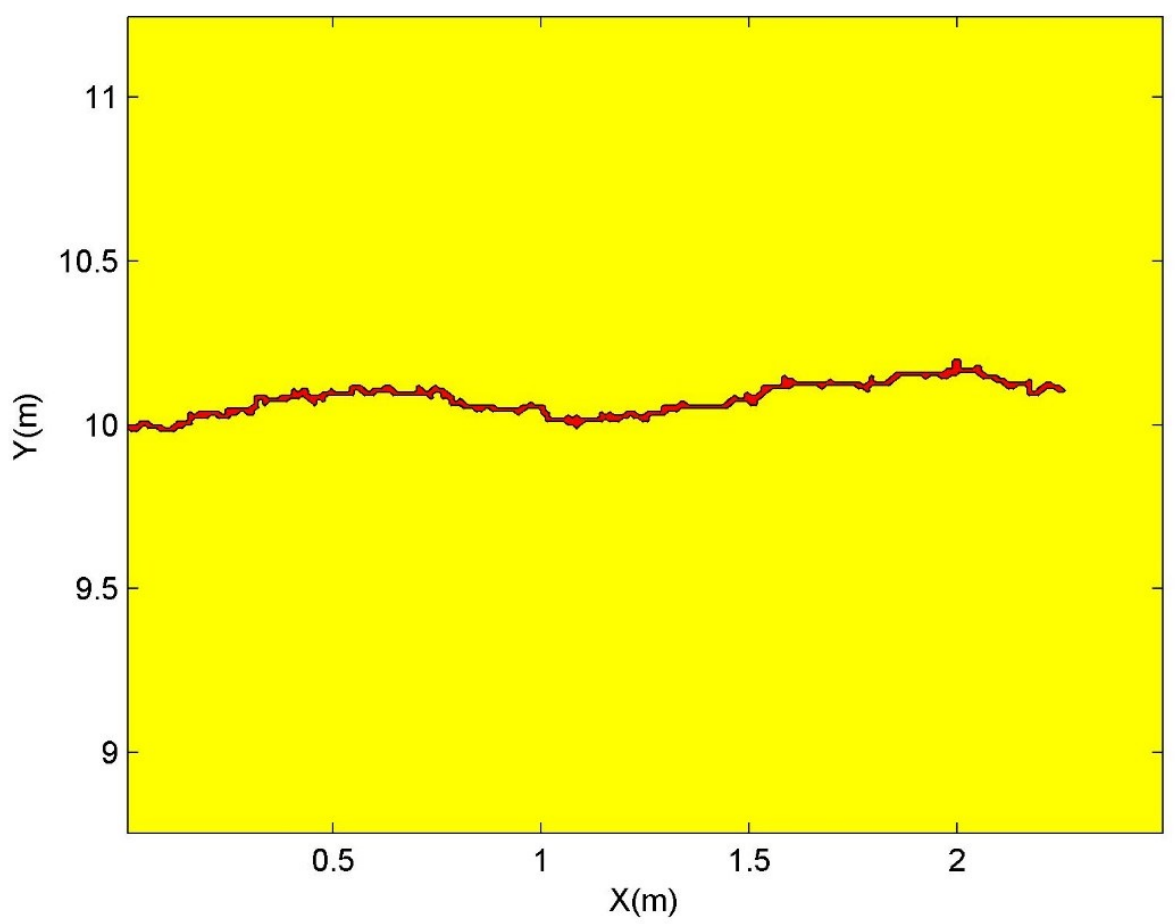

Figure A. 2 - Non-branched fracture 1.

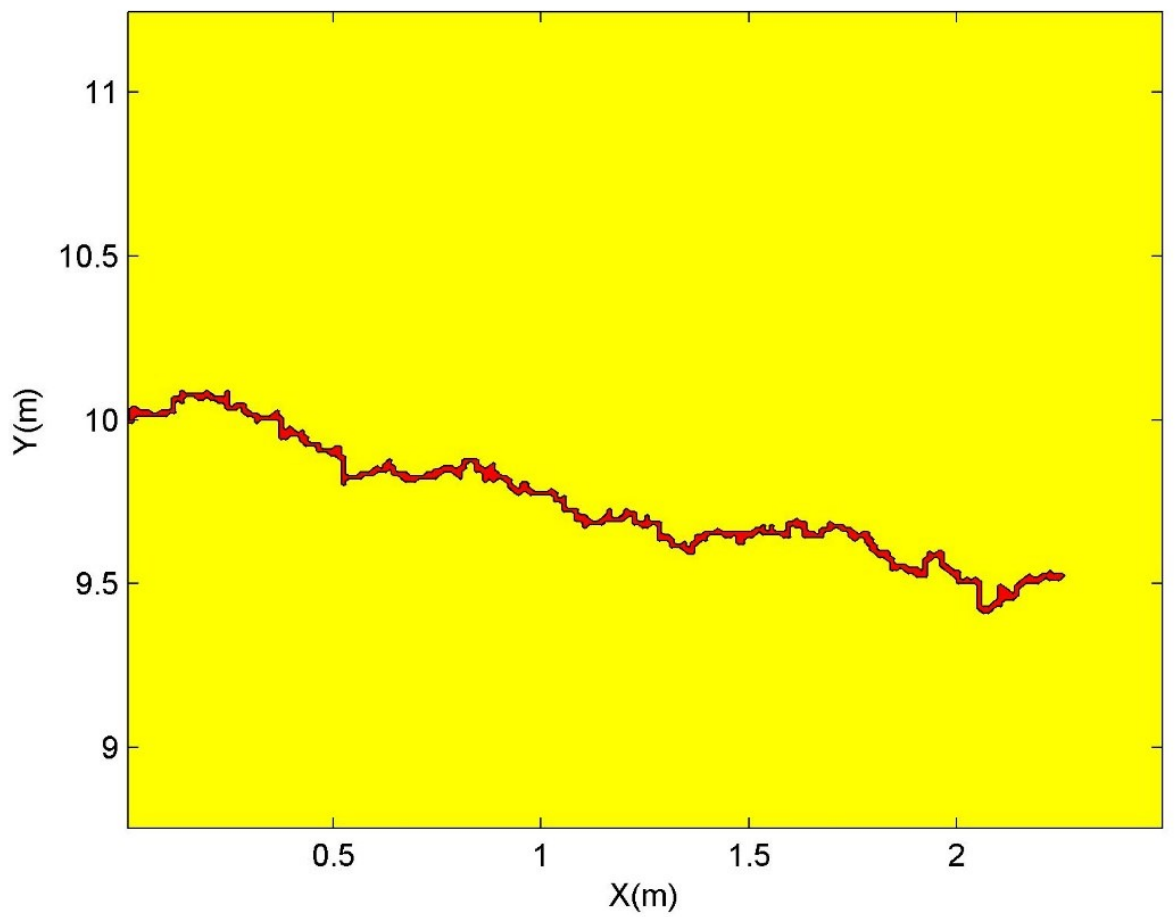

Figure A. 3 - Non-branched fracture 2. 


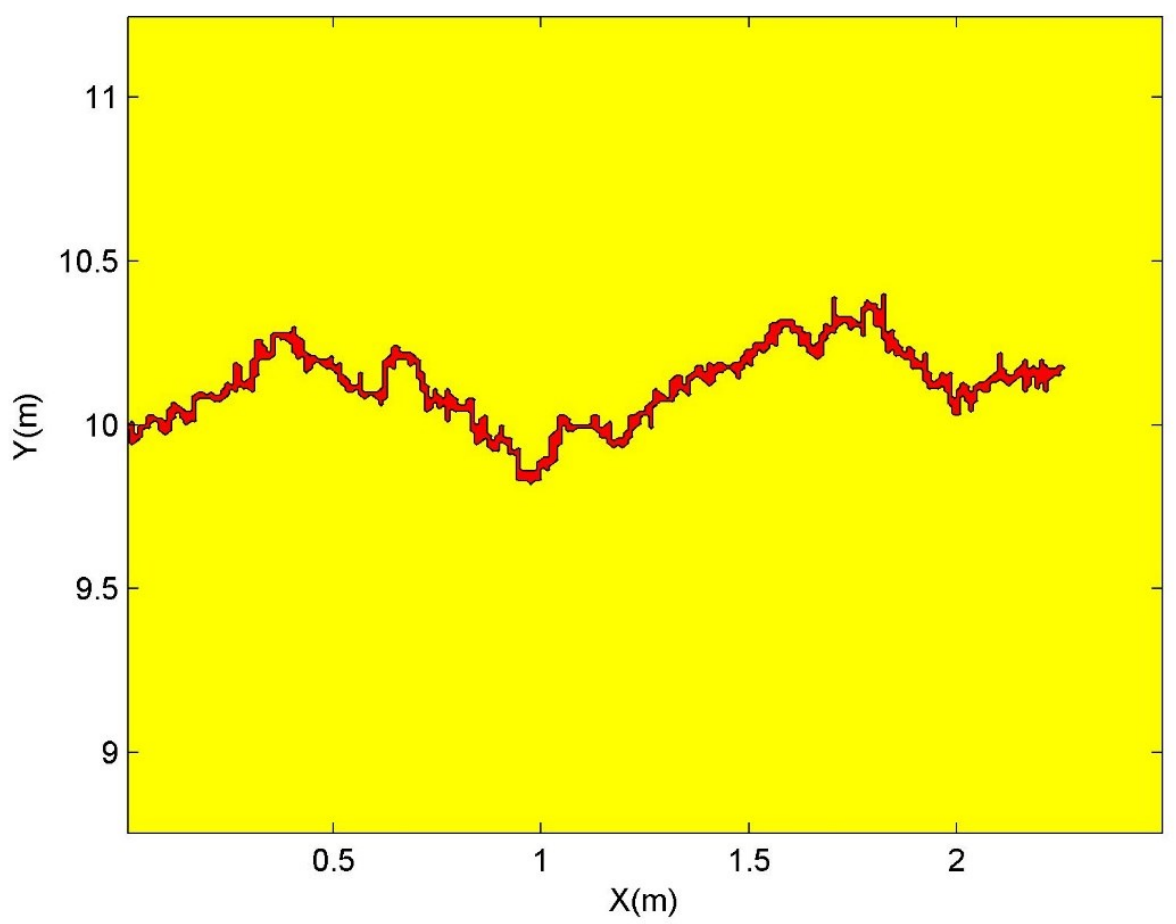

Figure A. 4 - Non-branched fracture 3.

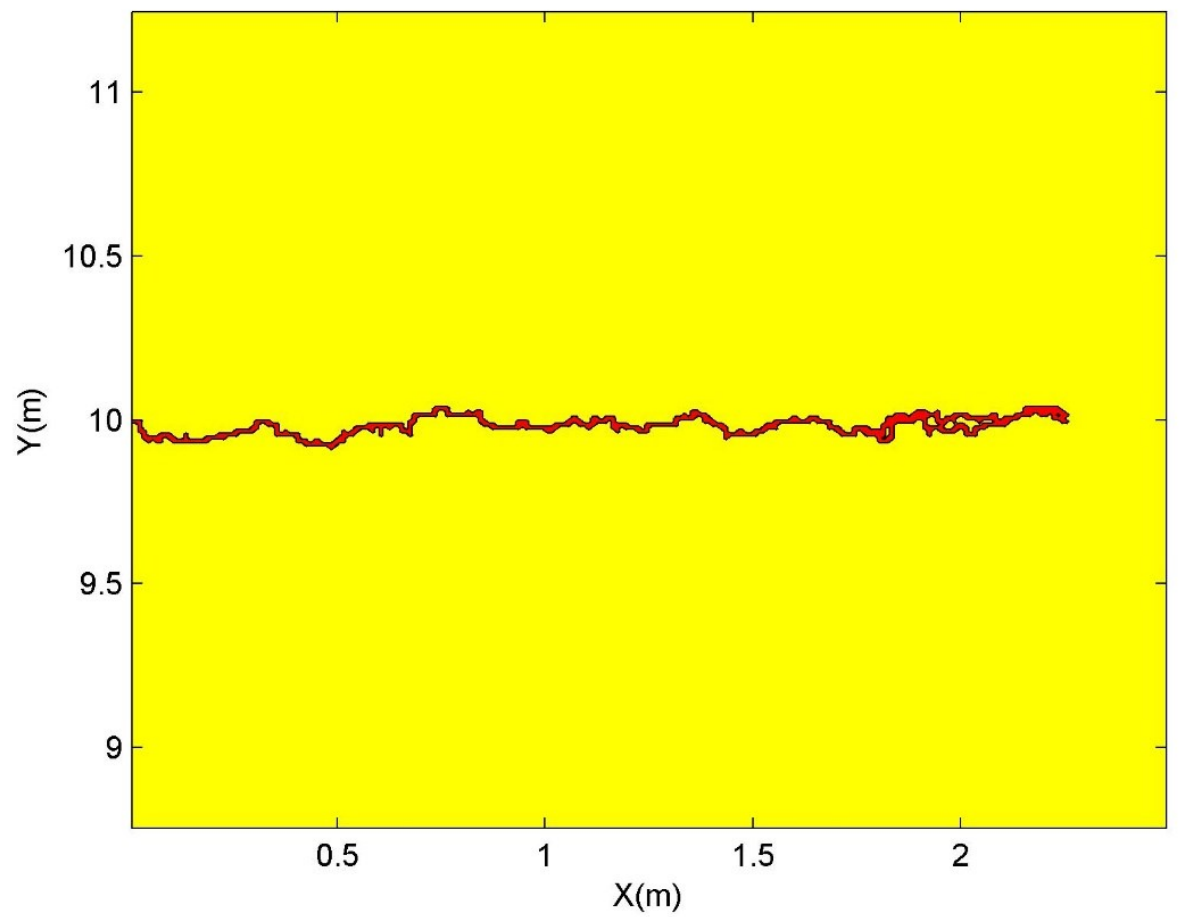

Figure A. 5 - Mono-branched fracture 1. 


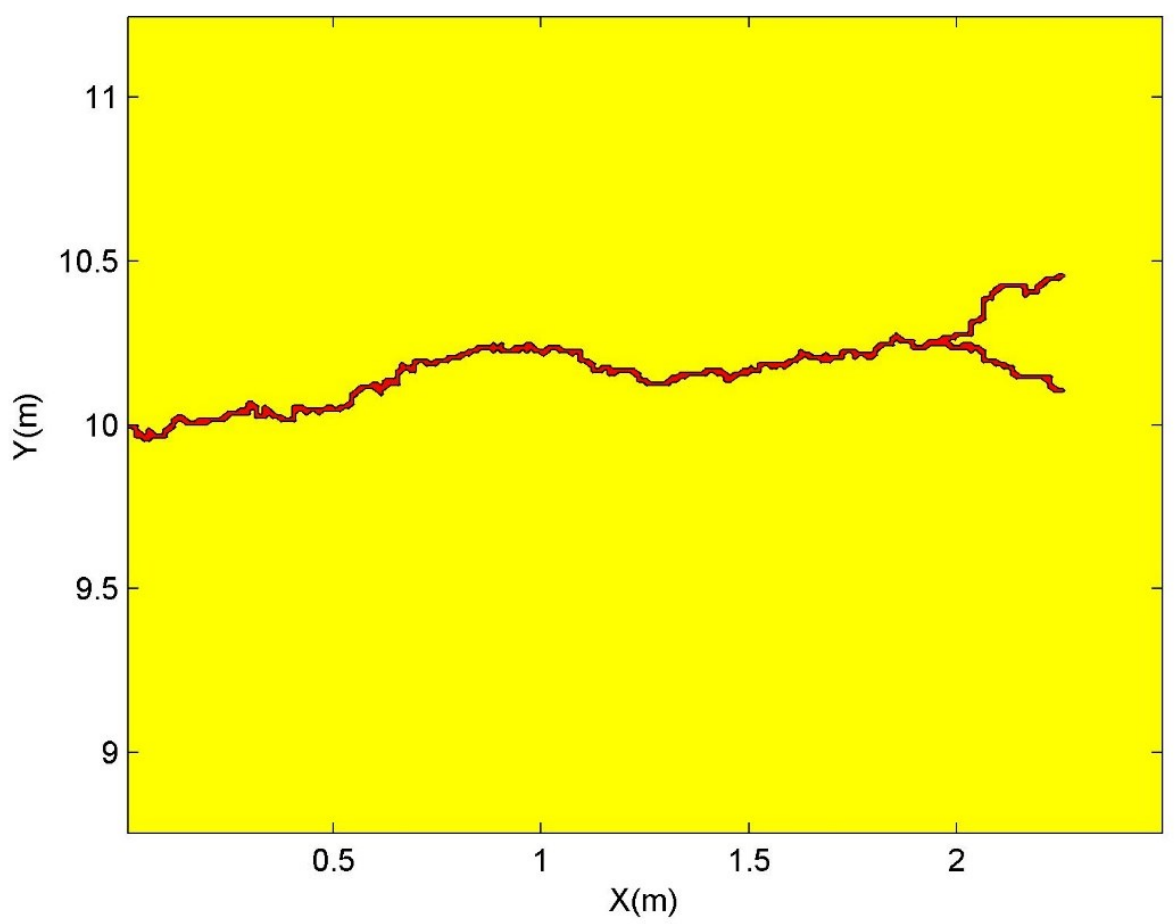

Figure A. 6 - Mono-branched fracture 2.

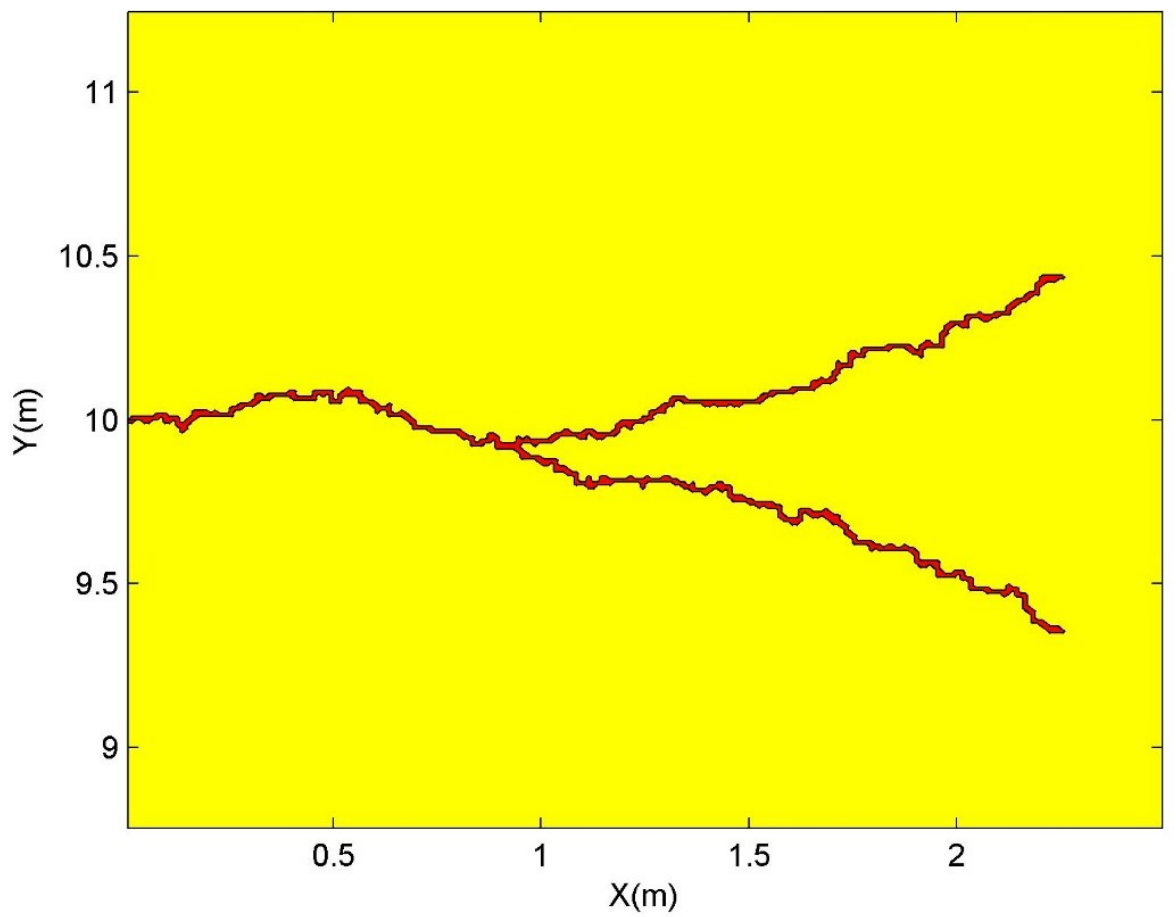

Figure A. 7 Mono-branched fracture 3. 


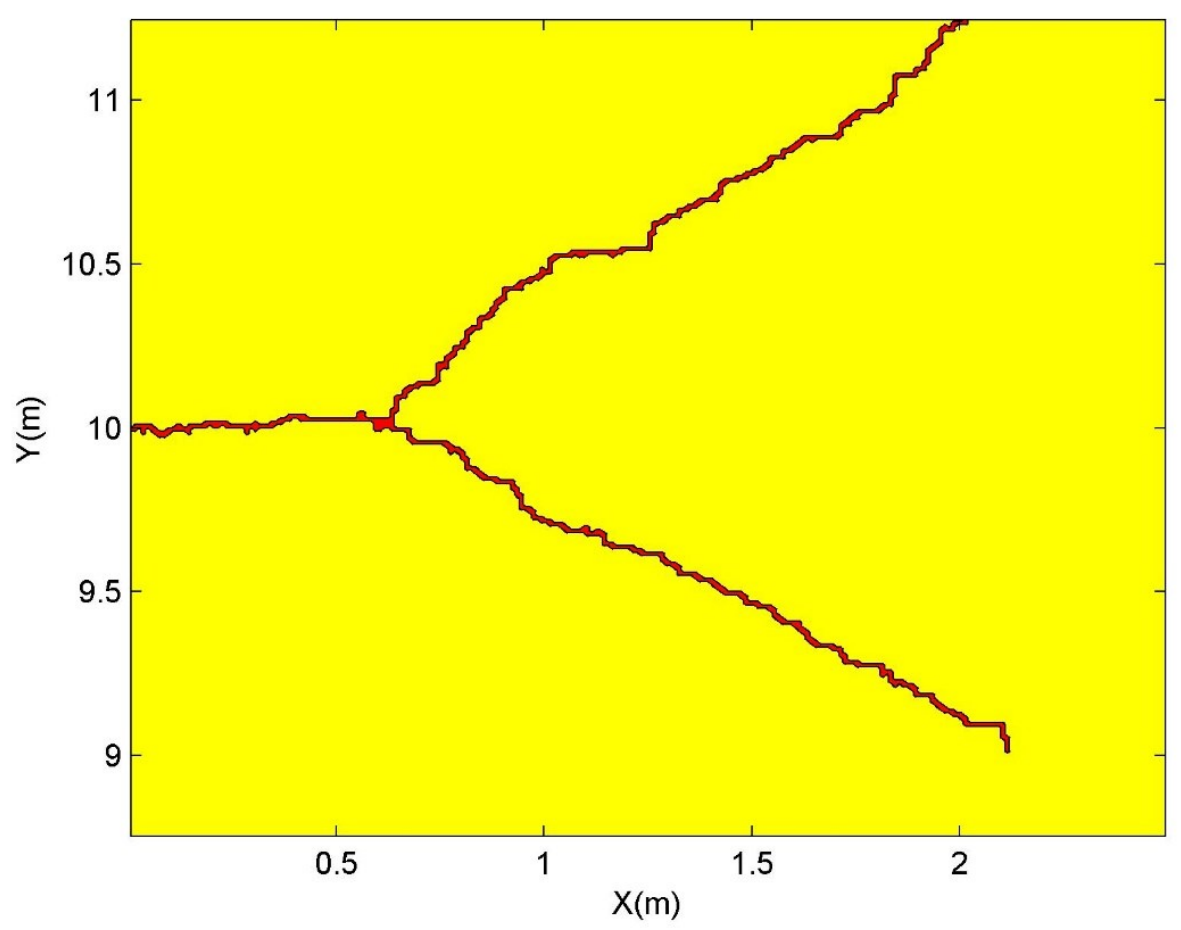

Figure A. 8 - Mono-branched fracture 4.

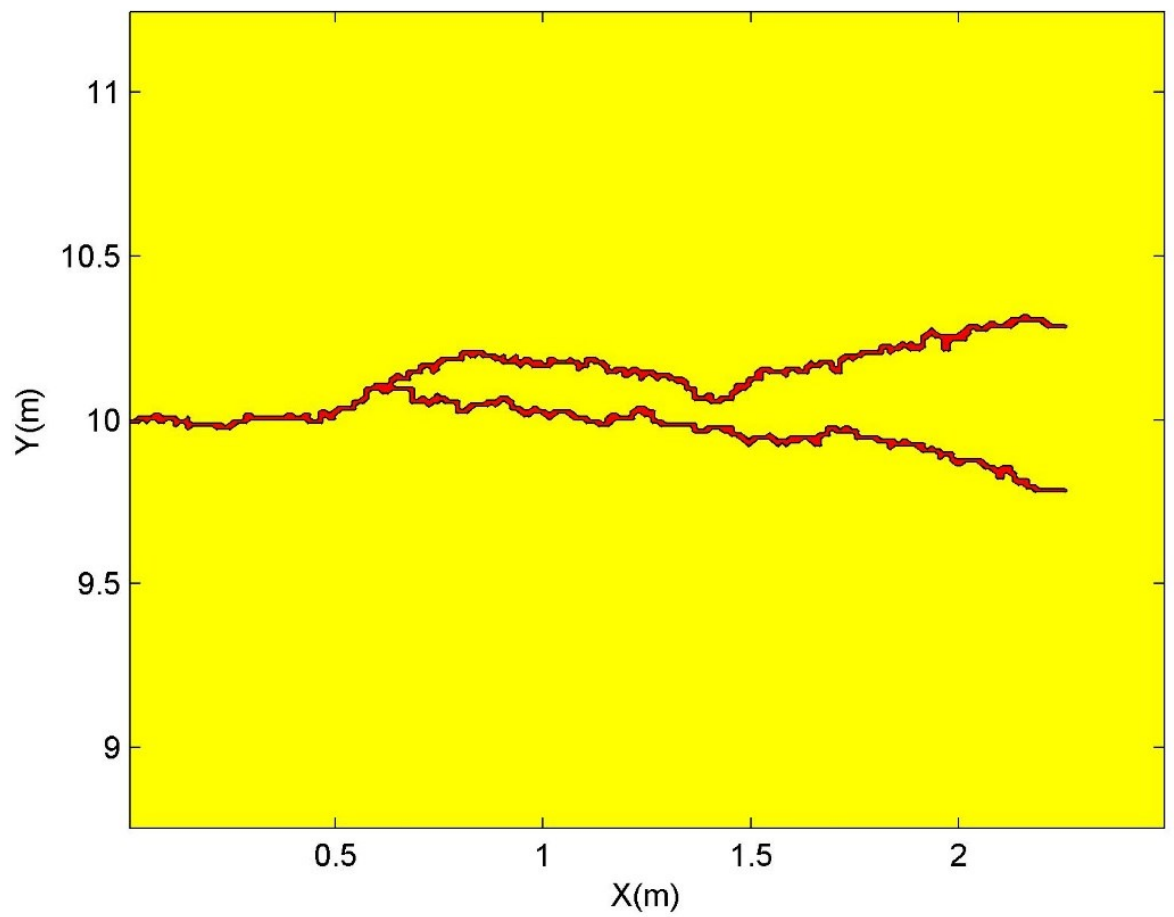

Figure A. 9 - Mono-branched fracture 5. 


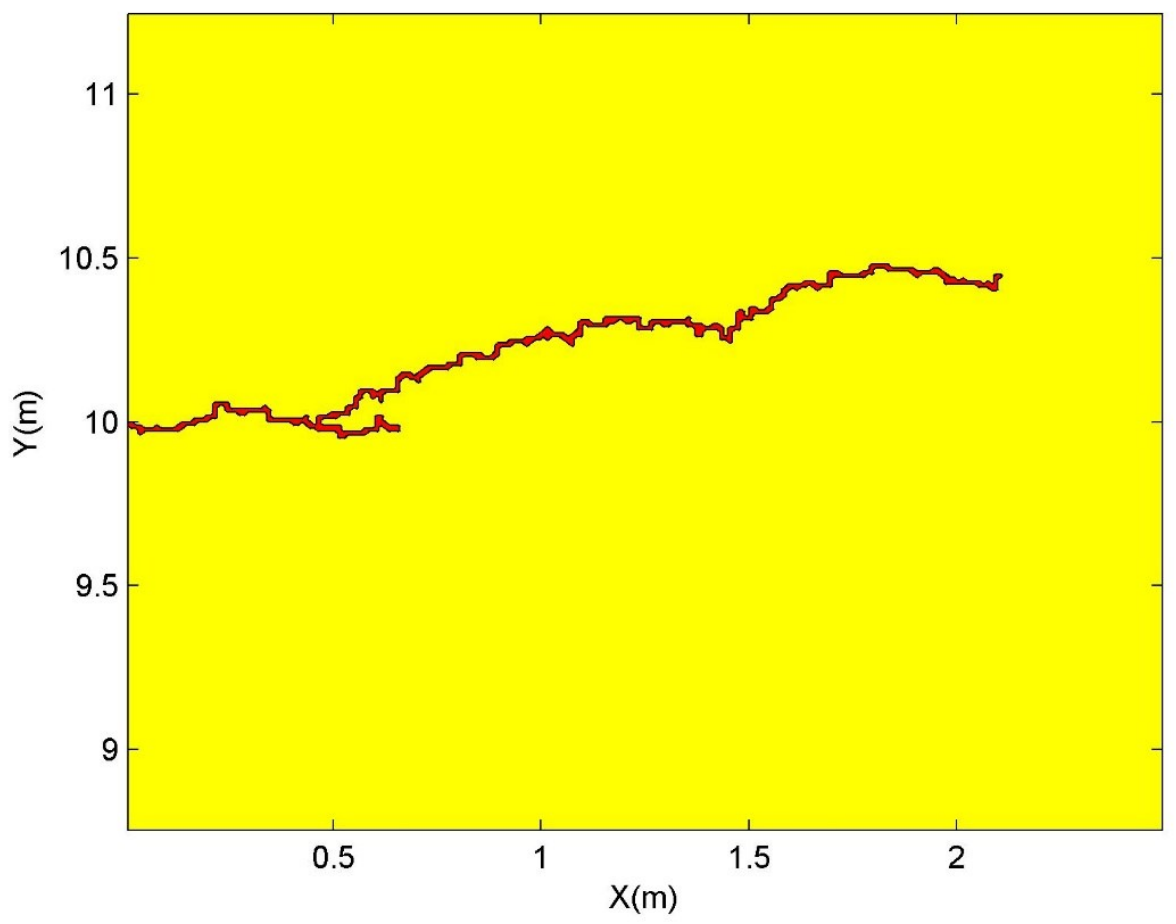

Figure A. 10 - Mono-branched fracture 6.

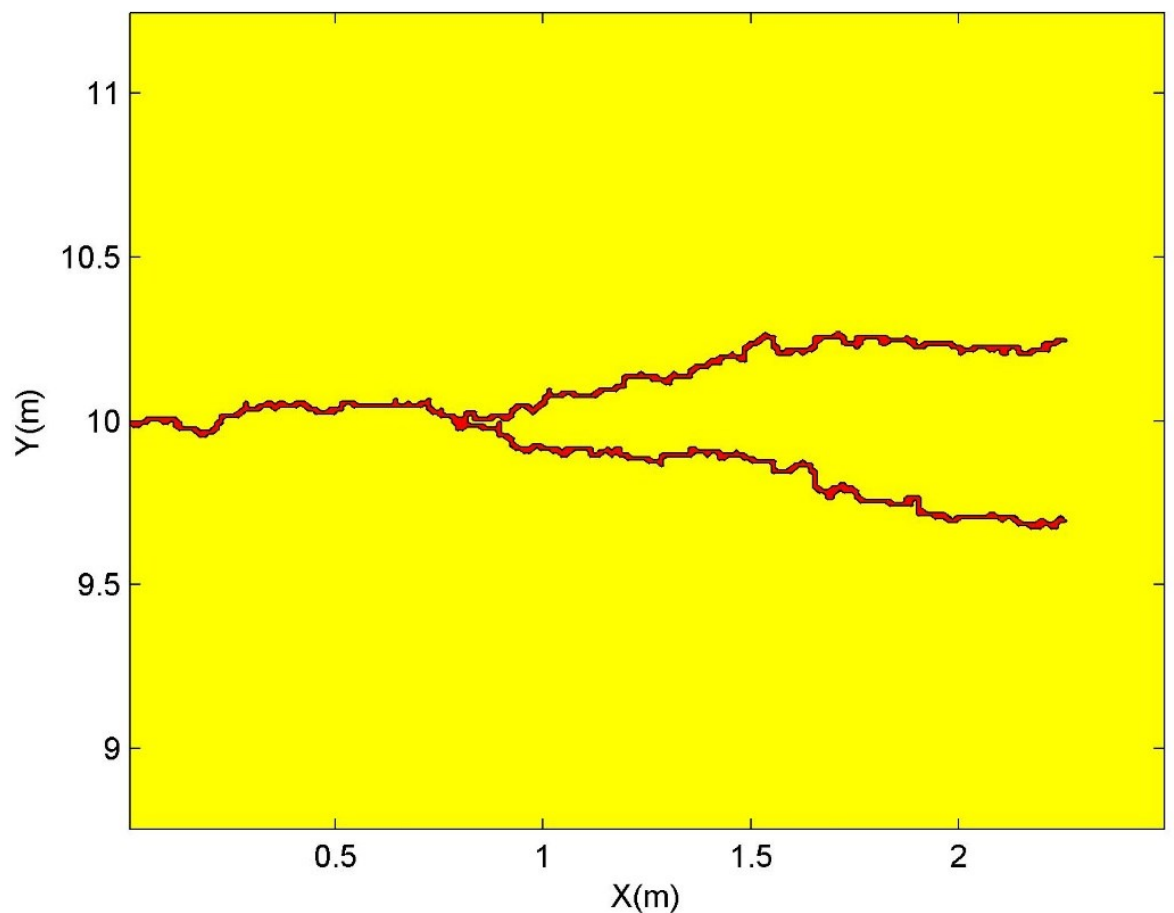

Figure A. 11 - Mono-branched fracture 7. 


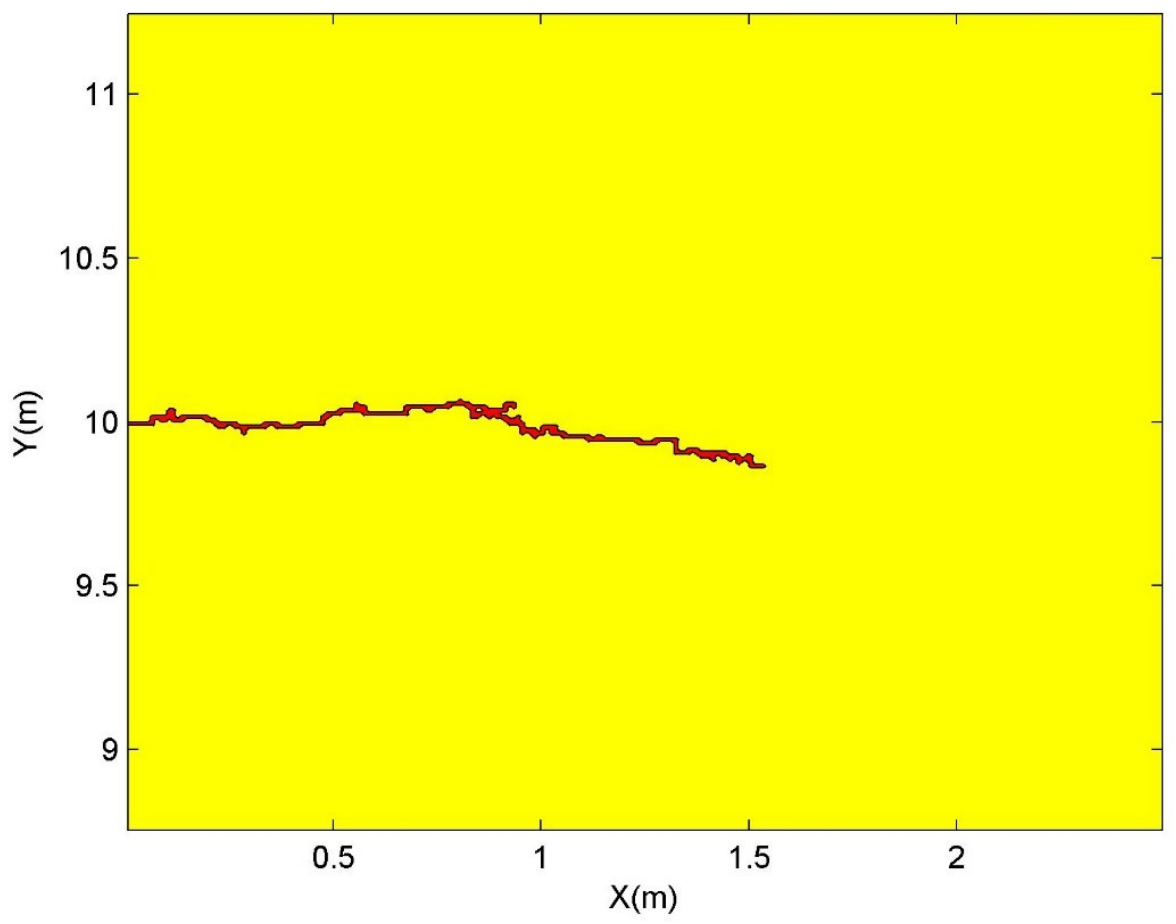

Figure A. 12 - Mono-branched fracture 8.

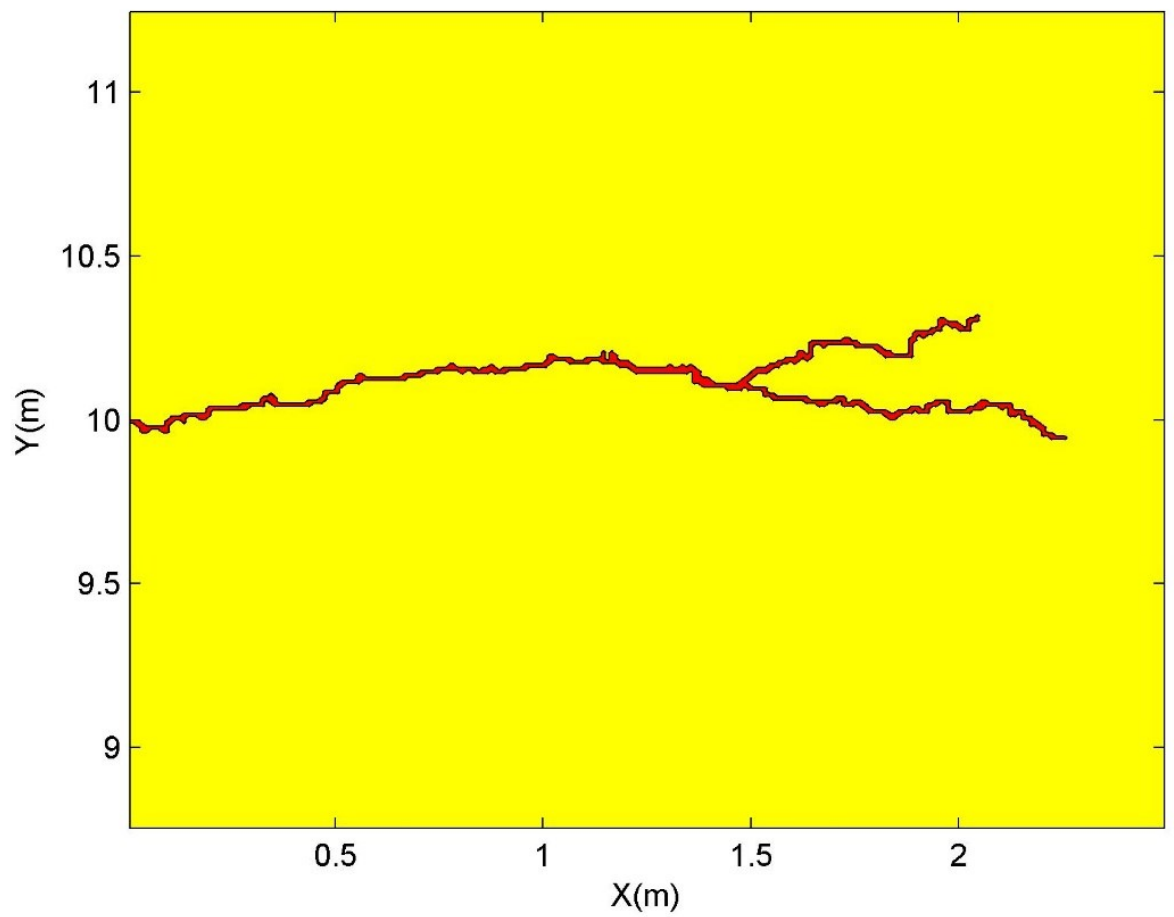

Figure A. 13 - Mono-branched fracture 9. 


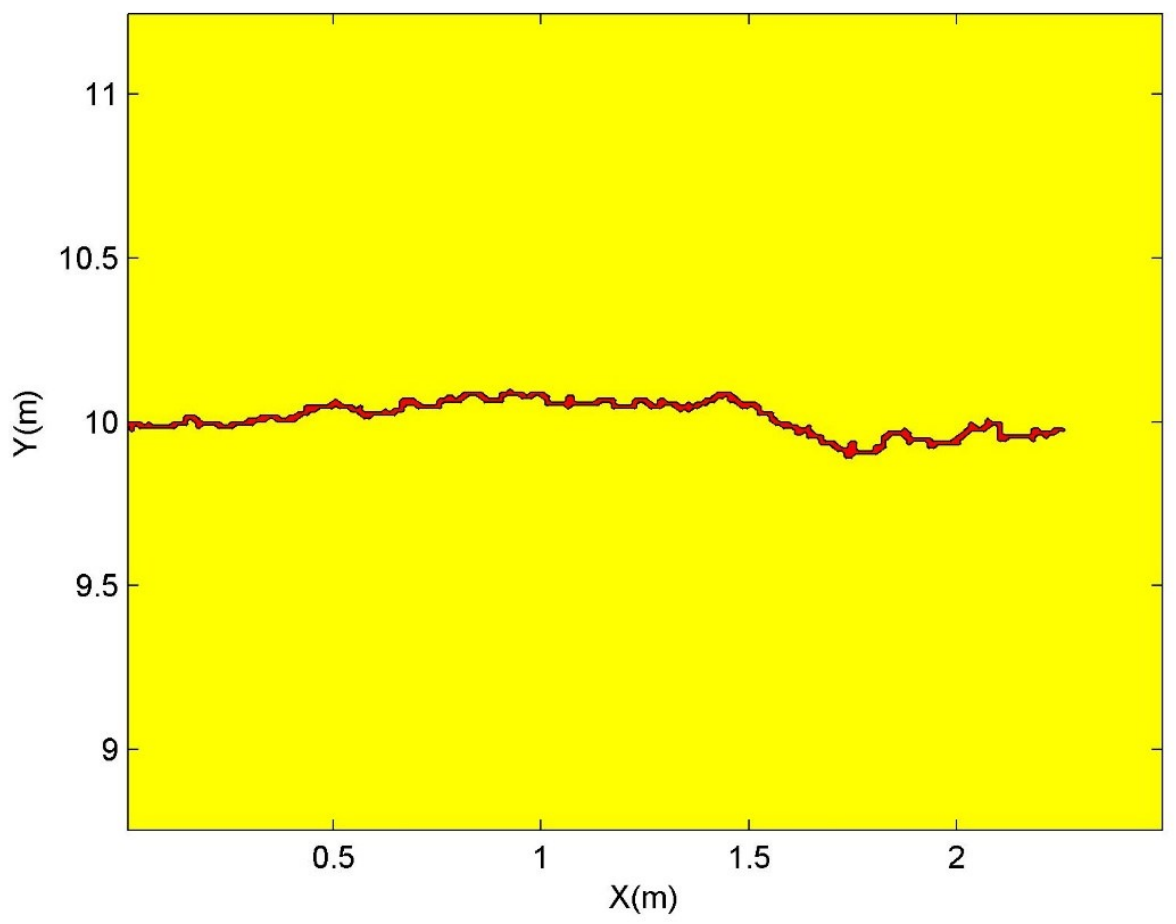

Figure A. 14 - Mono-branched fracture 10.

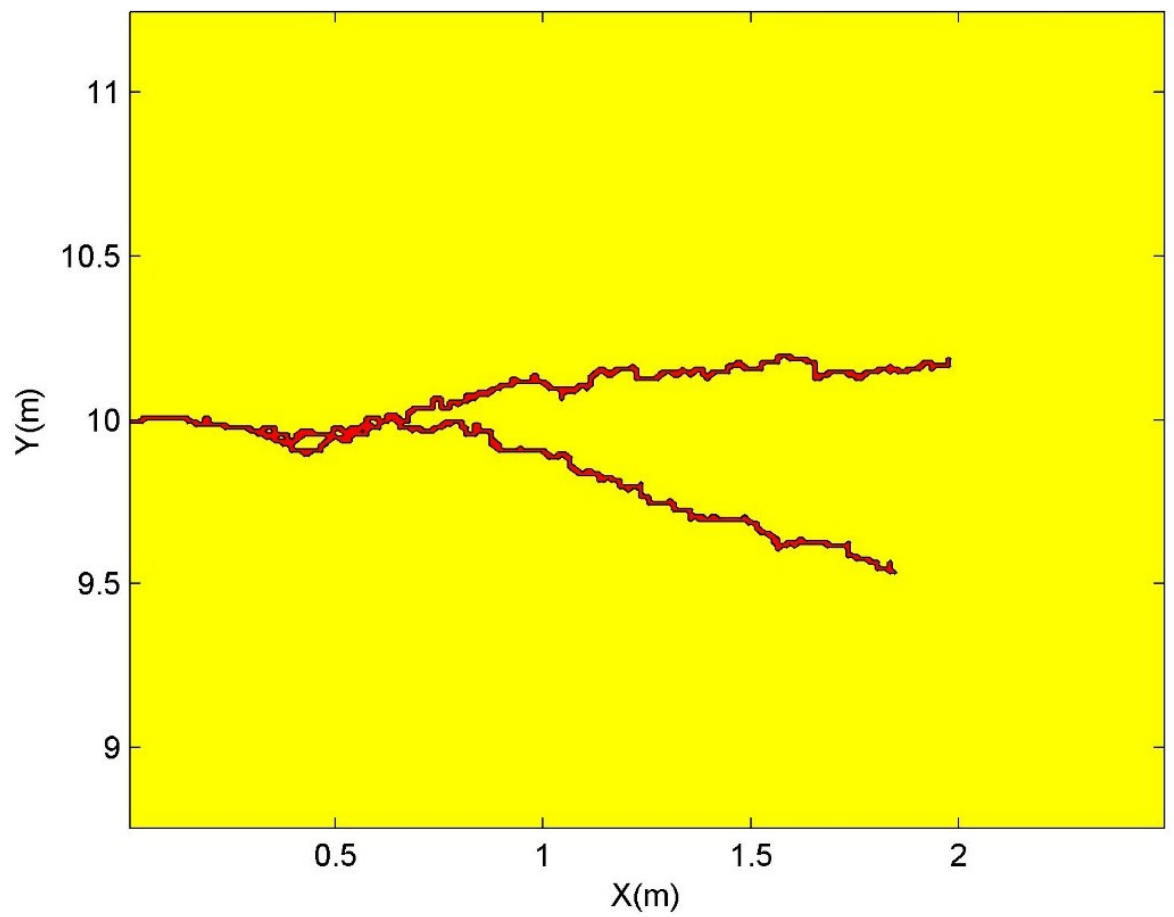

Figure A. 15 - Mono-branched fracture 11. 


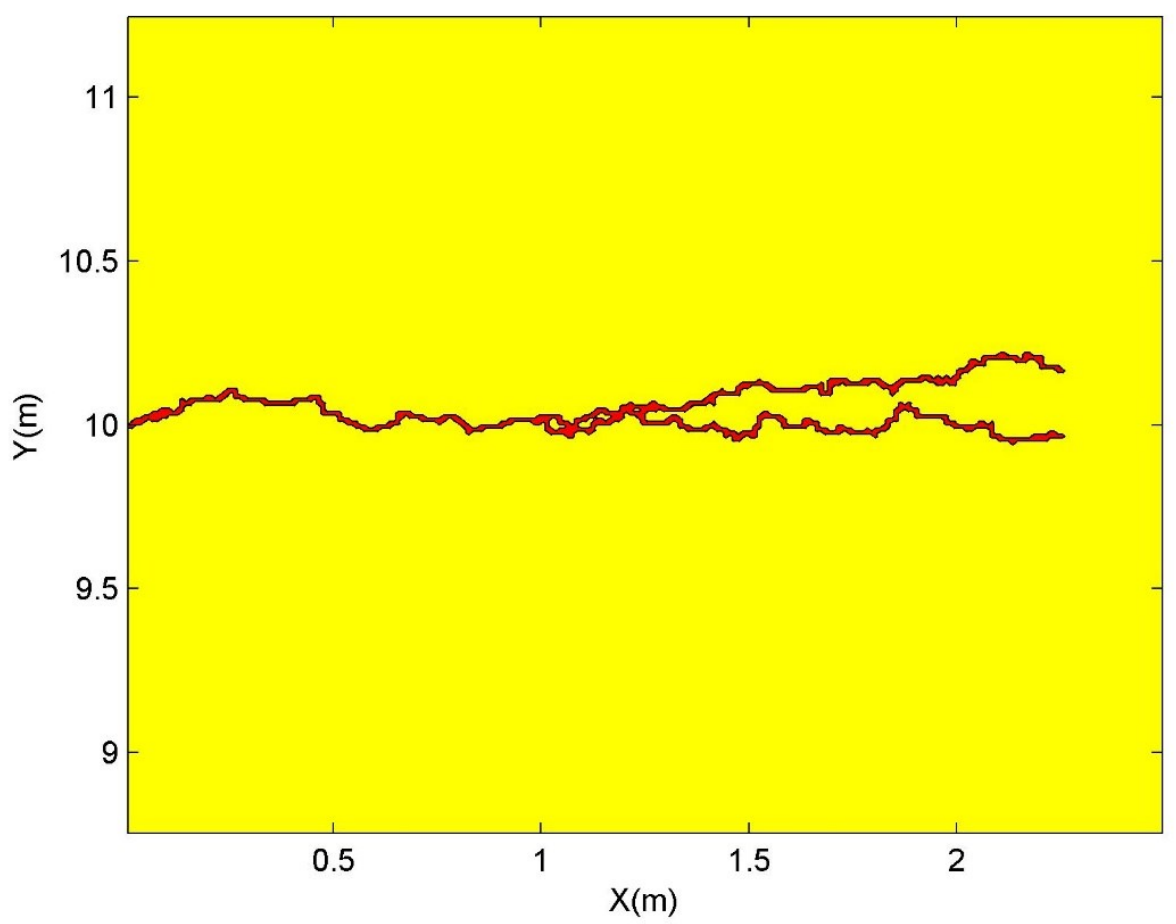

Figure A. 16 - Mono-branched fracture 12.

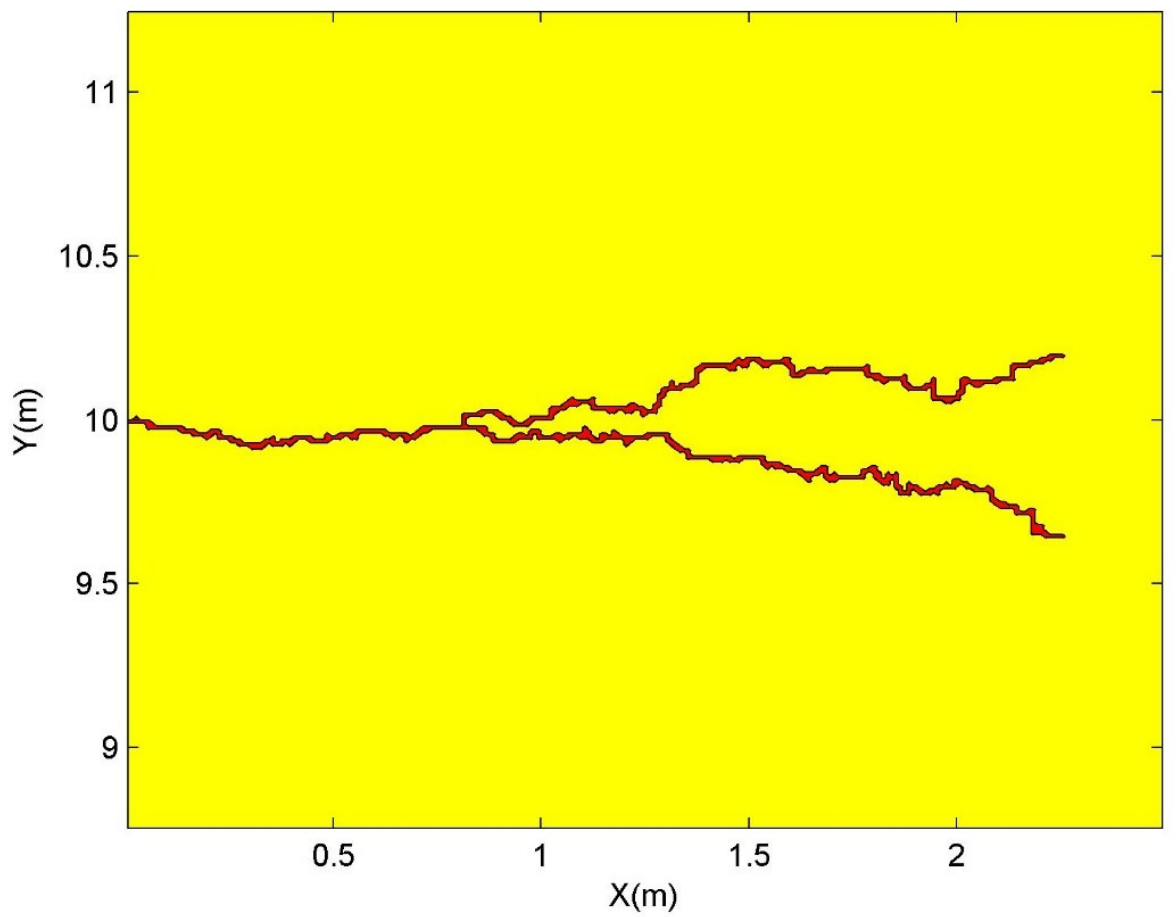

Figure A. 17 - Mono-branched fracture 13. 


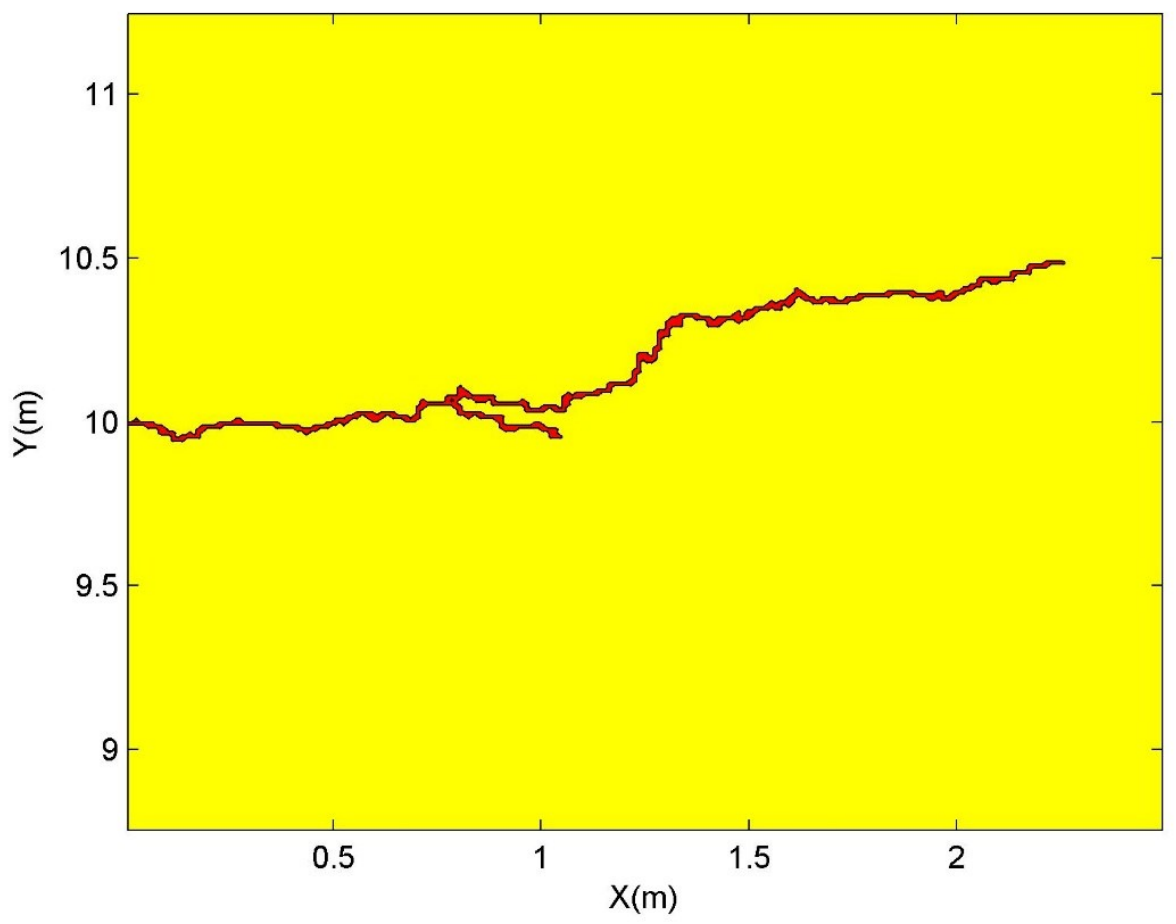

Figure A. 18 - Mono-branched fracture 14.

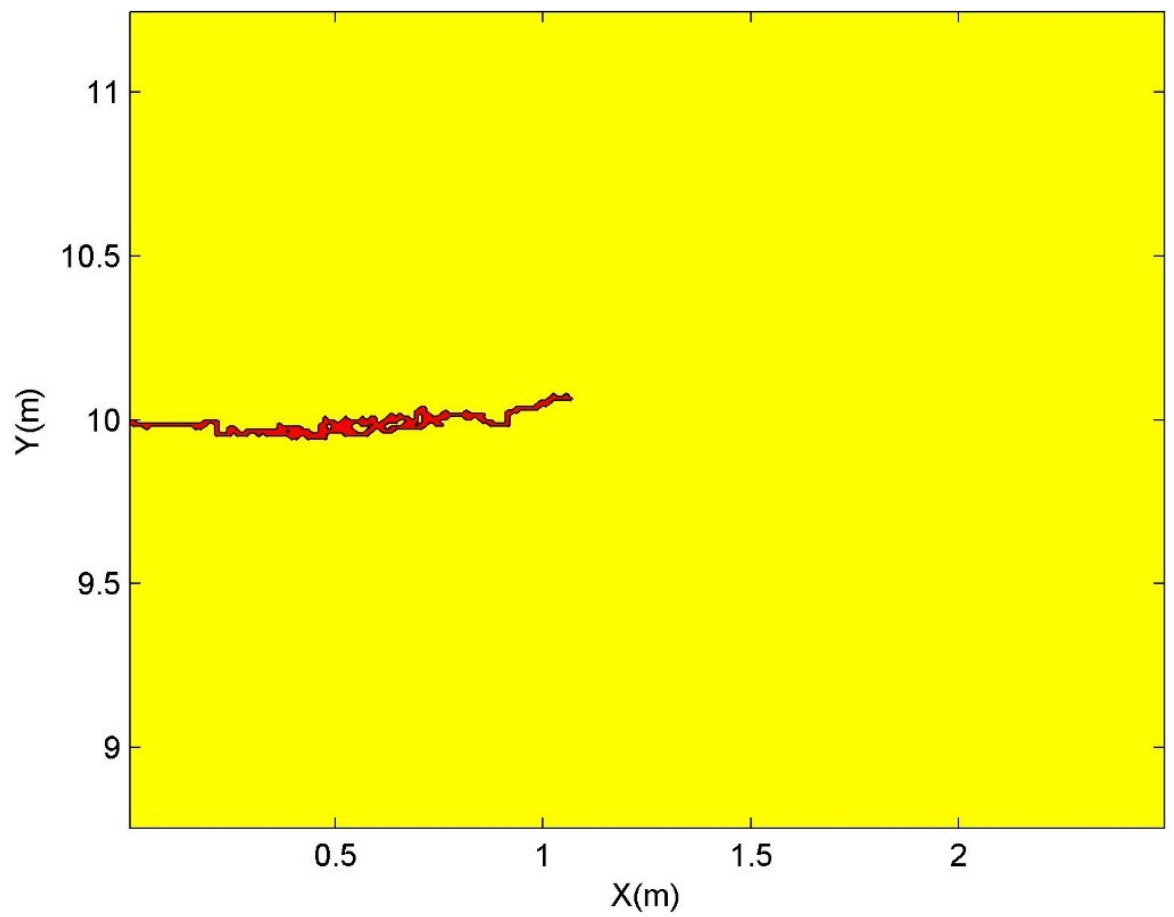

Figure A. 19 - Mono-branched fracture 15. 


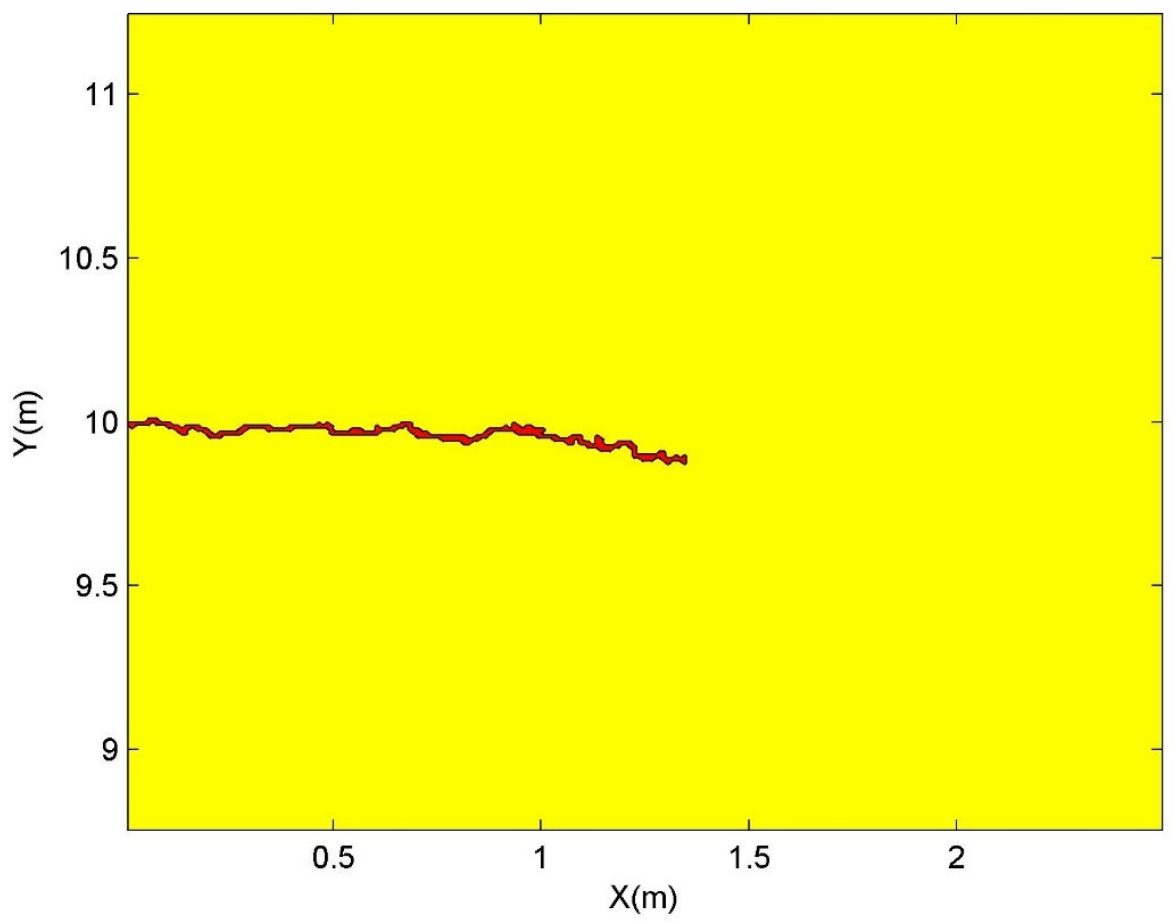

Figure A. 20 - Mono-branched fracture 16.

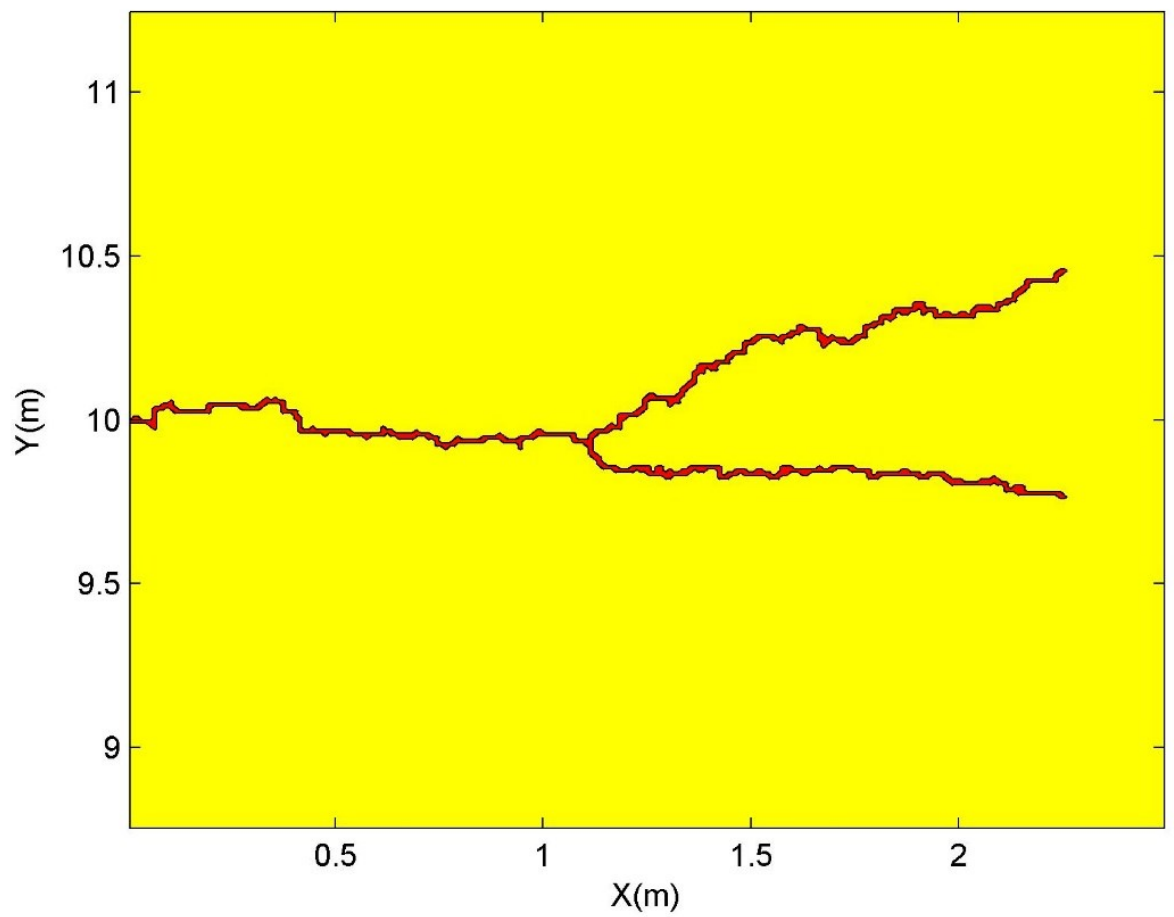

Figure A. 21 - Mono-branched fracture 17. 


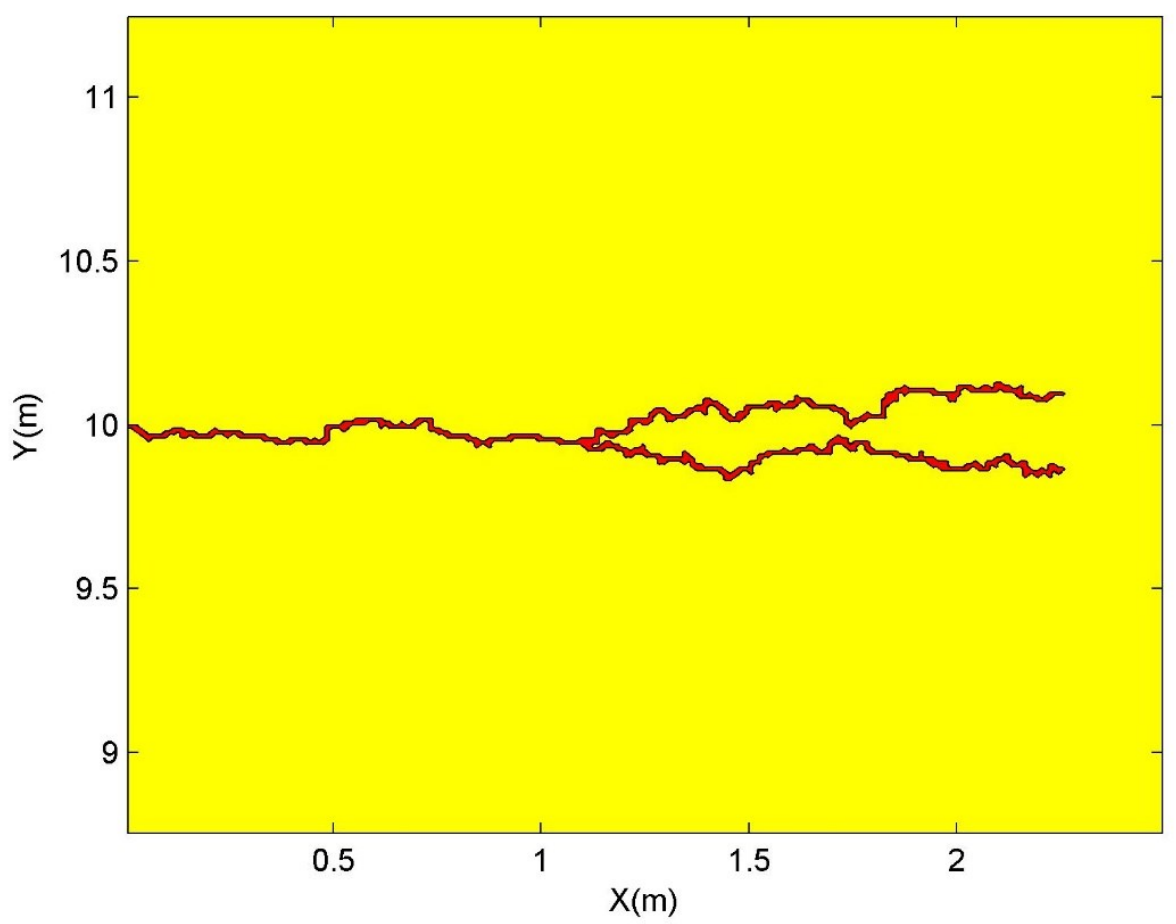

Figure A. 22 - Mono-branched fracture 18.

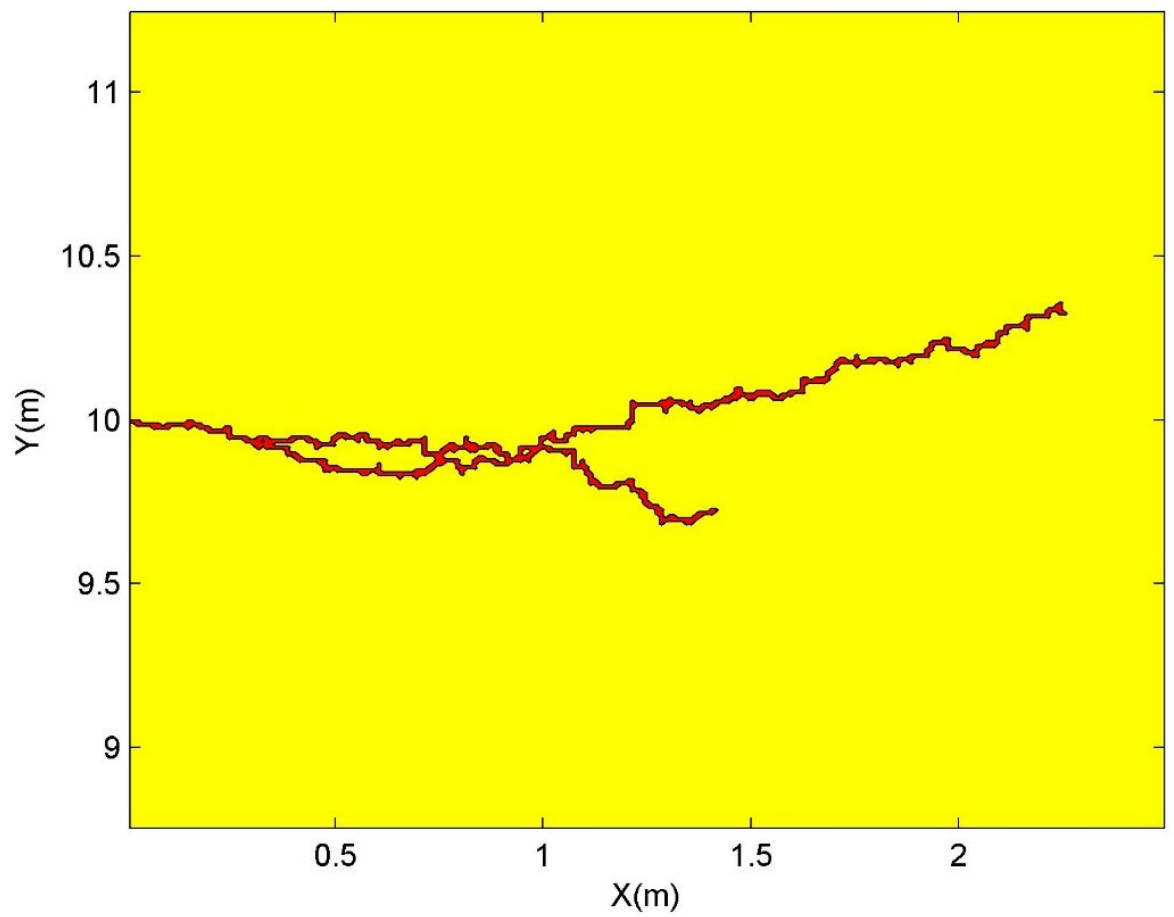

Figure A. 23 - Mono-branched fracture 19. 


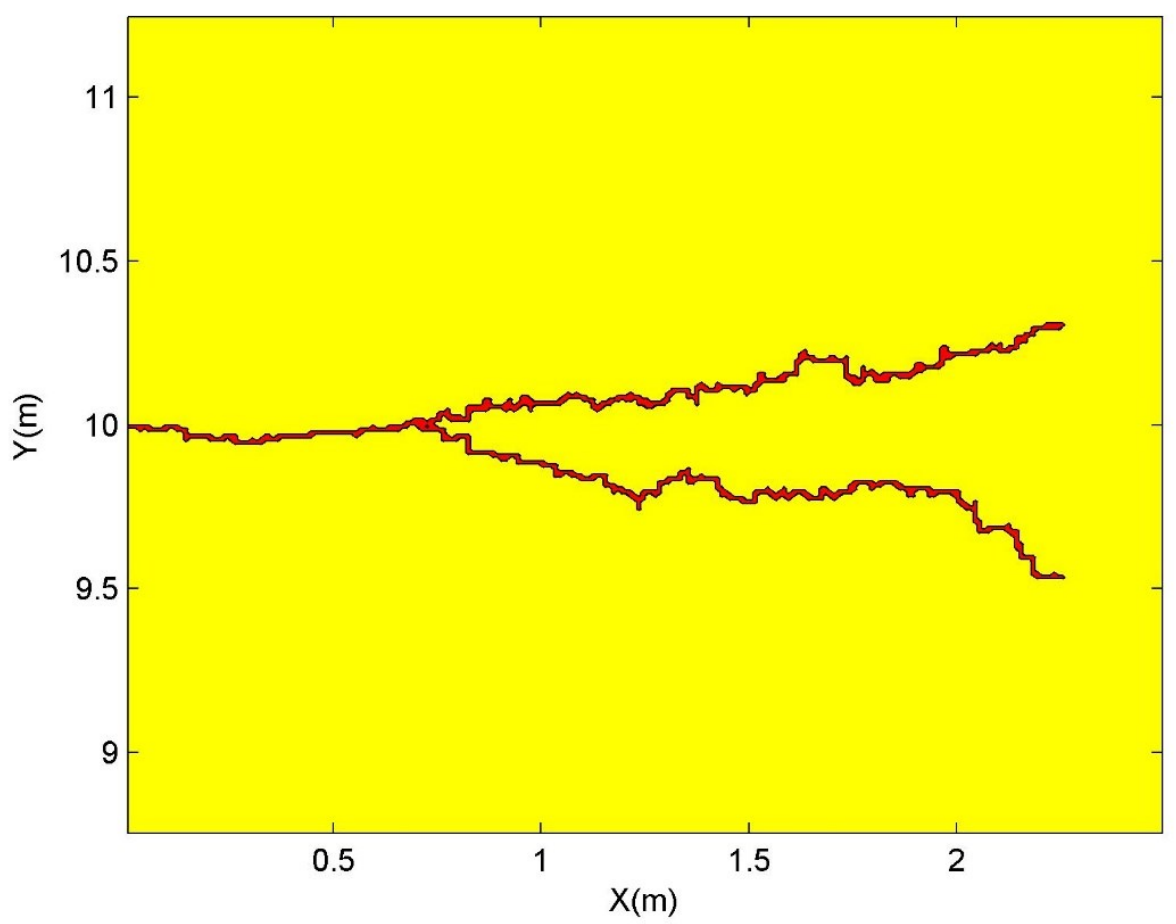

Figure A. 24 - Mono-branched fracture 20.

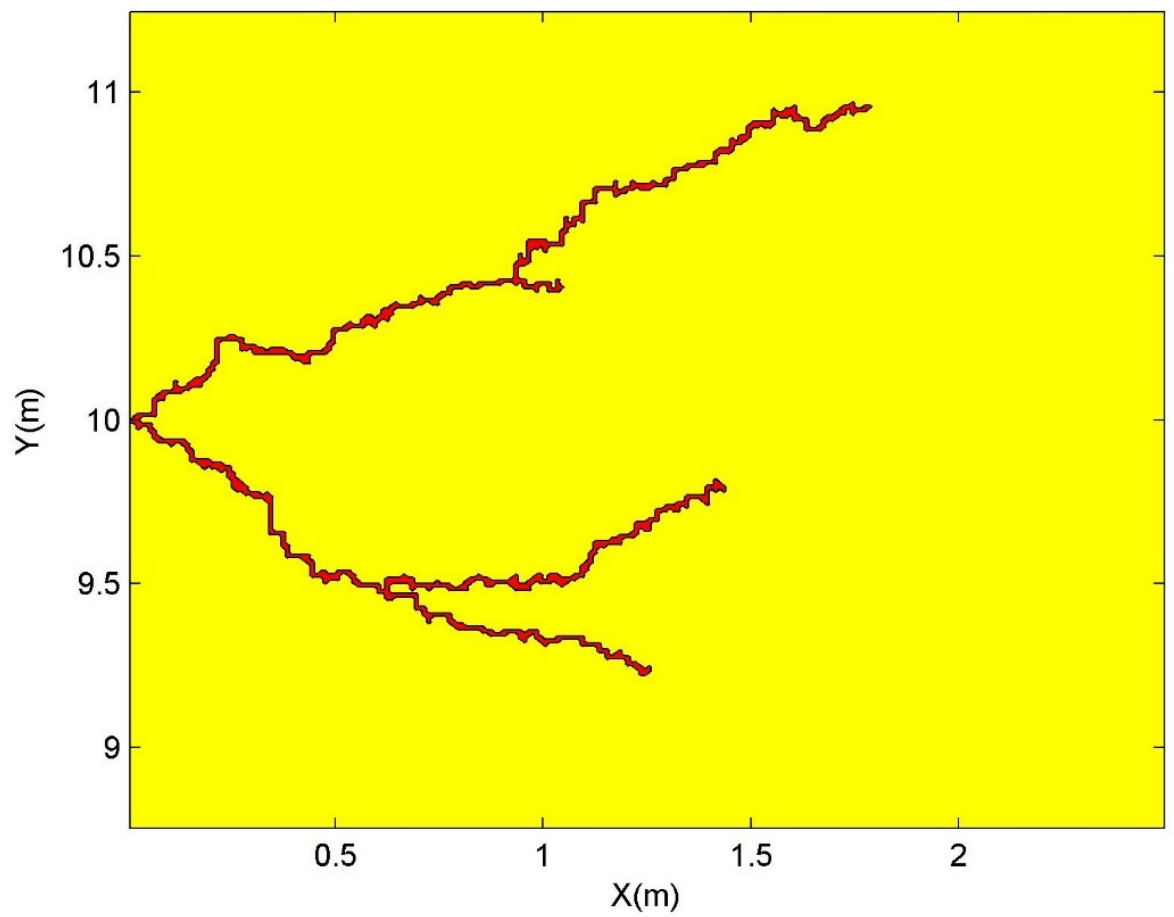

Figure A. 25 - Dual-branched fracture 1. 


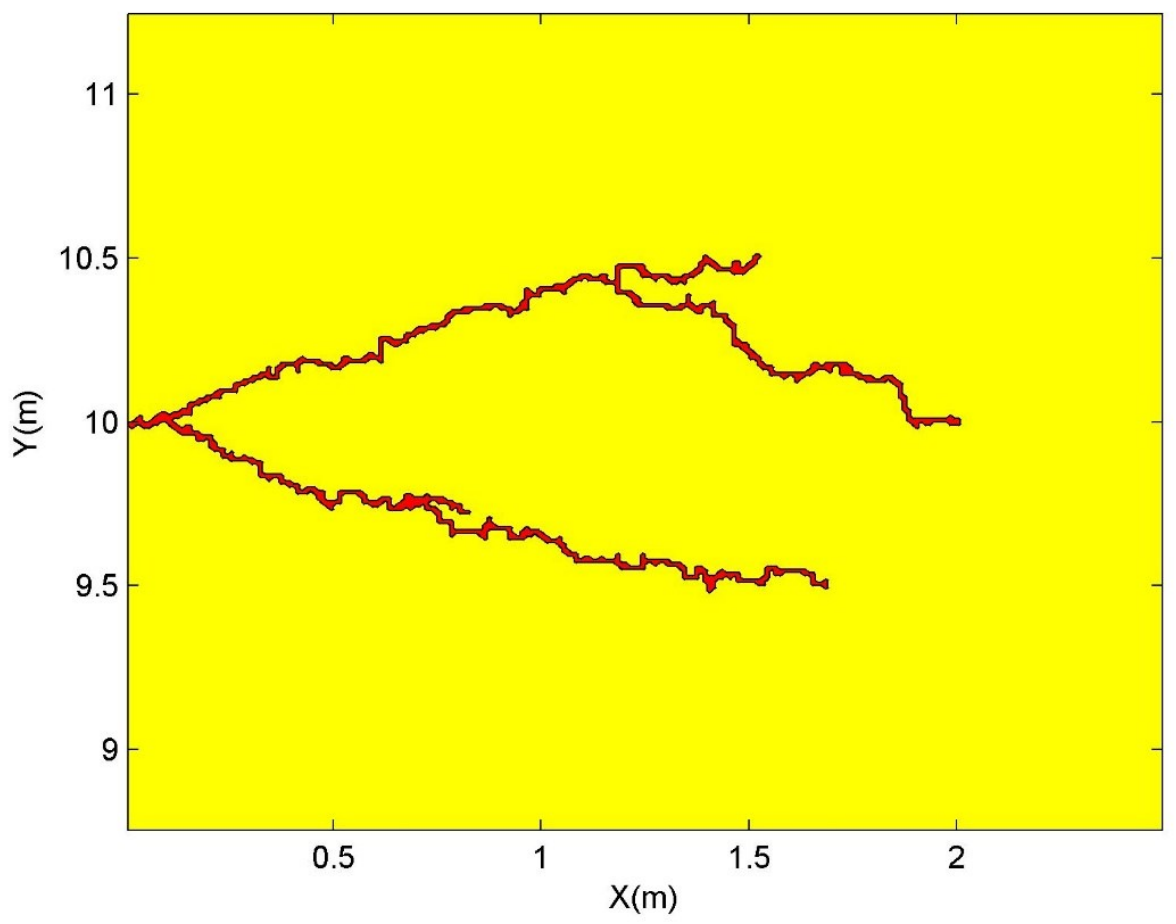

Figure A. 26 - Dual-branched fracture 2.

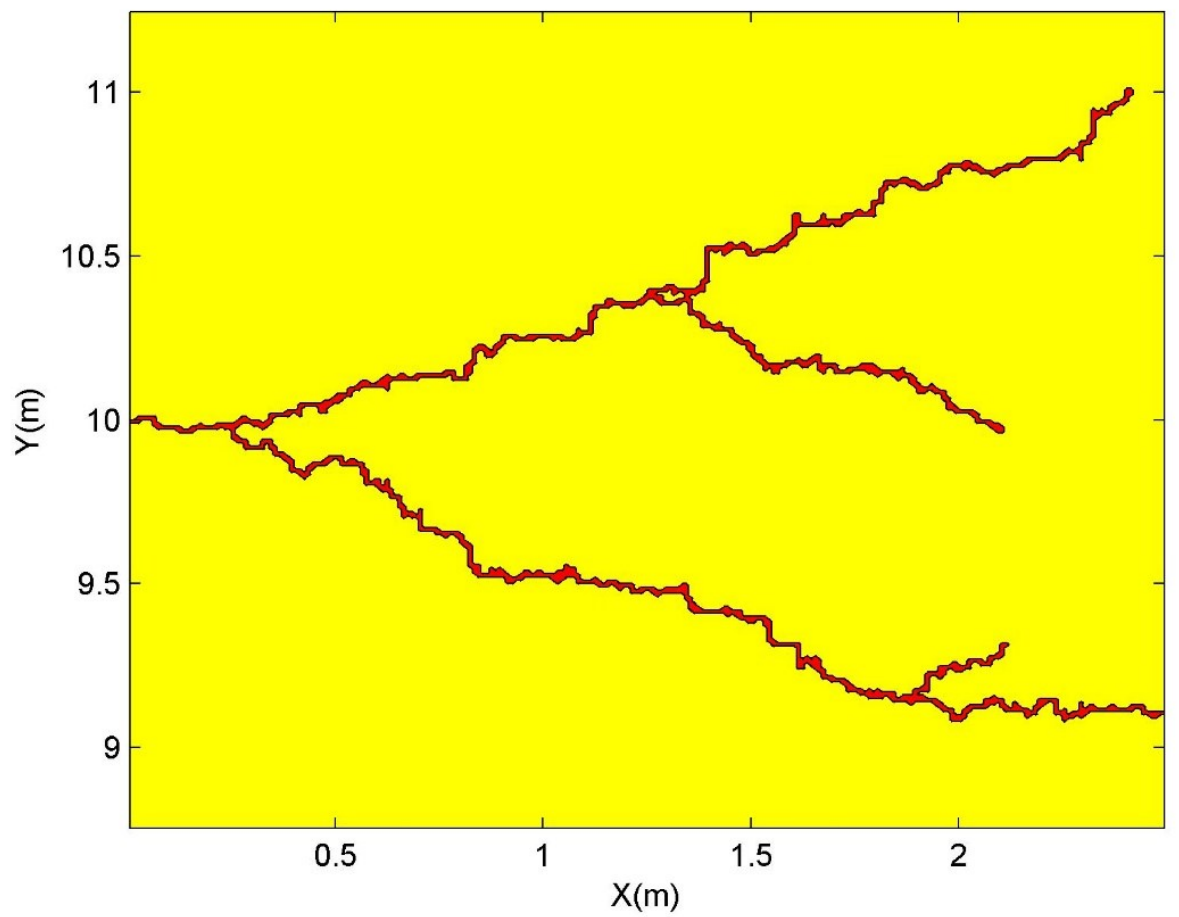

Figure A. 27 - Dual-branched fracture 3. 


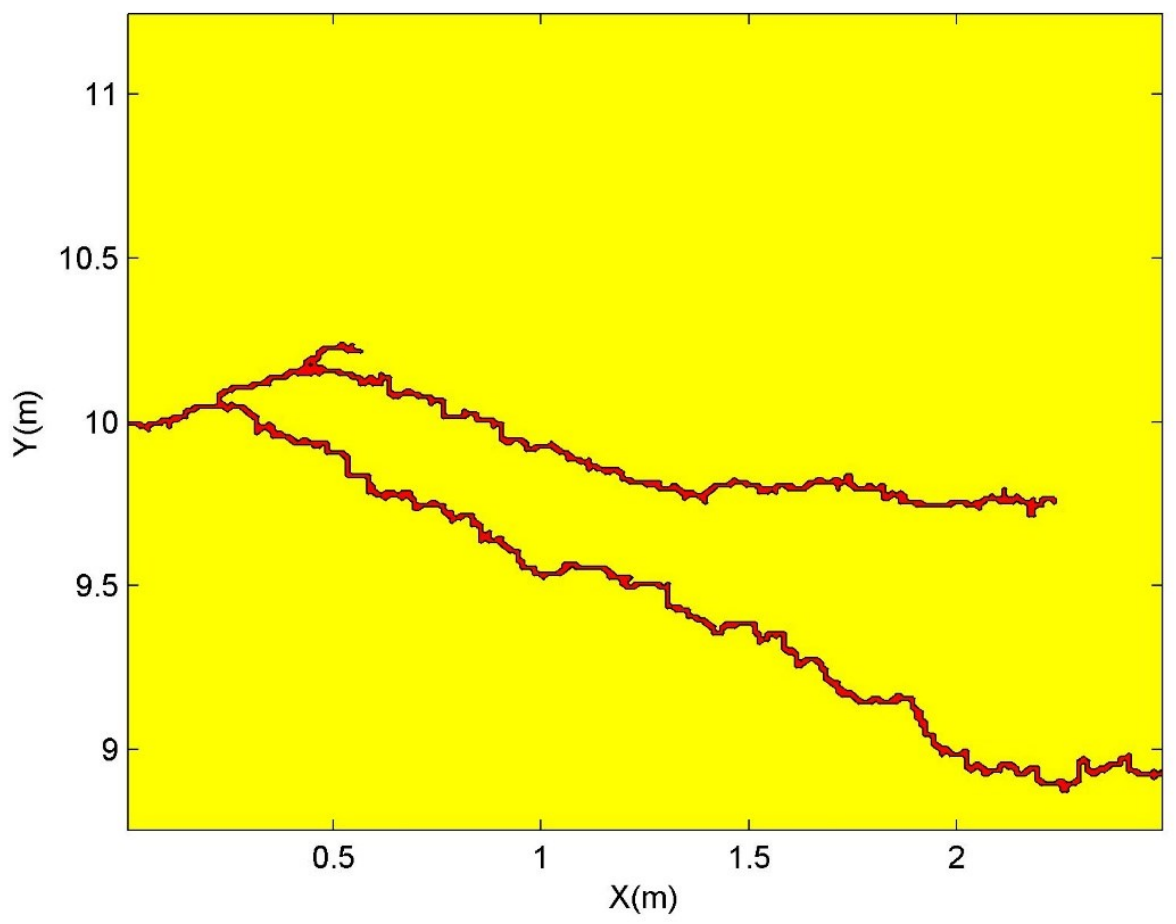

Figure A. 28 - Dual-branched fracture 4.

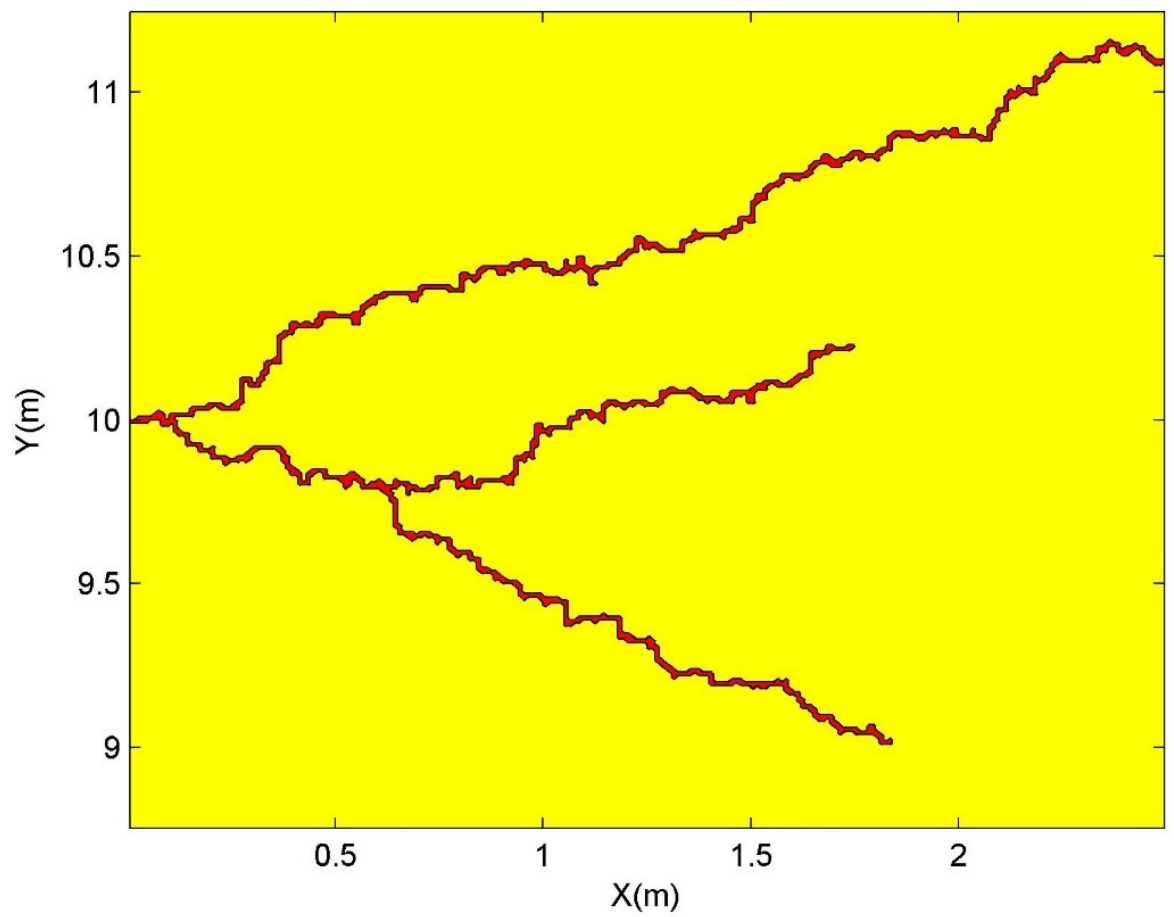

Figure A. 29 - Dual-branched fracture 5. 


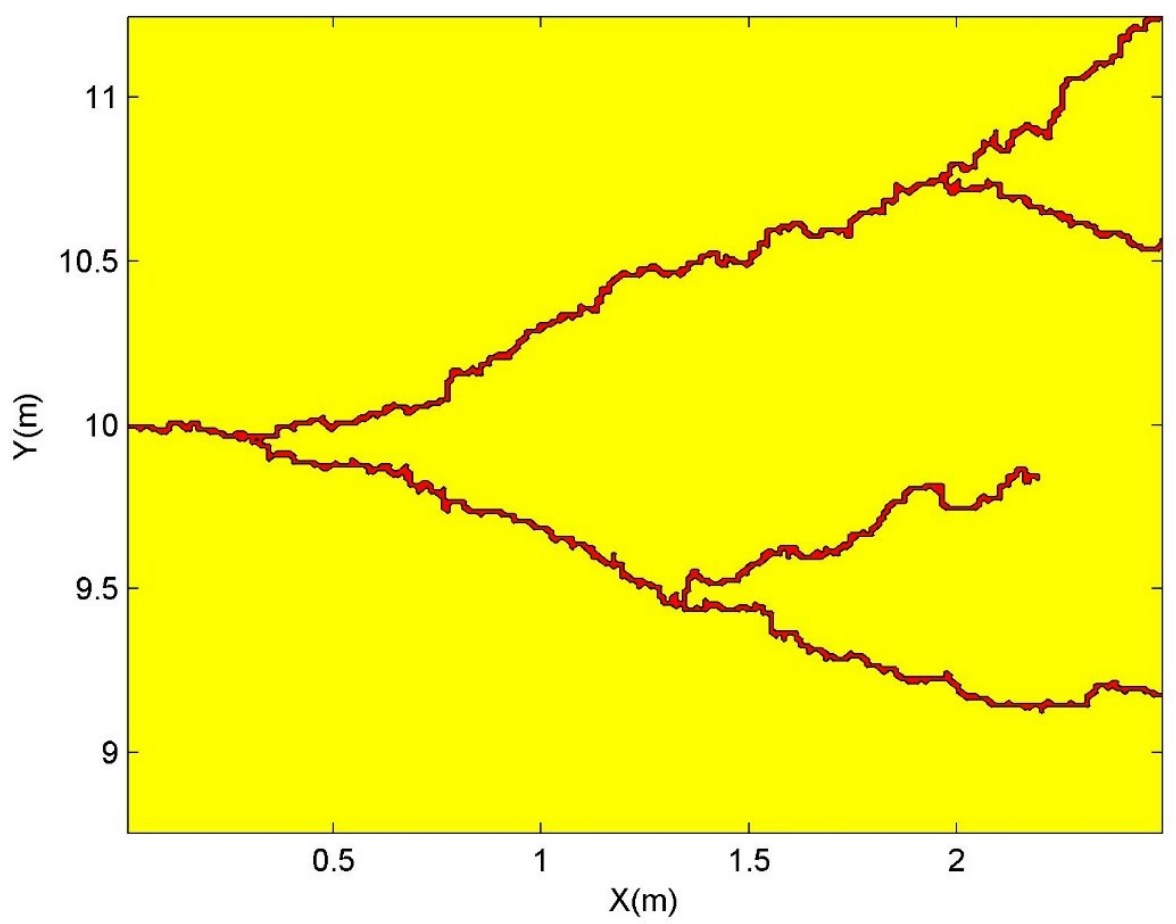

Figure A. 30 - Dual-branched fracture 6.

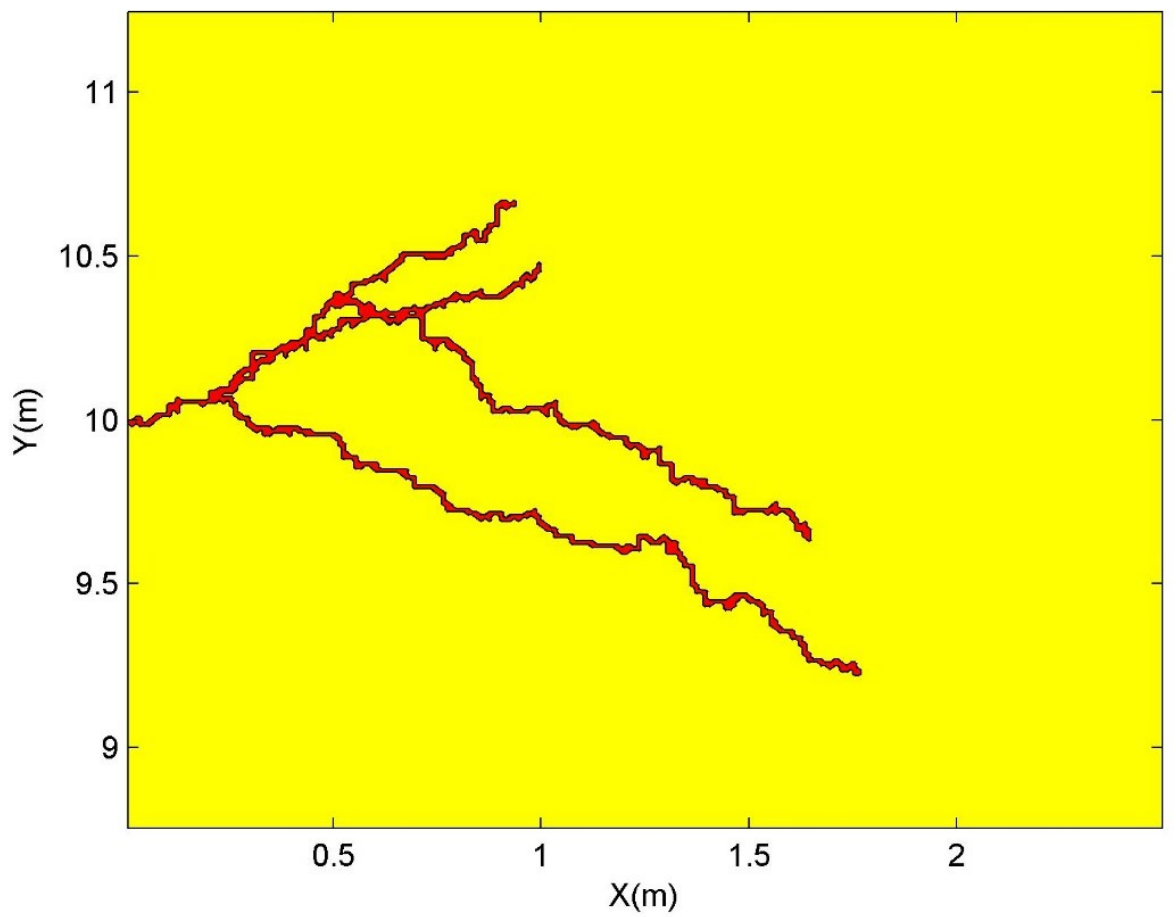

Figure A. 31 - Dual-branched fracture 7. 


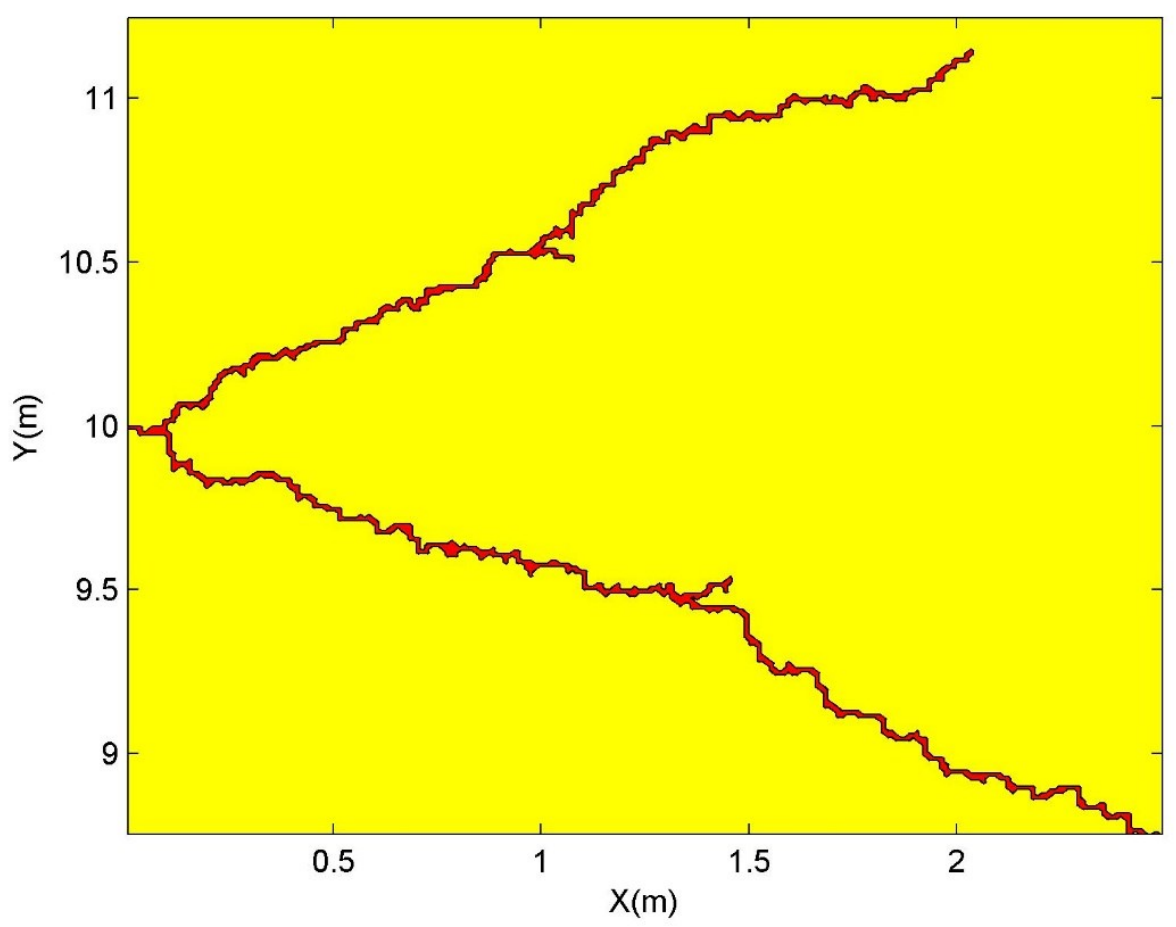

Figure A. 32 - Dual-branched fracture 8.

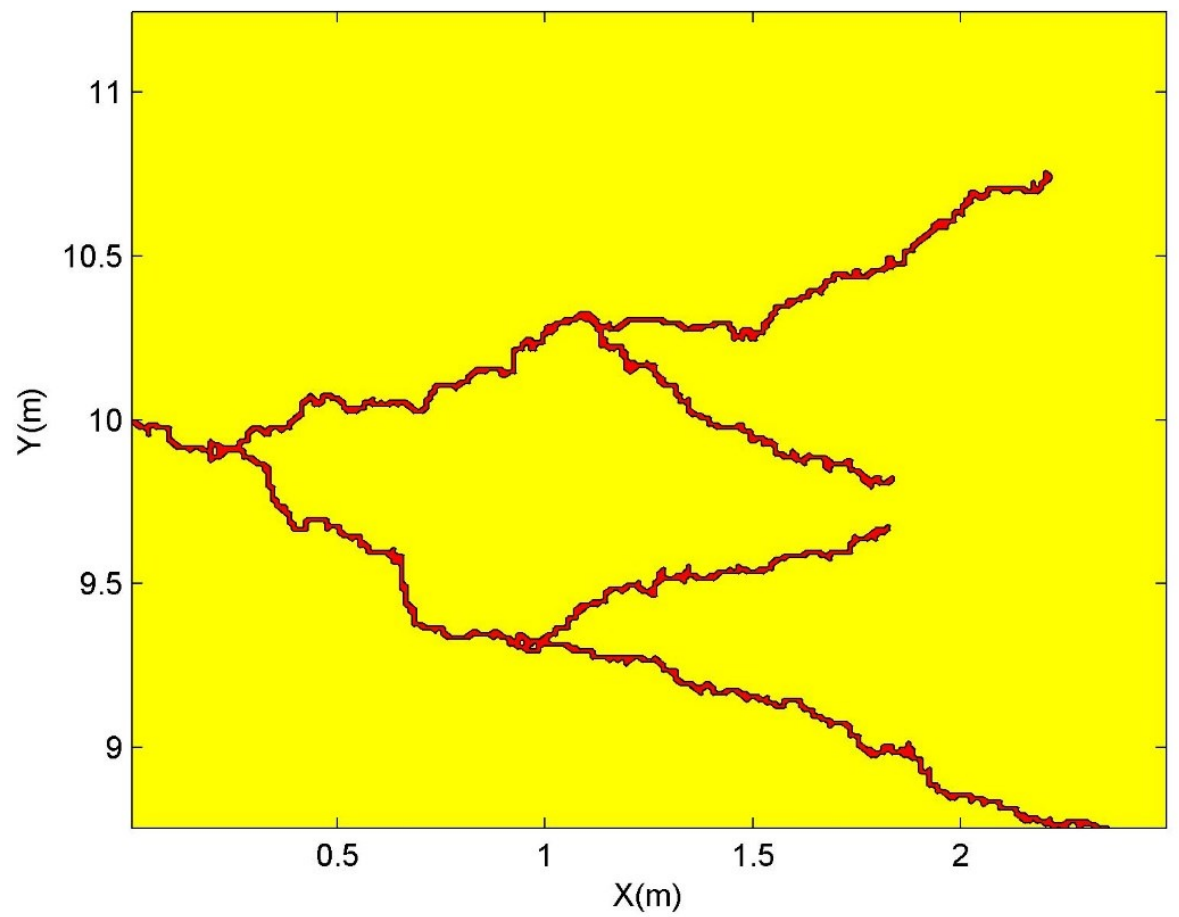

Figure A. 33 - Dual-branched fracture 9. 


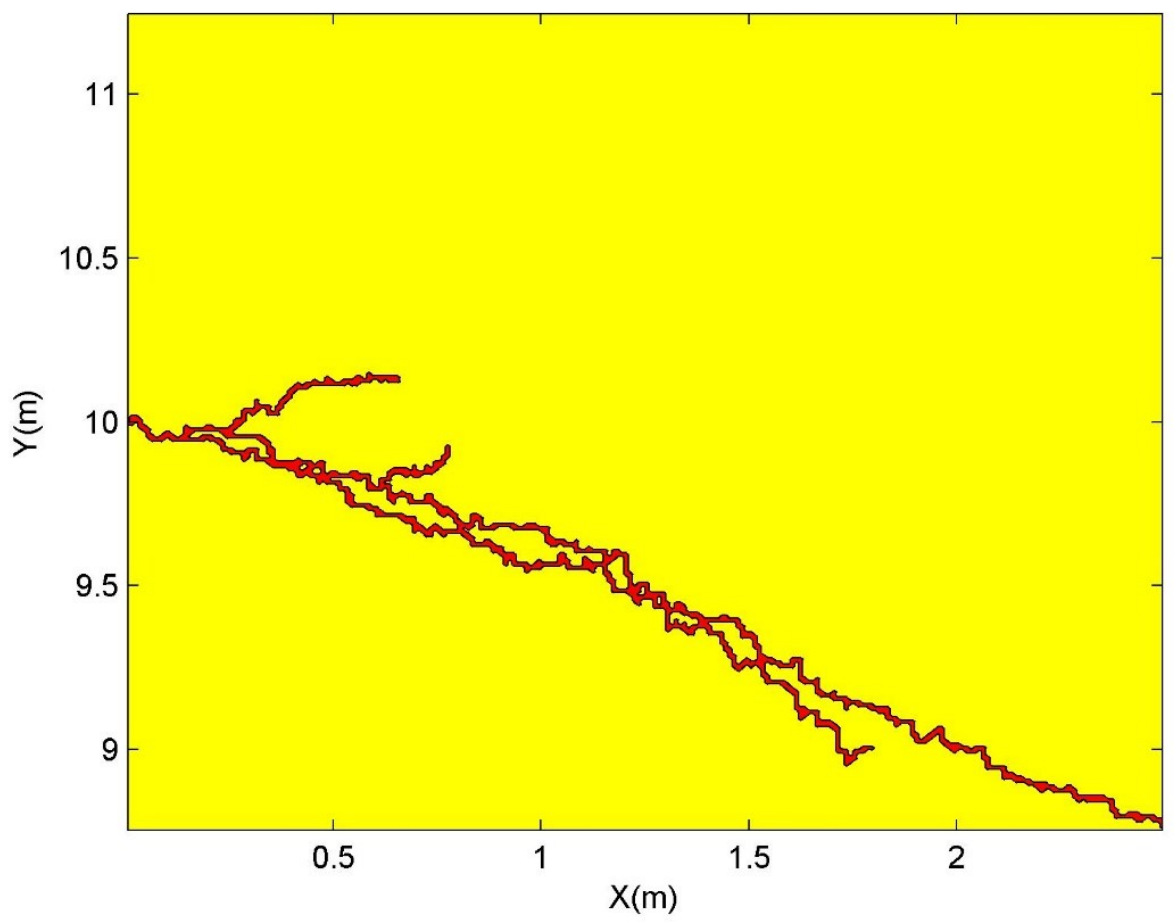

Figure A. 34 - Dual-branched fracture 10.

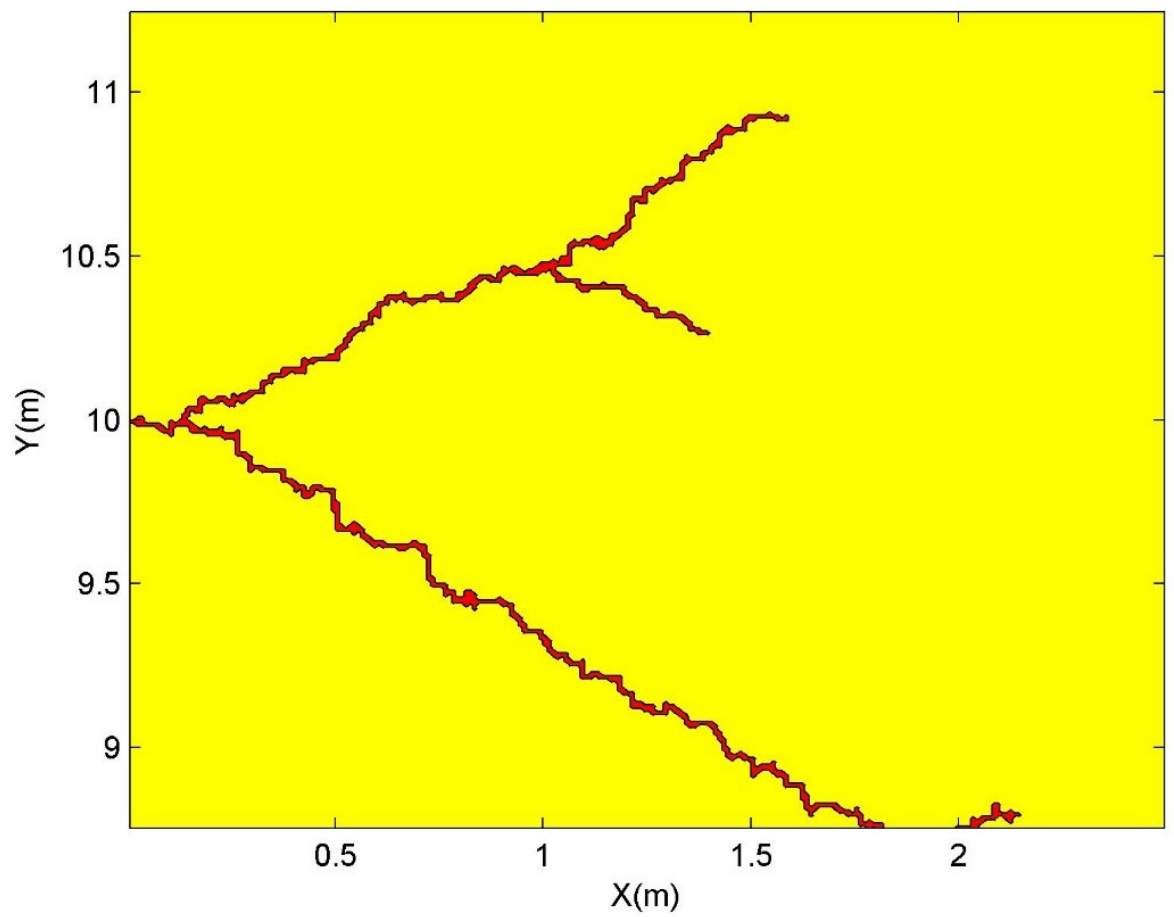

Figure A. 35 - Dual-branched fracture 11. 


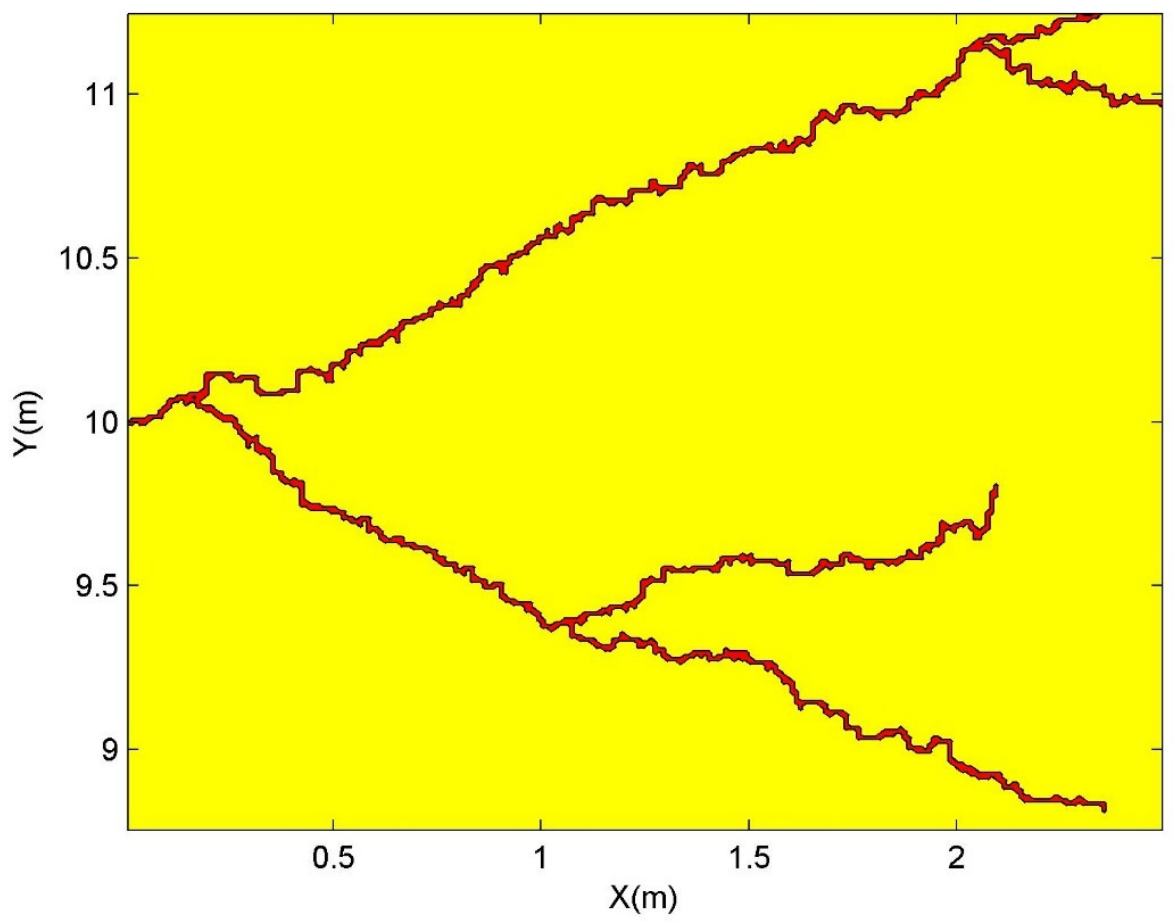

Figure A. 36 - Dual-branched fracture 12.

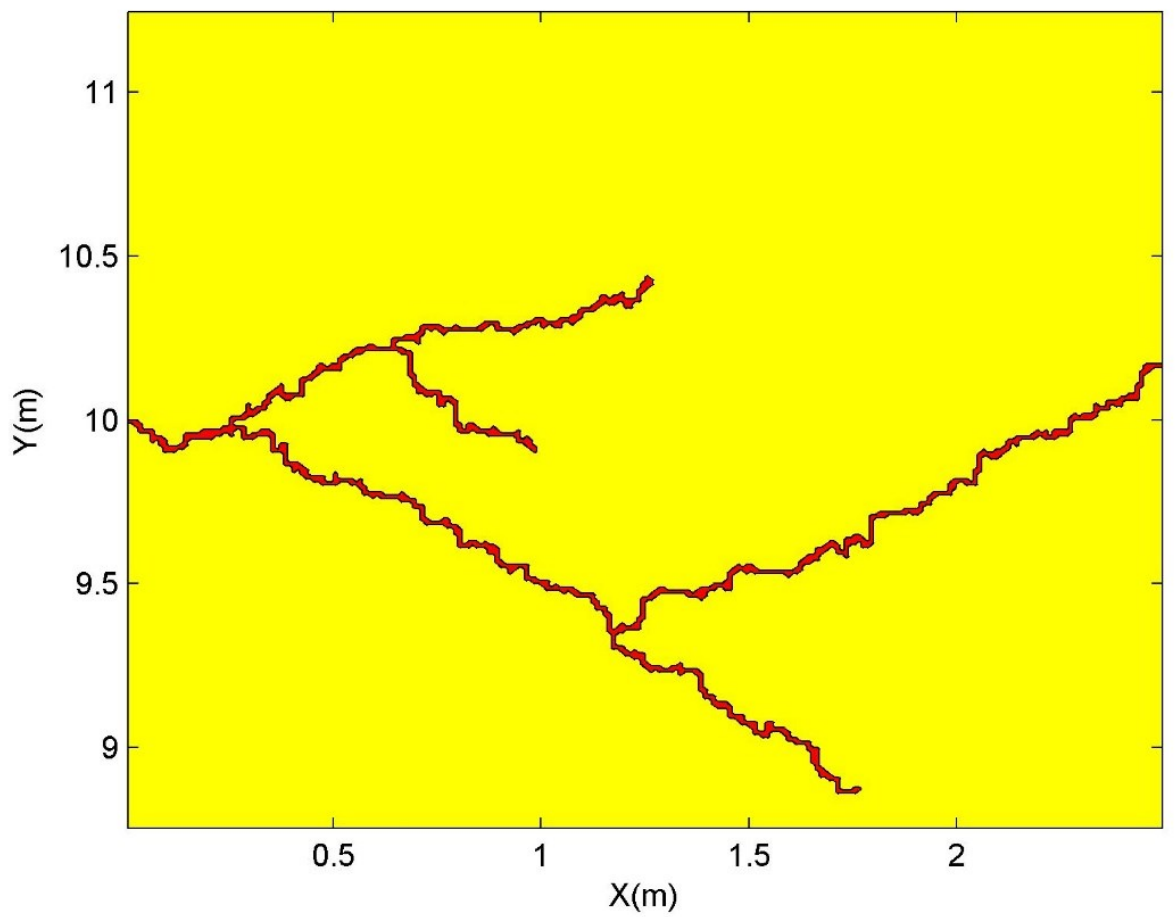

Figure A. 37 - Dual-branched fracture 13. 


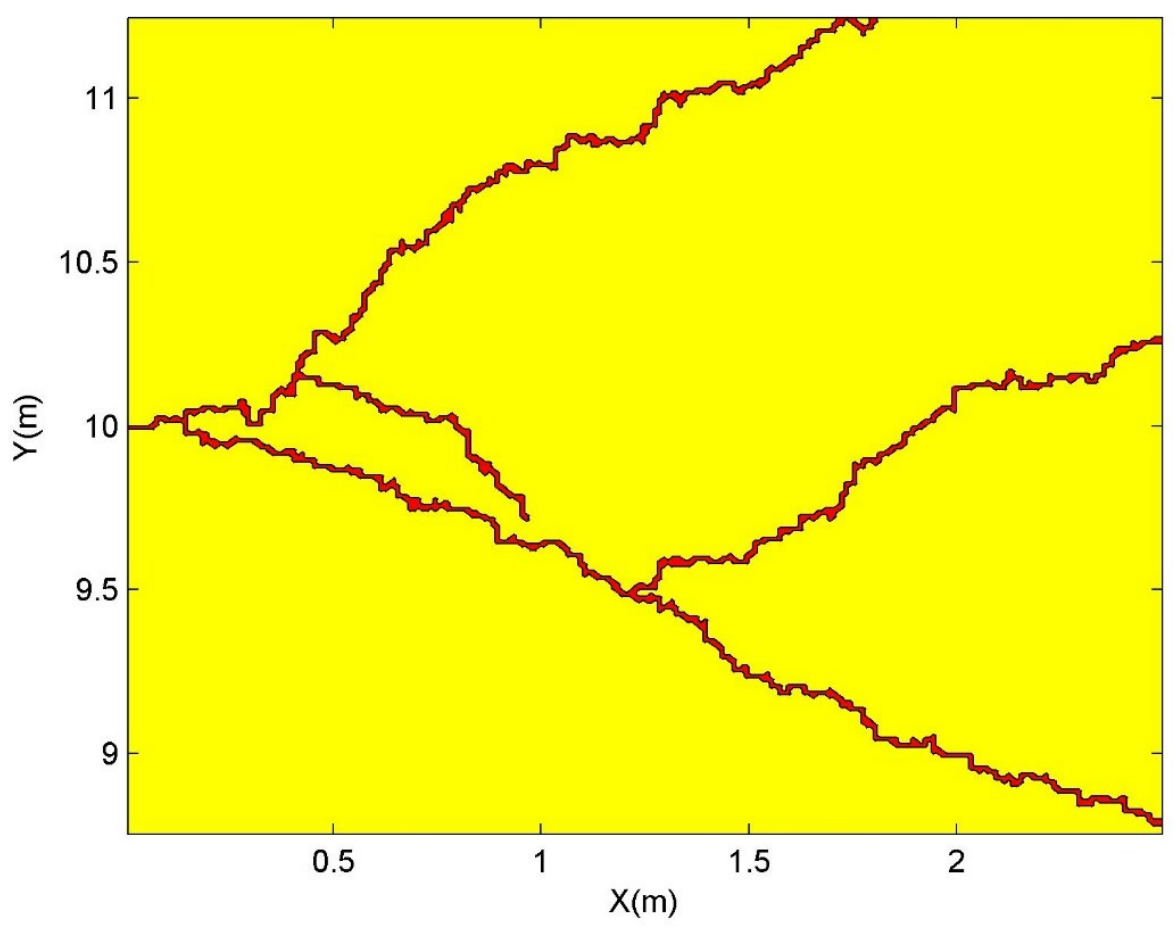

Figure A. 38 - Dual-branched fracture 14.

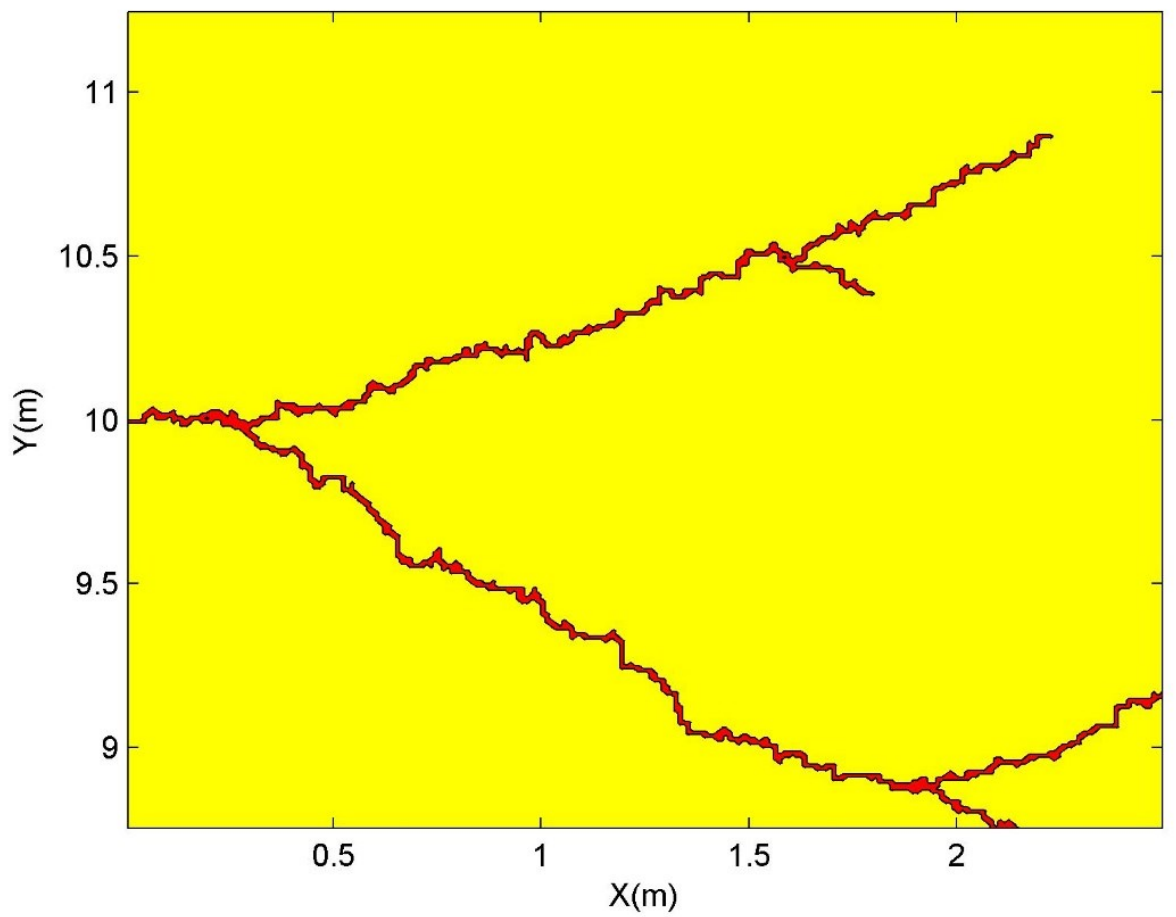

Figure A. 39 - Dual-branched fracture 15. 


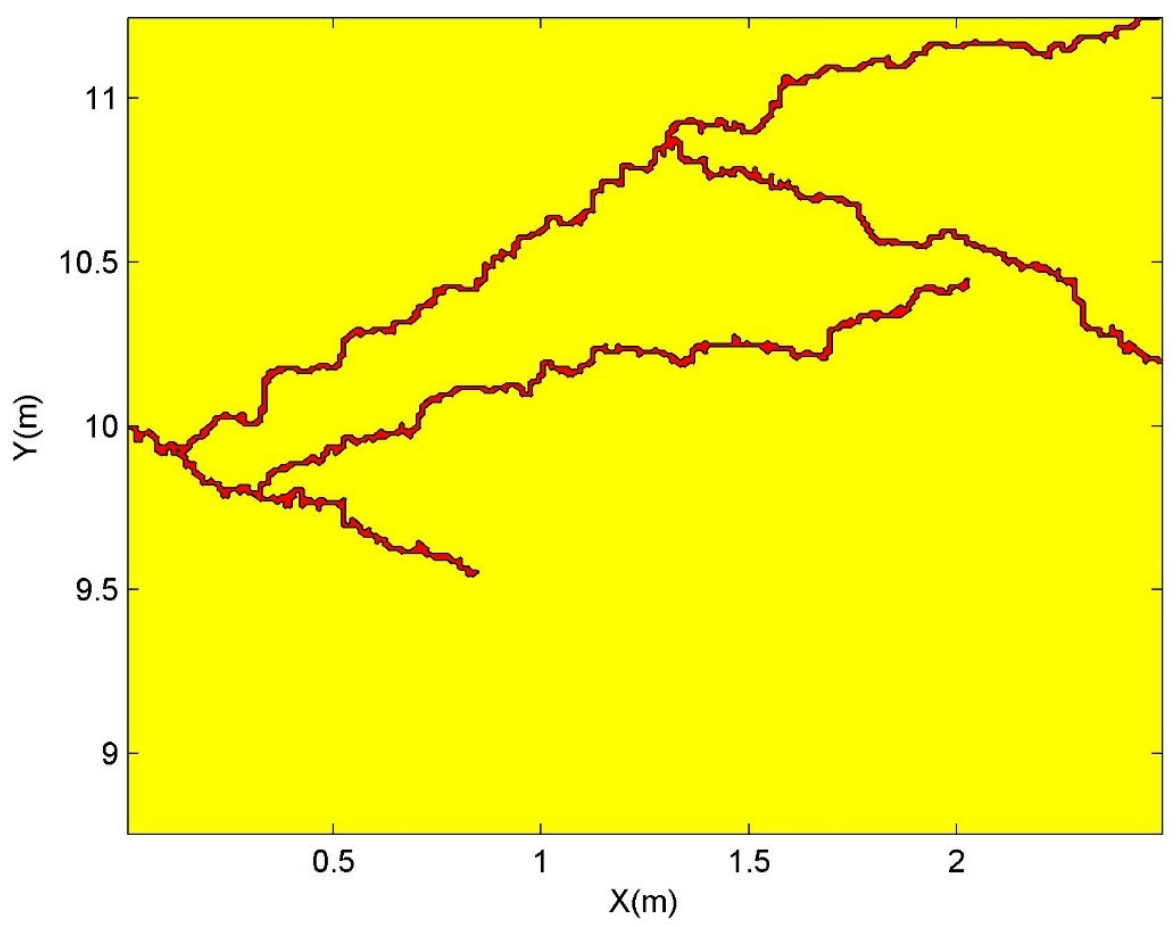

Figure A. 40 - Dual-branched fracture 16.

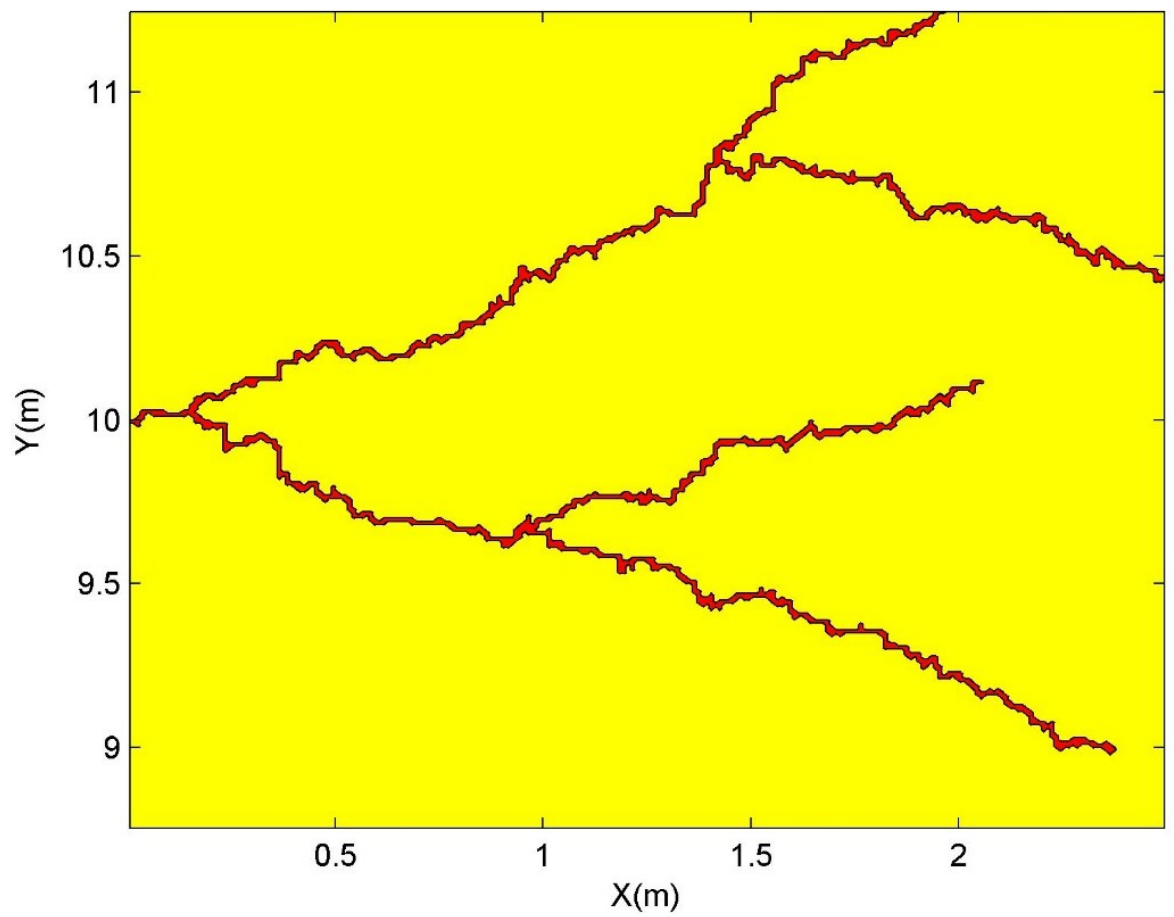

Figure A. 41 - Dual-branched fracture 17. 


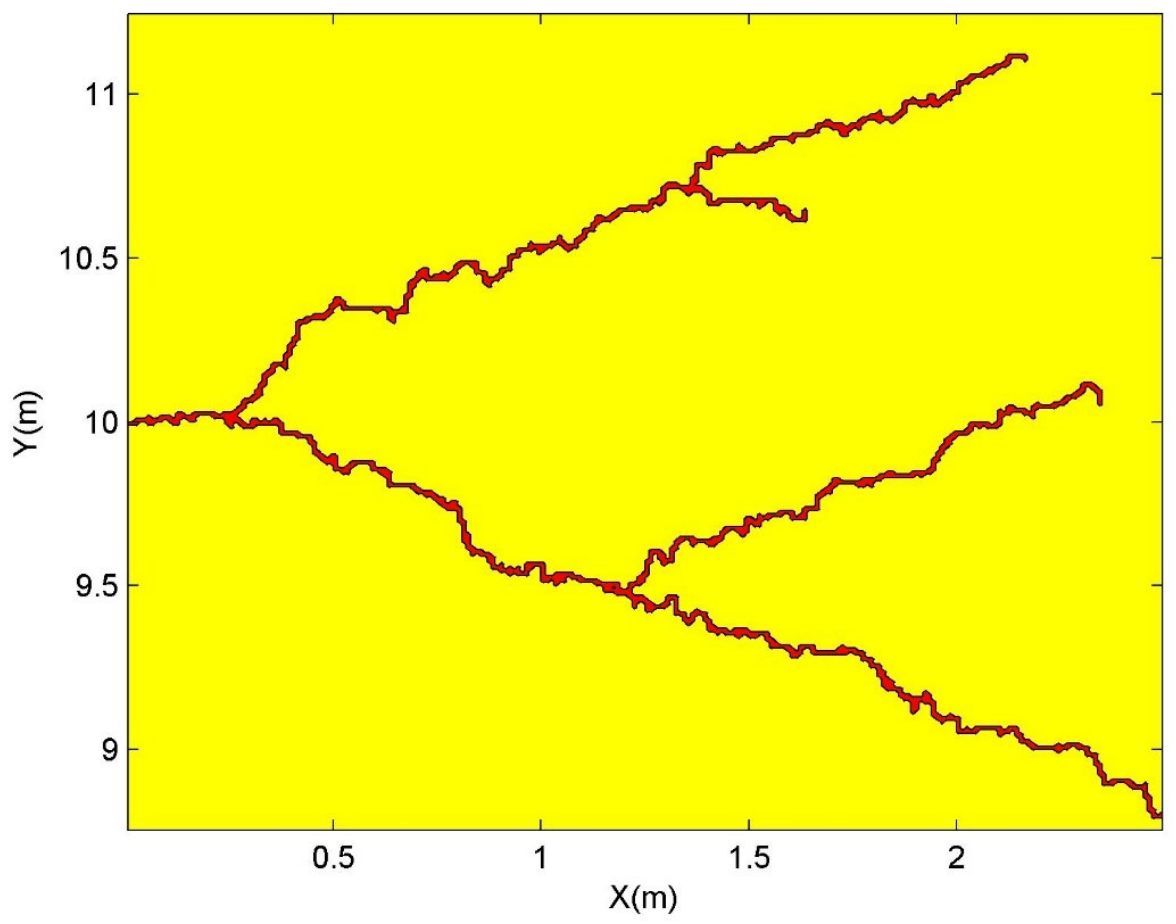

Figure A. 42 - Dual-branched fracture 18.

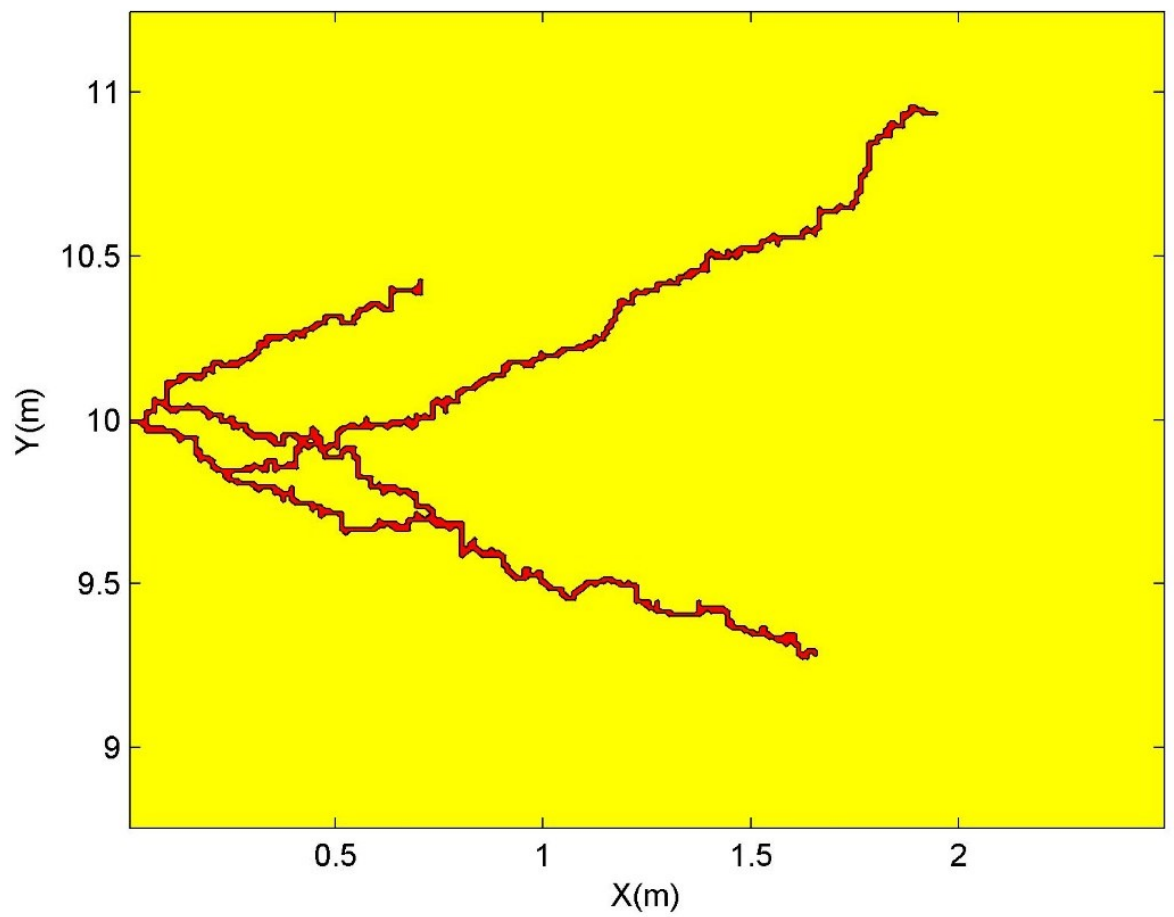

Figure A. 43 - Dual-branched fracture 19. 


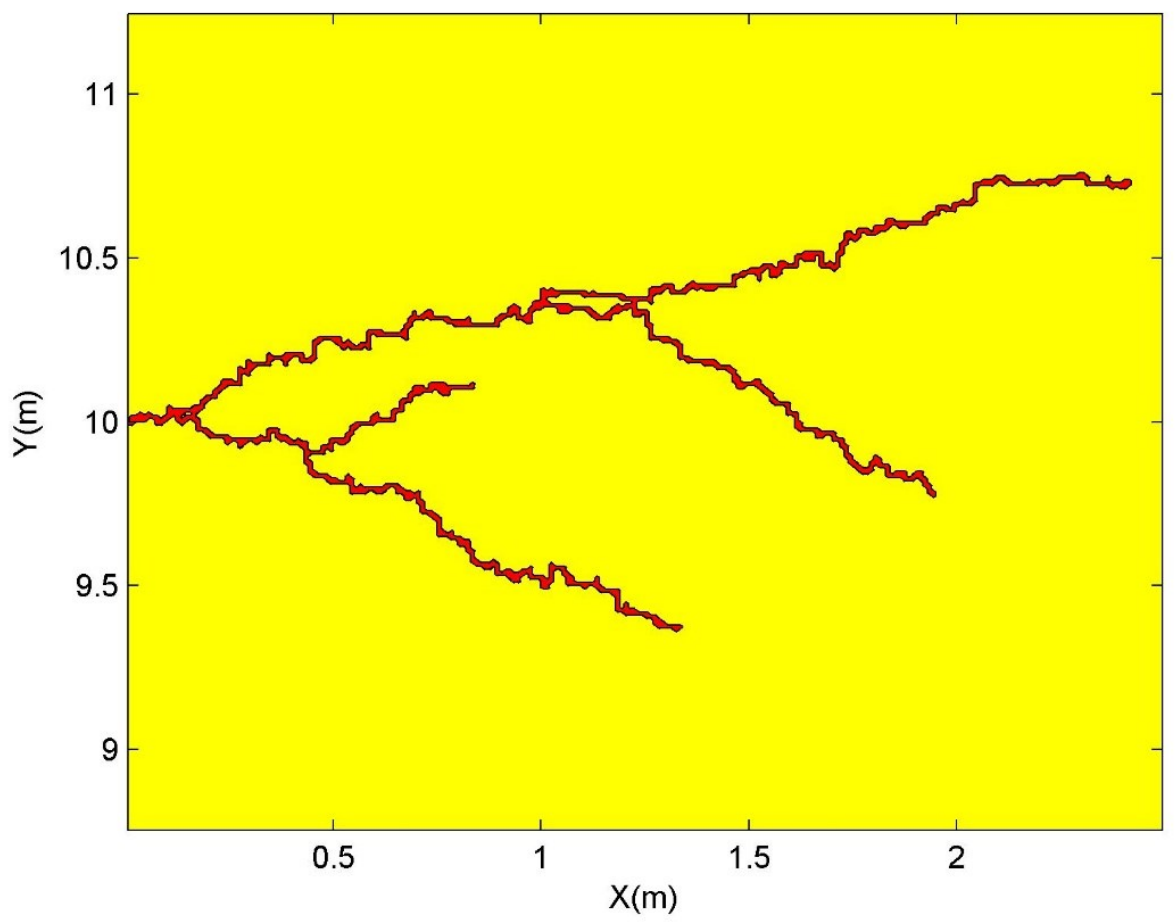

Figure A. 44 - Dual-branched fracture 20.

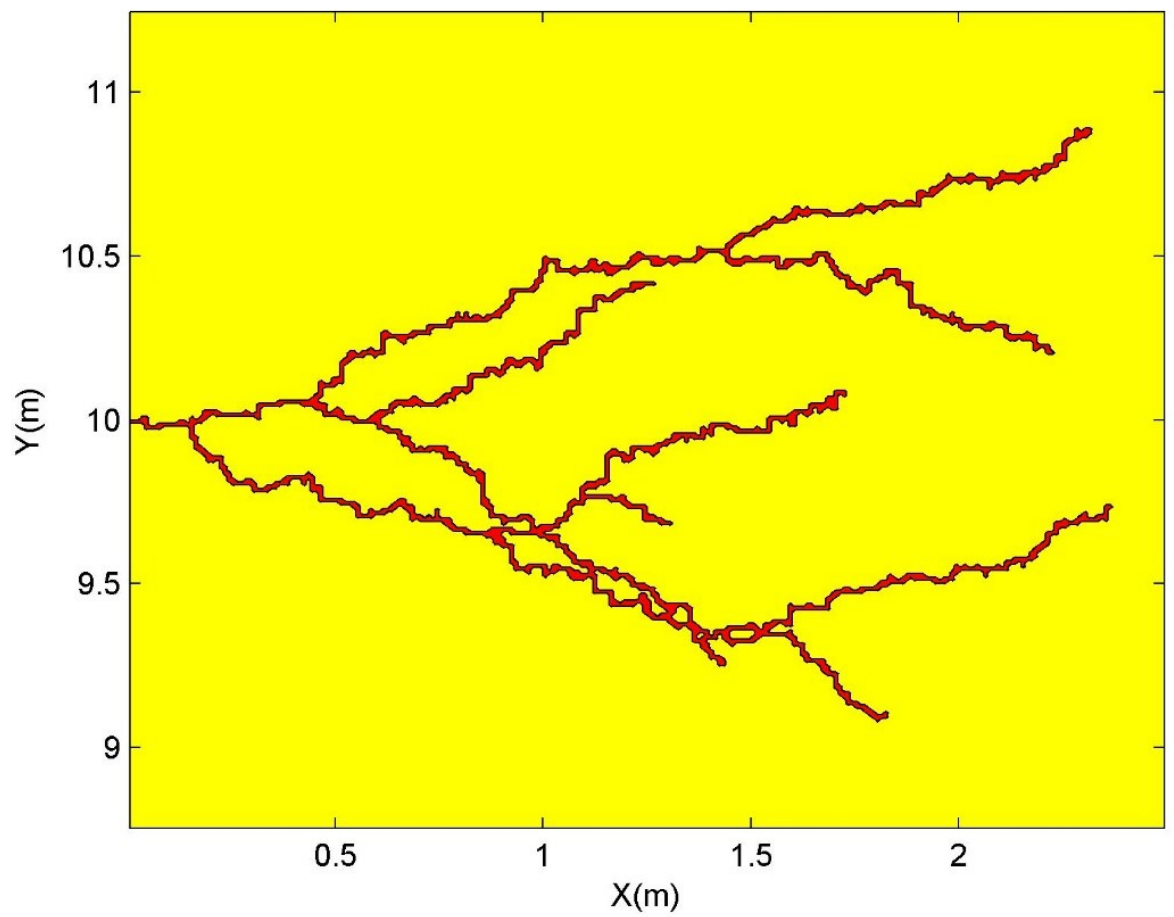

Figure A. 45 - Tri-branched fracture 1. 


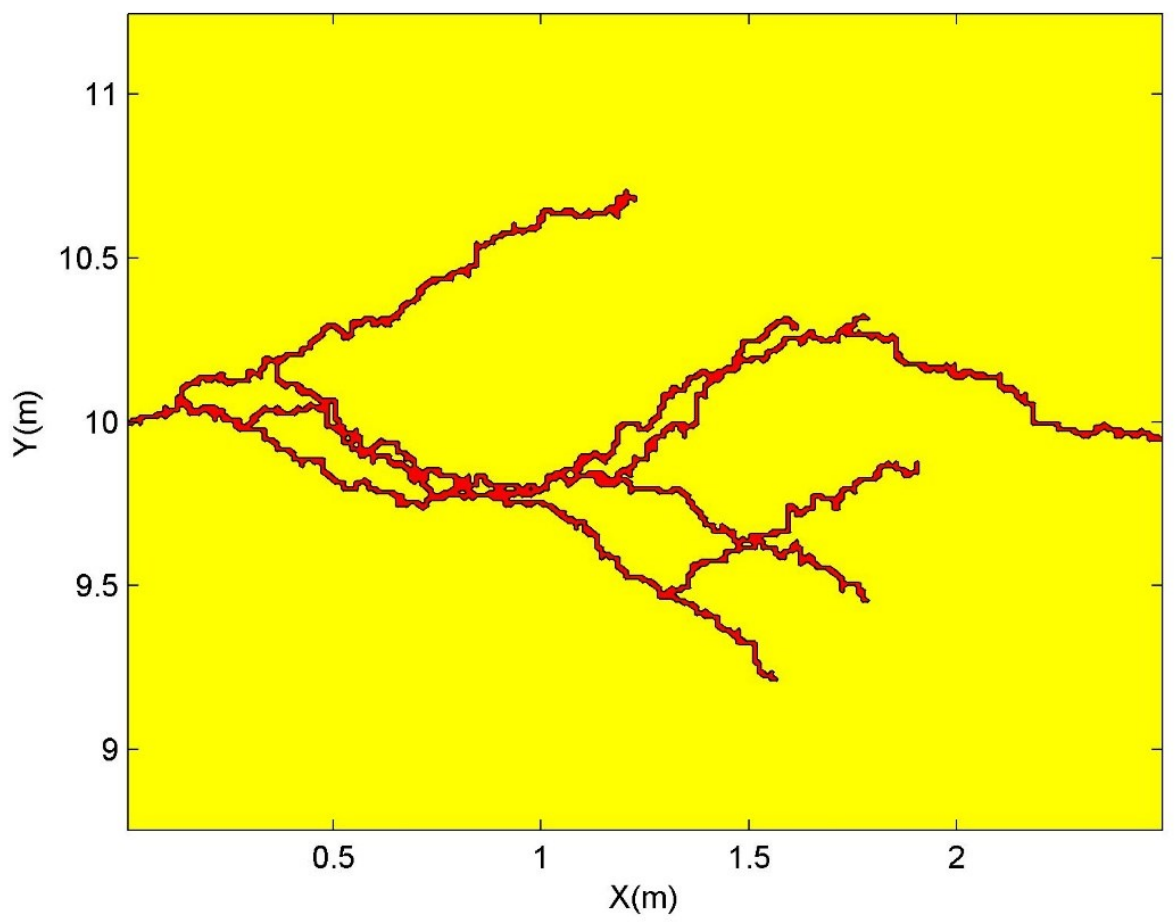

Figure A. 46 - Tri-branched fracture 2.

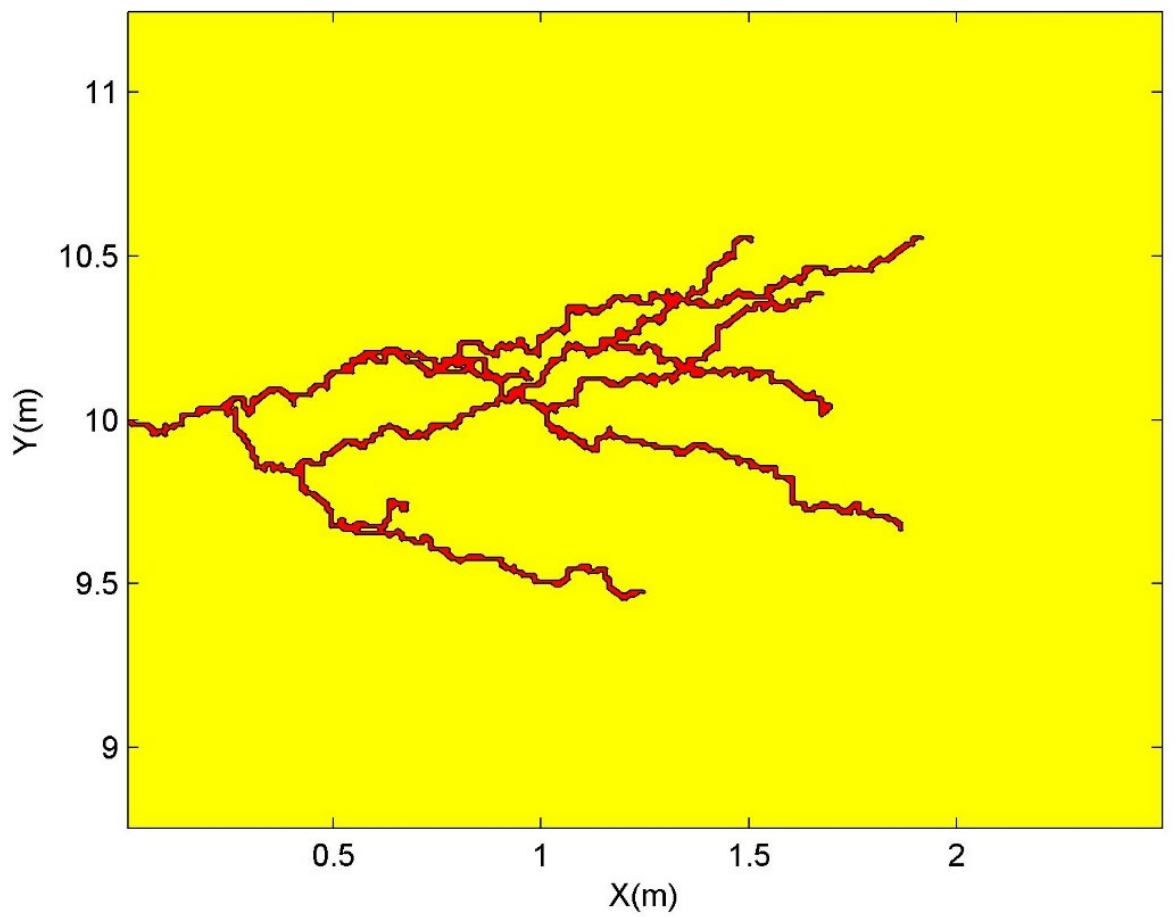

Figure A. 47 - Tri-branched fracture 3. 


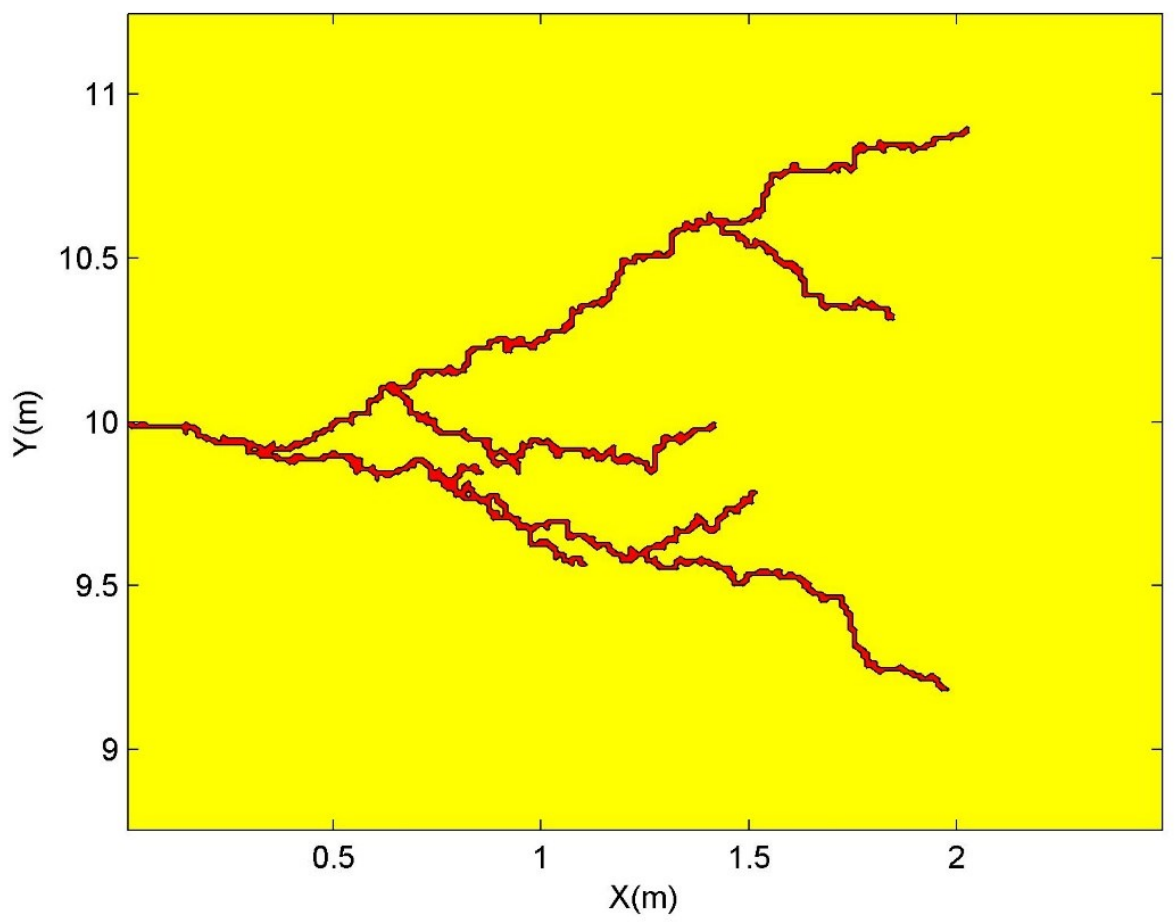

Figure A. 48 - Tri-branched fracture 4.

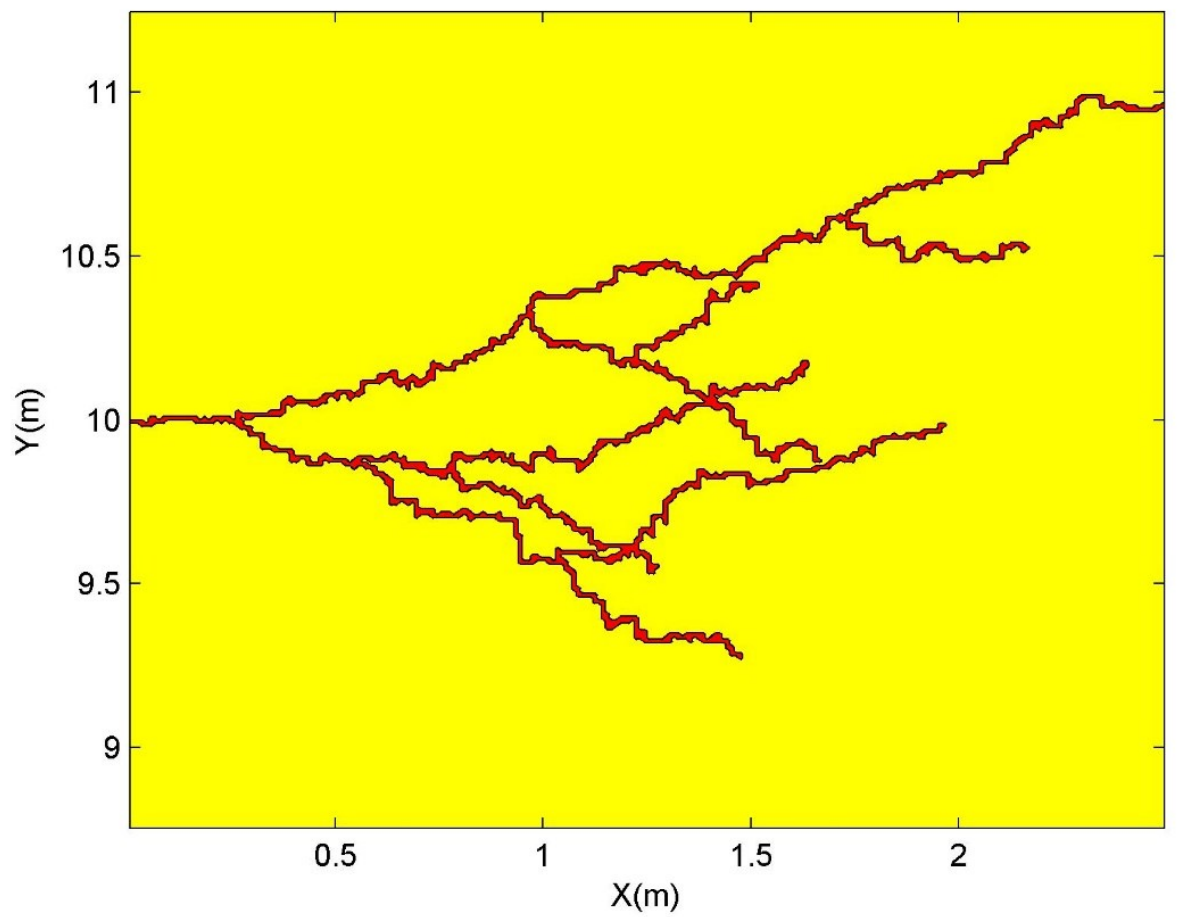

Figure A. 49 - Tri-branched fracture 5. 


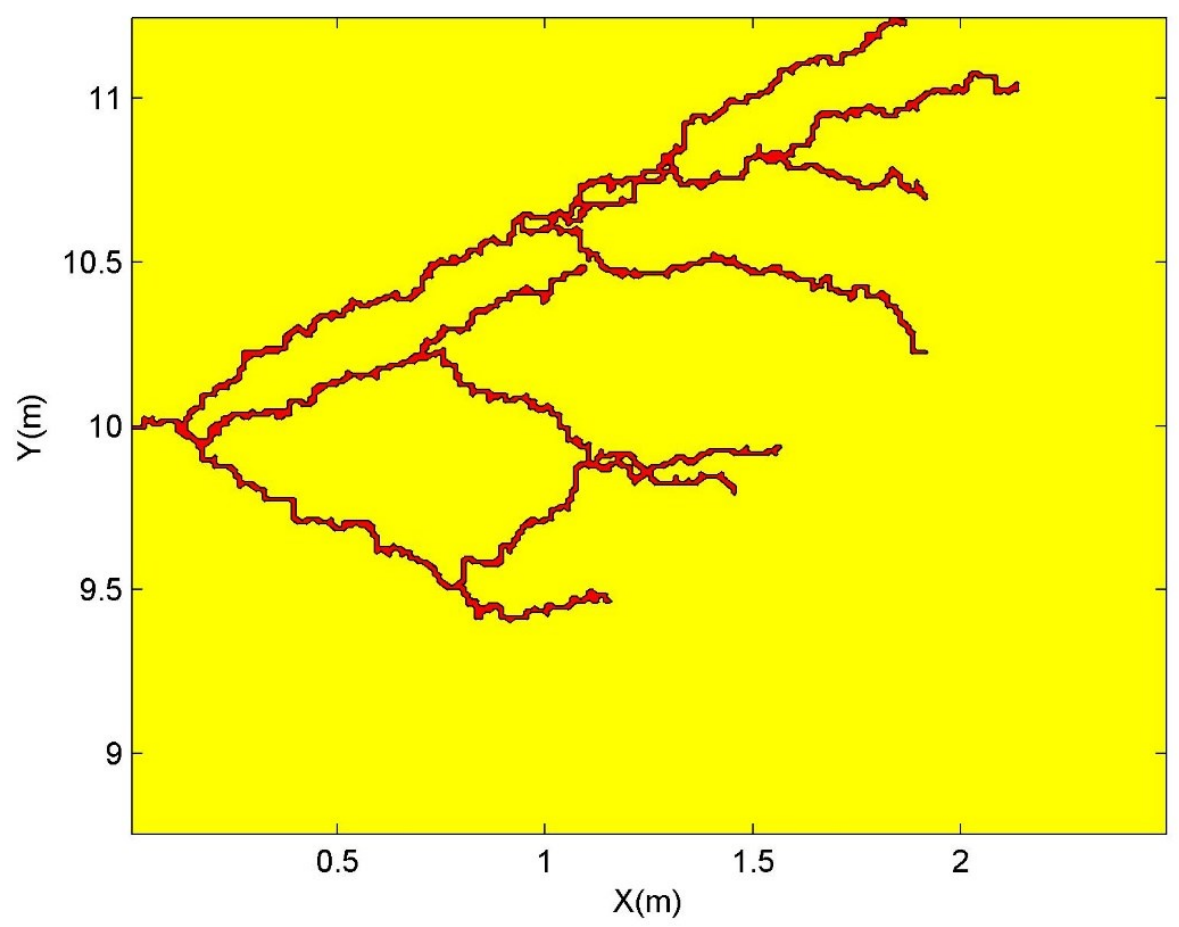

Figure A. 50 - Tri-branched fracture 6.

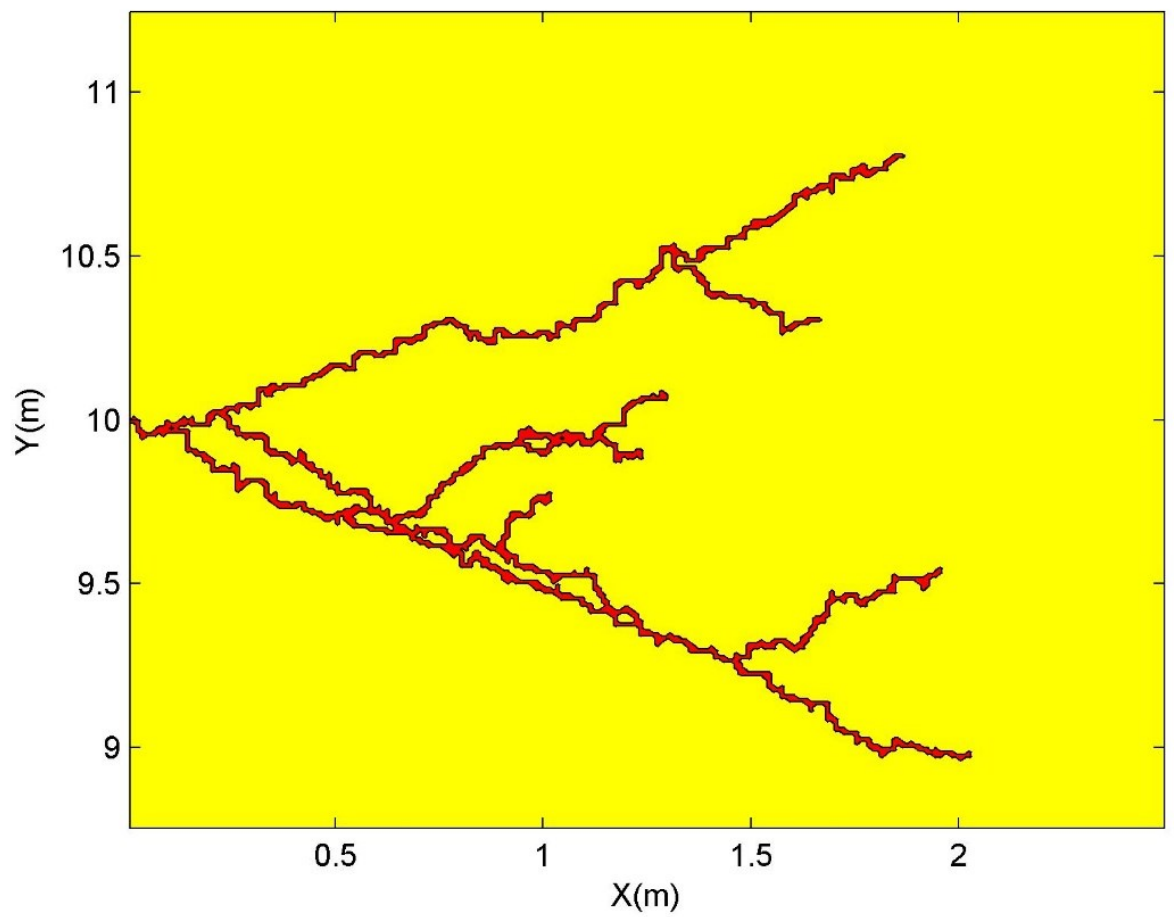

Figure A. 51 - Tri-branched fracture 7. 


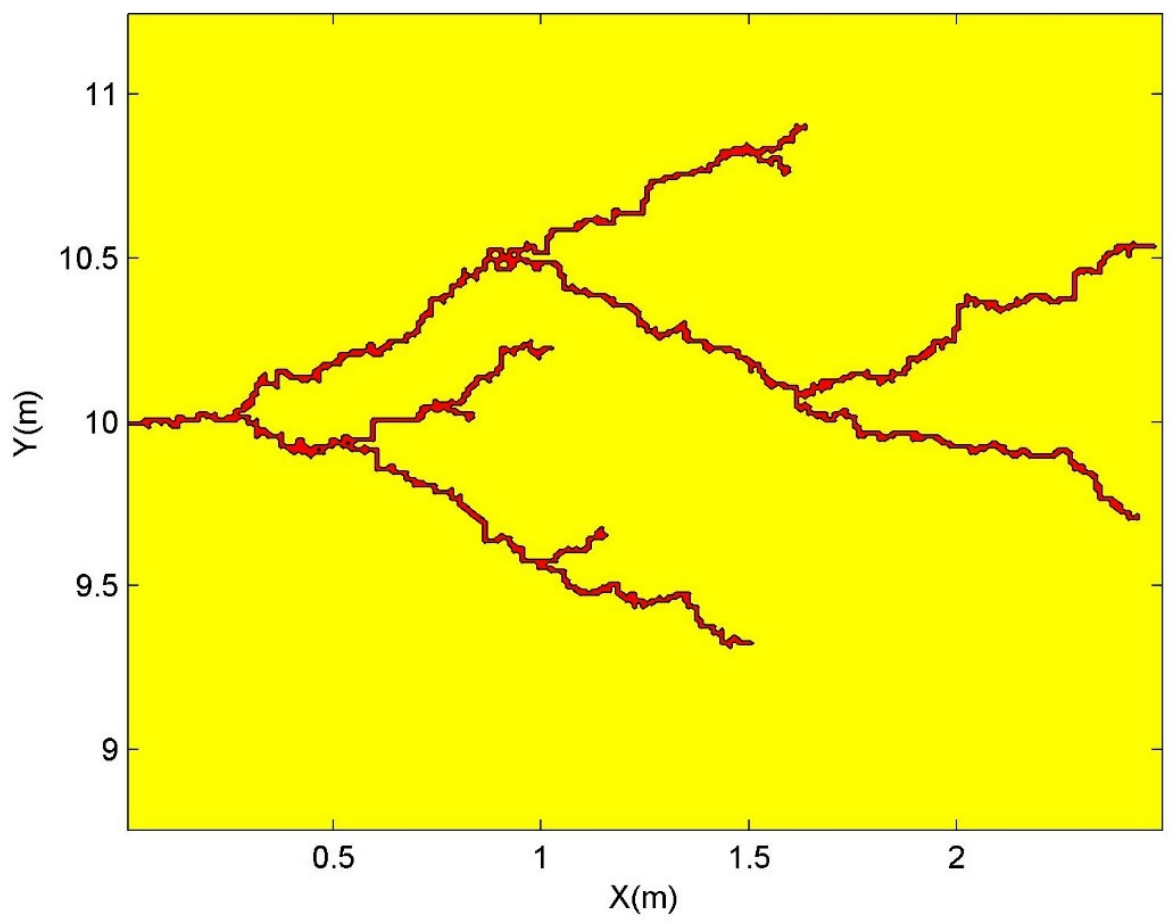

Figure A. $52-$ Tri-branched fracture 8.

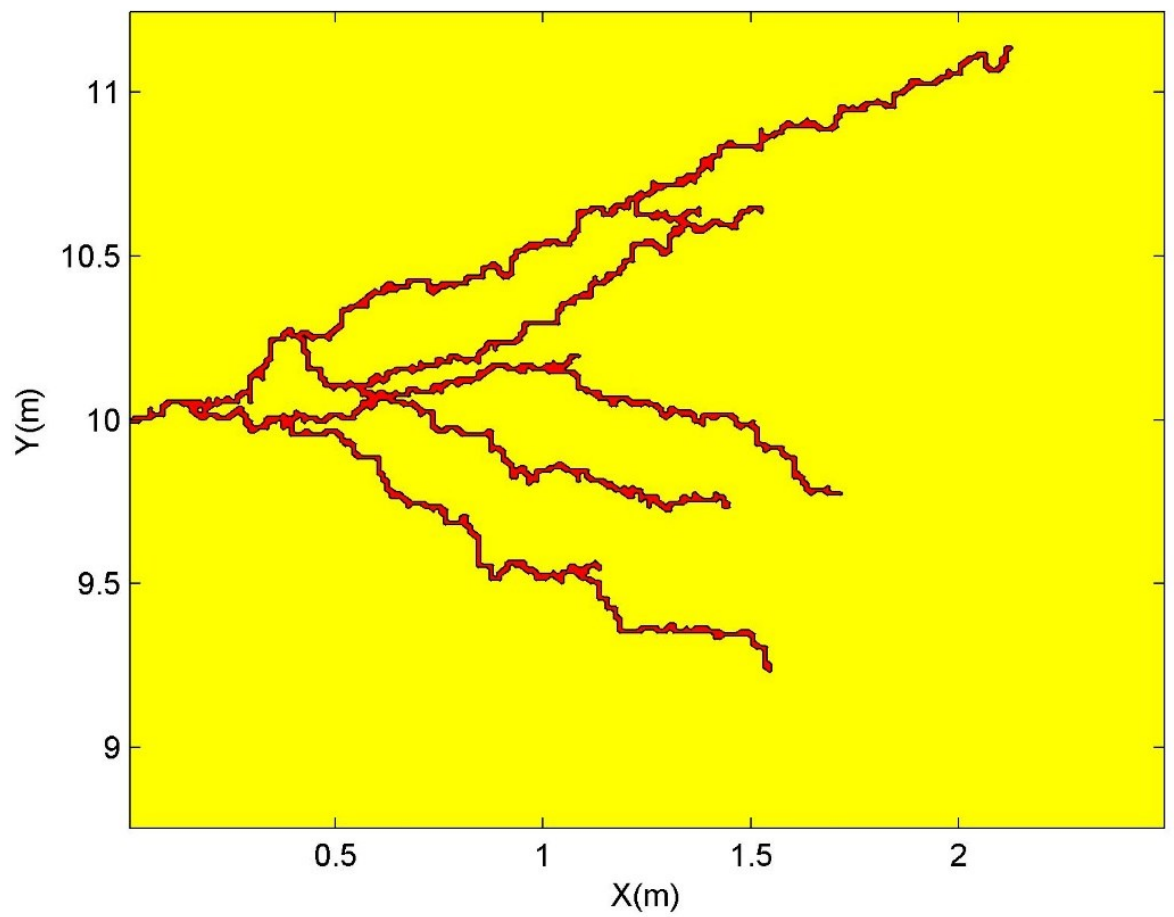

Figure A. $53 \quad-\quad$ Tri-branched fracture 9. 


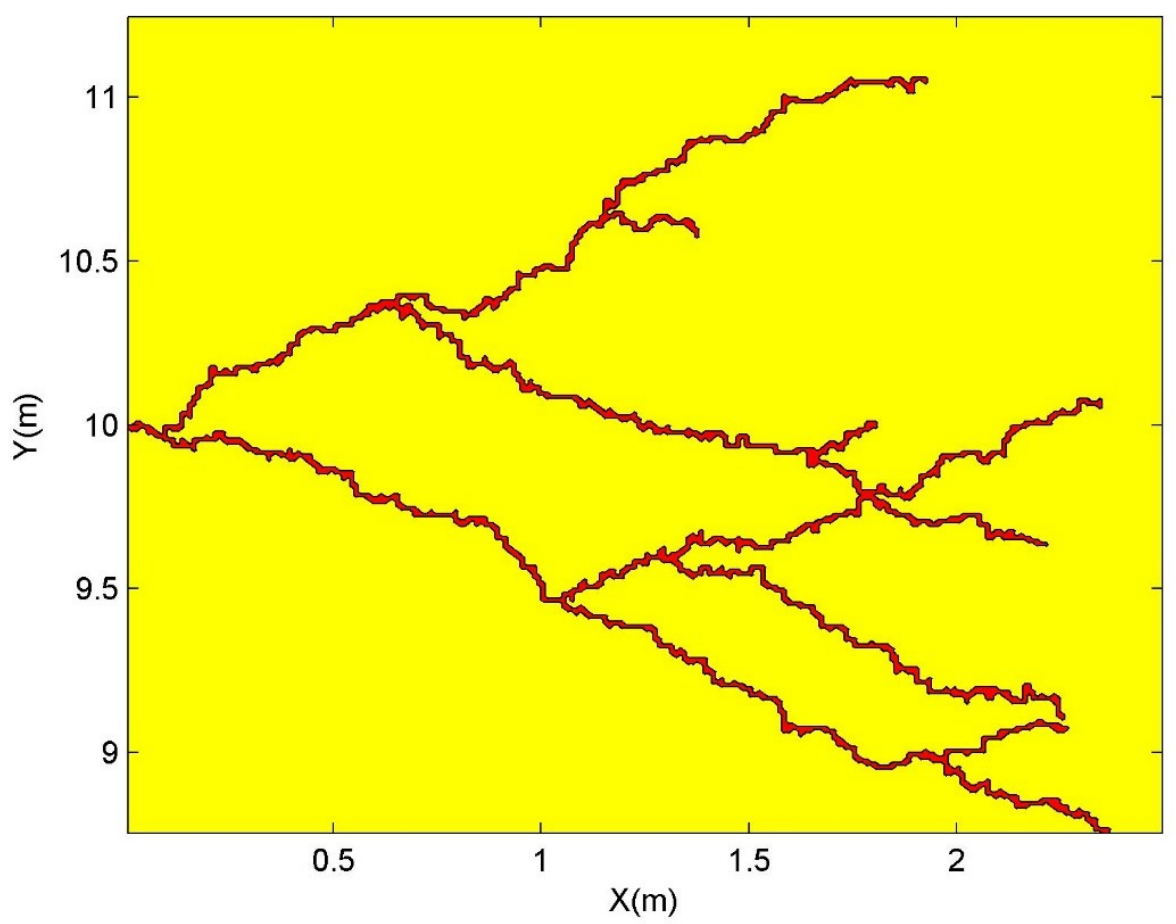

Figure A. 54 - Tri-branched fracture 10.

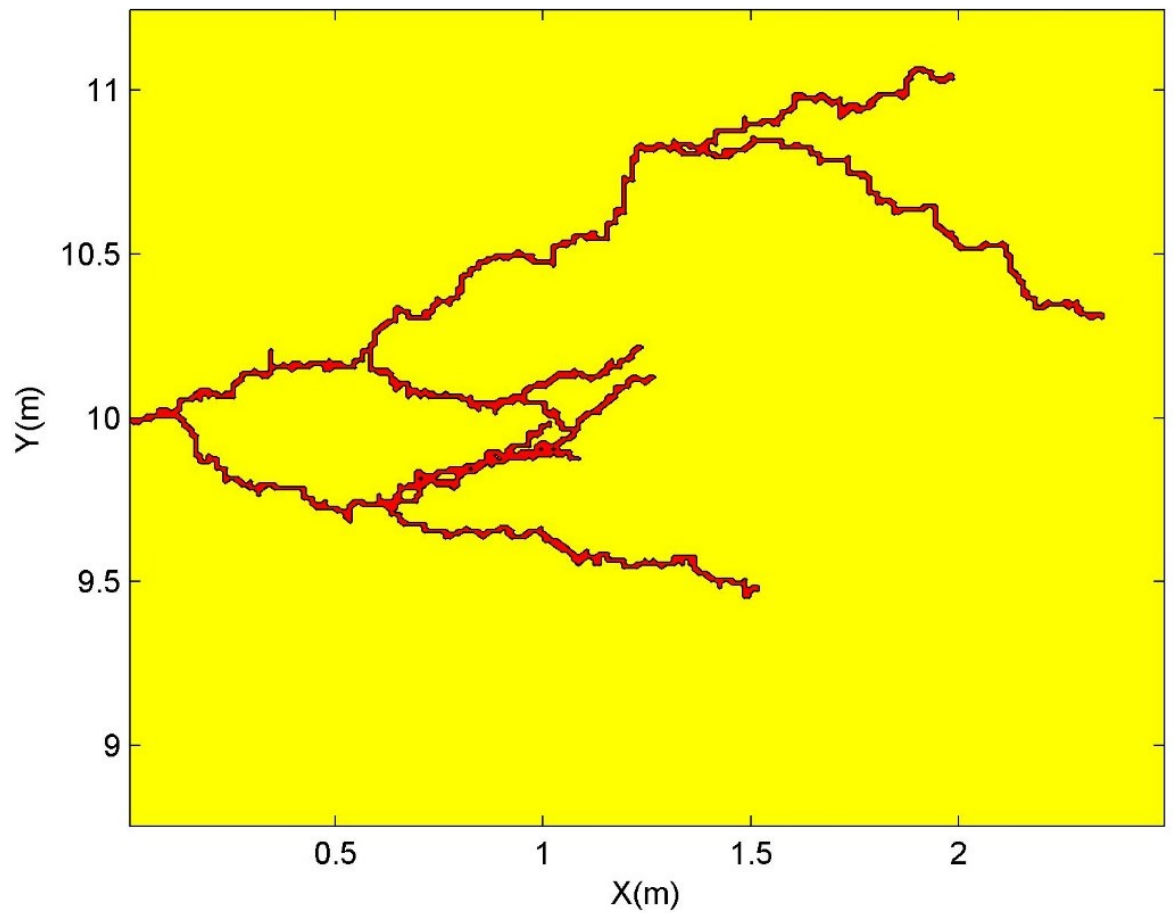

Figure A. 55 - Tri-branched fracture 11. 


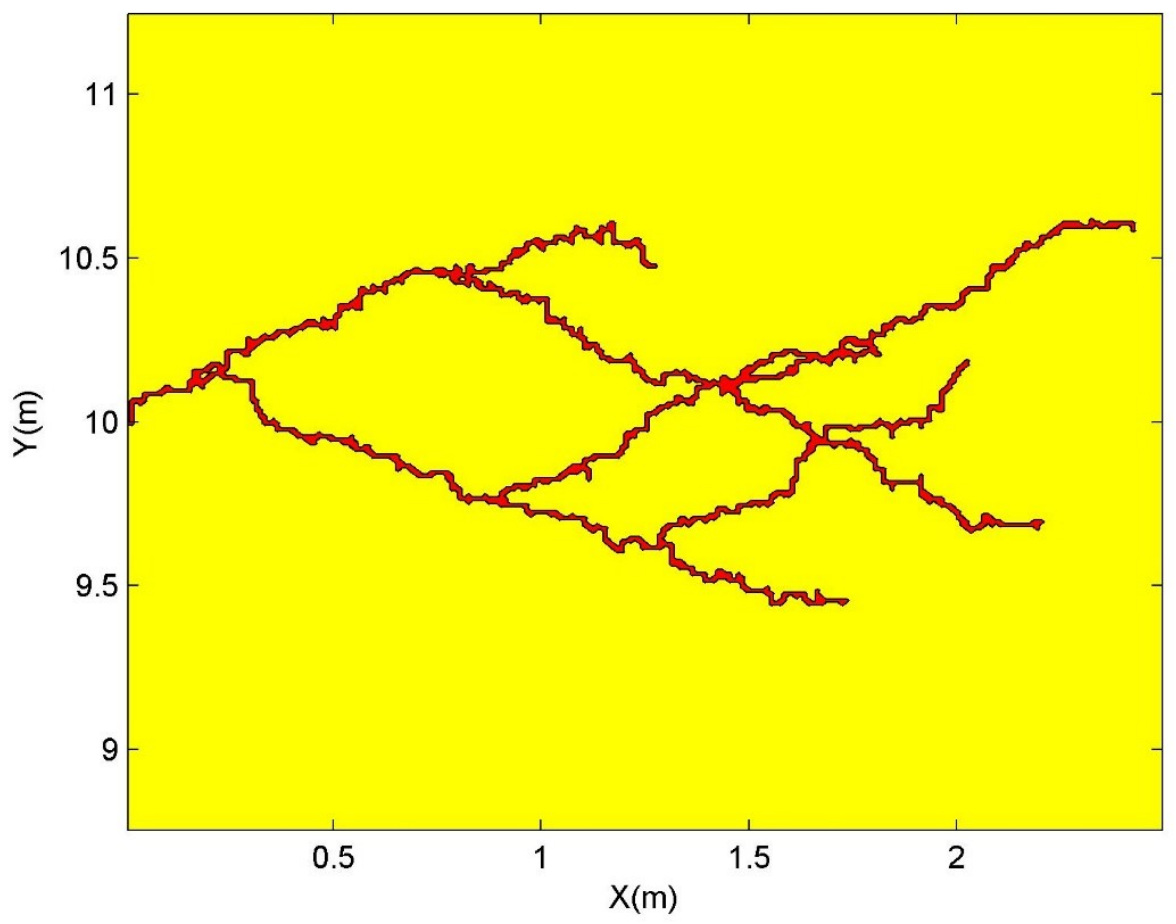

Figure A. 56 - Tri-branched fracture 12.

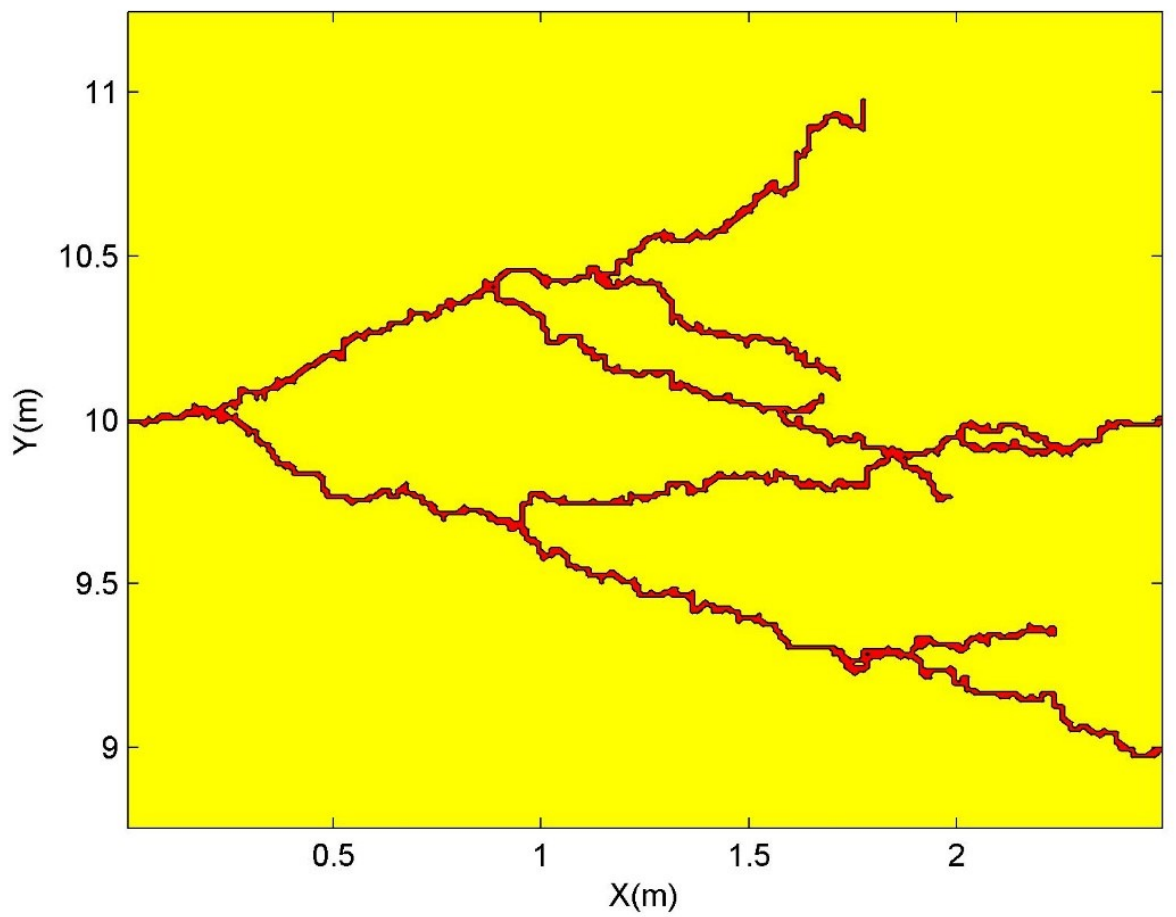

Figure A. 57 - Tri-branched fracture 13. 


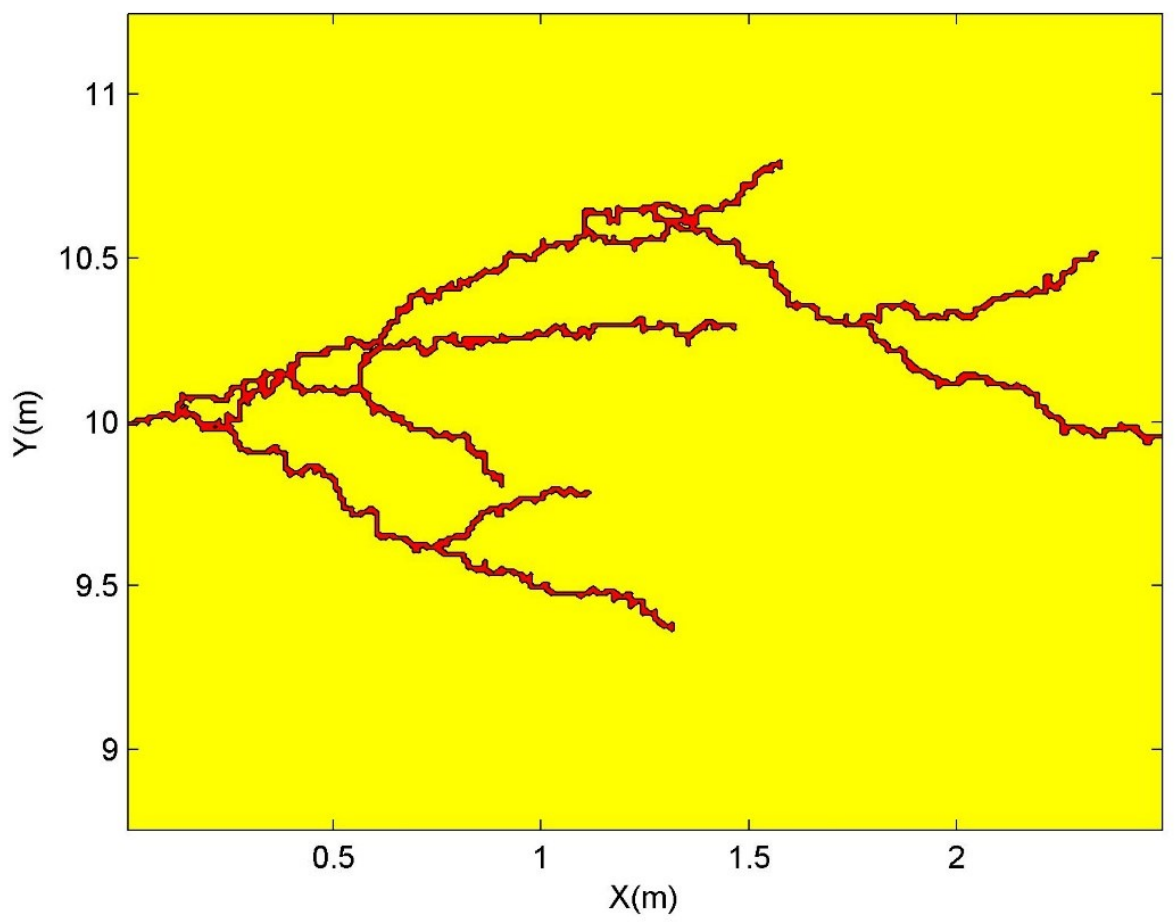

Figure A. 58 - Tri-branched fracture 14.

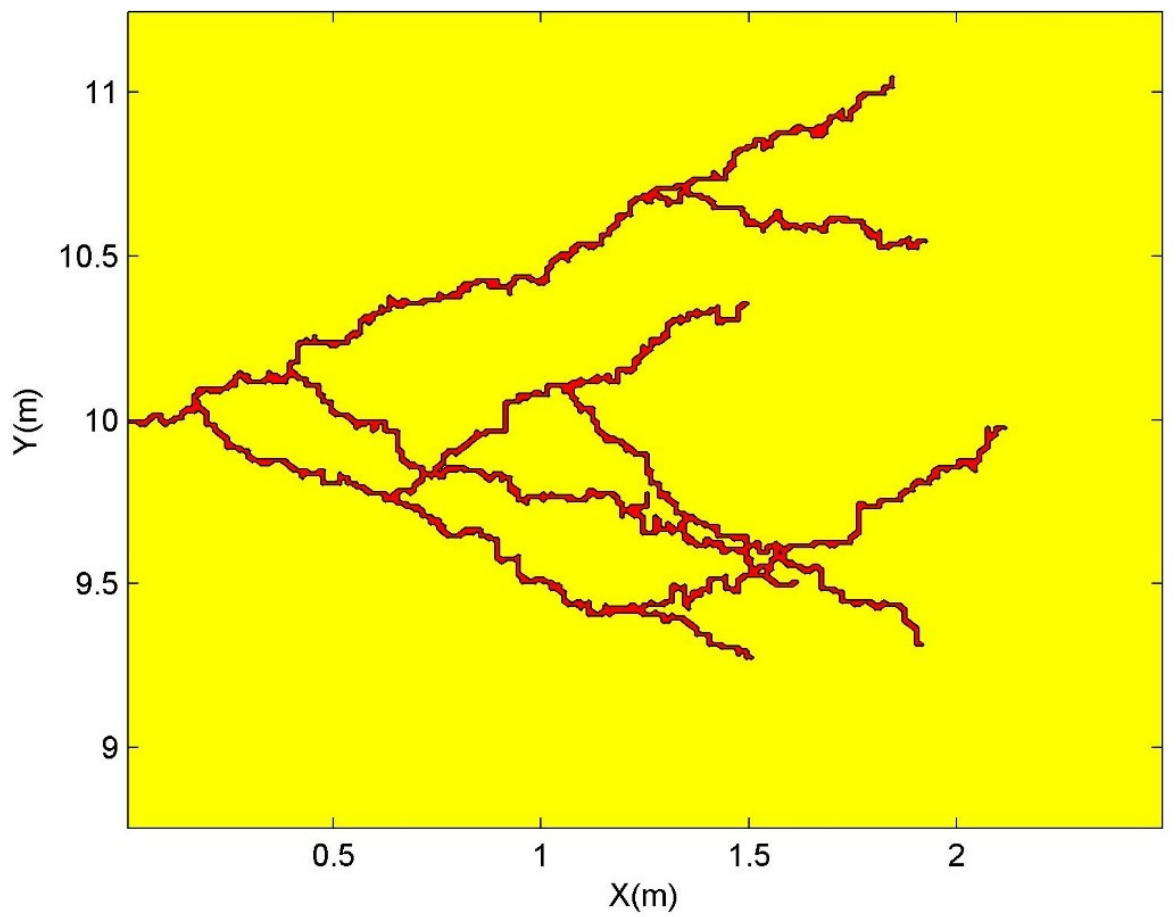

Figure A. 59 - Tri-branched fracture 15. 


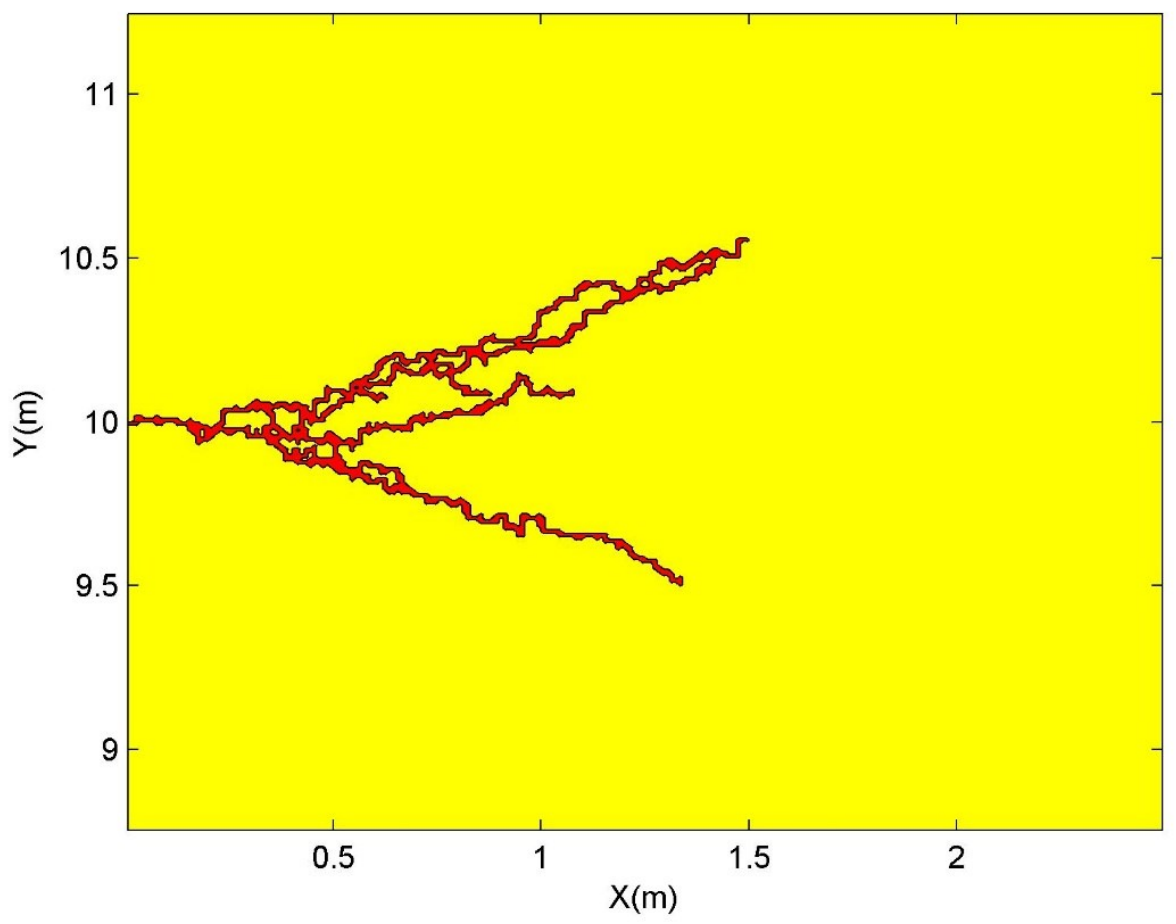

Figure A. 60 - Tri-branched fracture 16.

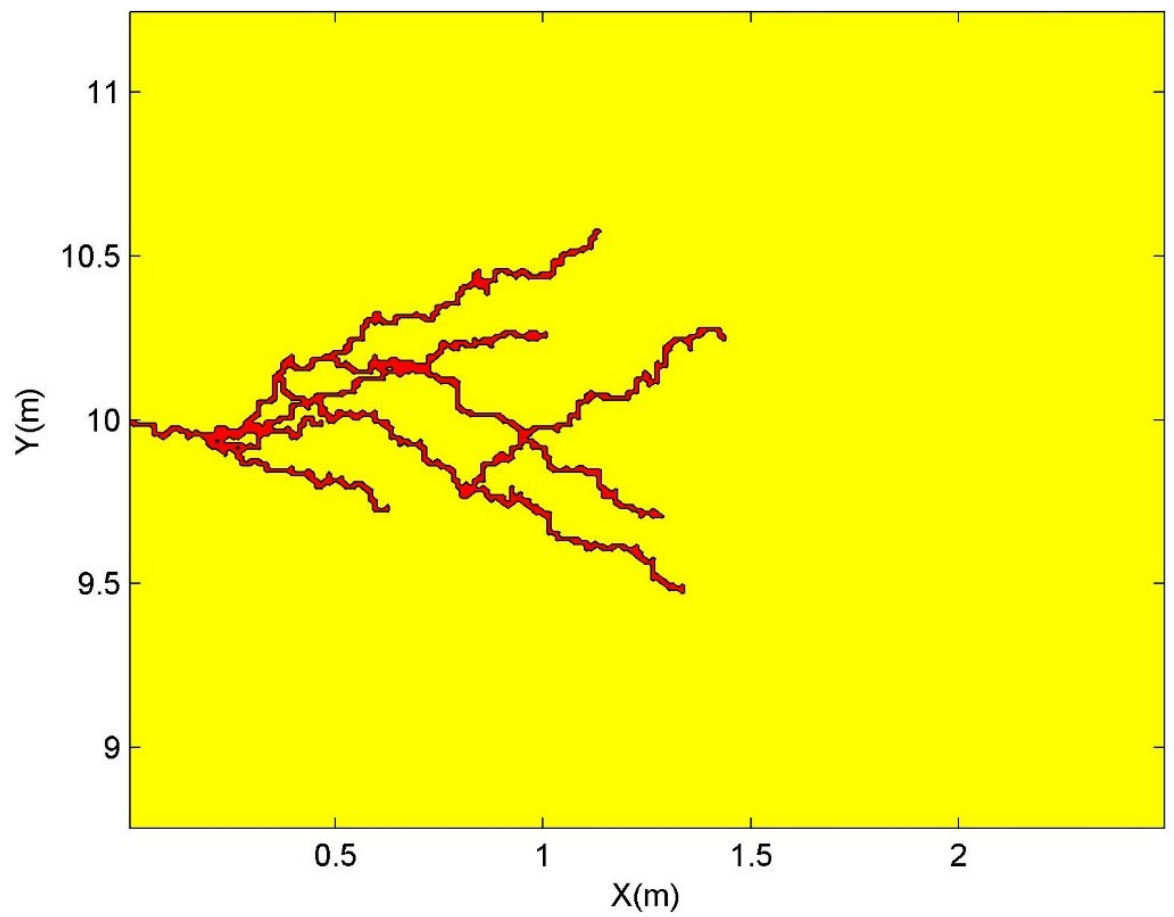

Figure A. 61 - Tri-branched fracture 17. 


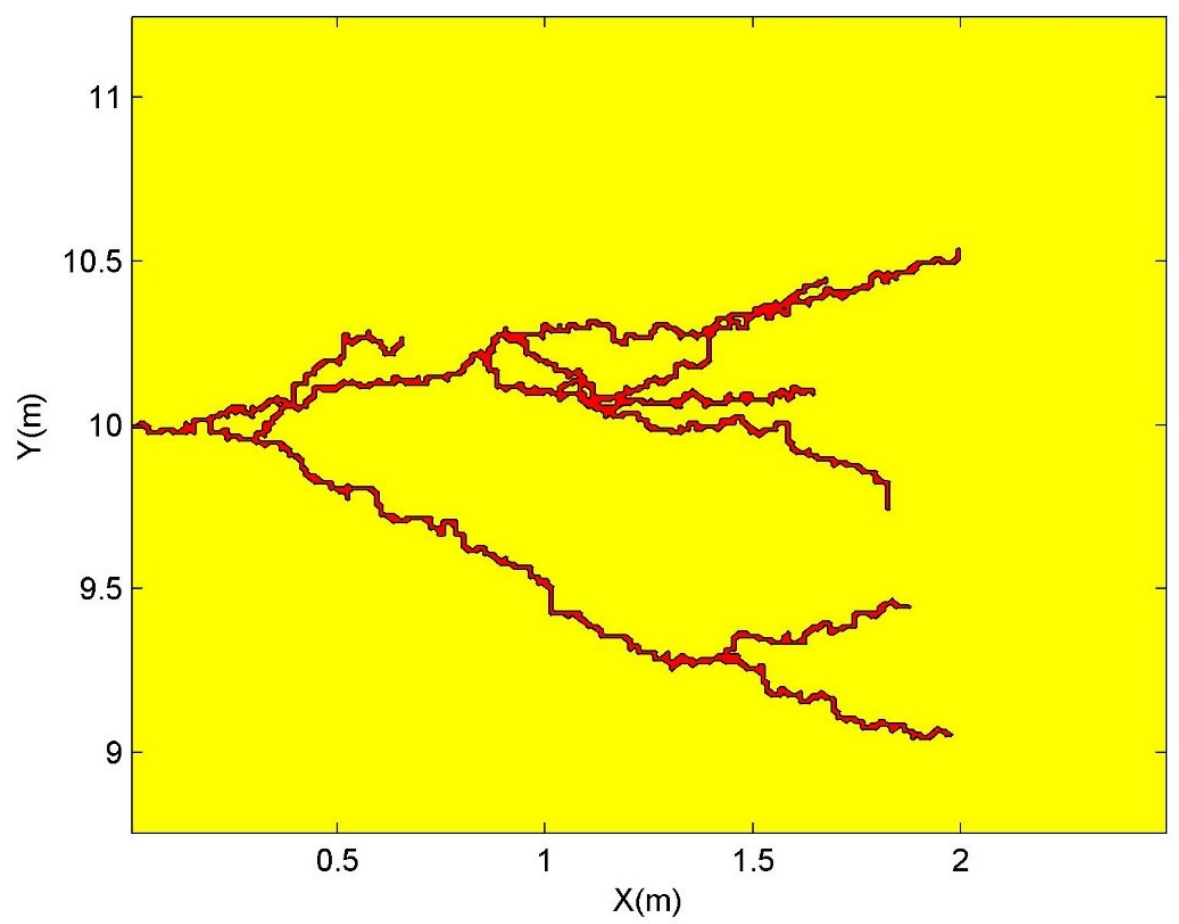

Figure A. 62 - Tri-branched fracture 18.

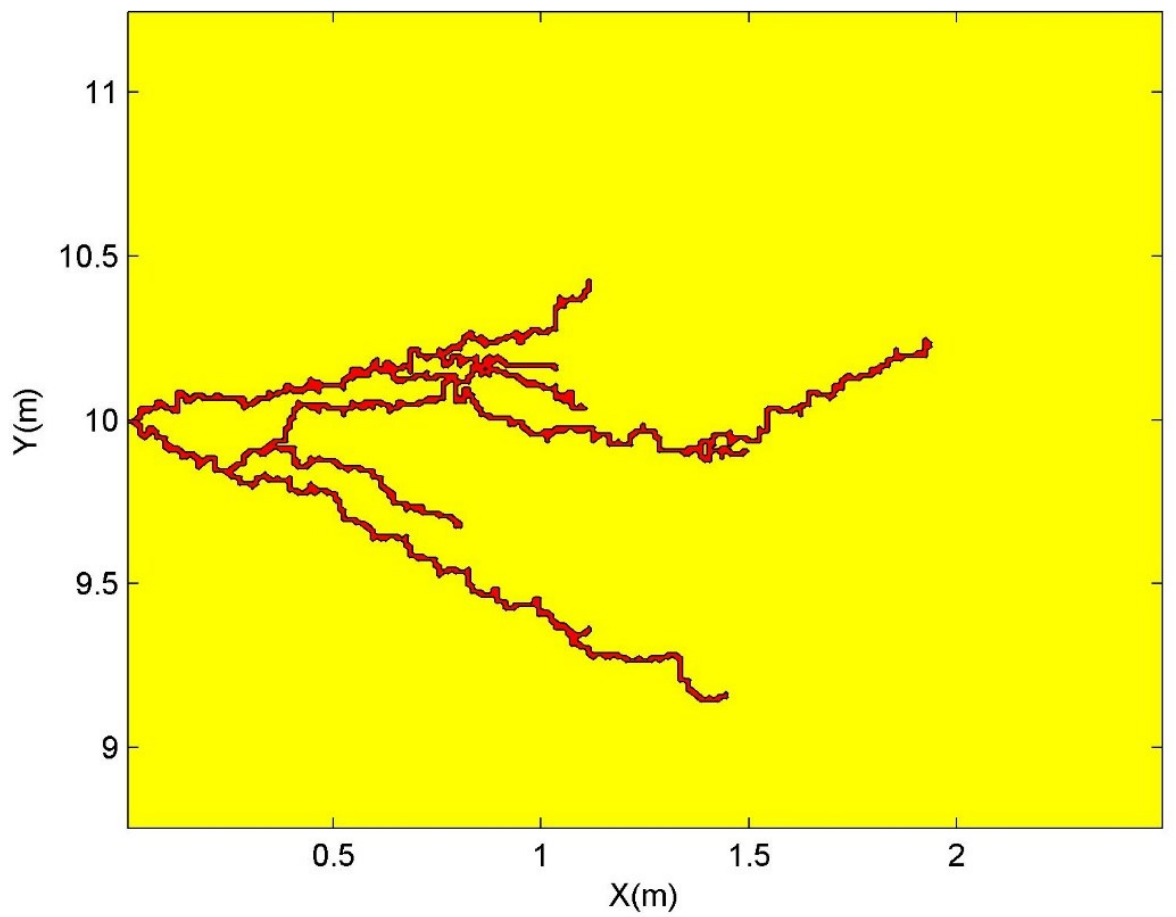

Figure A. 63 - Tri-branched fracture 19. 


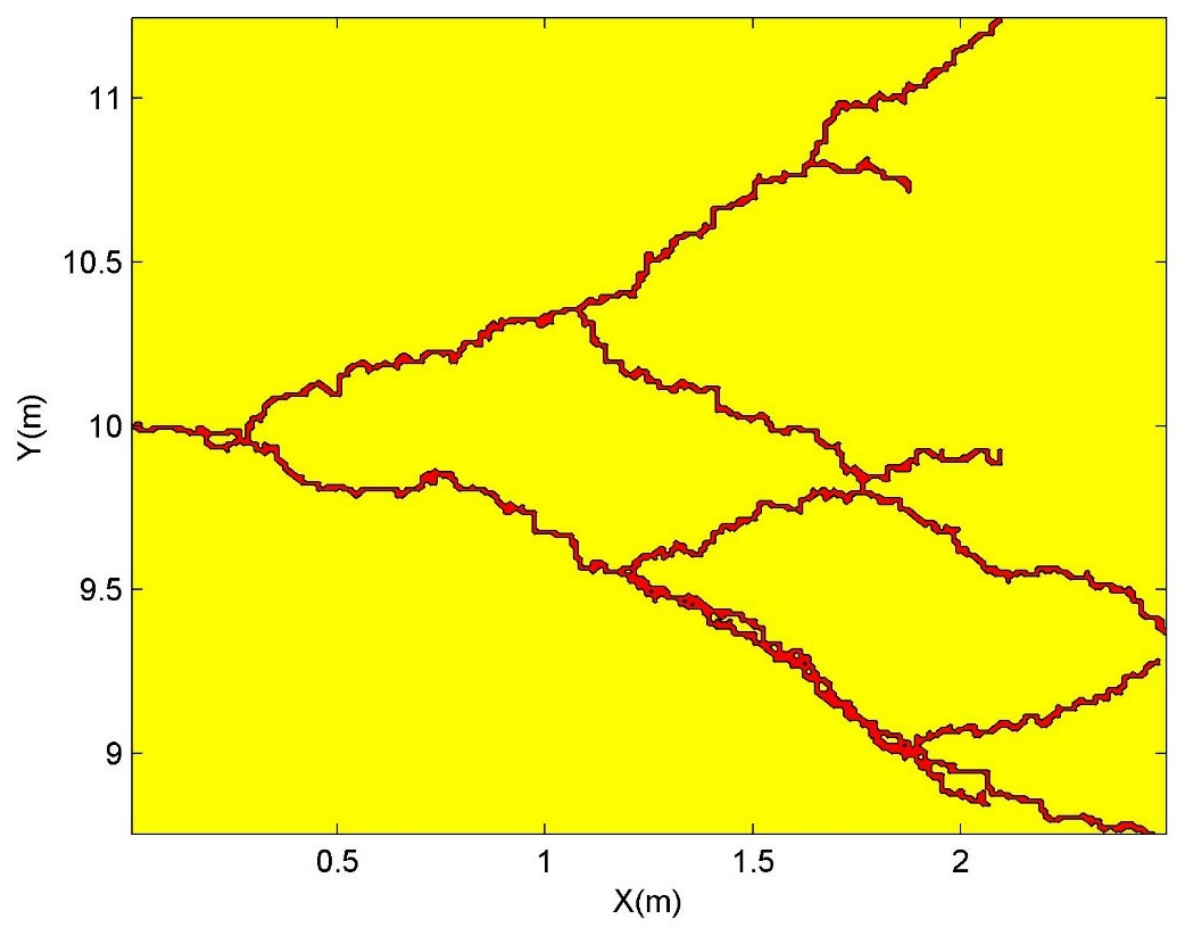

Figure A. 64 - Tri-branched fracture 20.

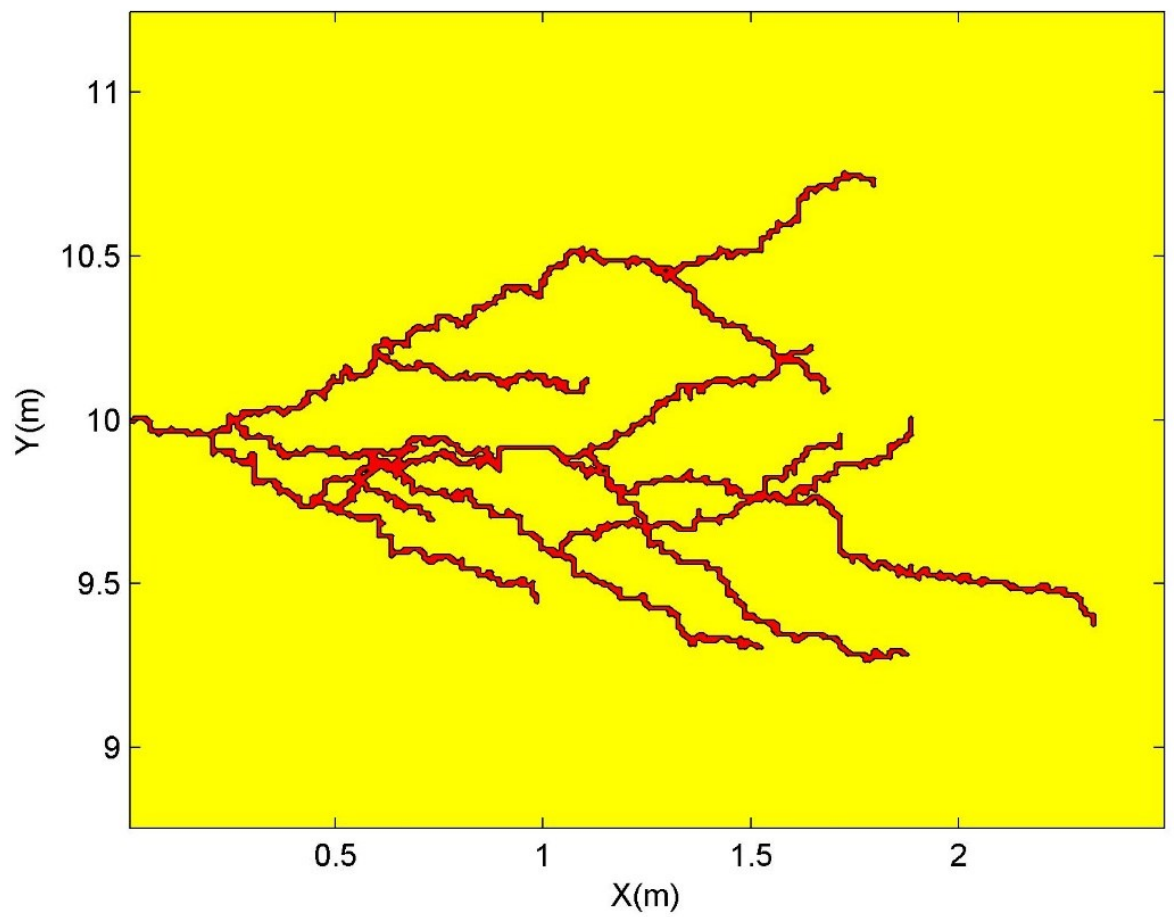

Figure A. 65 - Quad-branched fracture 1. 


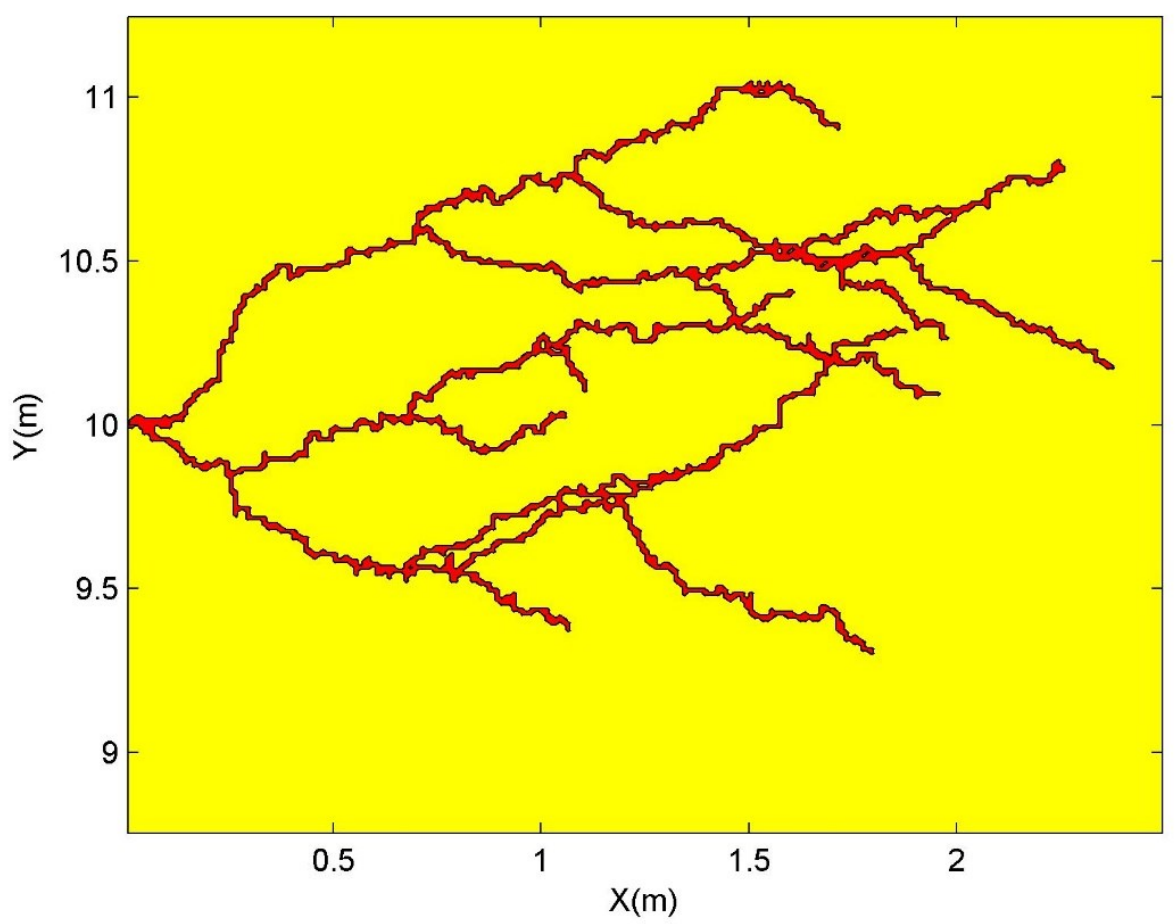

Figure A. 66 - Quad-branched fracture 2.

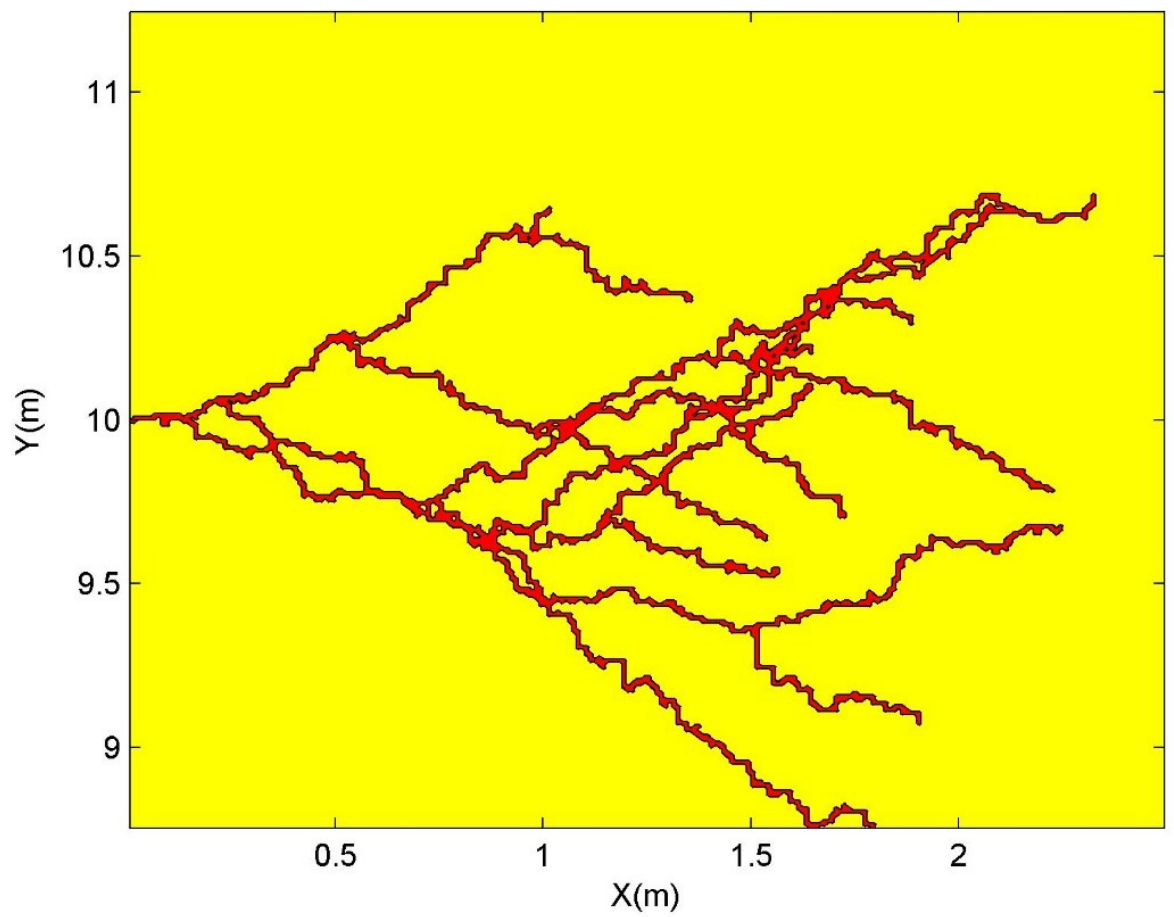

Figure A. 67 - Quad-branched fracture 3. 


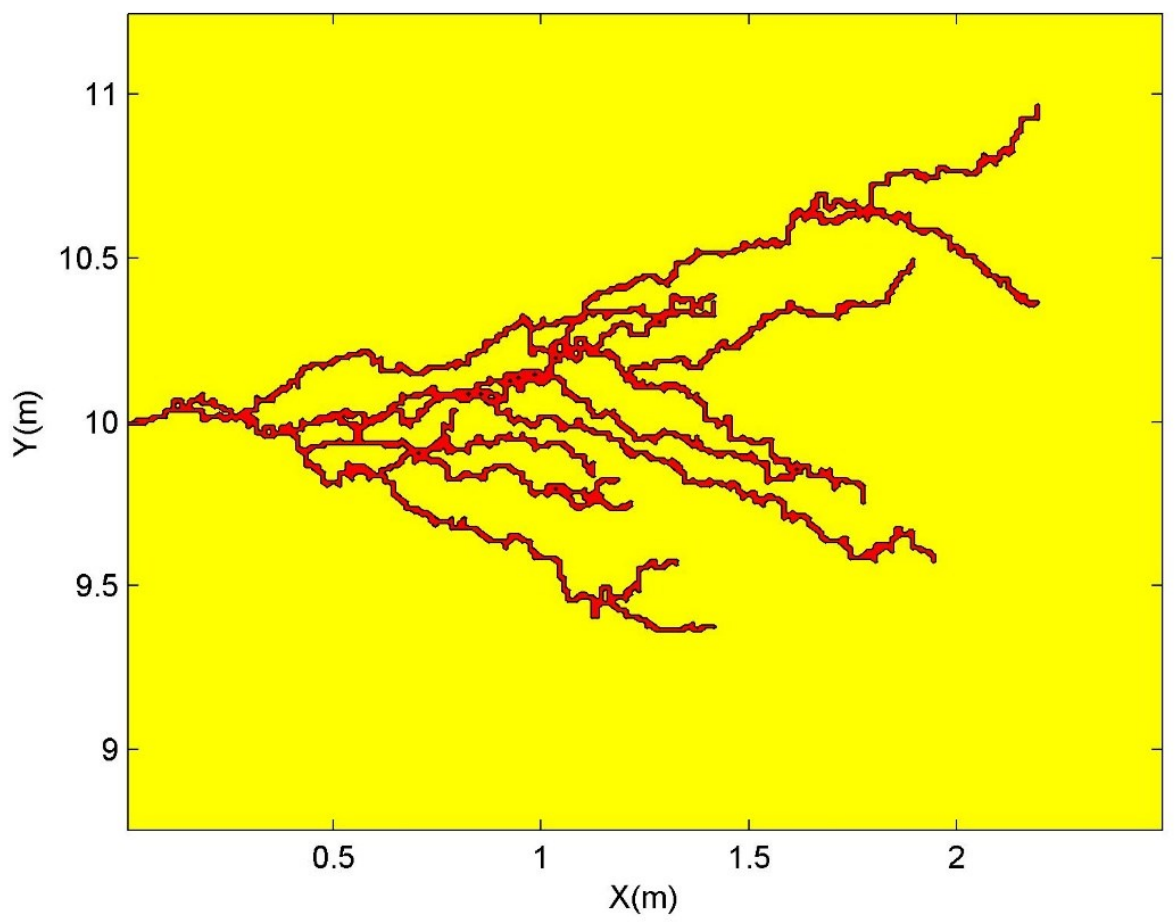

Figure A. 68 - Quad-branched fracture 4.

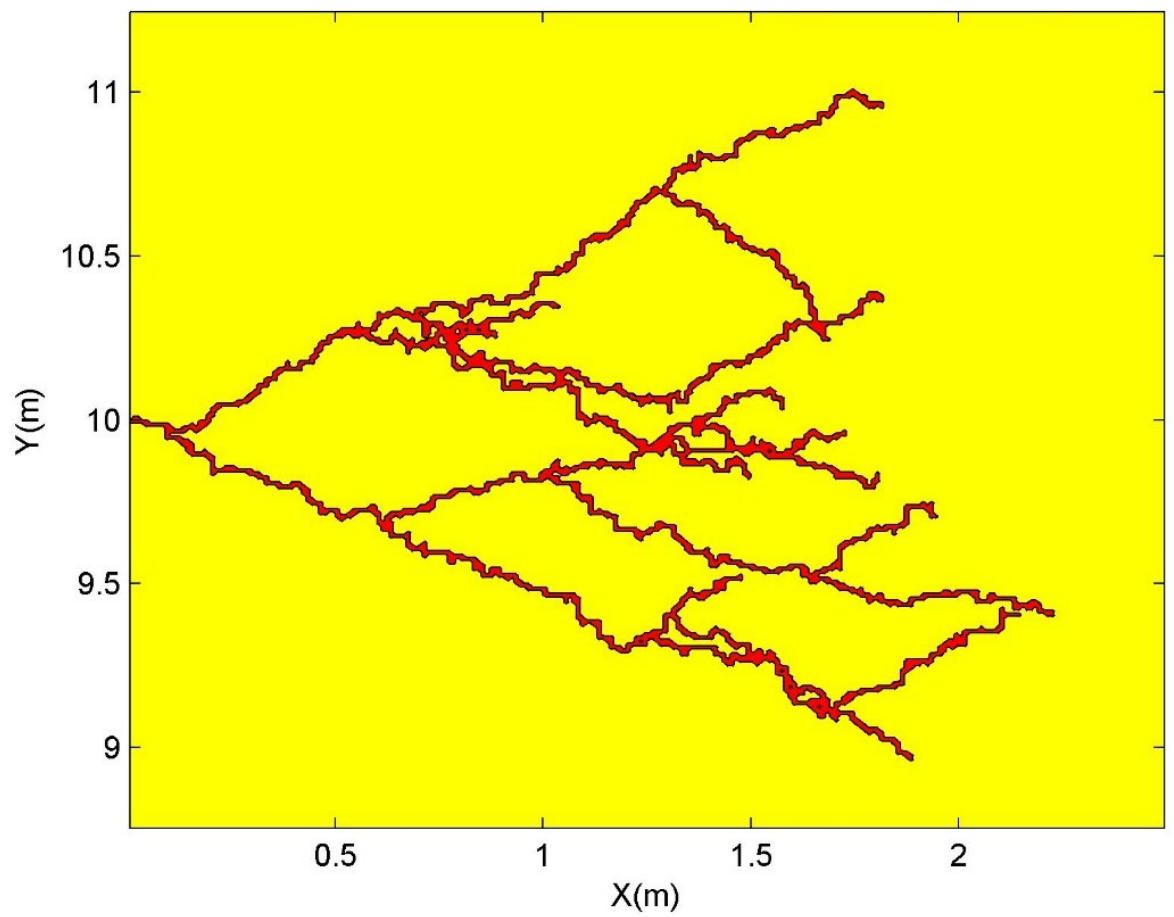

Figure A. 69 - Quad-branched fracture 5. 


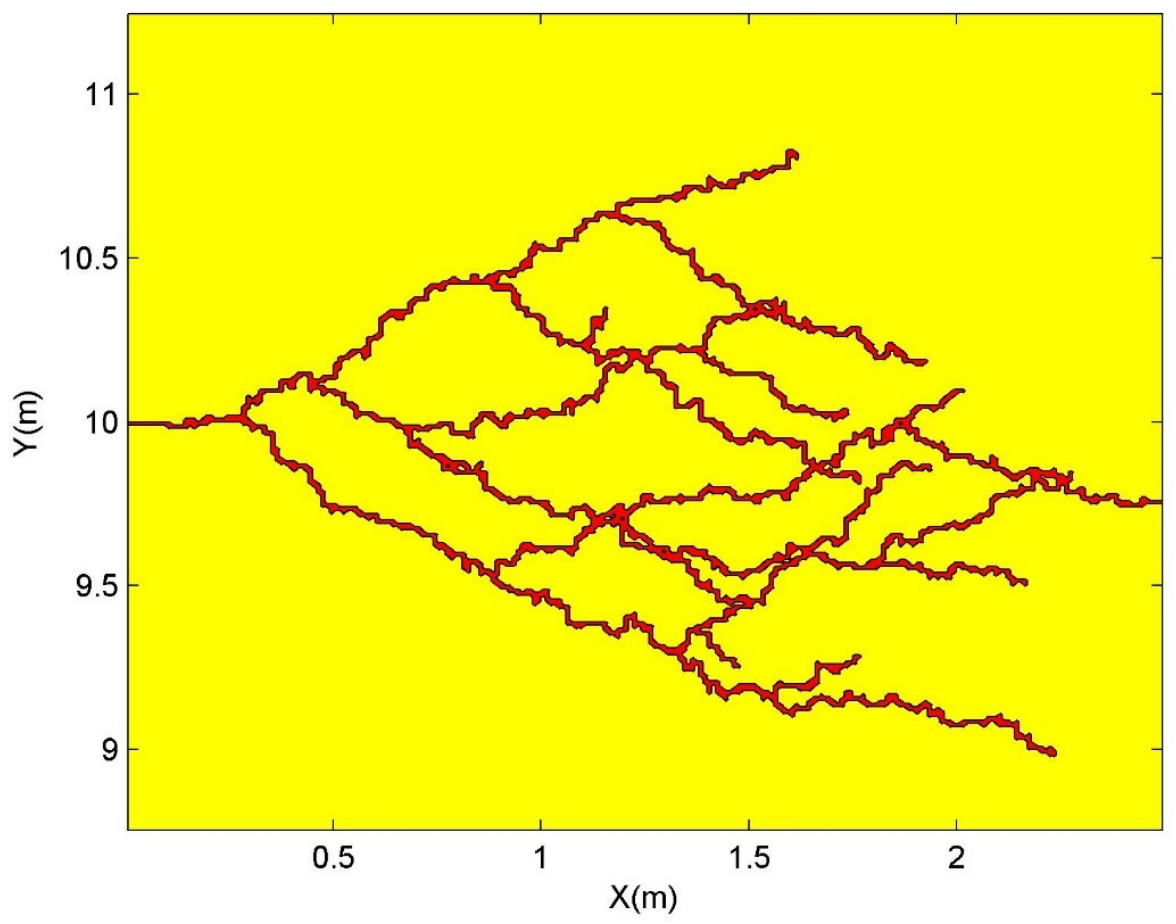

Figure A. 70 - Quad-branched fracture 6.

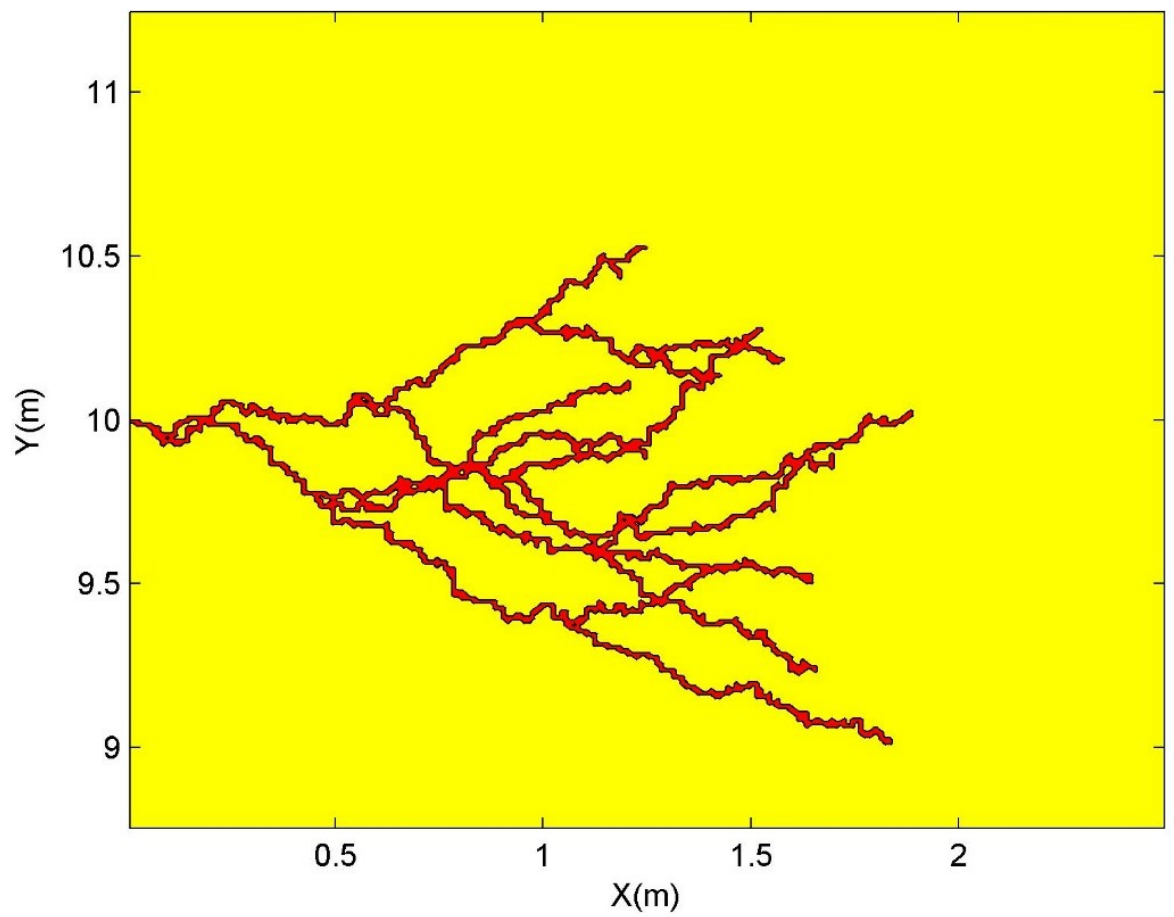

Figure A. 71 - Quad-branched fracture 7. 


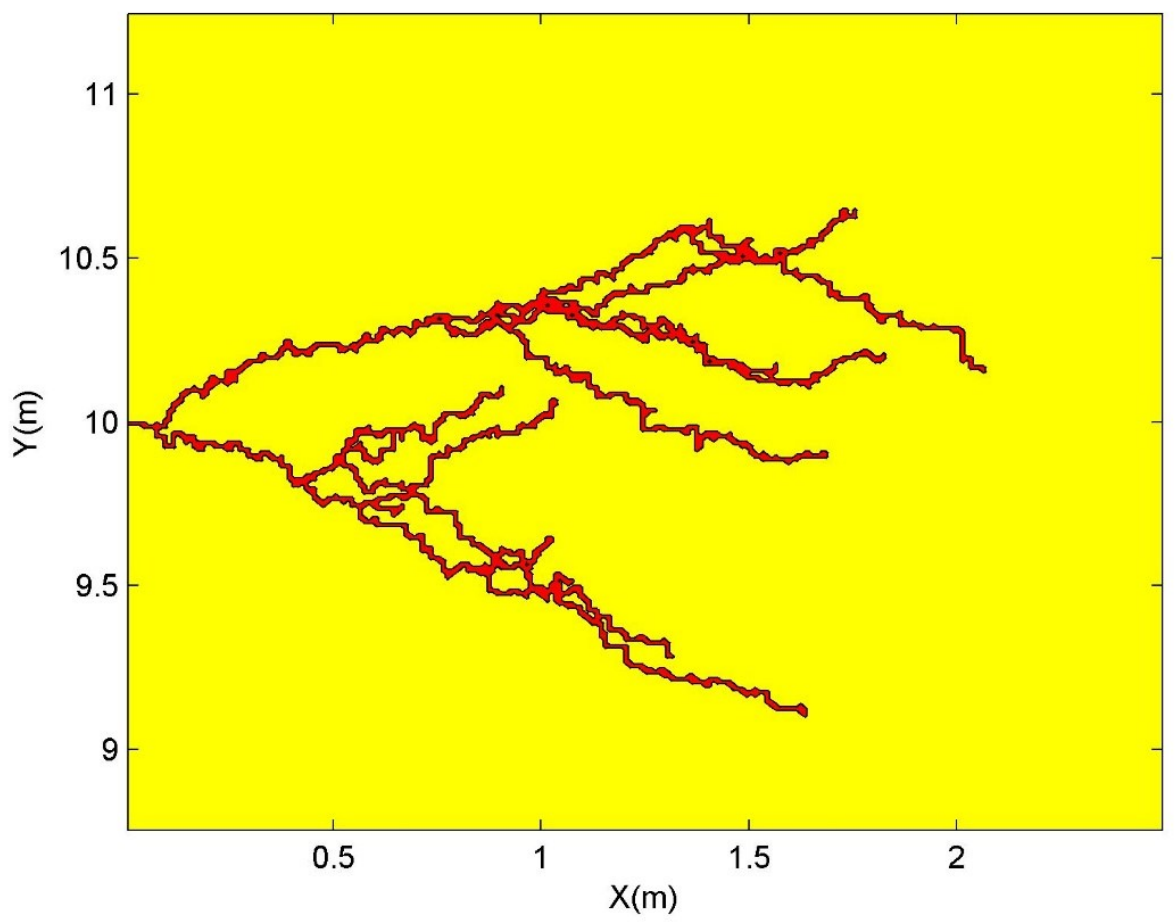

Figure A. 72 - Quad-branched fracture 8.

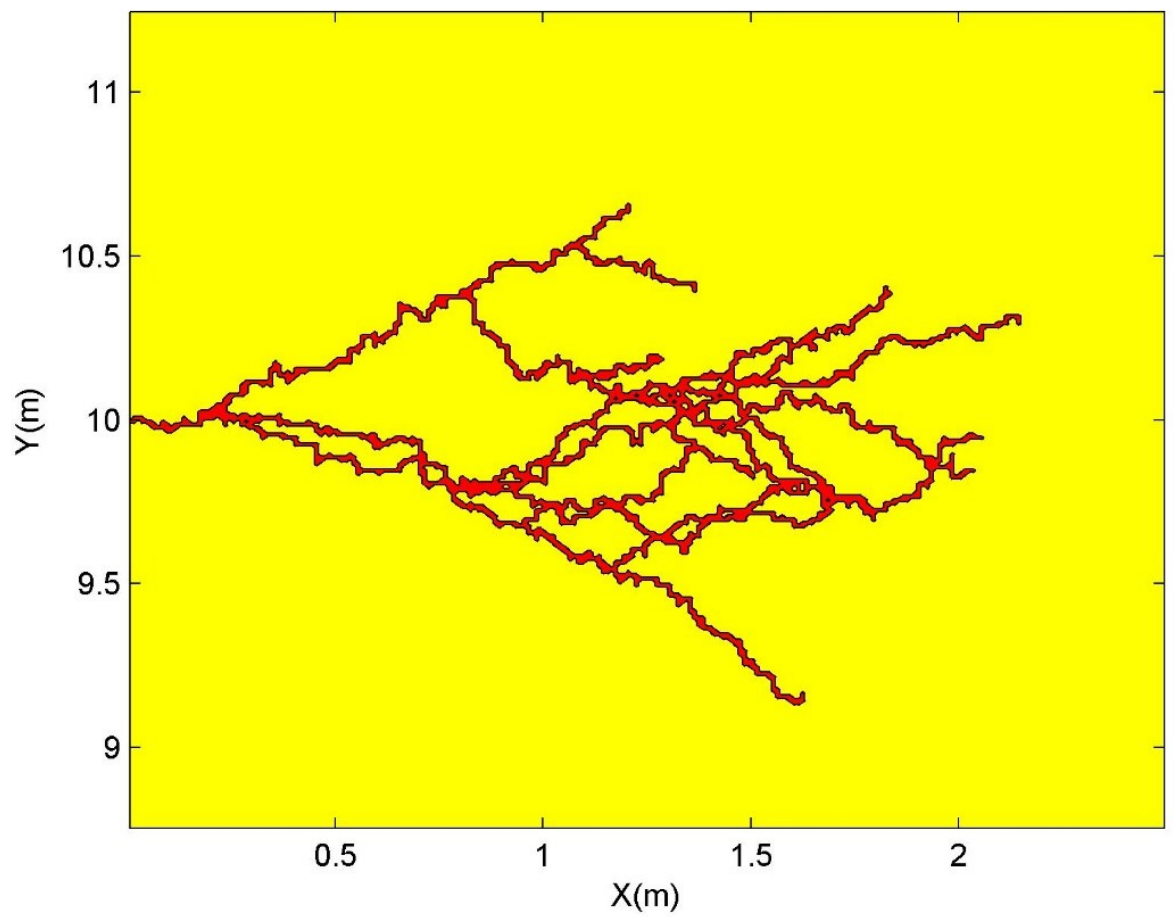

Figure A. 73 - Quad-branched fracture 9. 


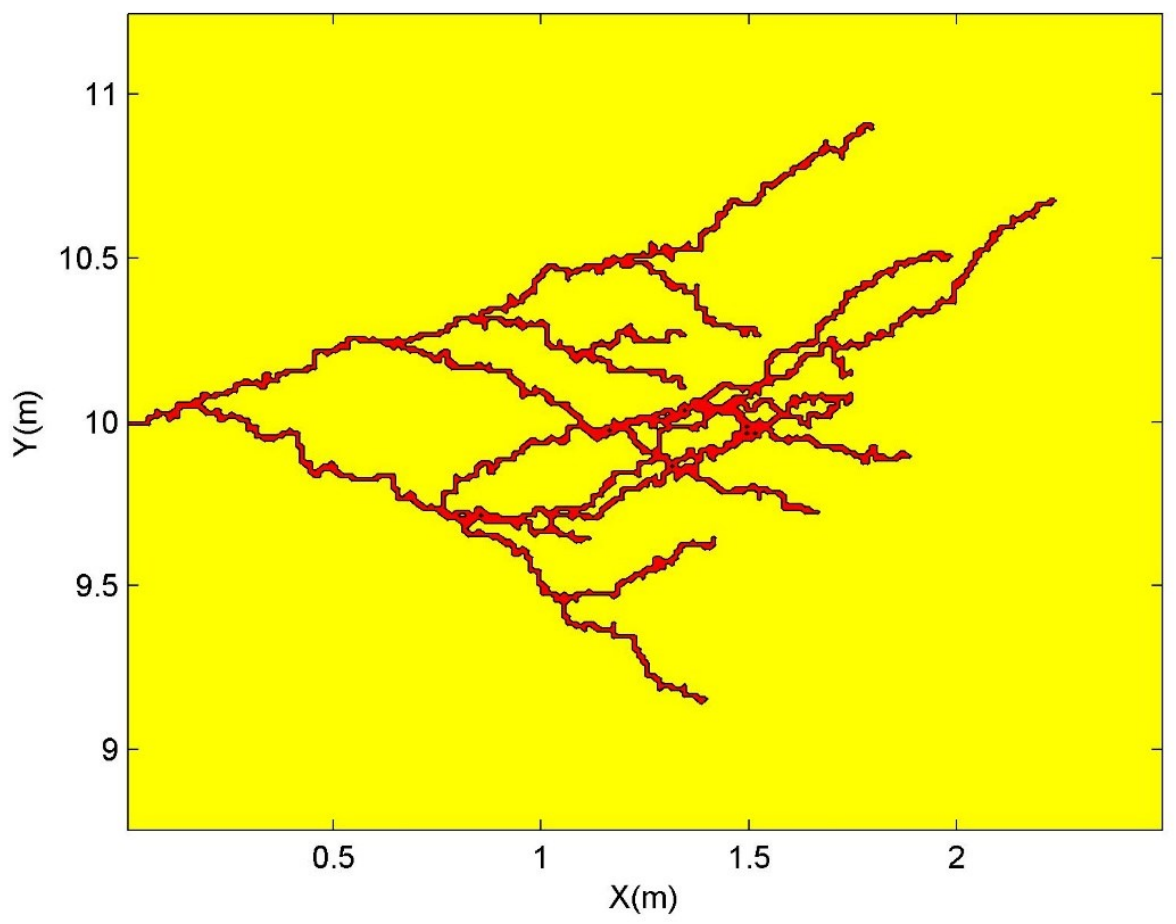

Figure A. 74 - Quad-branched fracture 10.

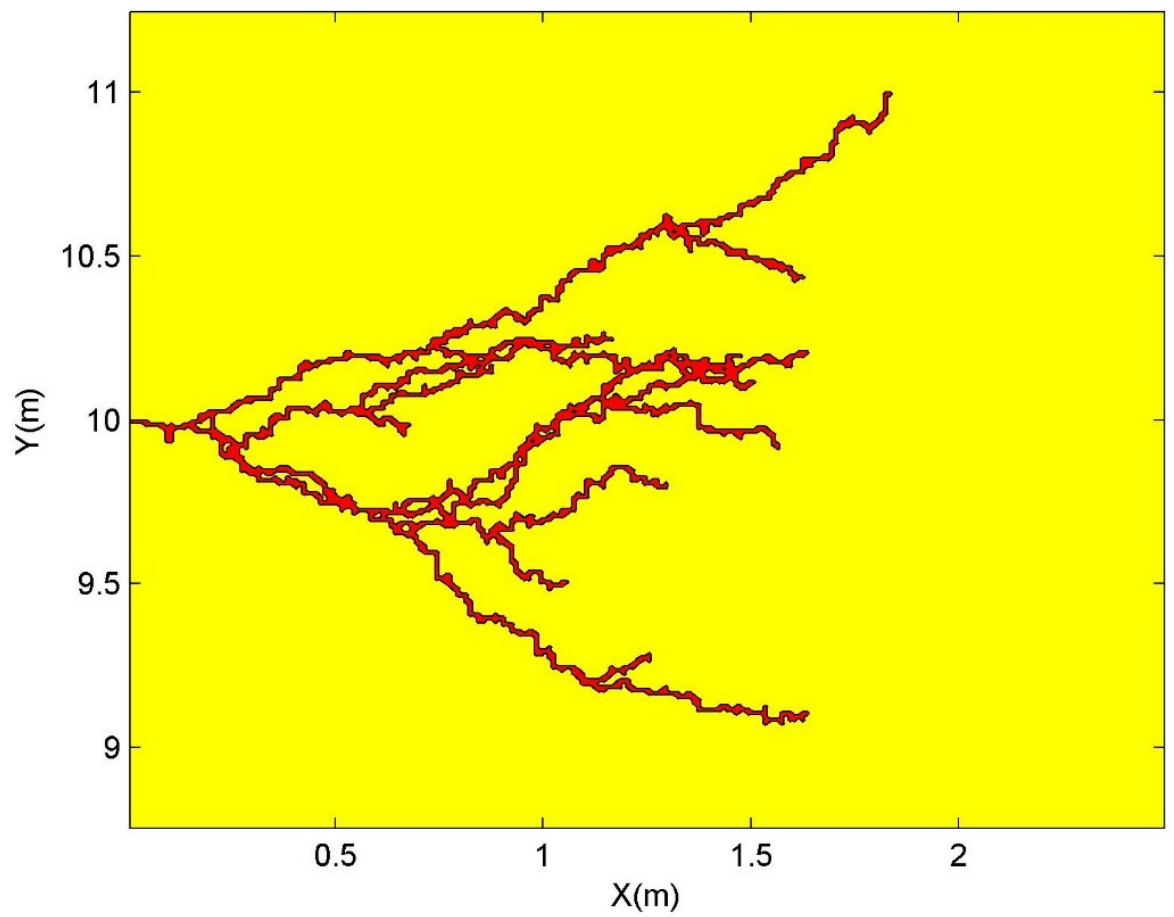

Figure A. 75 - Quad-branched fracture 11. 


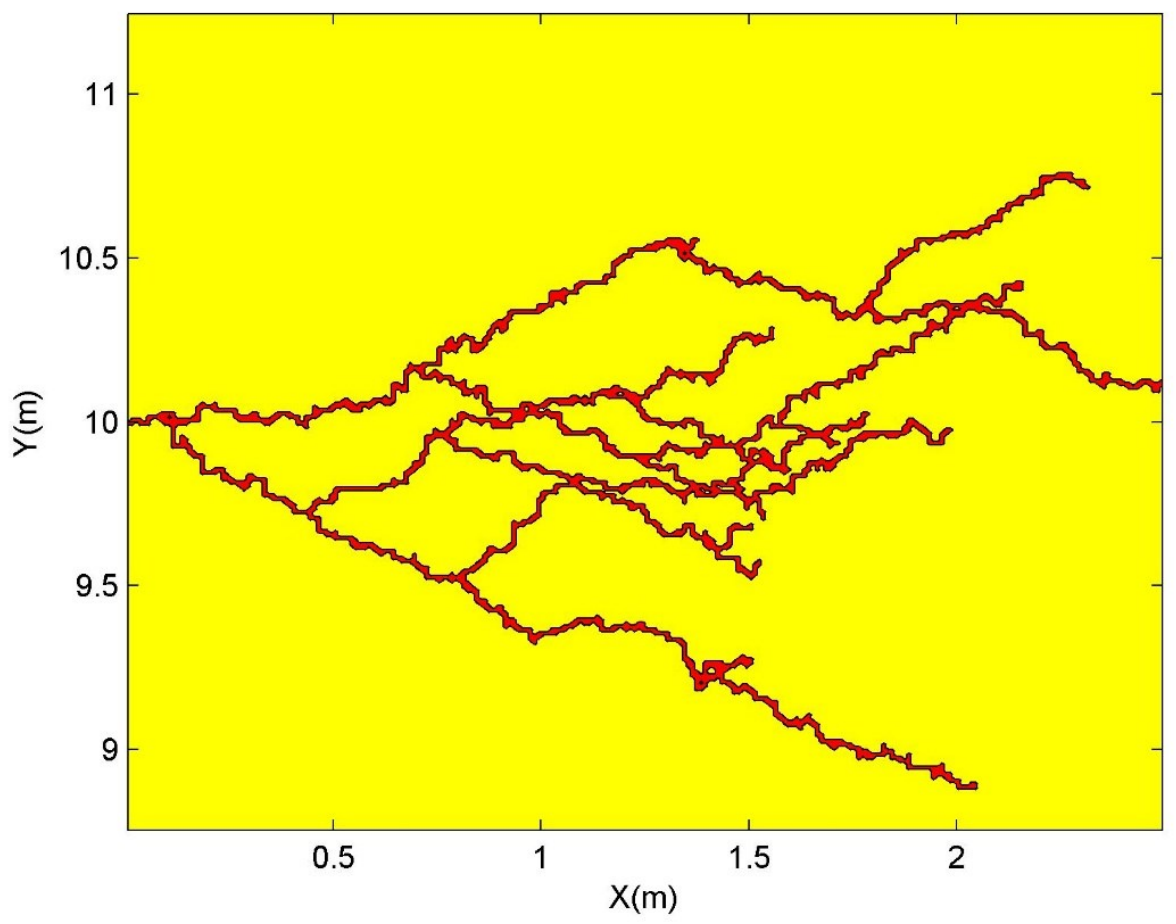

Figure A. 76 - Quad-branched fracture 12.

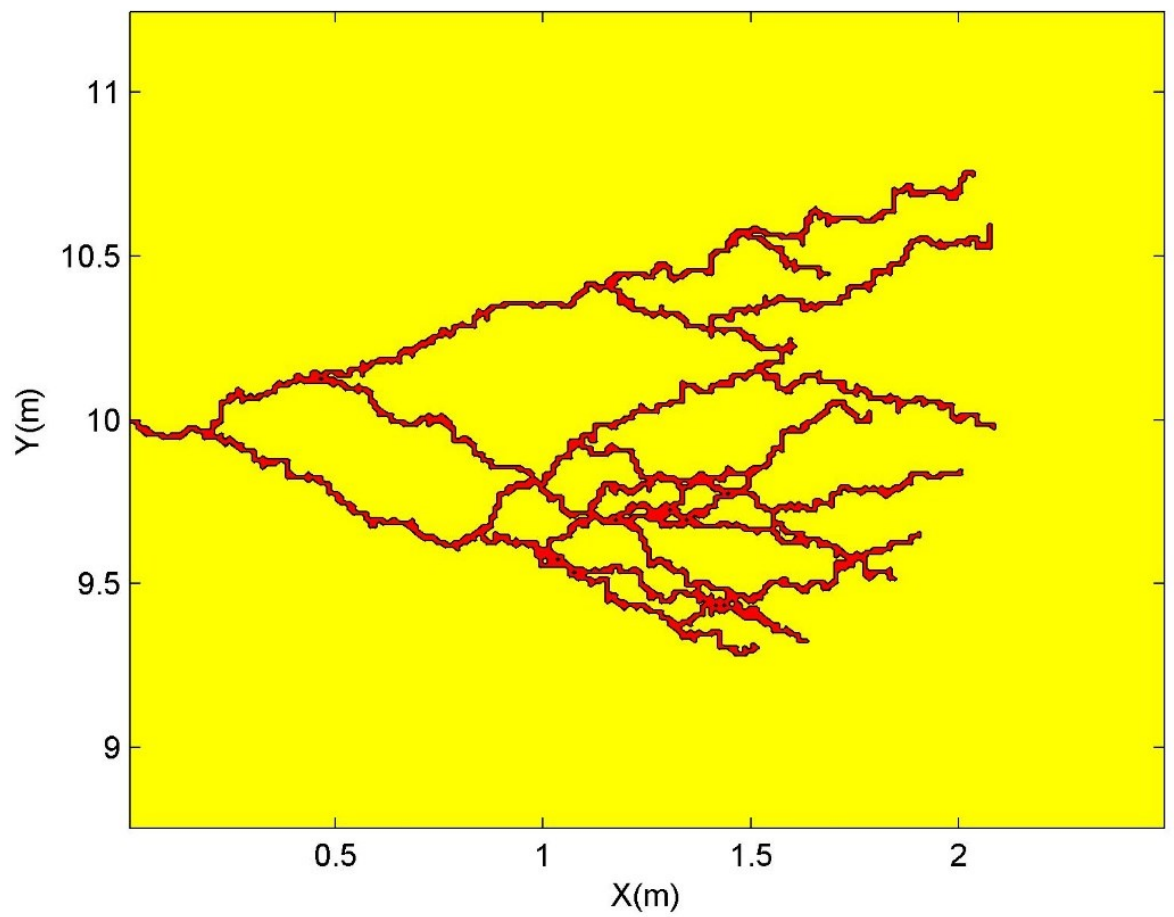

Figure A. 77 - Quad-branched fracture 13. 


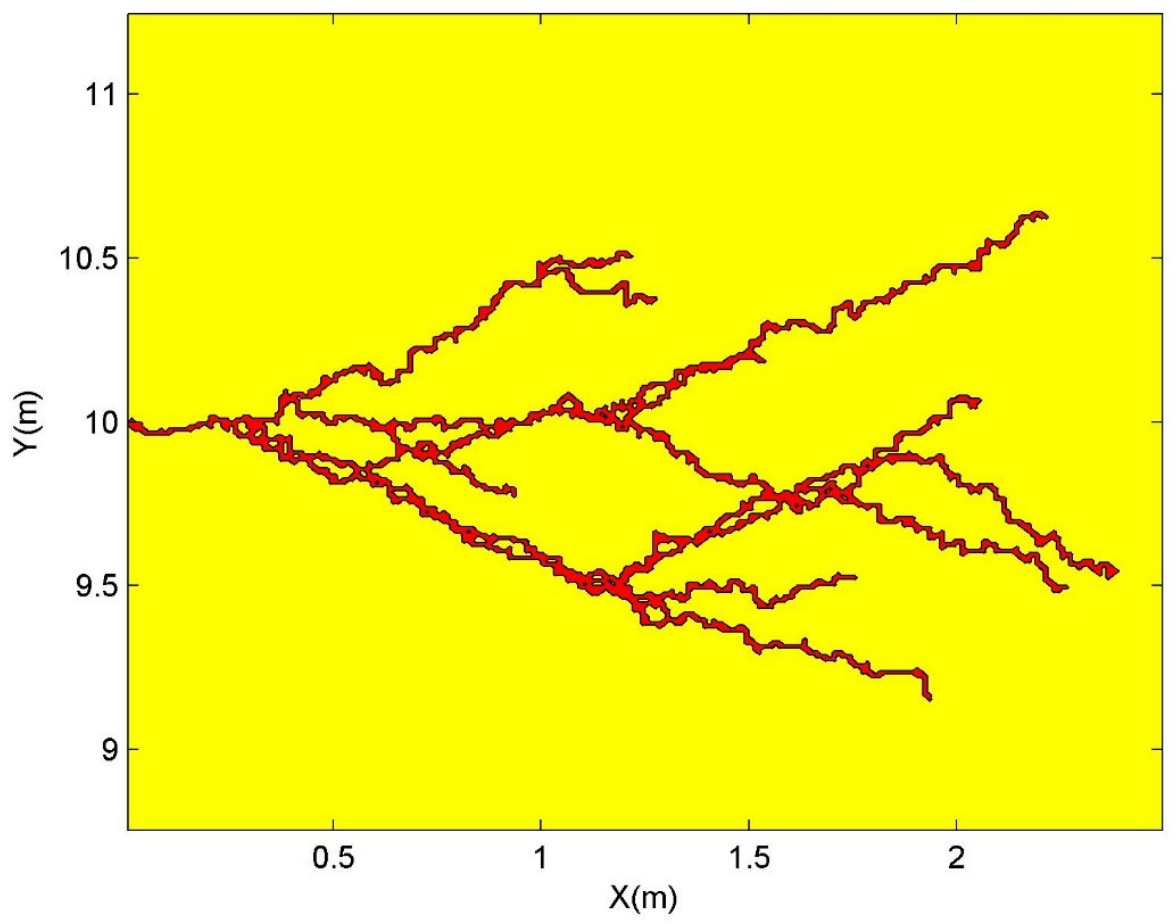

Figure A. 78 - Quad-branched fracture 14.

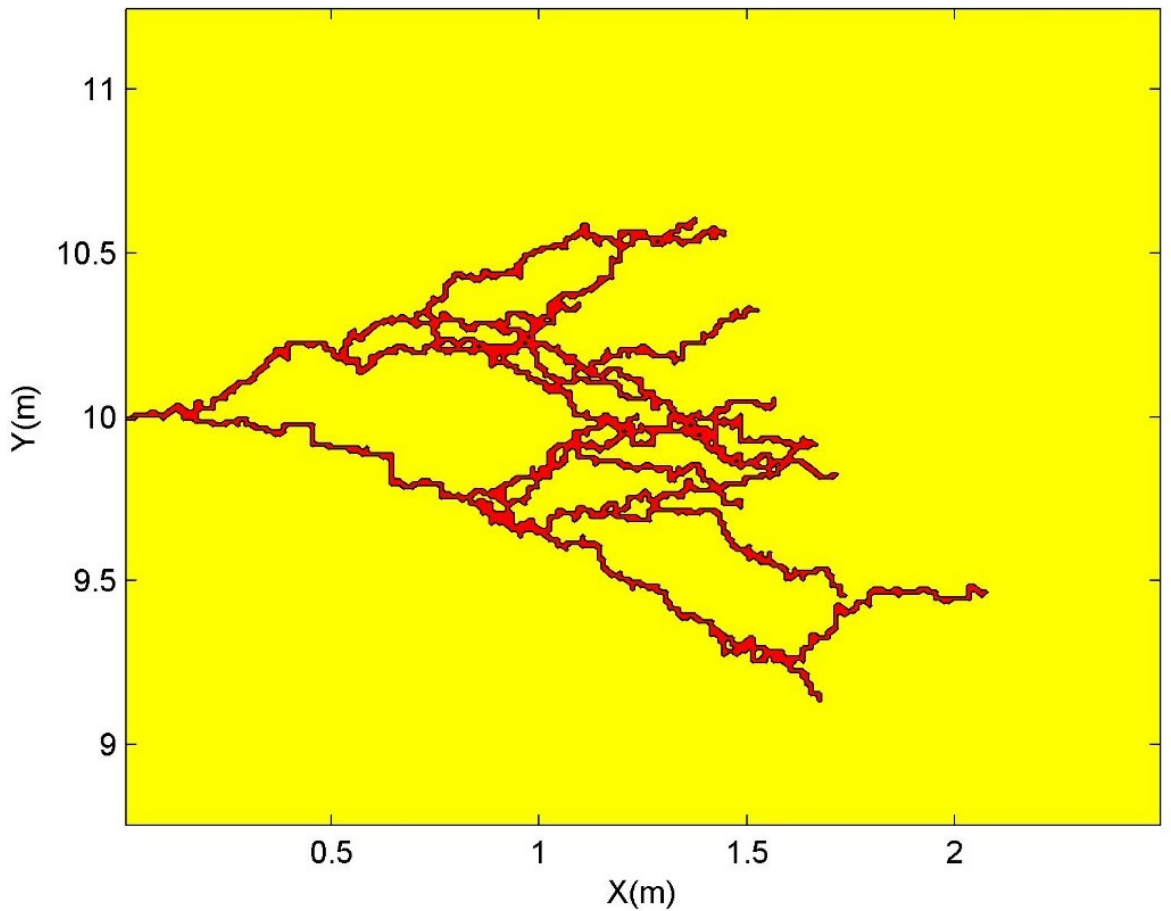

Figure A. 79 - Quad-branched fracture 15. 


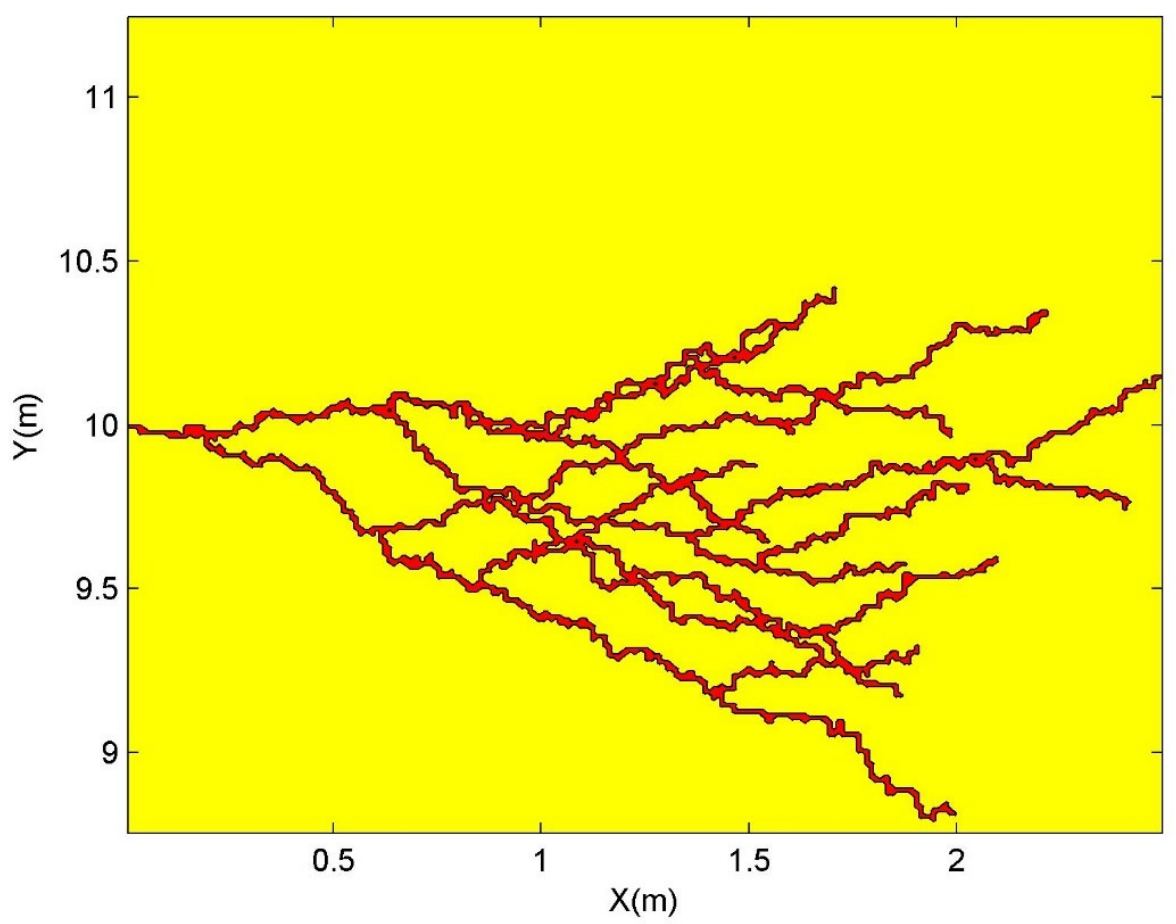

Figure A. 80 - Quad-branched fracture 16.

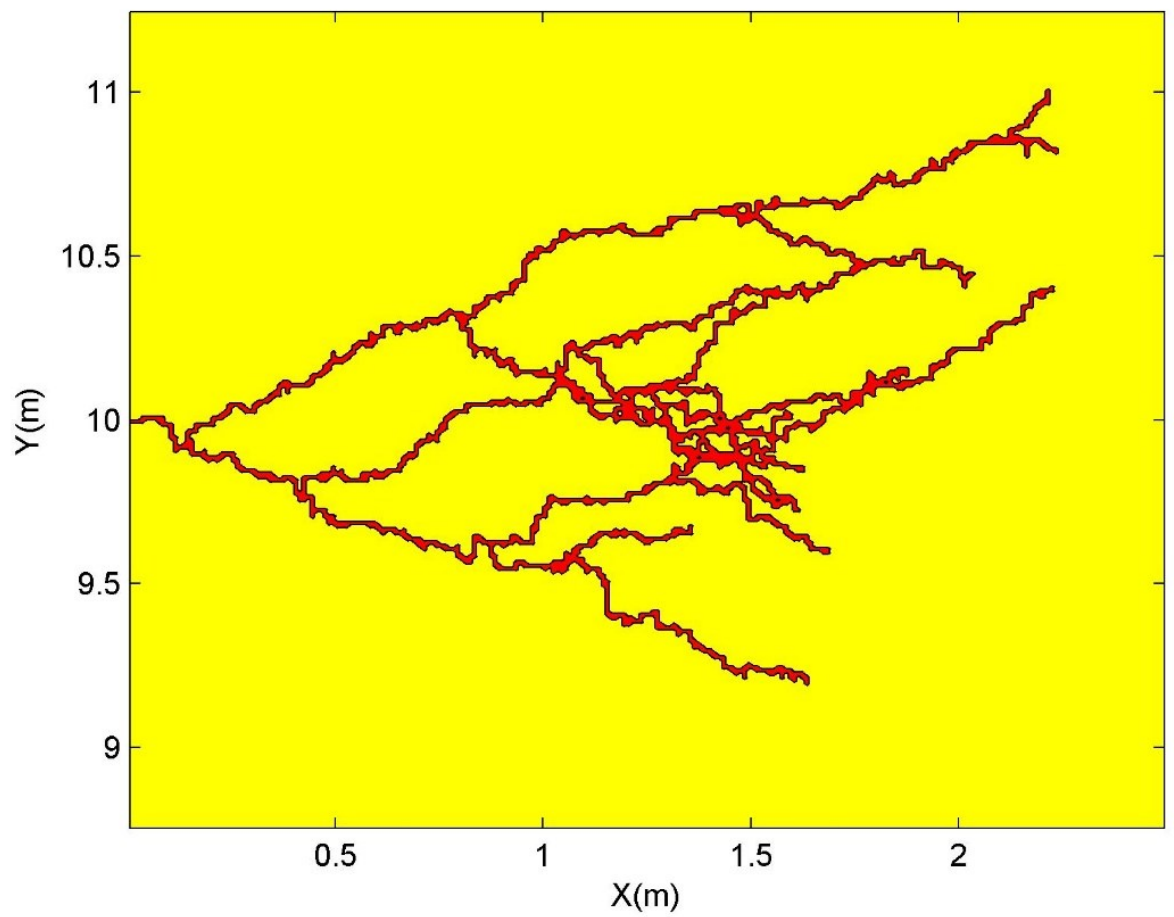

Figure A. 81 - Quad-branched fracture 17. 


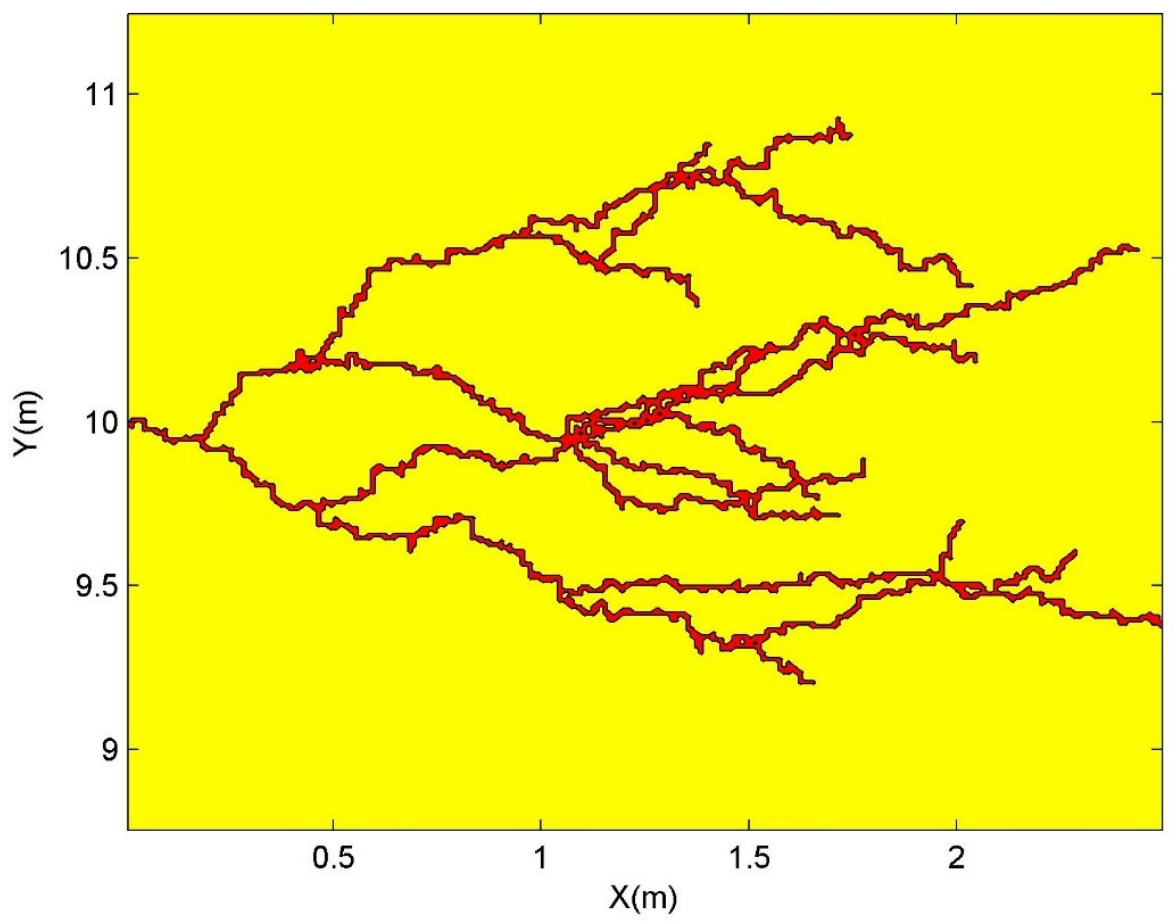

Figure A. 82 - Quad-branched fracture 18.

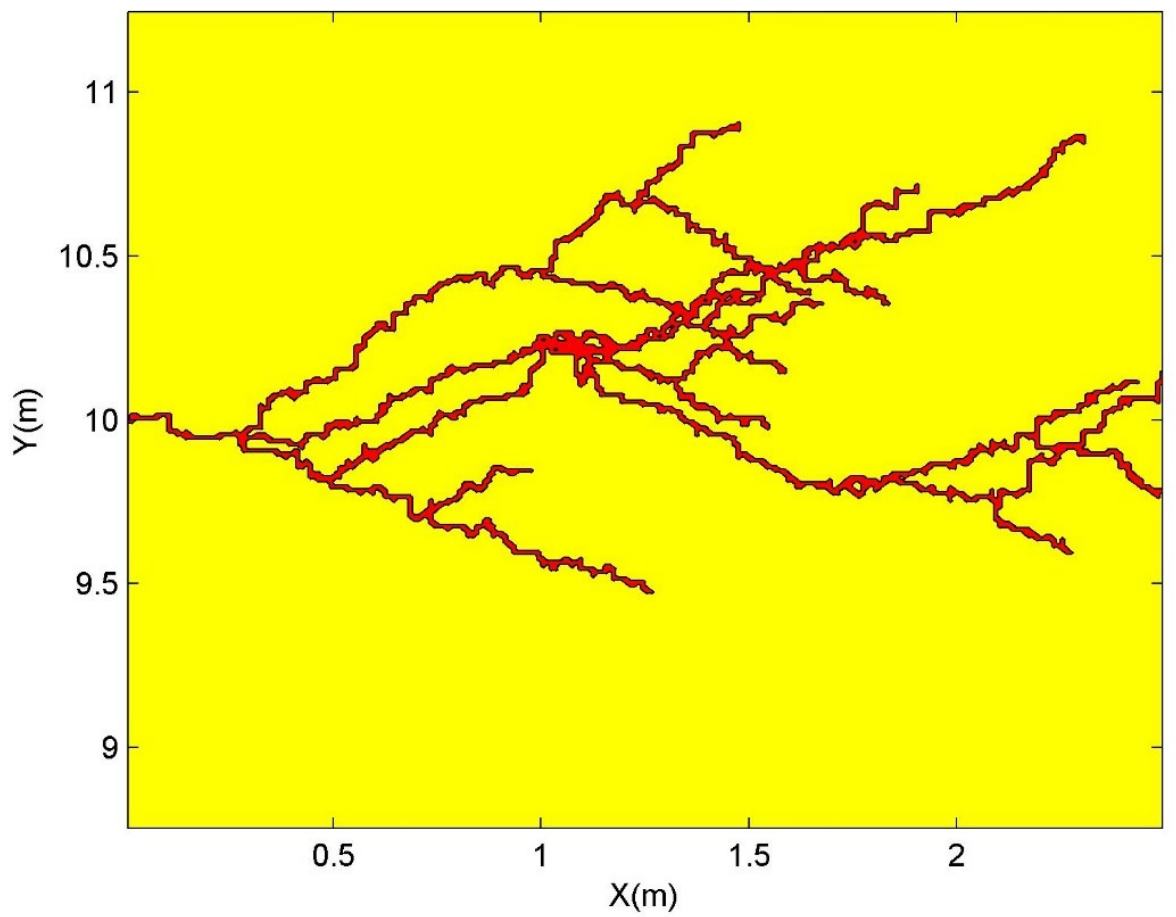

Figure A. 83 - Quad-branched fracture 19. 


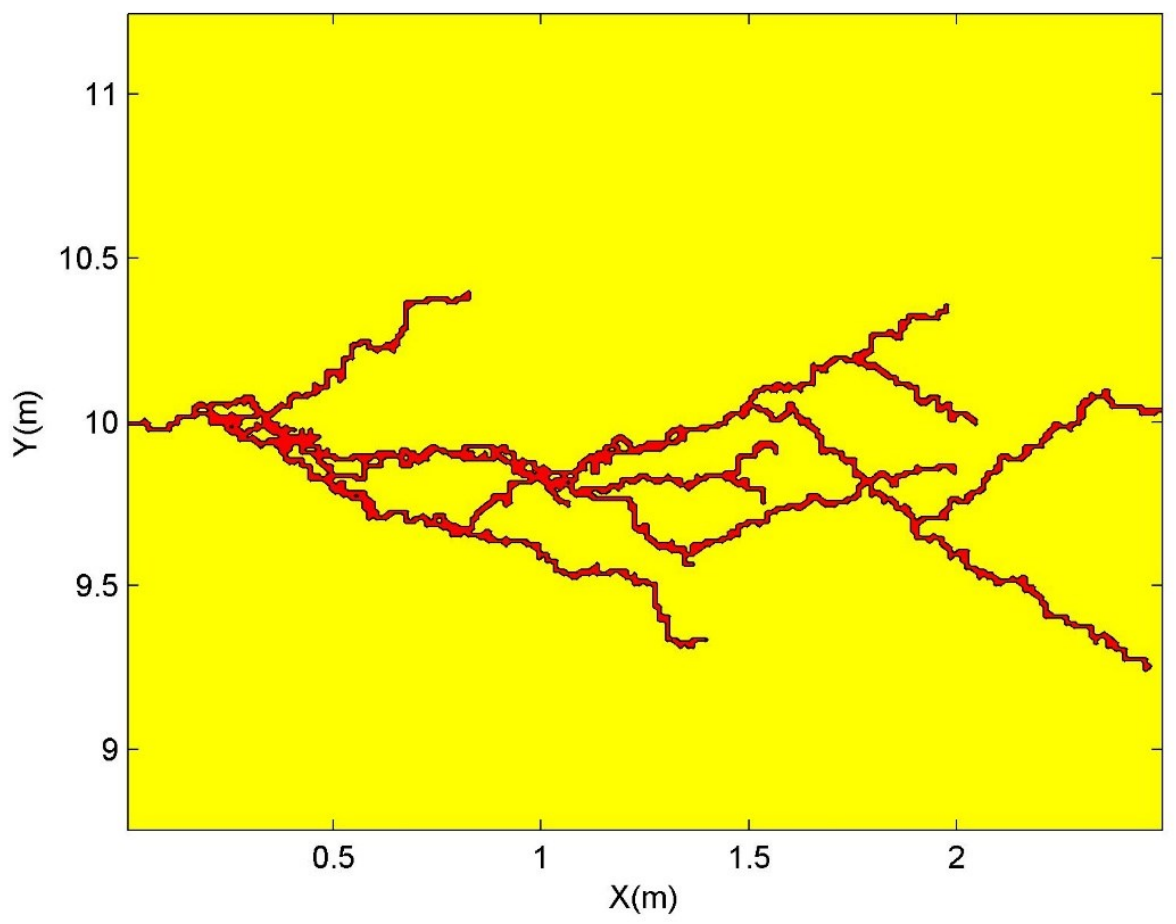

Figure A. 84 - Quad-branched fracture 20. 
MASS RATE DECLINE CURVES FOR SIMULATED STOCHASTIC FRACTURE PATTERNS

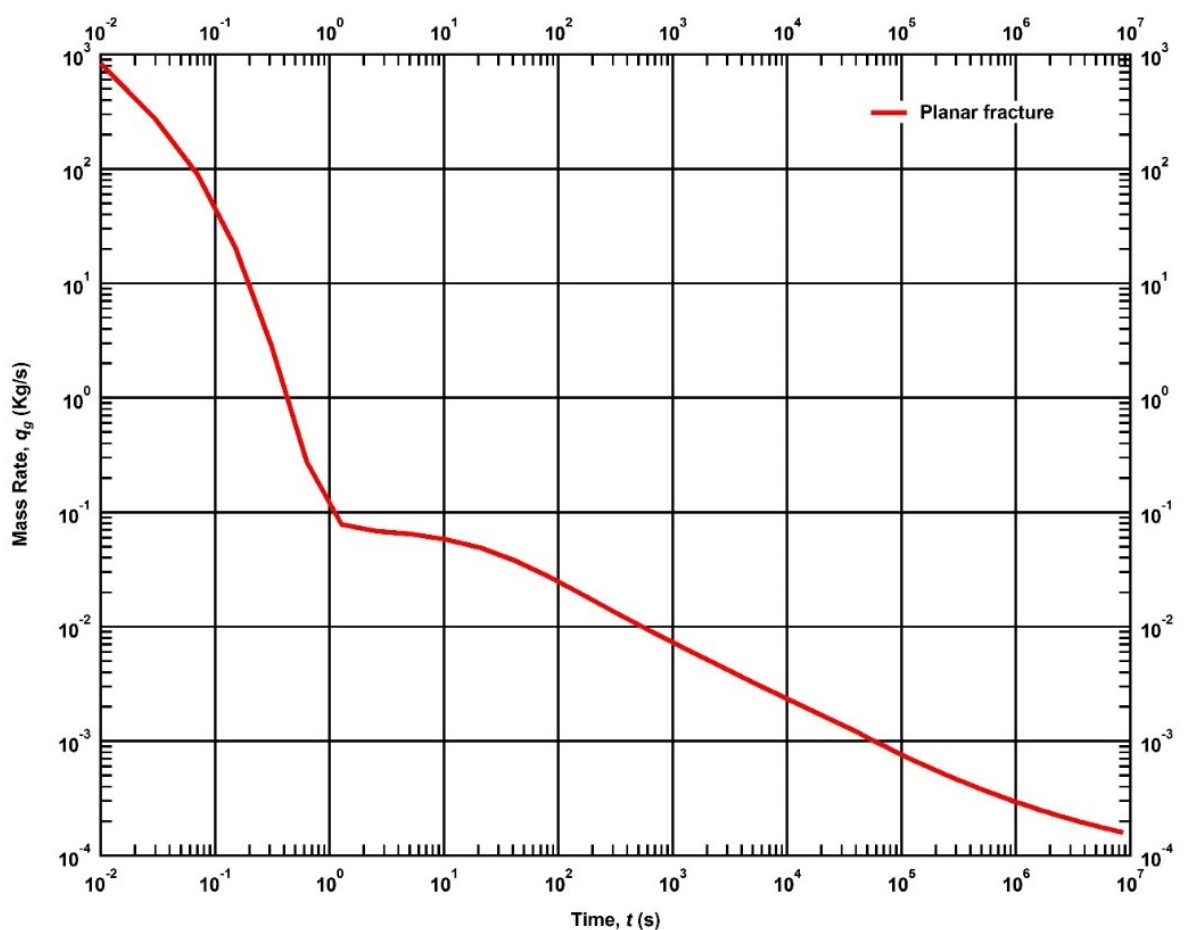

Figure B. 1 - Mass rate evolution for a shale gas reservoir produced from a planar fracture until 100 days of production. 


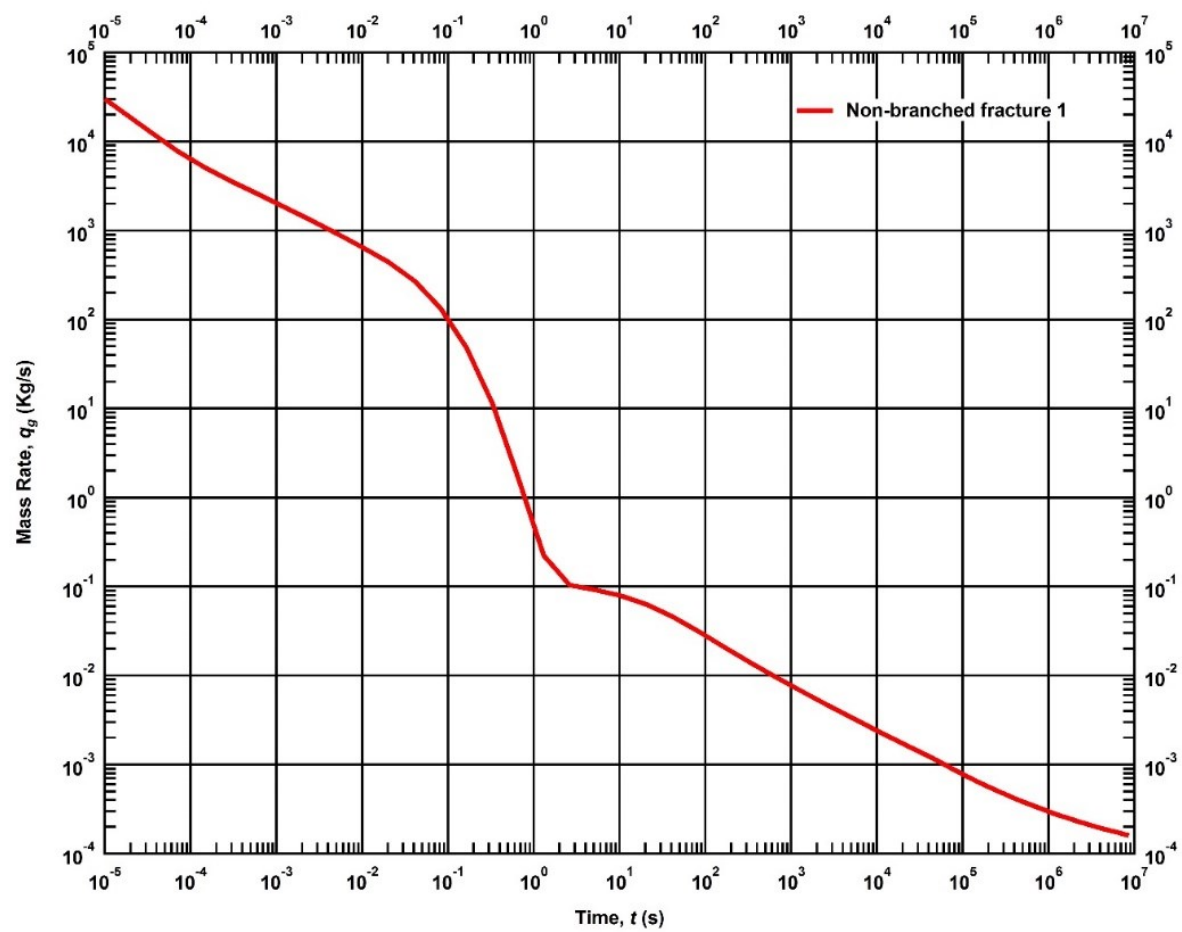

Figure B. 2 - Mass rate evolution for a shale gas reservoir produced from the non-branched fracture 1 until 100 days of production.

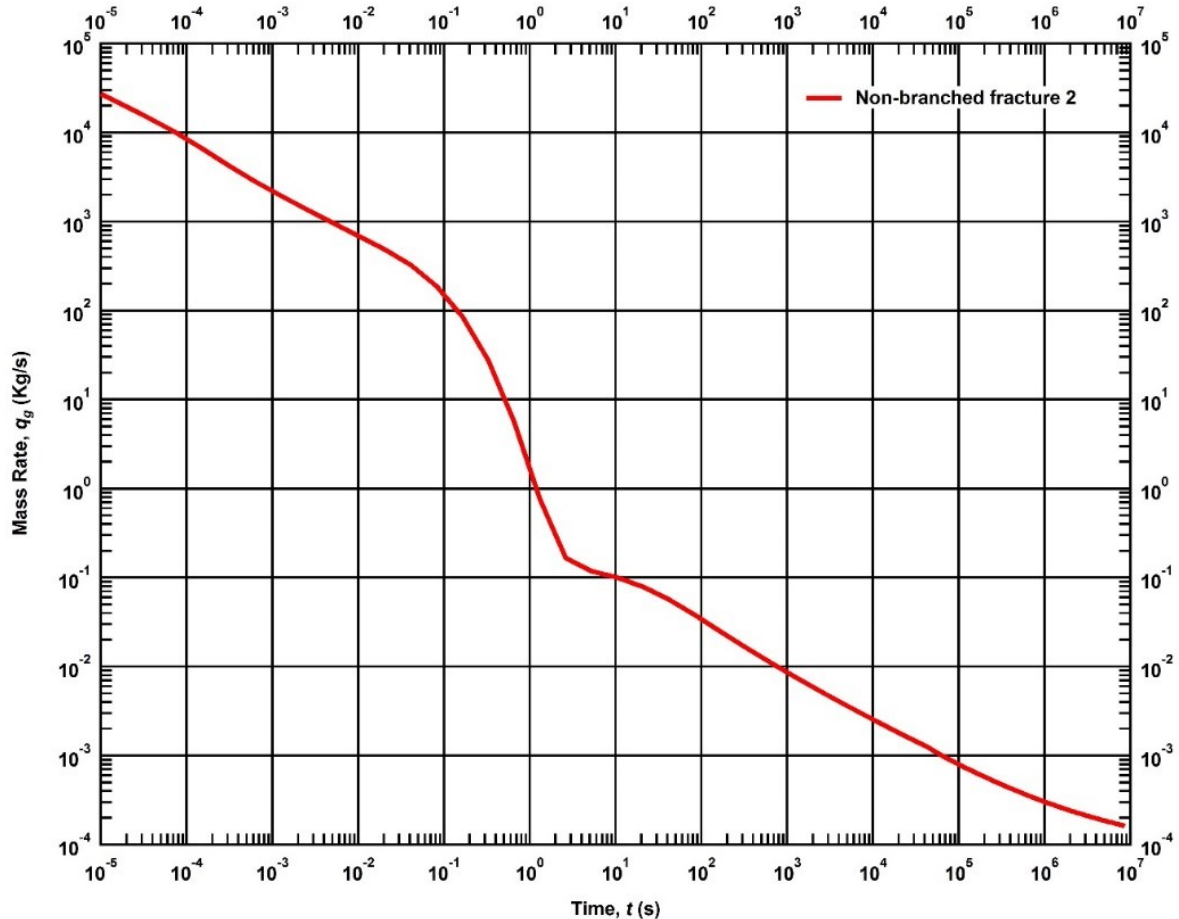

Figure B. 3 - Mass rate evolution for a shale gas reservoir produced from the non-branched fracture 2 until 100 days of production. 


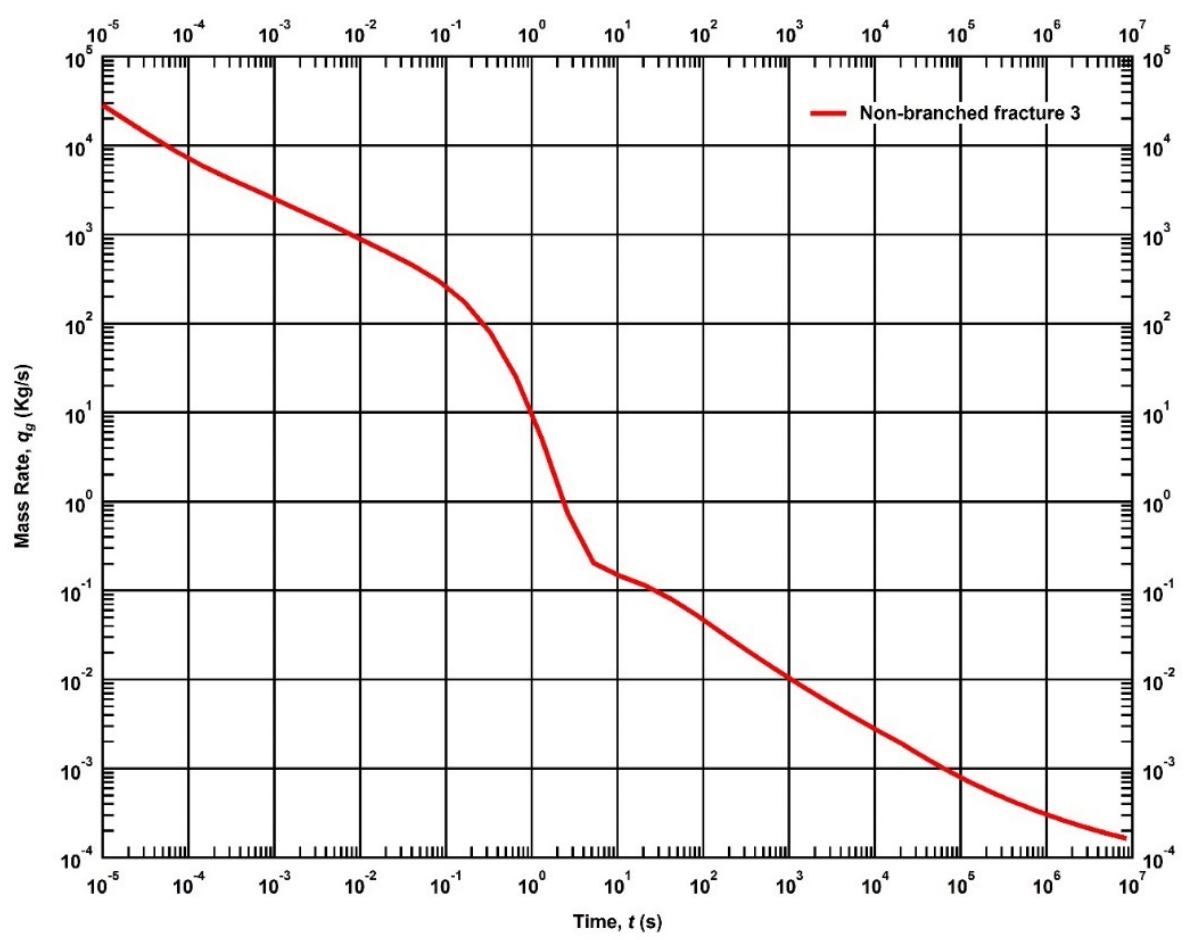

Figure B. 4 - Mass rate evolution for a shale gas reservoir produced from the non-branched fracture 3 until 100 days of production.

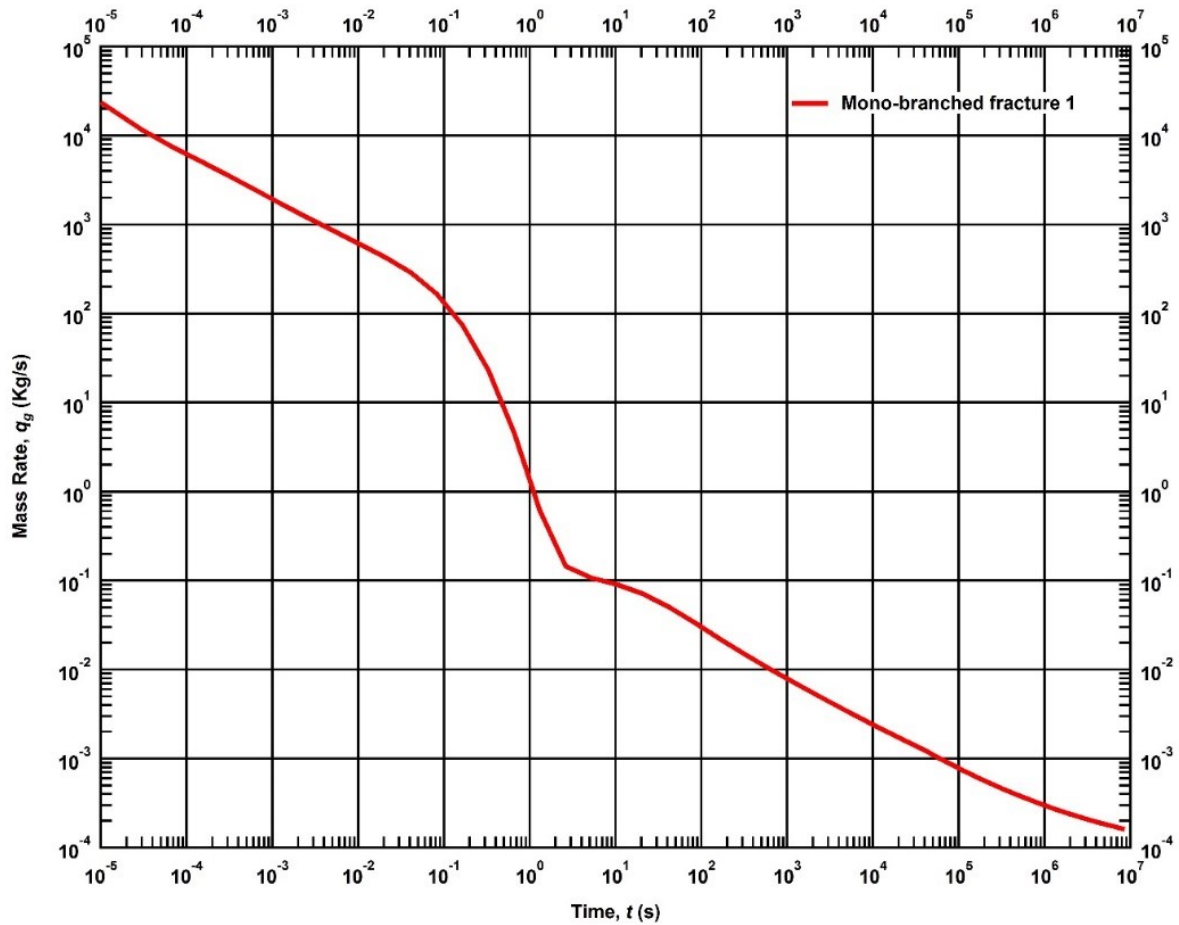

Figure B. 5 - Mass rate evolution for a shale gas reservoir produced from the mono-branched fracture 1 until 100 days of production. 


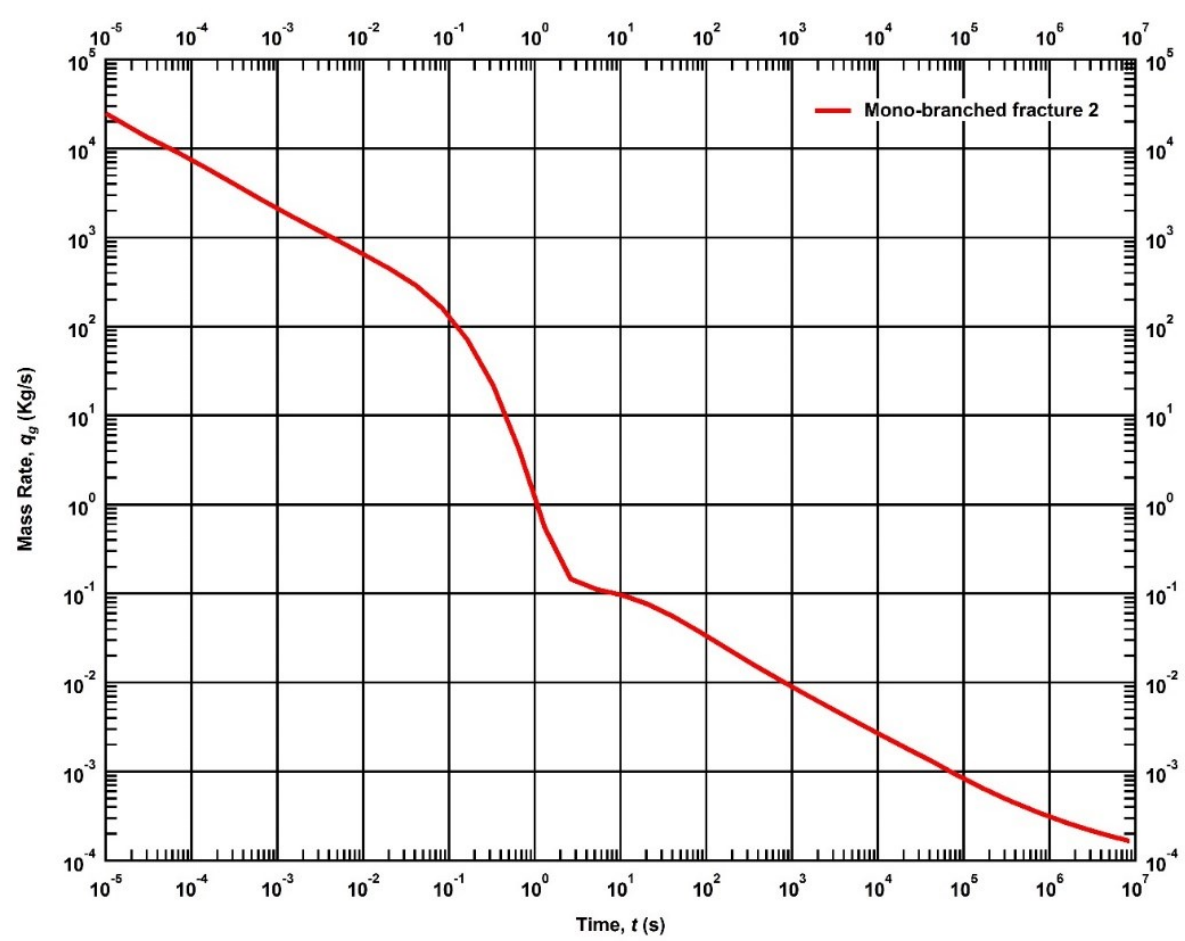

Figure B. 6 - Mass rate evolution for a shale gas reservoir produced from the mono-branched fracture 2 until 100 days of production.

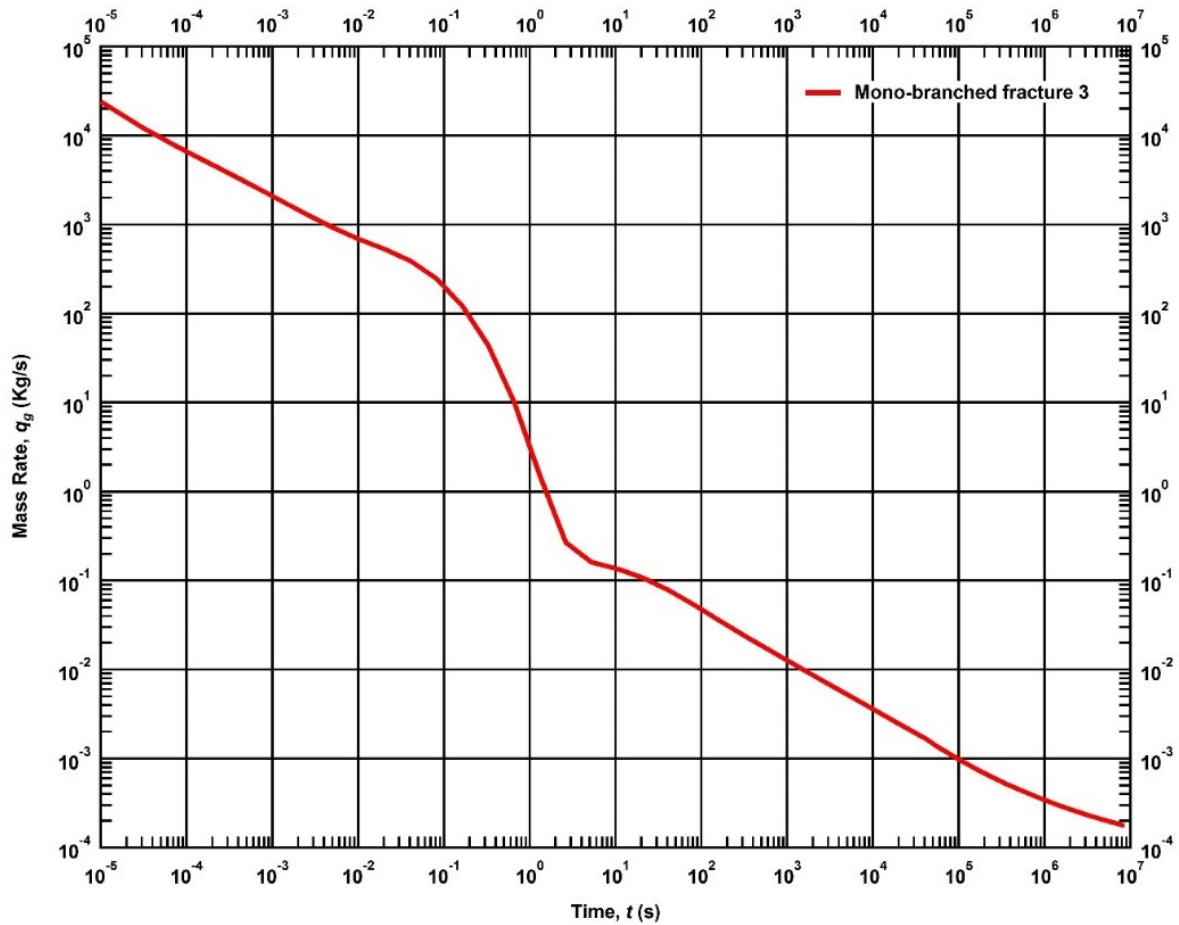

Figure B. 7 Mass rate evolution for a shale gas reservoir produced from the mono-branched fracture 3 until 100 days of production. 


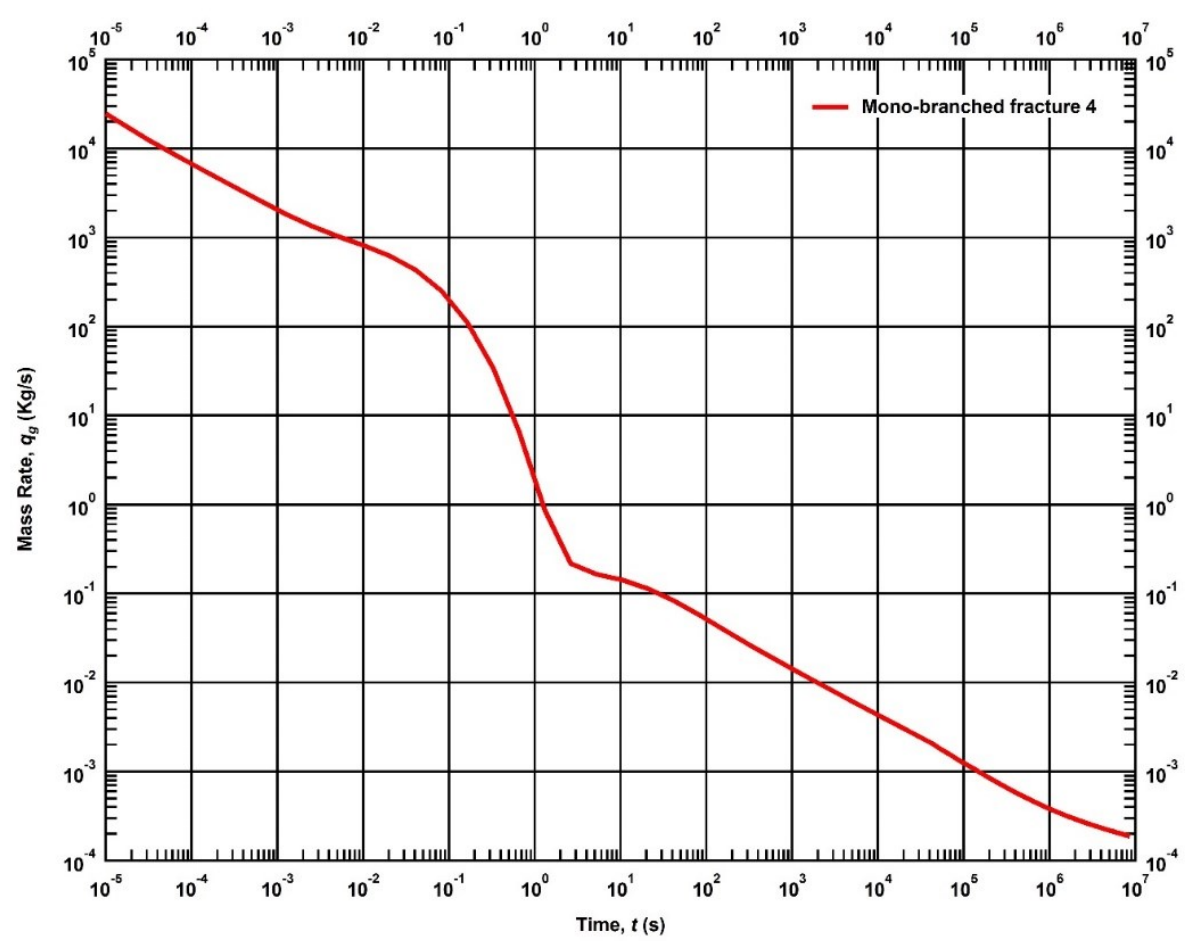

Figure B. 8 - Mass rate evolution for a shale gas reservoir produced from the mono-branched fracture 4 until 100 days of production.

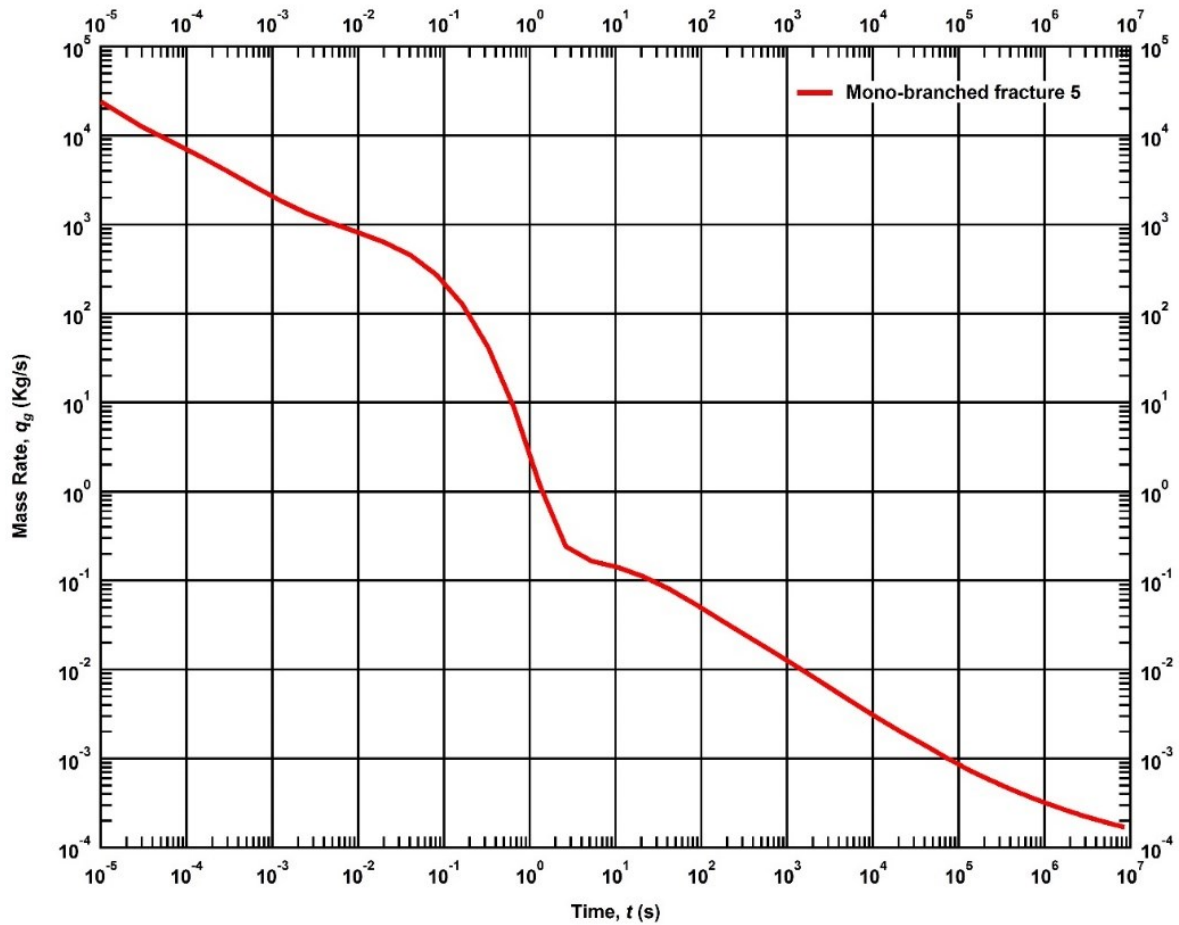

Figure B. 9 - Mass rate evolution for a shale gas reservoir produced from the mono-branched fracture 5 until 100 days of production. 


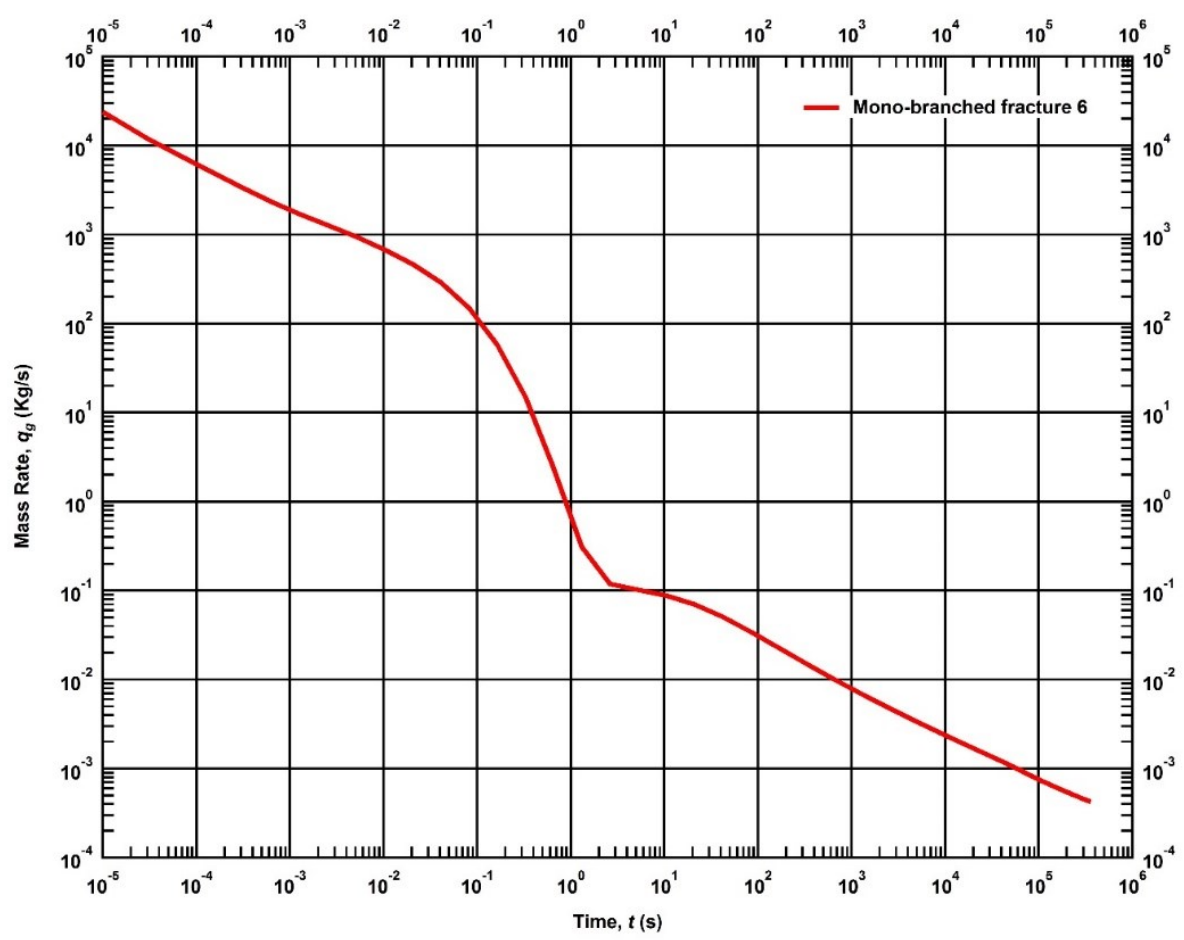

Figure B. 10 - Mass rate evolution for a shale gas reservoir produced from the mono-branched fracture 6 until 100 hours of production.

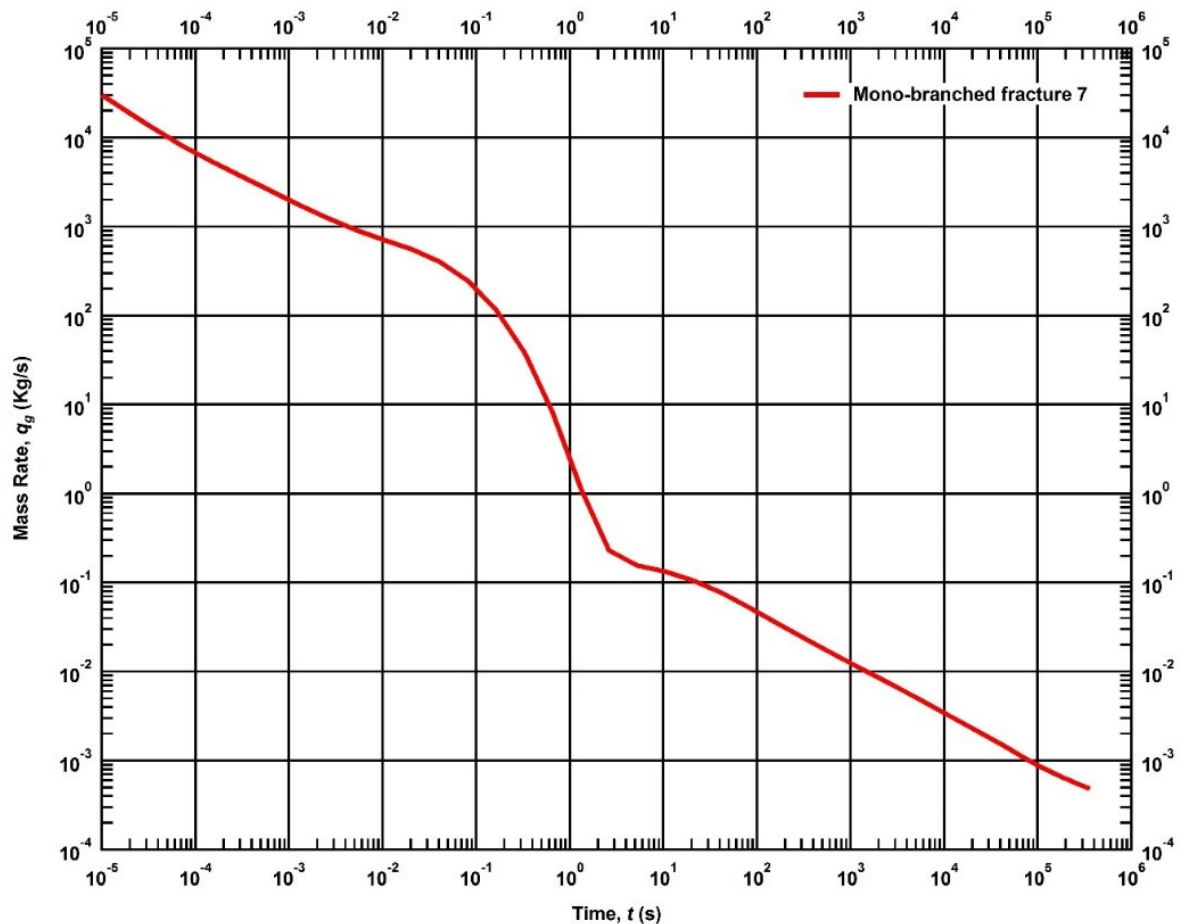

Figure B. 11 - Mass rate evolution for a shale gas reservoir produced from the mono-branched fracture 7 until 100 hours of production. 


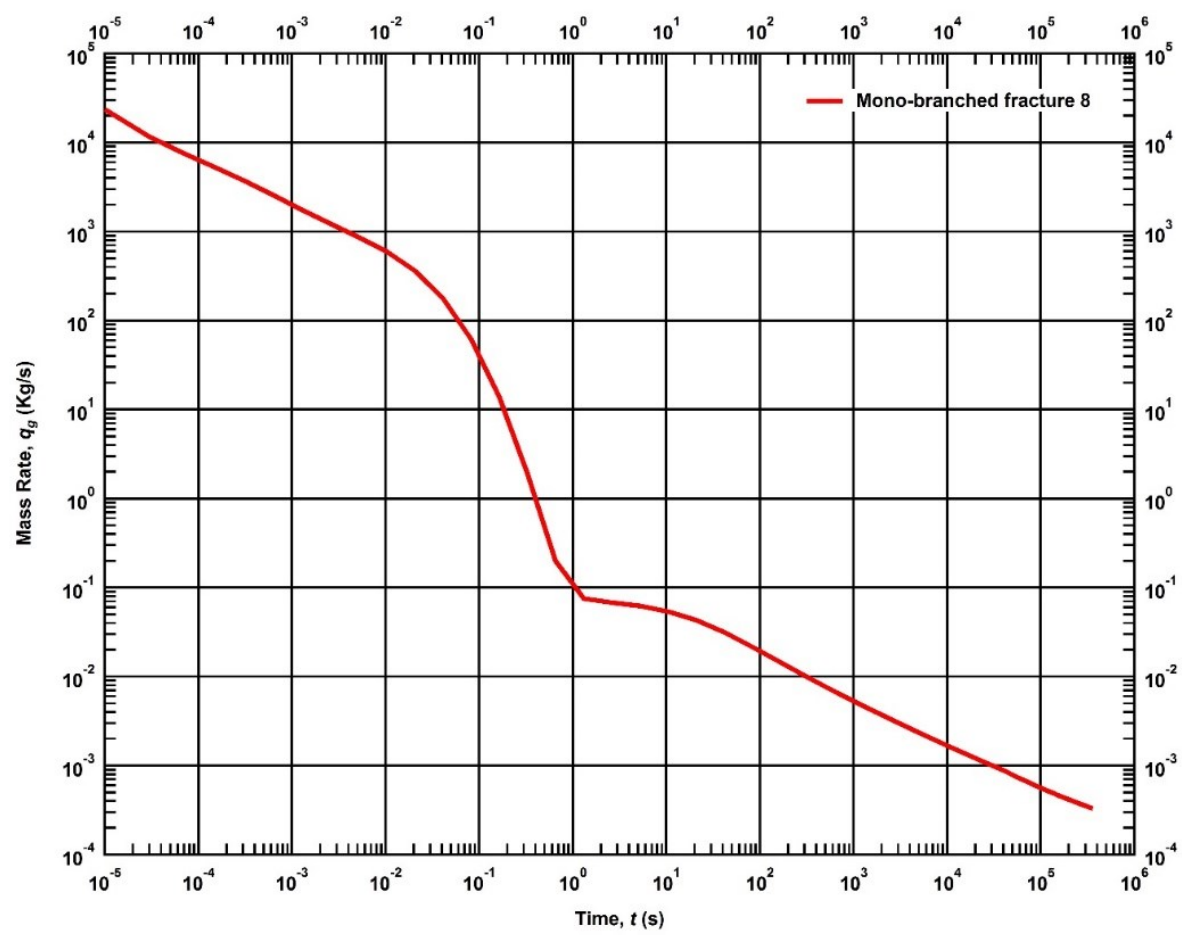

Figure B. 12 - Mass rate evolution for a shale gas reservoir produced from the mono-branched fracture 8 until 100 hours of production.

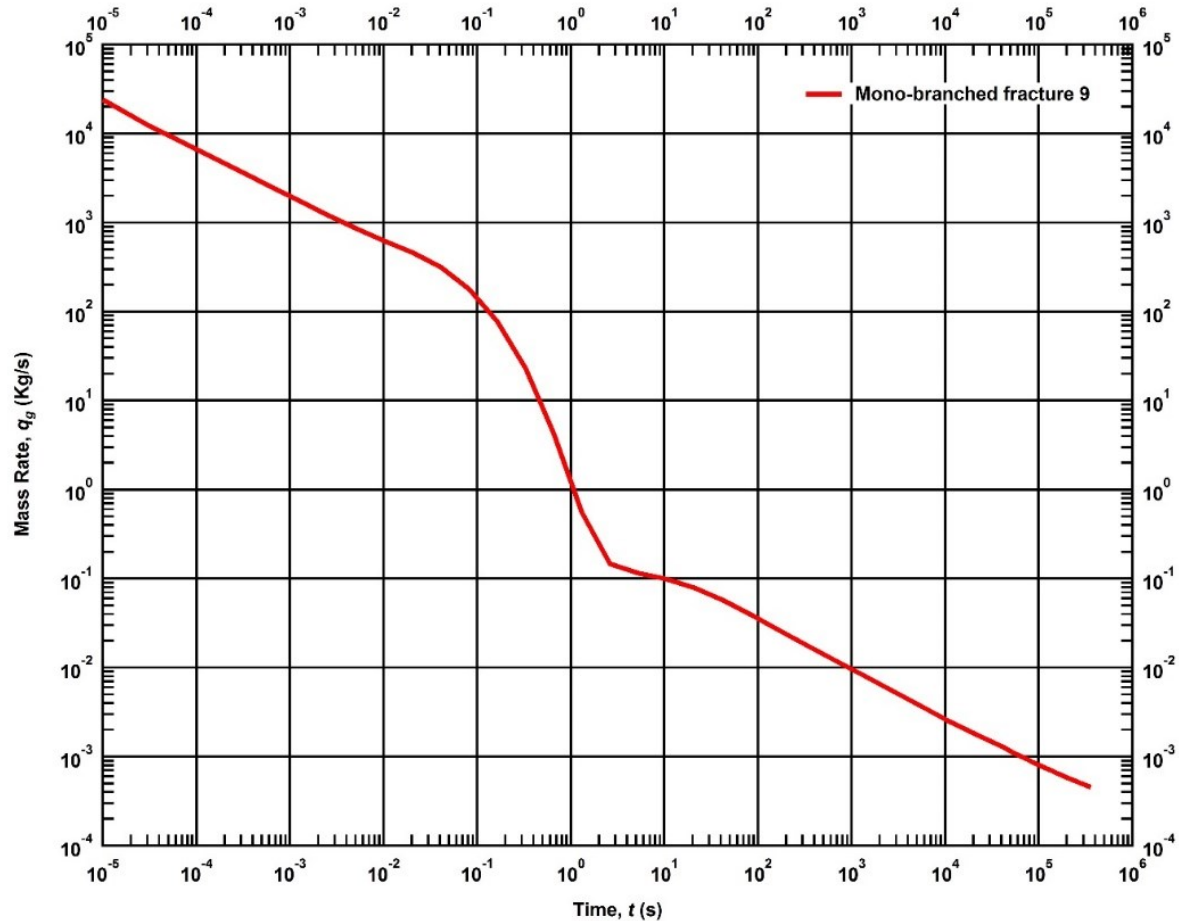

Figure B. 13 - Mass rate evolution for a shale gas reservoir produced from the mono-branched fracture 9 until 100 hours of production. 


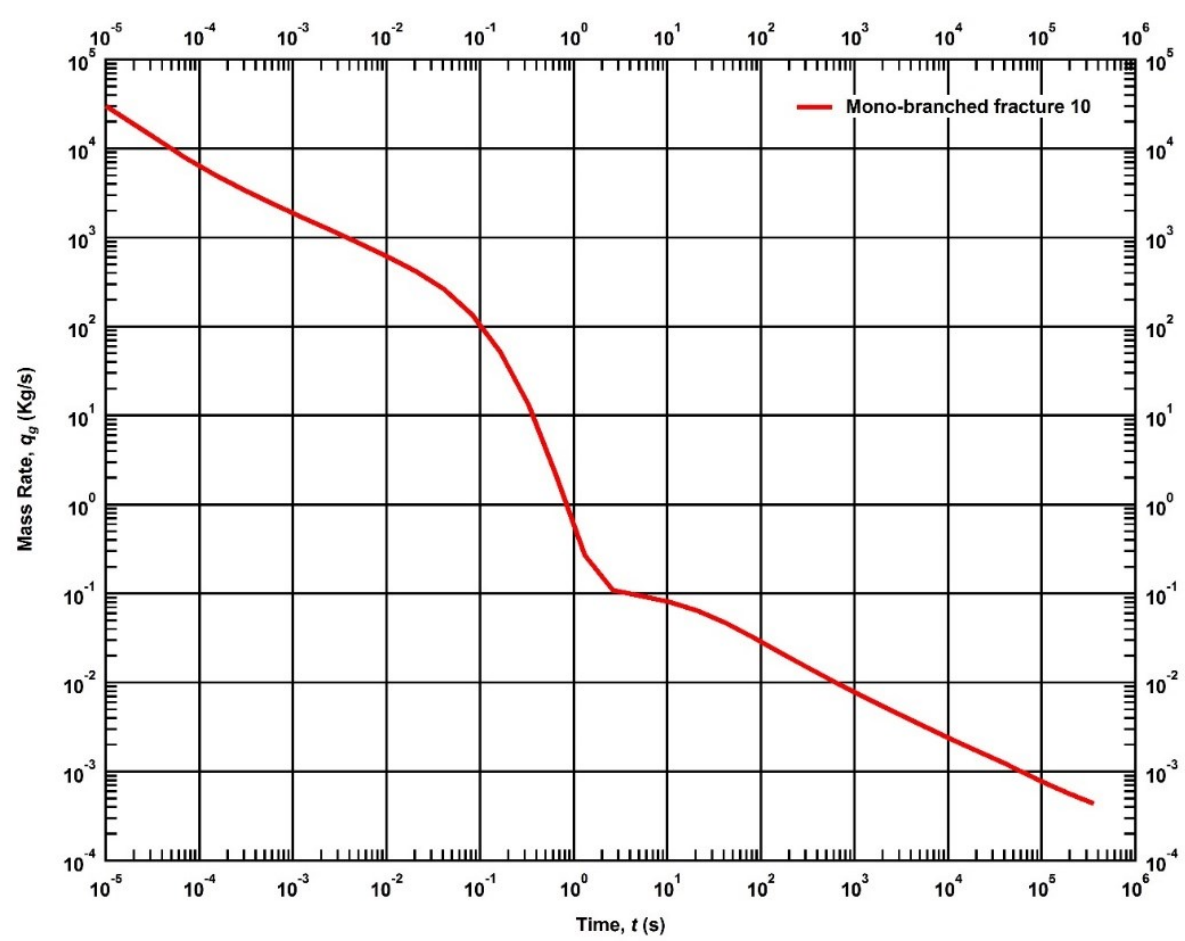

Figure B. 14 - Mass rate evolution for a shale gas reservoir produced from the mono-branched fracture 10 until 100 hours of production.

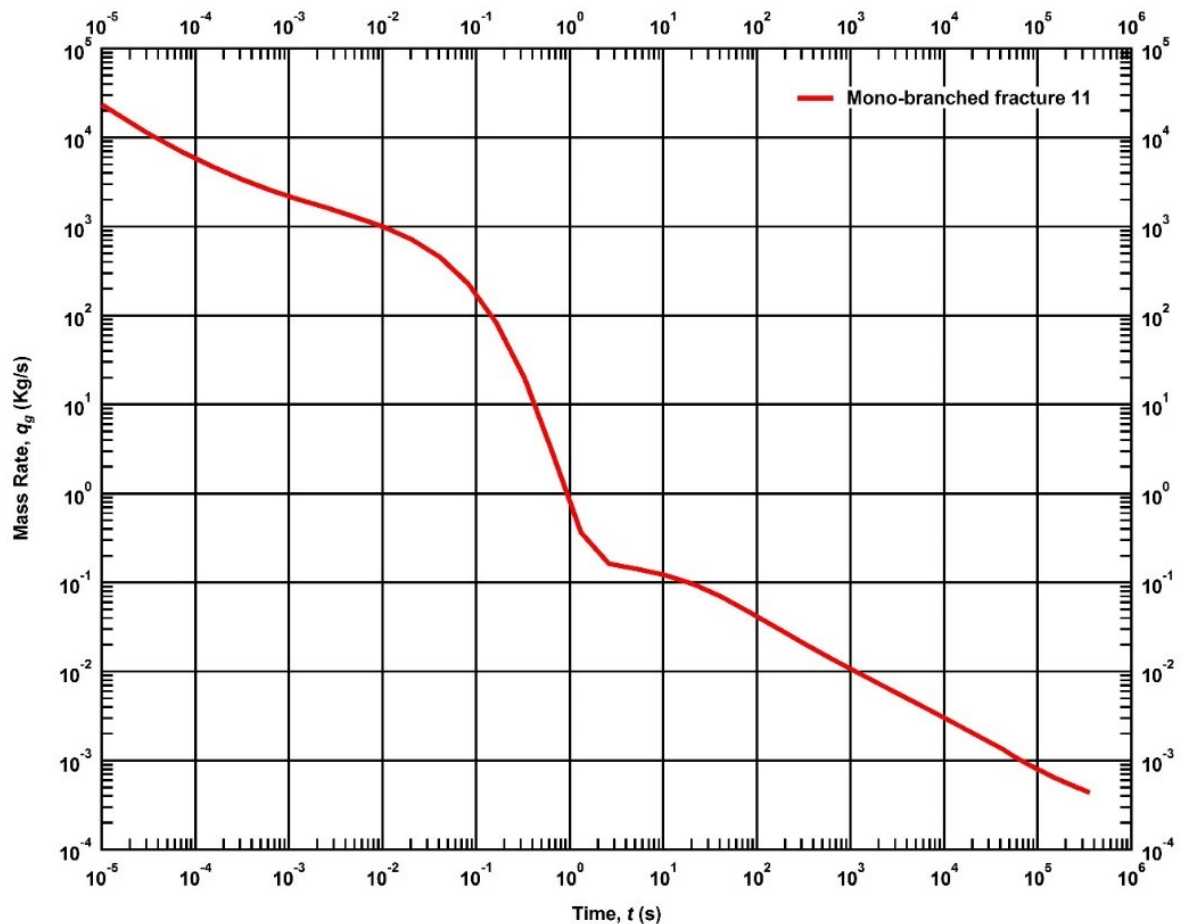

Figure B. 15 - Mass rate evolution for a shale gas reservoir produced from the mono-branched fracture 11 until 100 hours of production. 


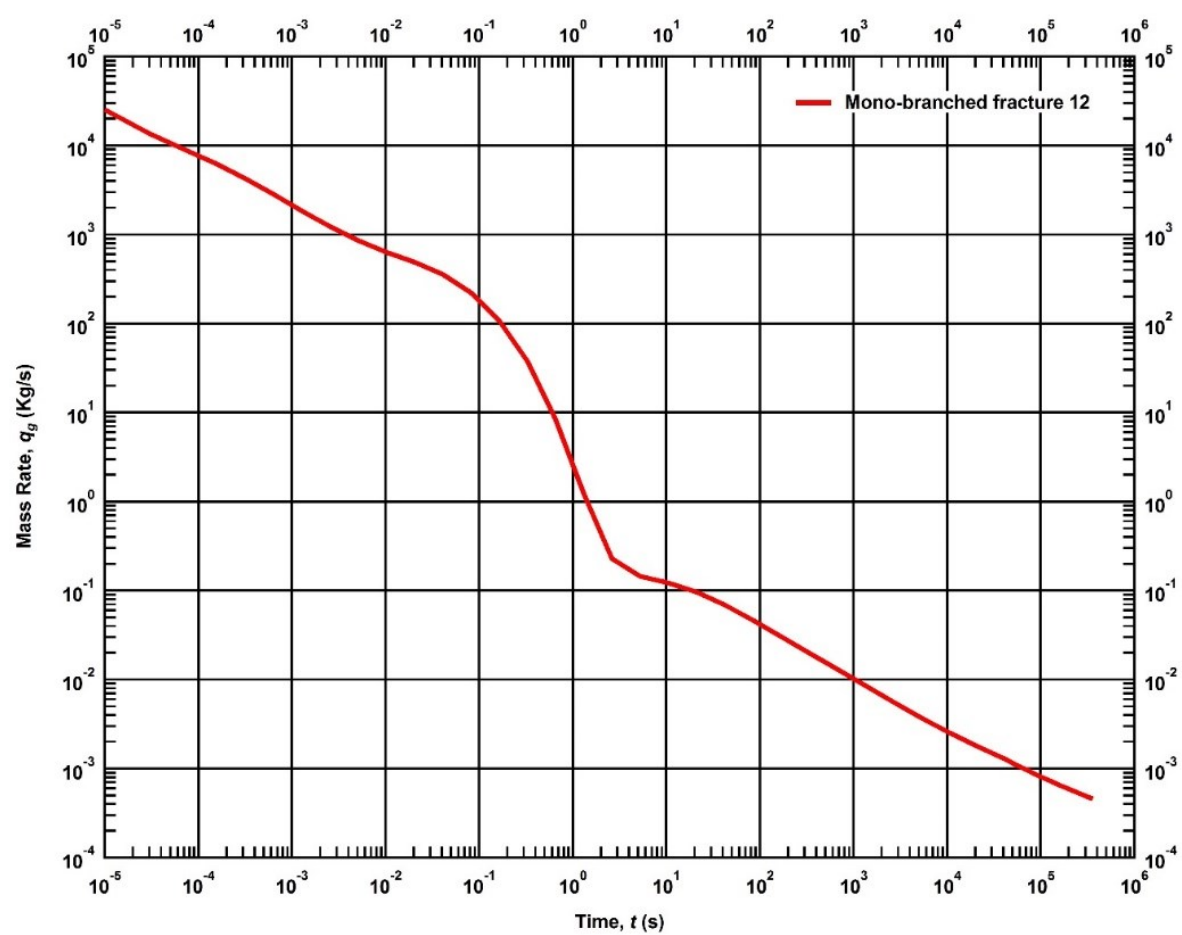

Figure B. 16 - Mass rate evolution for a shale gas reservoir produced from the mono-branched fracture 12 until 100 hours of production.

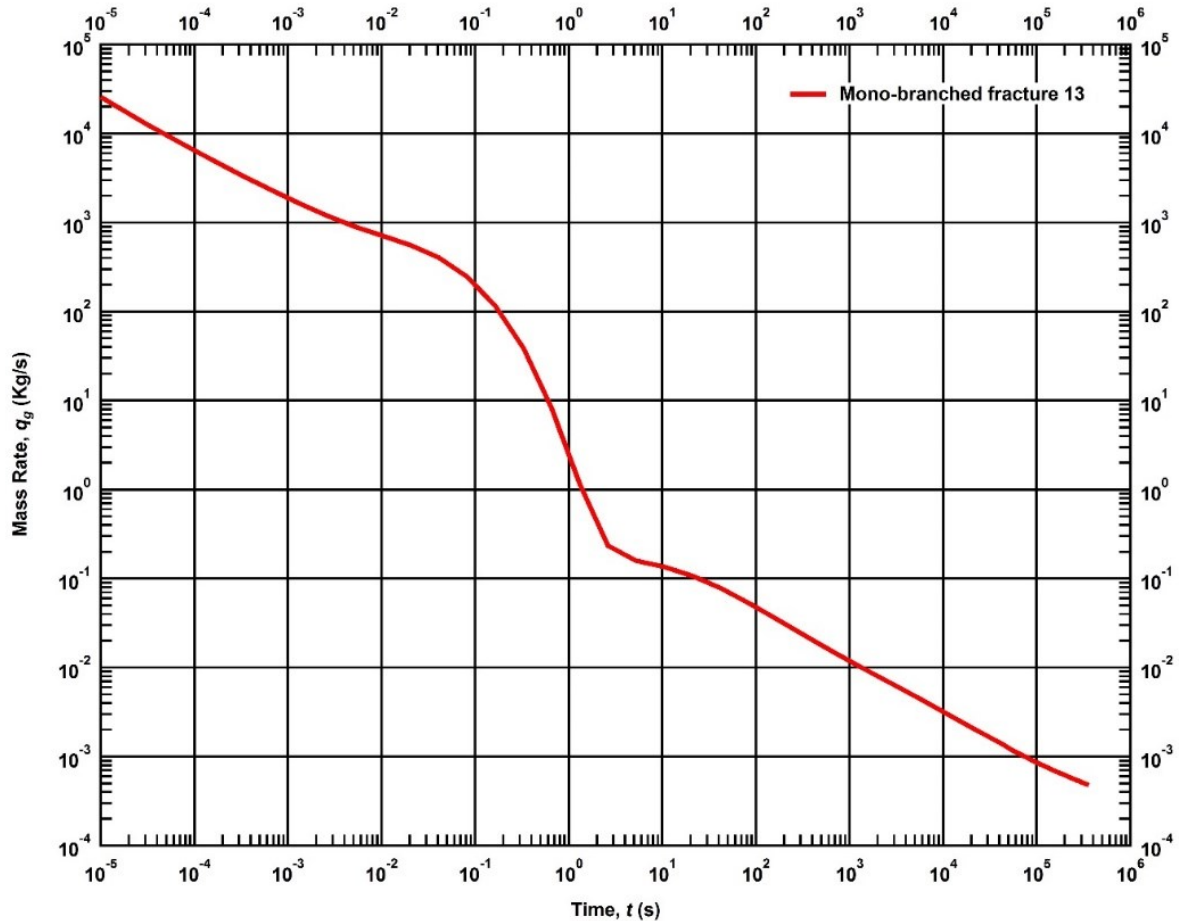

Figure B. 17 - Mass rate evolution for a shale gas reservoir produced from the mono-branched fracture 13 until 100 hours of production. 


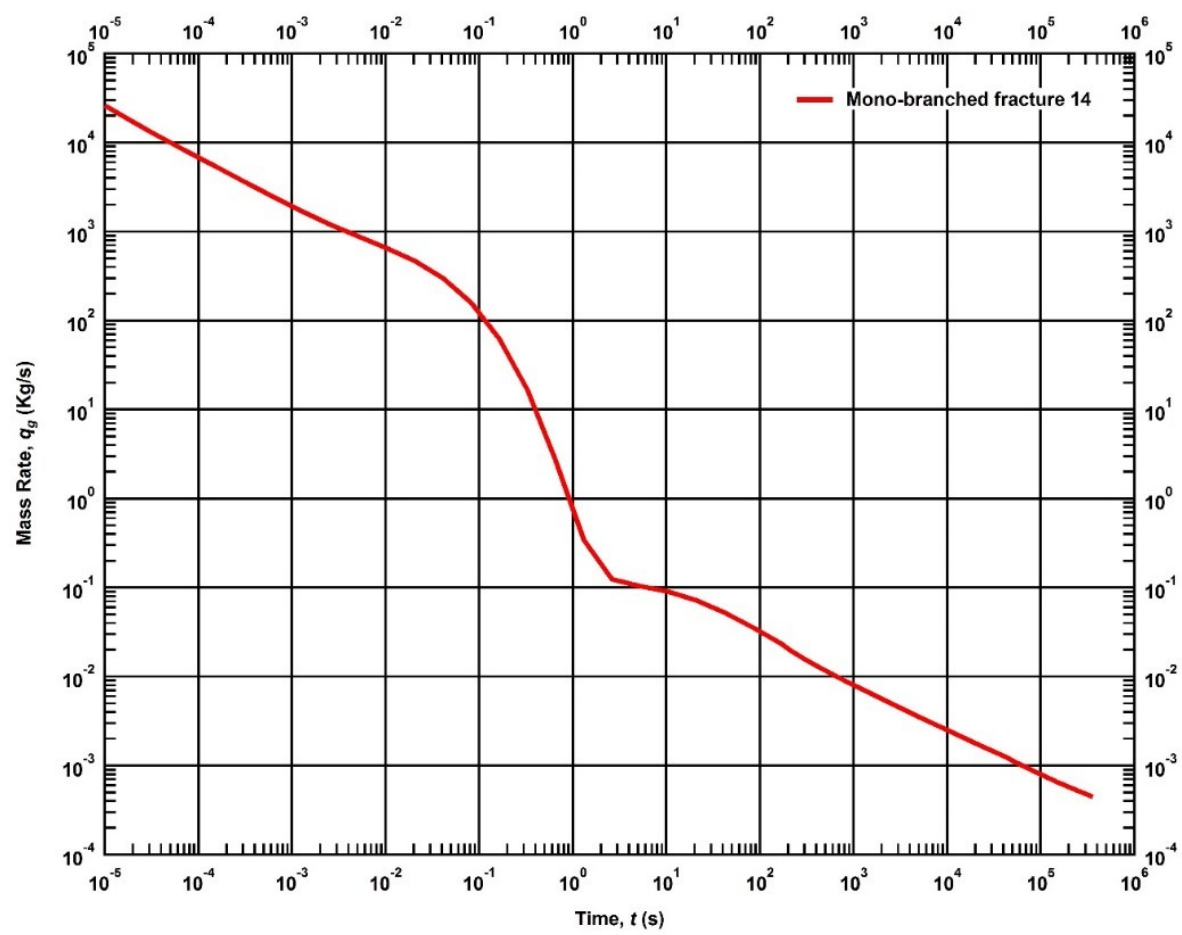

Figure B. 18 - Mass rate evolution for a shale gas reservoir produced from the mono-branched fracture 14 until 100 hours of production.

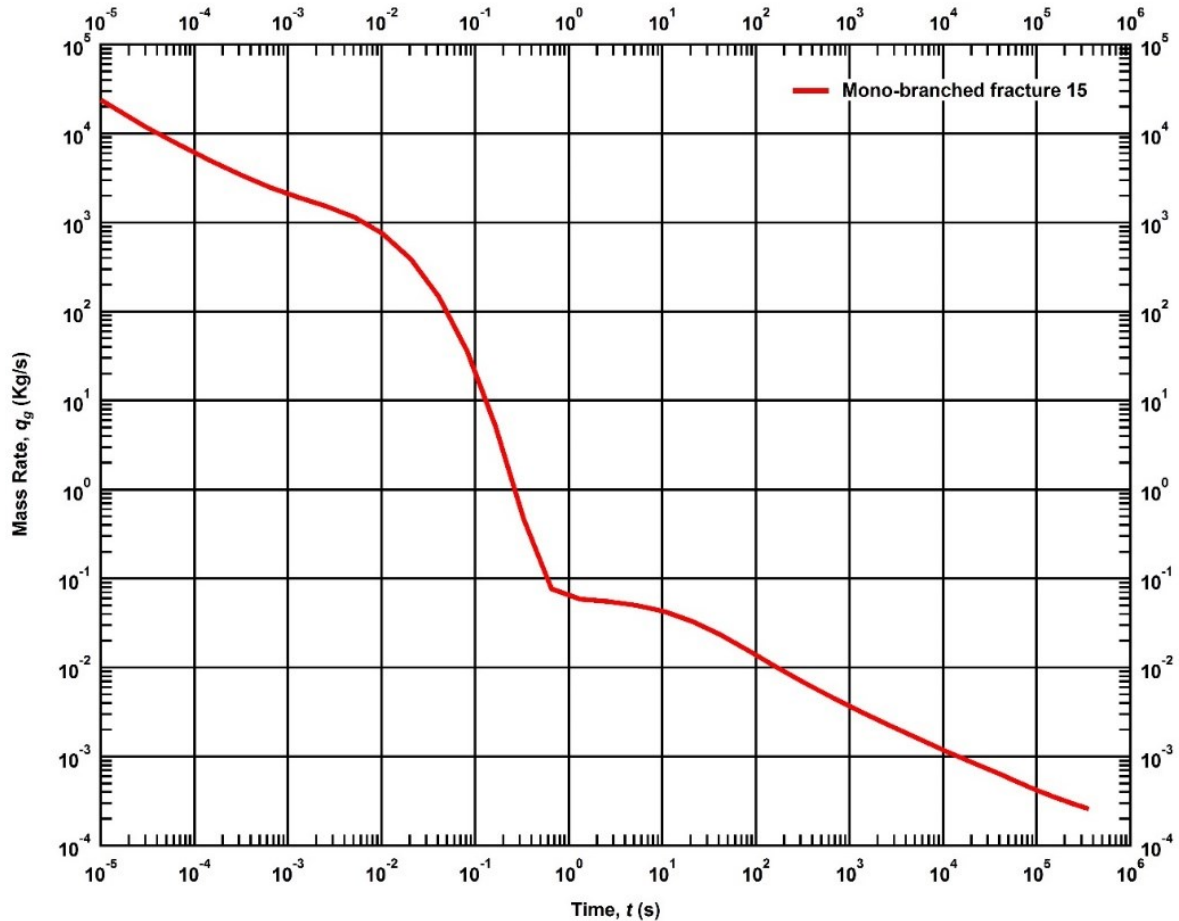

Figure B. 19 - Mass rate evolution for a shale gas reservoir produced from the mono-branched fracture 15 until 100 hours of production. 


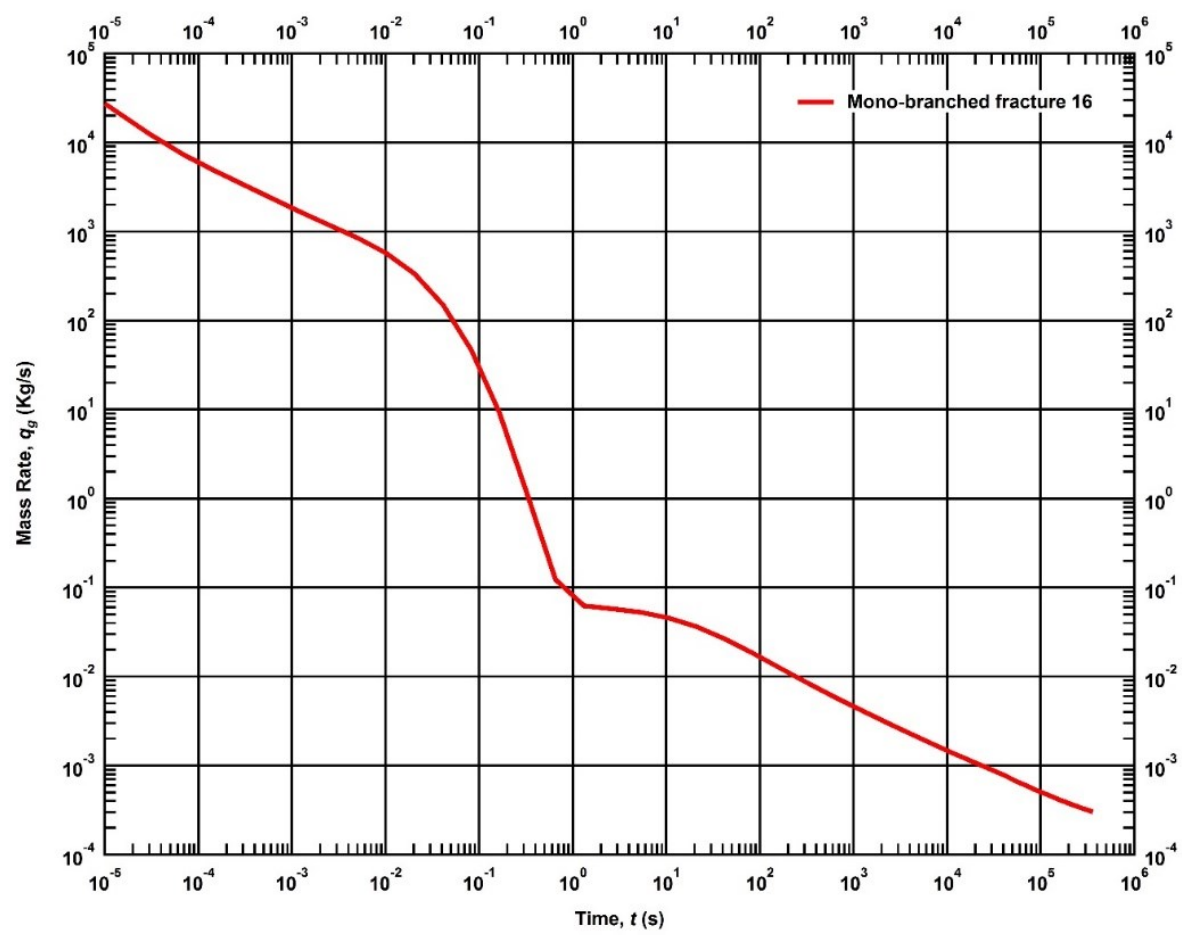

Figure B. 20 - Mass rate evolution for a shale gas reservoir produced from the mono-branched fracture 16 until 100 hours of production.

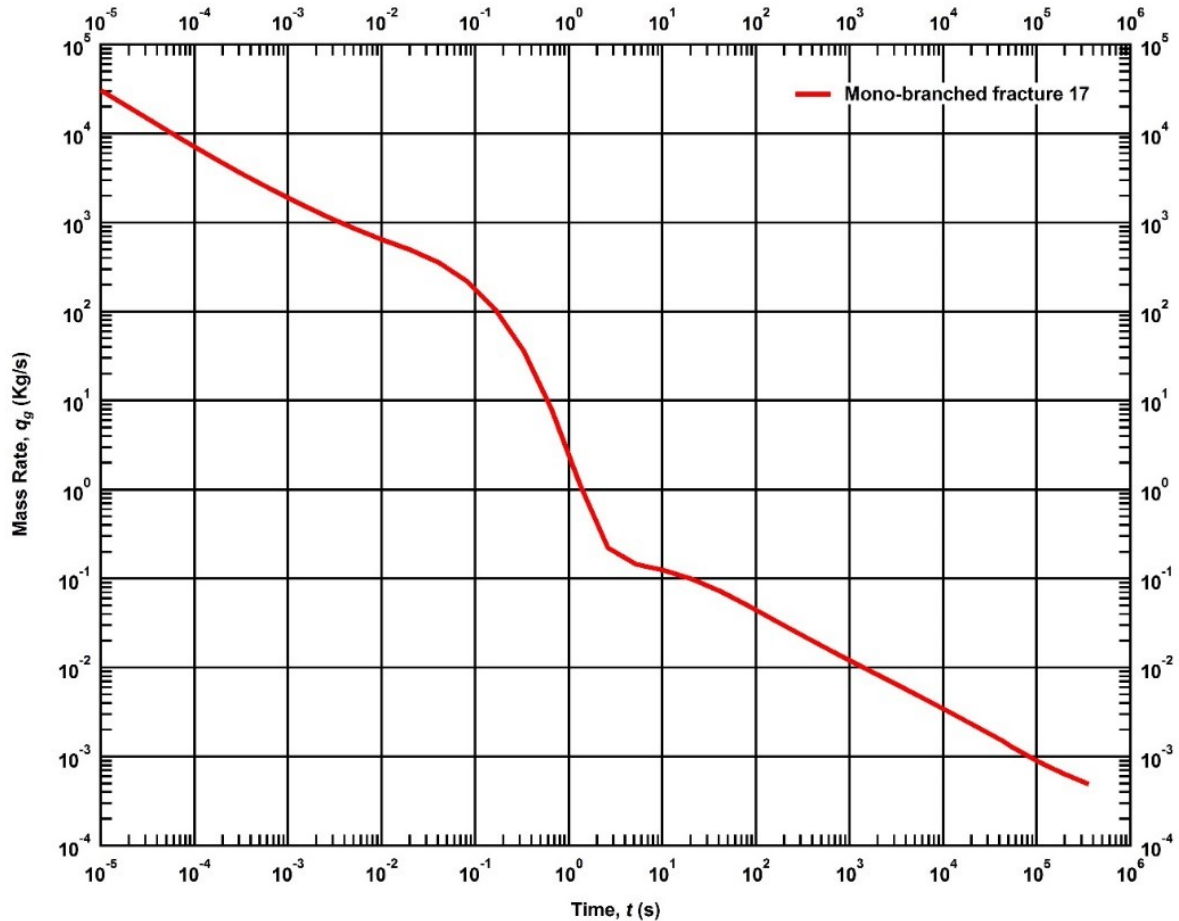

Figure B. 21 - Mass rate evolution for a shale gas reservoir produced from the mono-branched fracture 17 until 100 hours of production. 


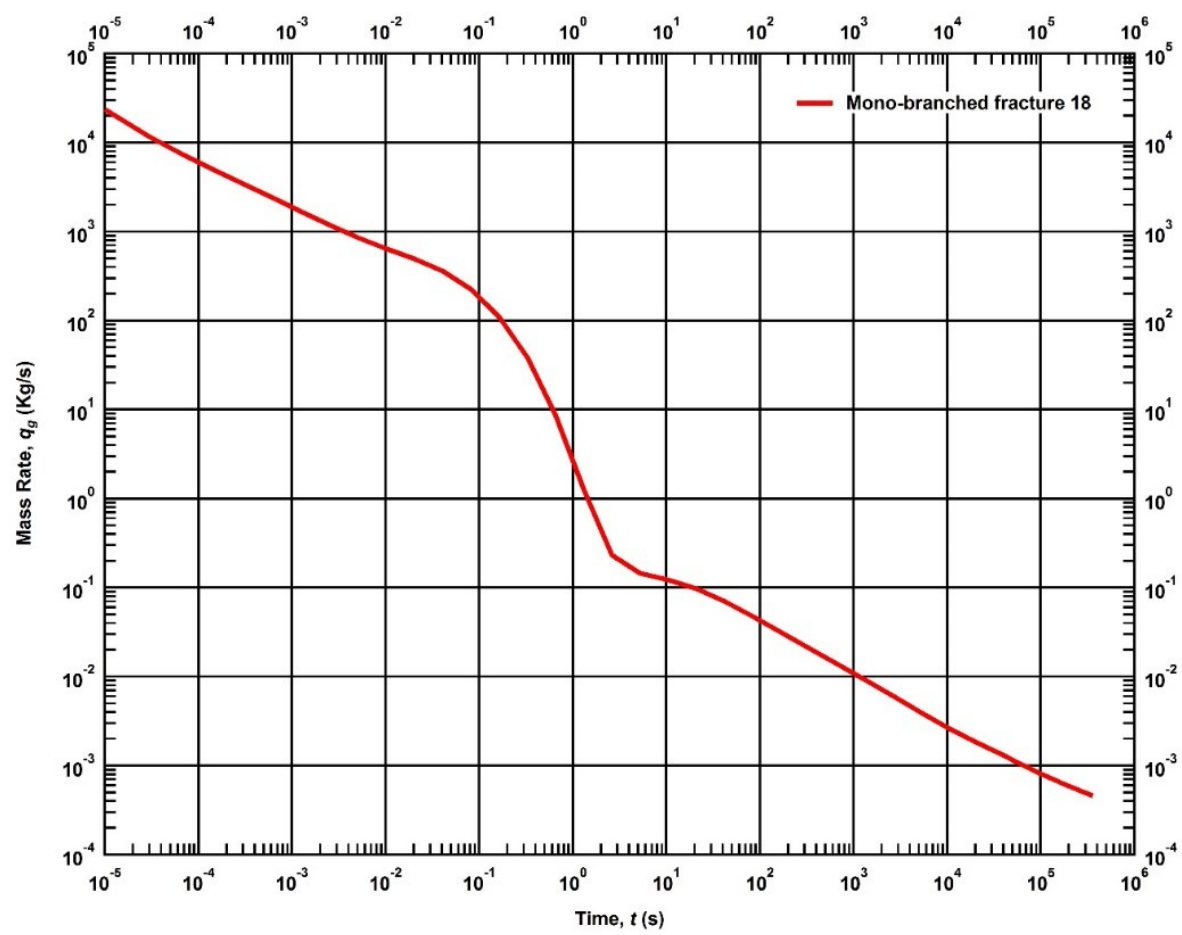

Figure B. 22 - Mass rate evolution for a shale gas reservoir produced from the mono-branched fracture 18 until 100 hours of production.

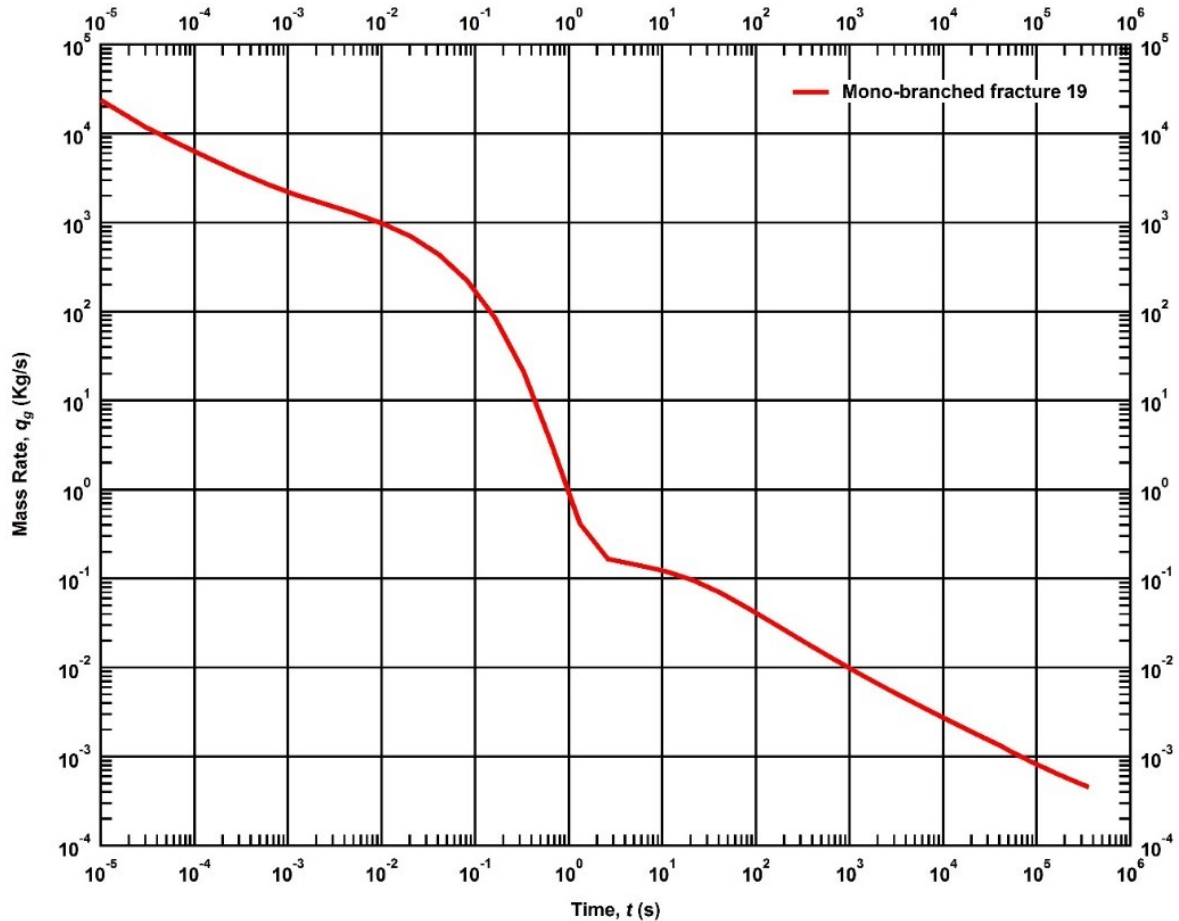

Figure B. 23 - Mass rate evolution for a shale gas reservoir produced from the mono-branched fracture 19 until 100 hours of production. 


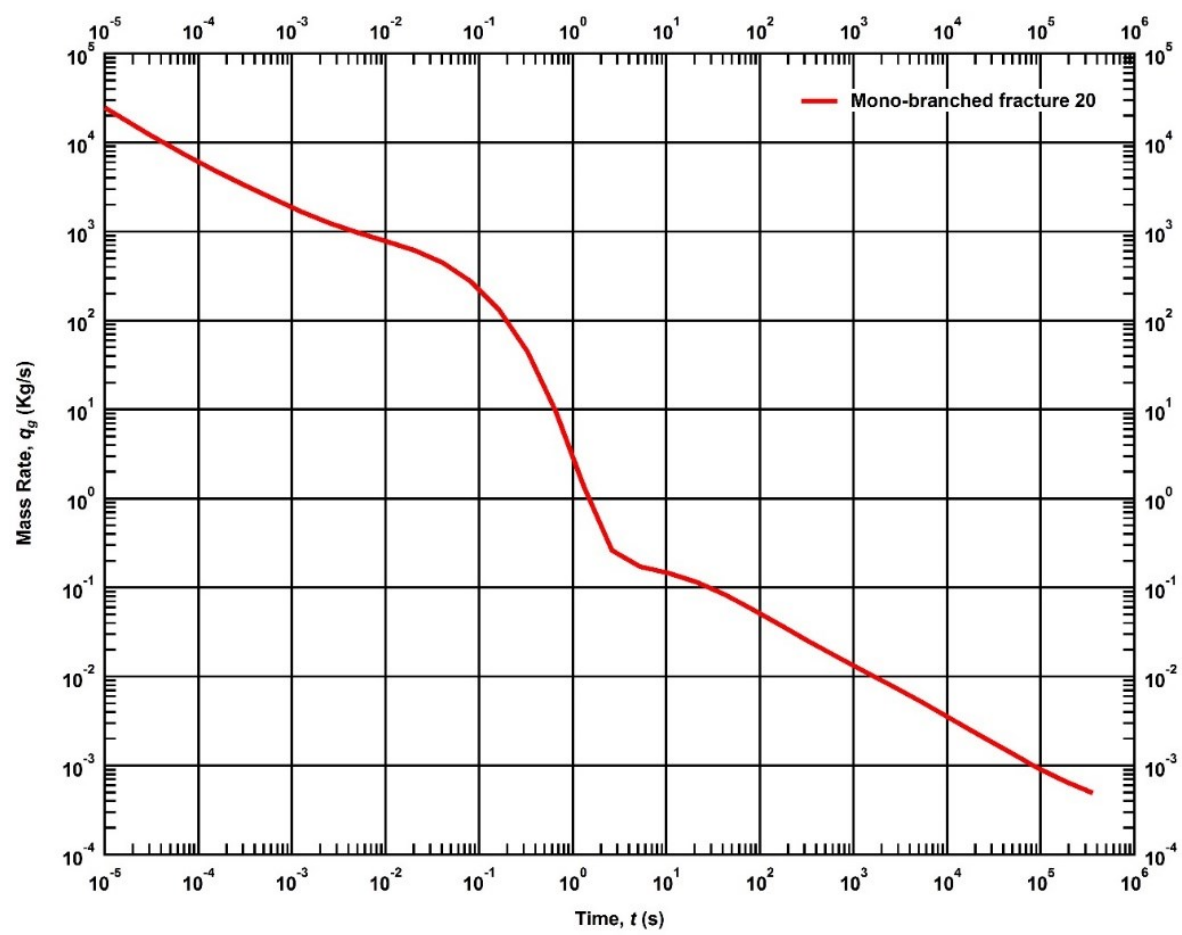

Figure B. 24 - Mass rate evolution for a shale gas reservoir produced from the mono-branched fracture 20 until 100 hours of production.

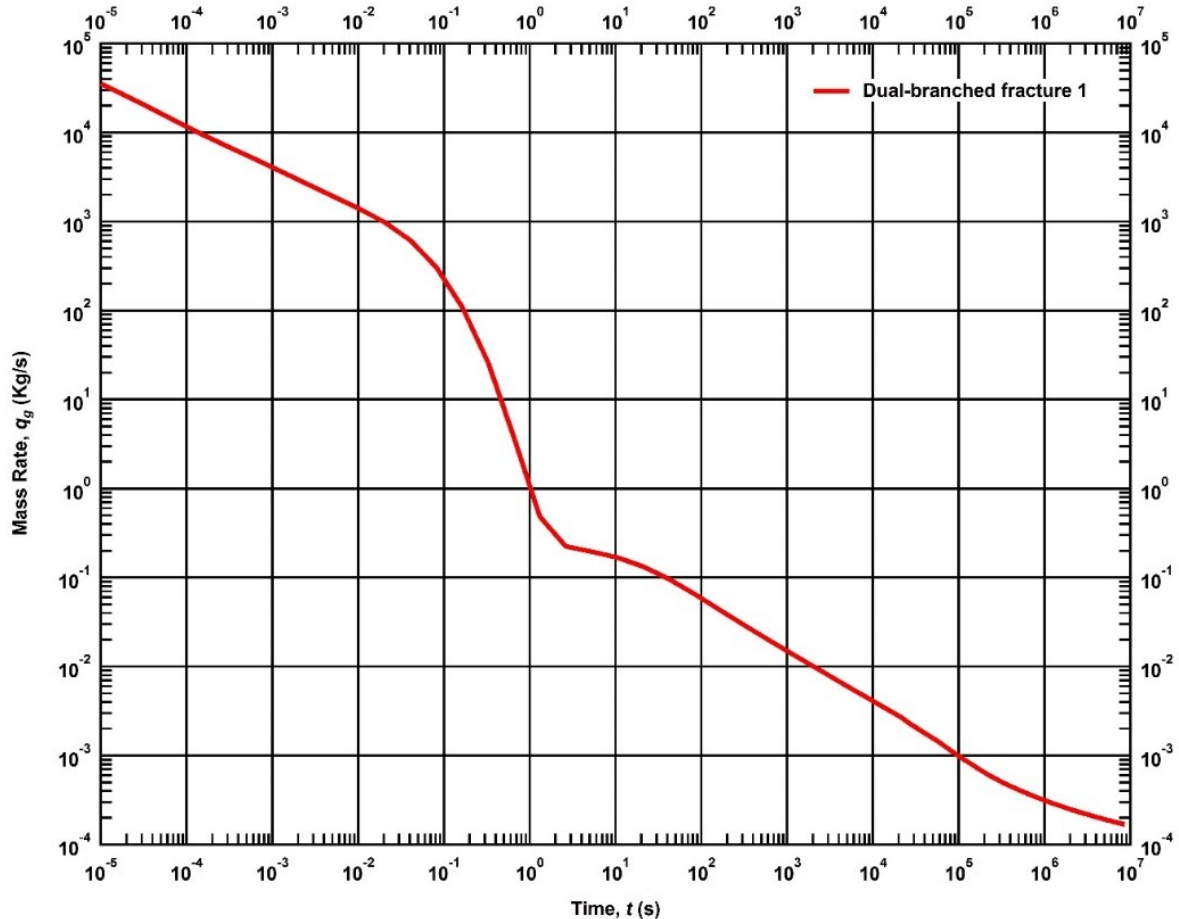

Figure B. 25 - Mass rate evolution for a shale gas reservoir produced from the dual-branched fracture 1 until 100 days of production. 


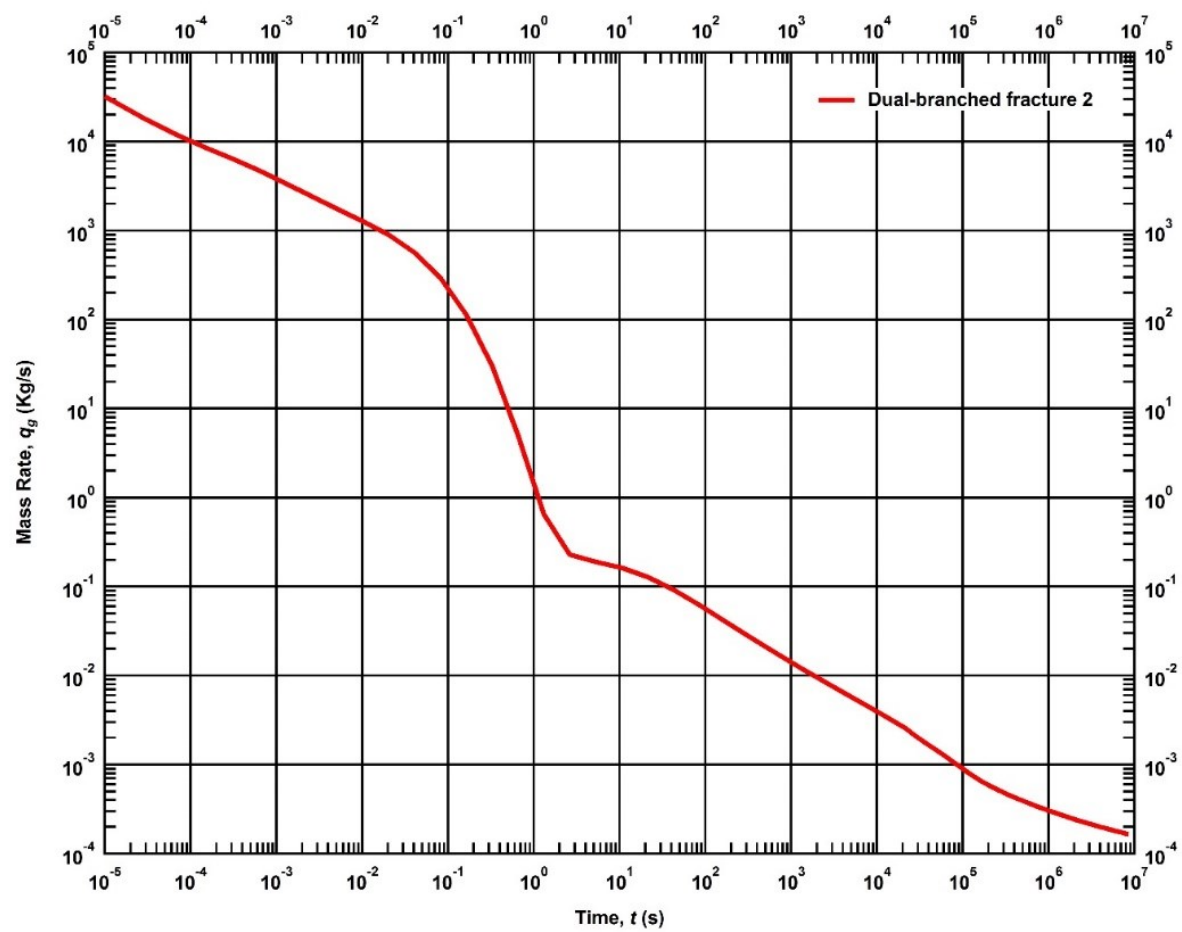

Figure B. 26 - Mass rate evolution for a shale gas reservoir produced from the dual-branched fracture 2 until 100 days of production.

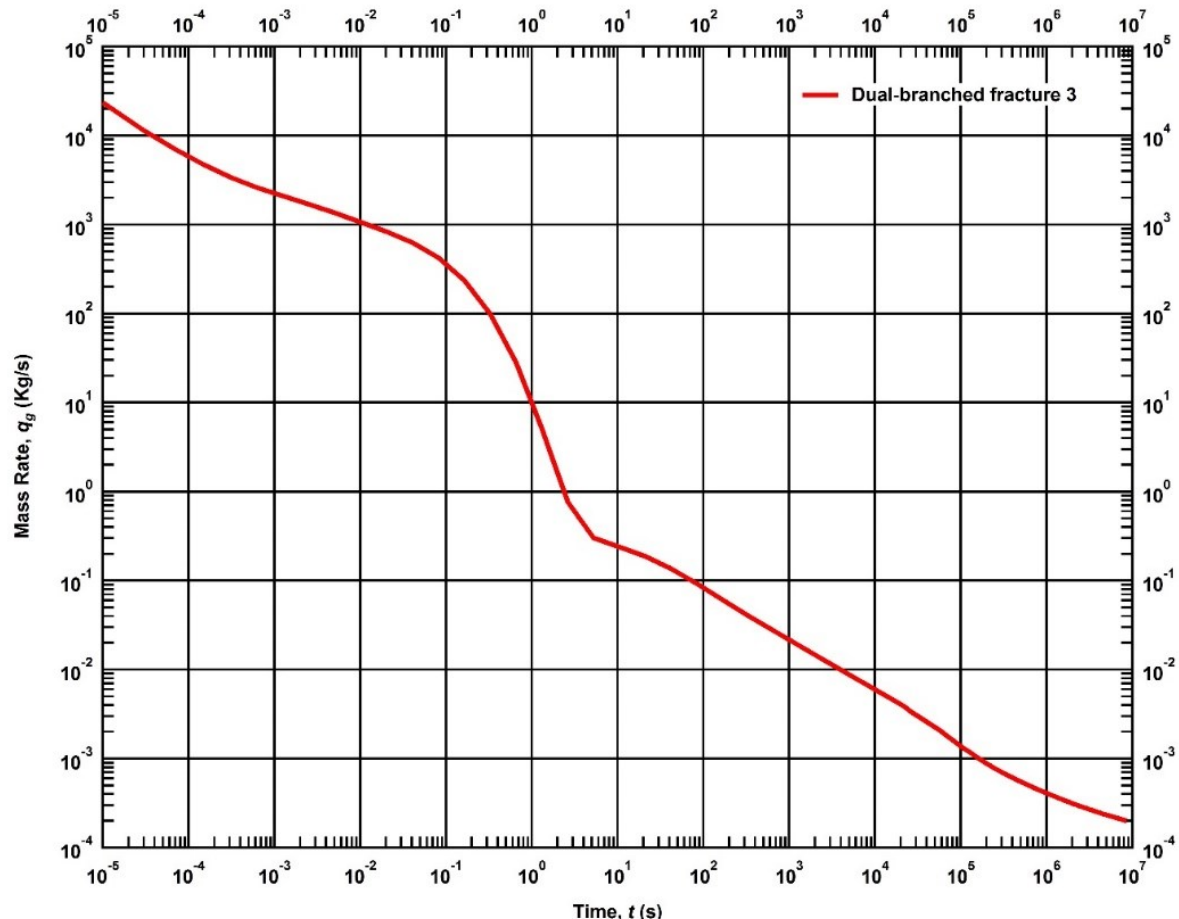

Figure B. 27 - Mass rate evolution for a shale gas reservoir produced from the dual-branched fracture 3 until 100 days of production. 


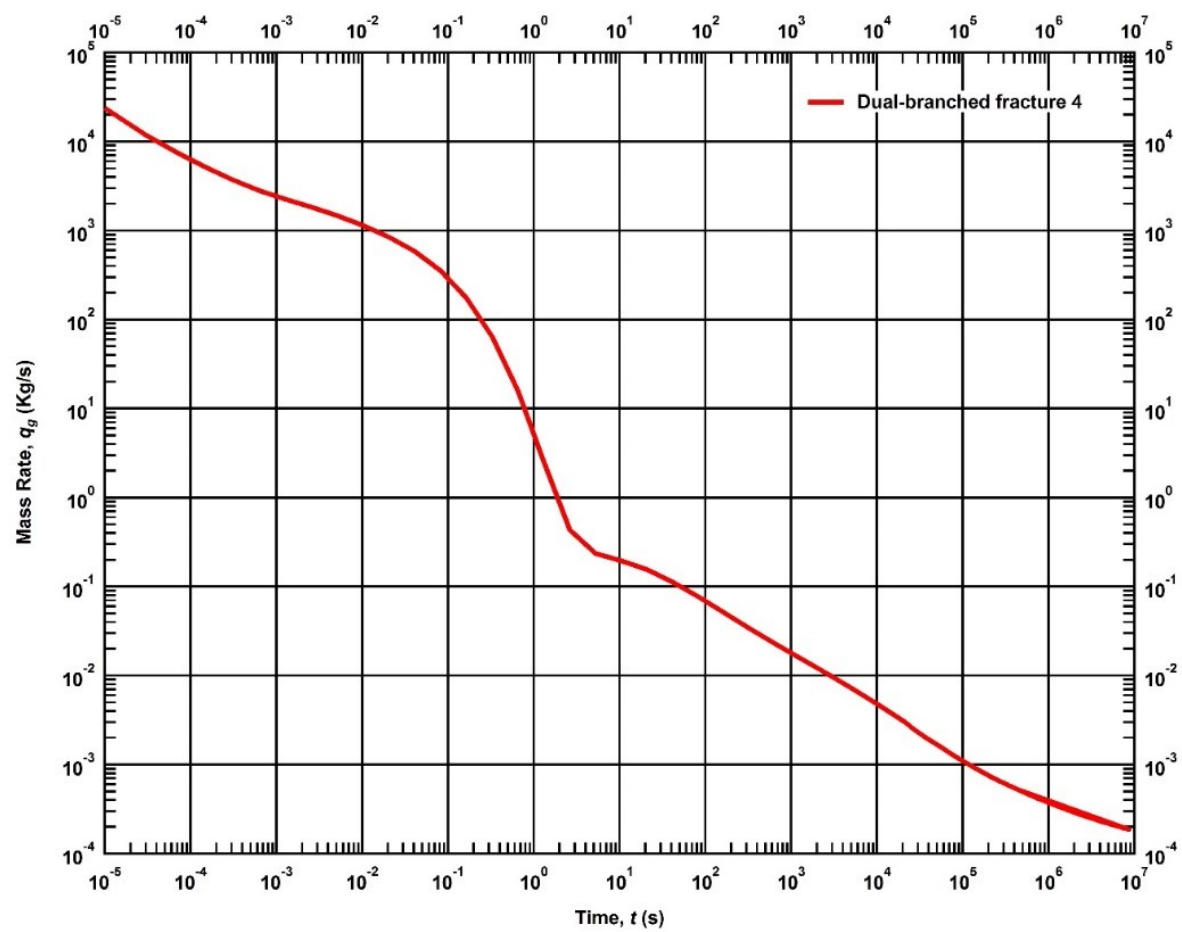

Figure B. 28 - Mass rate evolution for a shale gas reservoir produced from the dual-branched fracture 4 until 100 days of production.

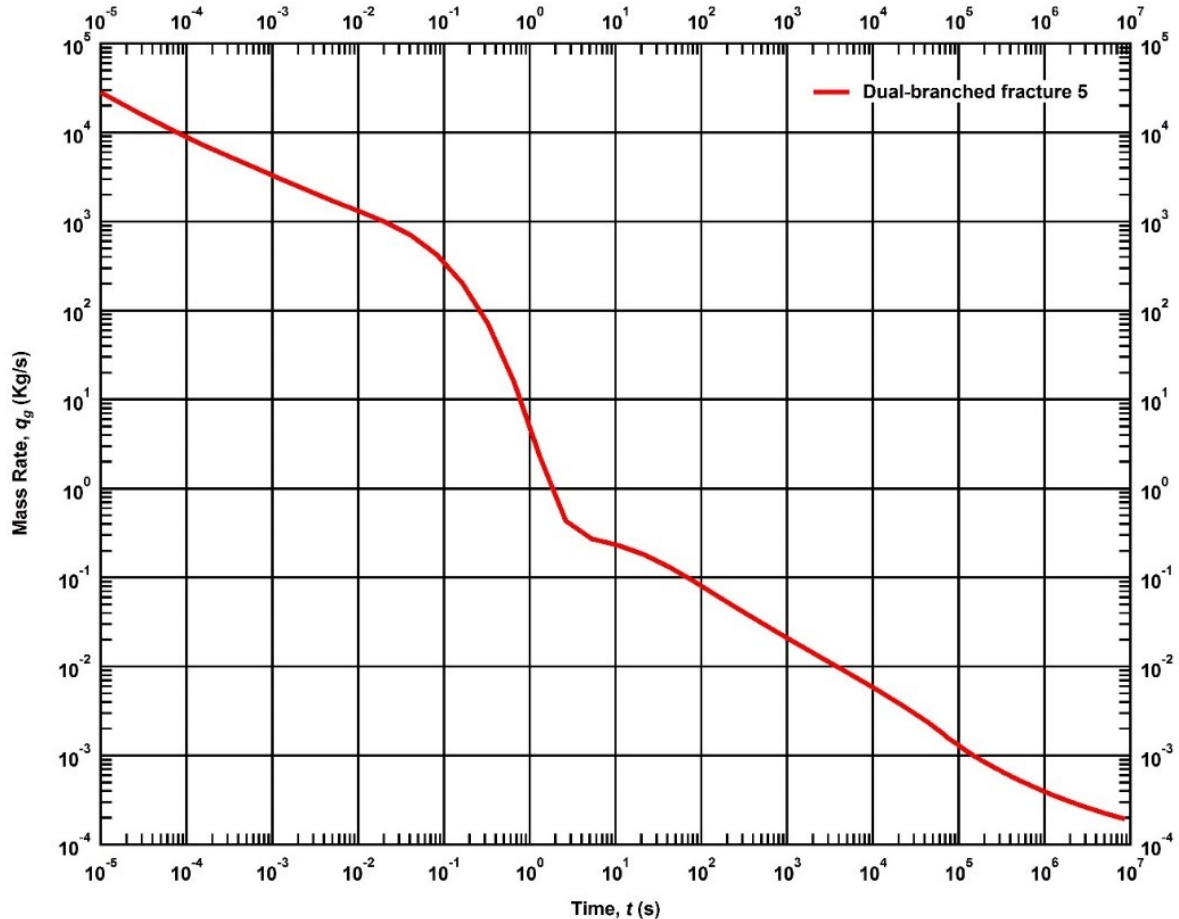

Figure B. 29 - Mass rate evolution for a shale gas reservoir produced from the dual-branched fracture 5 until 100 days of production. 


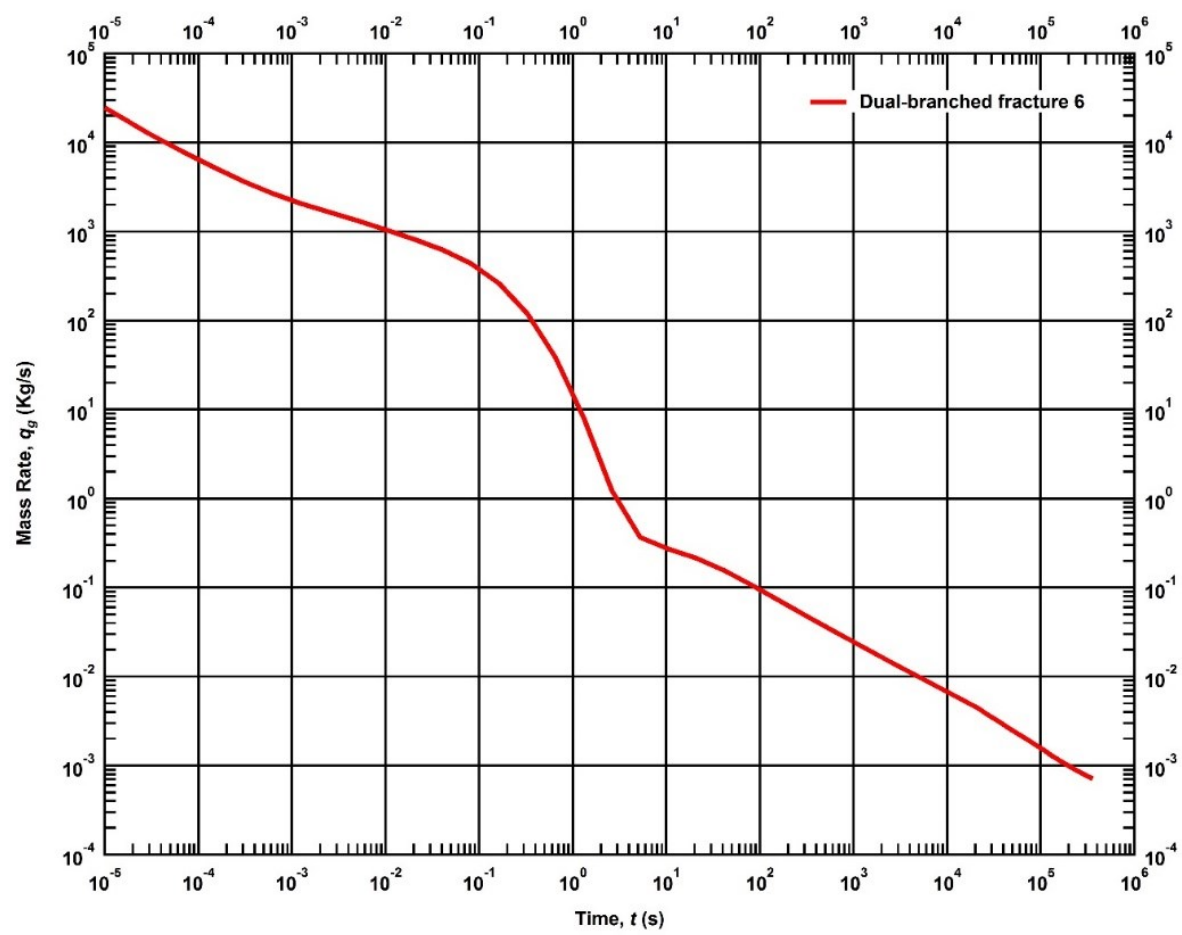

Figure B. 30 - Mass rate evolution for a shale gas reservoir produced from the dual-branched fracture 6 until 100 hours of production.

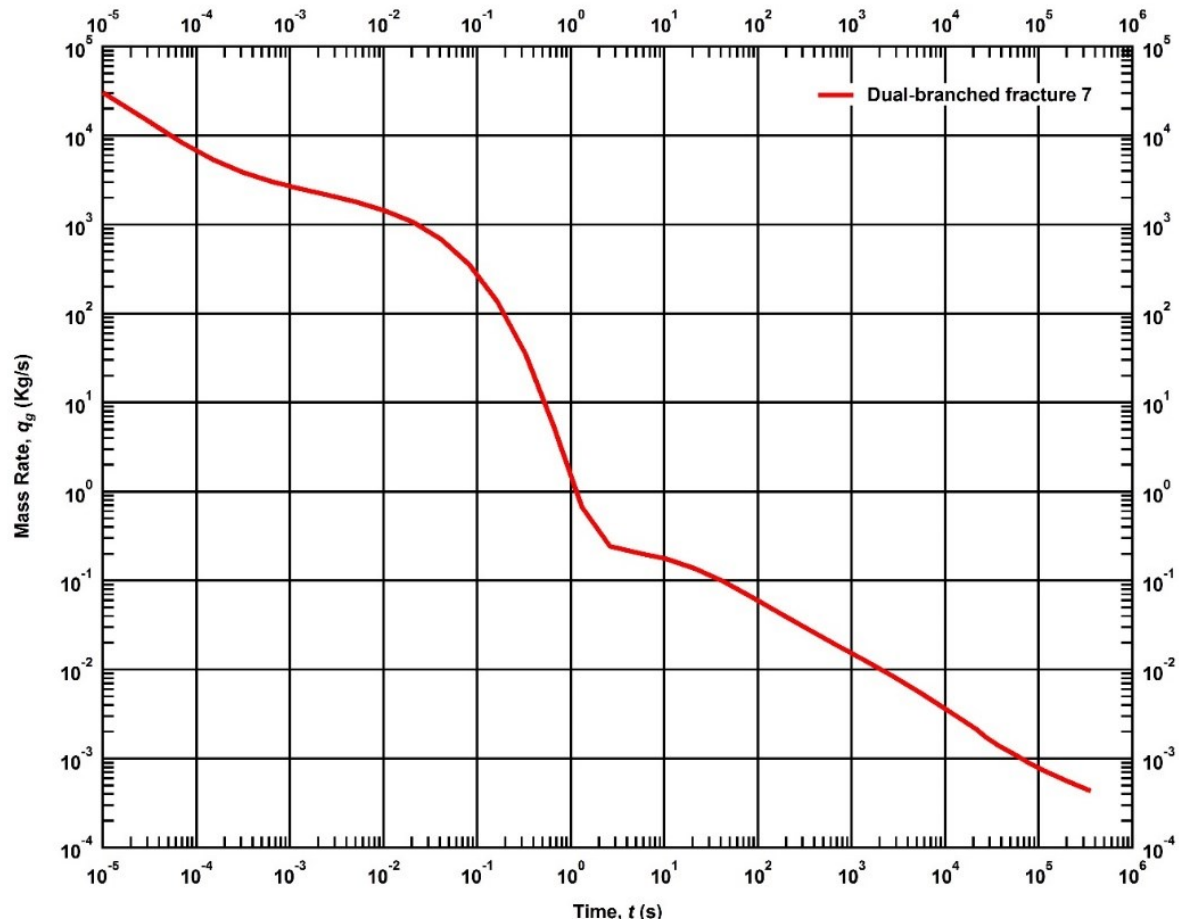

Figure B. 31 - Mass rate evolution for a shale gas reservoir produced from the dual-branched fracture 7 until 100 hours of production. 


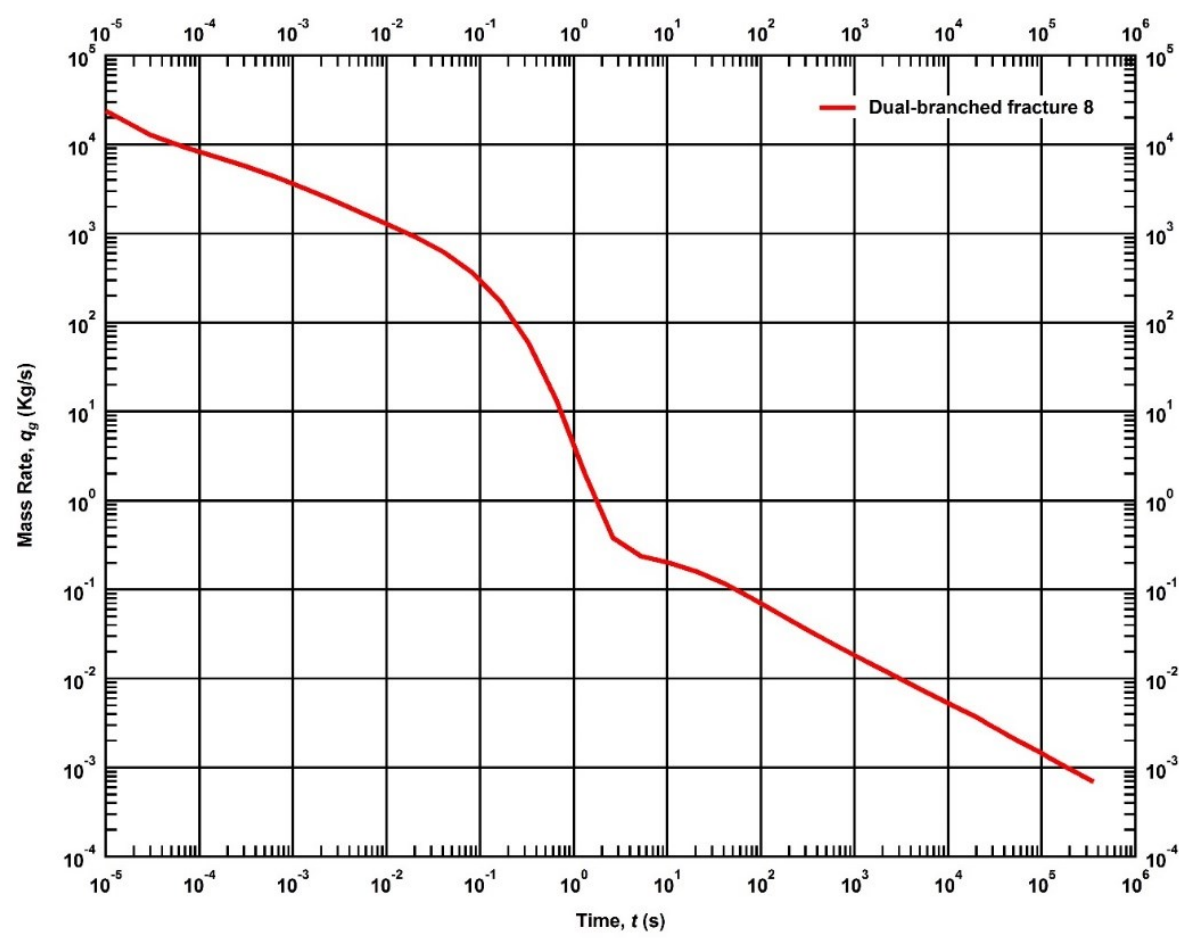

Figure B. 32 - Mass rate evolution for a shale gas reservoir produced from the dual-branched fracture 8 until 100 hours of production.

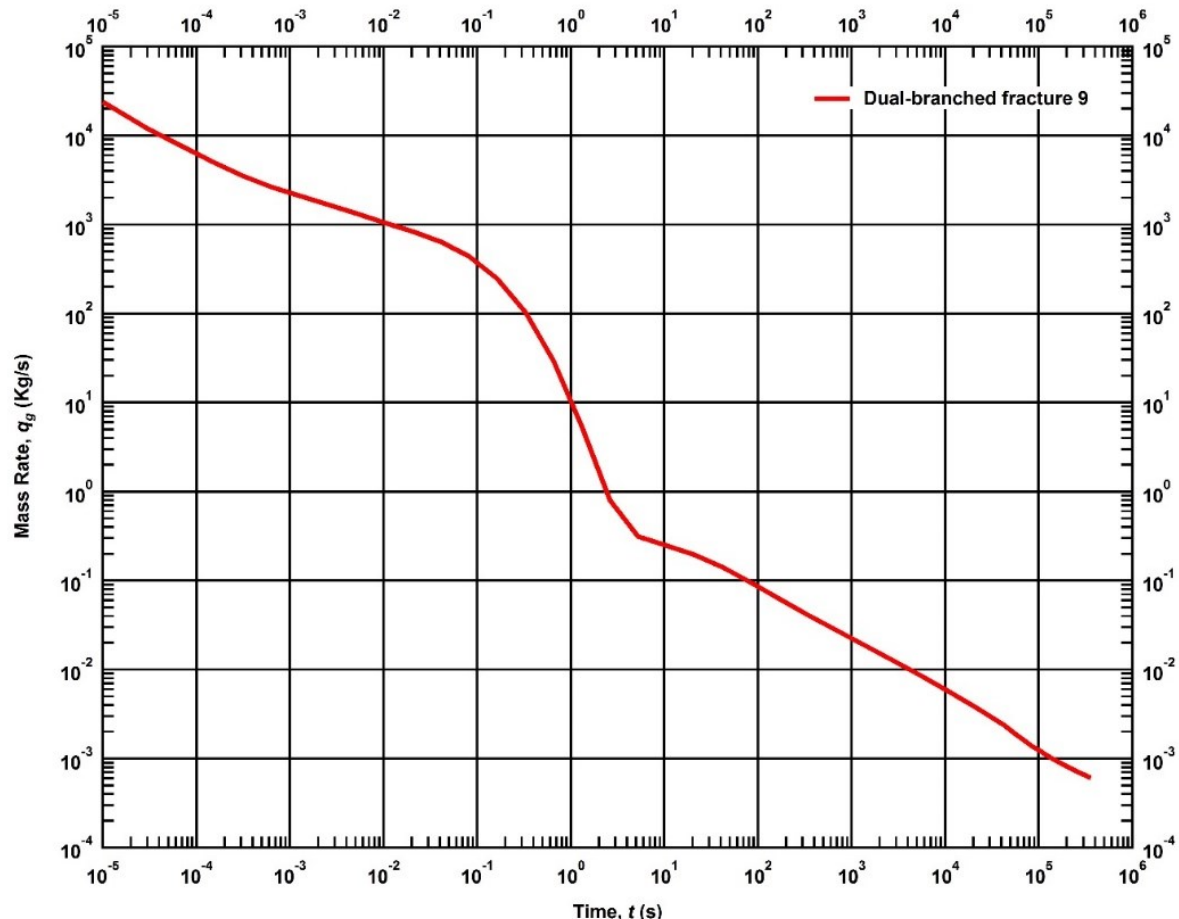

Figure B. 33 - Mass rate evolution for a shale gas reservoir produced from the dual-branched fracture 9 until 100 hours of production. 


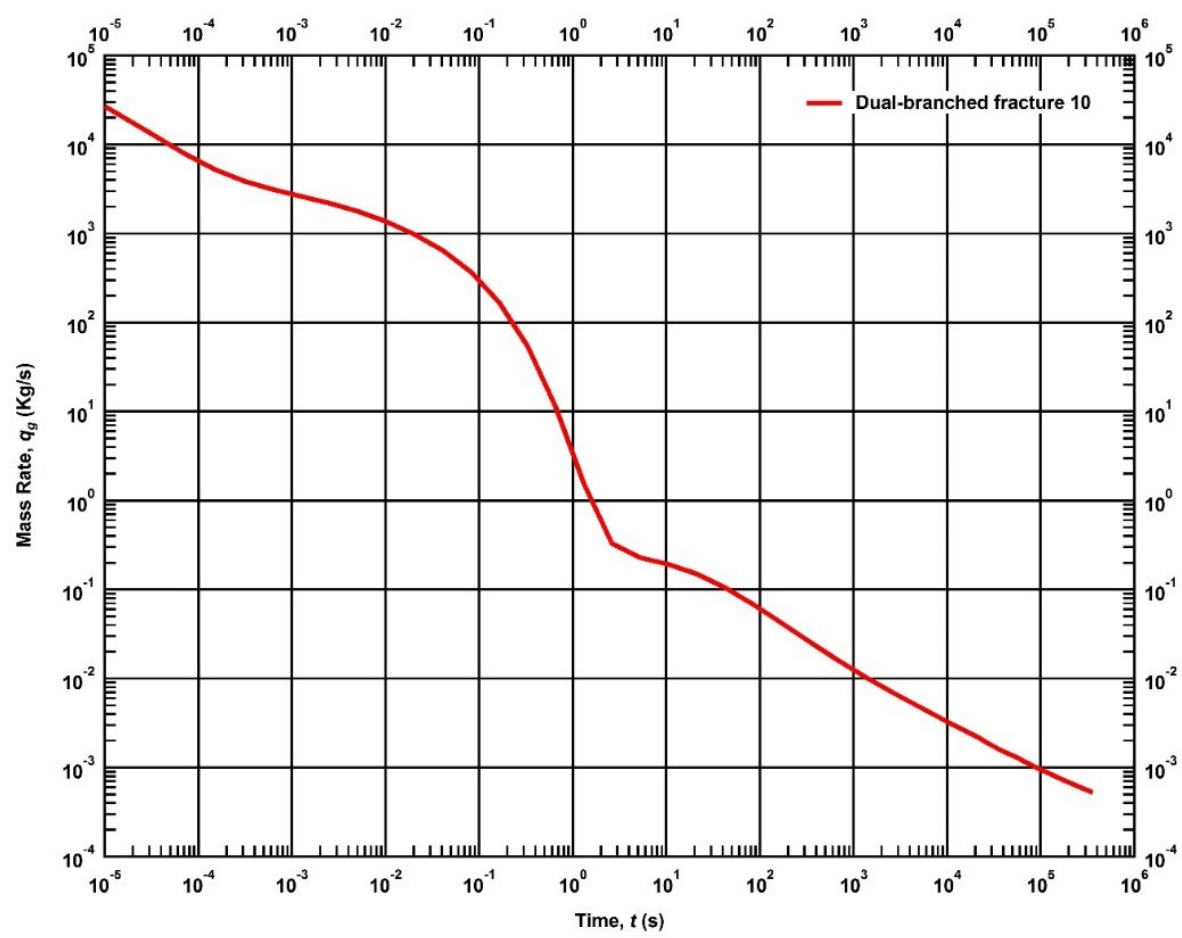

Figure B. 34 - Mass rate evolution for a shale gas reservoir produced from the dual-branched fracture 10 until 100 hours of production.

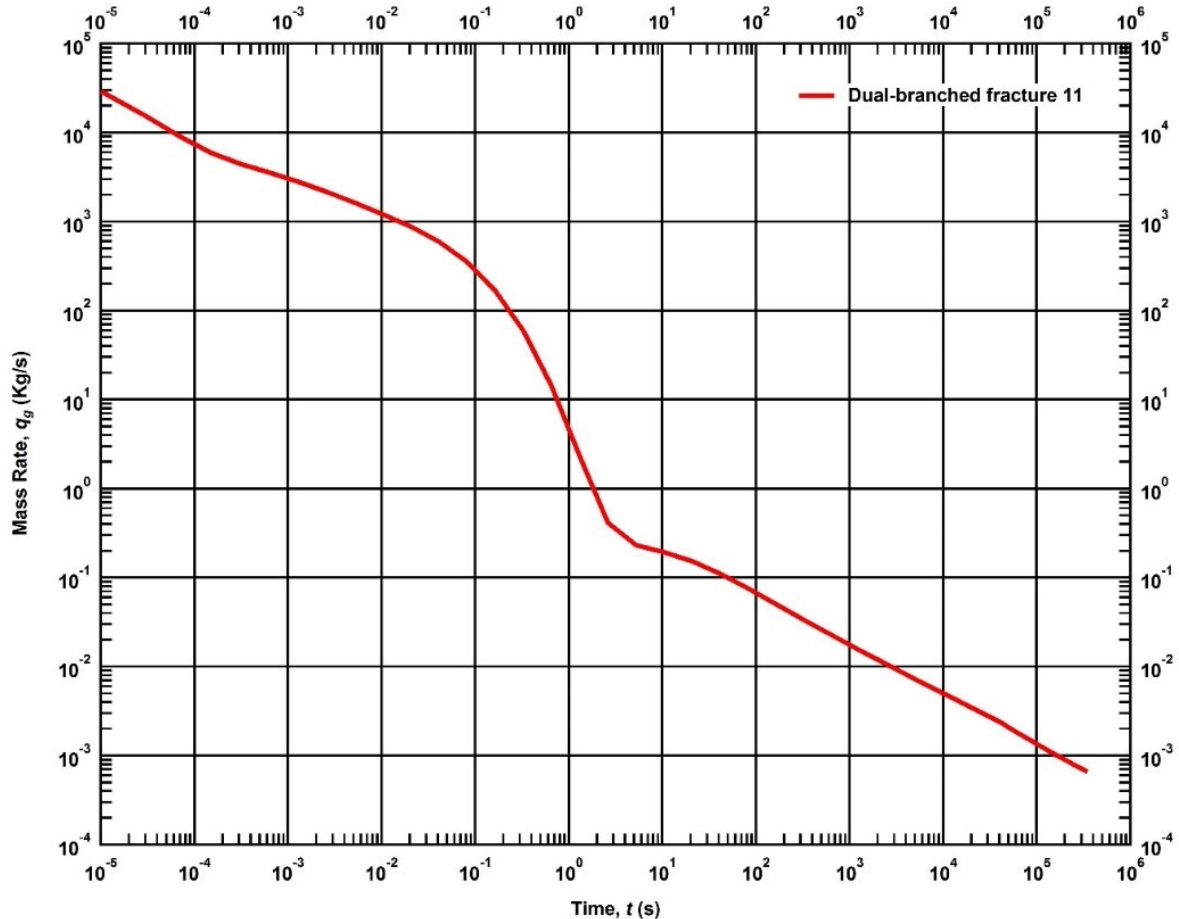

Figure B. 35 - Mass rate evolution for a shale gas reservoir produced from the dual-branched fracture 11 until 100 hours of production. 


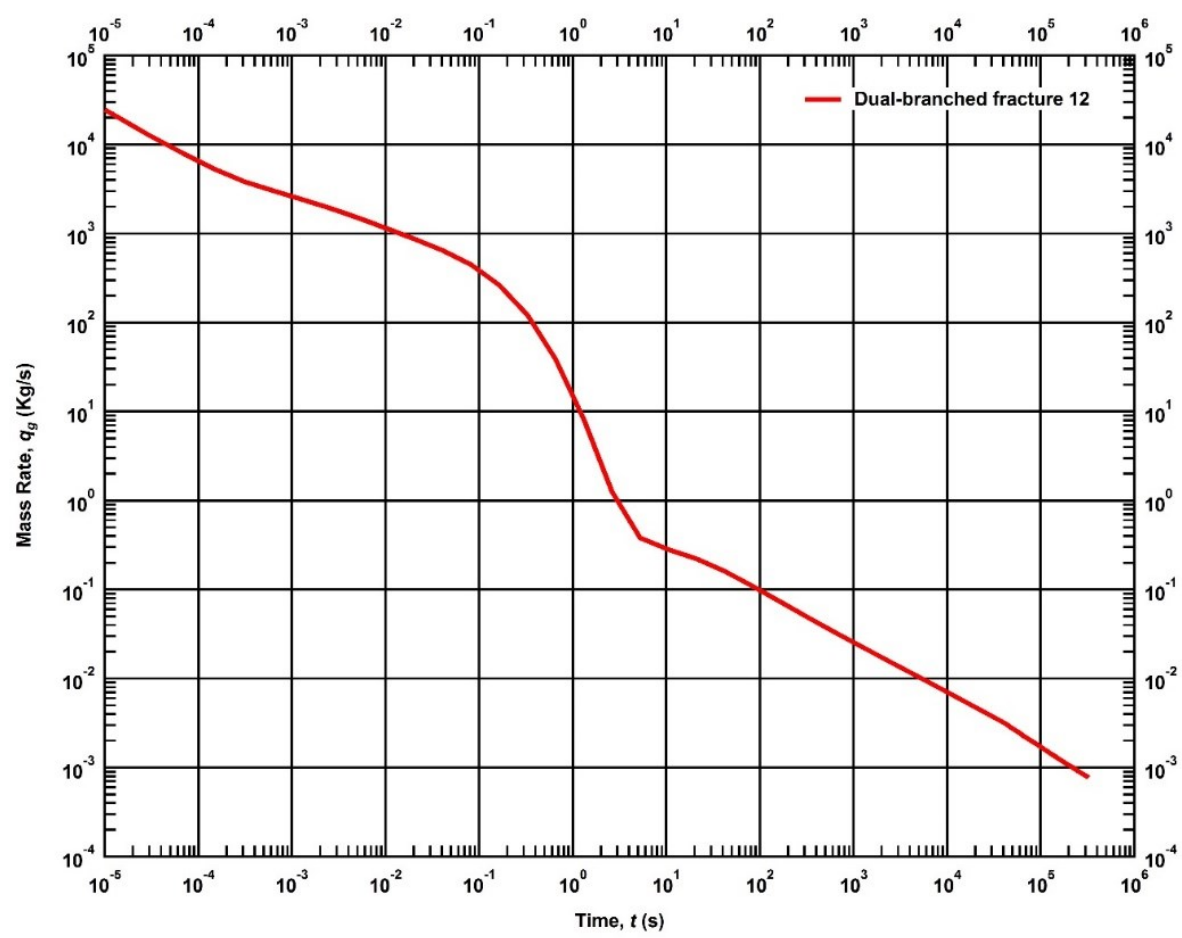

Figure B. 36 - Mass rate evolution for a shale gas reservoir produced from the dual-branched fracture 12 until 100 hours of production.

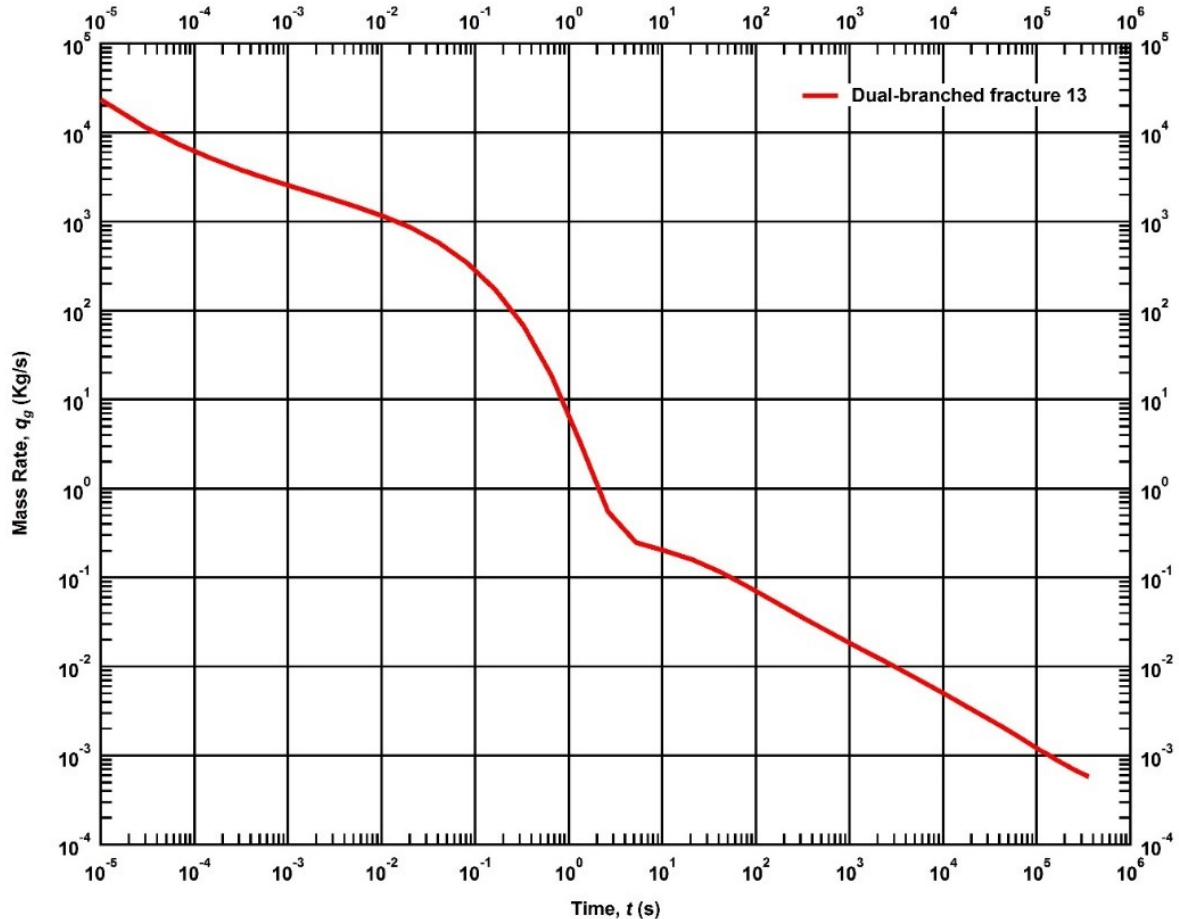

Figure B. 37 - Mass rate evolution for a shale gas reservoir produced from the dual-branched fracture 13 until 100 hours of production. 


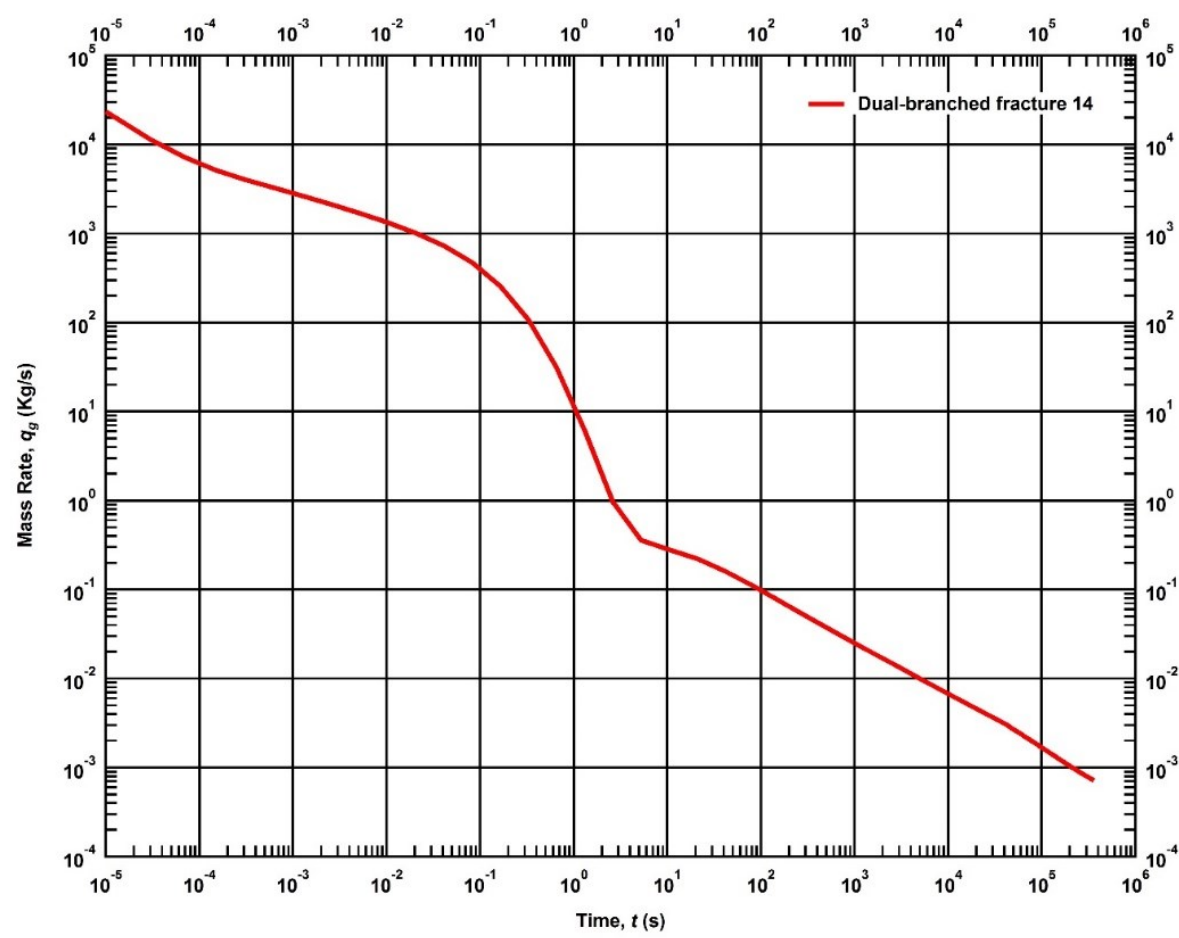

Figure B. 38 - Mass rate evolution for a shale gas reservoir produced from the dual-branched fracture 14 until 100 hours of production.

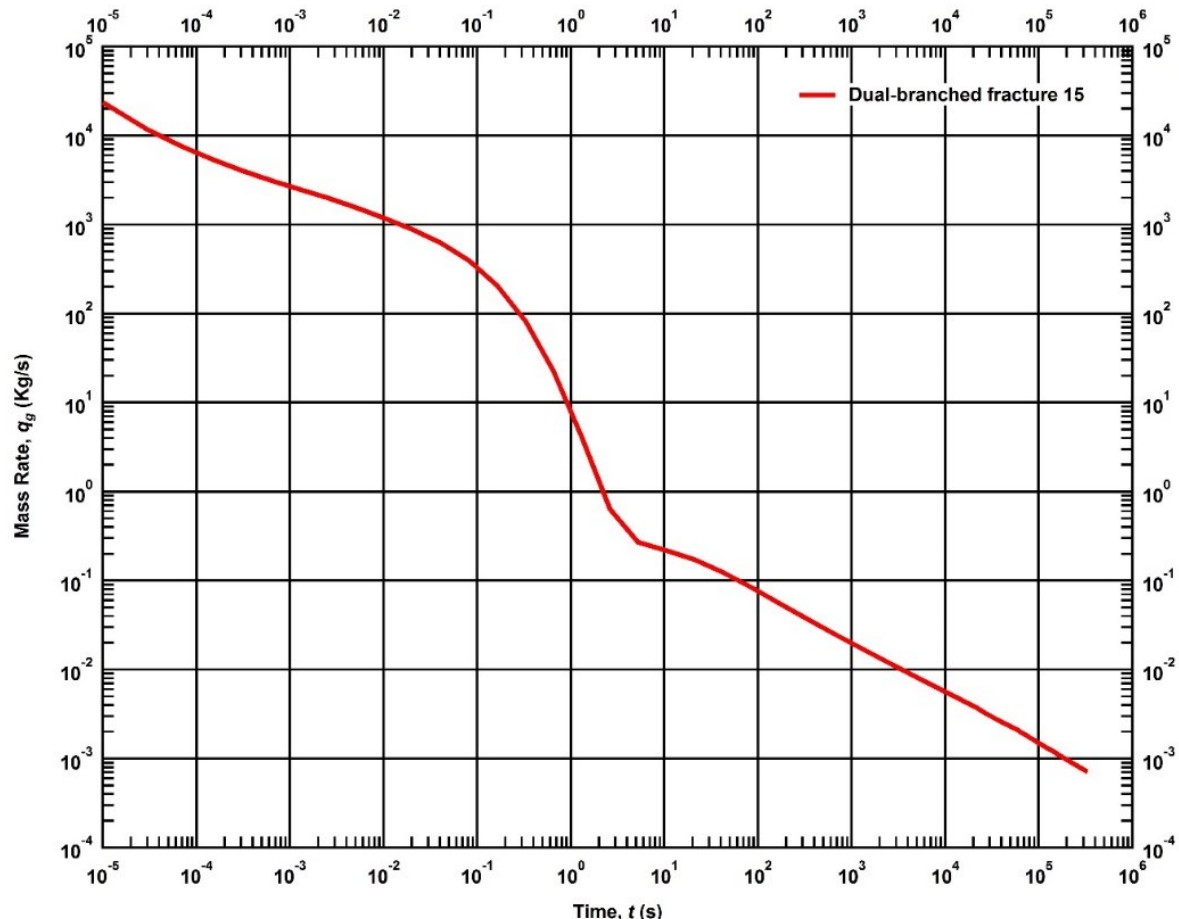

Figure B. 39 - Mass rate evolution for a shale gas reservoir produced from the dual-branched fracture 15 until 100 hours of production. 


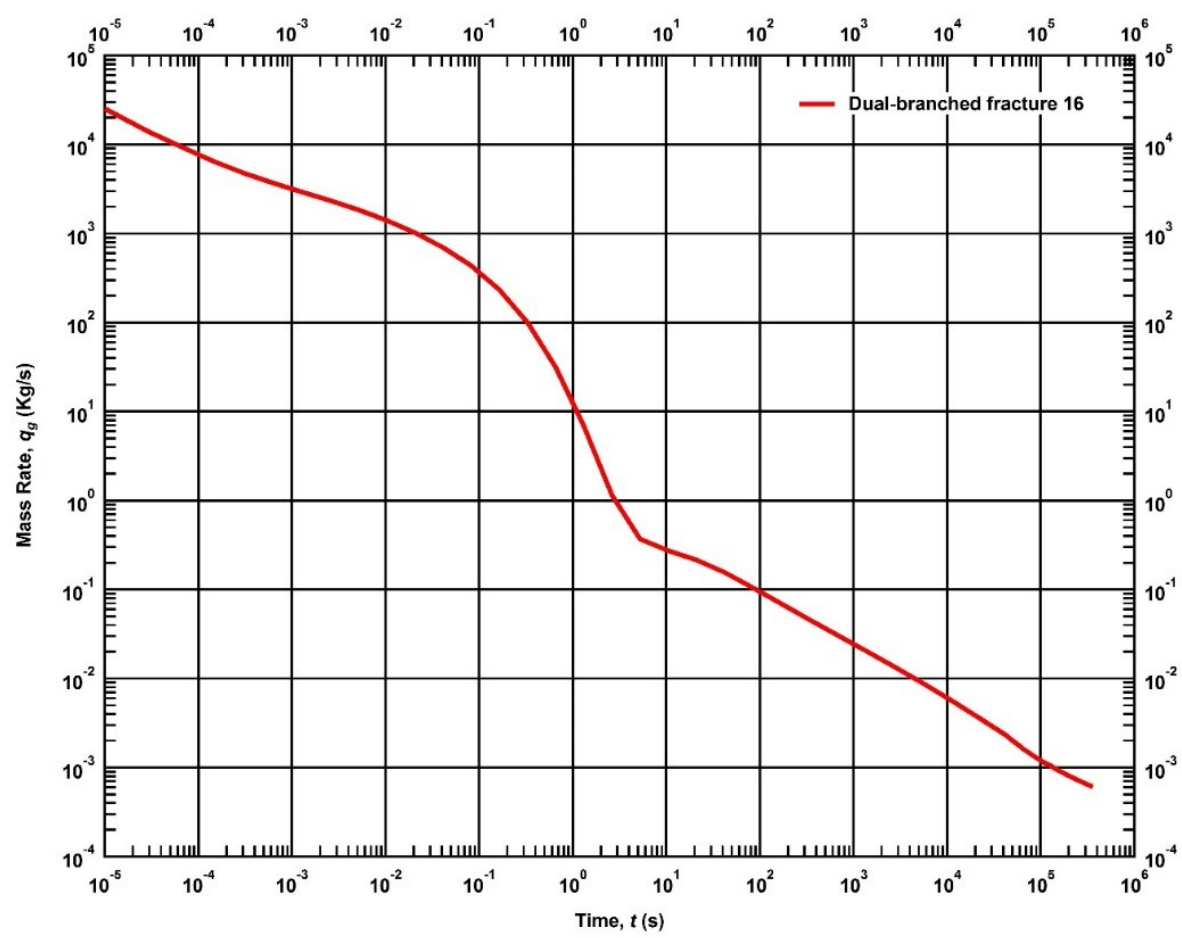

Figure B. 40 - Mass rate evolution for a shale gas reservoir produced from the dual-branched fracture 16 until 100 hours of production.

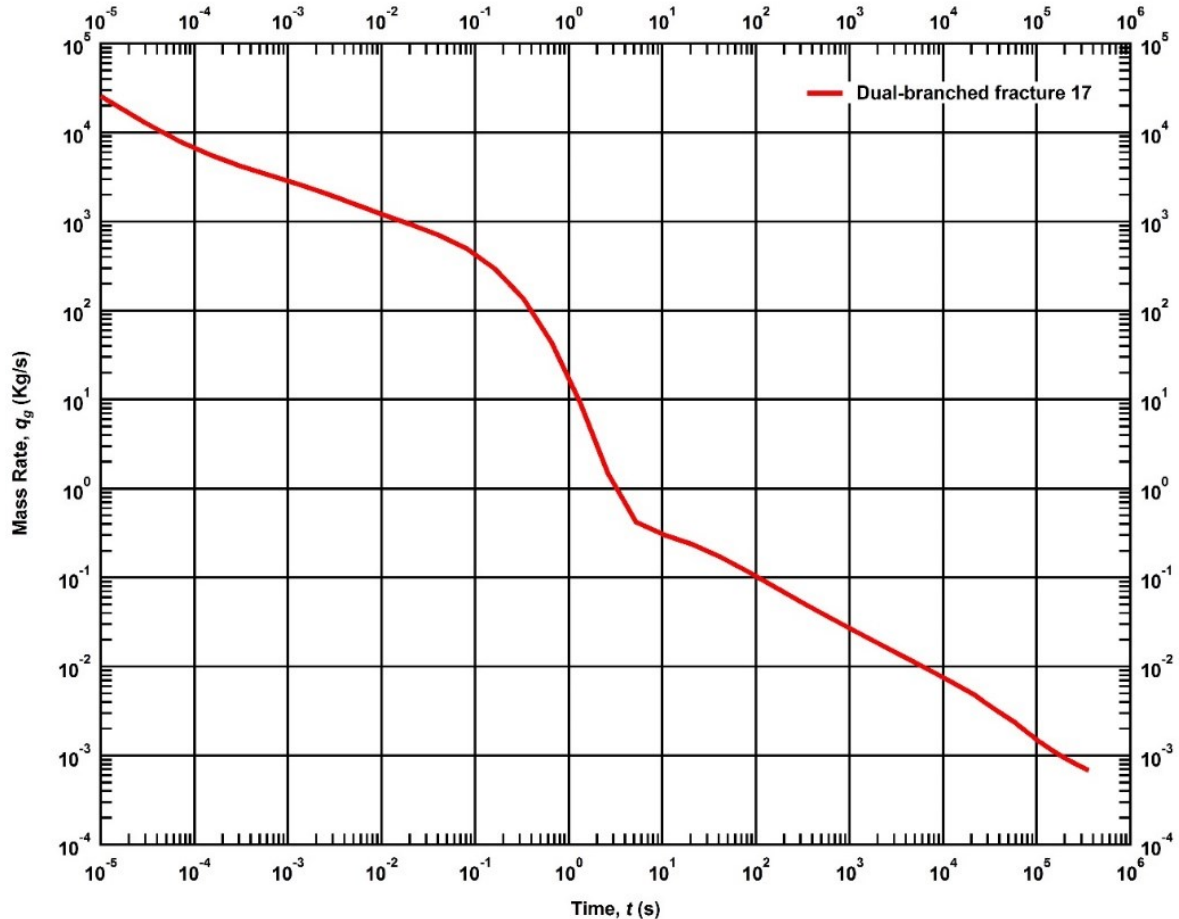

Figure B. 41 - Mass rate evolution for a shale gas reservoir produced from the dual-branched fracture 17 until 100 hours of production. 


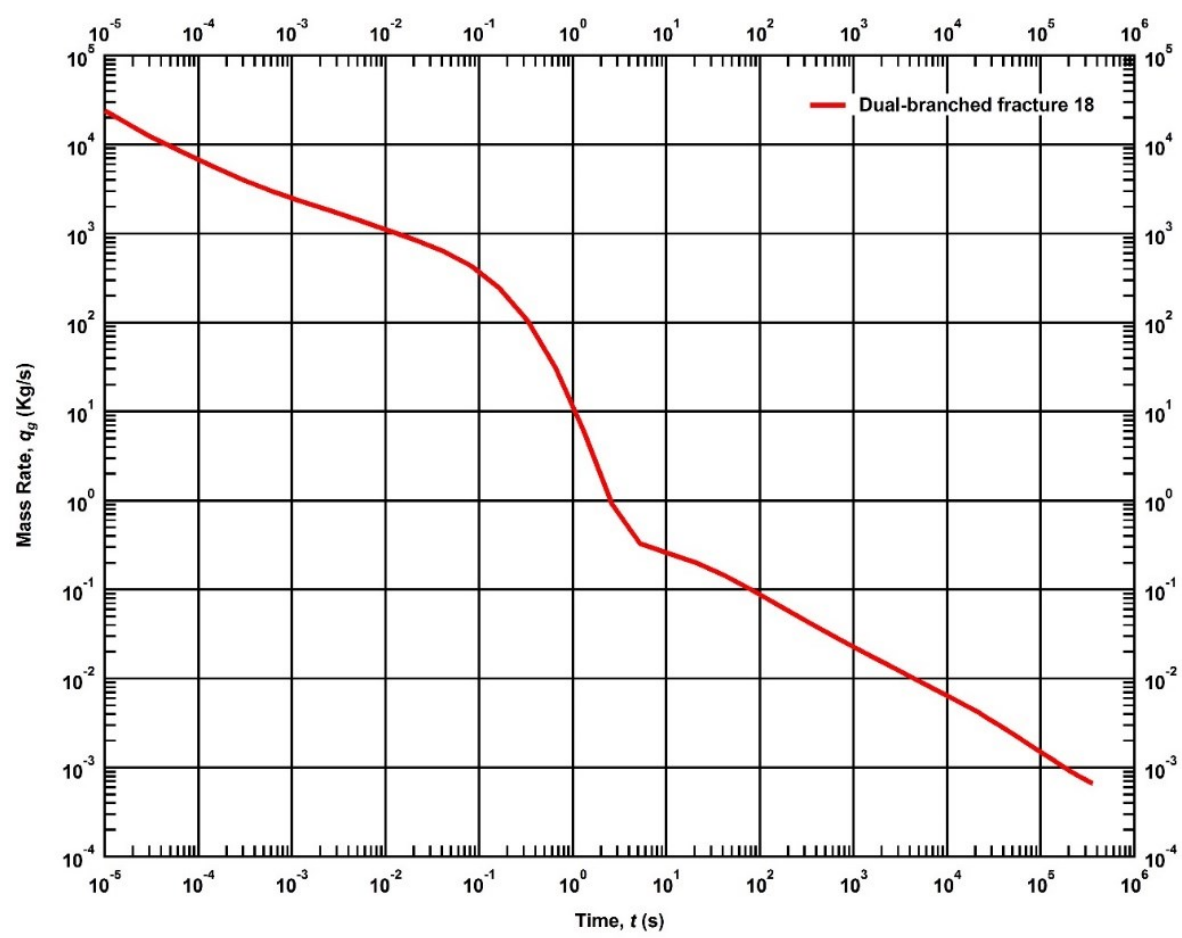

Figure B. 42 - Mass rate evolution for a shale gas reservoir produced from the dual-branched fracture 18 until 100 hours of production.

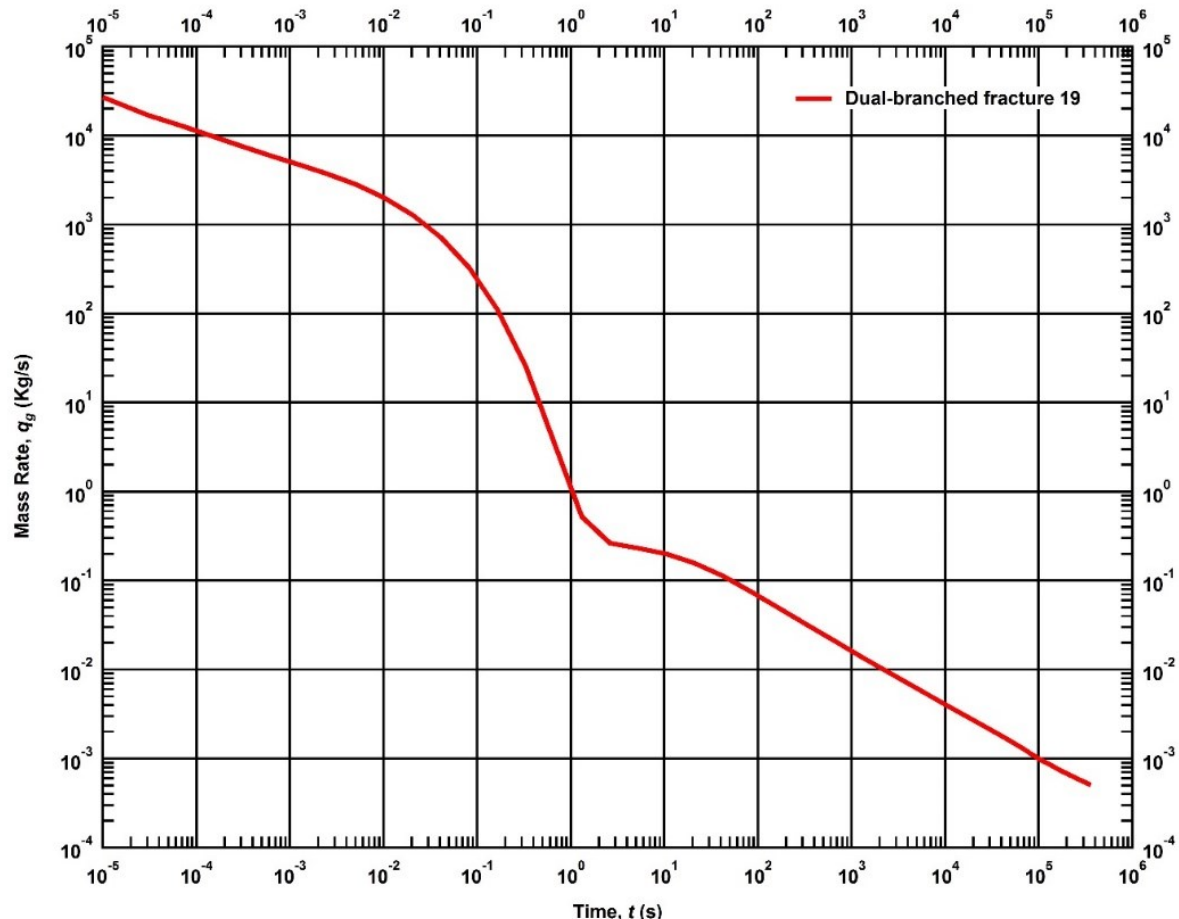

Figure B. 43 - Mass rate evolution for a shale gas reservoir produced from the dual-branched fracture 19 until 100 hours of production. 


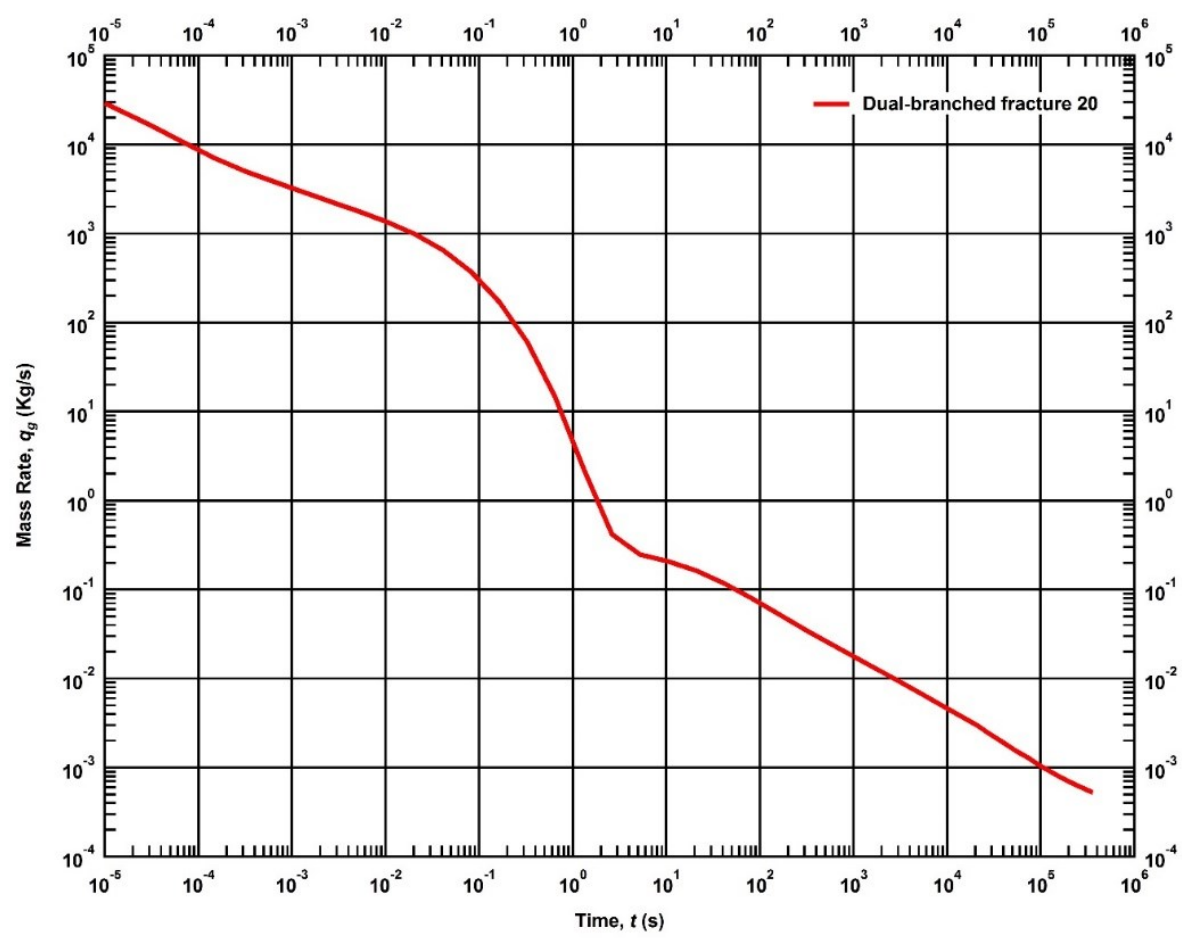

Figure B. 44 - Mass rate evolution for a shale gas reservoir produced from the dual-branched fracture 20 until 100 hours of production.

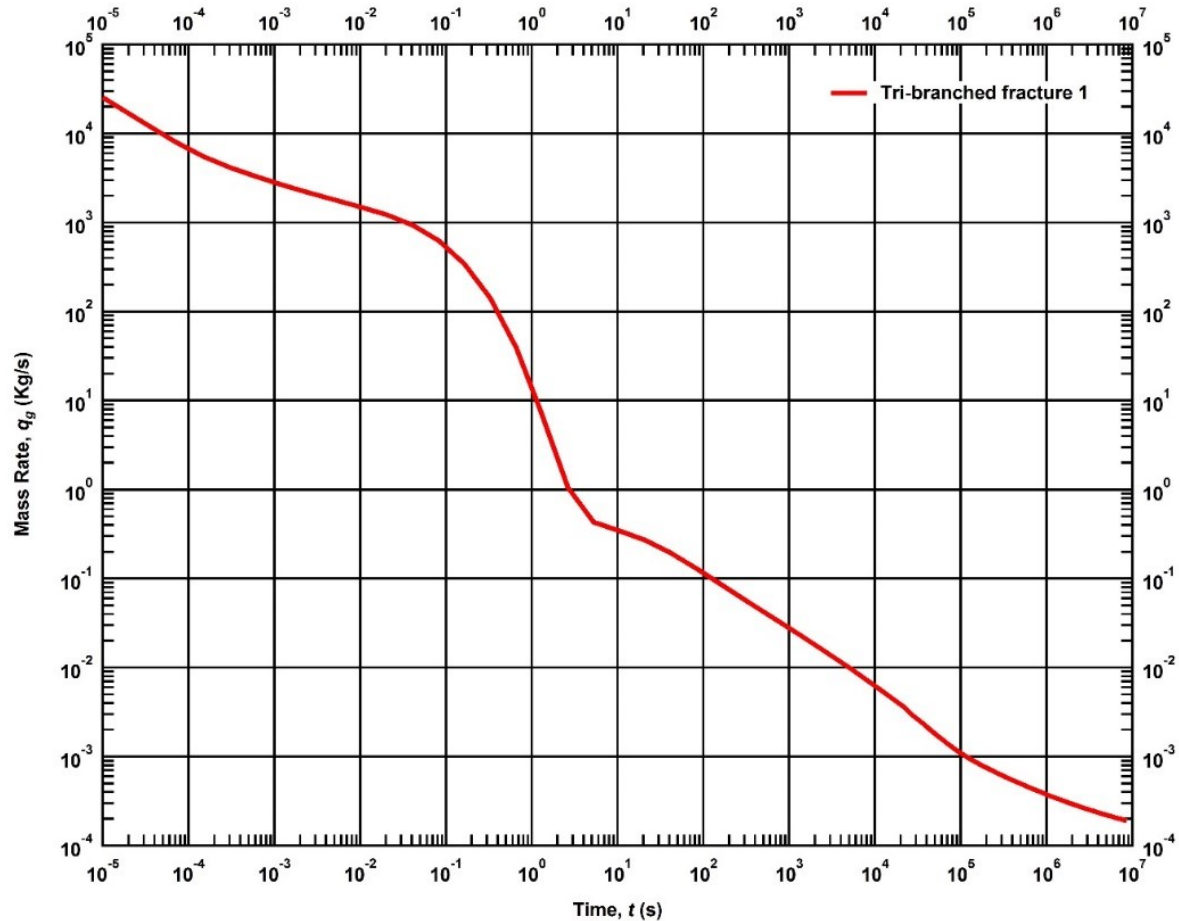

Figure B. 45 - Mass rate evolution for a shale gas reservoir produced from the tri-branched fracture 1 until 100 days of production. 


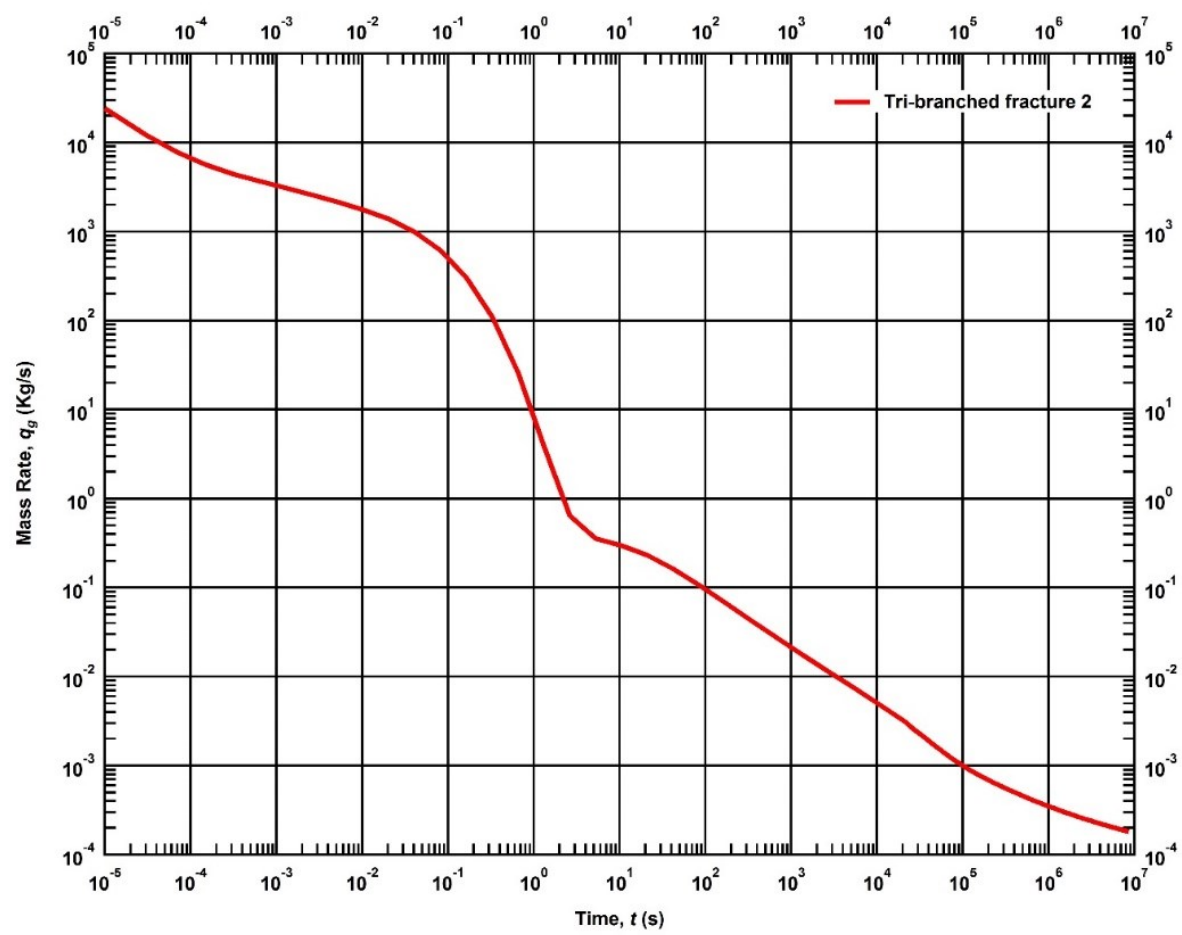

Figure B. 46 - Mass rate evolution for a shale gas reservoir produced from the tri-branched fracture 2 until 100 days of production.

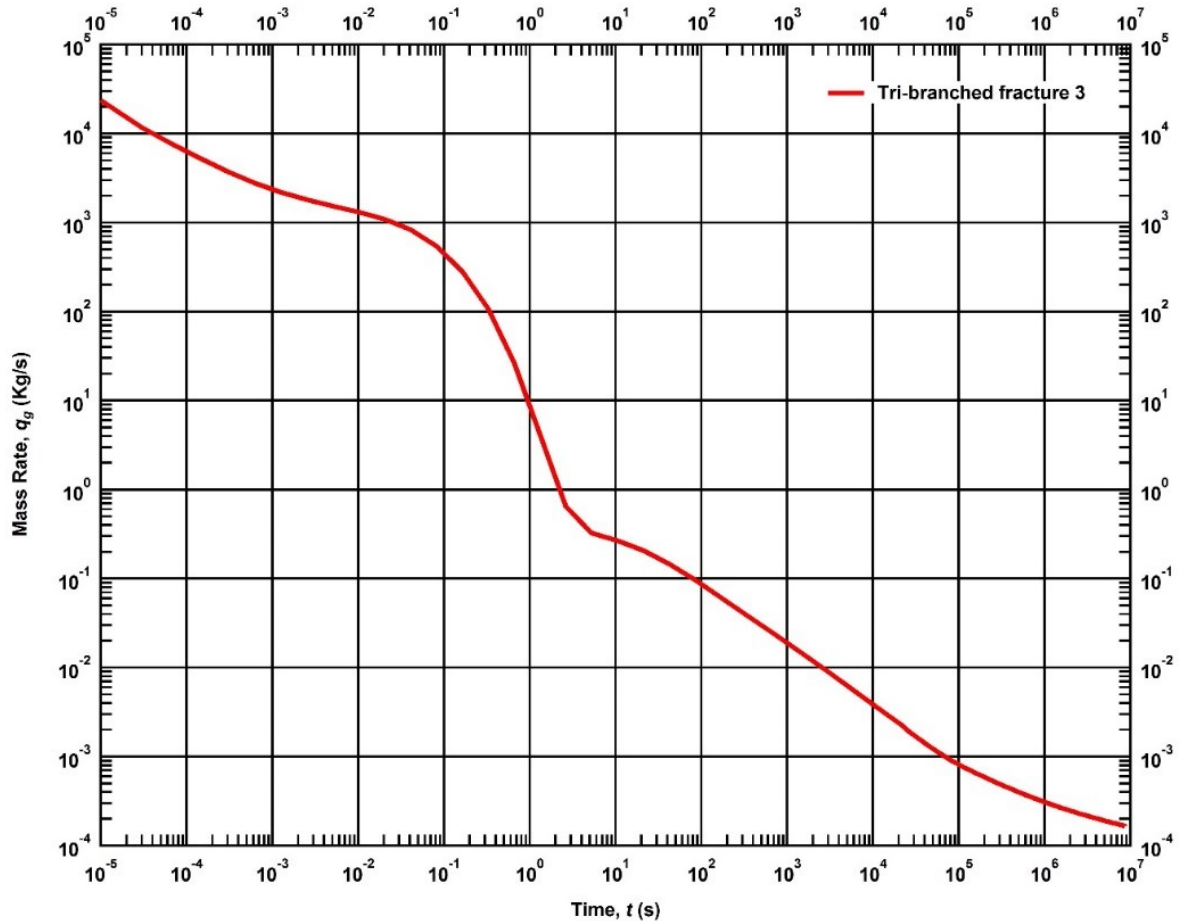

Figure B. 47 - Mass rate evolution for a shale gas reservoir produced from the tri-branched fracture 3 until 100 days of production. 


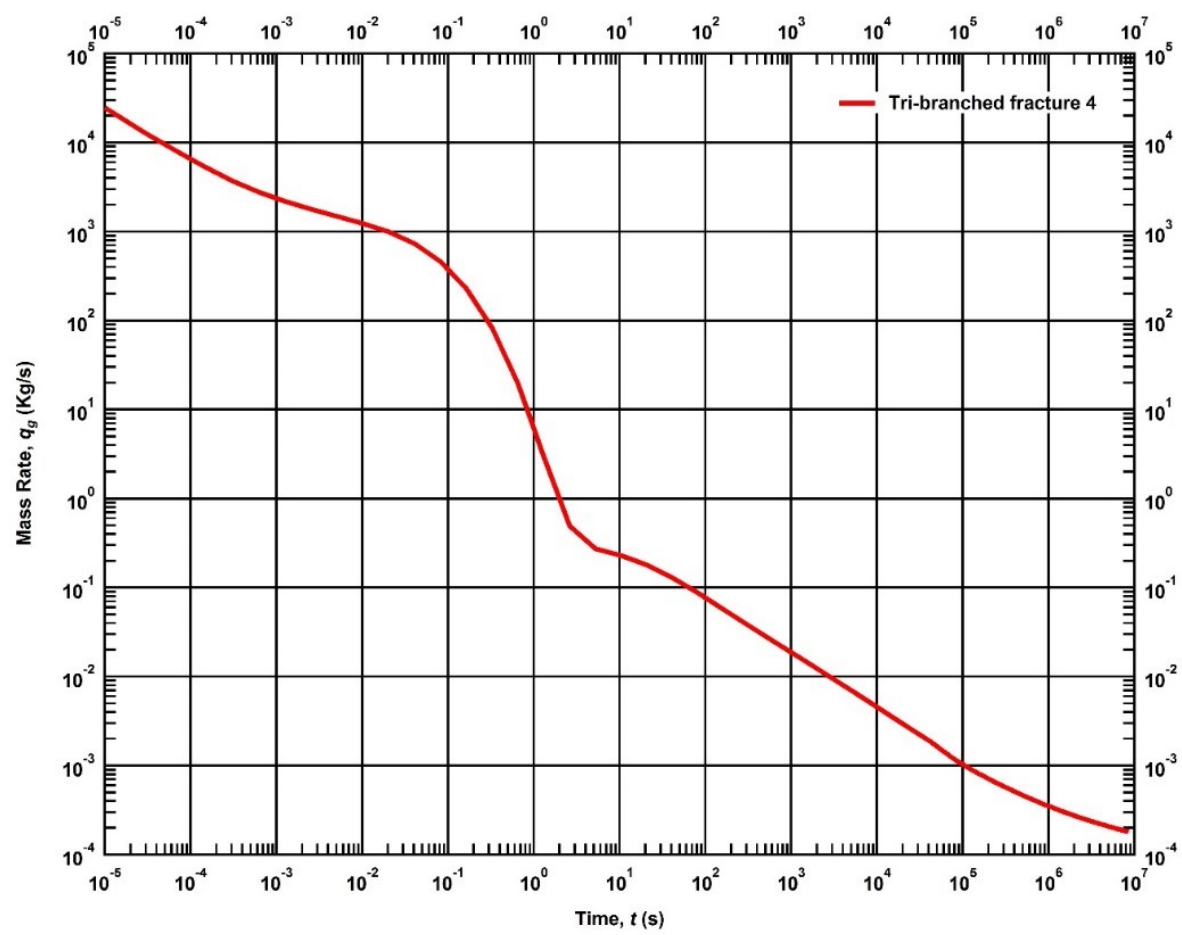

Figure B. 48 - Mass rate evolution for a shale gas reservoir produced from the tri-branched fracture 4 until 100 days of production.

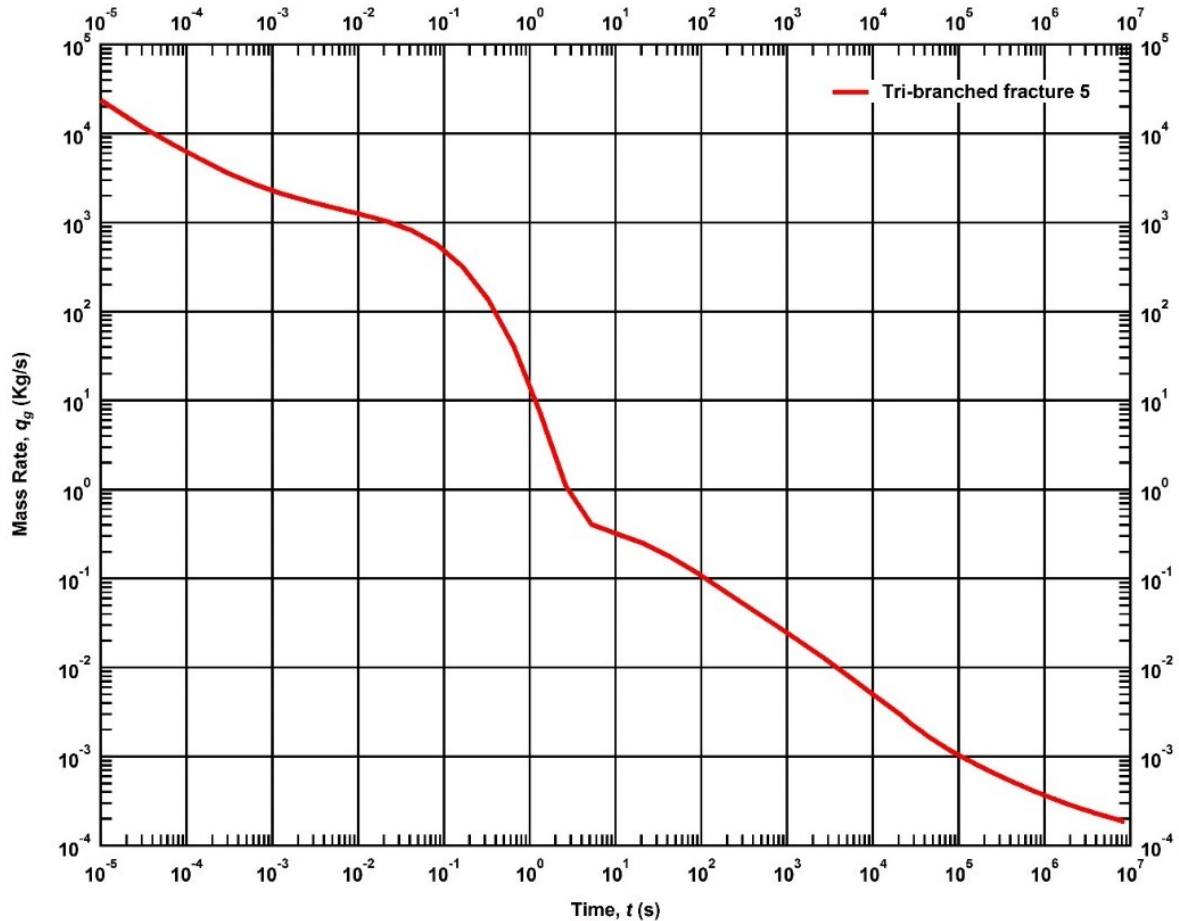

Figure B. 49 - Mass rate evolution for a shale gas reservoir produced from the tri-branched fracture 5 until 100 days of production. 


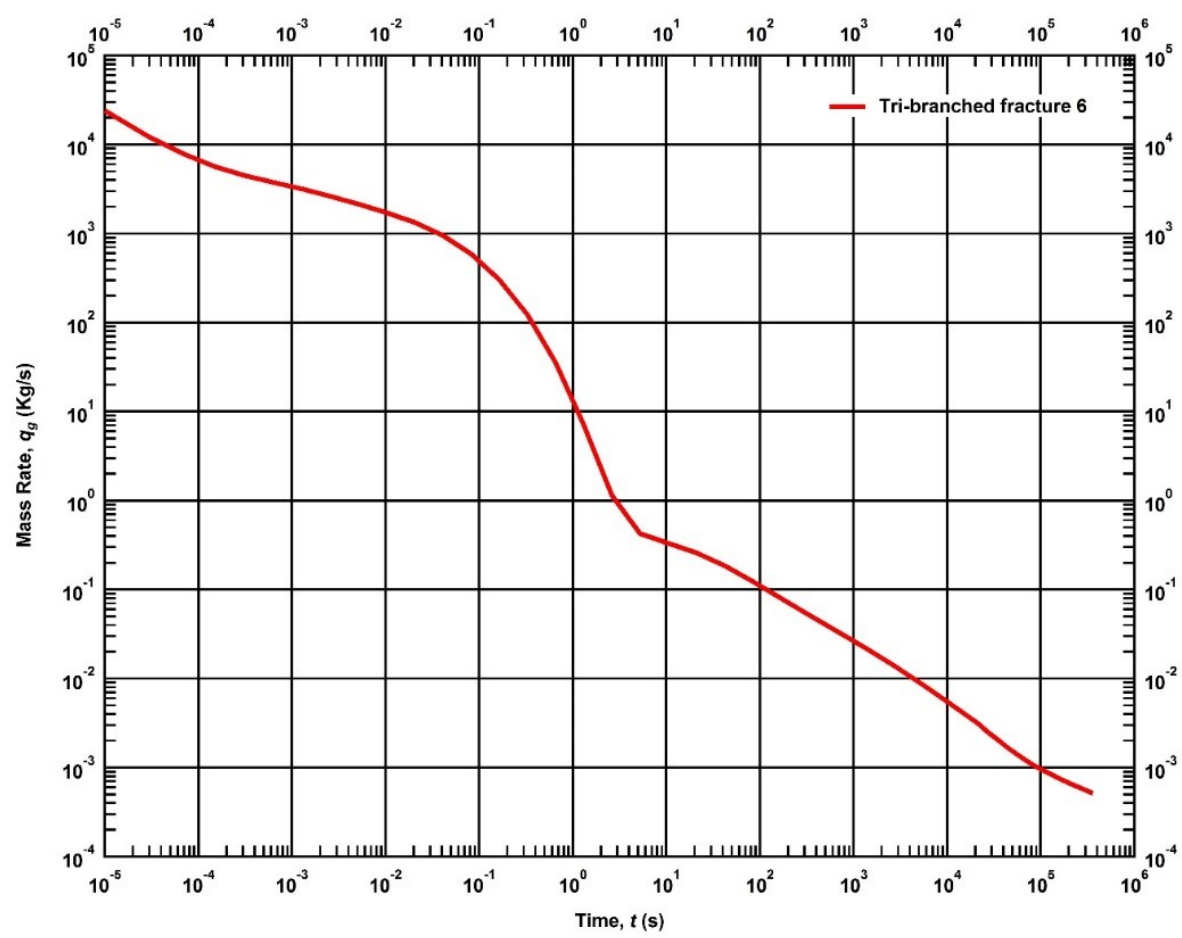

Figure B. 50 - Mass rate evolution for a shale gas reservoir produced from the tri-branched fracture 6 until 100 hours of production.

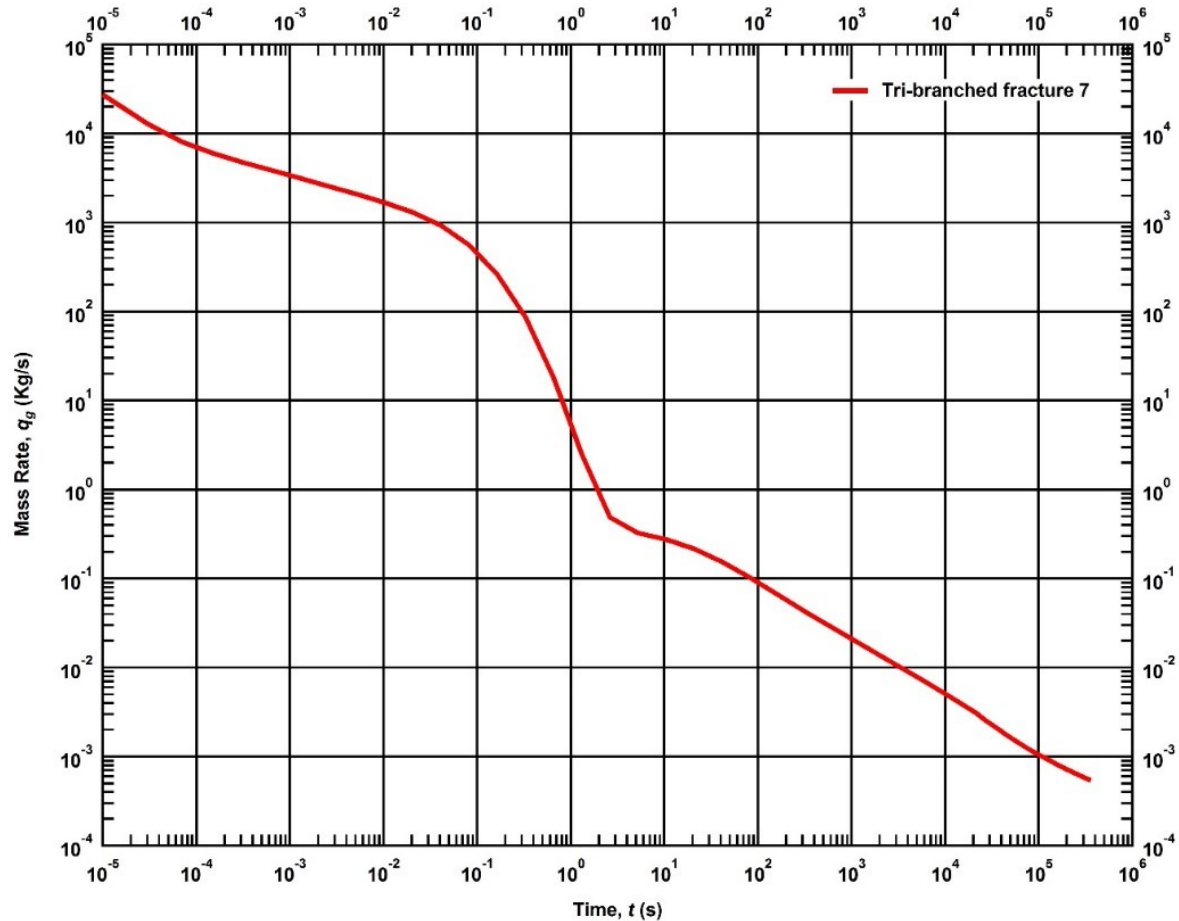

Figure B. 51 - Mass rate evolution for a shale gas reservoir produced from the tri-branched fracture 7 until 100 hours of production. 


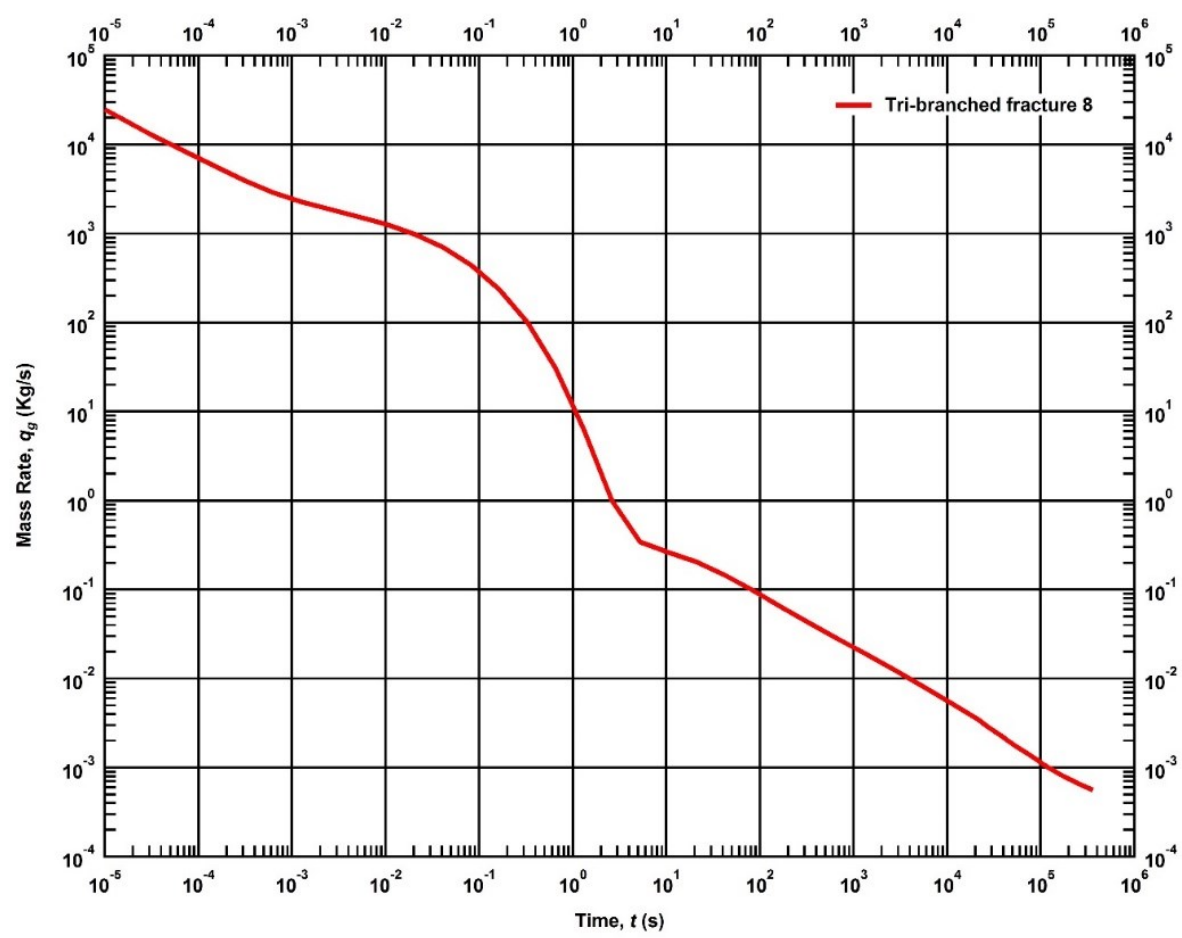

Figure B. 52 - Mass rate evolution for a shale gas reservoir produced from the tri-branched fracture 8 until 100 hours of production.

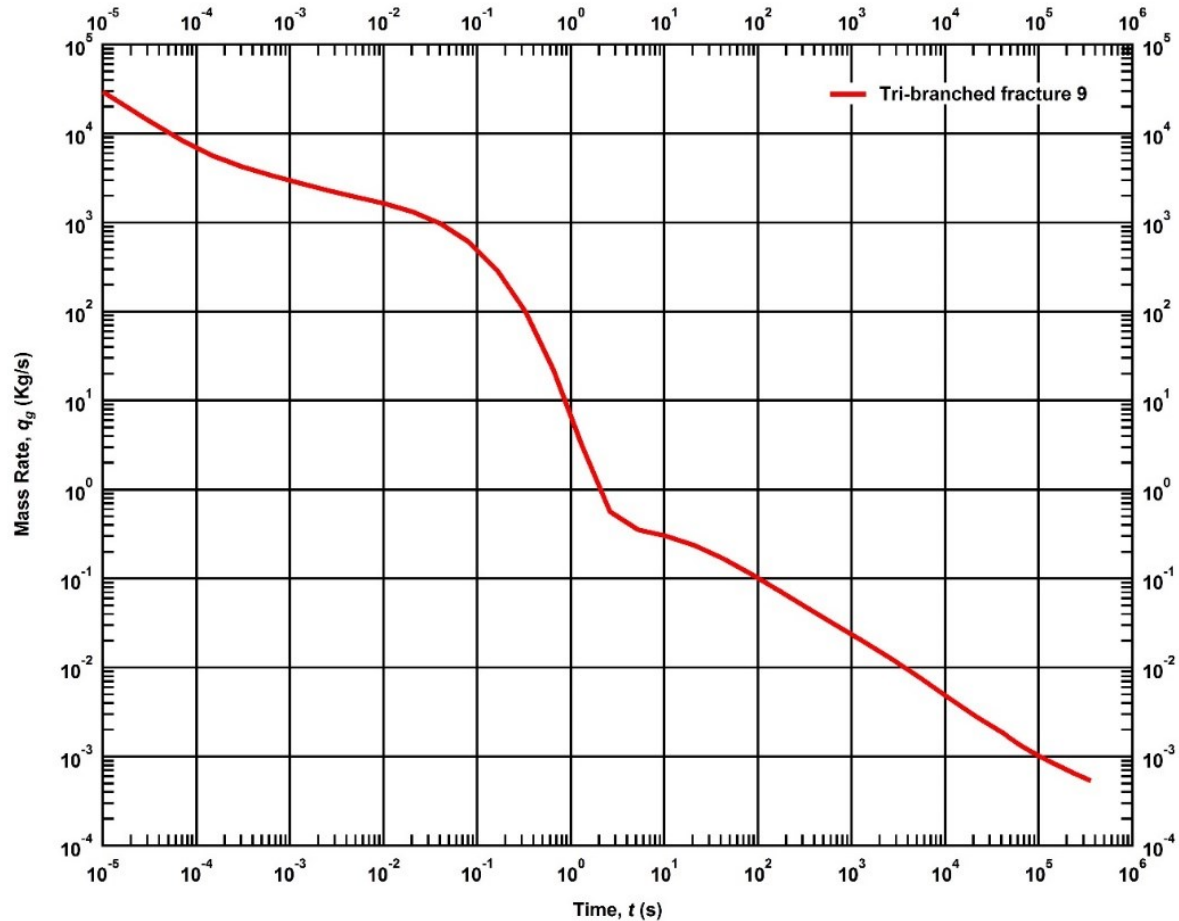

Figure B. 53 - Mass rate evolution for a shale gas reservoir produced from the tri-branched fracture 9 until 100 hours of production. 


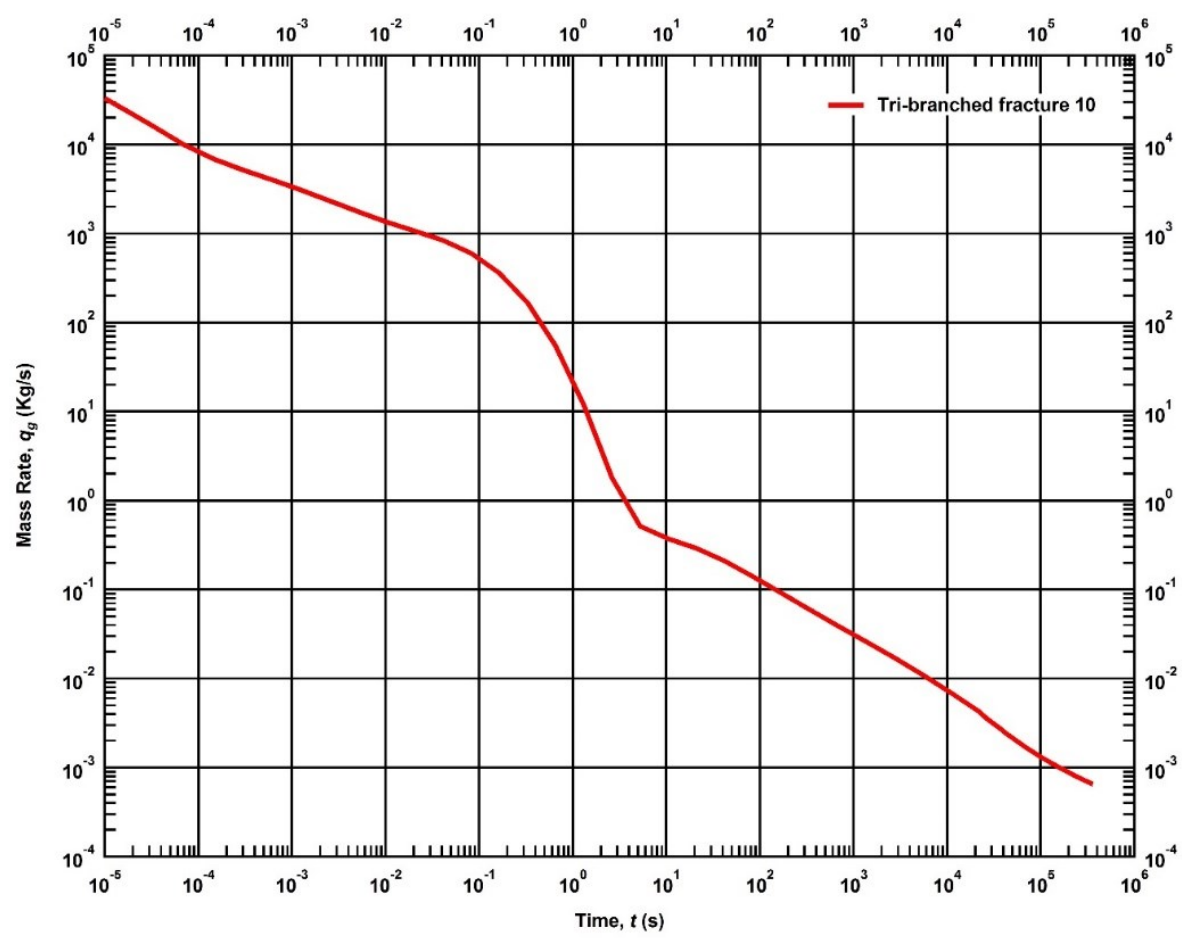

Figure B. 54 - Mass rate evolution for a shale gas reservoir produced from the tri-branched fracture 10 until 100 hours of production.

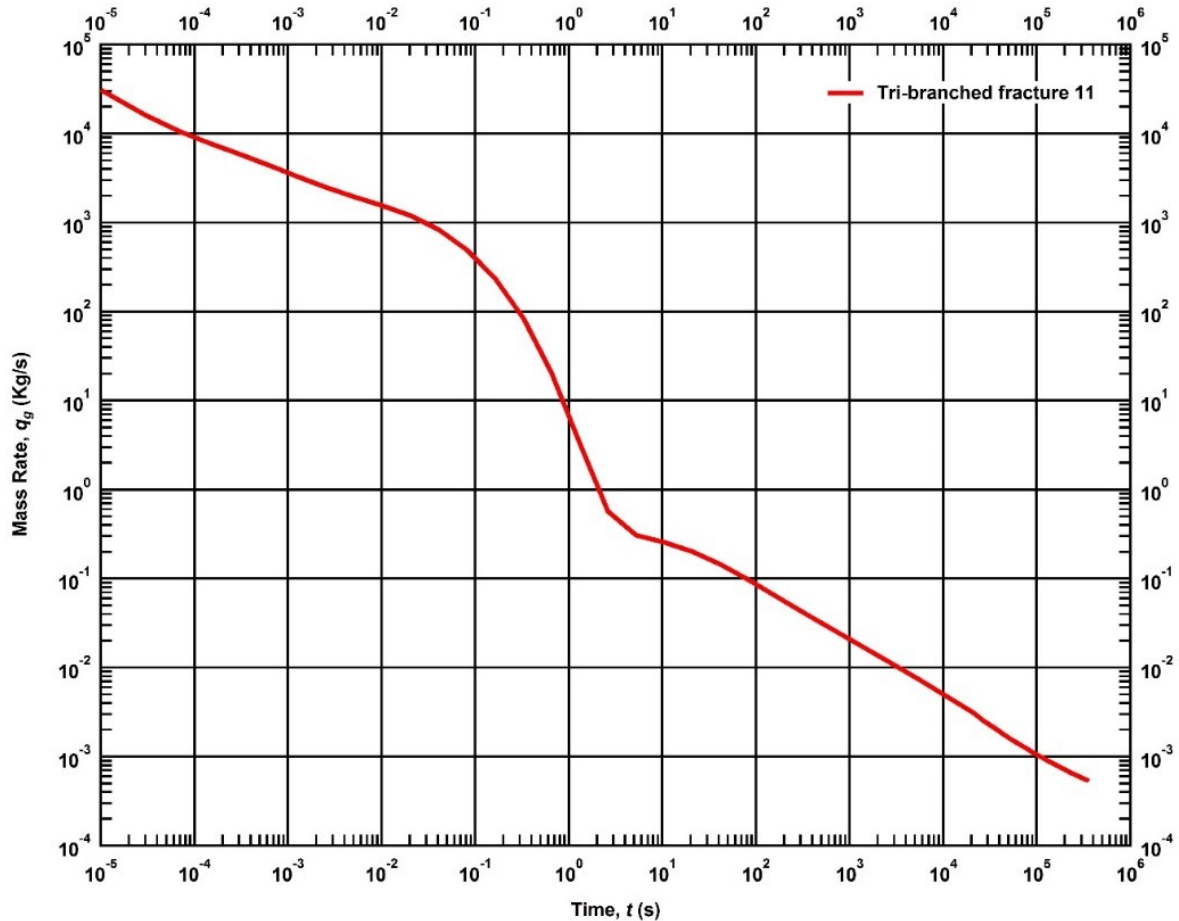

Figure B. 55 - Mass rate evolution for a shale gas reservoir produced from the tri-branched fracture 11 until 100 hours of production. 


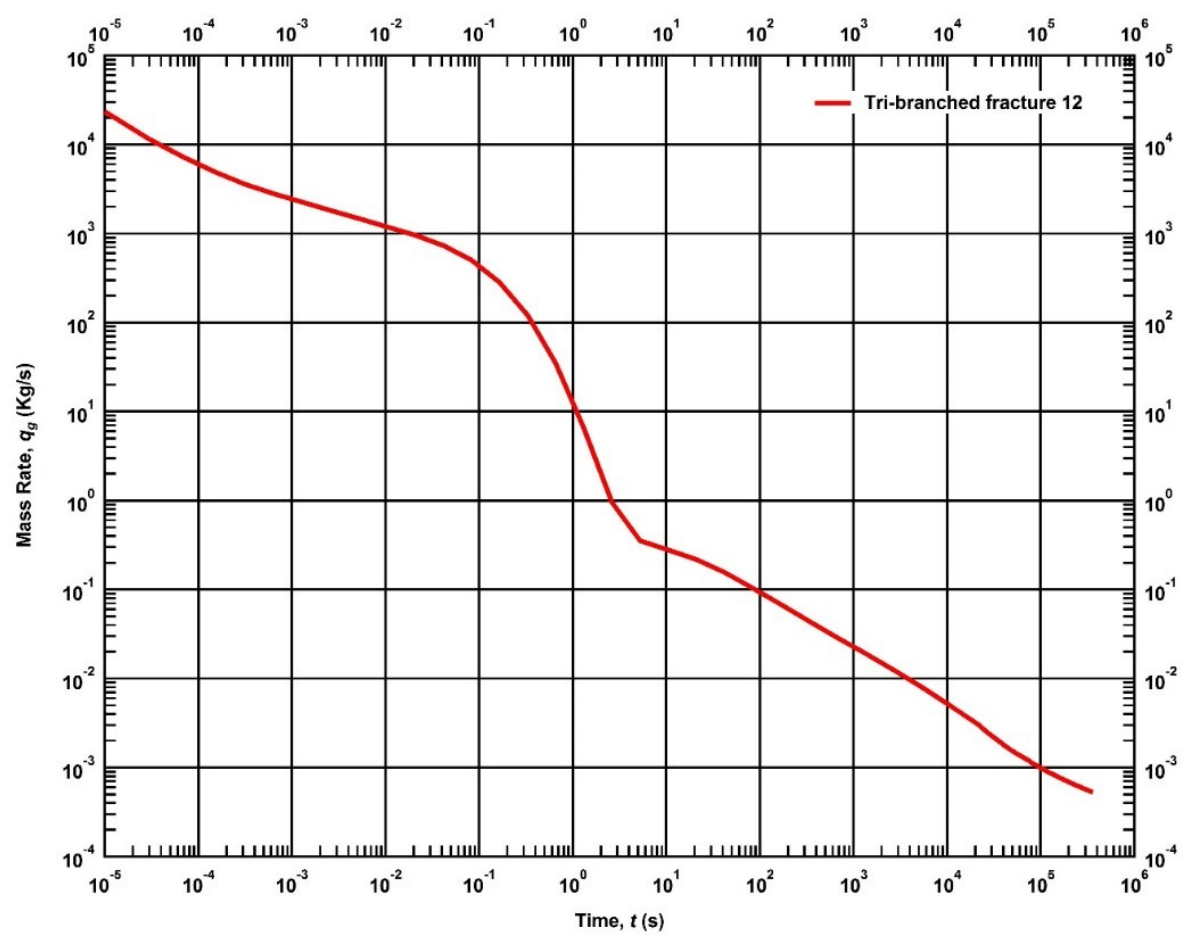

Figure B. 56 - Mass rate evolution for a shale gas reservoir produced from the tri-branched fracture 12 until 100 hours of production.

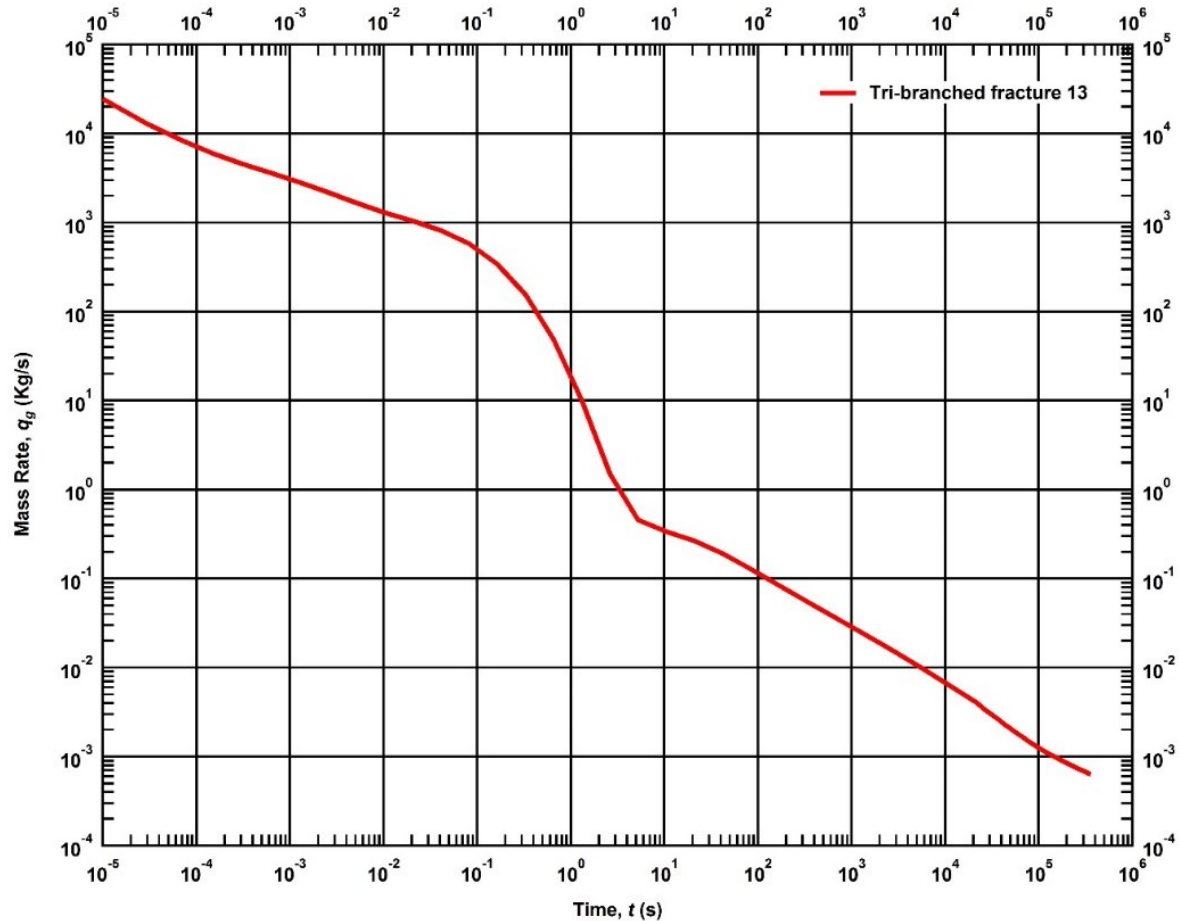

Figure B. 57 - Mass rate evolution for a shale gas reservoir produced from the tri-branched fracture 13 until 100 hours of production. 


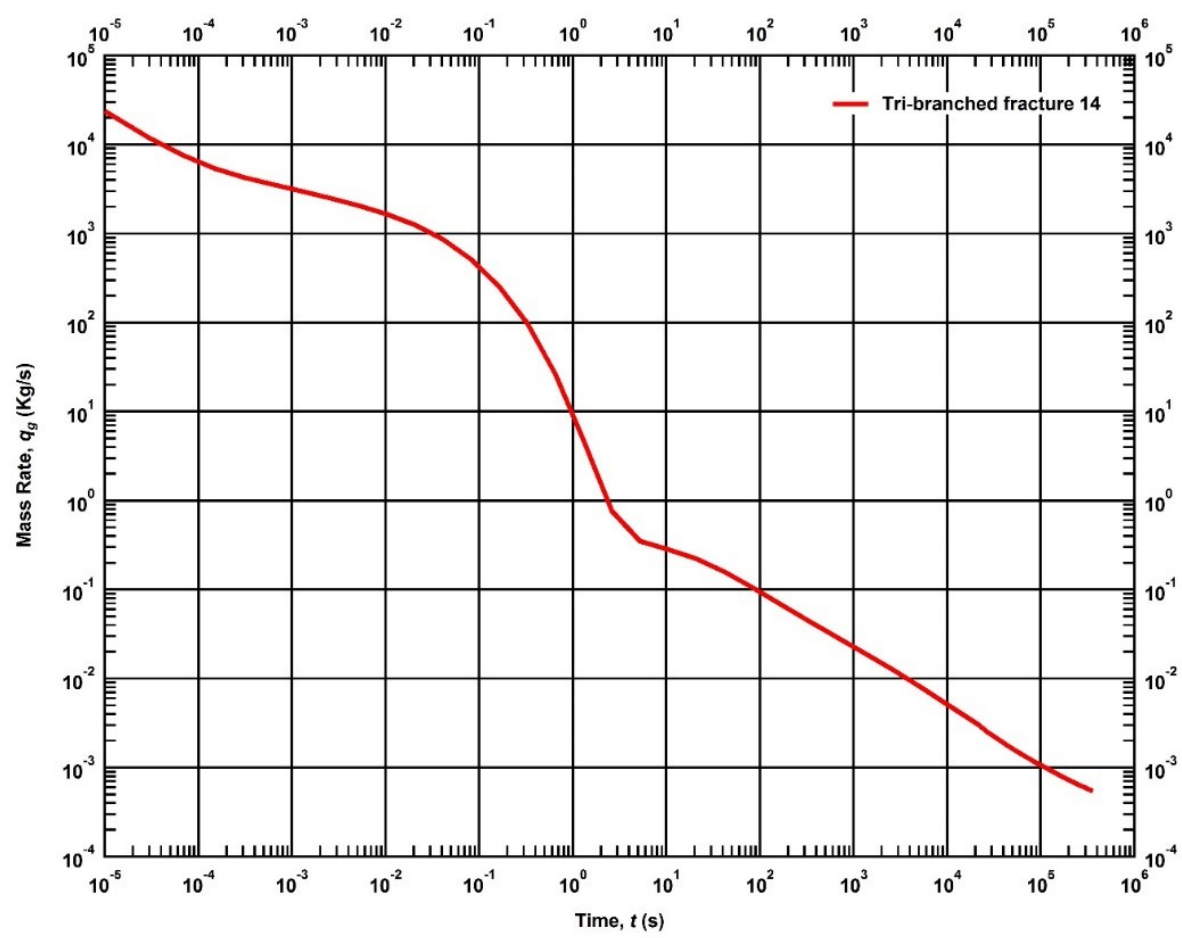

Figure B. 58 - Mass rate evolution for a shale gas reservoir produced from the tri-branched fracture 14 until 100 hours of production.

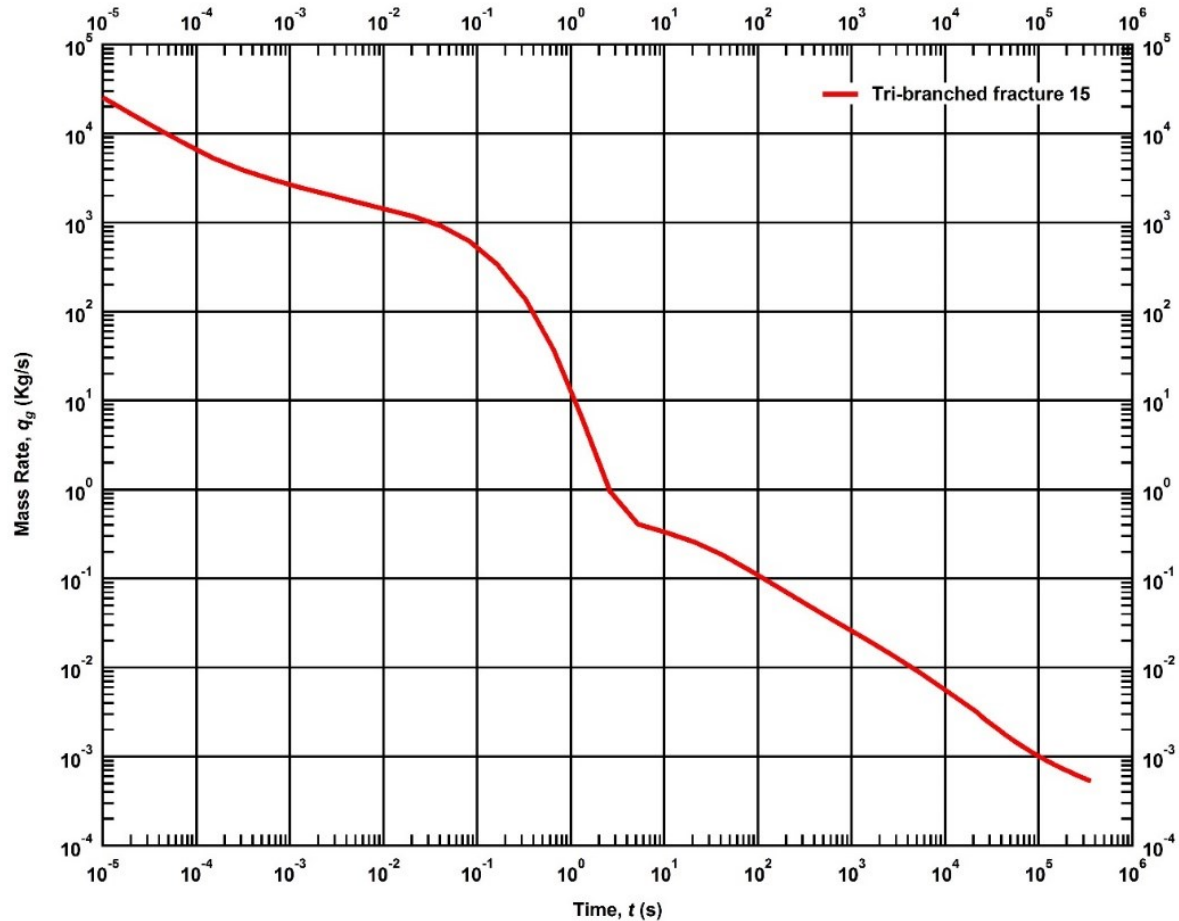

Figure B. 59 - Mass rate evolution for a shale gas reservoir produced from the tri-branched fracture 15 until 100 hours of production. 


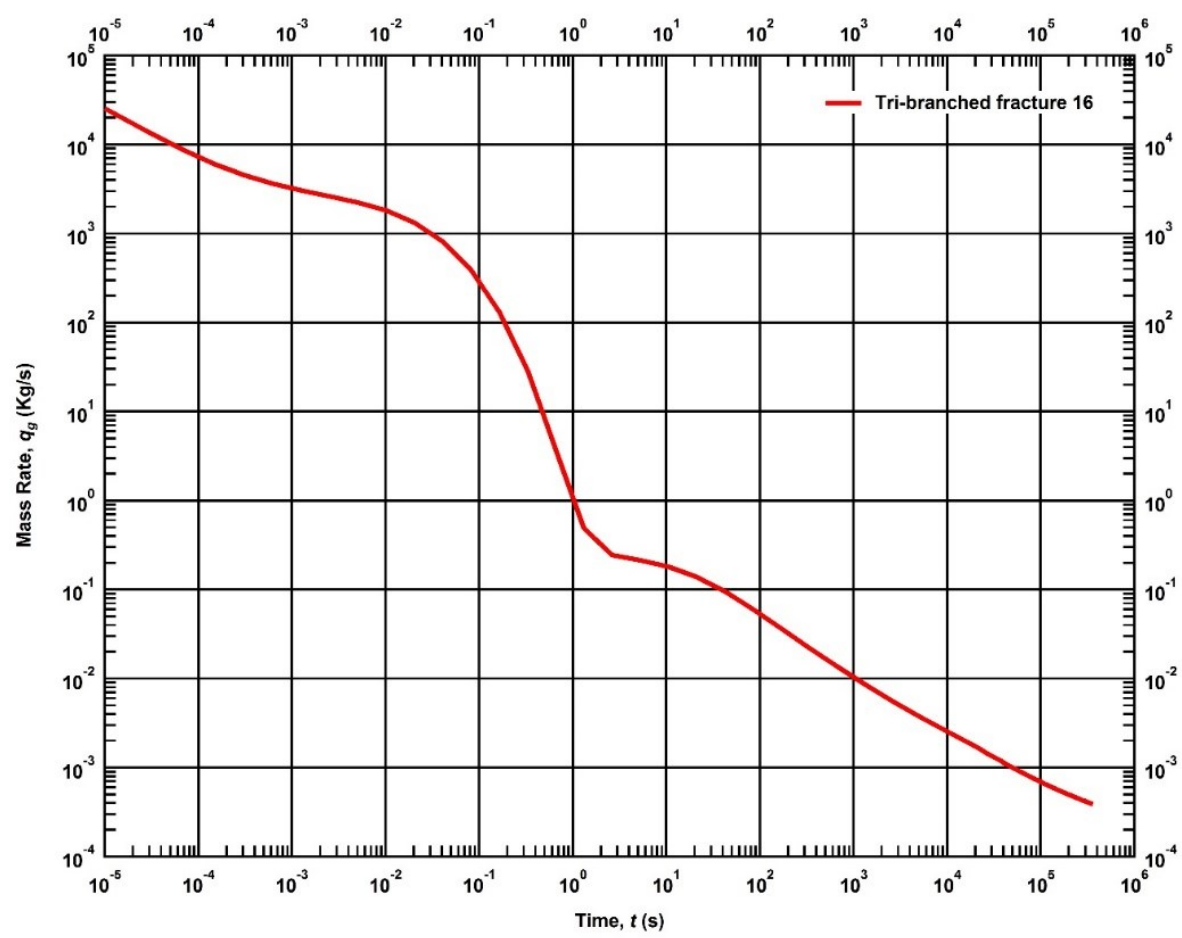

Figure B. 60 - Mass rate evolution for a shale gas reservoir produced from the tri-branched fracture 16 until 100 hours of production.

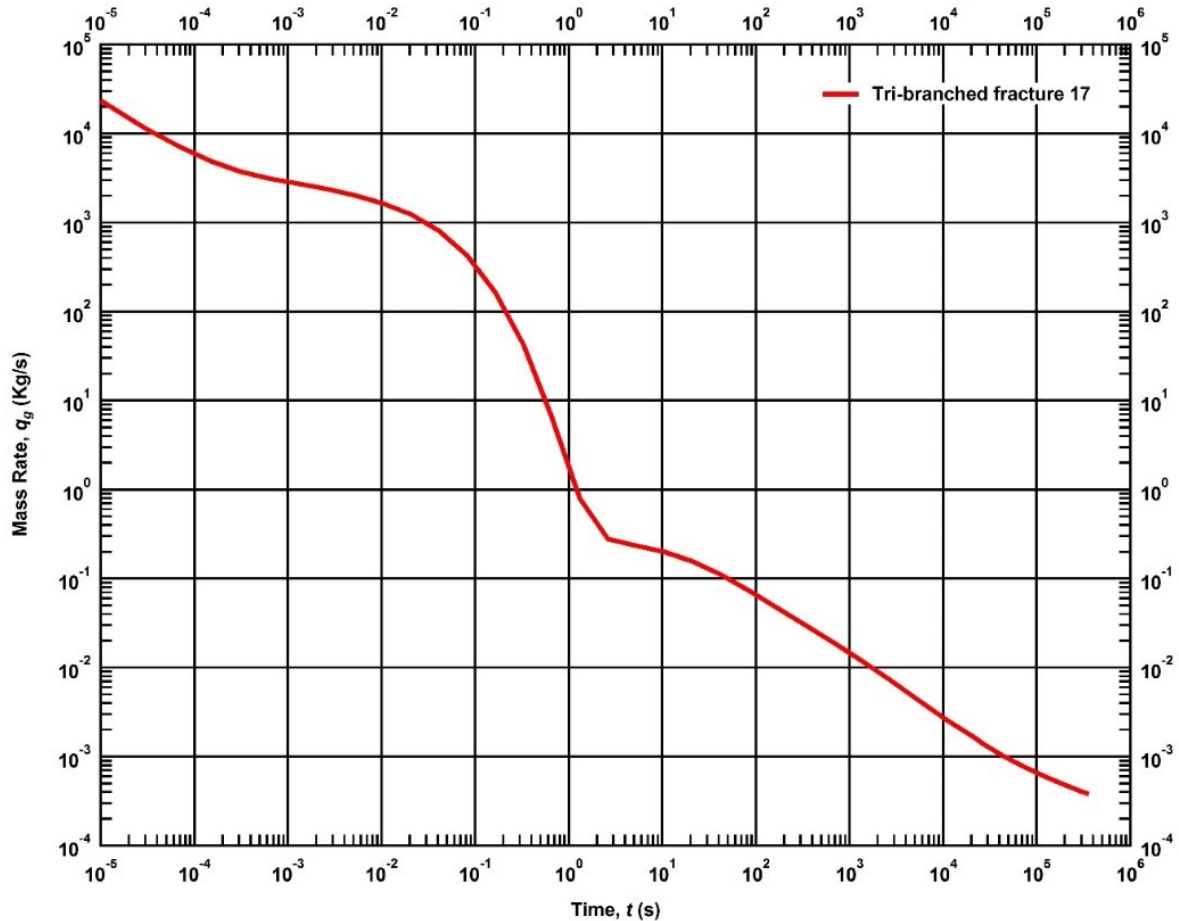

Figure B. 61 - Mass rate evolution for a shale gas reservoir produced from the tri-branched fracture 17 until 100 hours of production. 


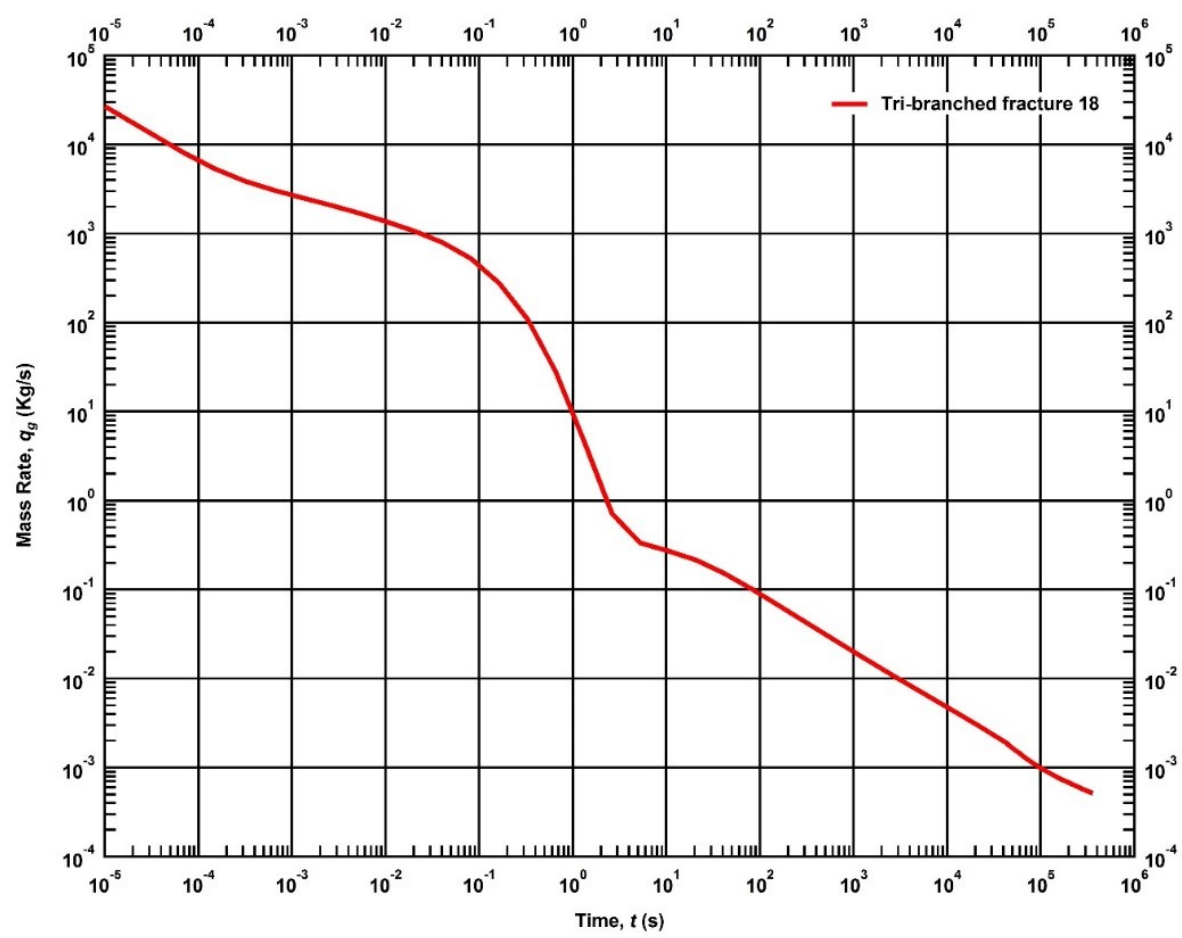

Figure B. 62 - Mass rate evolution for a shale gas reservoir produced from the tri-branched fracture 18 until 100 hours of production.

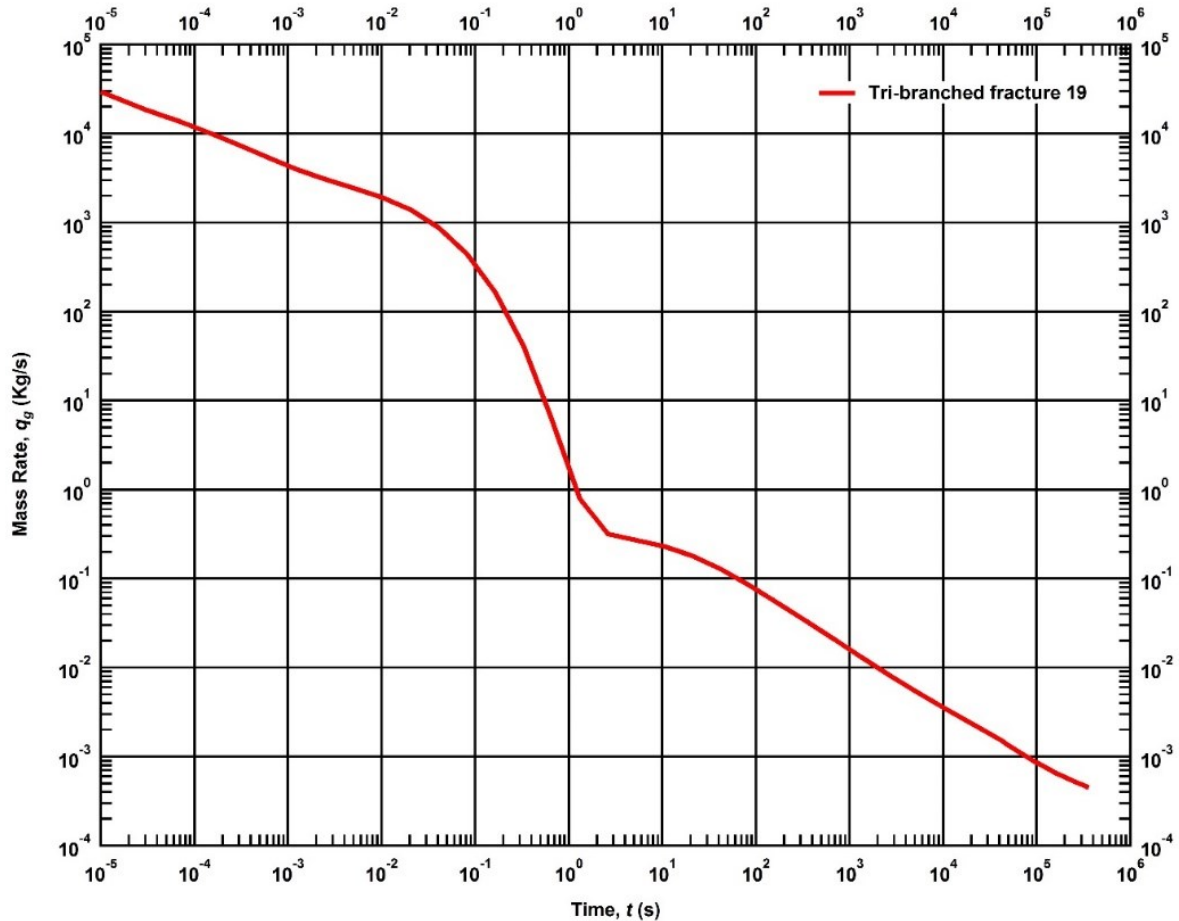

Figure B. 63 - Mass rate evolution for a shale gas reservoir produced from the tri-branched fracture 19 until 100 hours of production. 


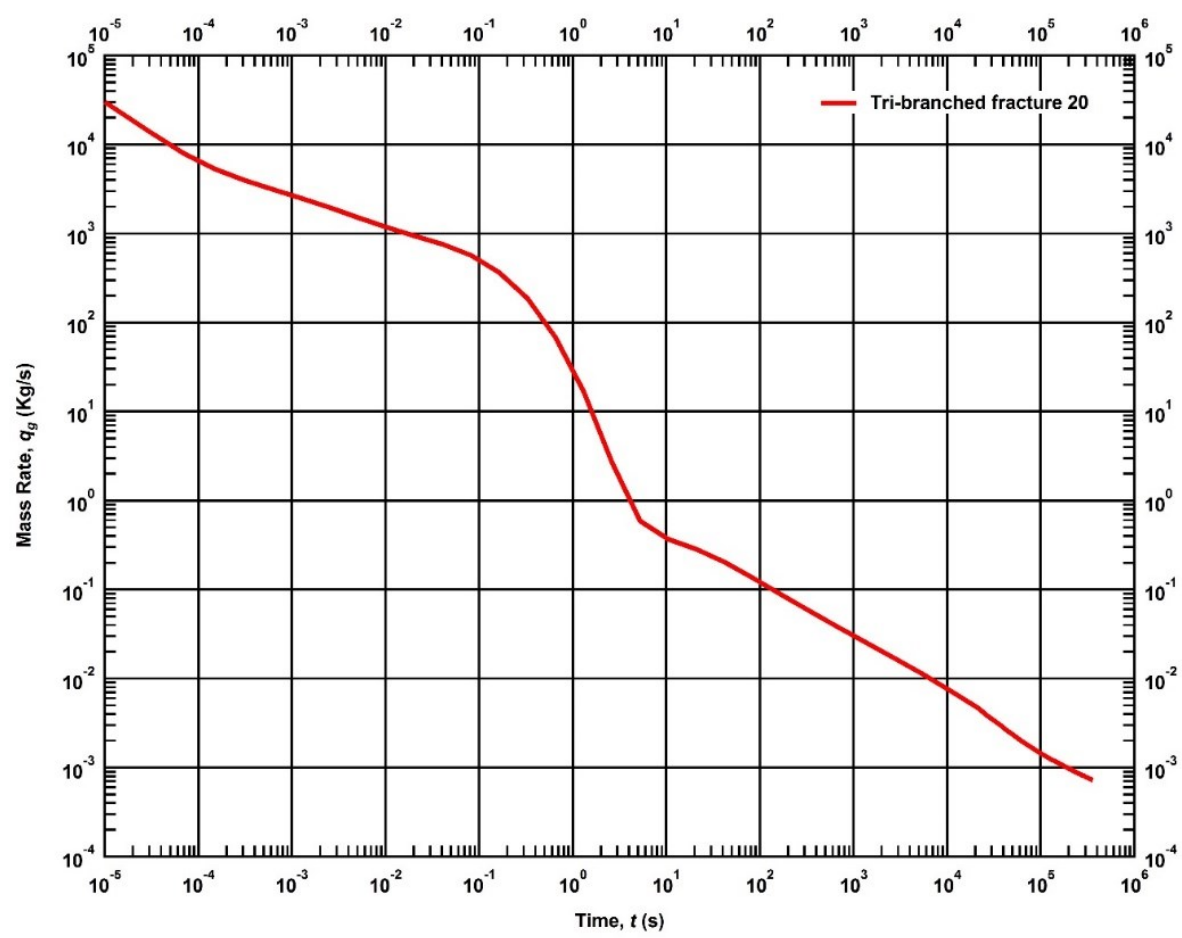

Figure B. 64 - Mass rate evolution for a shale gas reservoir produced from the tri-branched fracture 20 until 100 hours of production.

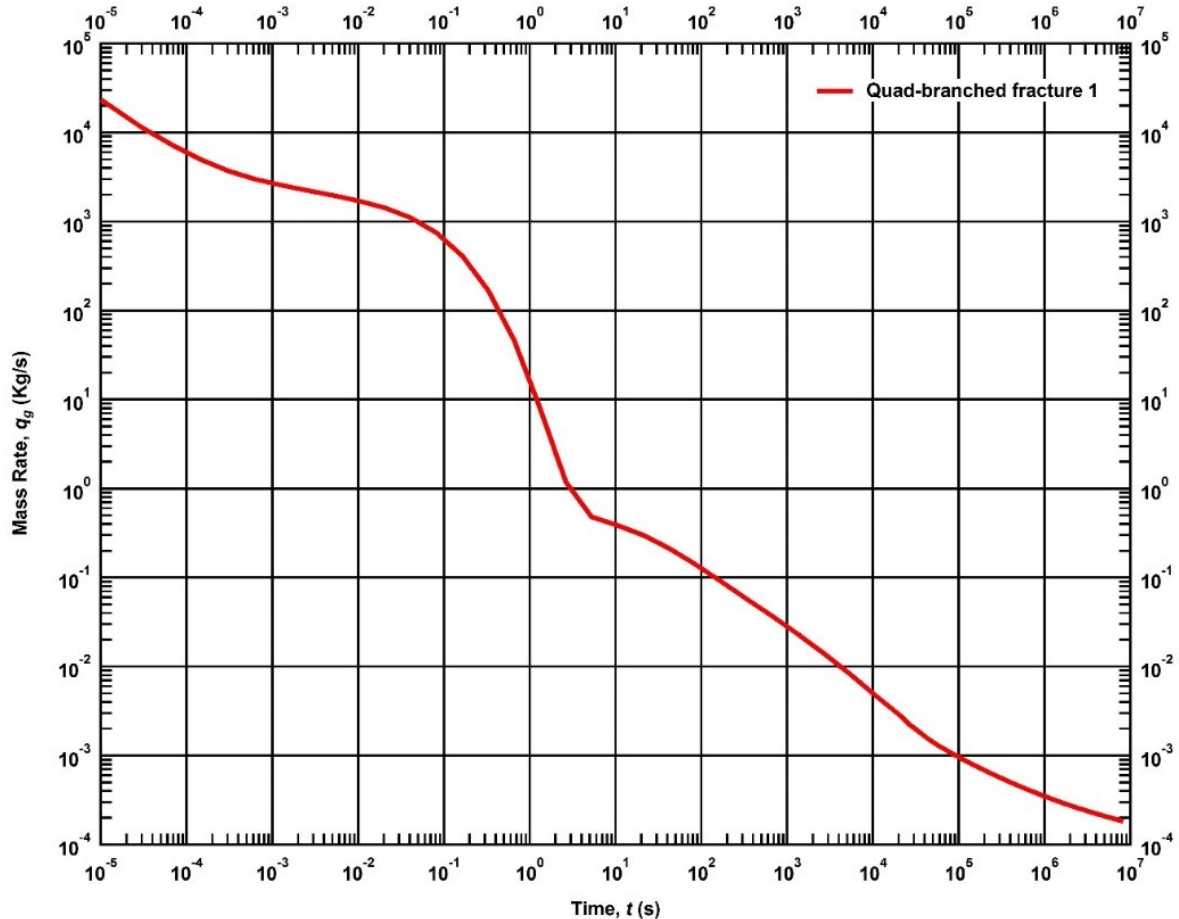

Figure B. 65 - Mass rate evolution for a shale gas reservoir produced from the quad-branched fracture 1 until 100 days of production. 


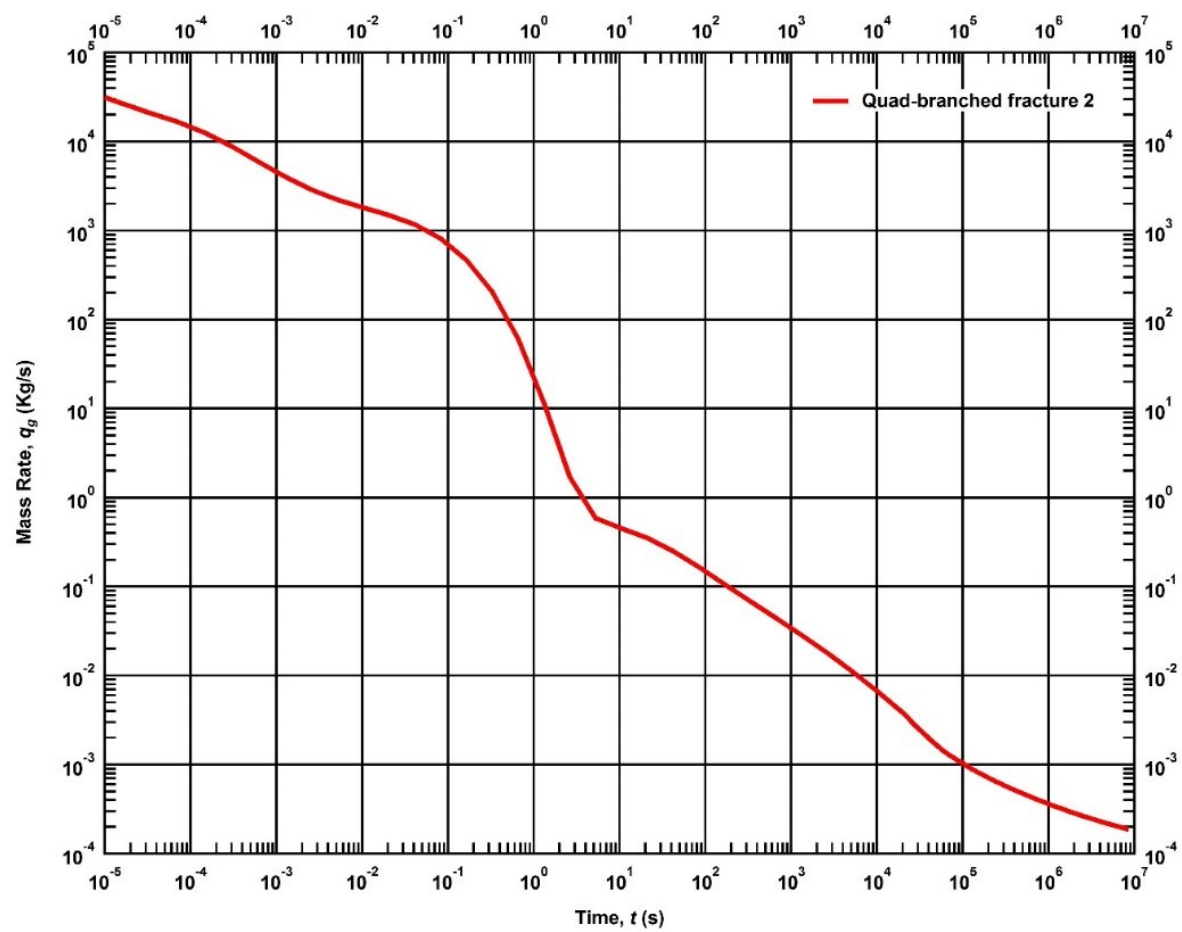

Figure B. 66 - Mass rate evolution for a shale gas reservoir produced from the quad-branched fracture 2 until 100 days of production.

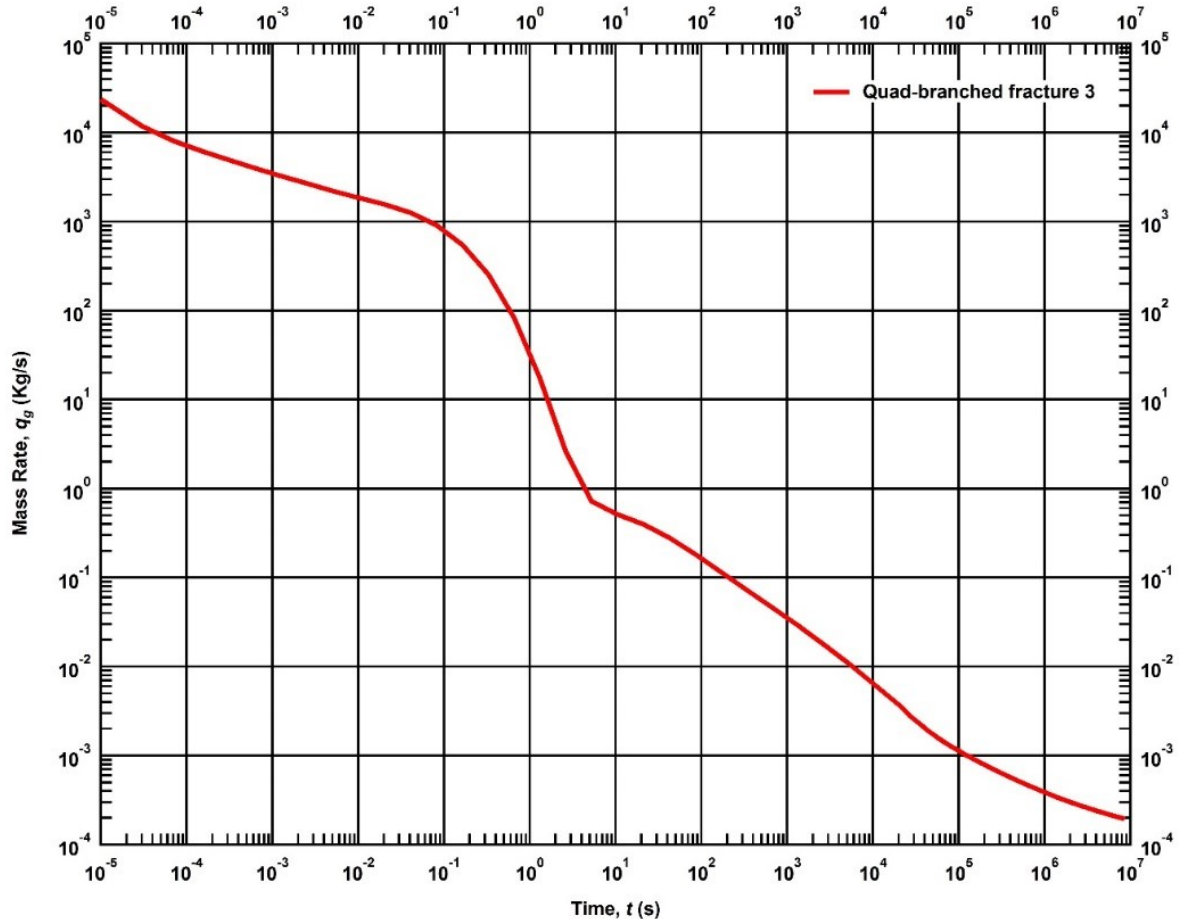

Figure B. 67 - Mass rate evolution for a shale gas reservoir produced from the quad-branched fracture 3 until 100 days of production. 


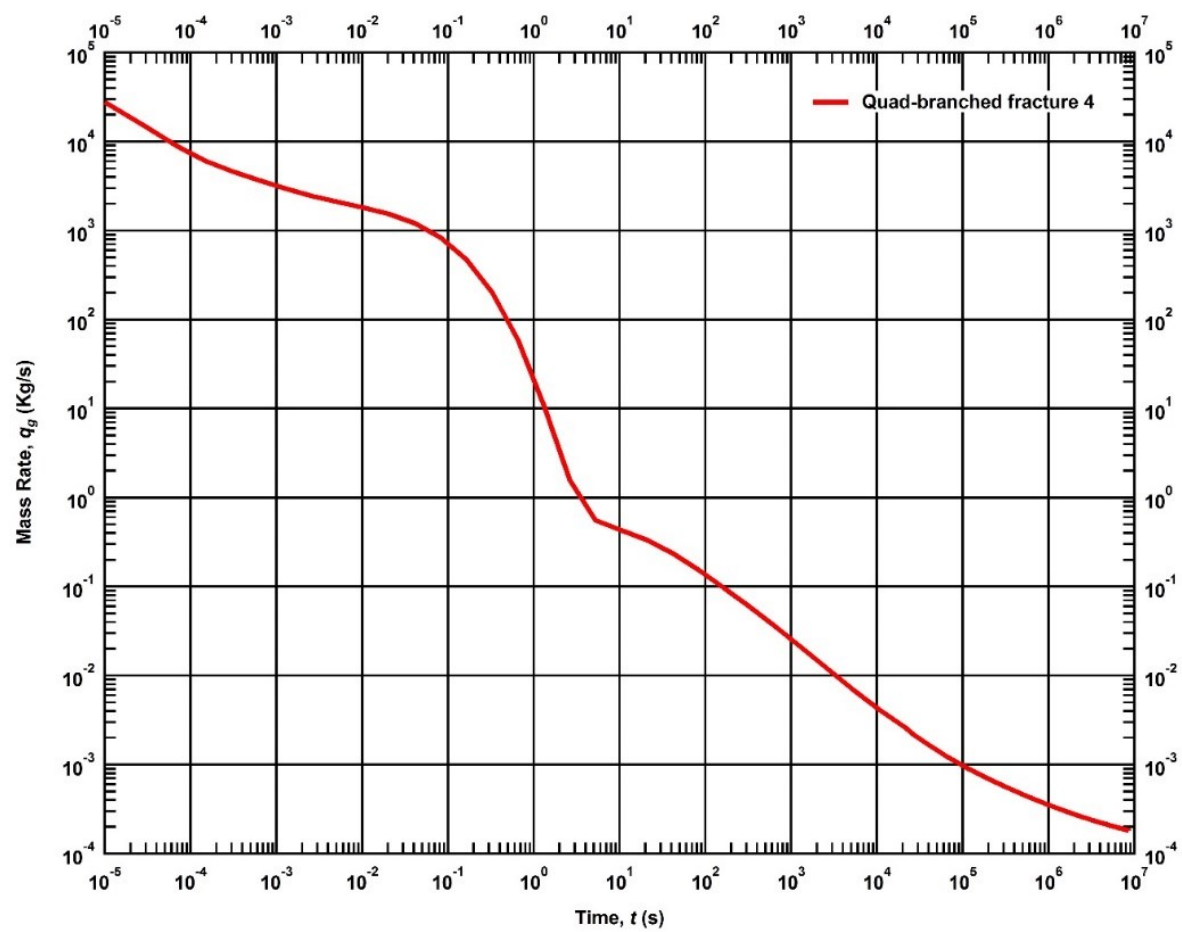

Figure B. 68 - Mass rate evolution for a shale gas reservoir produced from the quad-branched fracture 4 until 100 days of production.

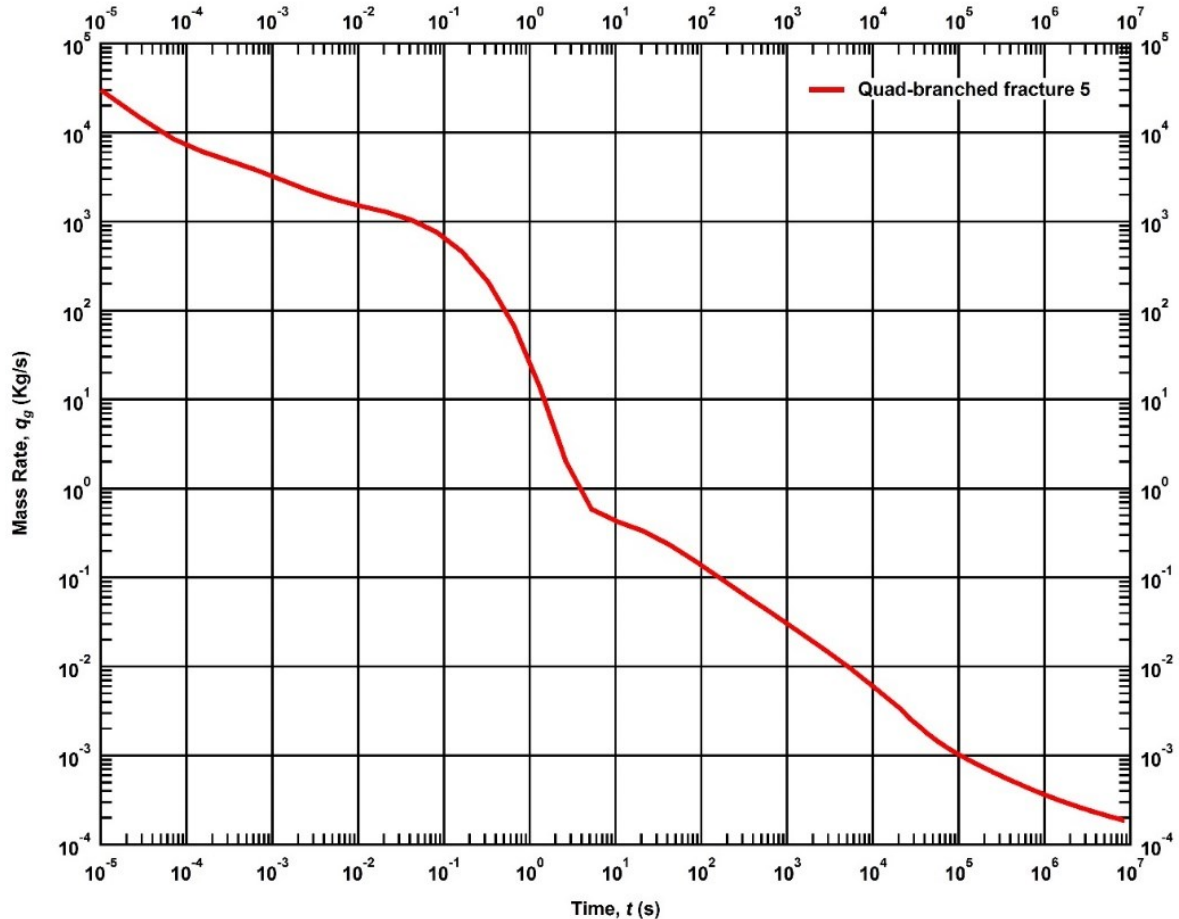

Figure B. 69 - Mass rate evolution for a shale gas reservoir produced from the quad-branched fracture 5 until 100 days of production. 


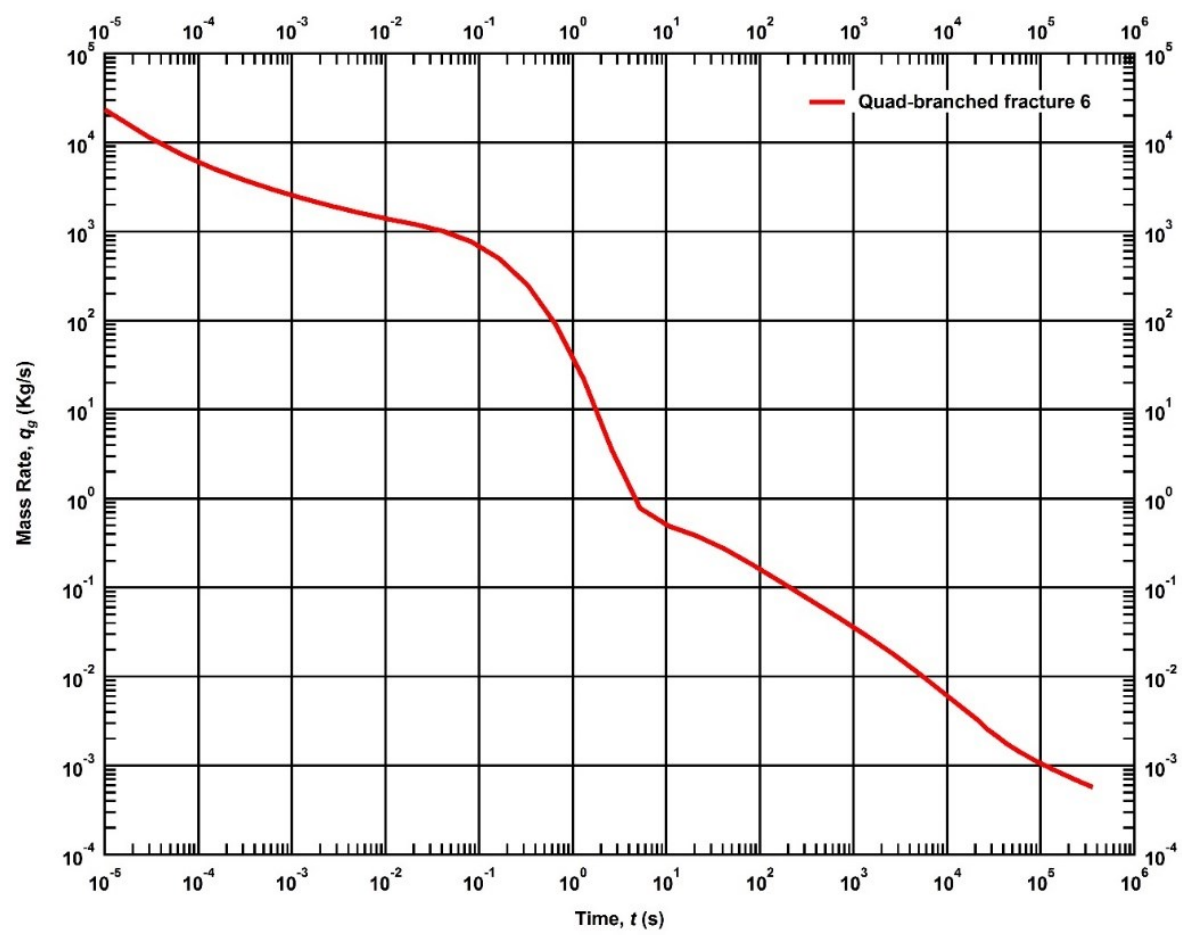

Figure B. 70 - Mass rate evolution for a shale gas reservoir produced from the quad-branched fracture 6 until 100 hours of production.

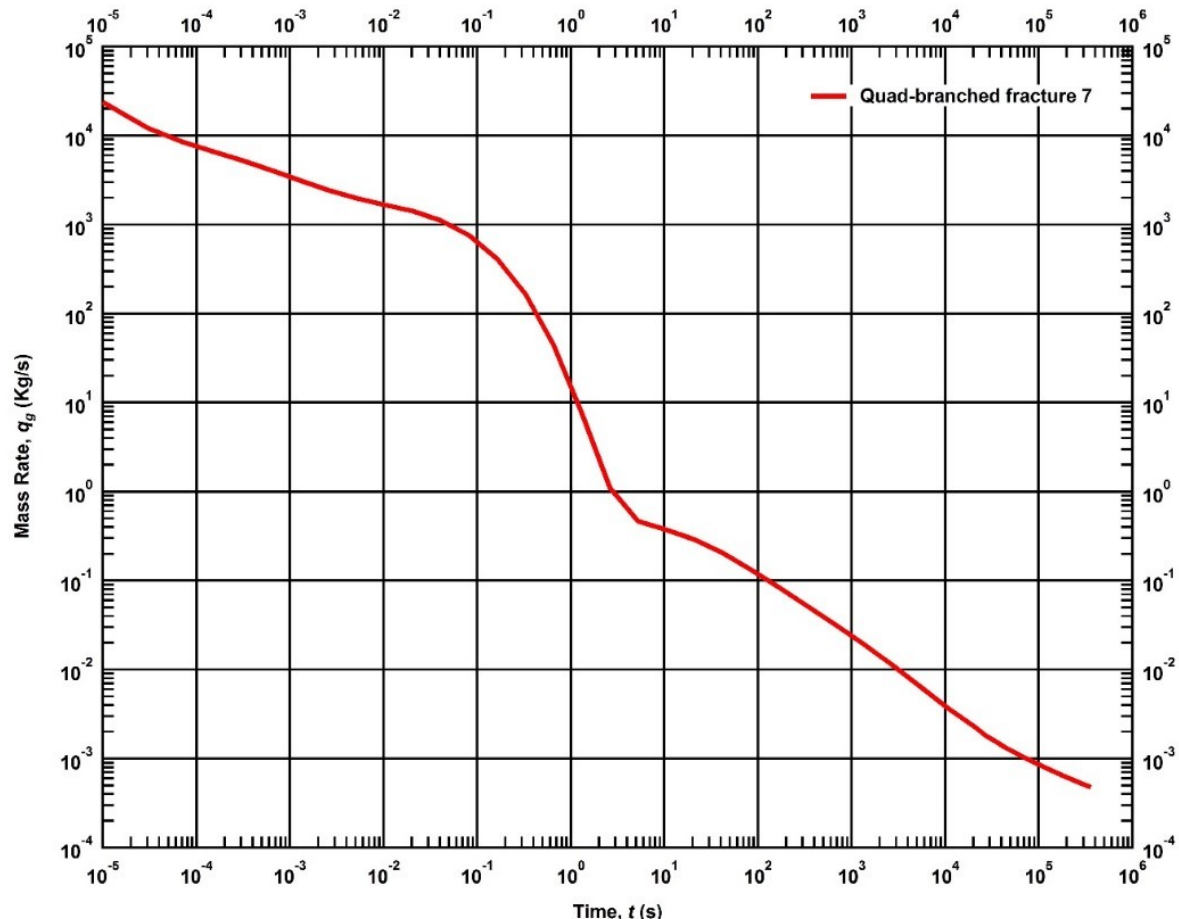

Figure B. 71 - Mass rate evolution for a shale gas reservoir produced from the quad-branched fracture 7 until 100 hours of production. 


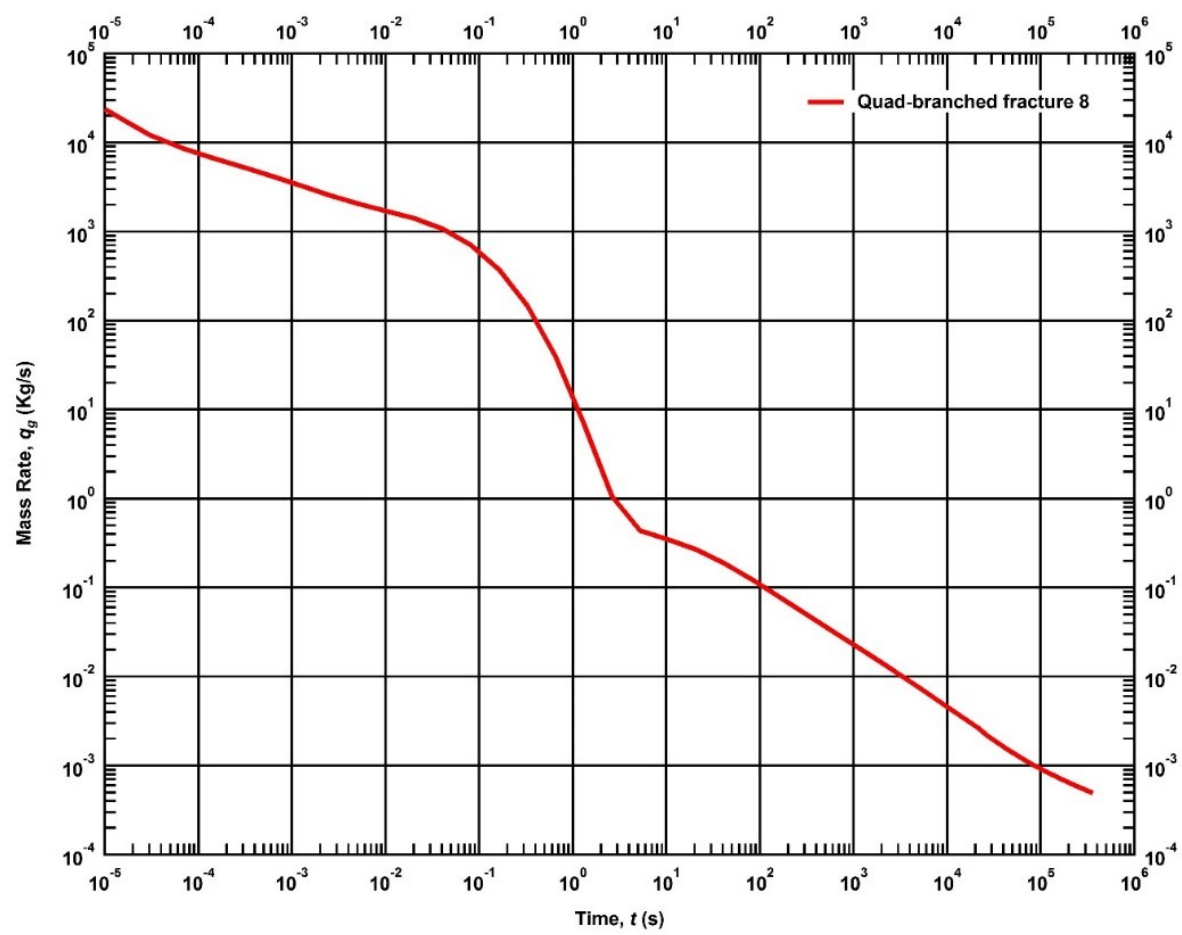

Figure B. 72 - Mass rate evolution for a shale gas reservoir produced from the quad-branched fracture 8 until 100 hours of production.

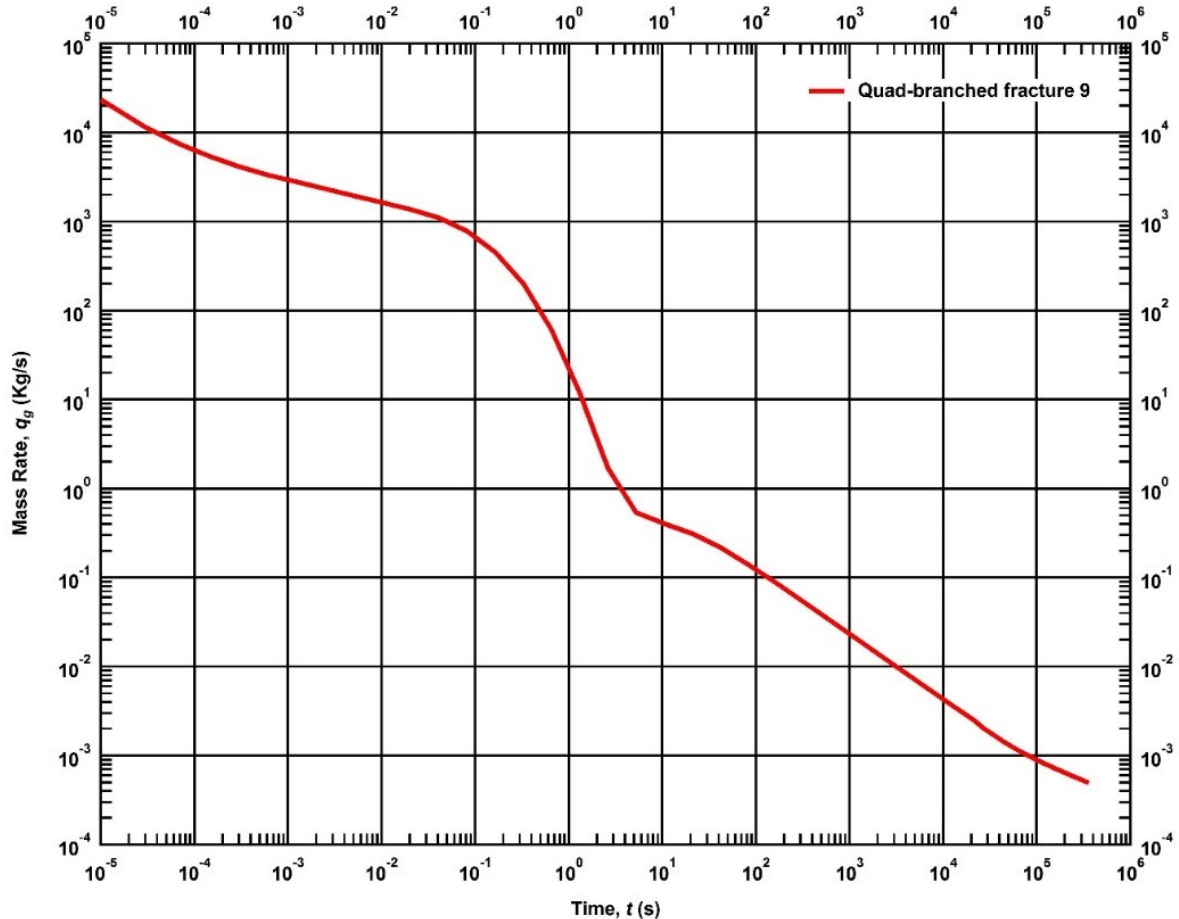

Figure B. 73 - Mass rate evolution for a shale gas reservoir produced from the quad-branched fracture 9 until 100 hours of production. 


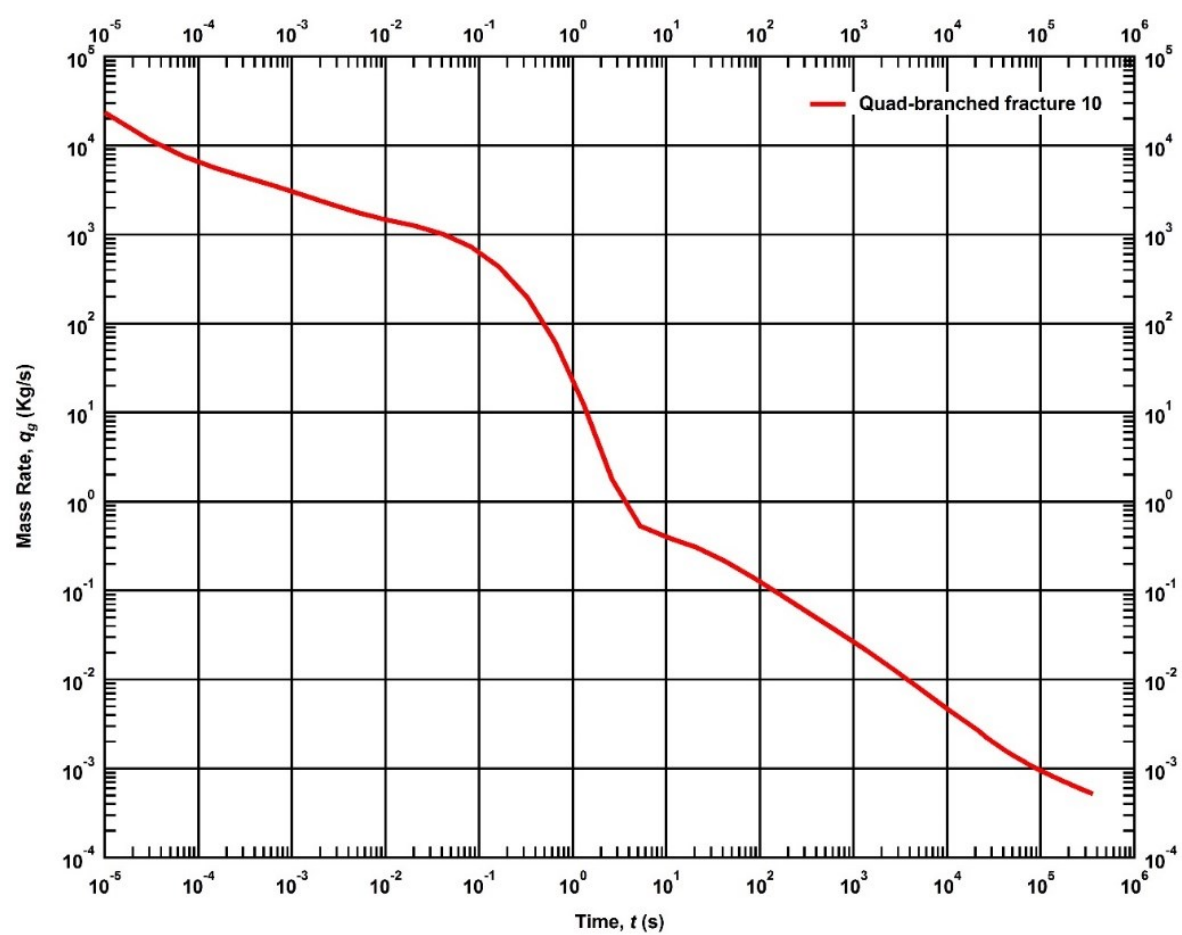

Figure B. 74 - Mass rate evolution for a shale gas reservoir produced from the quad-branched fracture 10 until 100 hours of production.

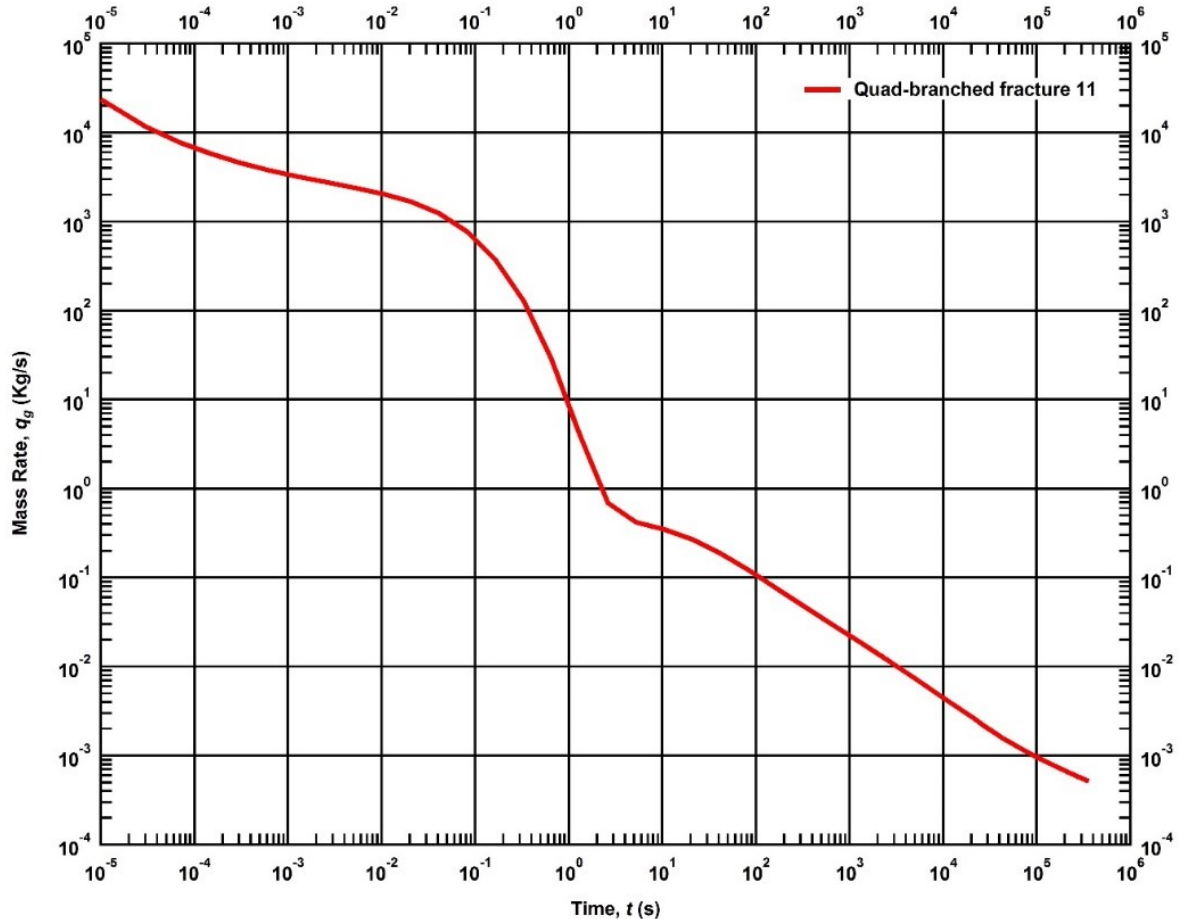

Figure B. 75 - Mass rate evolution for a shale gas reservoir produced from the quad-branched fracture 11 until 100 hours of production. 


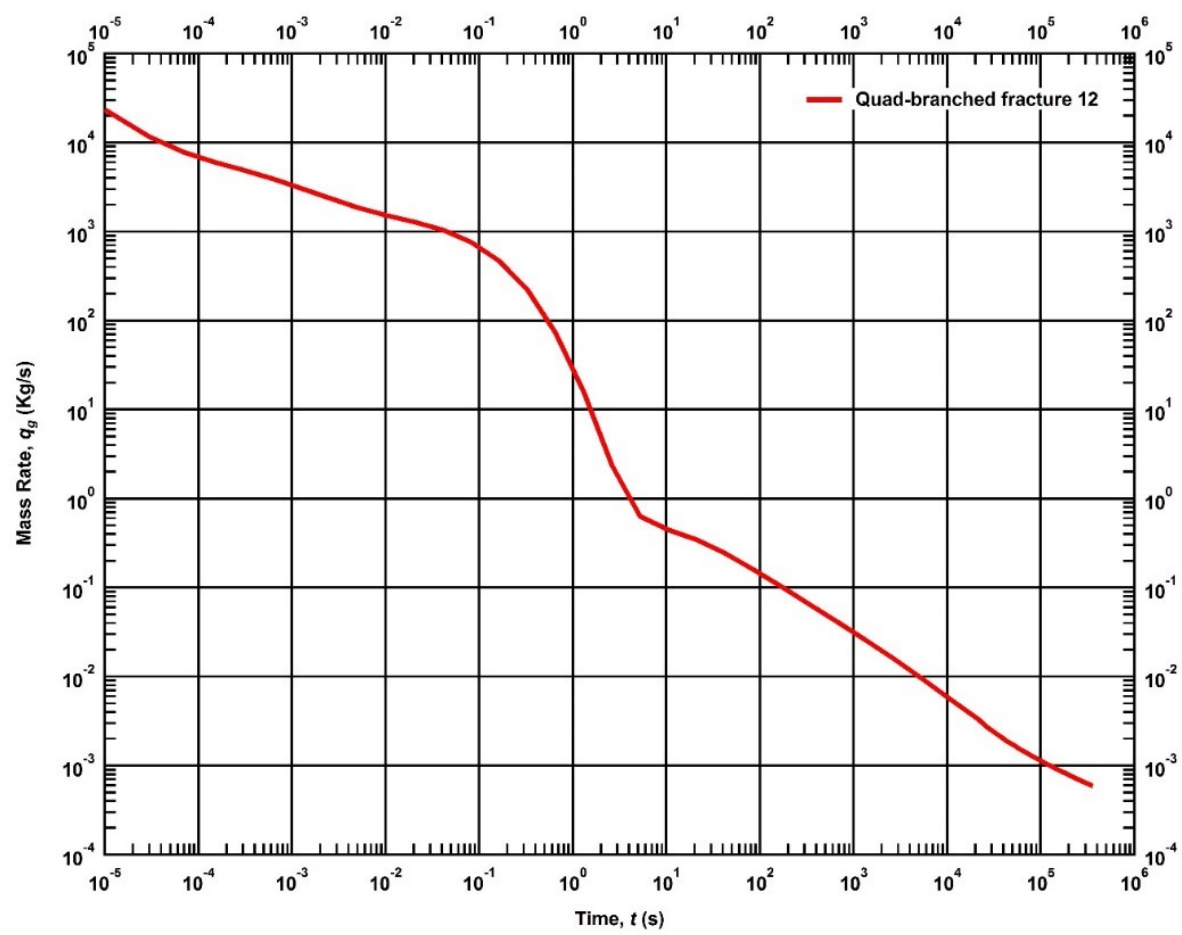

Figure B. 76 - Mass rate evolution for a shale gas reservoir produced from the quad-branched fracture 12 until 100 hours of production.

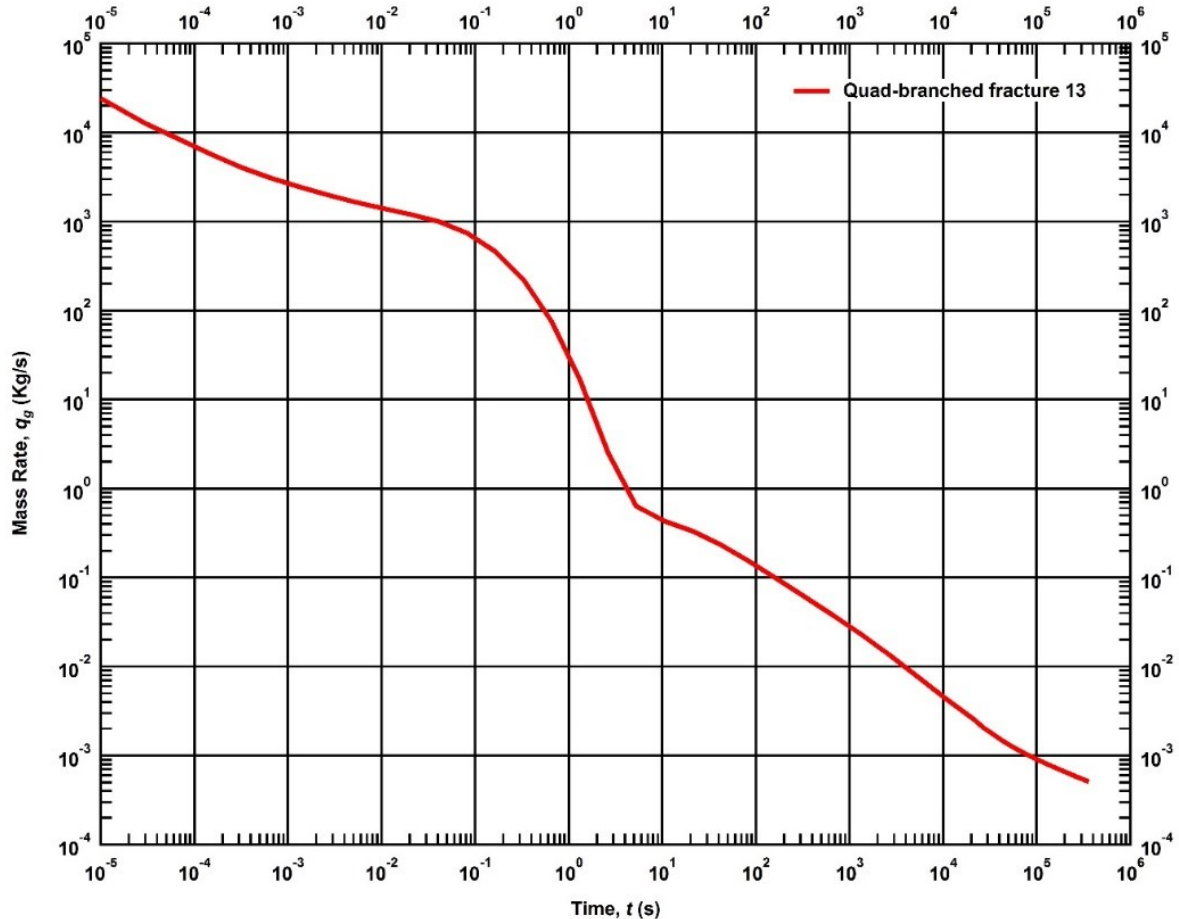

Figure B. 77 - Mass rate evolution for a shale gas reservoir produced from the quad-branched fracture 13 until 100 hours of production. 


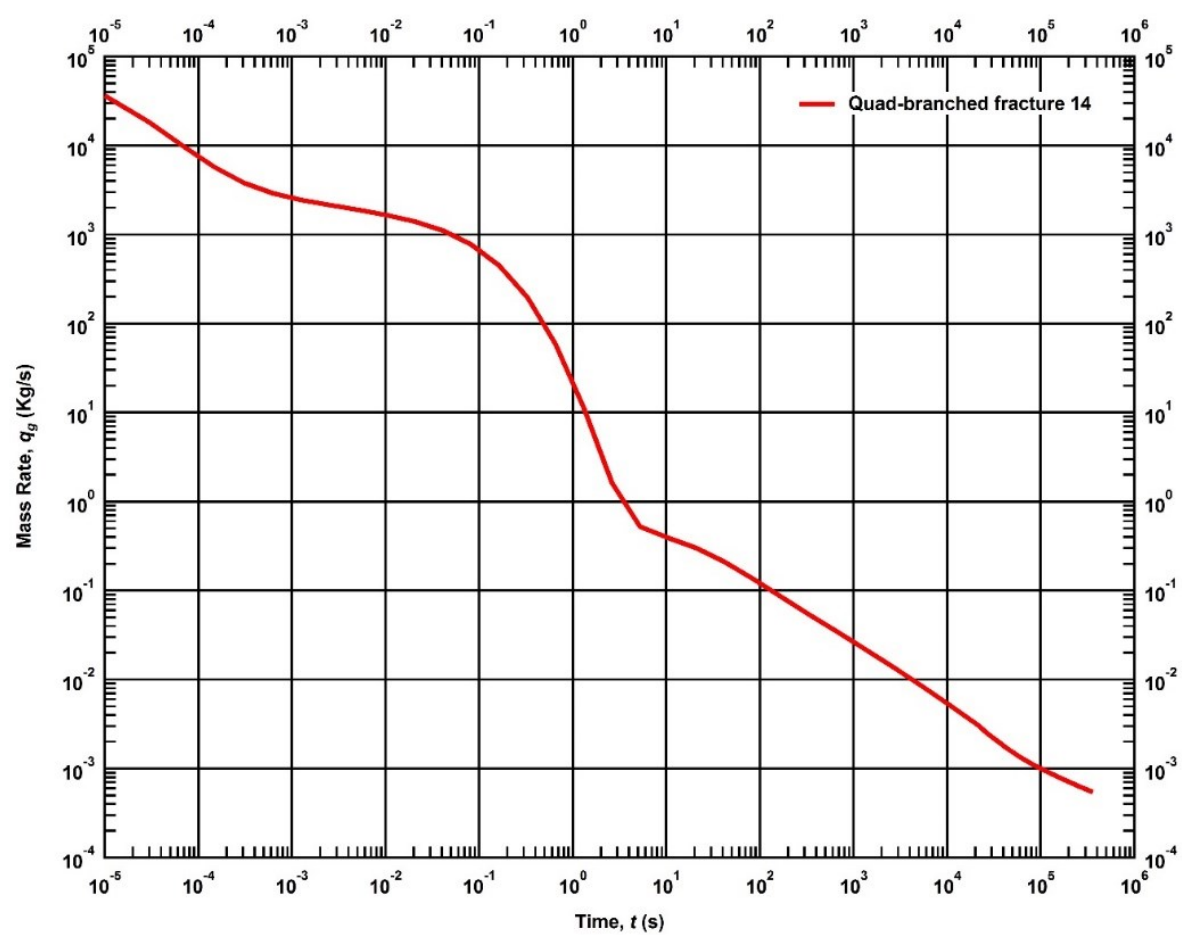

Figure B. 78 - Mass rate evolution for a shale gas reservoir produced from the quad-branched fracture 14 until 100 hours of production.

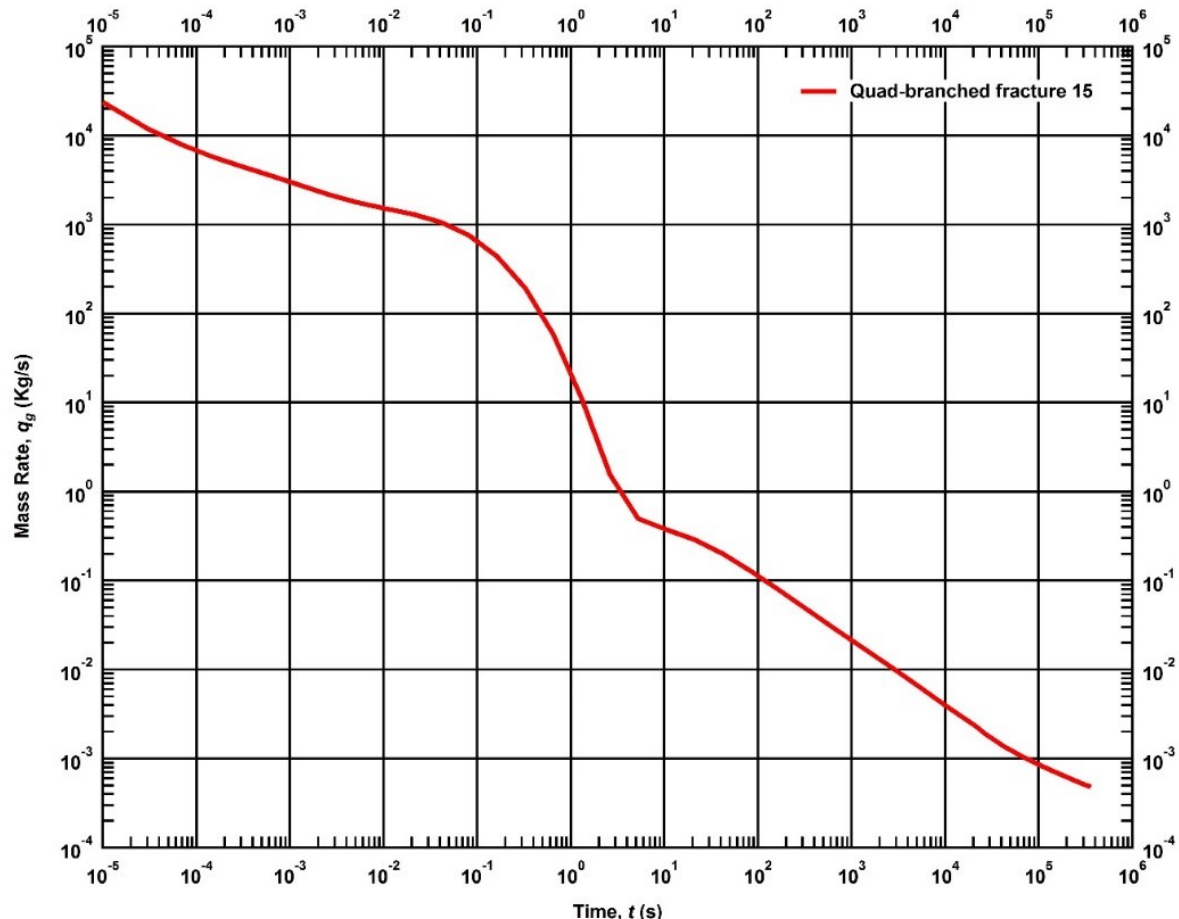

Figure B. 79 - Mass rate evolution for a shale gas reservoir produced from the quad-branched fracture 15 until 100 hours of production. 


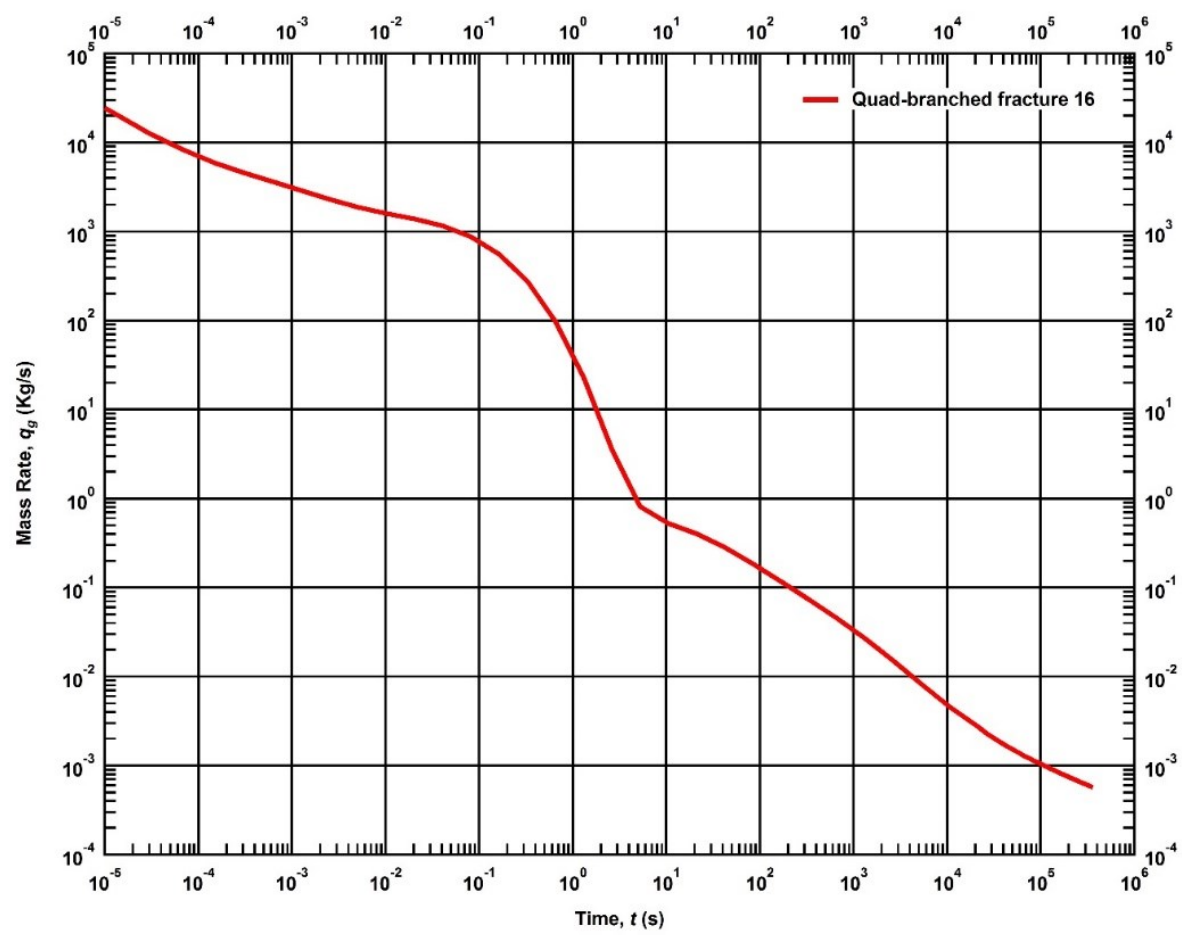

Figure B. 80 - Mass rate evolution for a shale gas reservoir produced from the quad-branched fracture 16 until 100 hours of production.

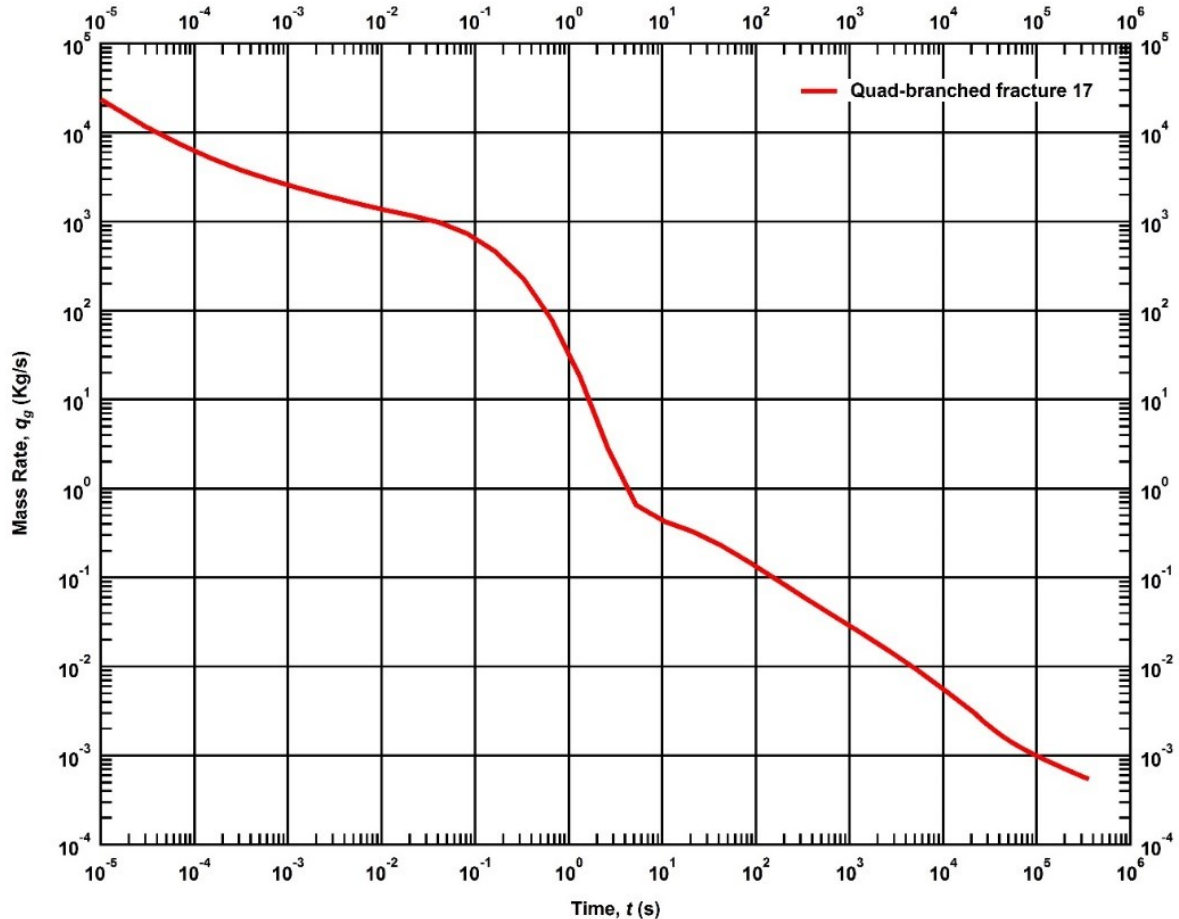

Figure B. 81 - Mass rate evolution for a shale gas reservoir produced from the quad-branched fracture 17 until 100 hours of production. 


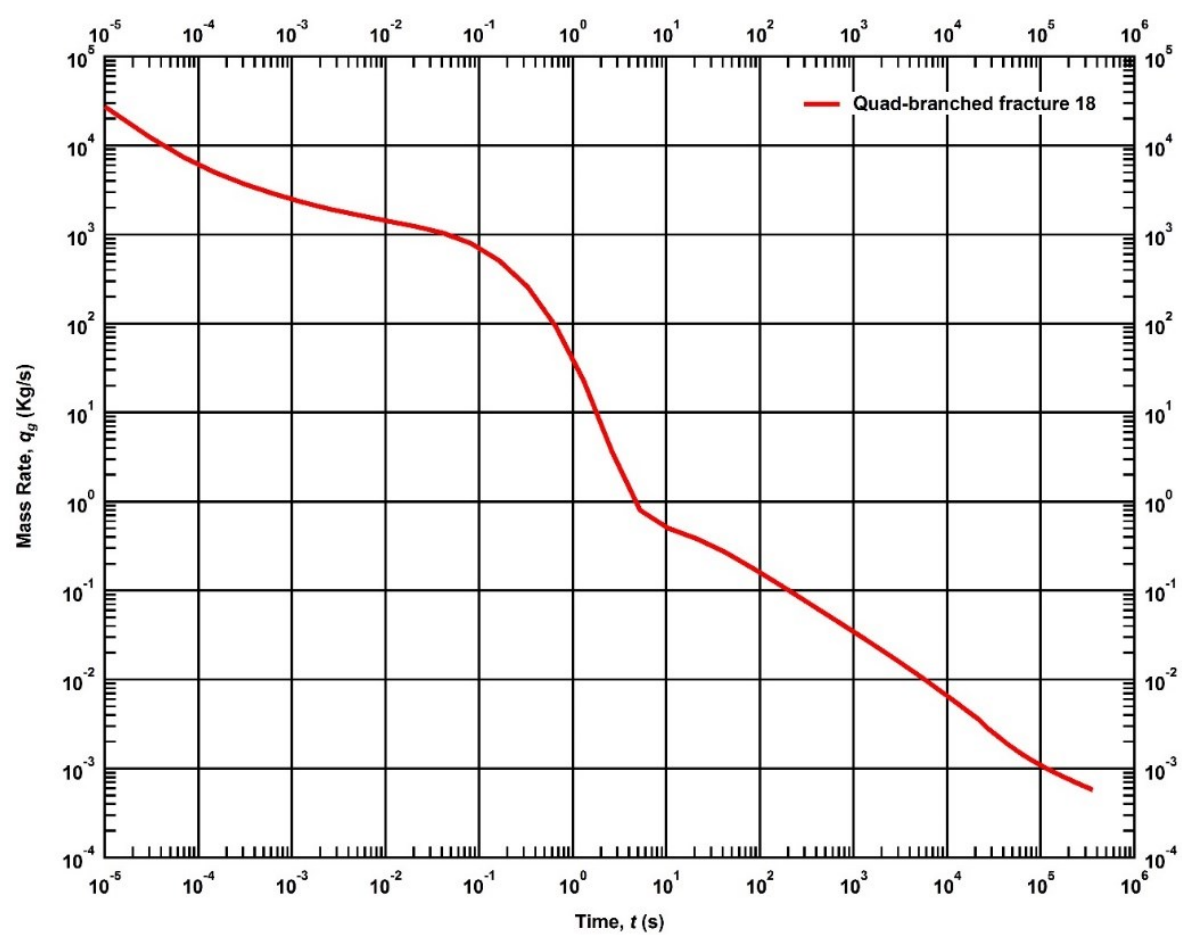

Figure B. 82 - Mass rate evolution for a shale gas reservoir produced from the quad-branched fracture 18 until 100 hours of production.

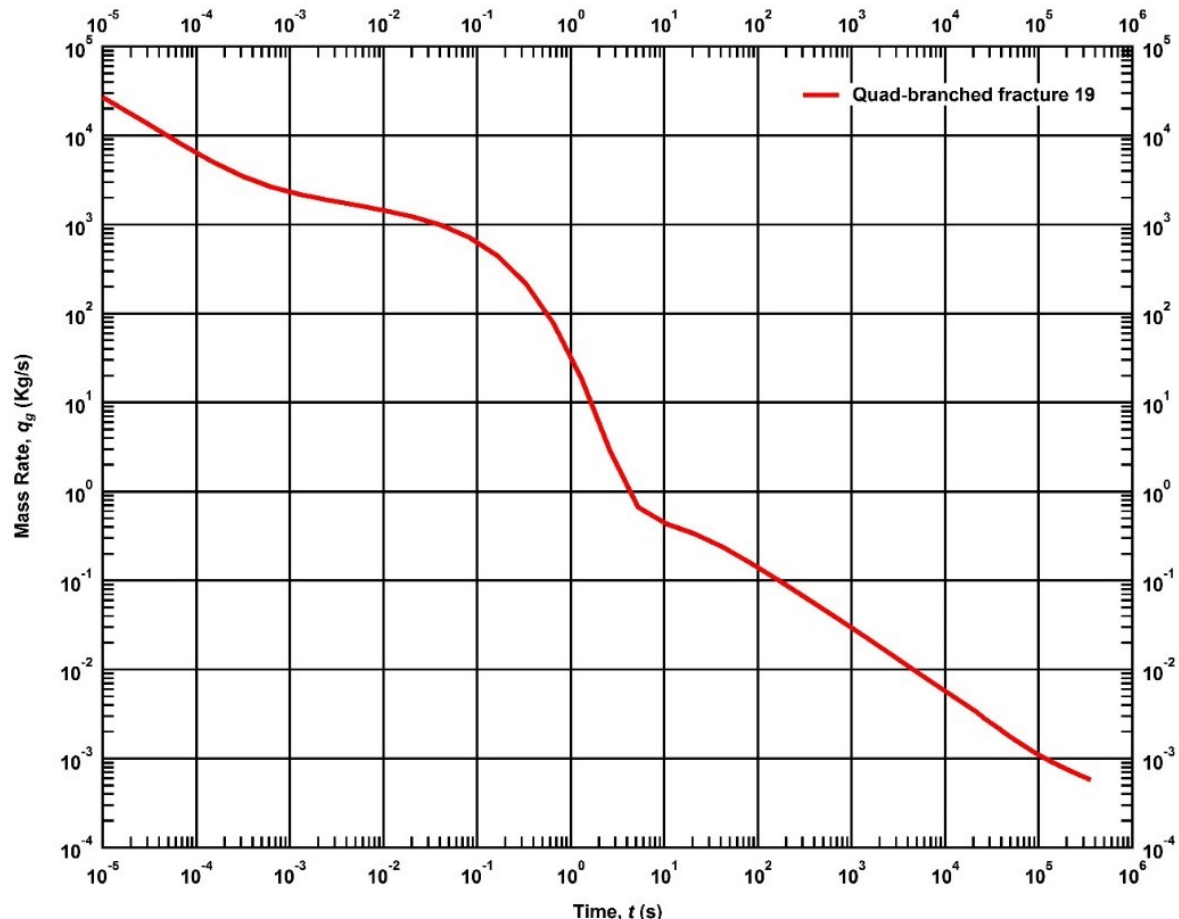

Figure B. 83 - Mass rate evolution for a shale gas reservoir produced from the quad-branched fracture 19 until 100 hours of production. 


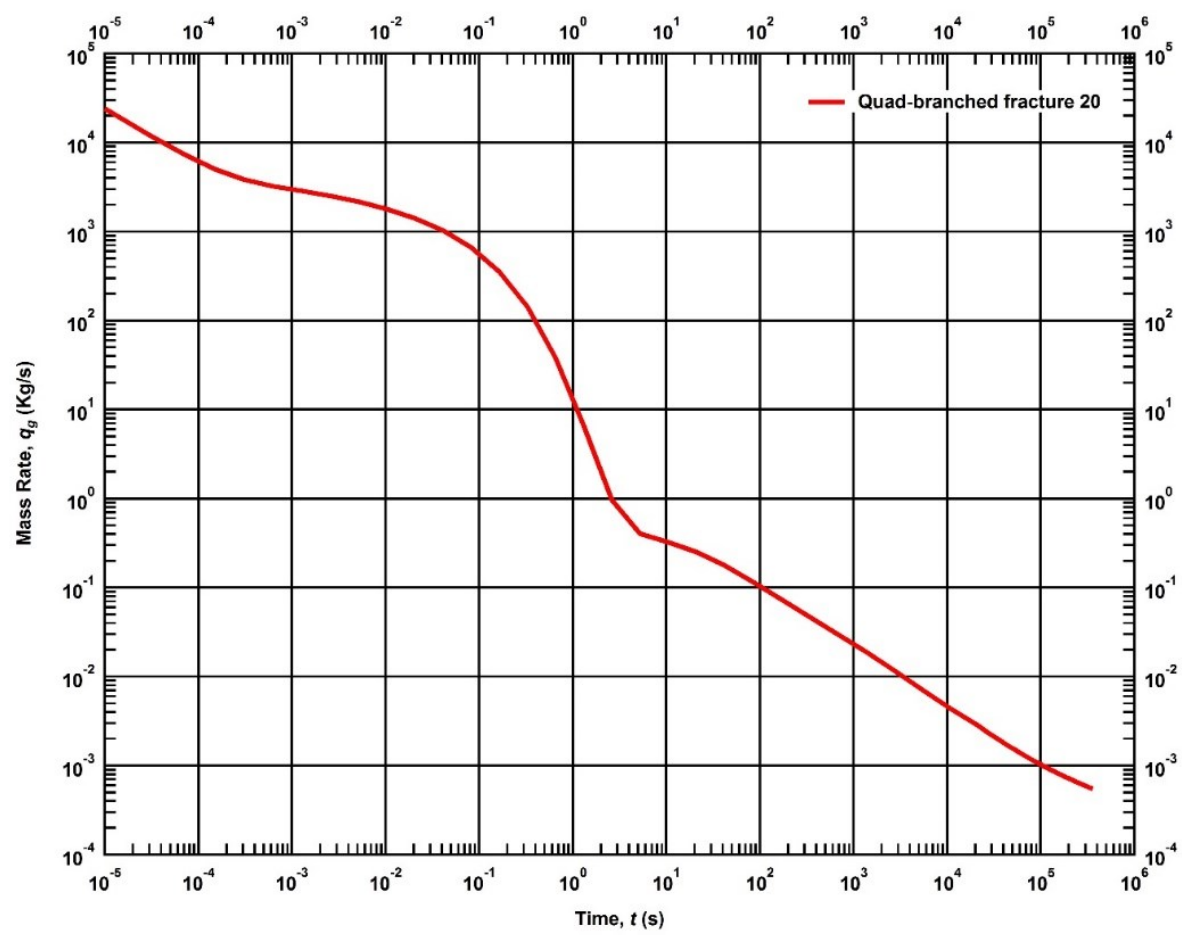

Figure B. 84 - Mass rate evolution for a shale gas reservoir produced from the quad-branched fracture 20 until 100 hours of production. 


\section{APPENDIX C}

\section{MASS RATE $\beta$-DERIVATIVE PLOTS FOR SIMULATED STOCHASTIC FRACTURE}

\section{PATTERNS}

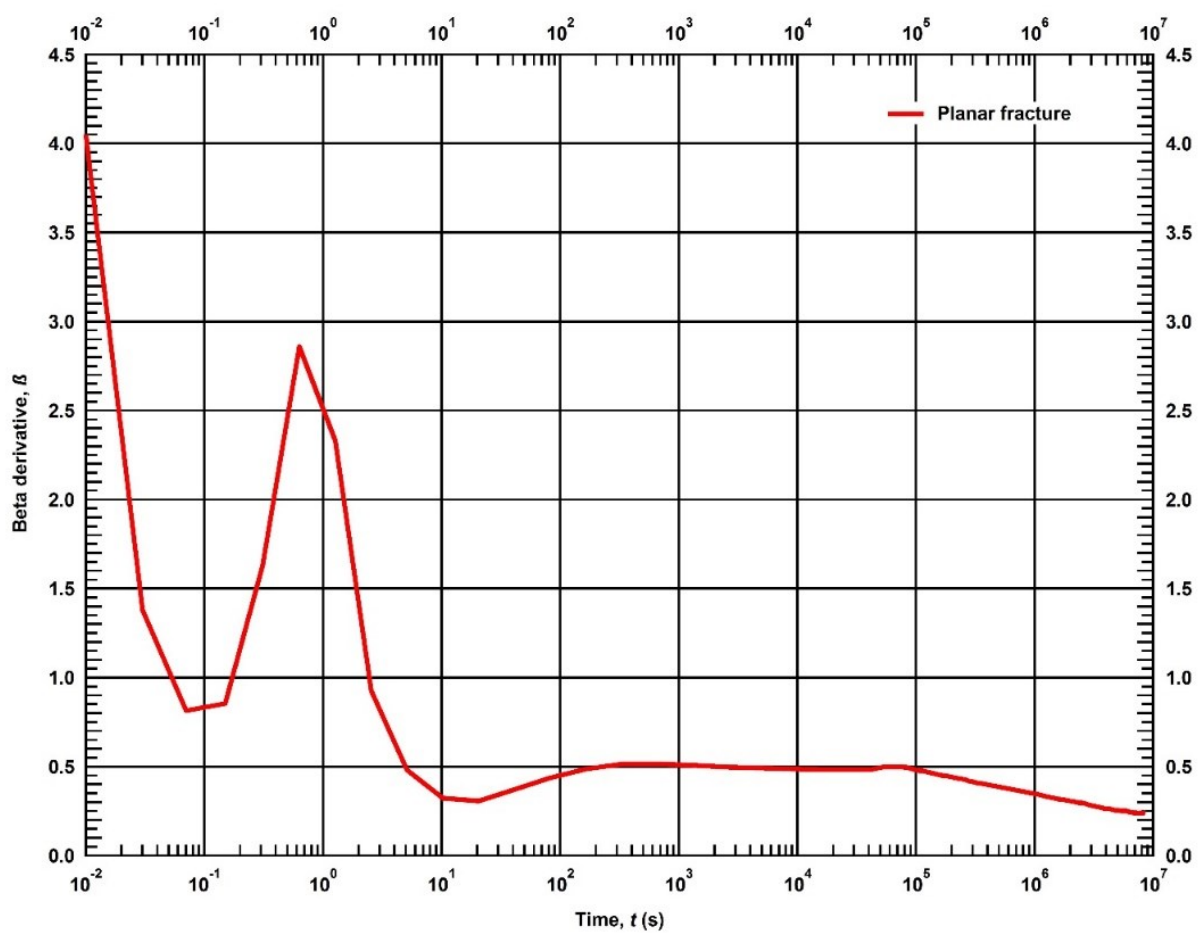

Figure C. 1 - $\beta$-derivative of the mass rate evolution for a shale gas reservoir produced from a planar fracture until 100 days of production. 


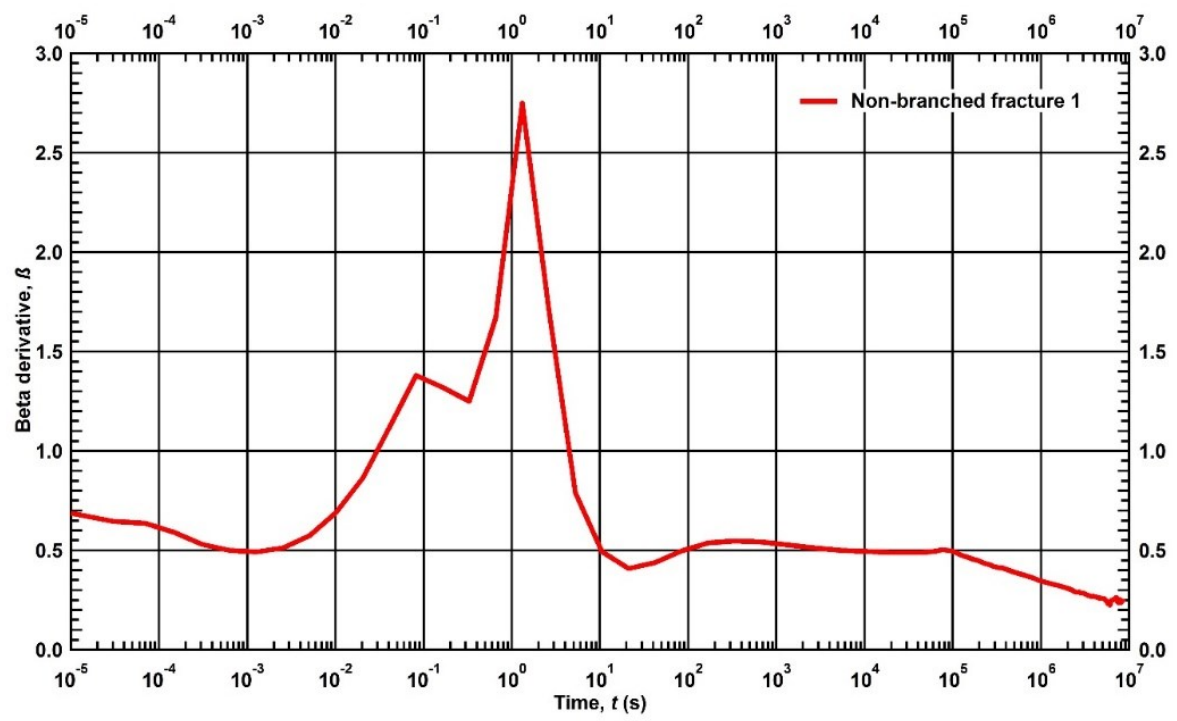

Figure C. 2 - $\beta$-derivative of the mass rate evolution for a shale gas reservoir produced from the non-branched fracture 1 until 100 days of production.

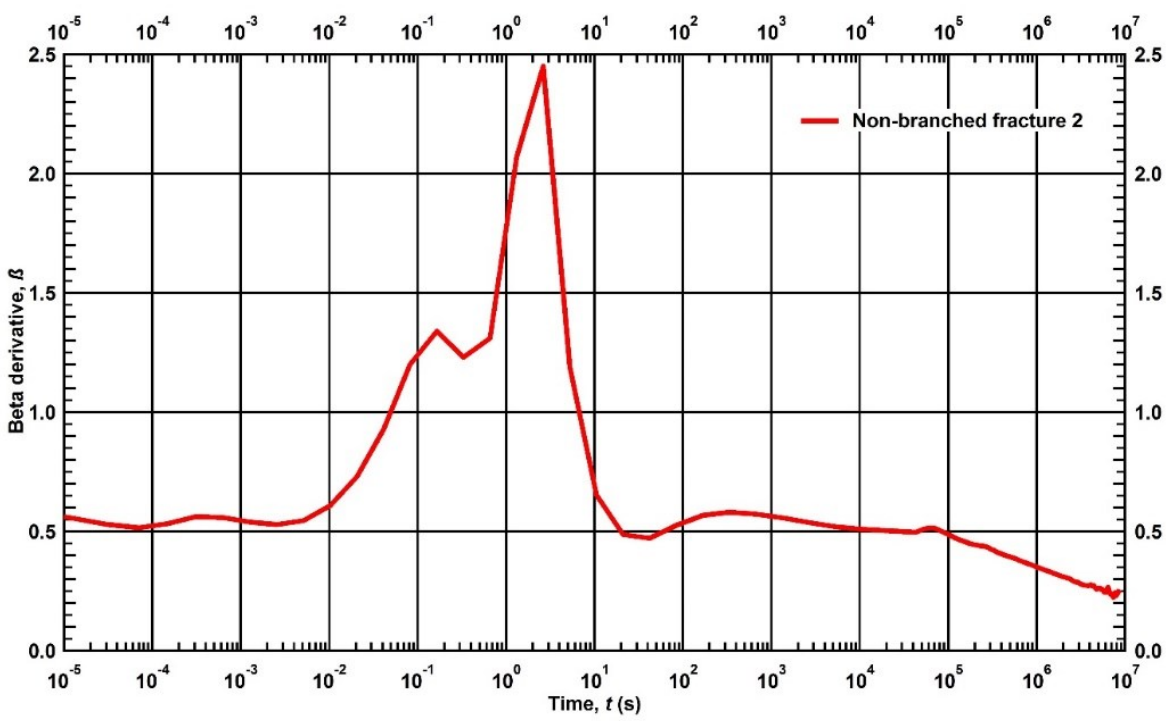

Figure C. 3 - $\beta$-derivative of the mass rate evolution for a shale gas reservoir produced from the non-branched fracture 2 until 100 days of production. 


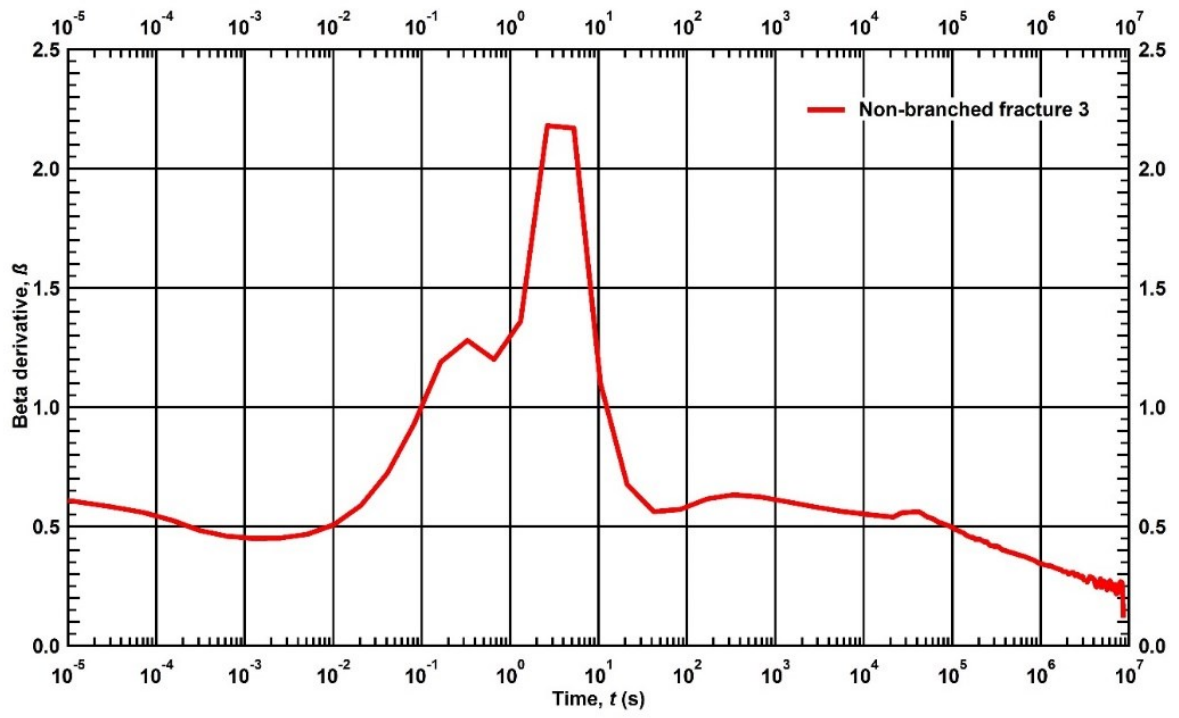

Figure C. 4 - $\beta$-derivative of the mass rate evolution for a shale gas reservoir produced from the non-branched fracture 3 until 100 days of production.

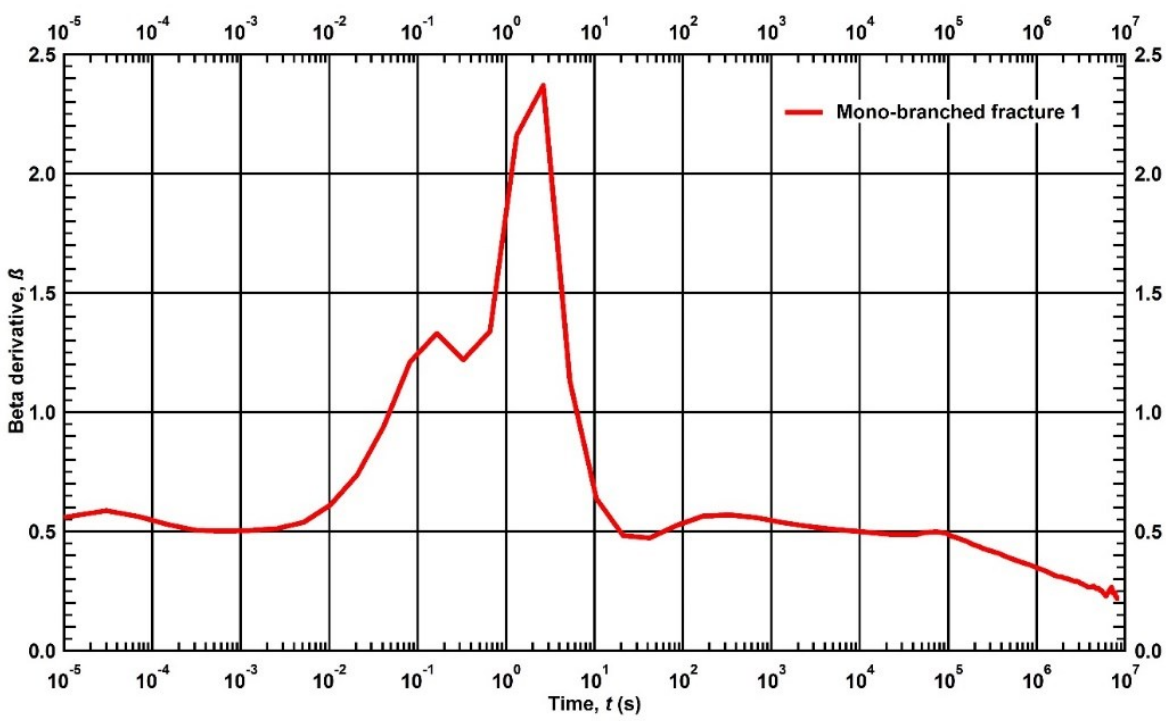

Figure C. 5 - $\beta$-derivative of the mass rate evolution for a shale gas reservoir produced from the mono-branched fracture 1 until 100 days of production. 


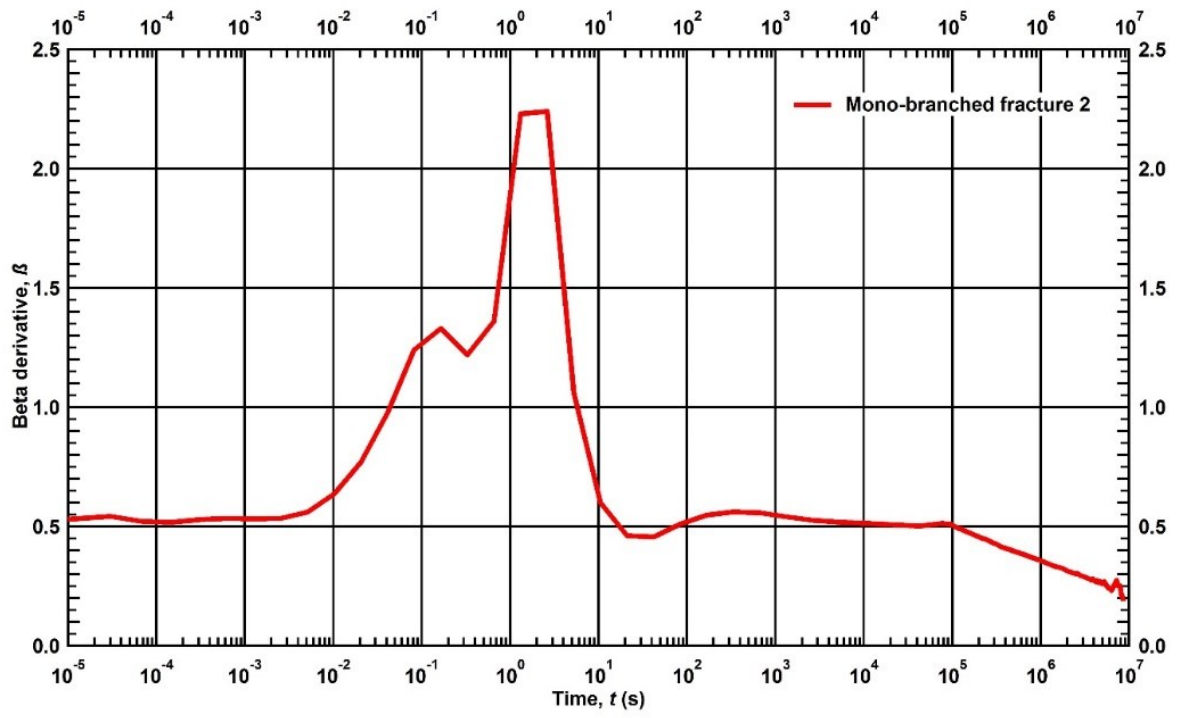

Figure C. 6 - $\beta$-derivative of the mass rate evolution for a shale gas reservoir produced from the mono-branched fracture 2 until 100 days of production.

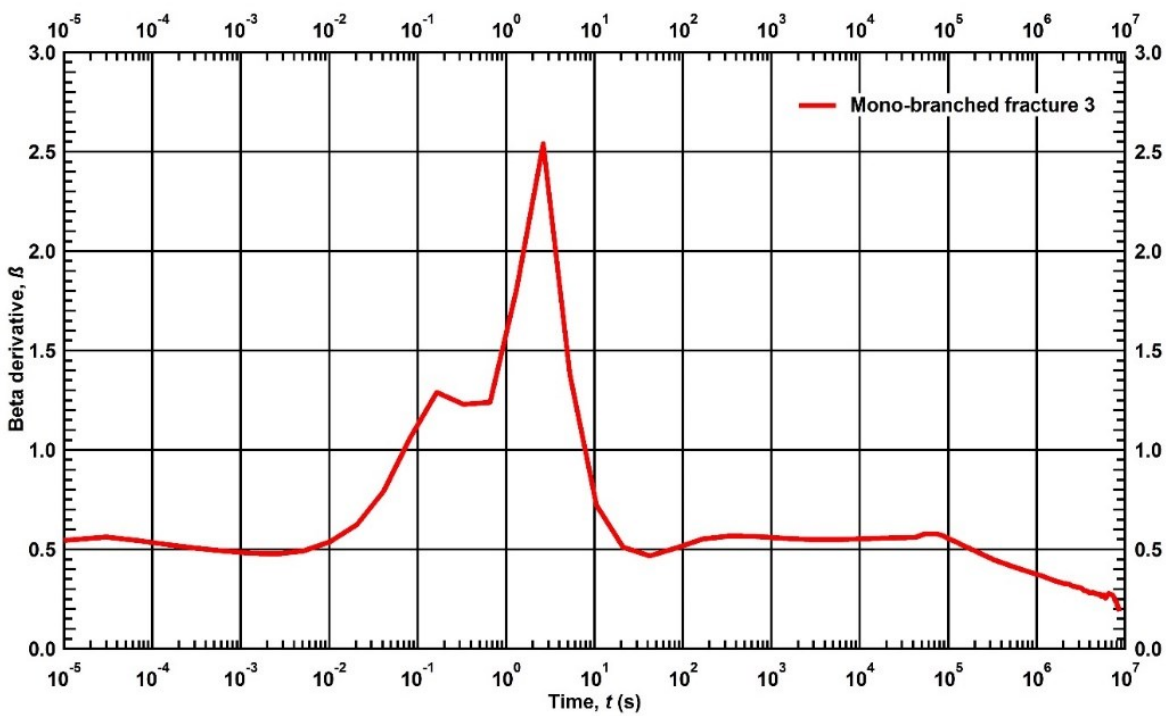

Figure C. 7 - $\beta$-derivative of the mass rate evolution for a shale gas reservoir produced from the mono-branched fracture 3 until 100 days of production. 


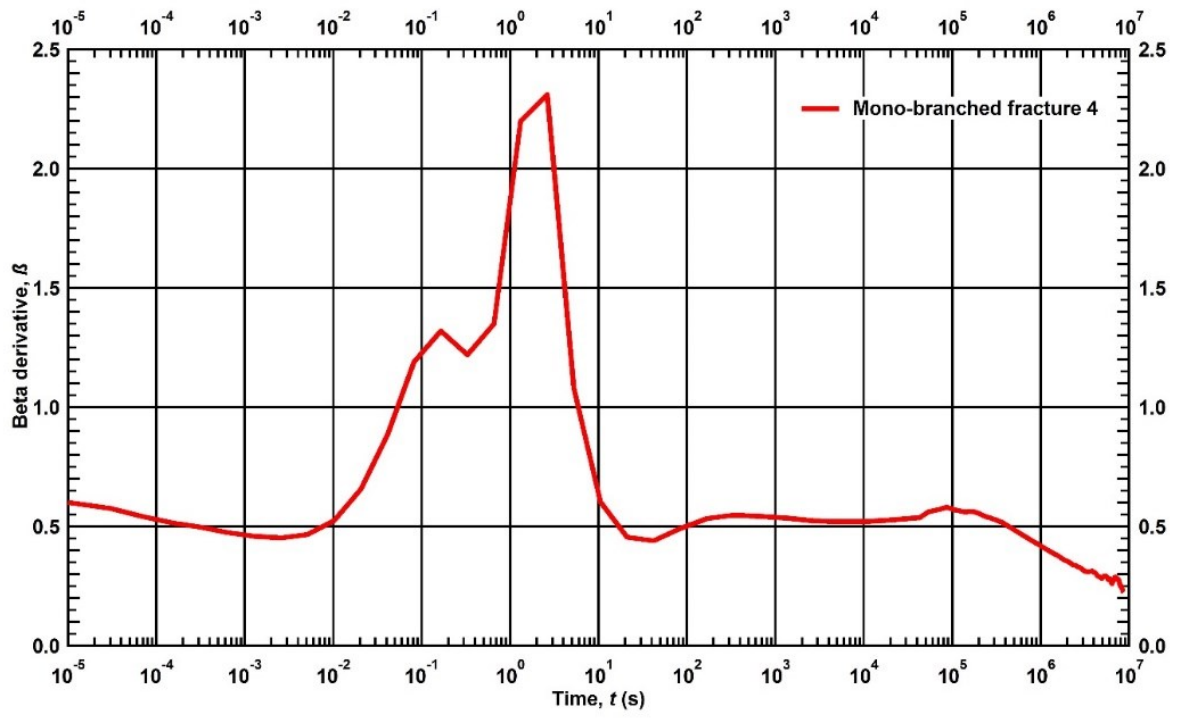

Figure C. 8 - $\beta$-derivative of the mass rate evolution for a shale gas reservoir produced from the mono-branched fracture 4 until 100 days of production.

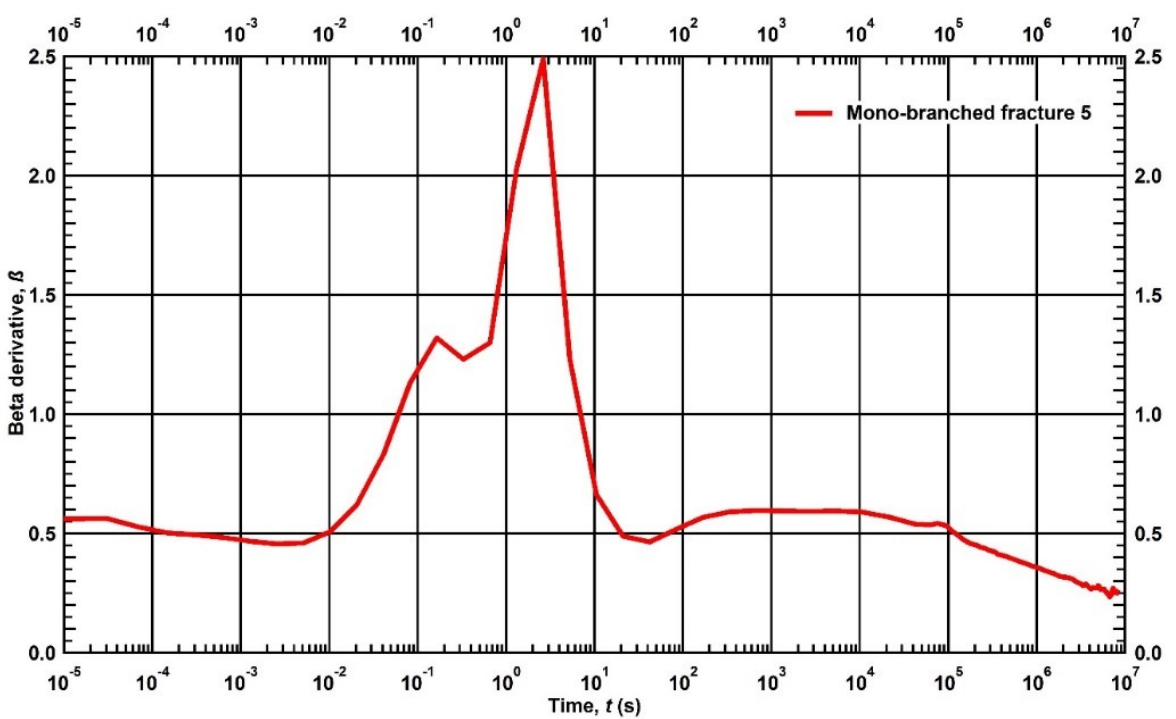

Figure C. 9 - $\beta$-derivative of the mass rate evolution for a shale gas reservoir produced from the mono-branched fracture 5 until 100 days of production. 


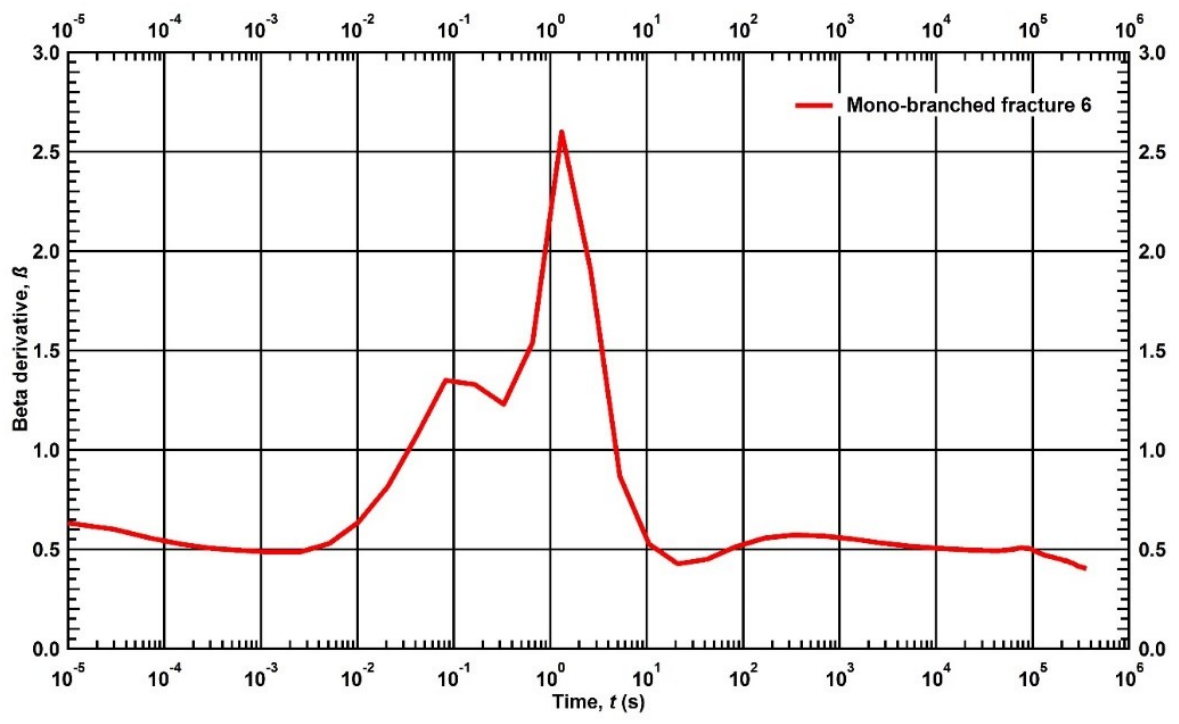

Figure C. 10 - $\beta$-derivative of the mass rate evolution for a shale gas reservoir produced from the mono-branched fracture 6 until 100 hours of production.

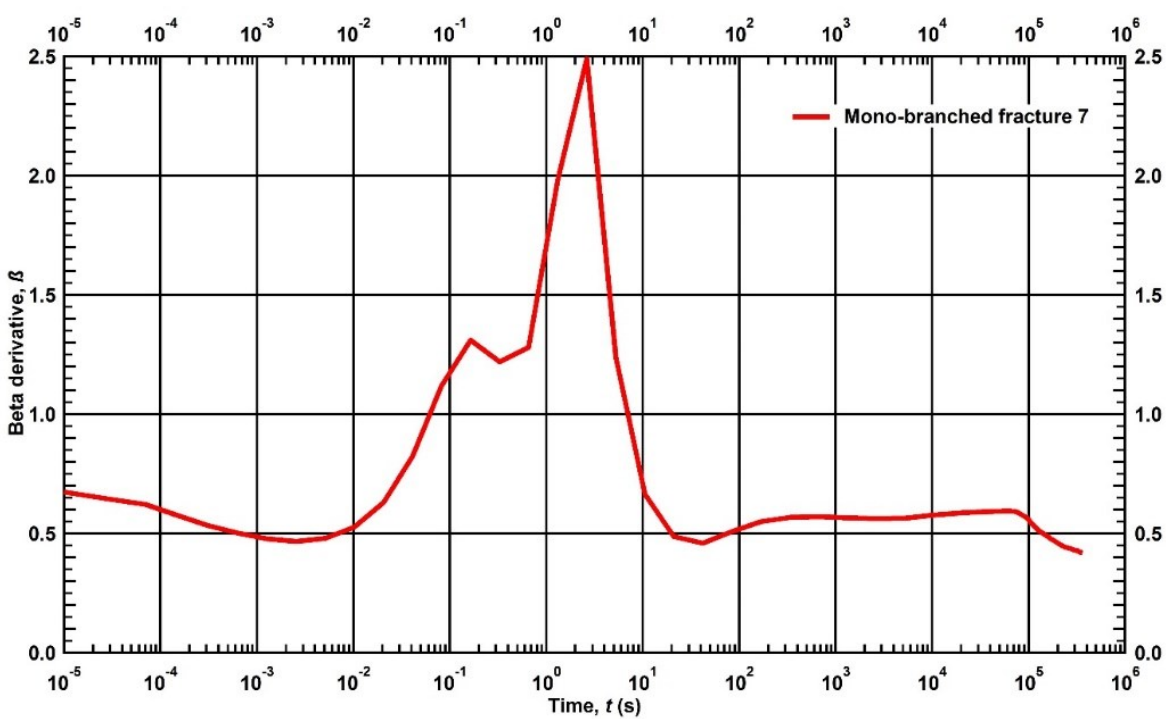

Figure C. 11 - $\beta$-derivative of the mass rate evolution for a shale gas reservoir produced from the mono-branched fracture 7 until 100 hours of production. 


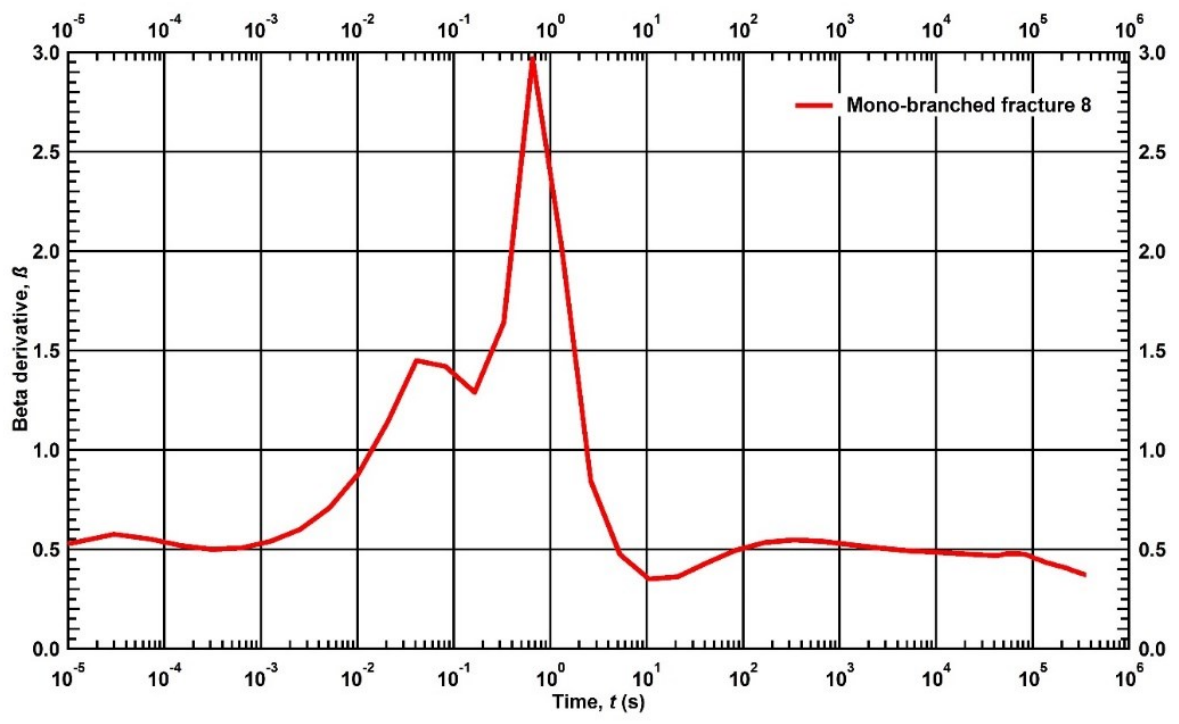

Figure C. 12 - $\beta$-derivative of the mass rate evolution for a shale gas reservoir produced from the mono-branched fracture 8 until 100 hours of production.

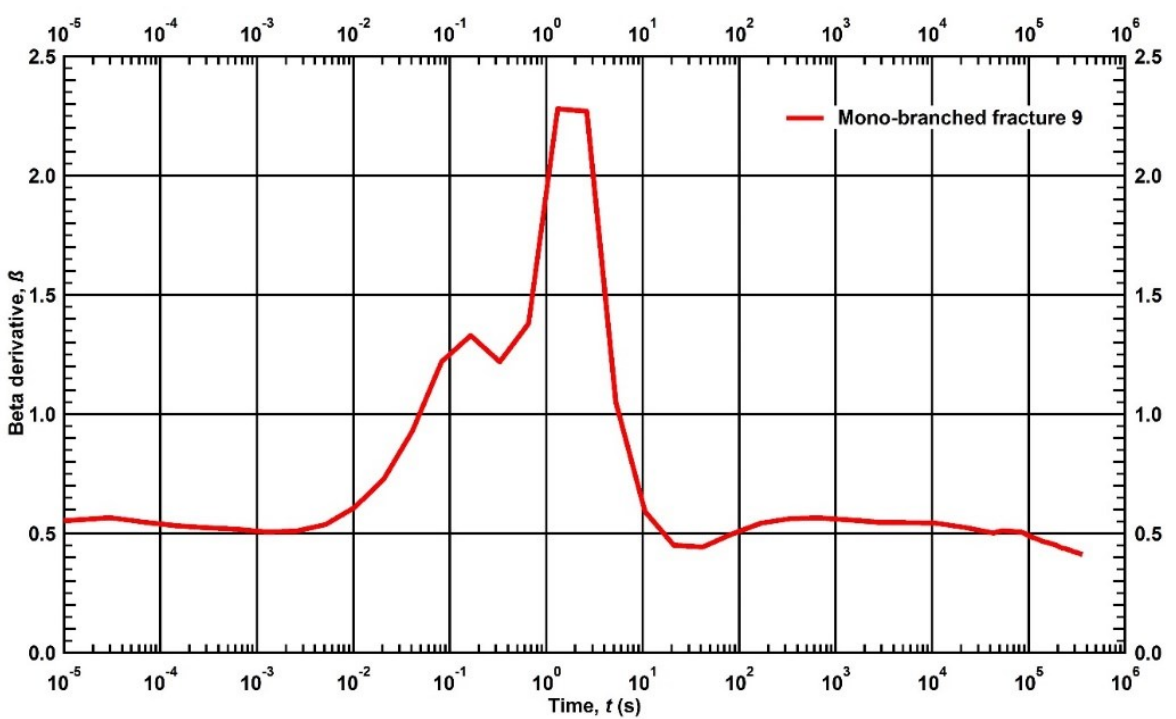

Figure C. 13 - $\beta$-derivative of the mass rate evolution for a shale gas reservoir produced from the mono-branched fracture 9 until 100 hours of production. 


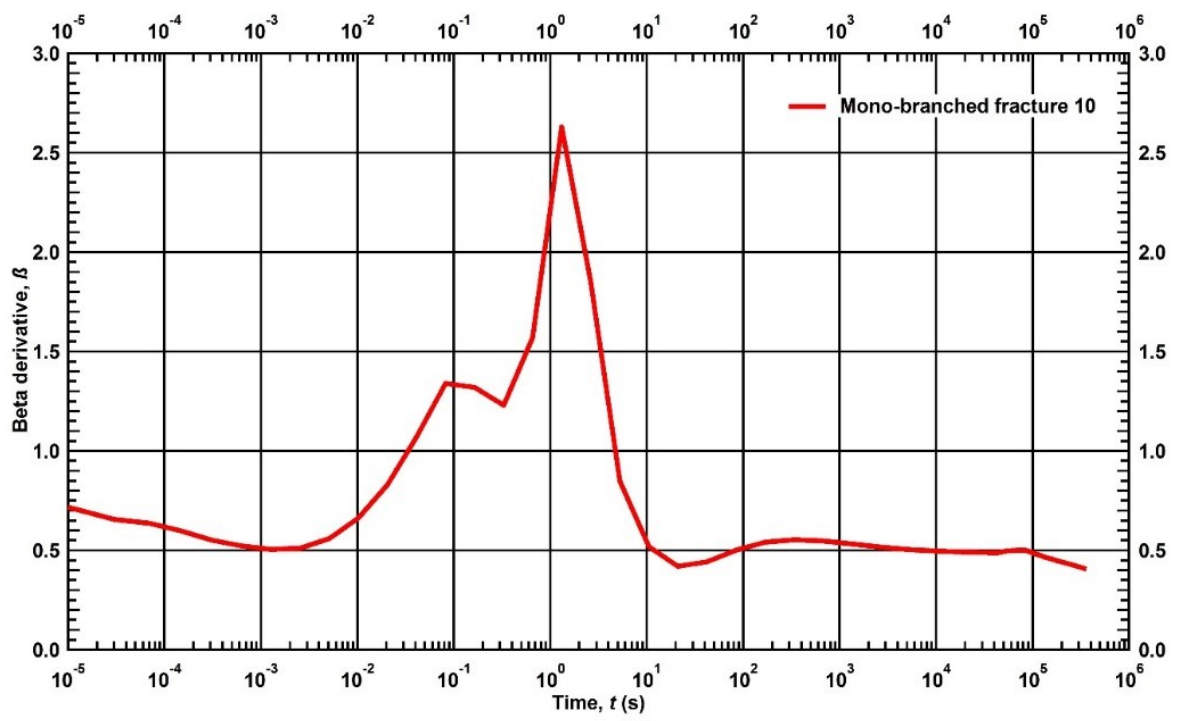

Figure C. 14 - $\beta$-derivative of the mass rate evolution for a shale gas reservoir produced from the mono-branched fracture 10 until 100 hours of production.

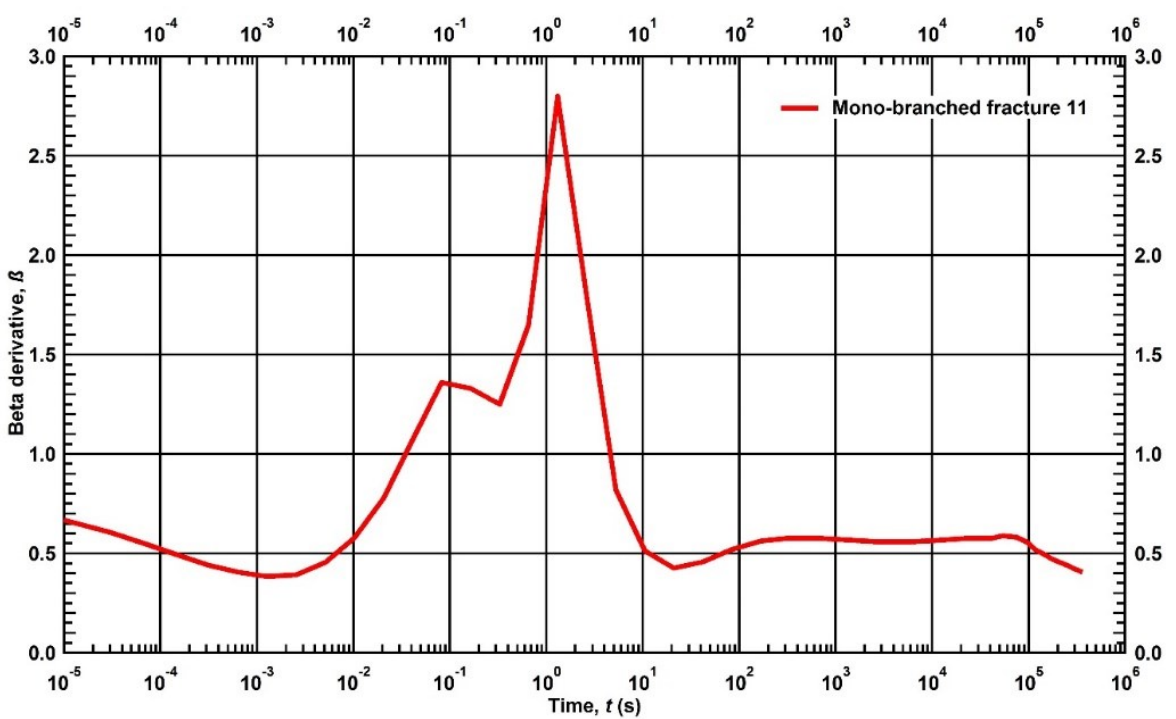

Figure C. 15 - $\beta$-derivative of the mass rate evolution for a shale gas reservoir produced from the mono-branched fracture 11 until 100 hours of production. 


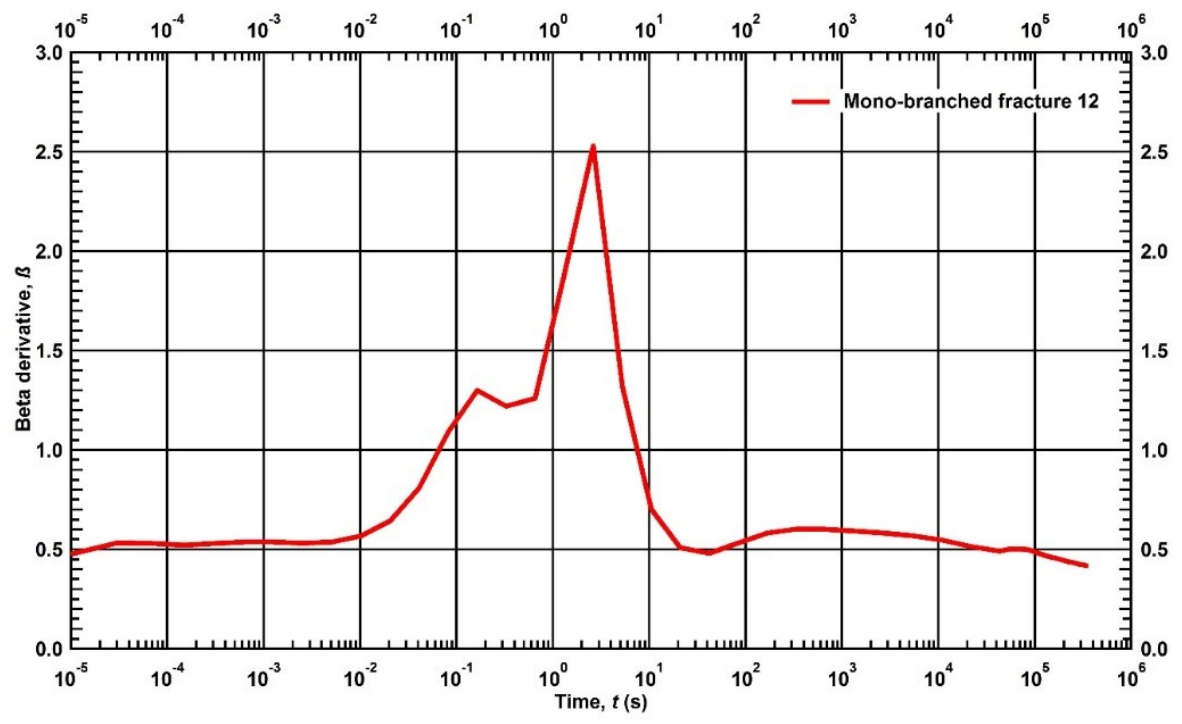

Figure C. 16 - $\beta$-derivative of the mass rate evolution for a shale gas reservoir produced from the mono-branched fracture 12 until 100 hours of production.

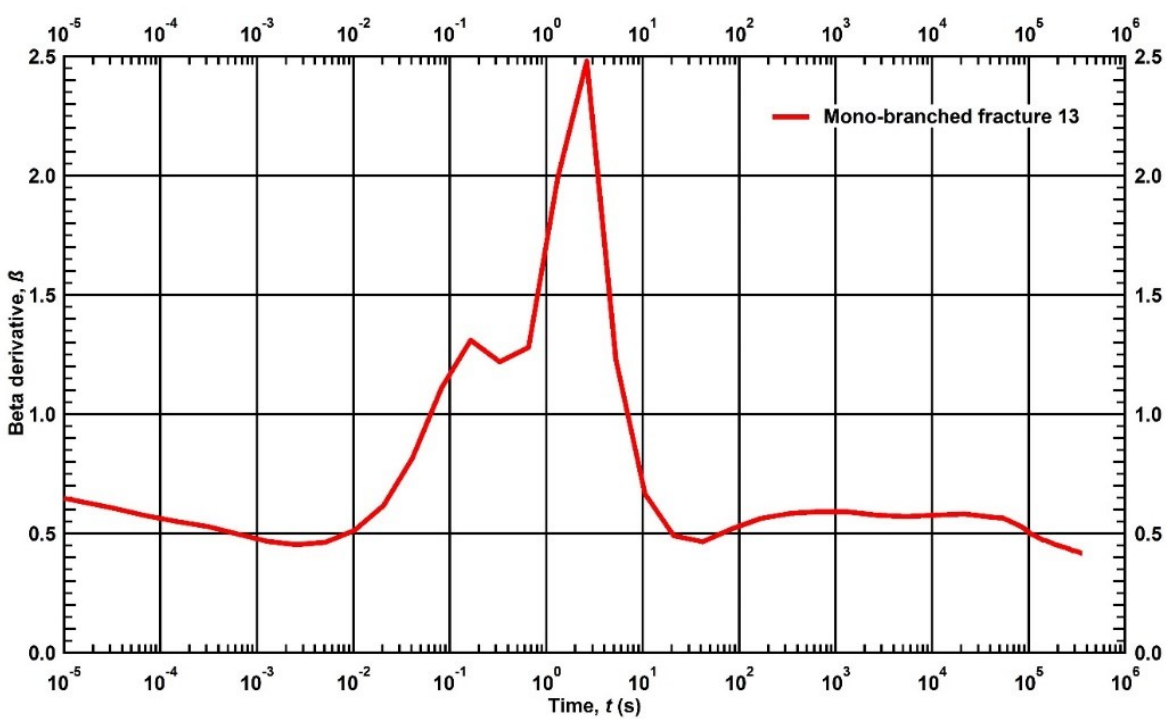

Figure C. 17 - $\beta$-derivative of the mass rate evolution for a shale gas reservoir produced from the mono-branched fracture 13 until 100 hours of production. 


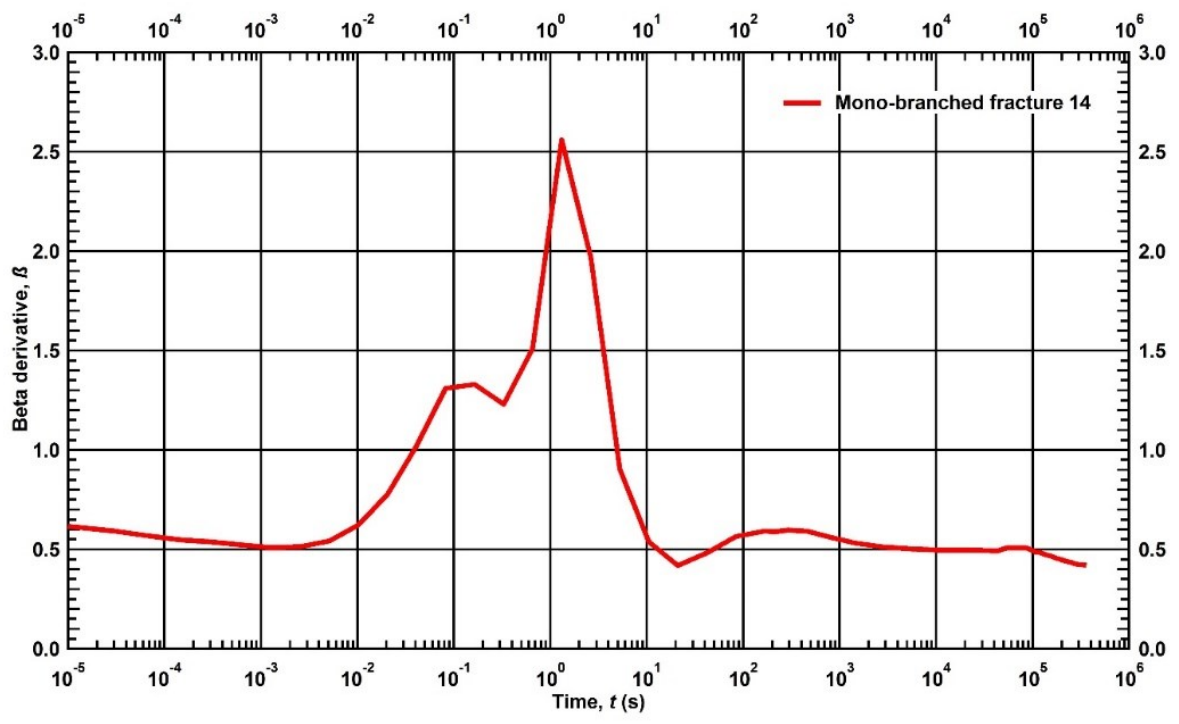

Figure C. 18 - $\beta$-derivative of the mass rate evolution for a shale gas reservoir produced from the mono-branched fracture 14 until 100 hours of production.

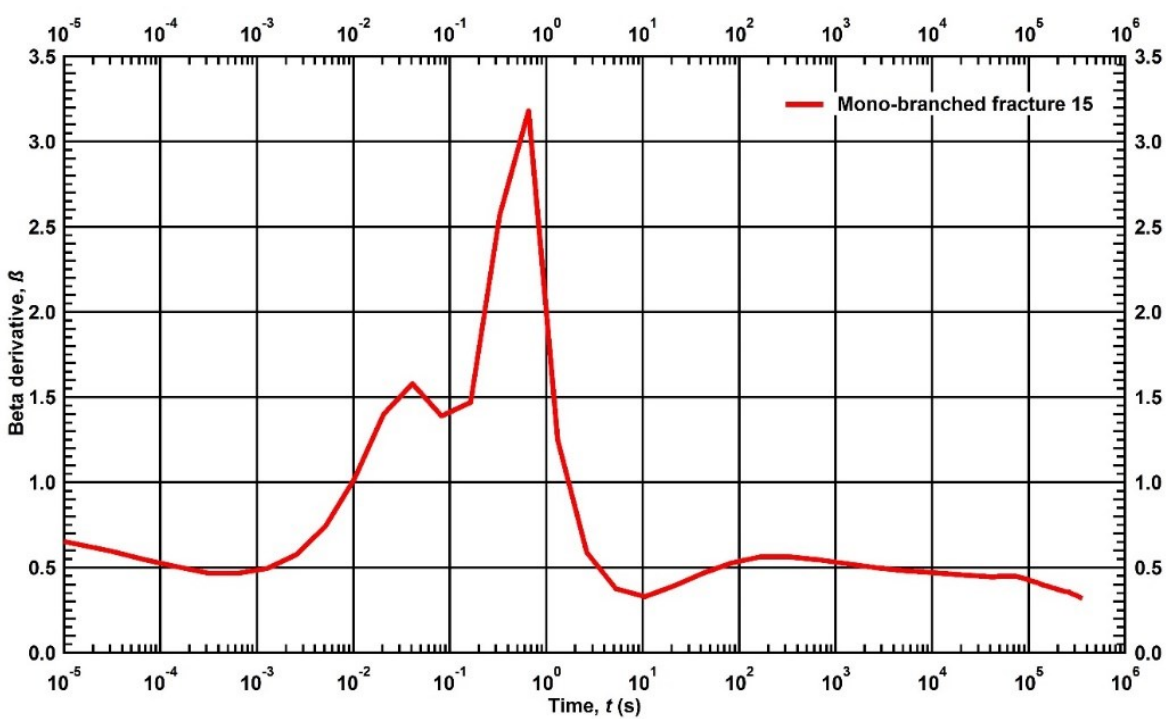

Figure C. 19 - $\beta$-derivative of the mass rate evolution for a shale gas reservoir produced from the mono-branched fracture 15 until 100 hours of production. 


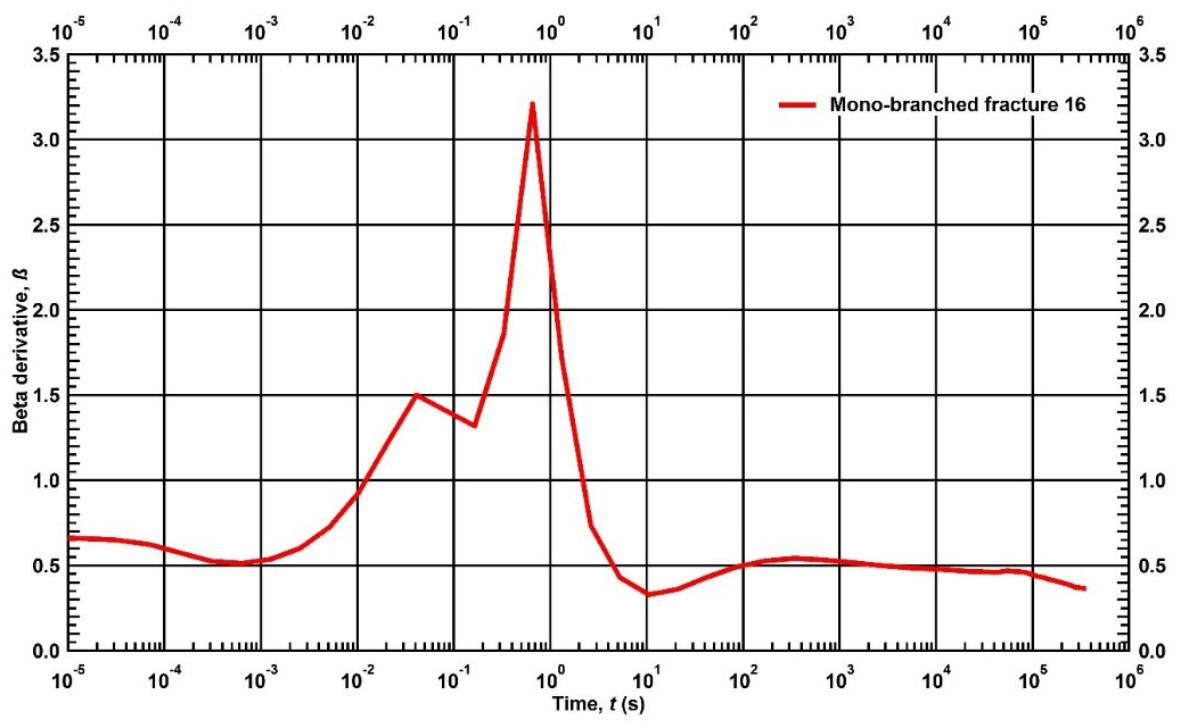

Figure C. 20 - $\beta$-derivative of the mass rate evolution for a shale gas reservoir produced from the mono-branched fracture 16 until 100 hours of production.

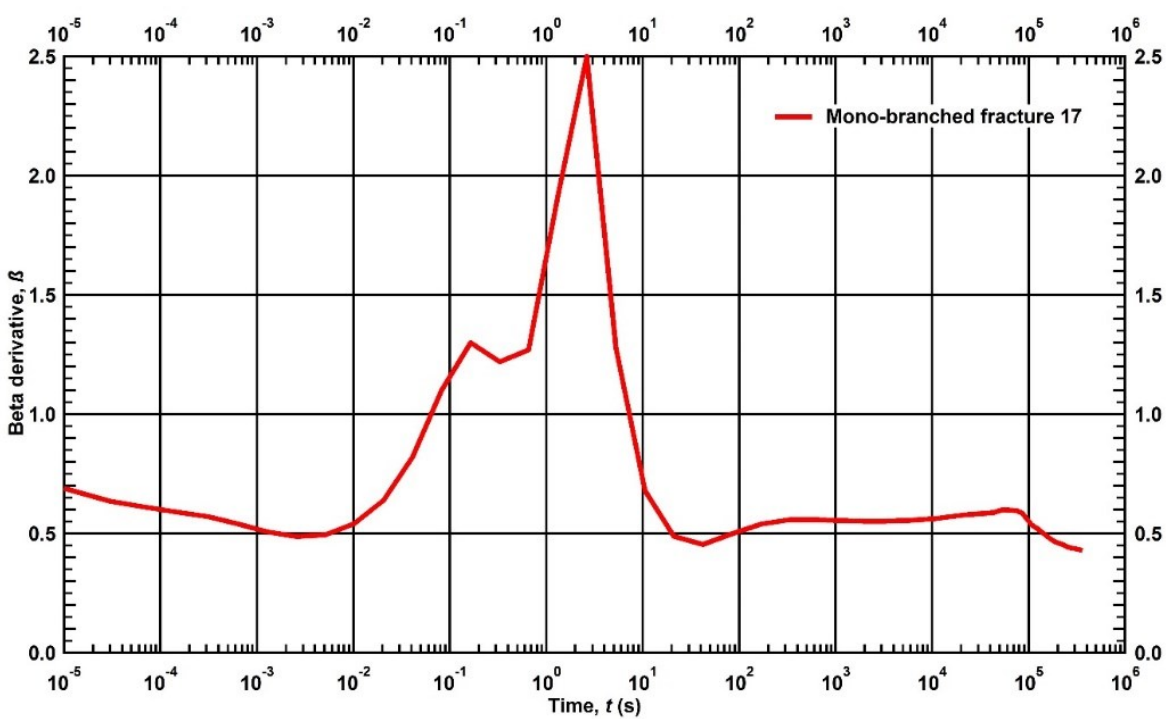

Figure C. 21 - $\beta$-derivative of the mass rate evolution for a shale gas reservoir produced from the mono-branched fracture 17 until 100 hours of production. 


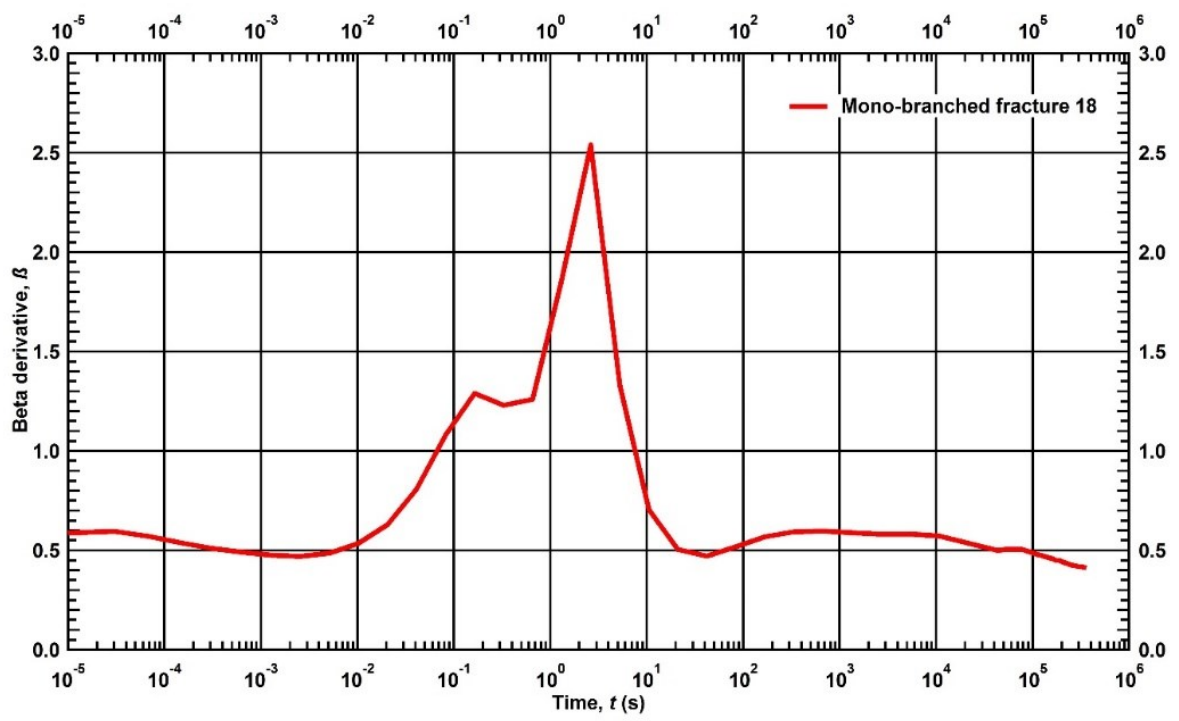

Figure C. 22 - $\beta$-derivative of the mass rate evolution for a shale gas reservoir produced from the mono-branched fracture 18 until 100 hours of production.

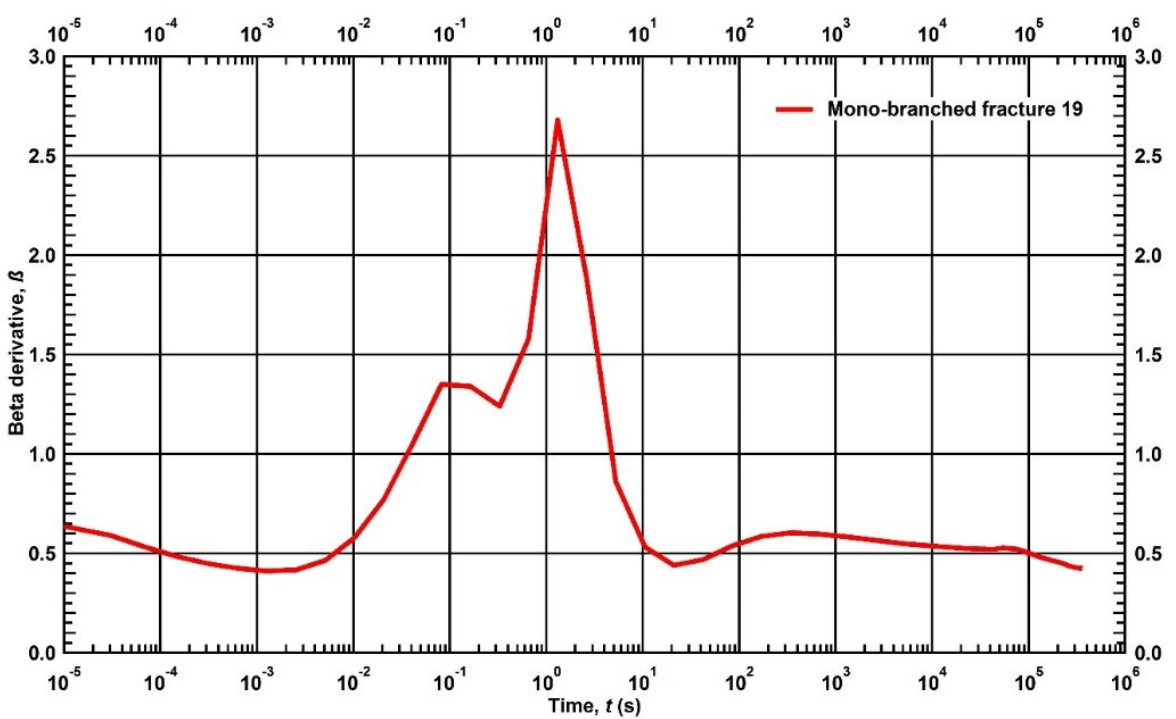

Figure C. 23 - $\beta$-derivative of the mass rate evolution for a shale gas reservoir produced from the mono-branched fracture 19 until 100 hours of production. 


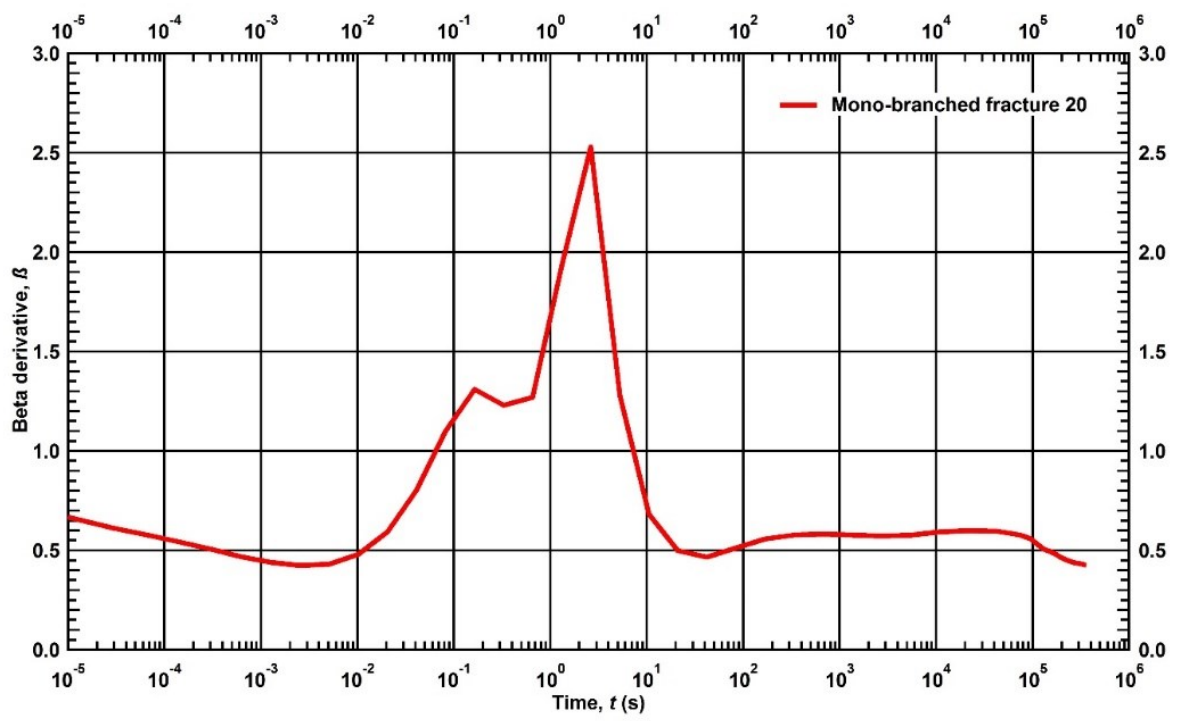

Figure C. 24 - $\beta$-derivative of the mass rate evolution for a shale gas reservoir produced from the mono-branched fracture 20 until 100 hours of production.

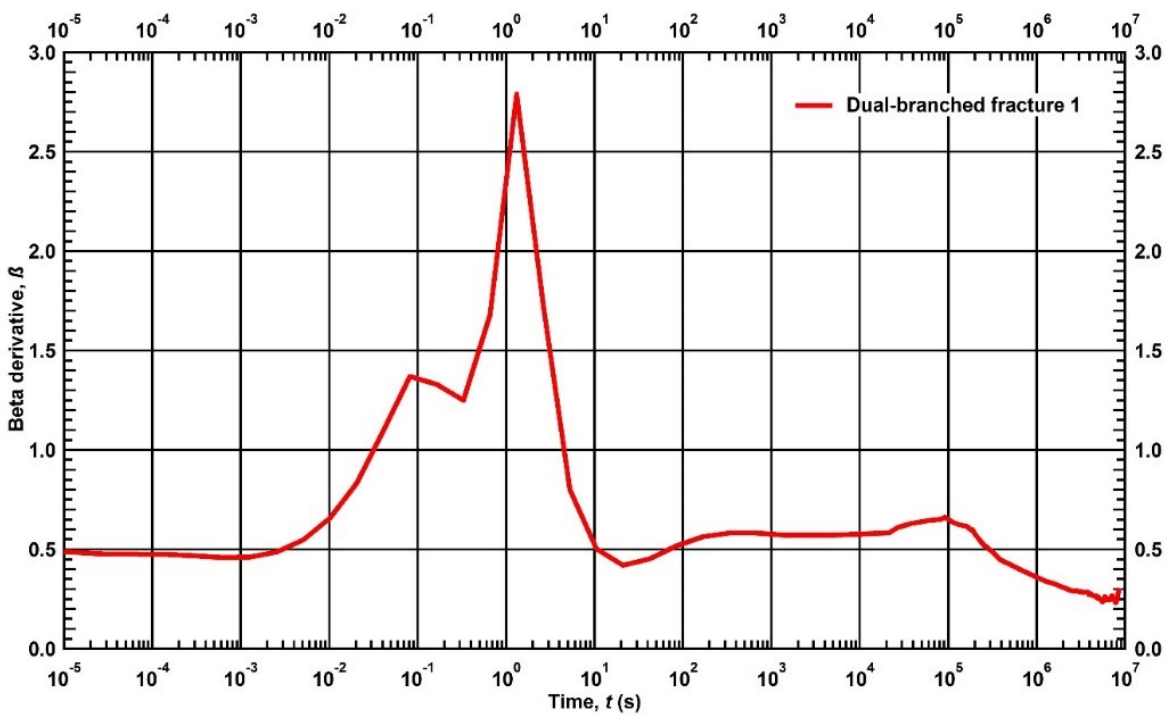

Figure C. 25 - $\beta$-derivative of the mass rate evolution for a shale gas reservoir produced from the dual-branched fracture 1 until 100 days of production. 


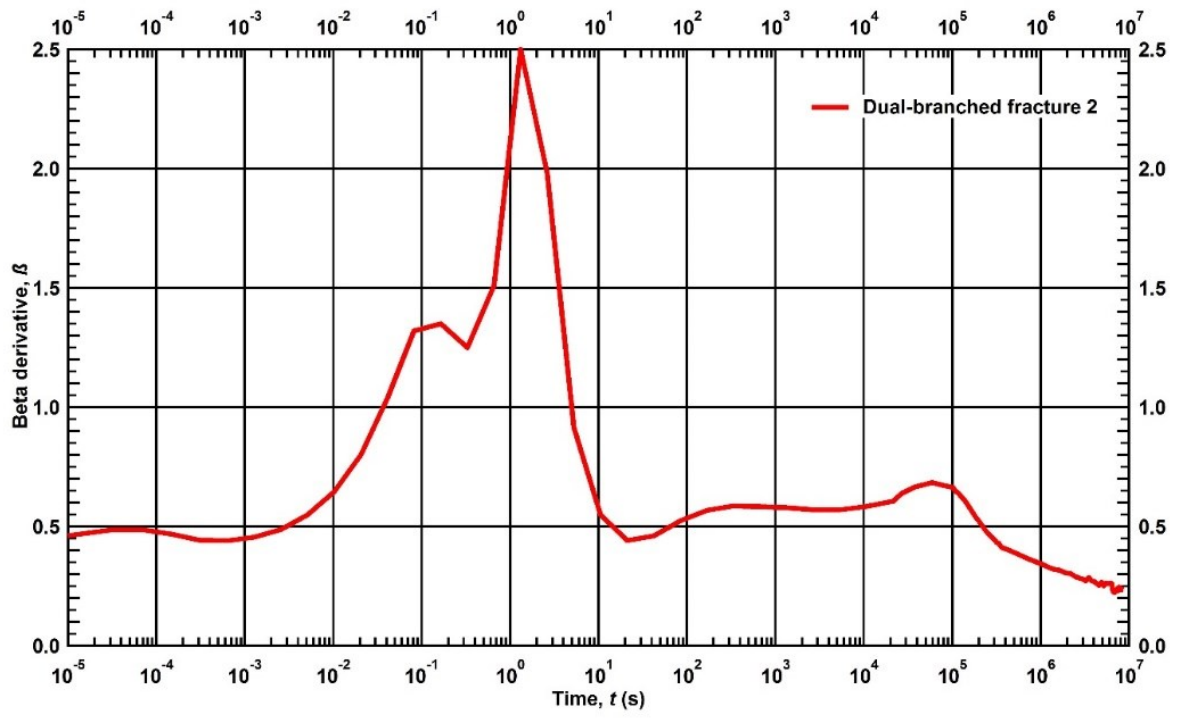

Figure C. 26 - $\beta$-derivative of the mass rate evolution for a shale gas reservoir produced from the dual-branched fracture 2 until 100 days of production.

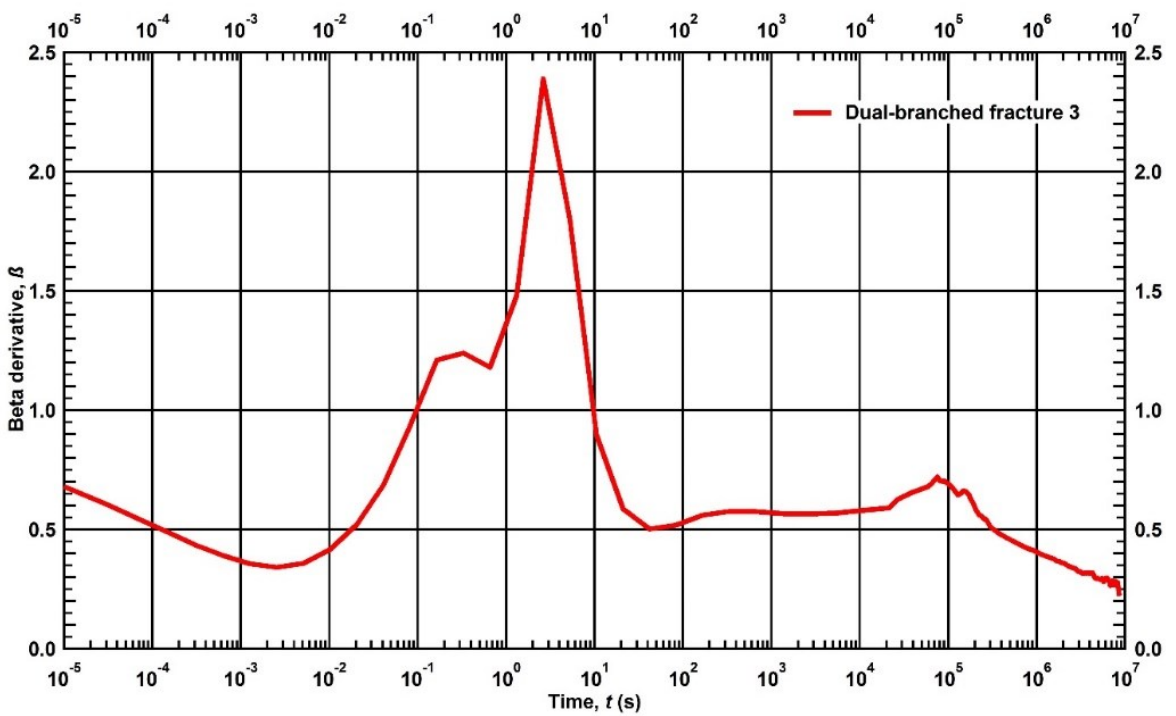

Figure C. 27 - $\beta$-derivative of the mass rate evolution for a shale gas reservoir produced from the dual-branched fracture 3 until 100 days of production. 


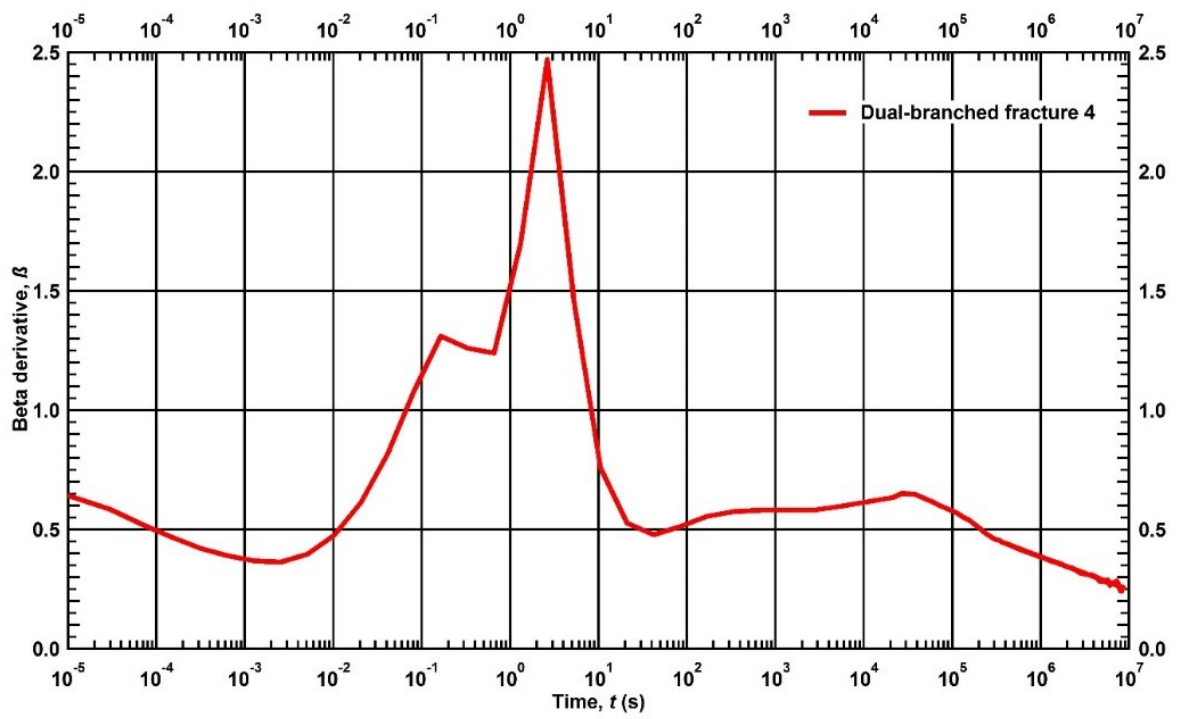

Figure C. 28 - $\beta$-derivative of the mass rate evolution for a shale gas reservoir produced from the dual-branched fracture 4 until 100 days of production.

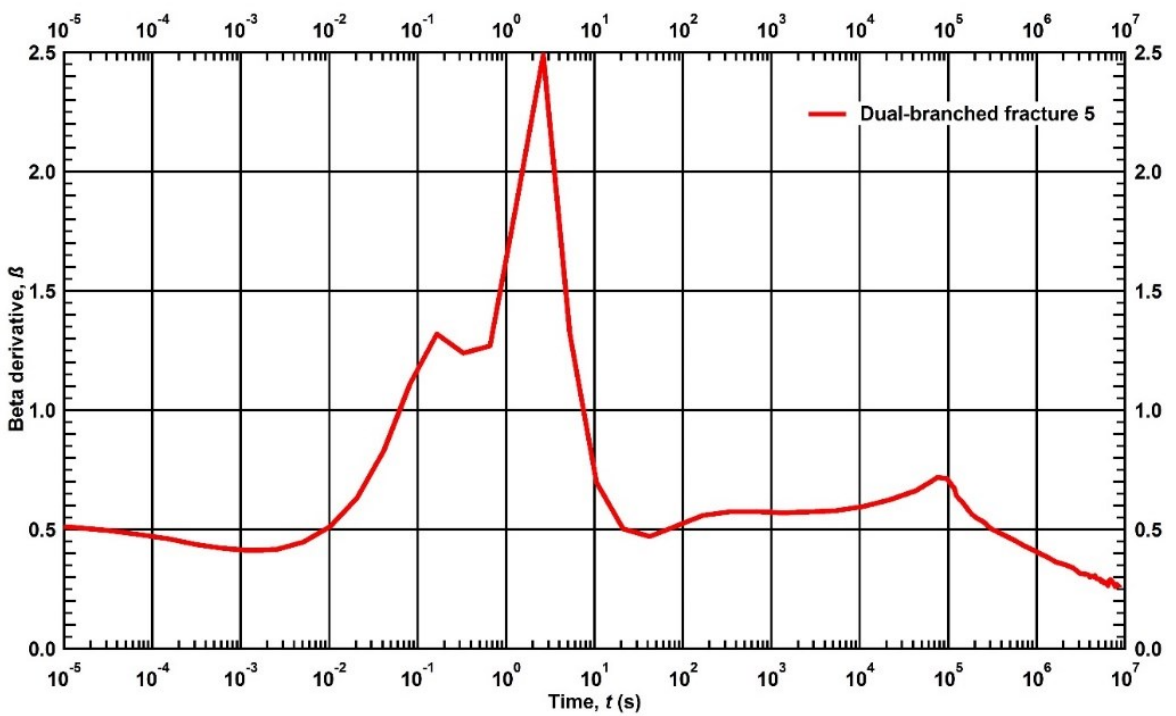

Figure C. 29 - $\beta$-derivative of the mass rate evolution for a shale gas reservoir produced from the dual-branched fracture 5 until 100 days of production. 


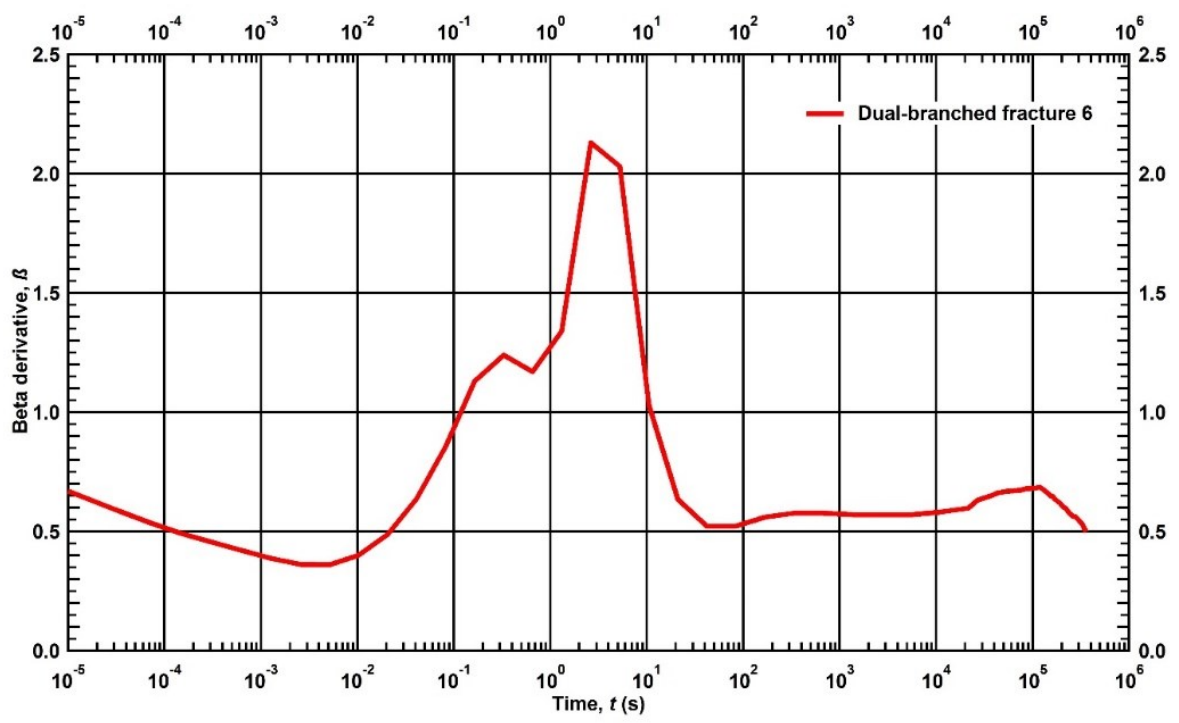

Figure C. 30 - $\beta$-derivative of the mass rate evolution for a shale gas reservoir produced from the dual-branched fracture 6 until 100 hours of production.

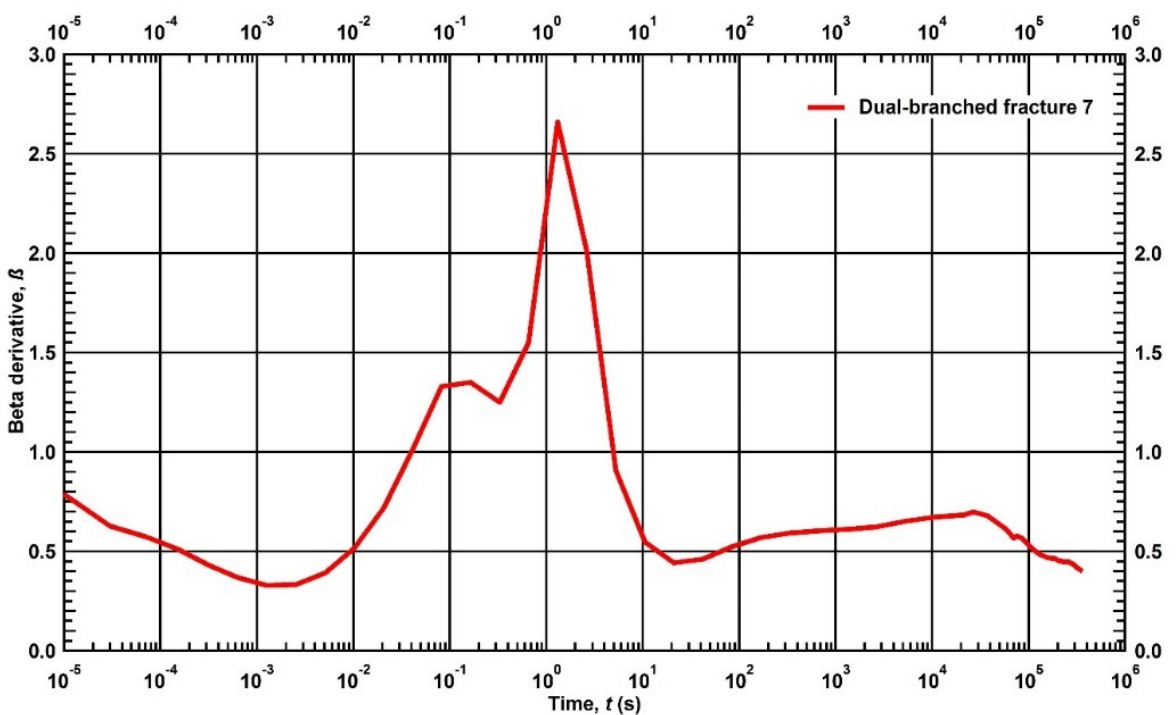

Figure C. 31 - $\beta$-derivative of the mass rate evolution for a shale gas reservoir produced from the dual-branched fracture 7 until 100 hours of production. 


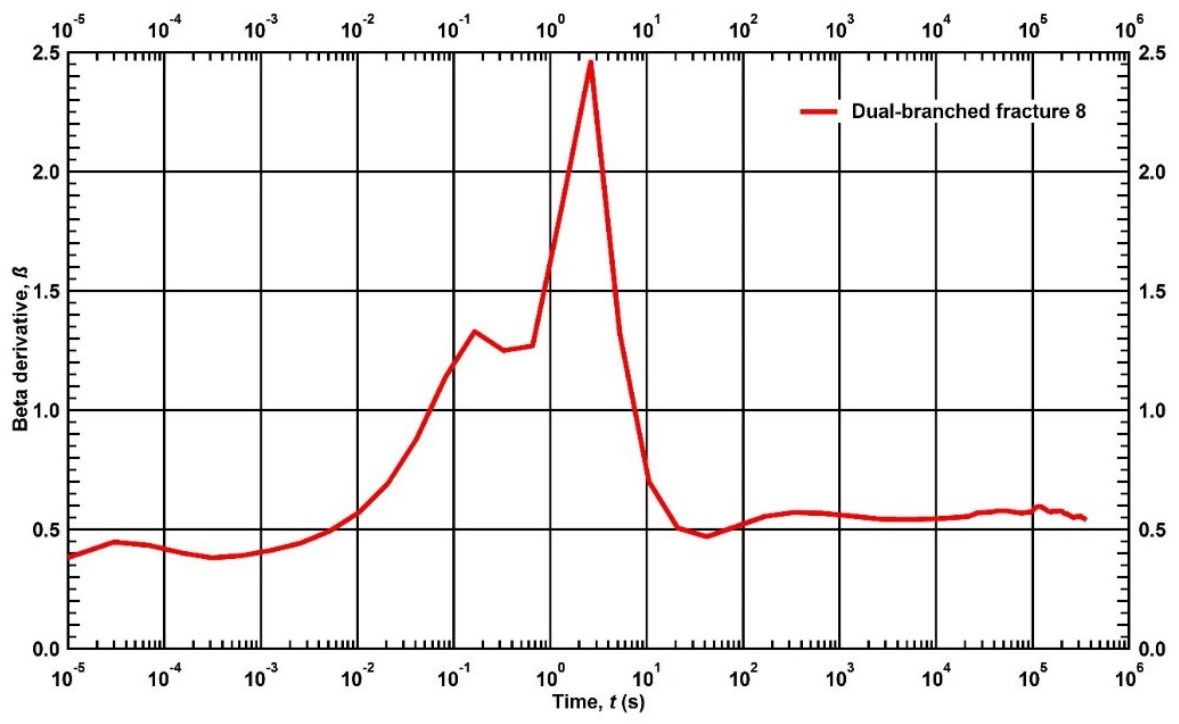

Figure C. 32 - $\beta$-derivative of the mass rate evolution for a shale gas reservoir produced from the dual-branched fracture 8 until 100 hours of production.

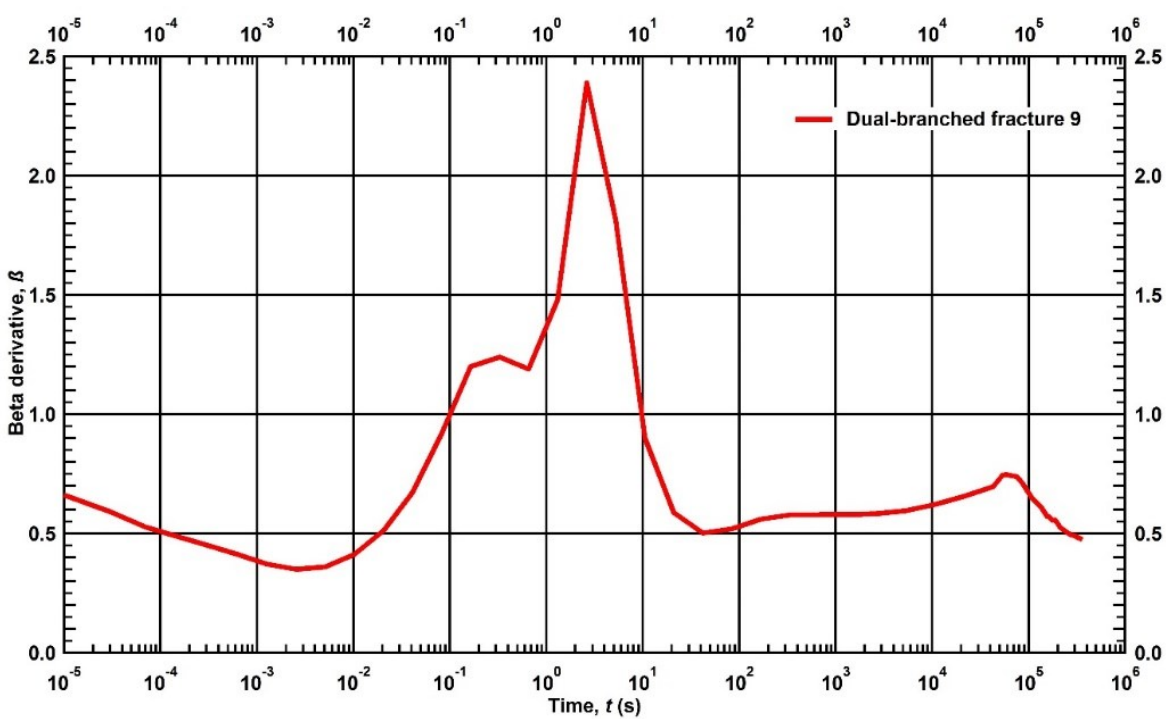

Figure C. 33 - $\beta$-derivative of the mass rate evolution for a shale gas reservoir produced from the dual-branched fracture 9 until 100 hours of production. 


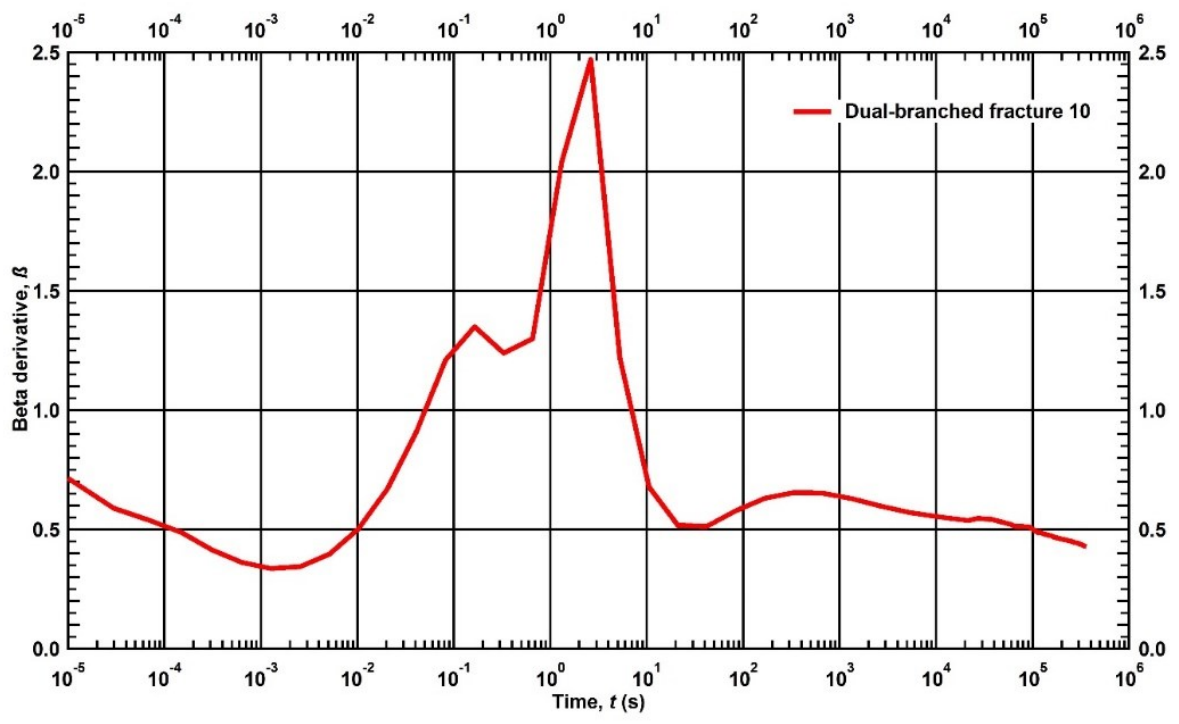

Figure C. 34 - $\beta$-derivative of the mass rate evolution for a shale gas reservoir produced from the dual-branched fracture 10 until 100 hours of production.

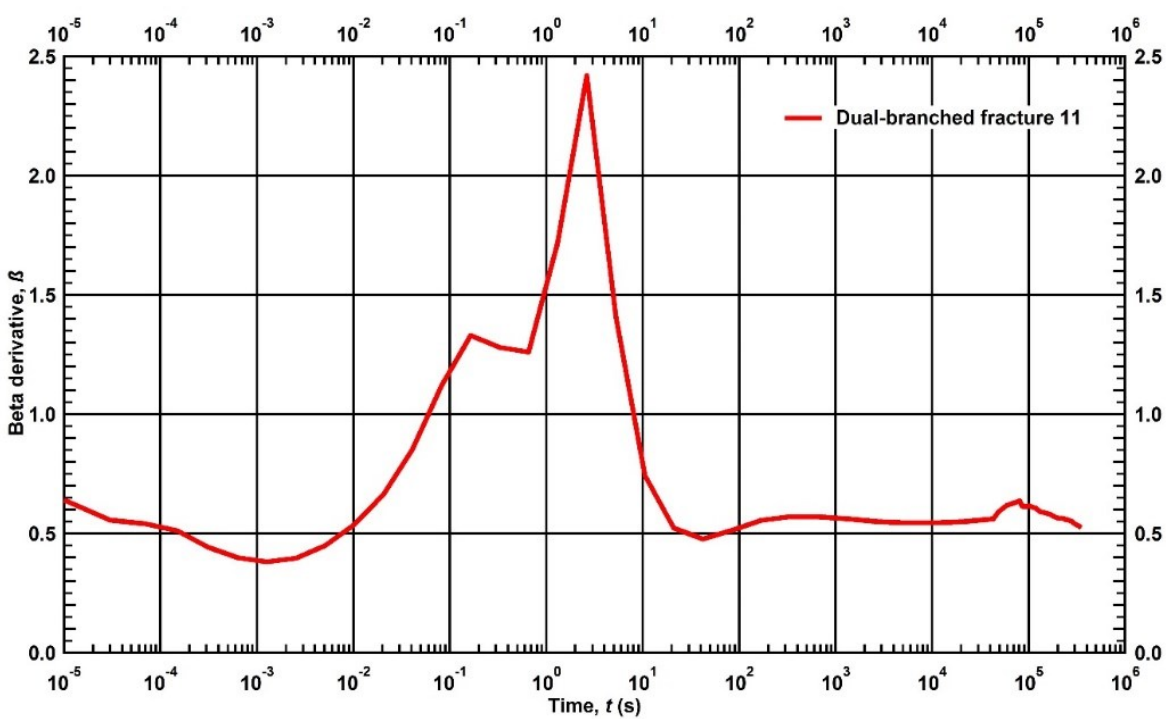

Figure C. 35 - $\beta$-derivative of the mass rate evolution for a shale gas reservoir produced from the dual-branched fracture 11 until 100 hours of production. 


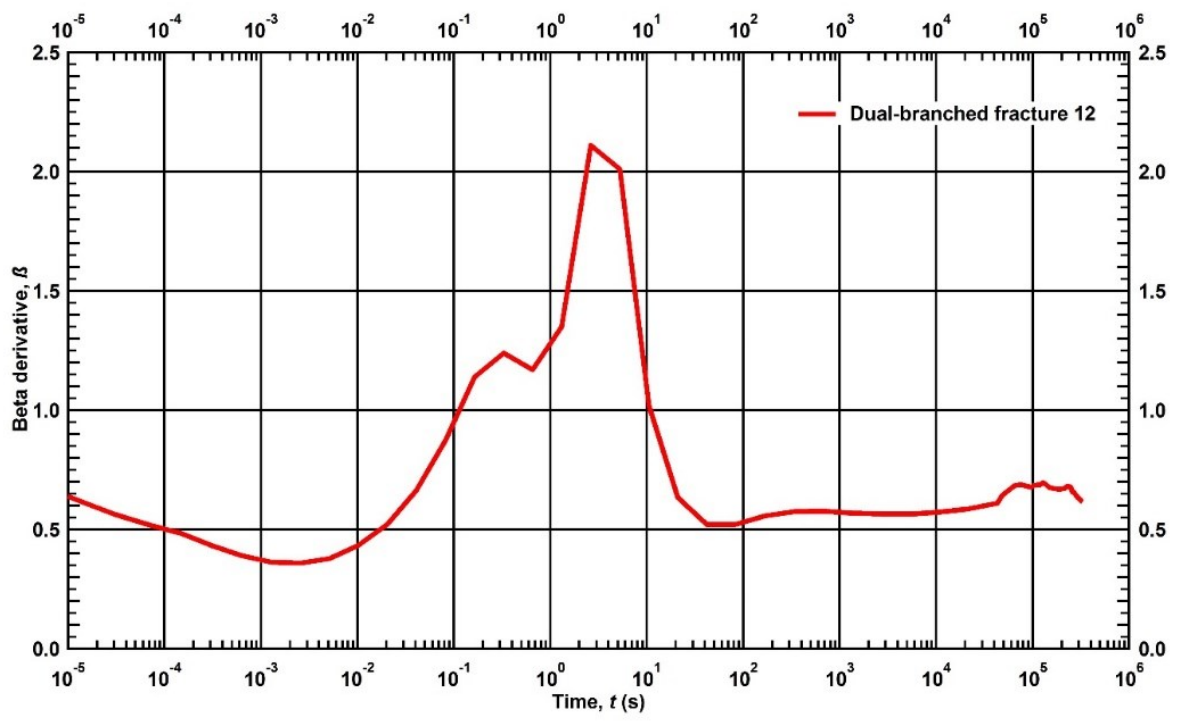

Figure C. 36 - $\beta$-derivative of the mass rate evolution for a shale gas reservoir produced from the dual-branched fracture 12 until 100 hours of production.

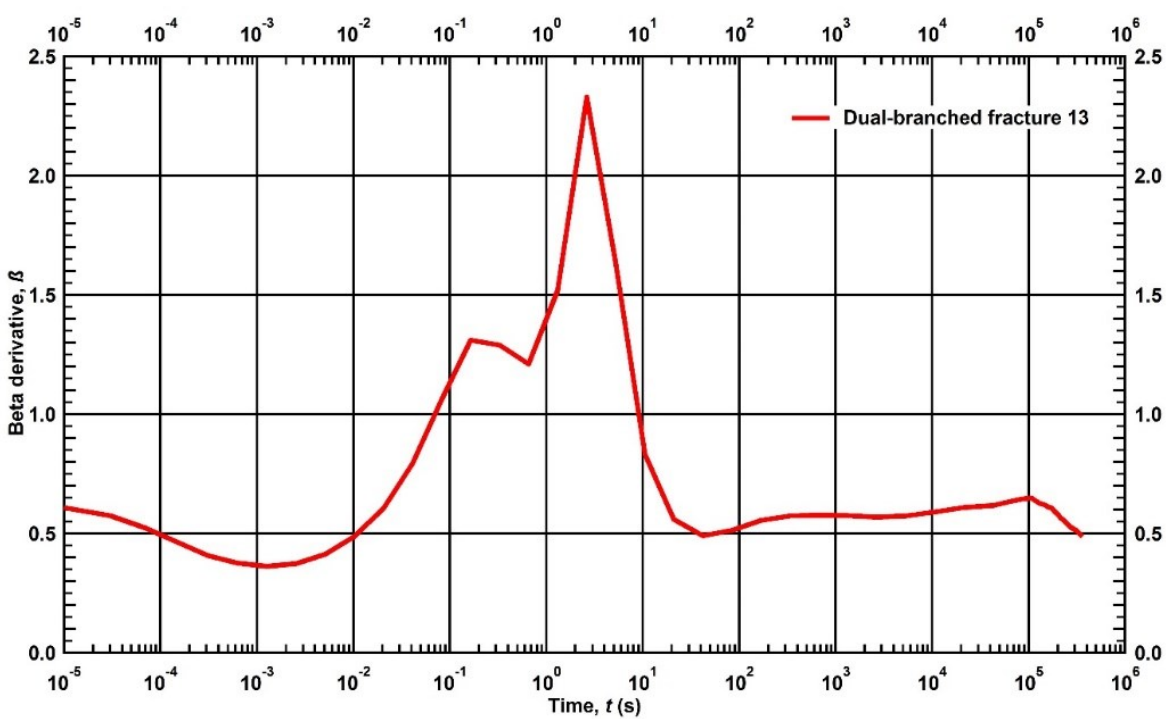

Figure C. 37 - $\beta$-derivative of the mass rate evolution for a shale gas reservoir produced from the dual-branched fracture 13 until 100 hours of production. 


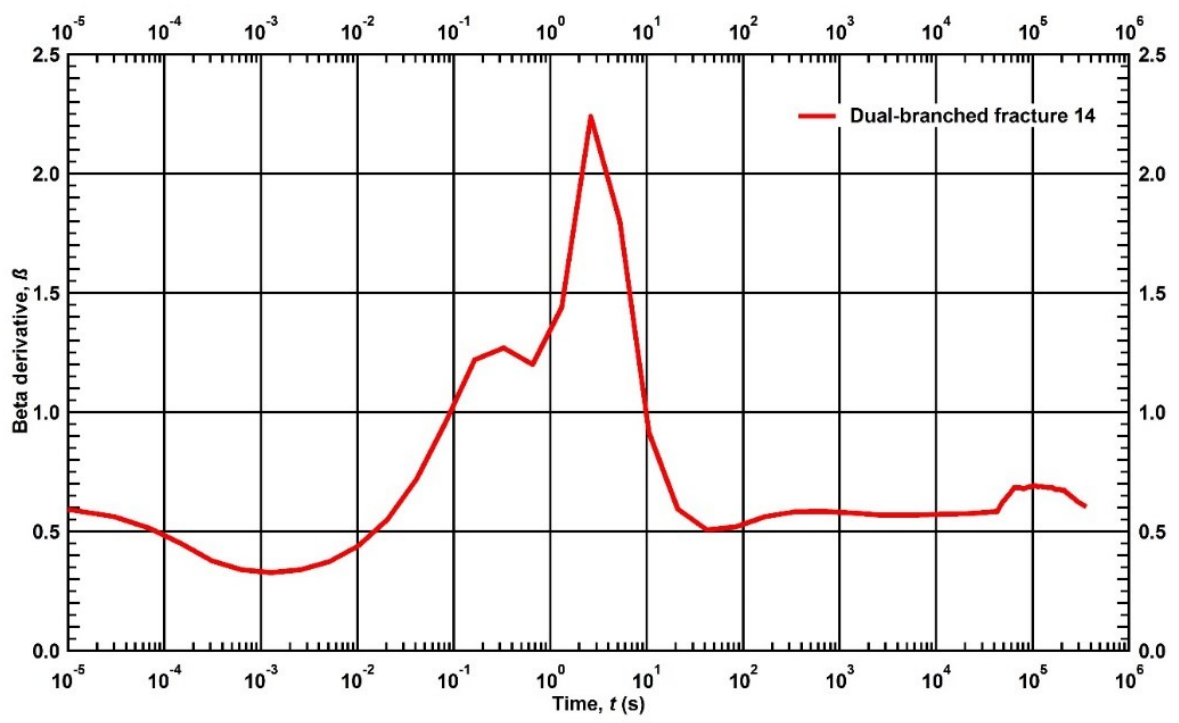

Figure C. 38 - $\beta$-derivative of the mass rate evolution for a shale gas reservoir produced from the dual-branched fracture 14 until 100 hours of production.

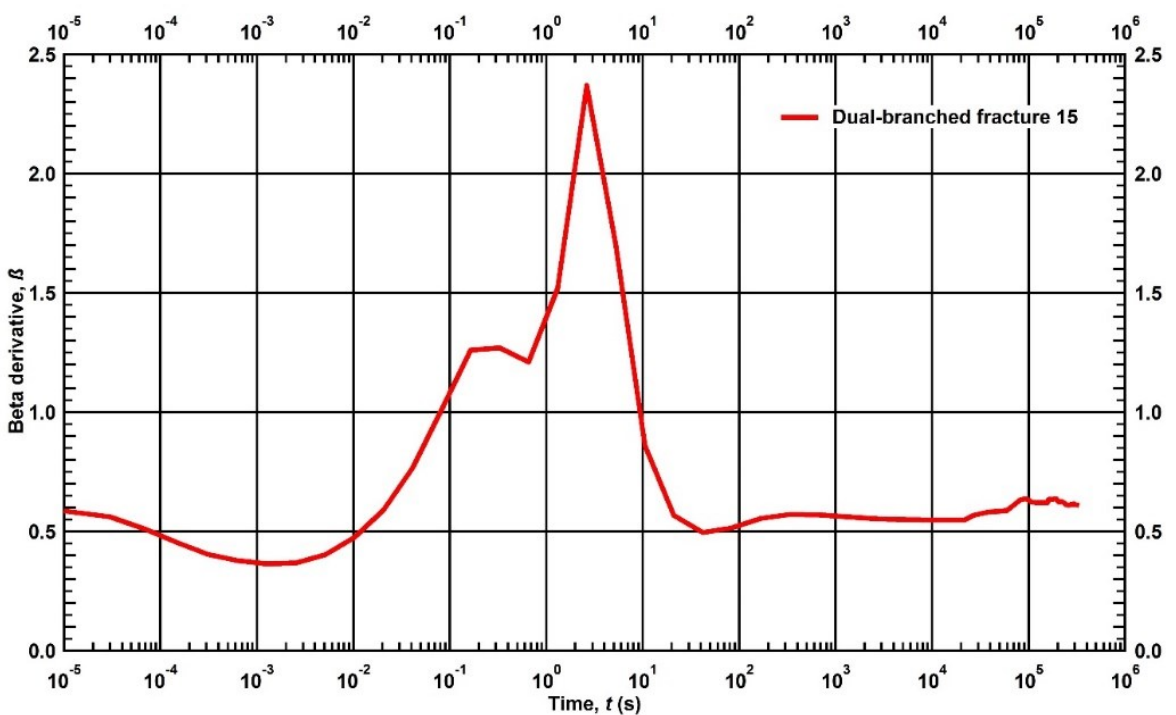

Figure C. 39 - $\beta$-derivative of the mass rate evolution for a shale gas reservoir produced from the dual-branched fracture 15 until 100 hours of production. 


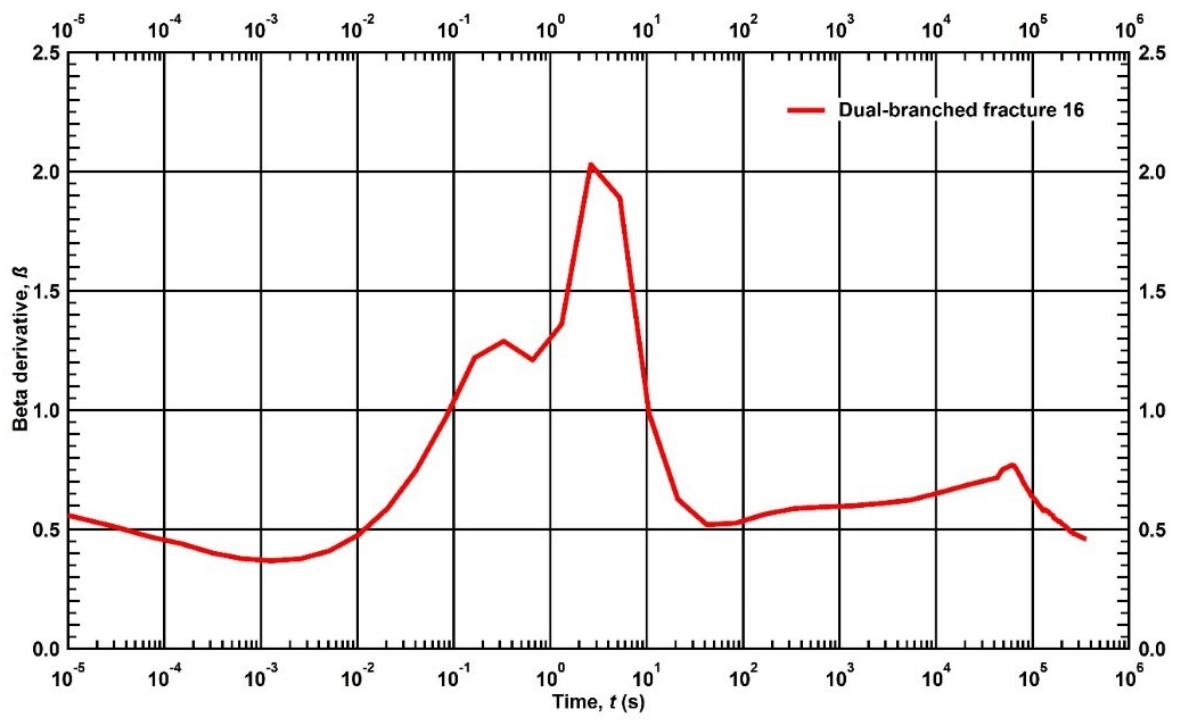

Figure C. 40 - $\beta$-derivative of the mass rate evolution for a shale gas reservoir produced from the dual-branched fracture 16 until 100 hours of production.

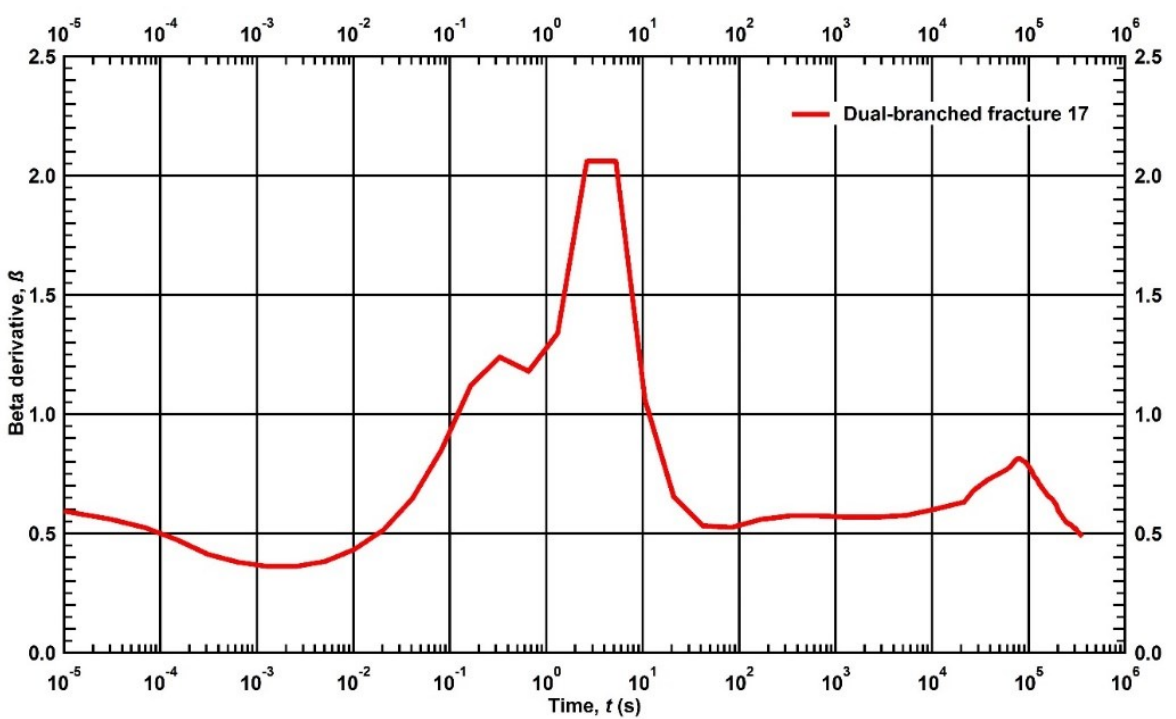

Figure C. 41 - $\beta$-derivative of the mass rate evolution for a shale gas reservoir produced from the dual-branched fracture 17 until 100 hours of production. 


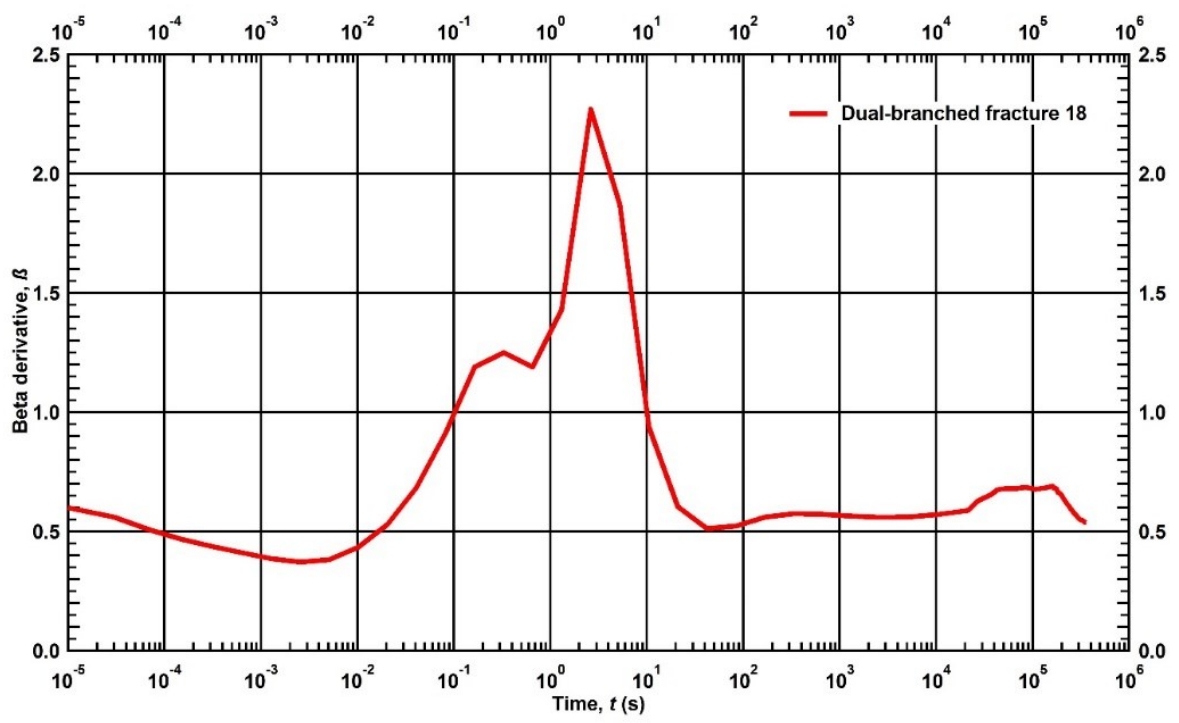

Figure C. 42 - $\beta$-derivative of the mass rate evolution for a shale gas reservoir produced from the dual-branched fracture 18 until 100 hours of production.

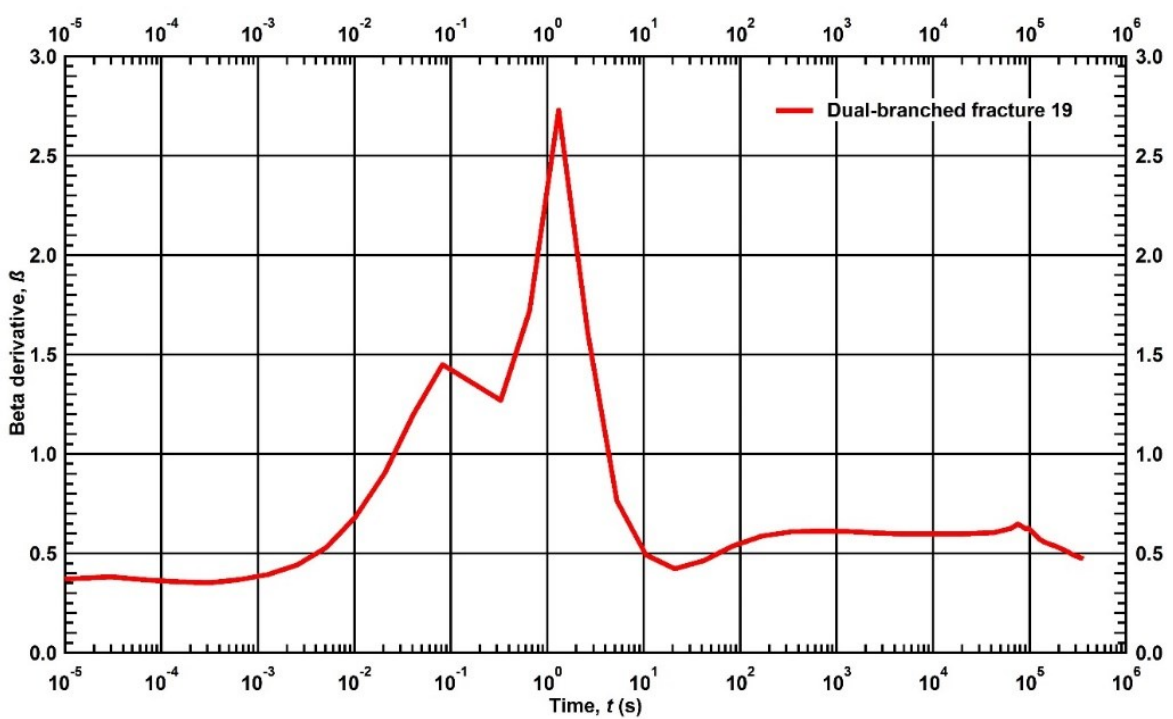

Figure C. 43 - $\beta$-derivative of the mass rate evolution for a shale gas reservoir produced from the dual-branched fracture 19 until 100 hours of production. 


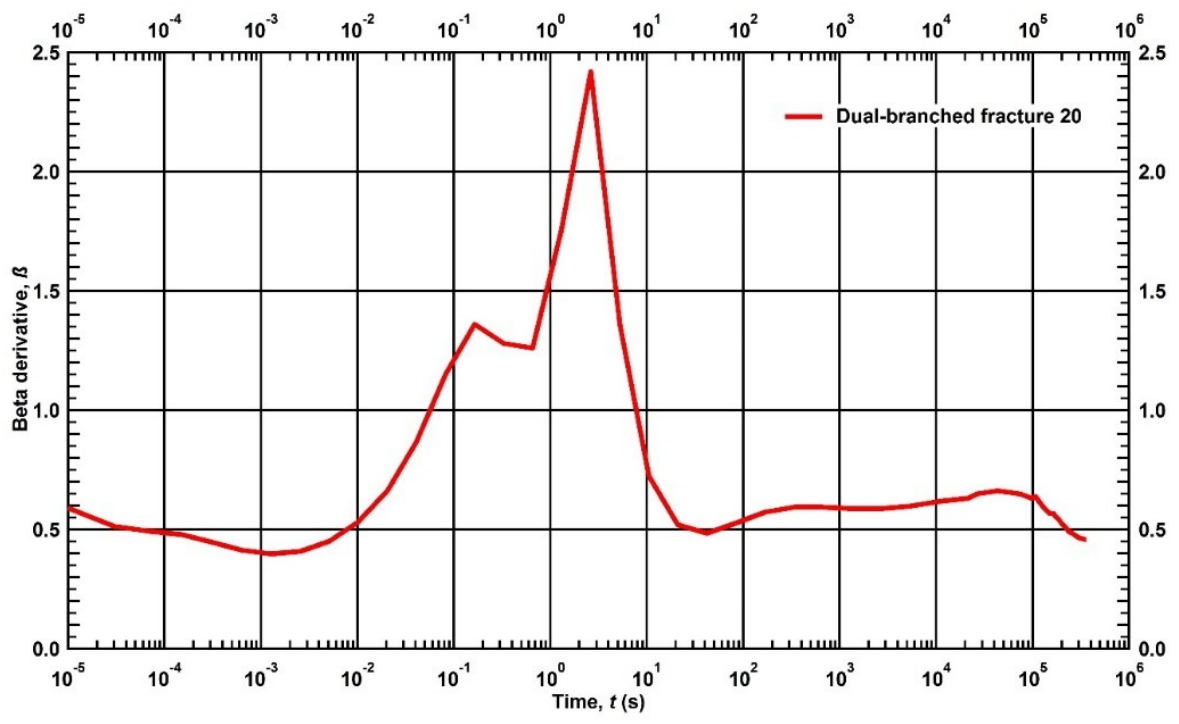

Figure C. 44 - $\beta$-derivative of the mass rate evolution for a shale gas reservoir produced from the dual-branched fracture 20 until 100 hours of production.

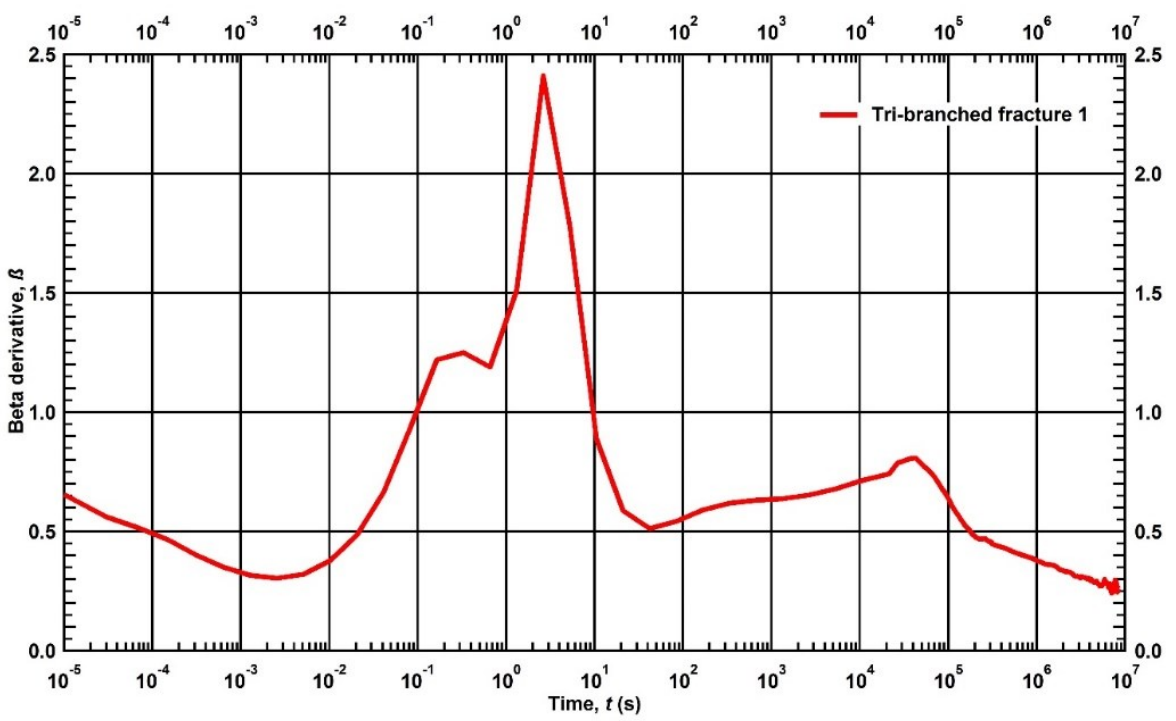

Figure C. 45 - $\beta$-derivative of the mass rate evolution for a shale gas reservoir produced from the tri-branched fracture 1 until 100 days of production. 


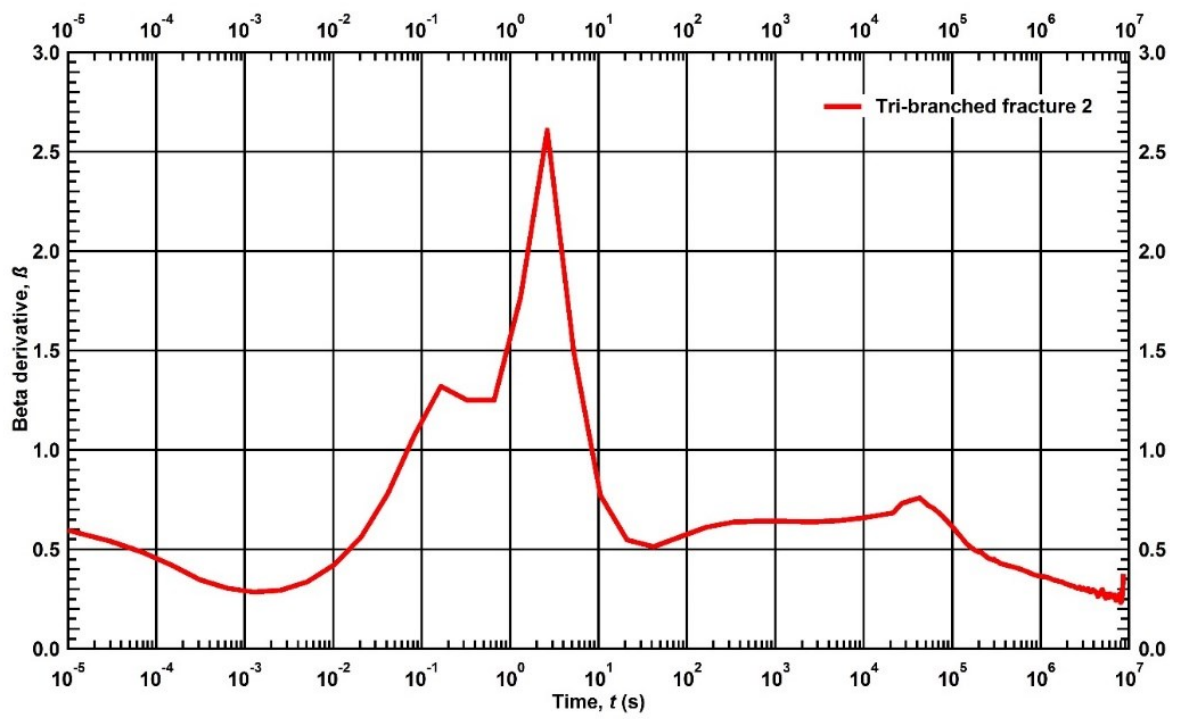

Figure C. 46 - $\beta$-derivative of the mass rate evolution for a shale gas reservoir produced from the tri-branched fracture 2 until 100 days of production.

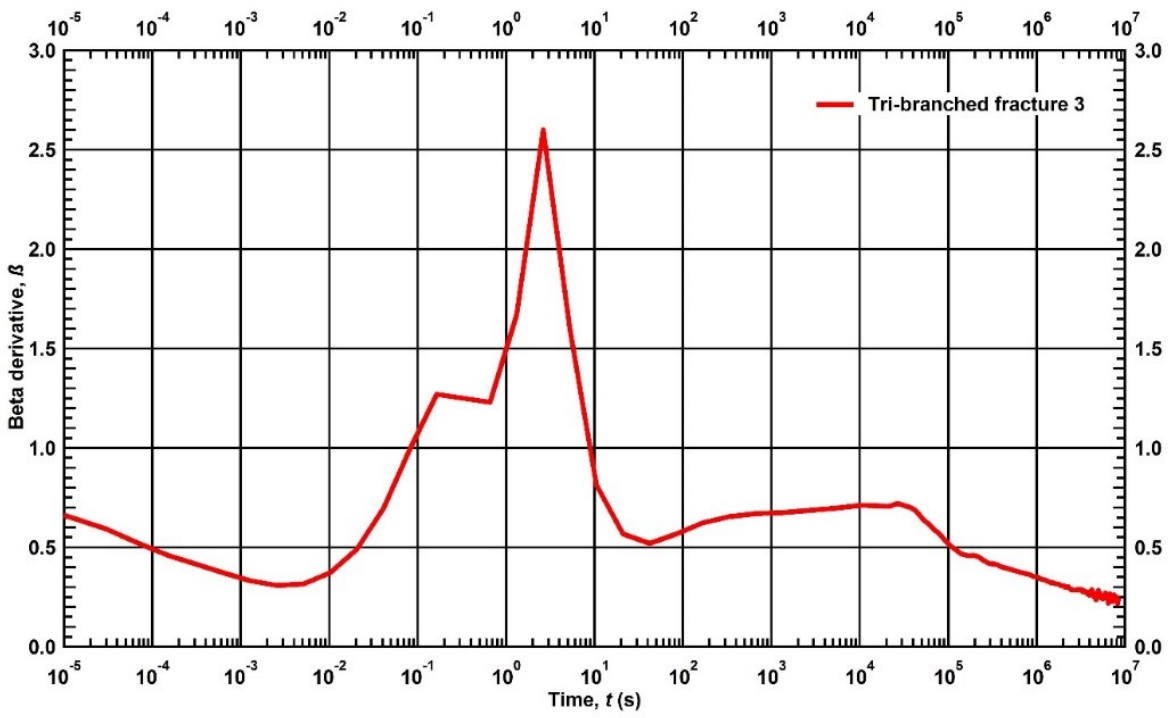

Figure C. 47 - $\beta$-derivative of the mass rate evolution for a shale gas reservoir produced from the tri-branched fracture 3 until 100 days of production. 


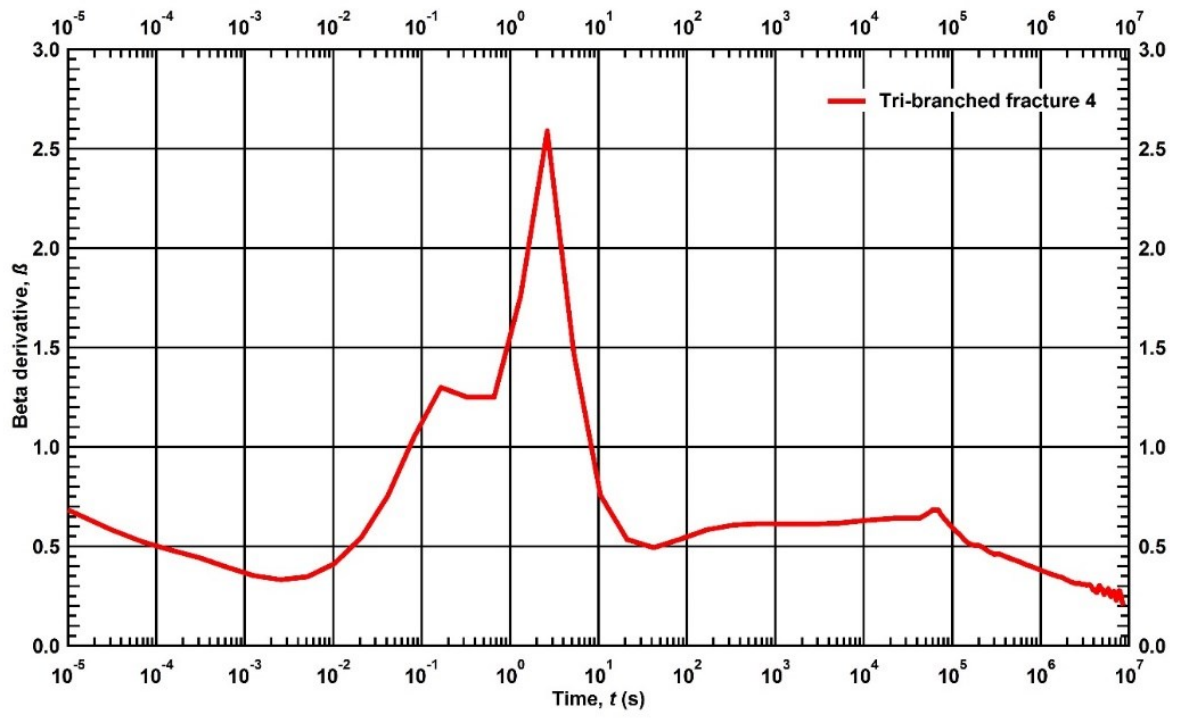

Figure C. 48 - $\beta$-derivative of the mass rate evolution for a shale gas reservoir produced from the tri-branched fracture 4 until 100 days of production.

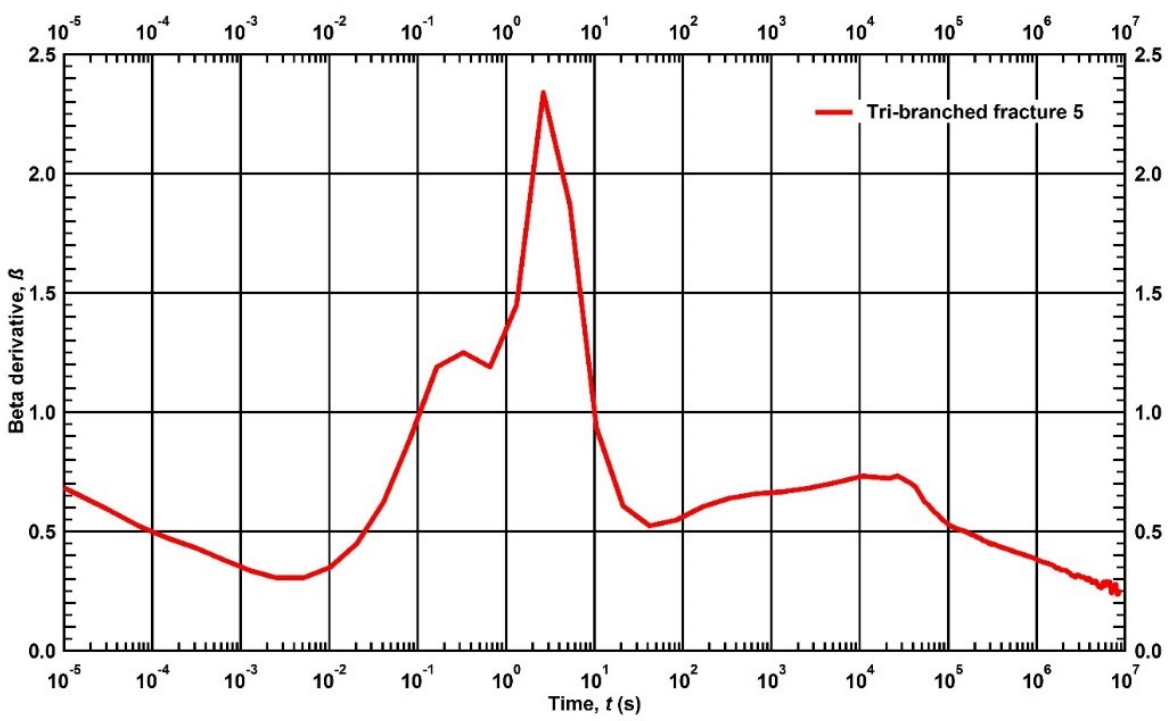

Figure C. 49 - $\beta$-derivative of the mass rate evolution for a shale gas reservoir produced from the tri-branched fracture 5 until 100 days of production. 


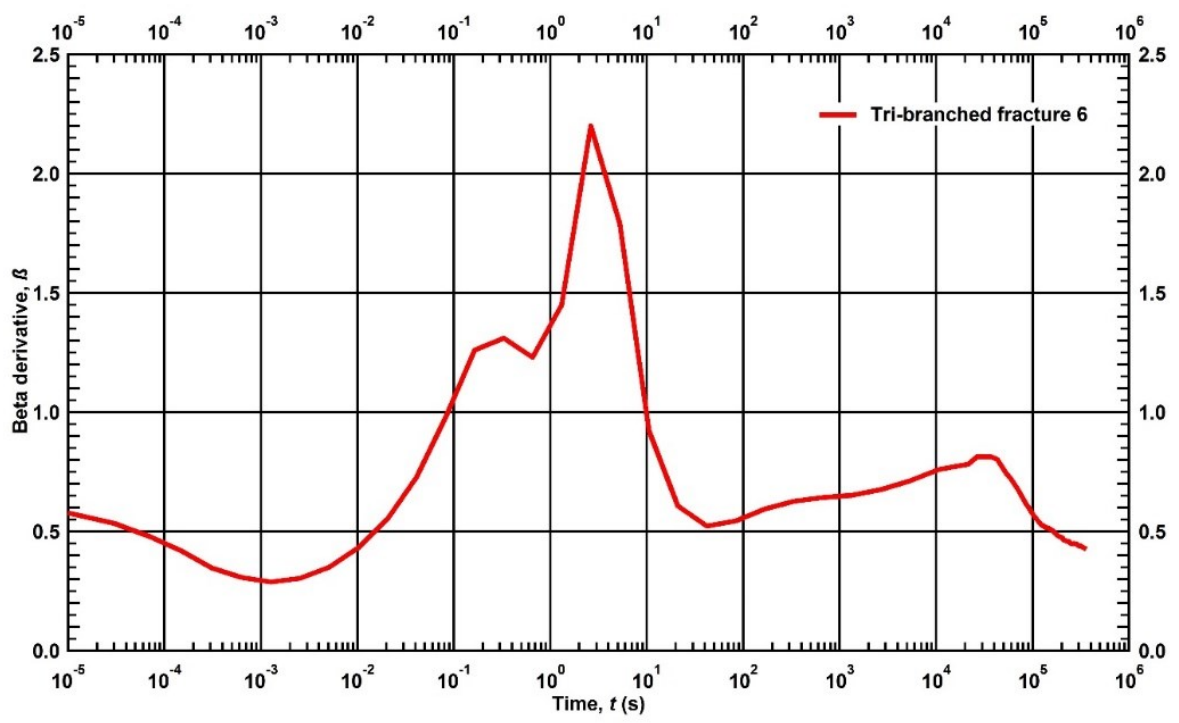

Figure C. 50 - $\beta$-derivative of the mass rate evolution for a shale gas reservoir produced from the tri-branched fracture 6 until 100 hours of production.

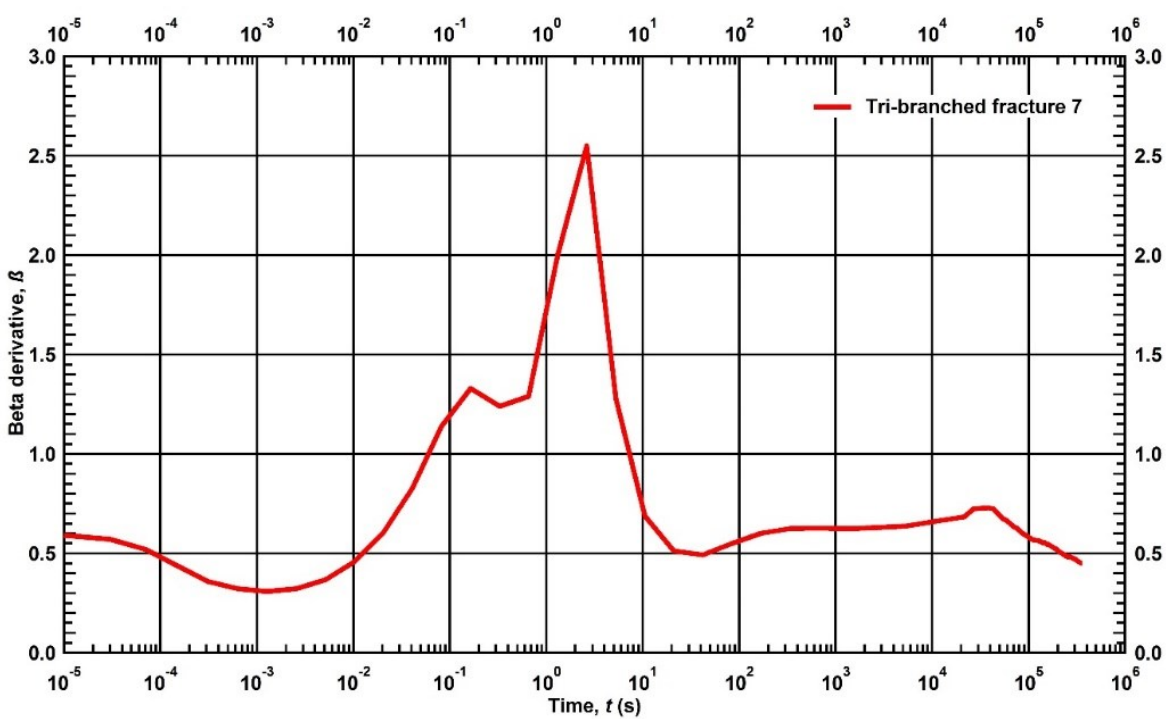

Figure C. 51 - $\beta$-derivative of the mass rate evolution for a shale gas reservoir produced from the tri-branched fracture 7 until 100 hours of production. 


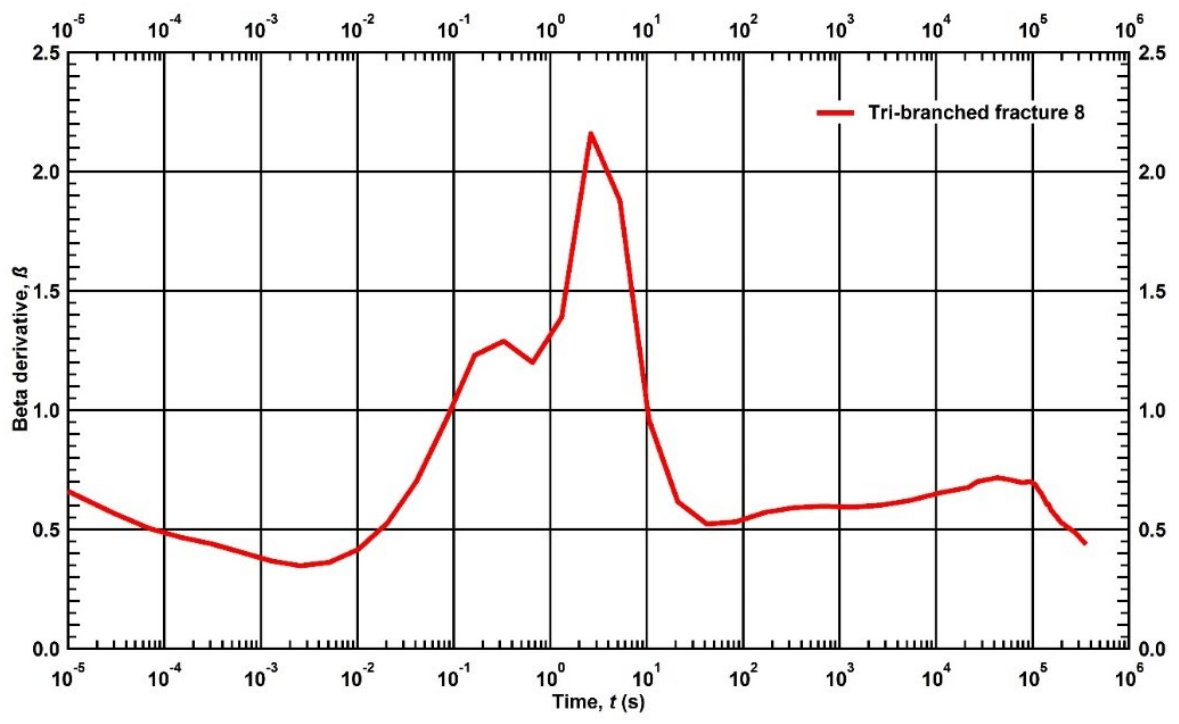

Figure C. 52 - $\beta$-derivative of the mass rate evolution for a shale gas reservoir produced from the tri-branched fracture 8 until 100 hours of production.

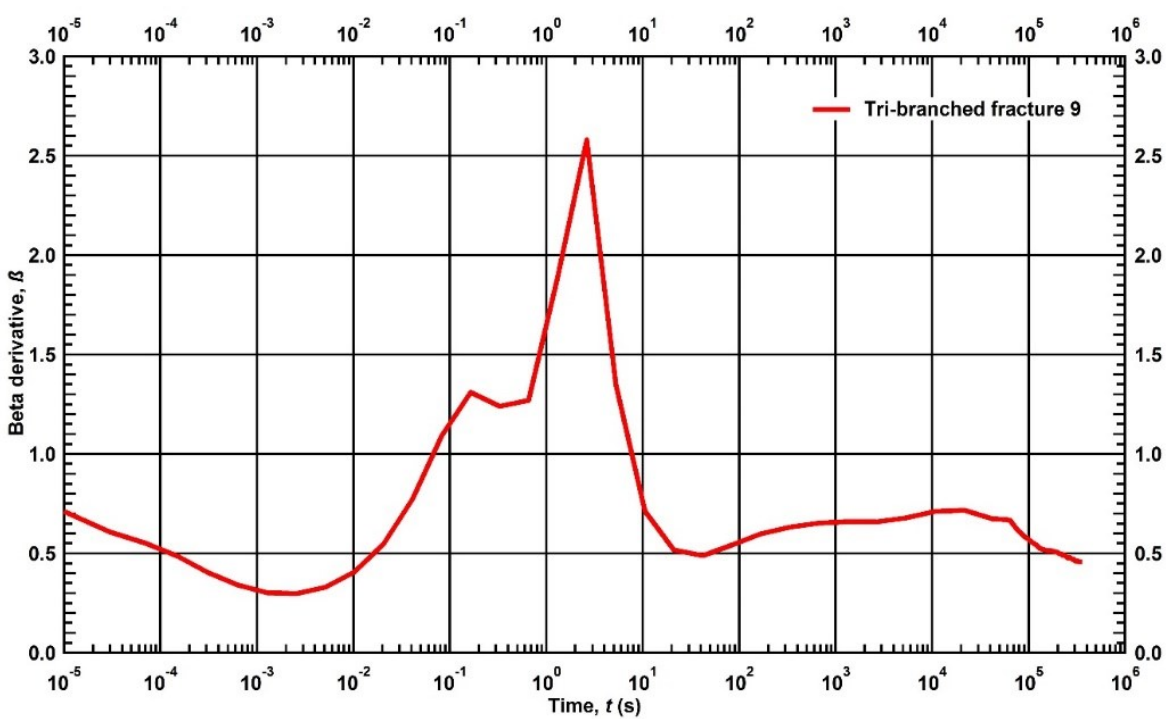

Figure C. 53 - $\beta$-derivative of the mass rate evolution for a shale gas reservoir produced from the tri-branched fracture 9 until 100 hours of production. 


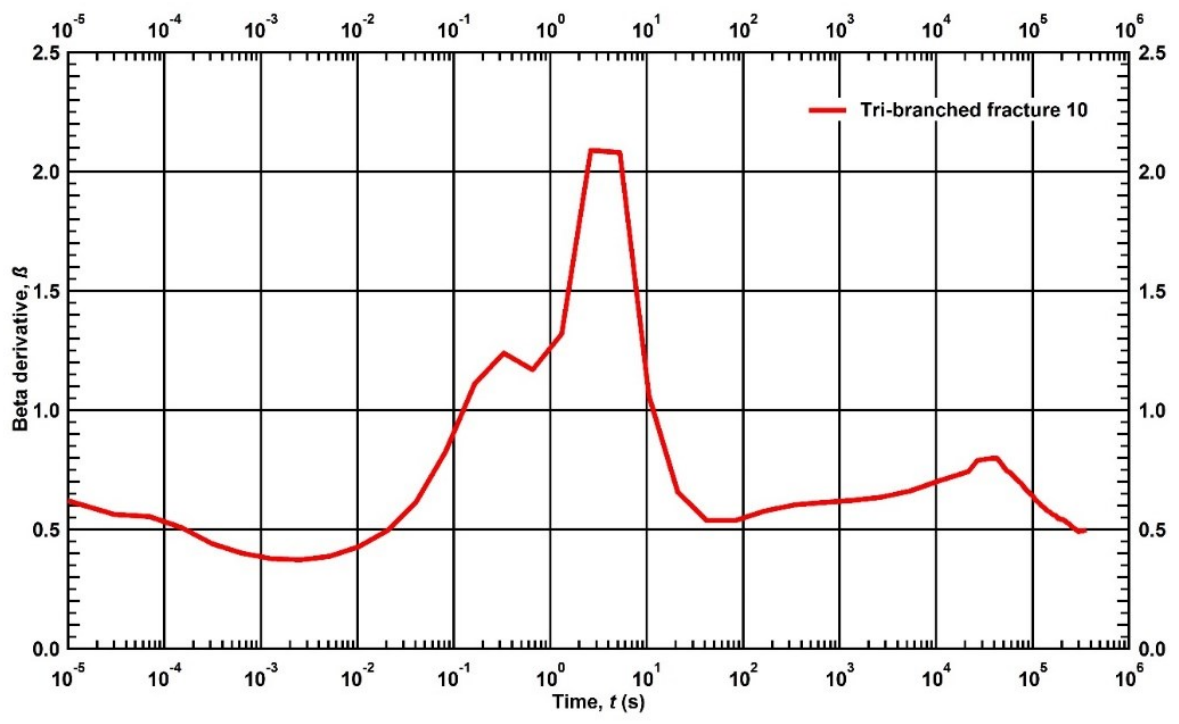

Figure C. 54 - $\beta$-derivative of the mass rate evolution for a shale gas reservoir produced from the tri-branched fracture 10 until 100 hours of production.

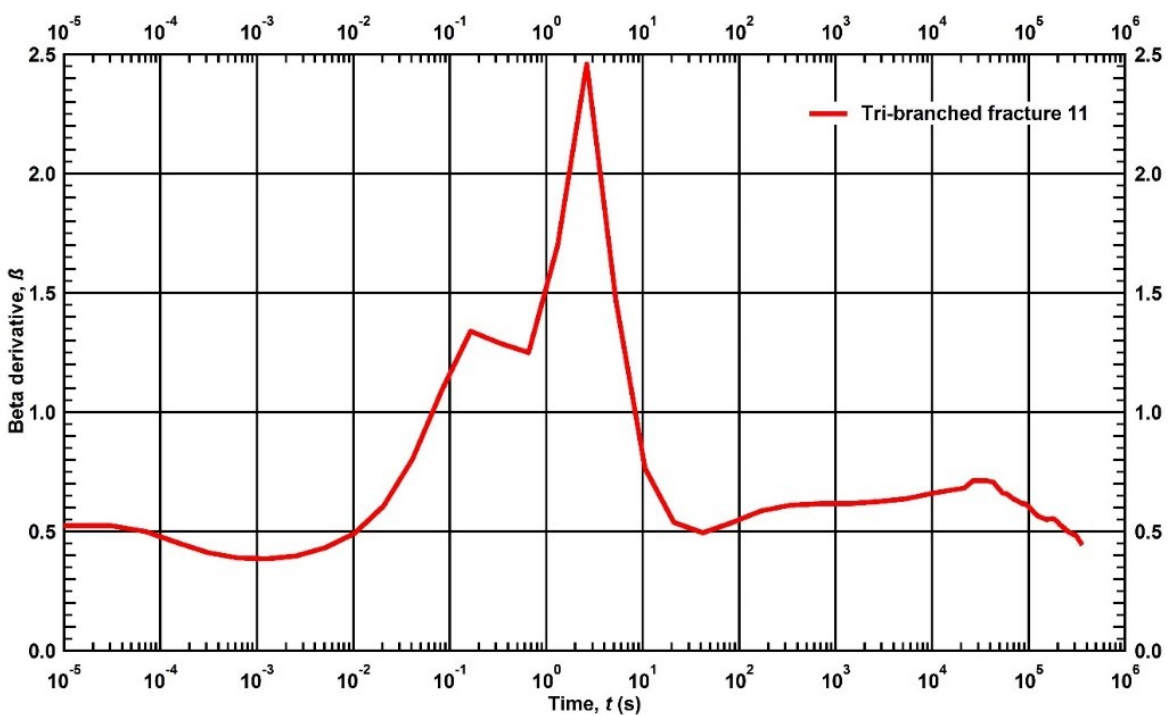

Figure C. 55 - $\beta$-derivative of the mass rate evolution for a shale gas reservoir produced from the tri-branched fracture 11 until 100 hours of production. 


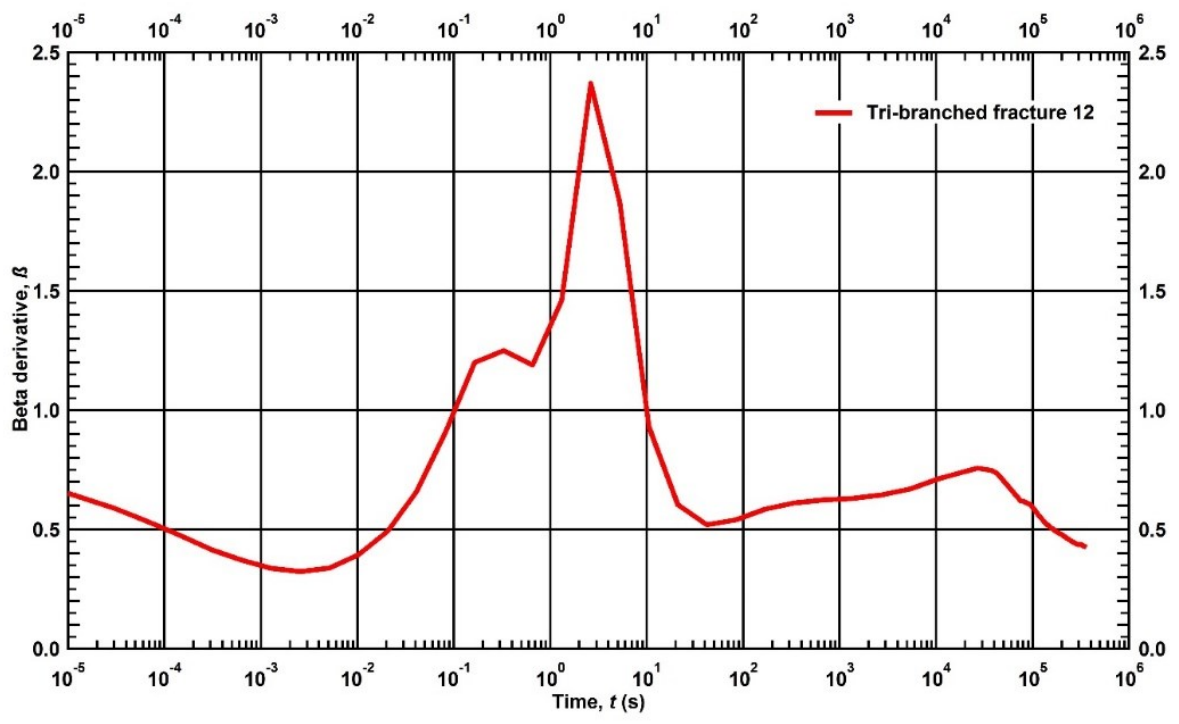

Figure C. 56 - $\beta$-derivative of the mass rate evolution for a shale gas reservoir produced from the tri-branched fracture 12 until 100 hours of production.

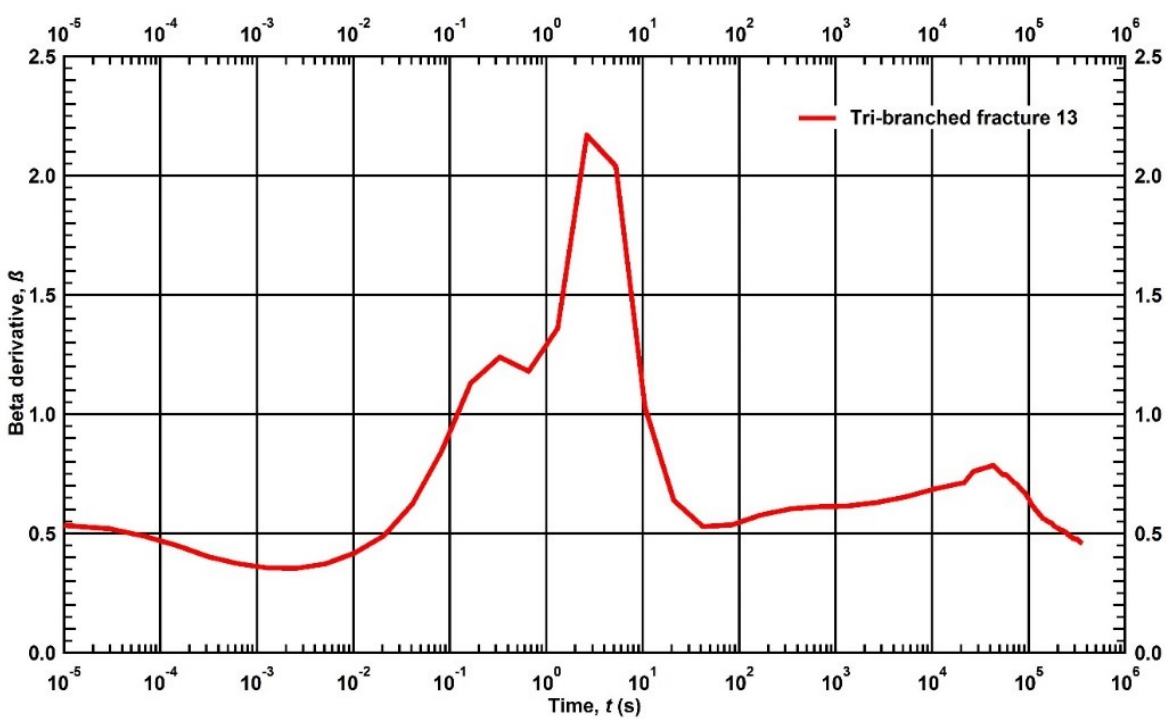

Figure C. 57 - $\beta$-derivative of the mass rate evolution for a shale gas reservoir produced from the tri-branched fracture 13 until 100 hours of production. 


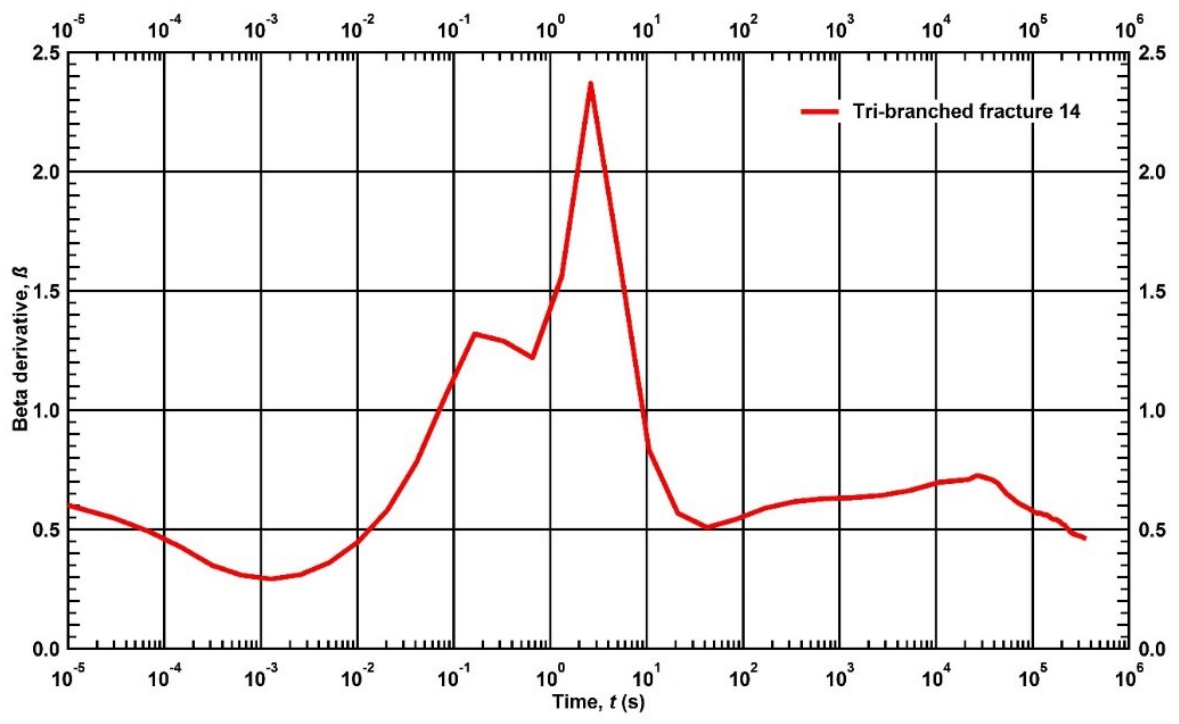

Figure C. 58 - $\beta$-derivative of the mass rate evolution for a shale gas reservoir produced from the tri-branched fracture 14 until 100 hours of production.

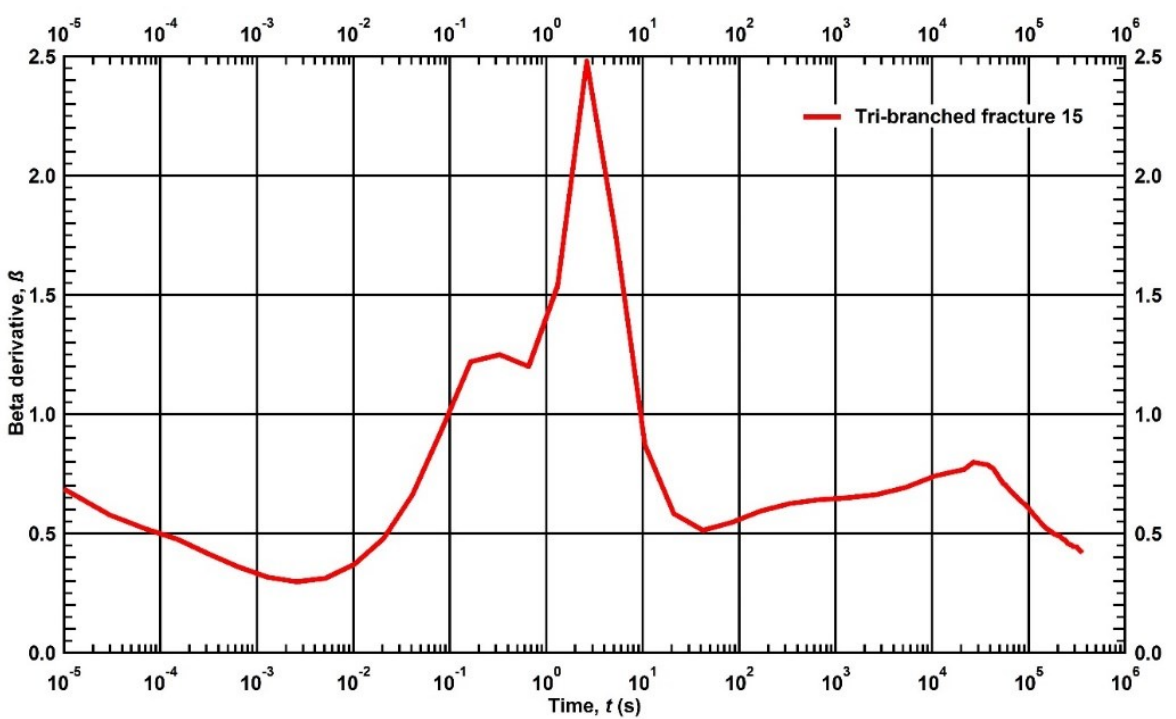

Figure C. 59 - $\beta$-derivative of the mass rate evolution for a shale gas reservoir produced from the tri-branched fracture 15 until 100 hours of production. 


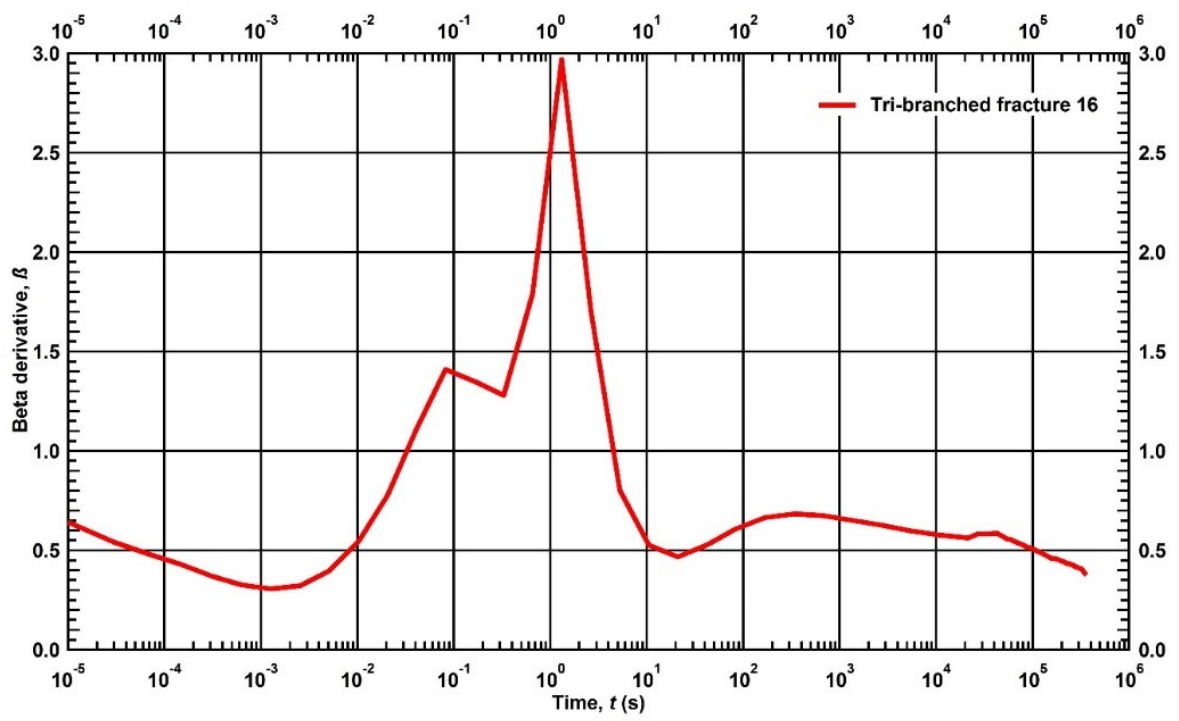

Figure C. 60 - $\beta$-derivative of the mass rate evolution for a shale gas reservoir produced from the tri-branched fracture 16 until 100 hours of production.

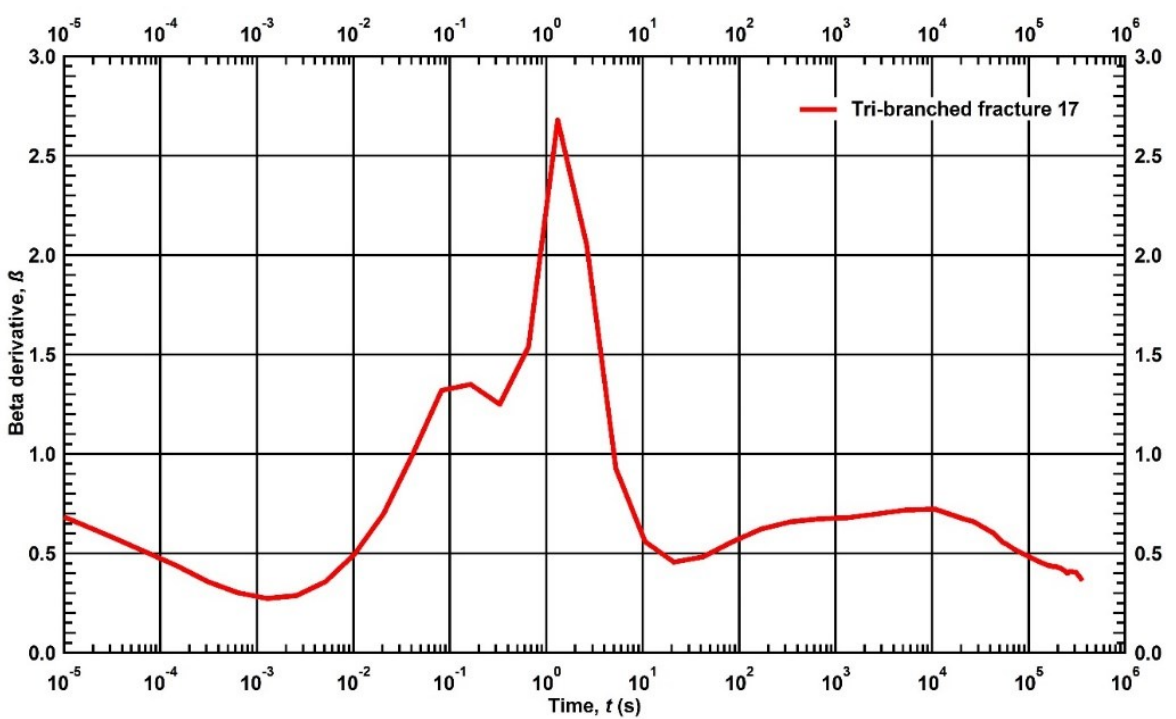

Figure C. 61 - $\beta$-derivative of the mass rate evolution for a shale gas reservoir produced from the tri-branched fracture 17 until 100 hours of production. 


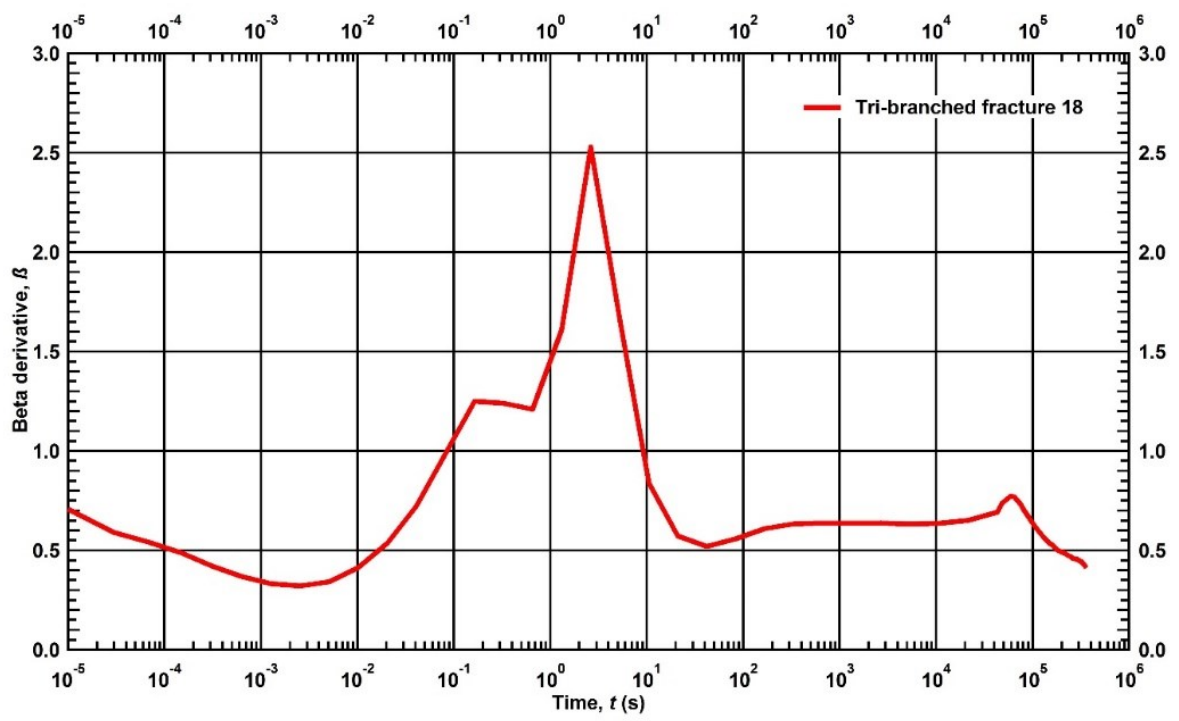

Figure C. 62 - $\beta$-derivative of the mass rate evolution for a shale gas reservoir produced from the tri-branched fracture 18 until 100 hours of production.

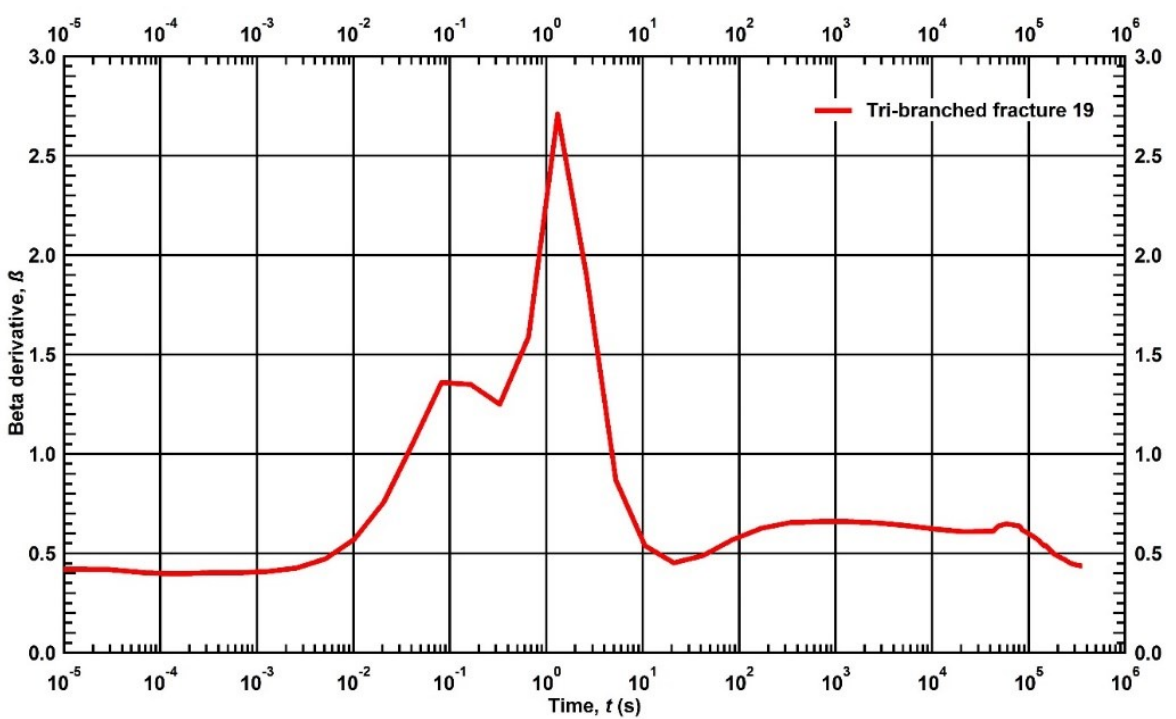

Figure C. 63 - $\beta$-derivative of the mass rate evolution for a shale gas reservoir produced from the tri-branched fracture 19 until 100 hours of production. 


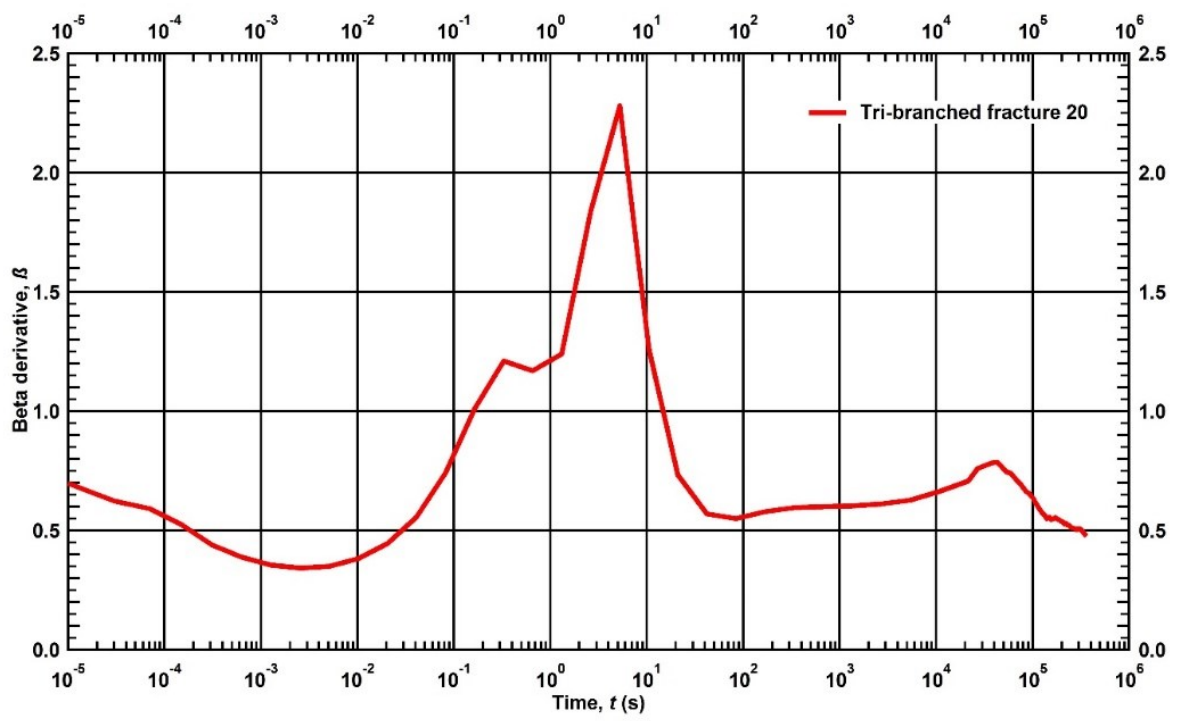

Figure C. 64 - $\beta$-derivative of the mass rate evolution for a shale gas reservoir produced from the tri-branched fracture 20 until 100 hours of production.

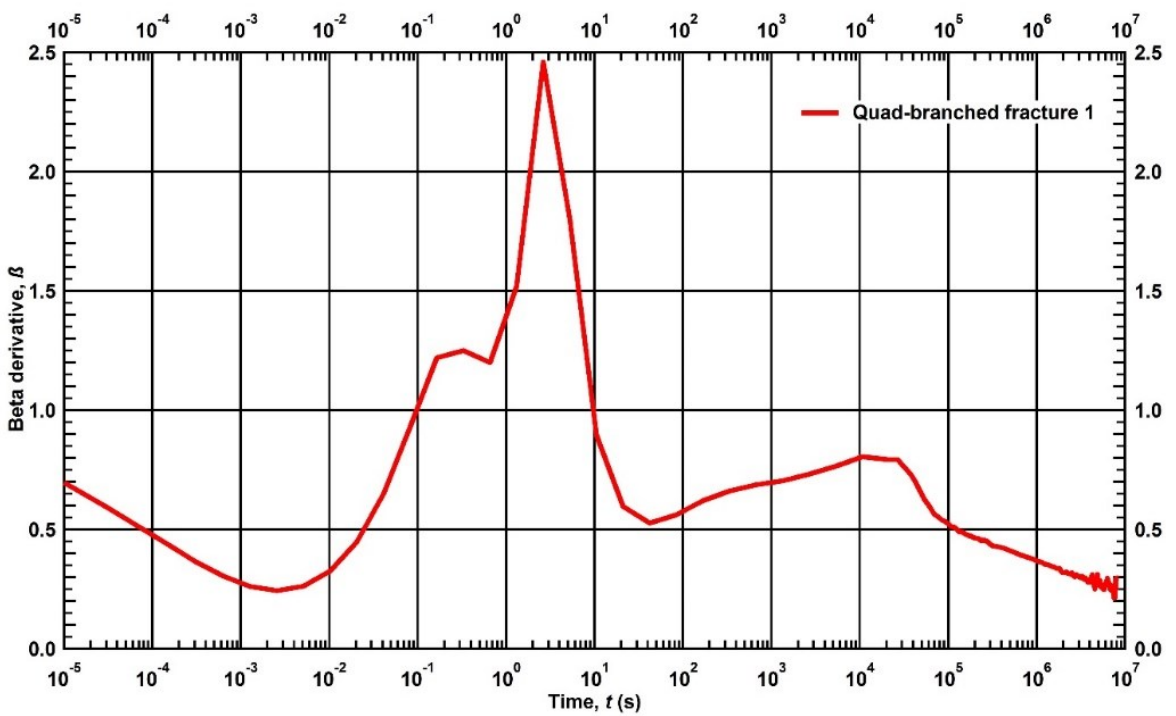

Figure C. 65 - $\beta$-derivative of the mass rate evolution for a shale gas reservoir produced from the quad-branched fracture 1 until 100 days of production. 


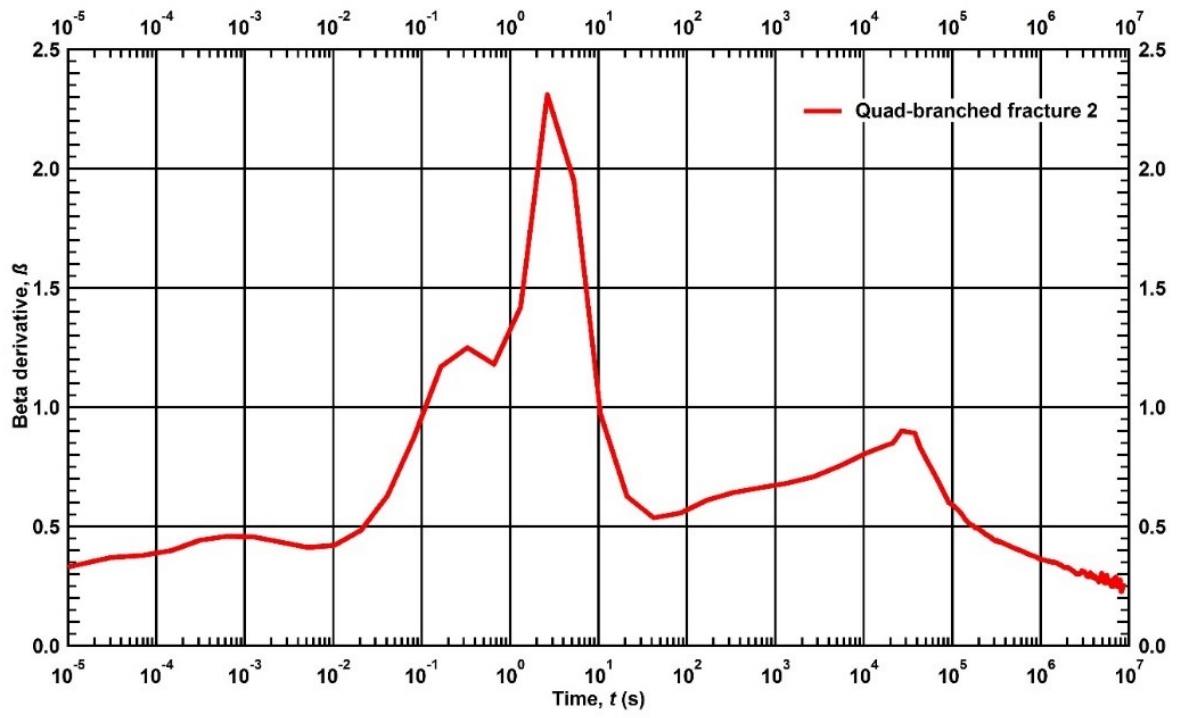

Figure C. 66 - $\beta$-derivative of the mass rate evolution for a shale gas reservoir produced from the quad-branched fracture 2 until 100 days of production.

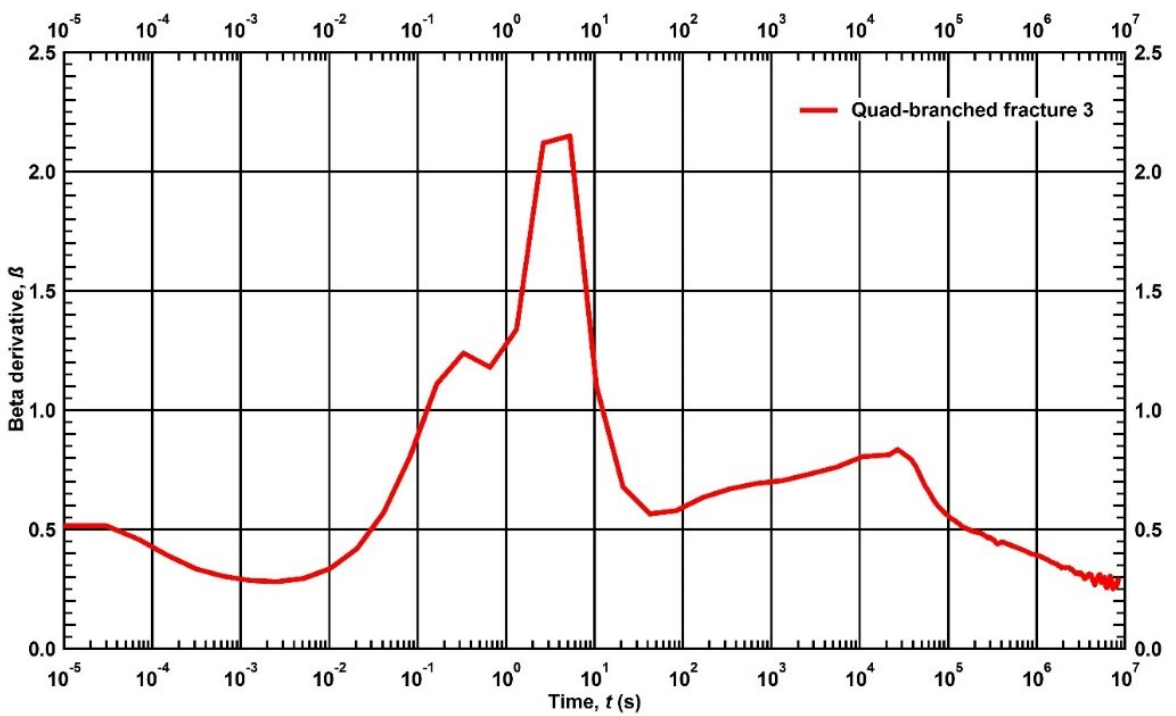

Figure C. 67 - $\beta$-derivative of the mass rate evolution for a shale gas reservoir produced from the quad-branched fracture 3 until 100 days of production. 


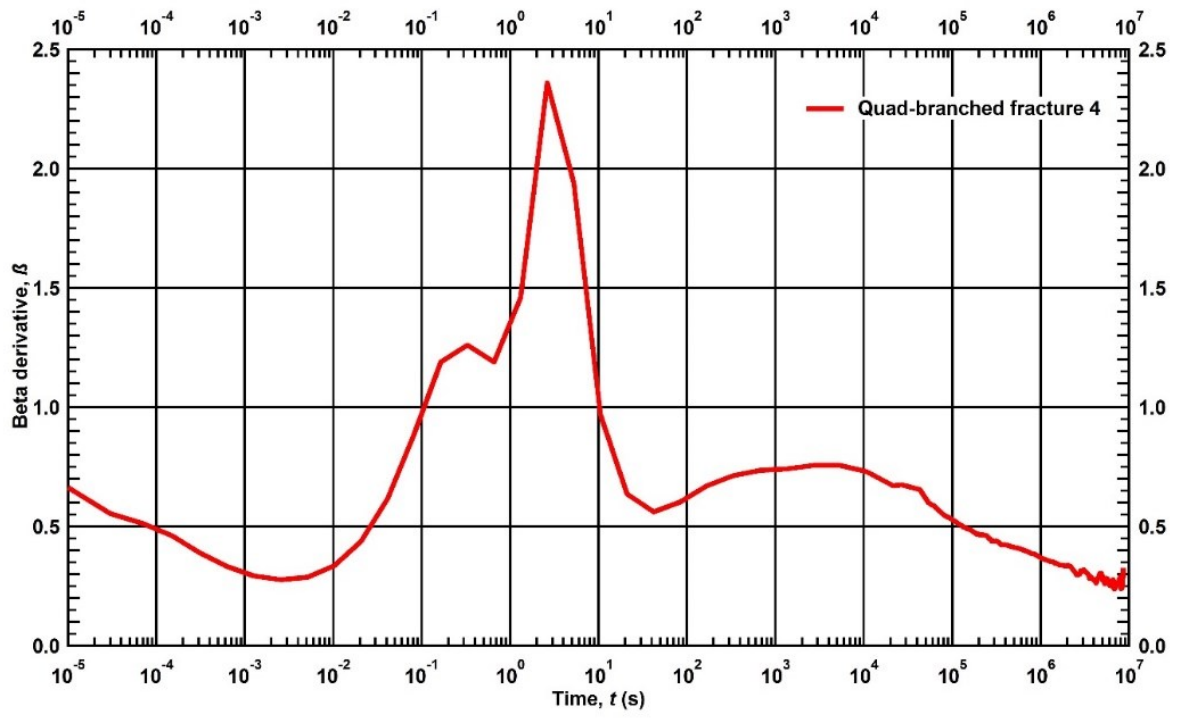

Figure C. 68 - $\beta$-derivative of the mass rate evolution for a shale gas reservoir produced from the quad-branched fracture 4 until 100 days of production.

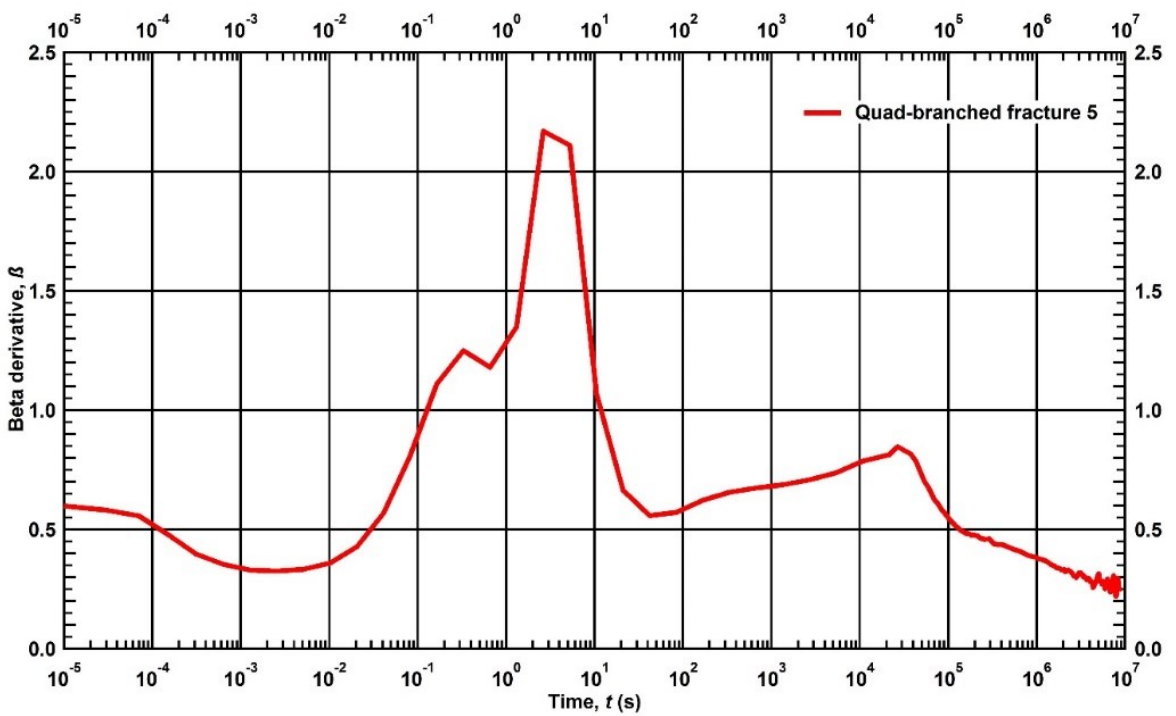

Figure C. 69 - $\beta$-derivative of the mass rate evolution for a shale gas reservoir produced from the quad-branched fracture 5 until 100 days of production. 


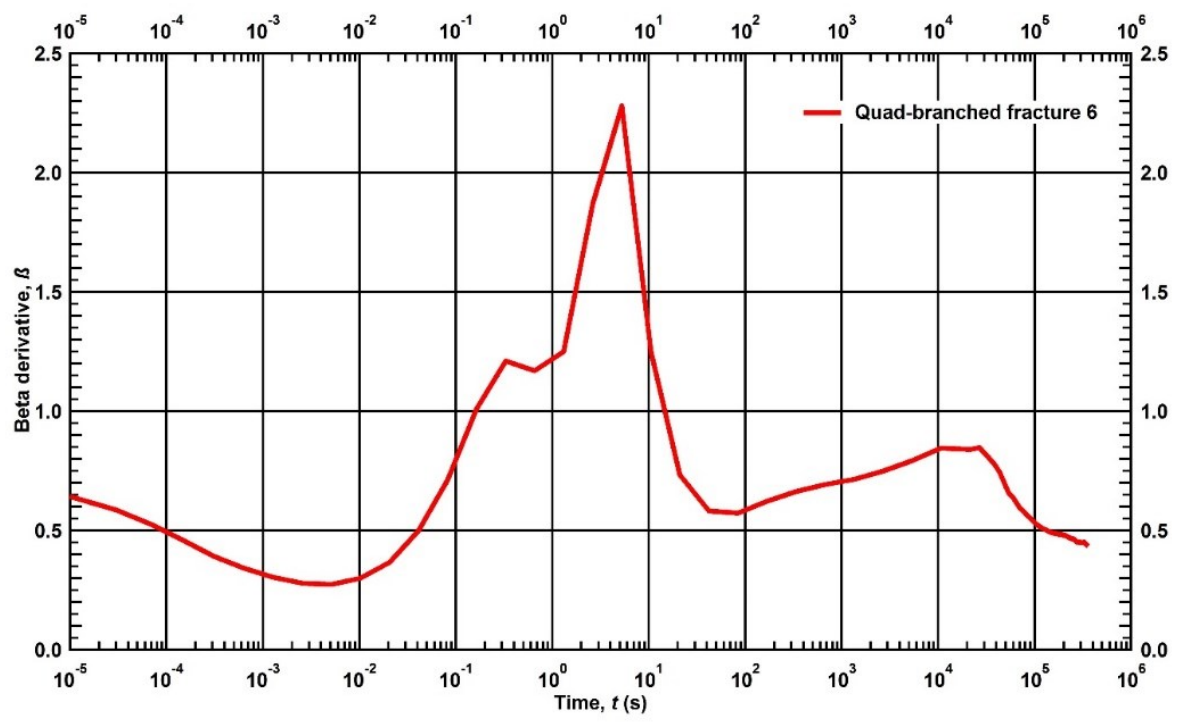

Figure C. 70 - $\beta$-derivative of the mass rate evolution for a shale gas reservoir produced from the quad-branched fracture 6 until 100 hours of production.

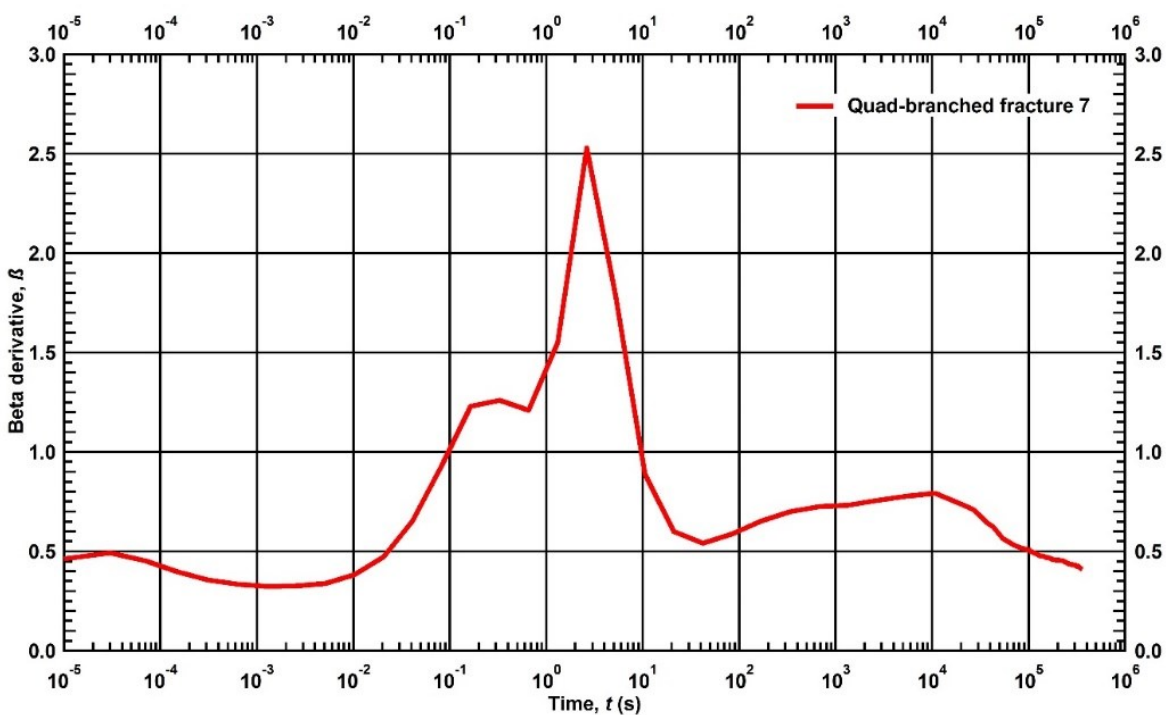

Figure C. 71 - $\beta$-derivative of the mass rate evolution for a shale gas reservoir produced from the quad-branched fracture 7 until 100 hours of production. 


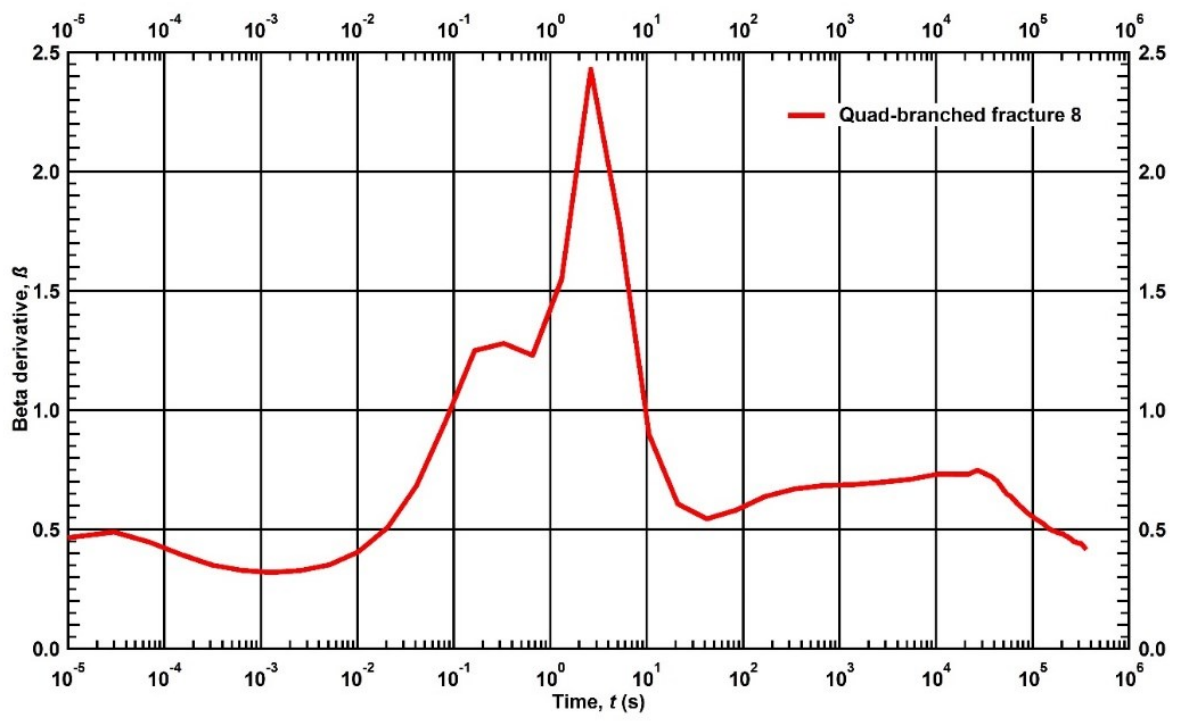

Figure C. 72 - $\beta$-derivative of the mass rate evolution for a shale gas reservoir produced from the quad-branched fracture 8 until 100 hours of production.

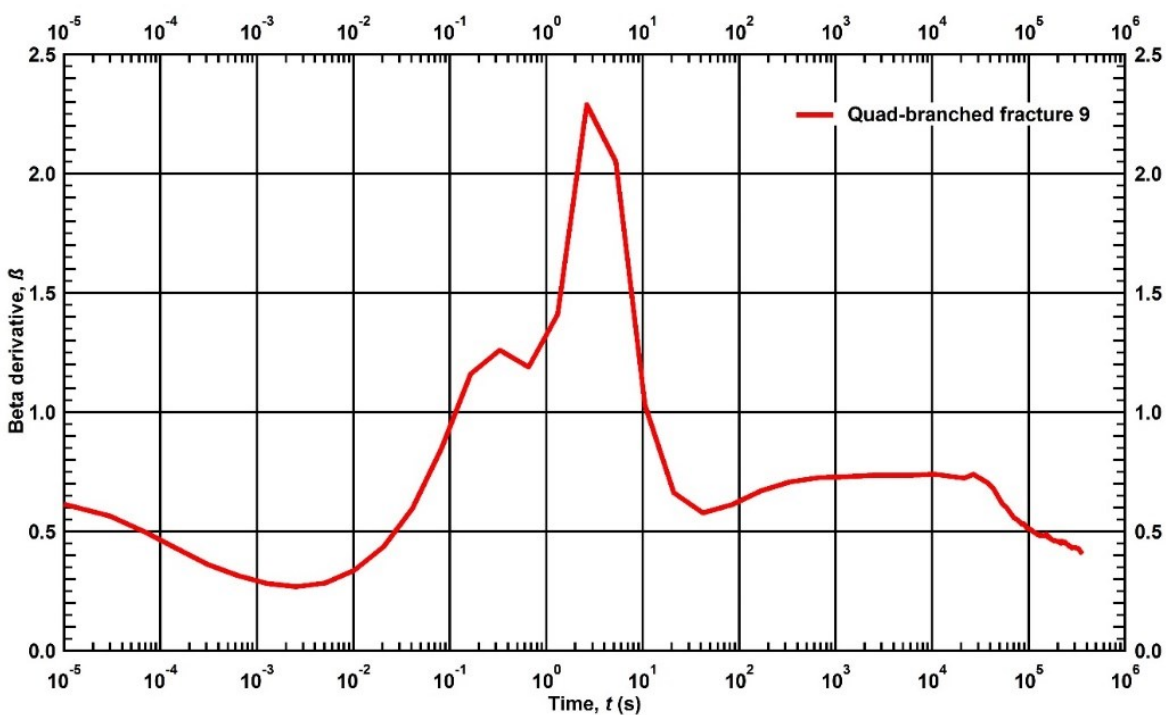

Figure C. 73 - $\beta$-derivative of the mass rate evolution for a shale gas reservoir produced from the quad-branched fracture 9 until 100 hours of production. 


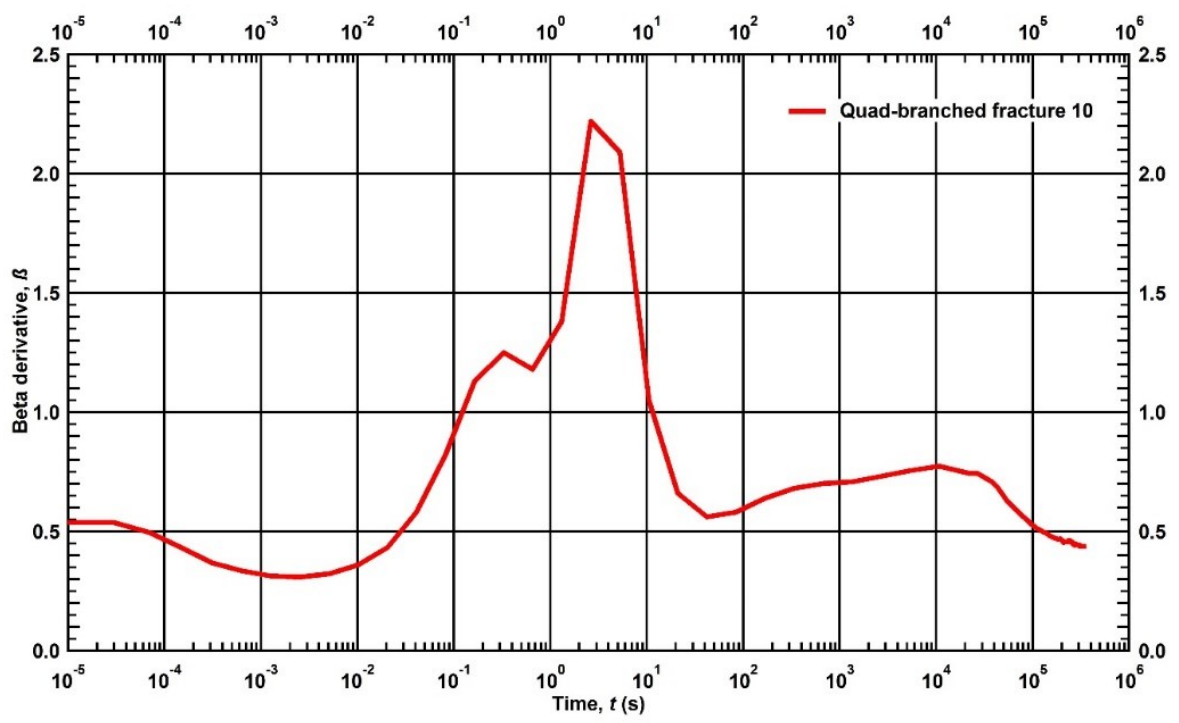

Figure C. 74 - $\beta$-derivative of the mass rate evolution for a shale gas reservoir produced from the quad-branched fracture 10 until 100 hours of production.

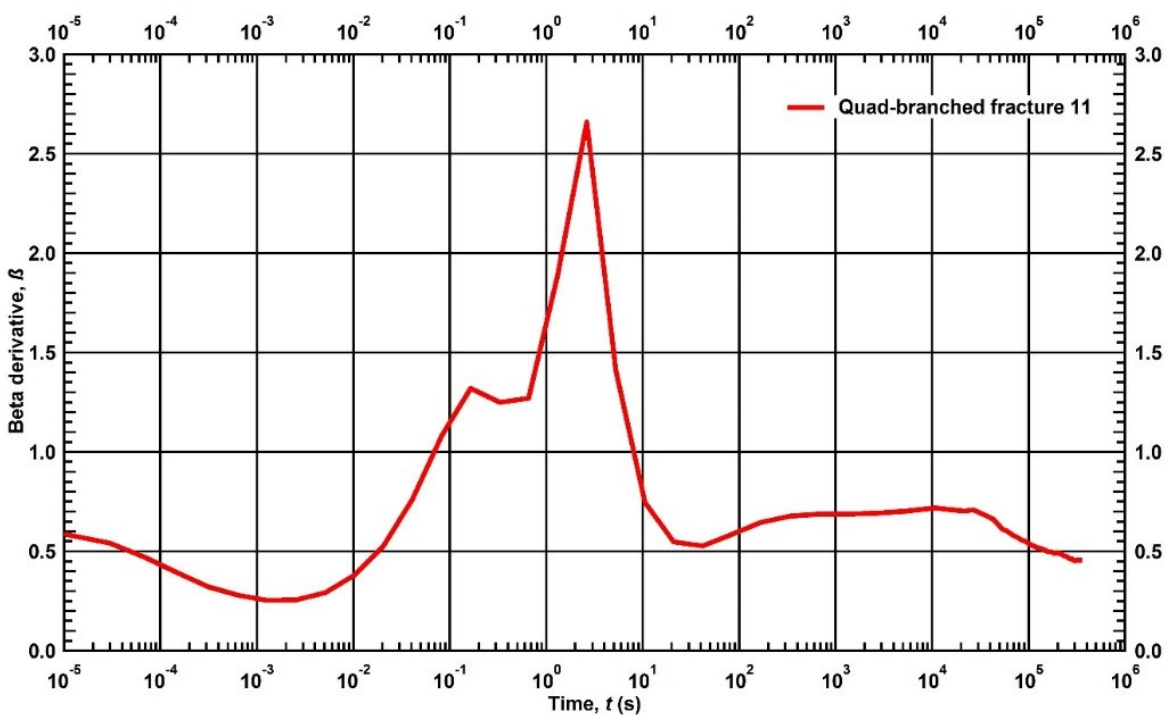

Figure C. 75 - $\beta$-derivative of the mass rate evolution for a shale gas reservoir produced from the quad-branched fracture 11 until 100 hours of production. 


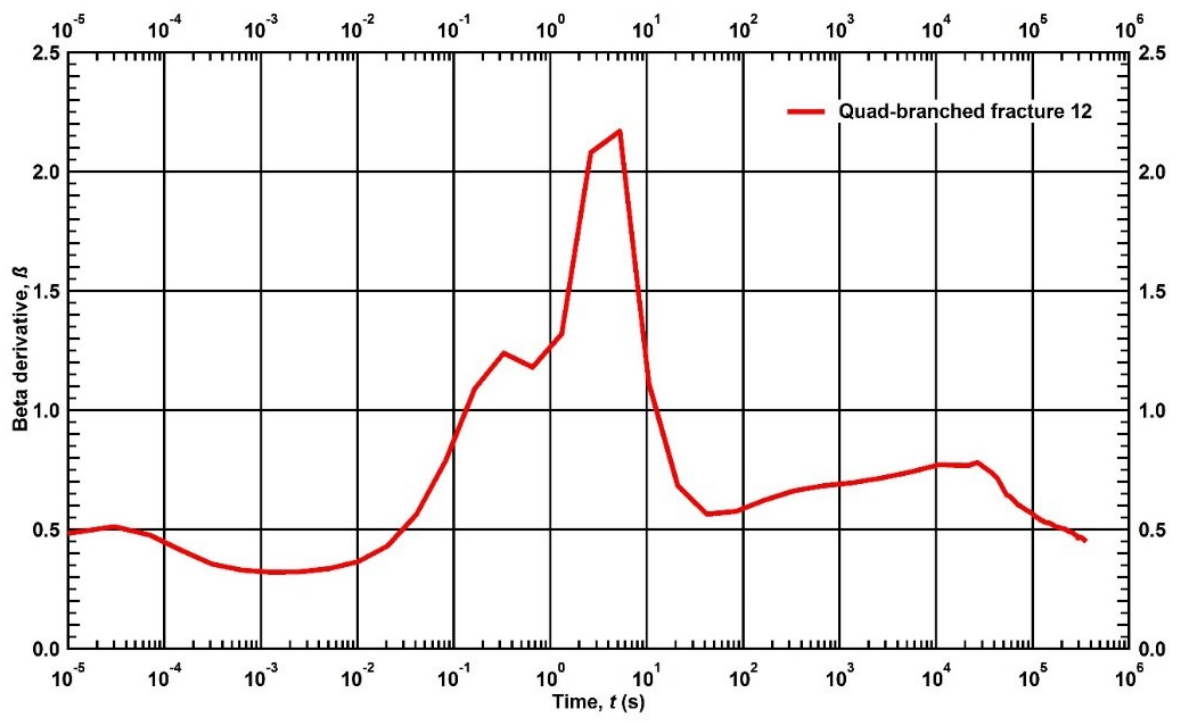

Figure C. 76 - $\beta$-derivative of the mass rate evolution for a shale gas reservoir produced from the quad-branched fracture 12 until 100 hours of production.

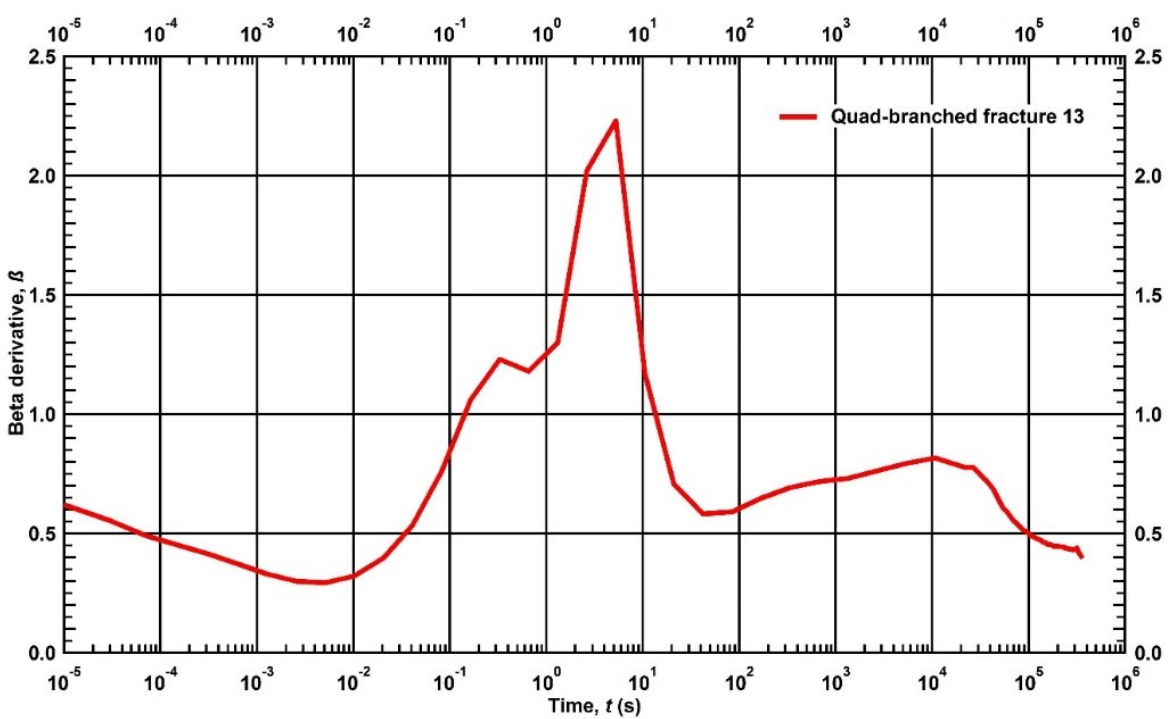

Figure C. 77 - $\beta$-derivative of the mass rate evolution for a shale gas reservoir produced from the quad-branched fracture 13 until 100 hours of production. 


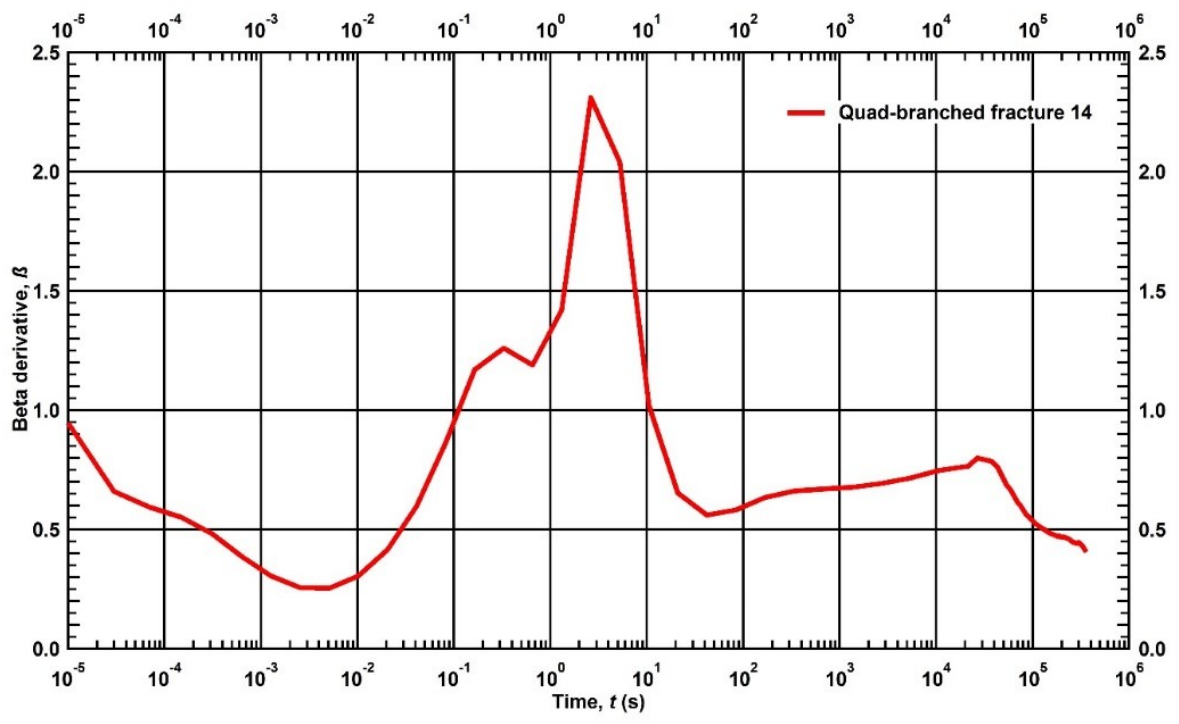

Figure C. 78 - $\beta$-derivative of the mass rate evolution for a shale gas reservoir produced from the quad-branched fracture 14 until 100 hours of production.

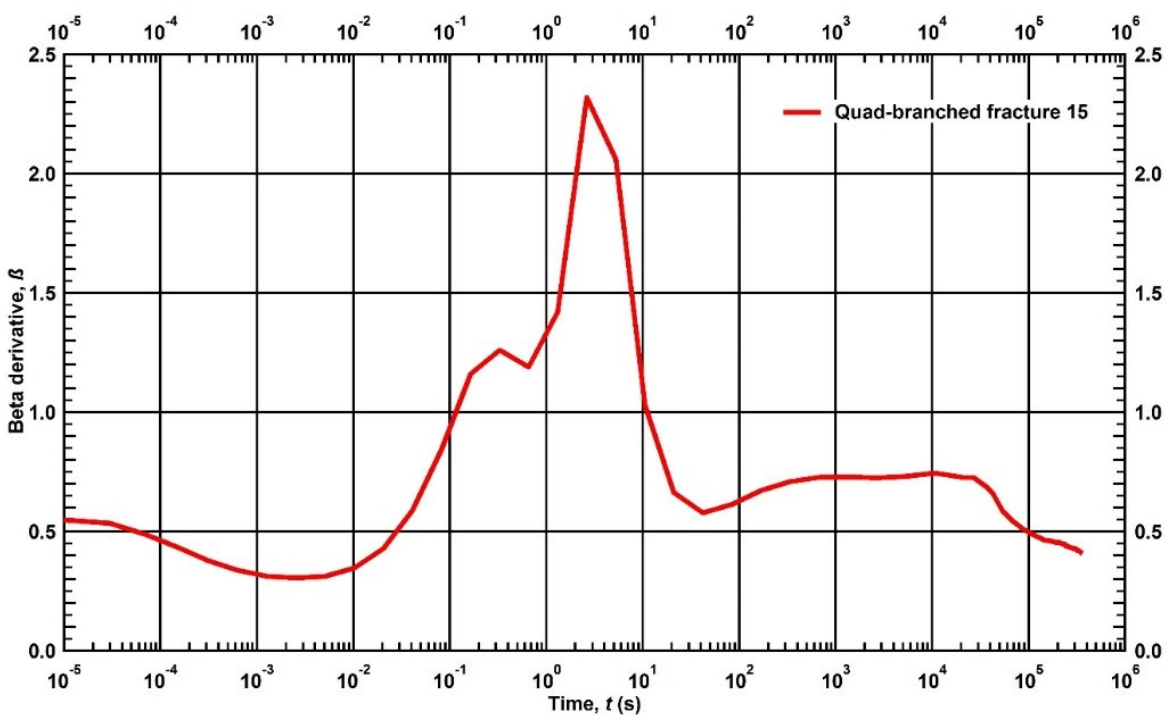

Figure C. 79 - $\beta$-derivative of the mass rate evolution for a shale gas reservoir produced from the quad-branched fracture 15 until 100 hours of production. 


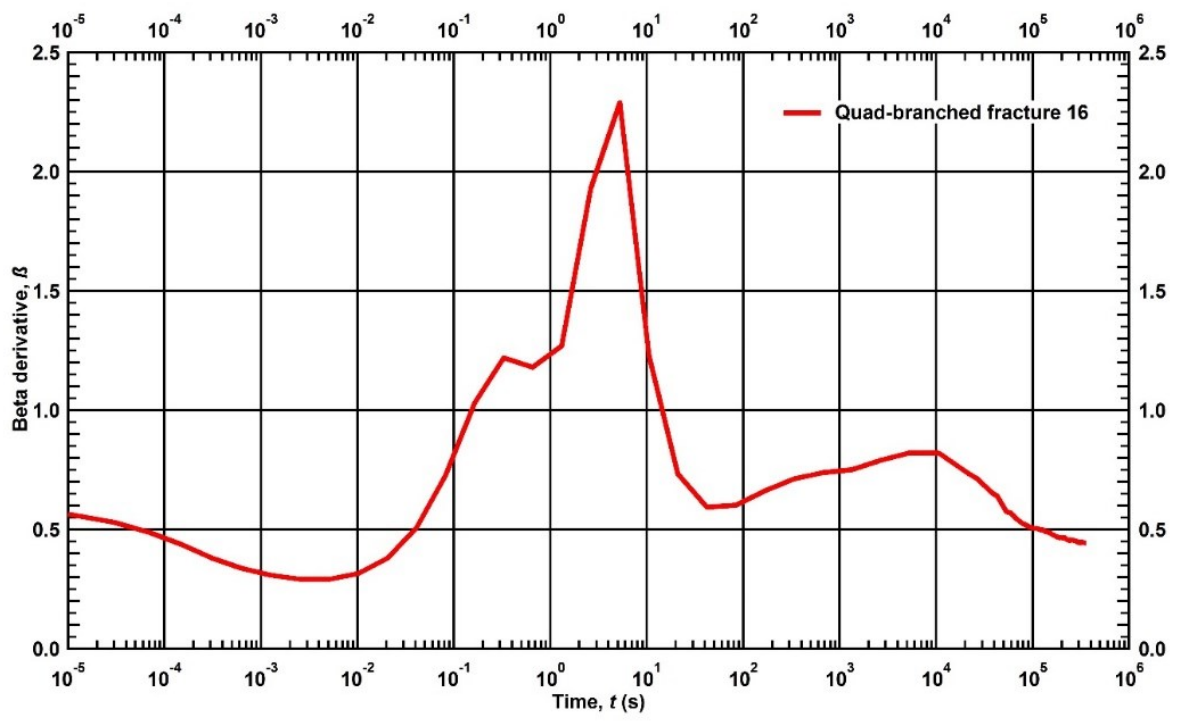

Figure C. 80 - $\beta$-derivative of the mass rate evolution for a shale gas reservoir produced from the quad-branched fracture 16 until 100 hours of production.

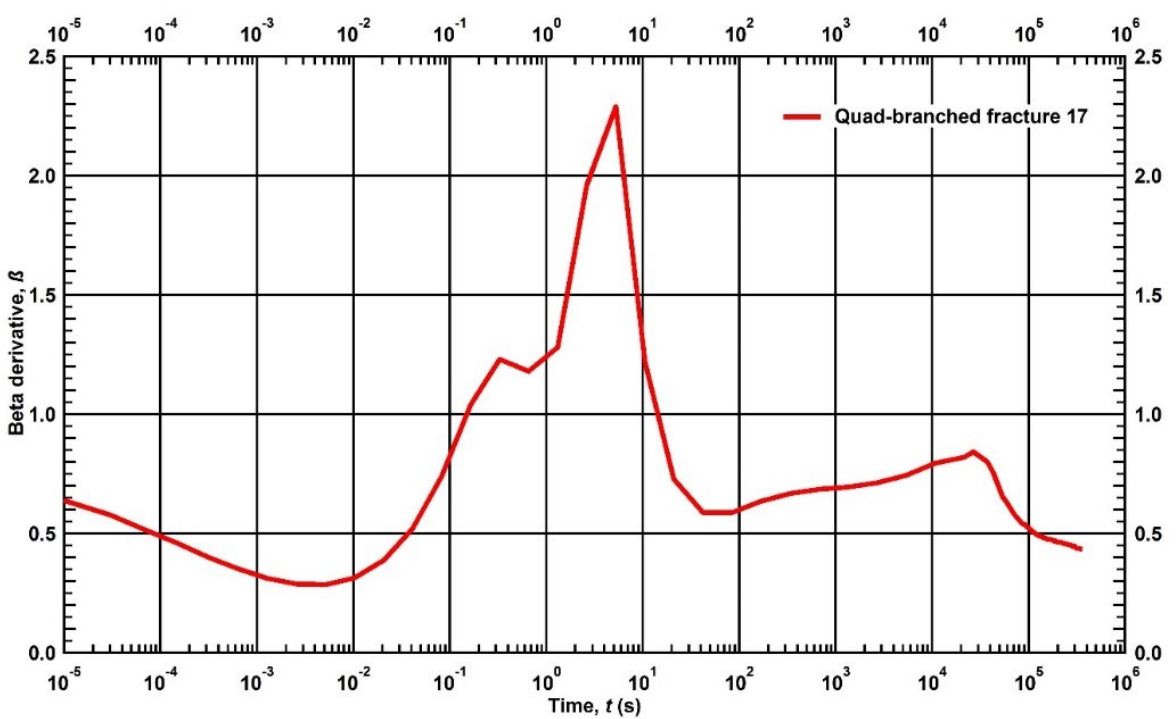

Figure C. 81 - $\beta$-derivative of the mass rate evolution for a shale gas reservoir produced from the quad-branched fracture 17 until 100 hours of production. 


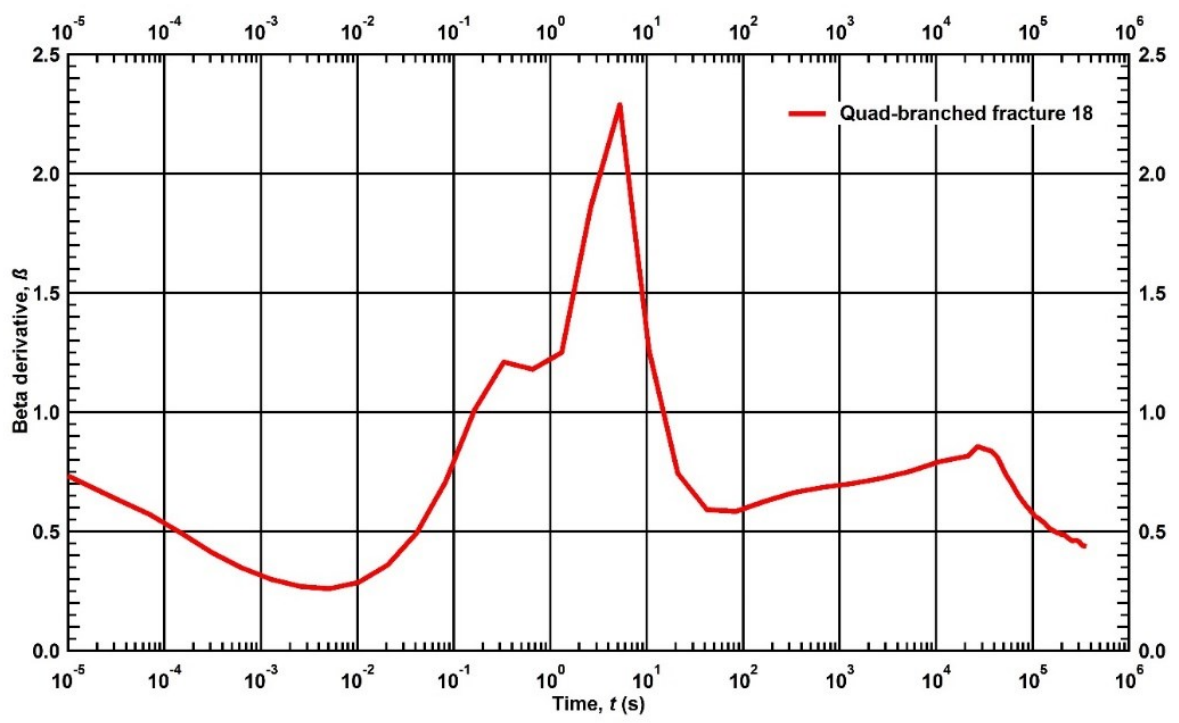

Figure C. 82 - $\beta$-derivative of the mass rate evolution for a shale gas reservoir produced from the quad-branched fracture 18 until 100 hours of production.

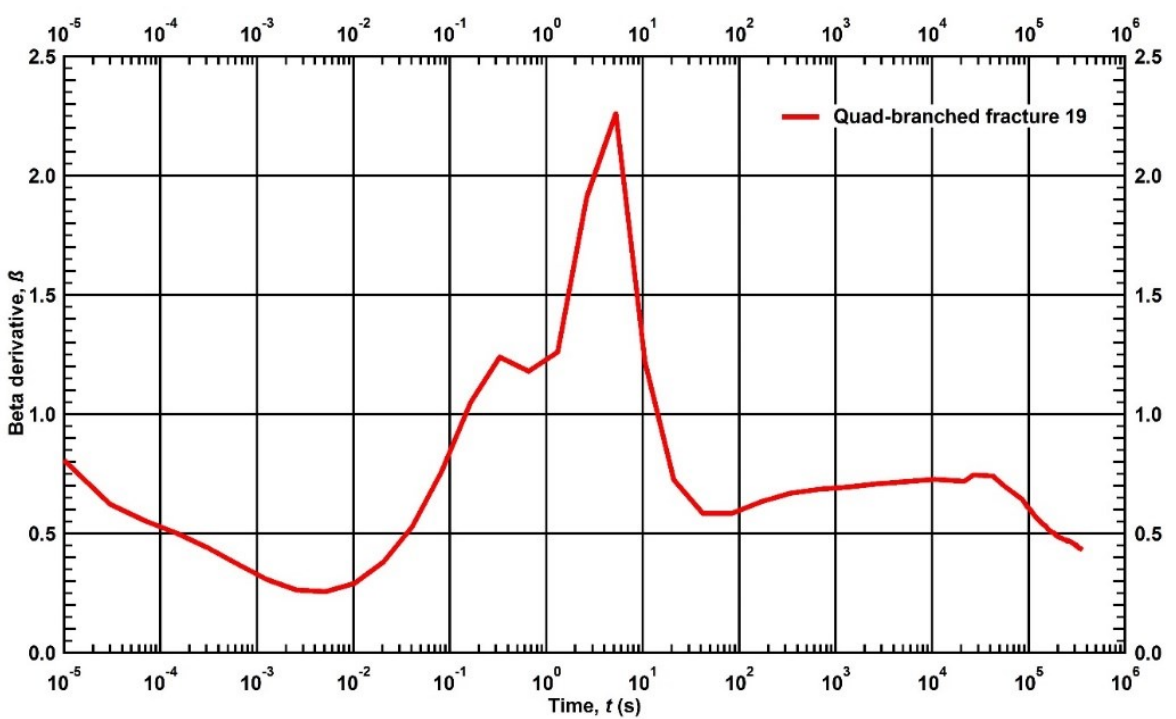

Figure C. 83 - $\beta$-derivative of the mass rate evolution for a shale gas reservoir produced from the quad-branched fracture 19 until 100 hours of production. 


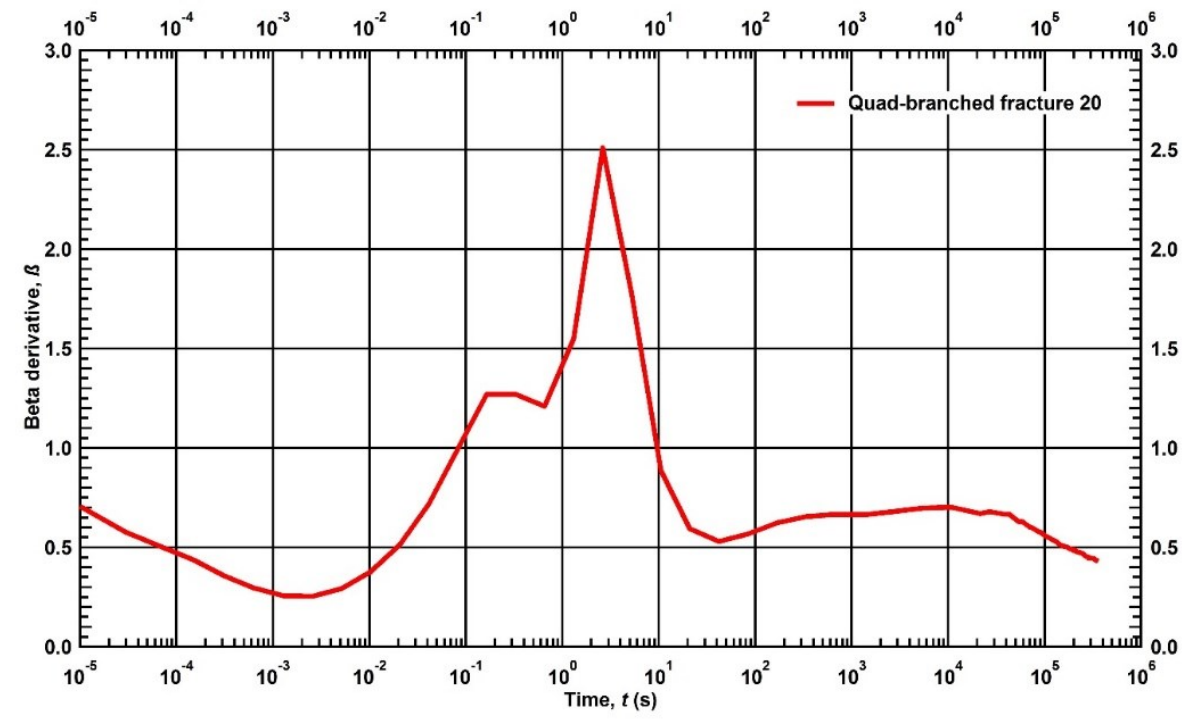

Figure C. 84 - $\beta$-derivative of the mass rate evolution for a shale gas reservoir produced from the quad-branched fracture 20 until 100 hours of production. 
PRESSURE EVOLUTION MAPS FOR SIMULATED STOCHASTIC FRACTURE PATTERNS
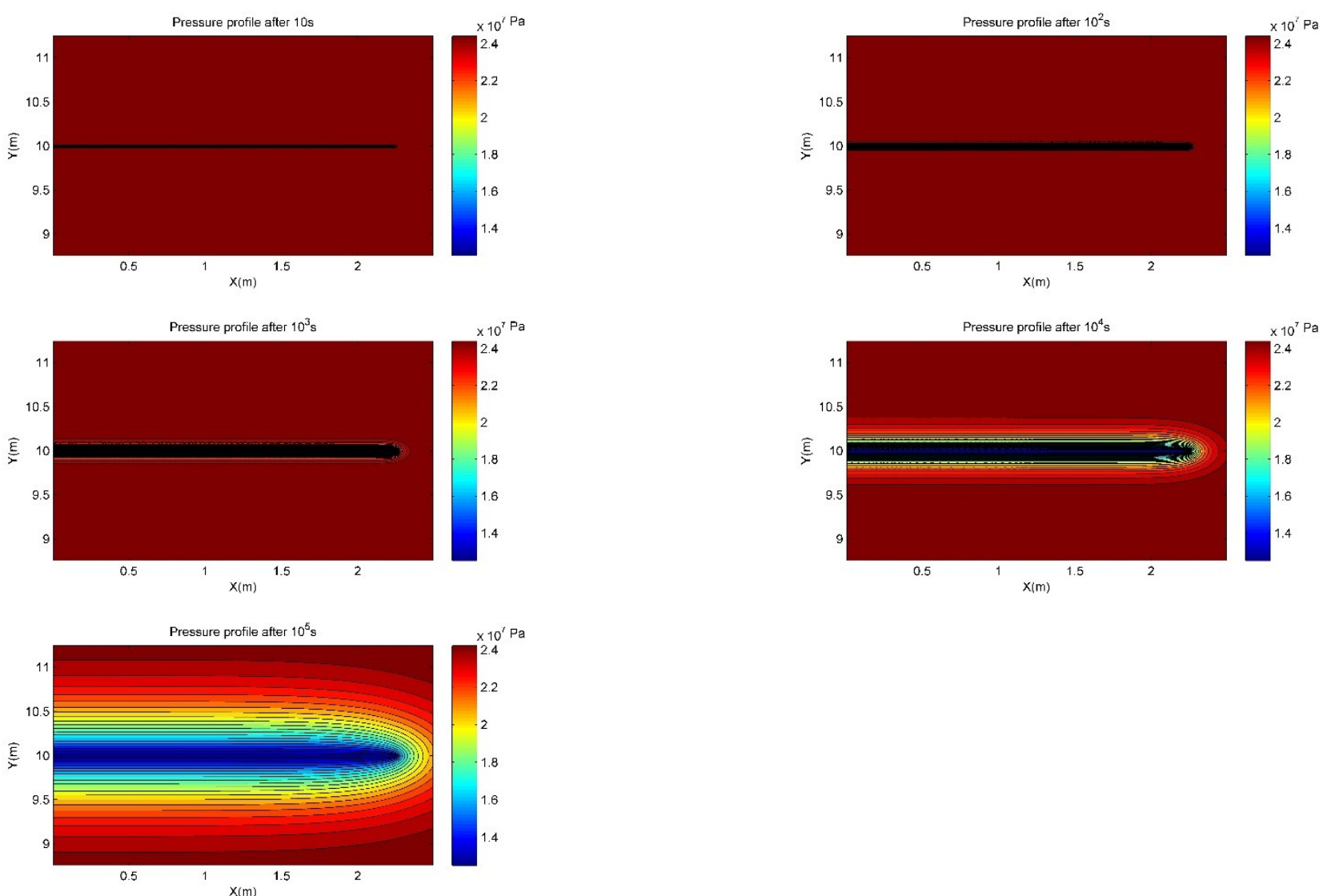

Figure D. 1 - Planar fracture pressure maps after $1 \times 10^{1} \mathrm{~s}, 1 \times 10^{2} \mathrm{~s}, 1 \times 10^{3} \mathrm{~s}, 1 \times 10^{4} \mathrm{~s}$ and $1 \times 10^{5} \mathrm{~s}$. 

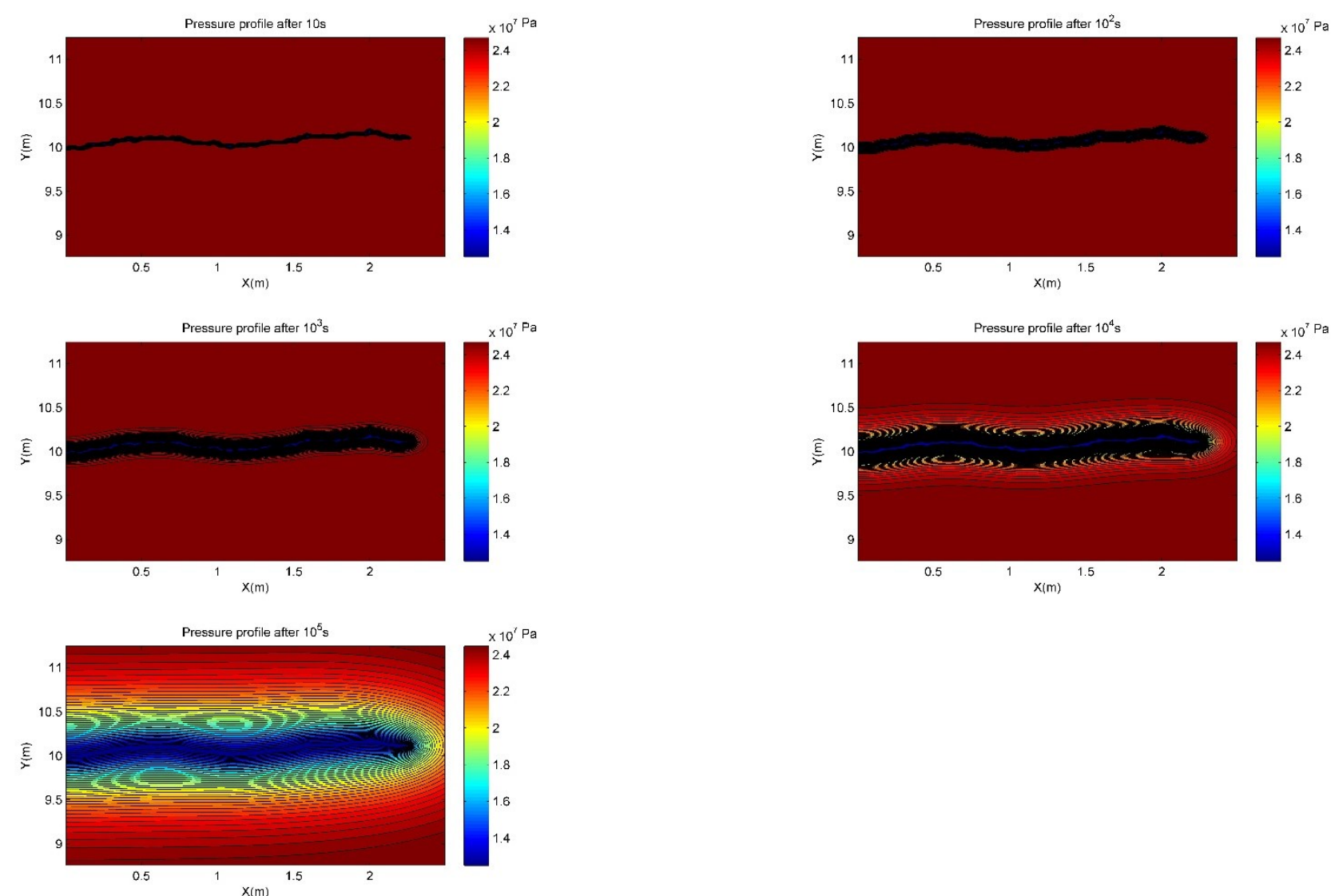

Figure D. 2 - Non-branched fracture 1 pressure maps after $1 \times 10^{1} \mathrm{~s}, 1 \times 10^{2} \mathrm{~s}, 1 \times 10^{3} \mathrm{~s}, 1 \times 10^{4} \mathrm{~s}$ and $1 \times 10^{5} \mathrm{~s}$. 

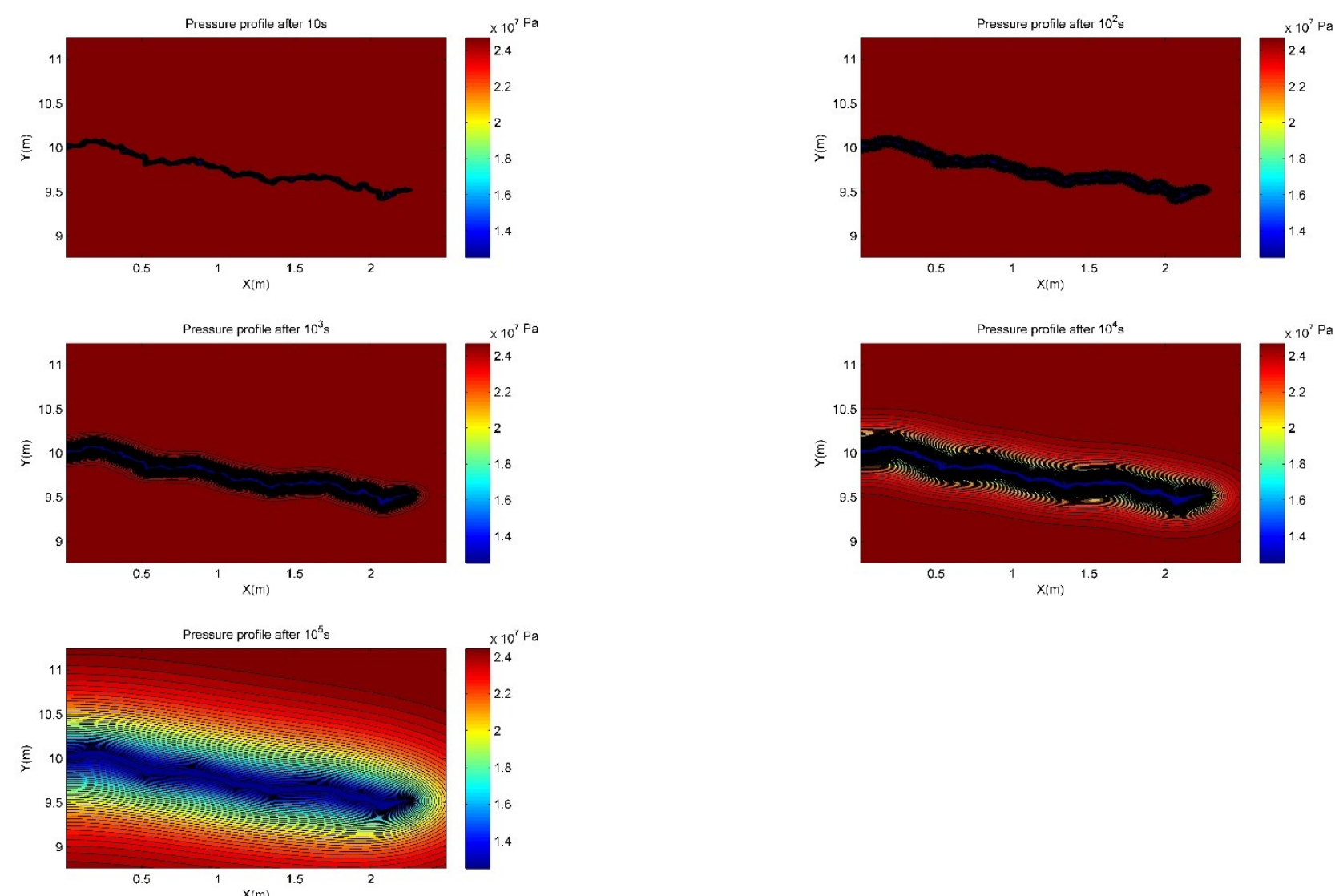

Figure D. 3 - Non-branched fracture 2 pressure maps after $1 \times 10^{1} \mathrm{~s}, 1 \times 10^{2} \mathrm{~s}, 1 \times 10^{3} \mathrm{~s}, 1 \times 10^{4} \mathrm{~s}$ and $1 \times 10^{5} \mathrm{~s}$. 

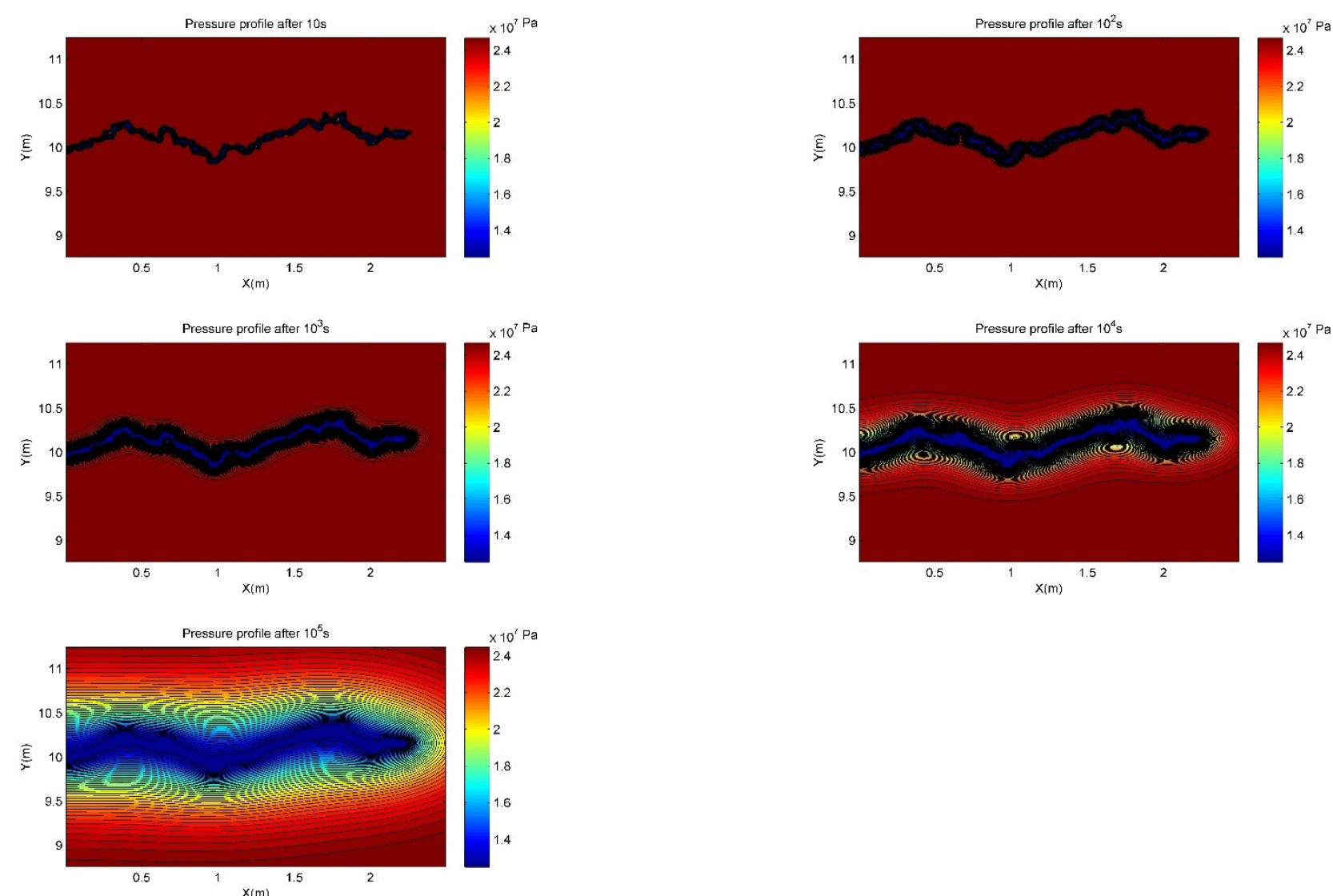

Figure D. 4 - Non-branched fracture 3 pressure maps after $1 \times 10^{1} \mathrm{~s}, 1 \times 10^{2} \mathrm{~s}, 1 \times 10^{3} \mathrm{~s}, 1 \times 10^{4} \mathrm{~s}$ and $1 \times 10^{5} \mathrm{~s}$. 

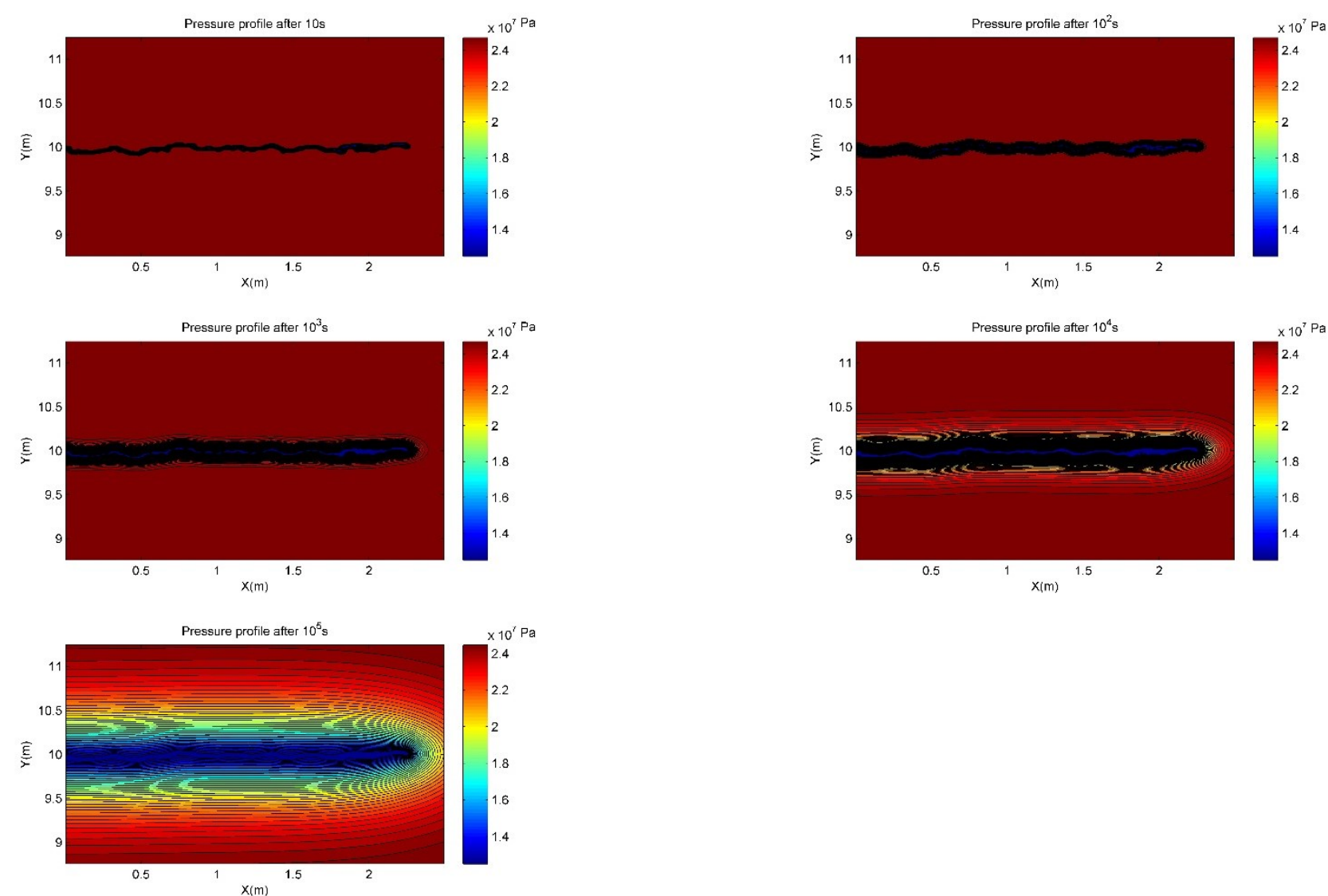

Figure D. 5 - Mono-branched fracture 1 pressure maps after $1 \times 10^{1} \mathrm{~s}, 1 \times 10^{2} \mathrm{~s}, 1 \times 10^{3} \mathrm{~s}, 1 \times 10^{4} \mathrm{~s}$ and $1 \times 10^{5} \mathrm{~s}$. 

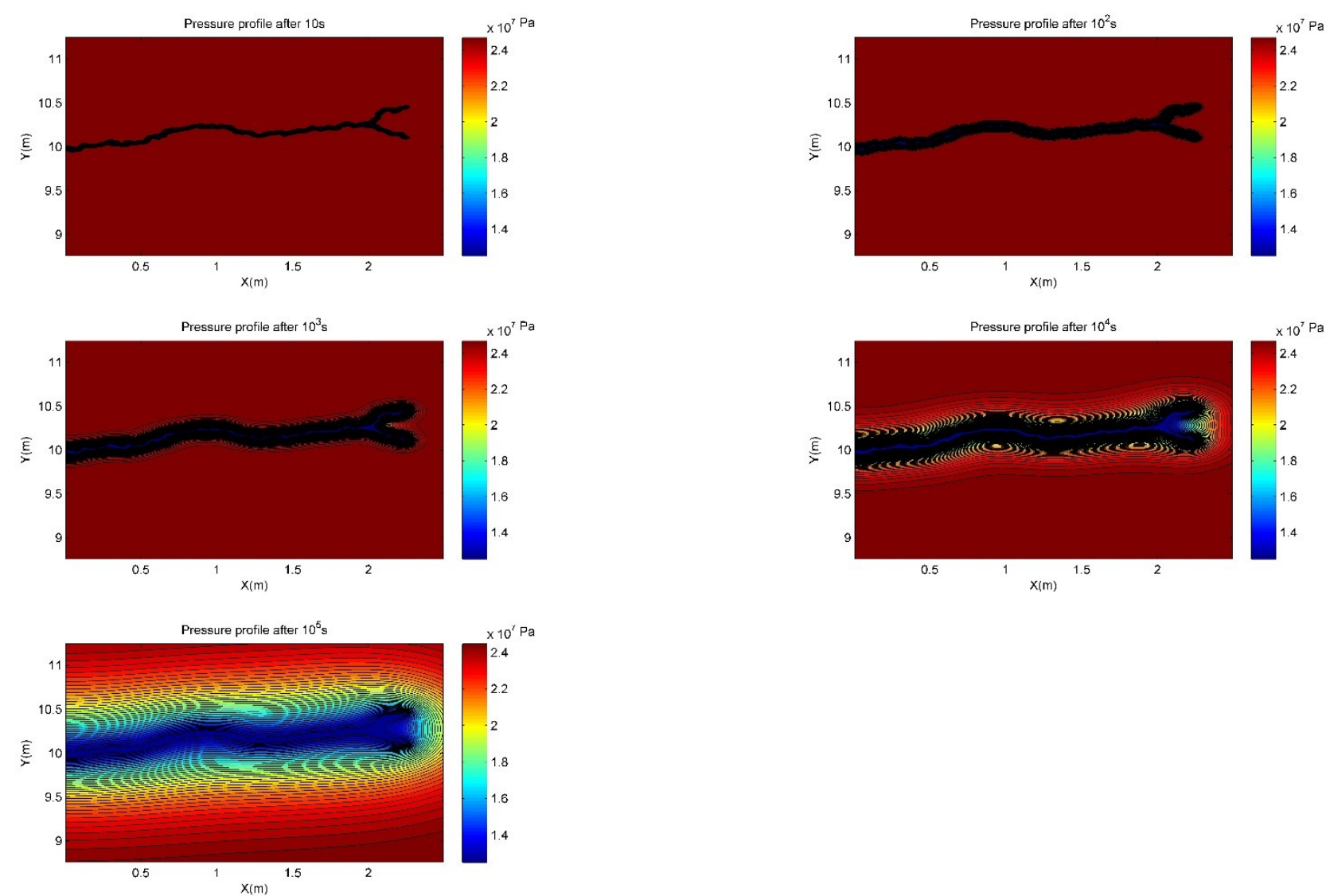

Figure D. 6 - Mono-branched fracture 2 pressure maps after $1 \times 10^{1} \mathrm{~s}, 1 \times 10^{2} \mathrm{~s}, 1 \times 10^{3} \mathrm{~s}, 1 \times 10^{4} \mathrm{~s}$ and $1 \times 10^{5} \mathrm{~s}$ 

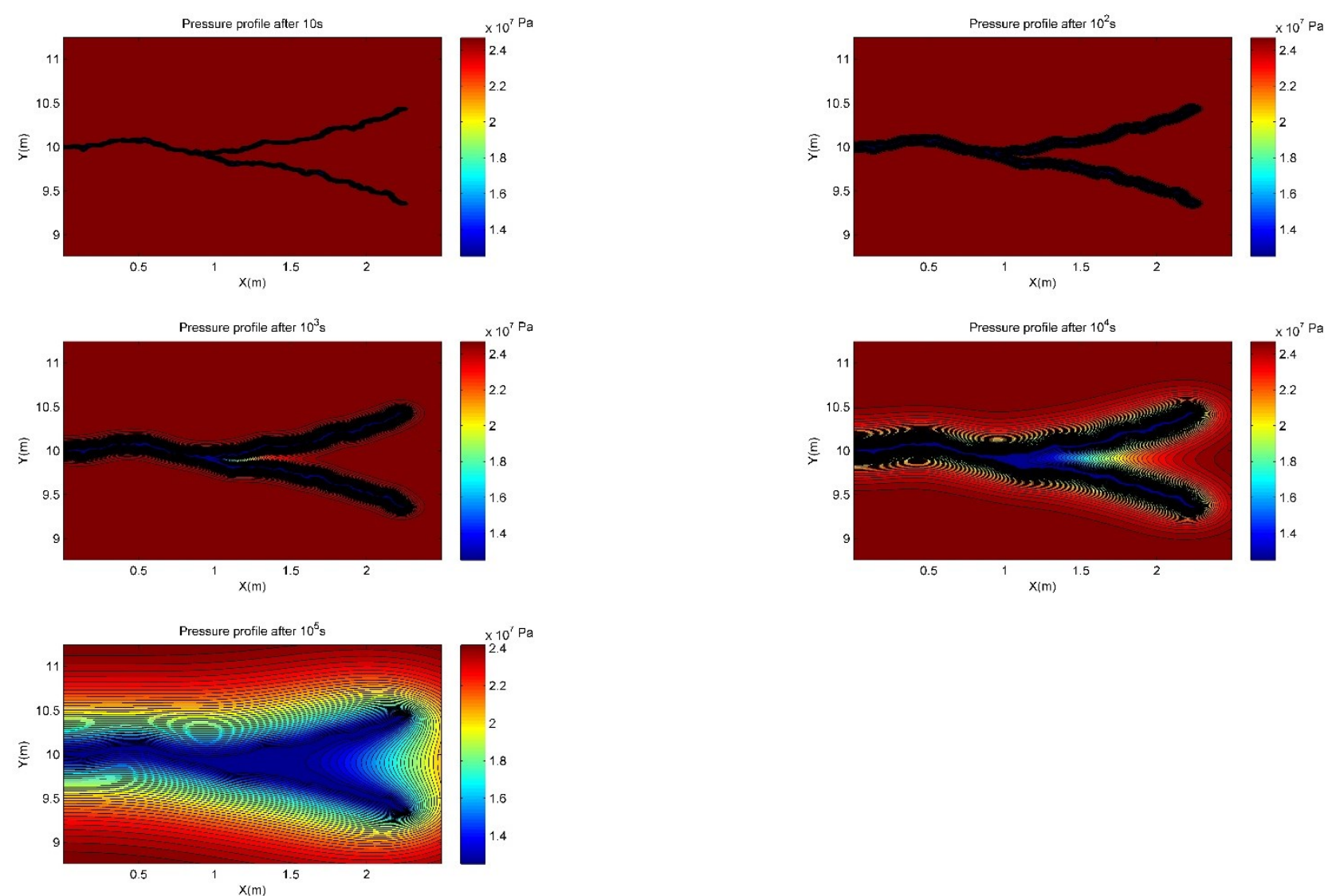

Figure D. 7 - Mono-branched fracture 3 pressure maps after $1 \times 10^{1} \mathrm{~s}, 1 \times 10^{2} \mathrm{~s}, 1 \times 10^{3} \mathrm{~s}, 1 \times 10^{4} \mathrm{~s}$ and $1 \times 10^{5} \mathrm{~s}$ 

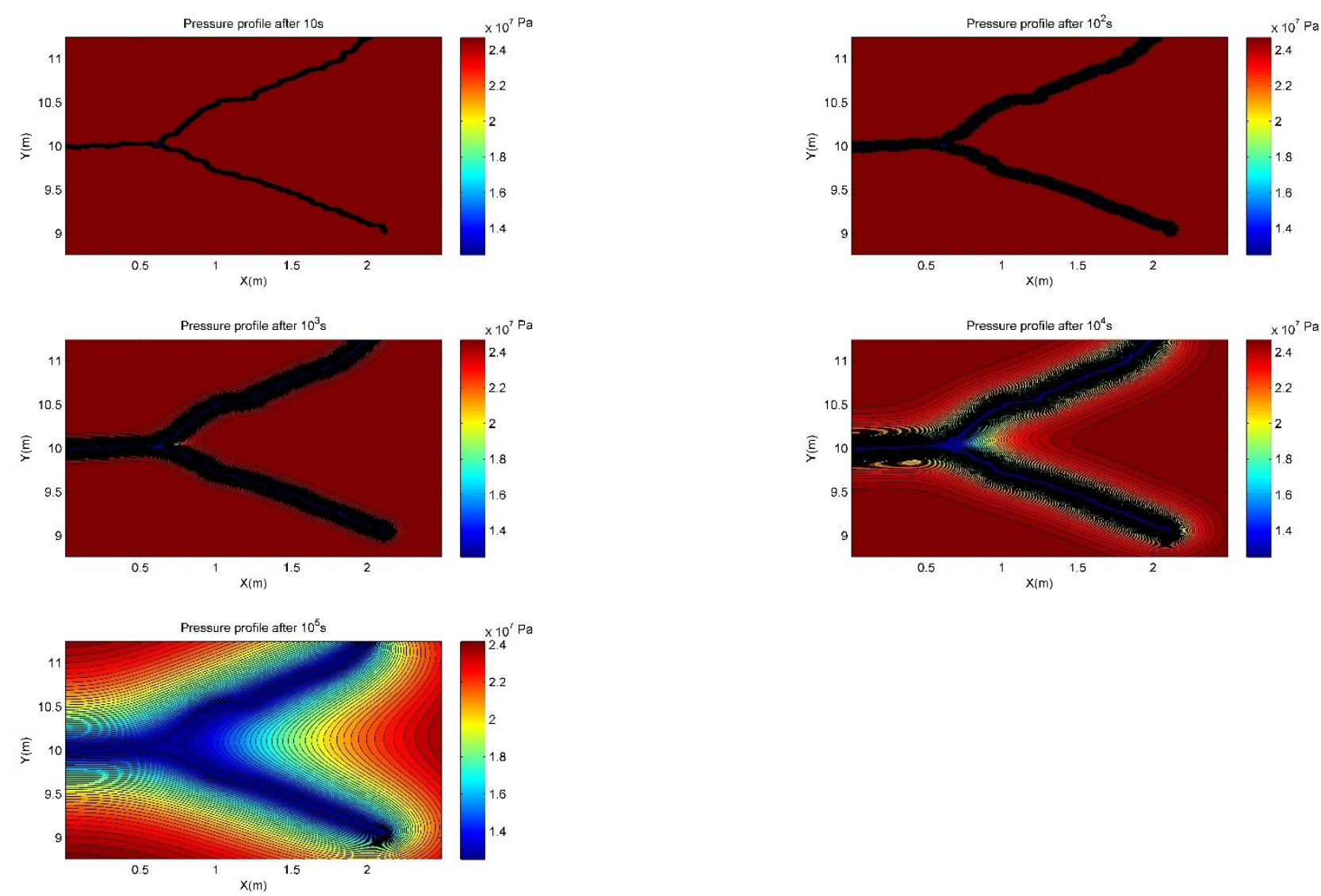

Figure D. 8 - Mono-branched fracture 4 pressure maps after $1 \times 10^{1} \mathrm{~s}, 1 \times 10^{2} \mathrm{~s}, 1 \times 10^{3} \mathrm{~s}, 1 \times 10^{4} \mathrm{~s}$ and $1 \times 10^{5} \mathrm{~s}$ 

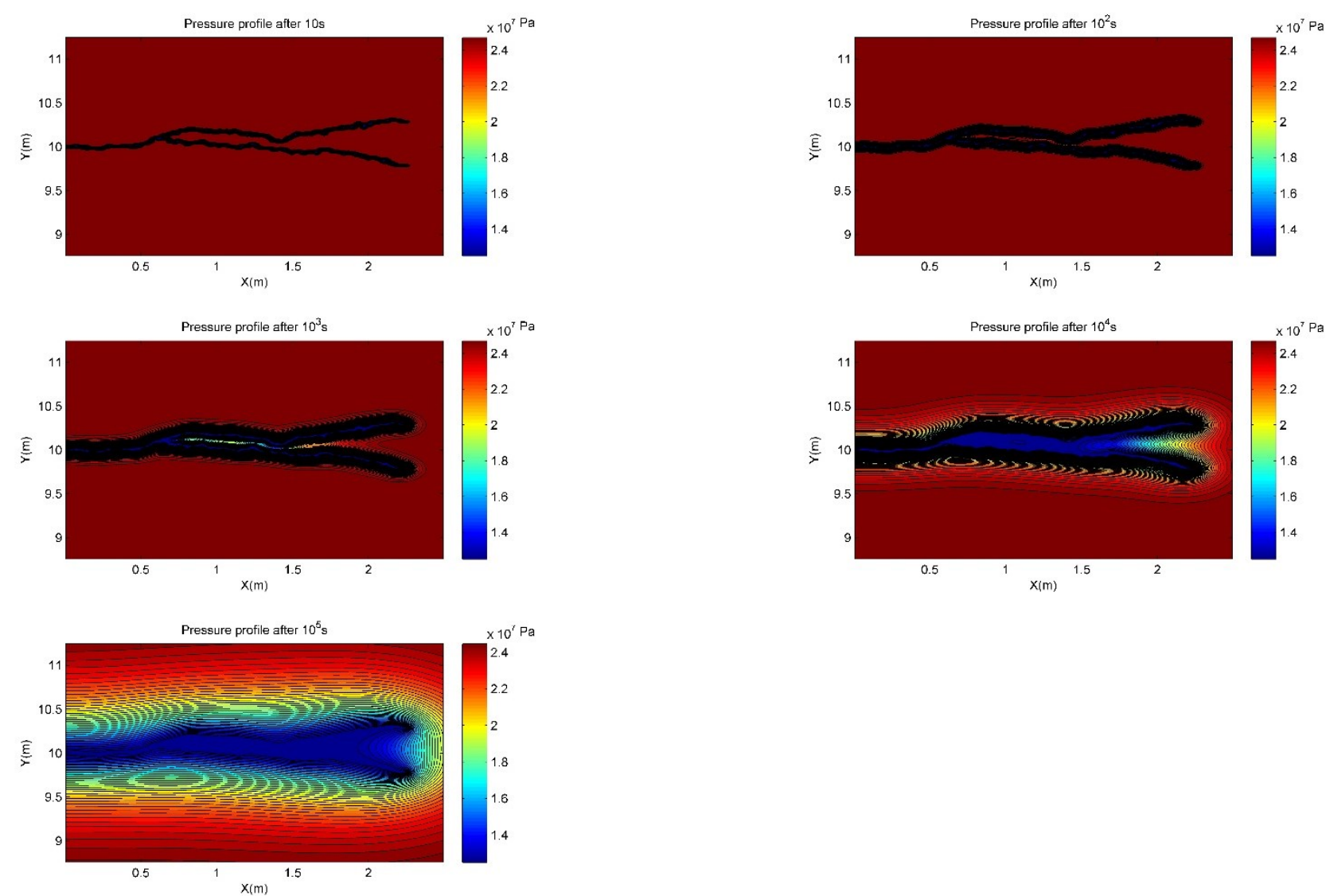

Figure D. 9 - Mono-branched fracture 5 pressure maps after $1 \times 10^{1} \mathrm{~s}, 1 \times 10^{2} \mathrm{~s}, 1 \times 10^{3} \mathrm{~s}, 1 \times 10^{4} \mathrm{~s}$ and $1 \times 10^{5} \mathrm{~s}$. 

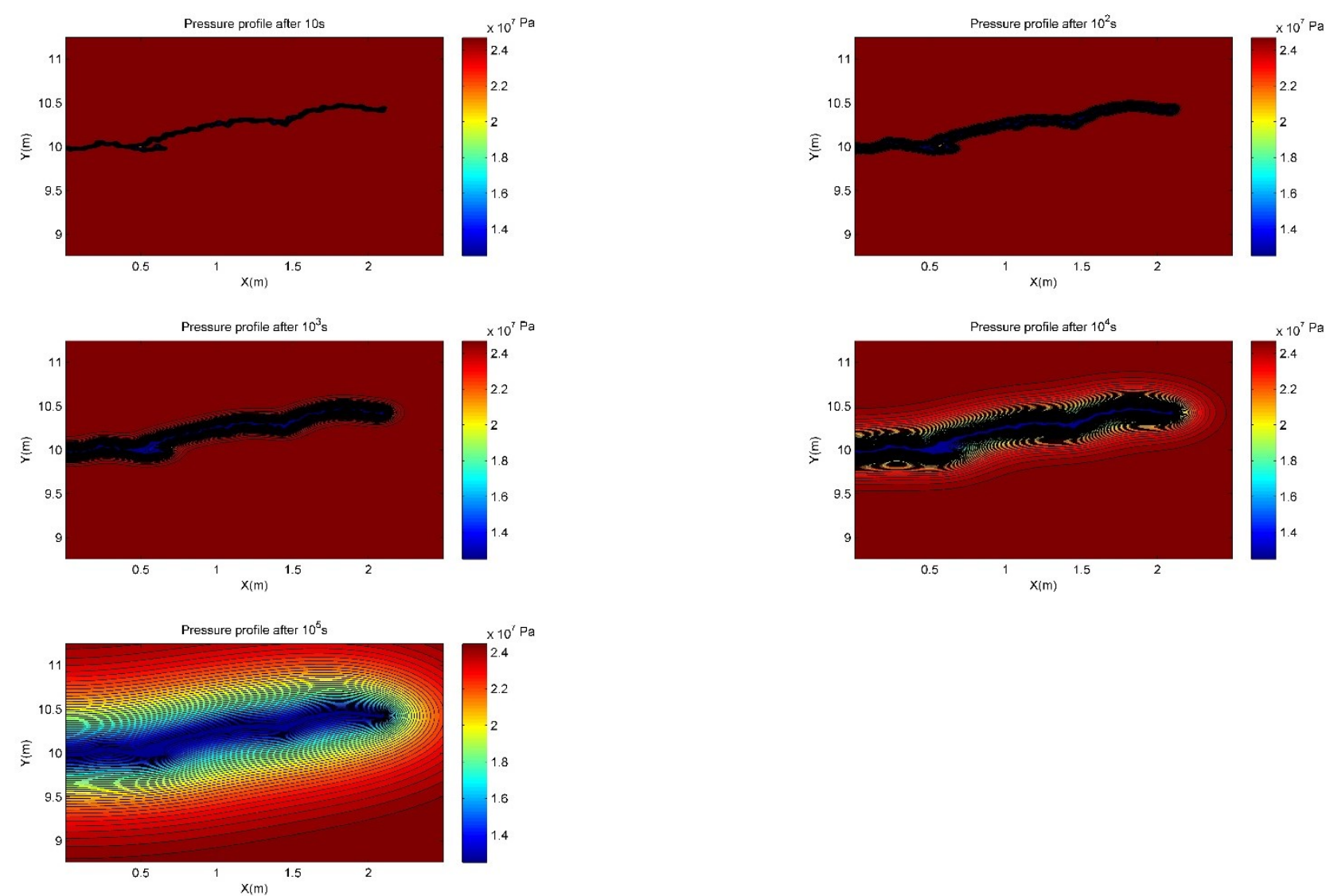

Figure D. 10 - Mono-branched fracture 6 pressure maps after $1 \times 10^{1} \mathrm{~s}, 1 \times 10^{2} \mathrm{~s}, 1 \times 10^{3} \mathrm{~s}, 1 \times 10^{4} \mathrm{~s}$ and $1 \times 10^{5} \mathrm{~s}$ 

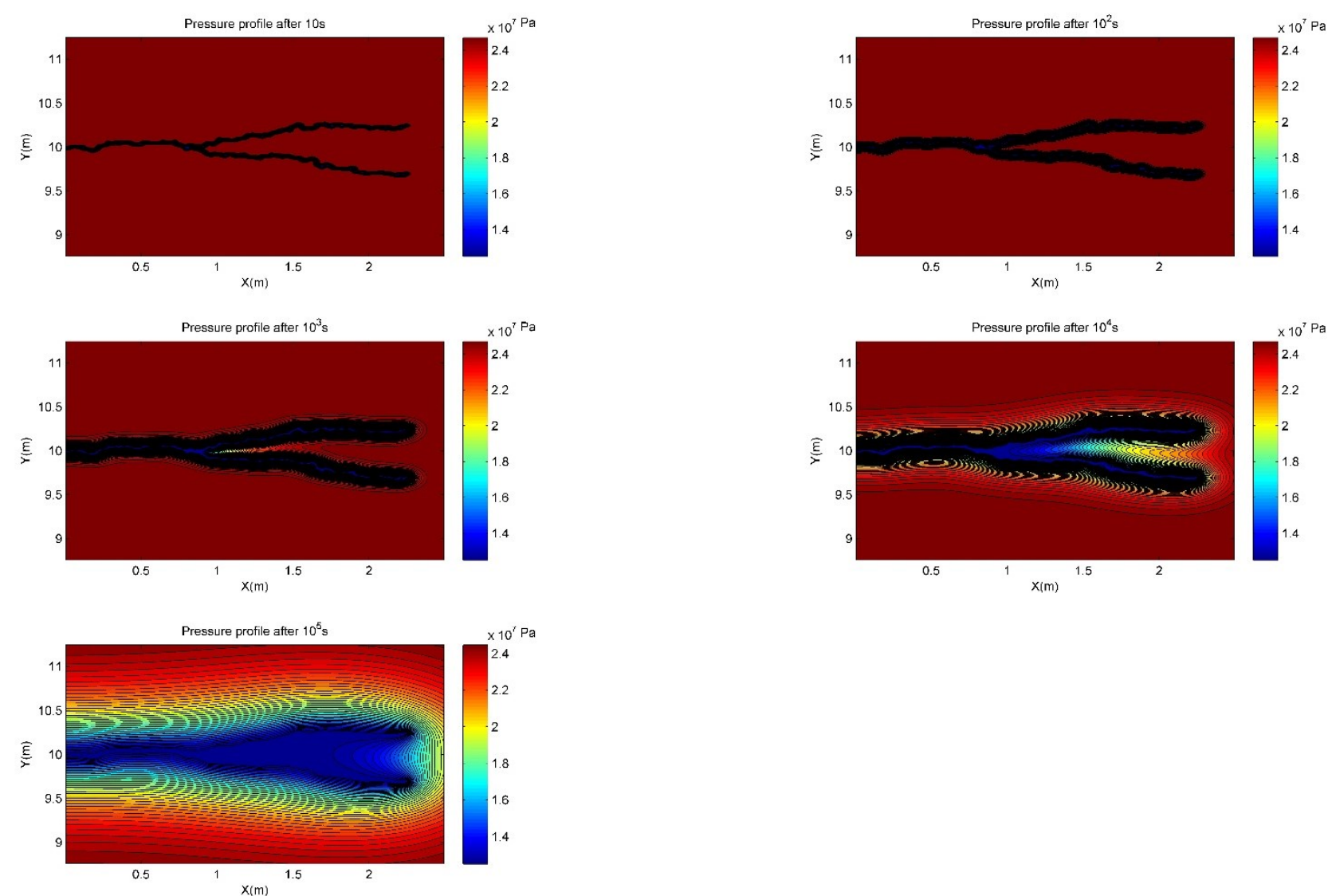

Figure D. 11 - Mono-branched fracture 7 pressure maps after $1 \times 10^{1} \mathrm{~s}, 1 \times 10^{2} \mathrm{~s}, 1 \times 10^{3} \mathrm{~s}, 1 \times 10^{4} \mathrm{~s}$ and $1 \times 10^{5} \mathrm{~s}$ 

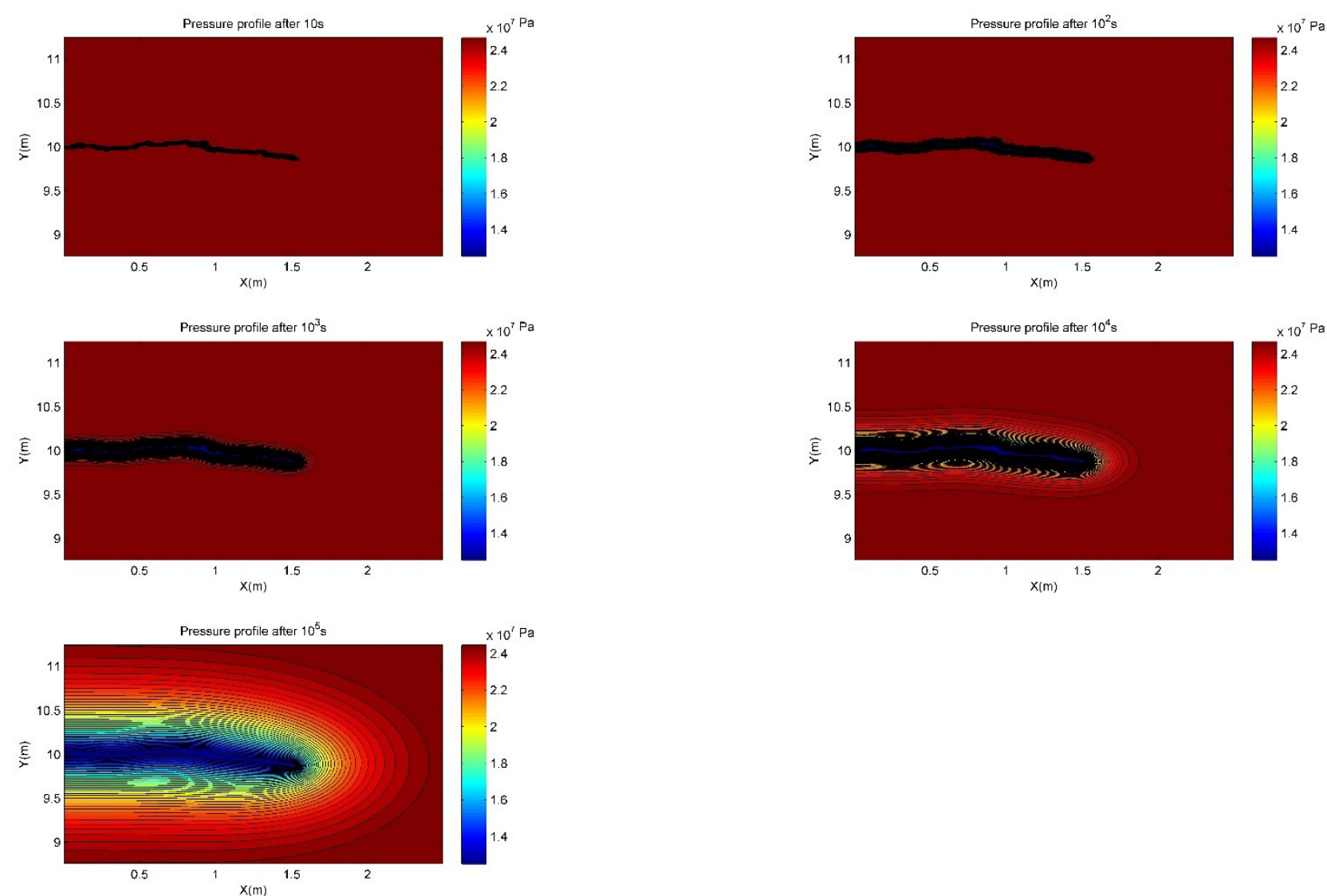

Figure D. 12 - Mono-branched fracture 8 pressure maps after $1 \times 10^{1} \mathrm{~s}, 1 \times 10^{2} \mathrm{~s}, 1 \times 10^{3} \mathrm{~s}, 1 \times 10^{4} \mathrm{~s}$ and $1 \times 10^{5} \mathrm{~s}$ 

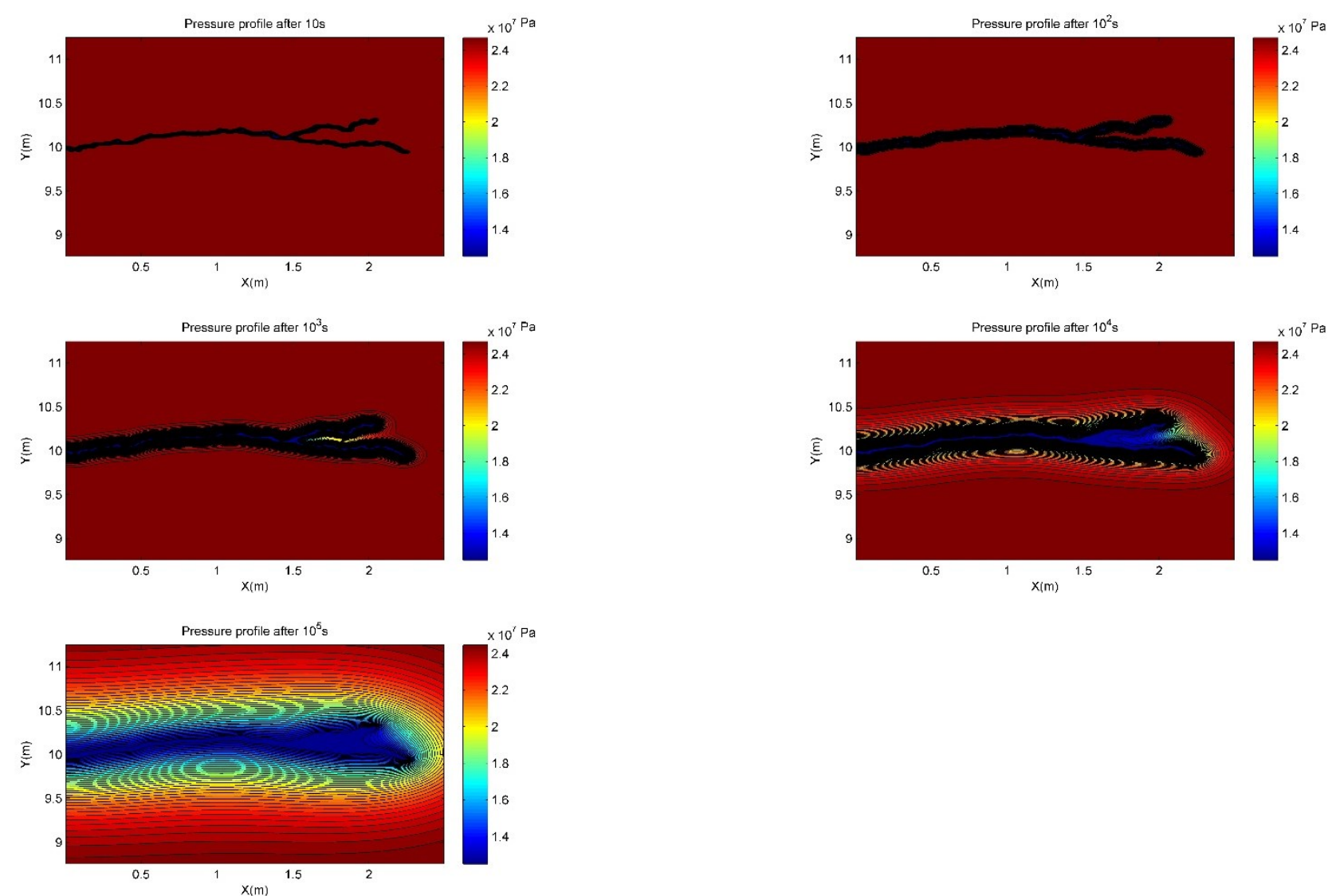

Figure D. 13 - Mono-branched fracture 9 pressure maps after $1 \times 10^{1} \mathrm{~s}, 1 \times 10^{2} \mathrm{~s}, 1 \times 10^{3} \mathrm{~s}, 1 \times 10^{4} \mathrm{~s}$ and $1 \times 10^{5} \mathrm{~s}$ 

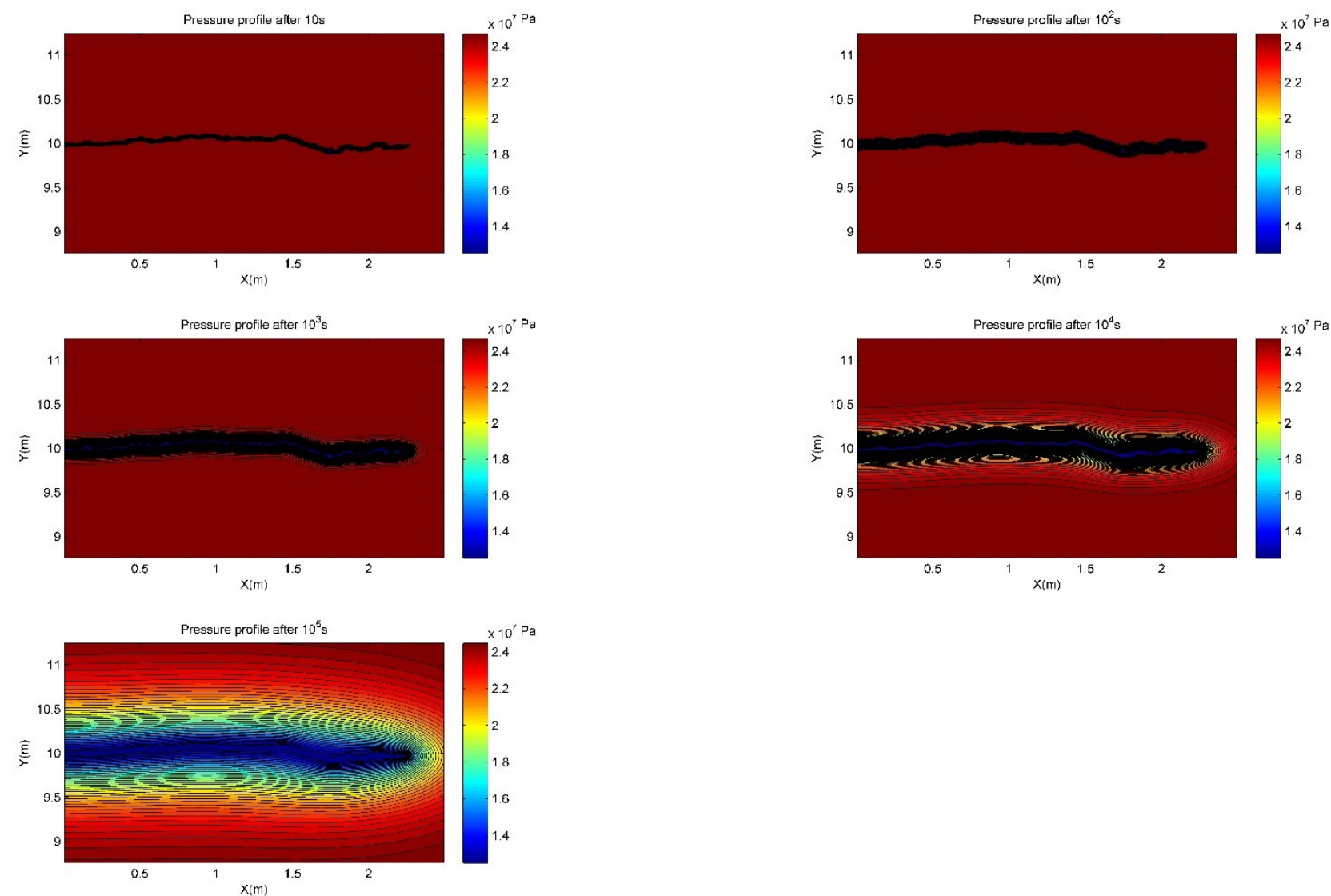

Figure D. 14 - Mono-branched fracture 10 pressure maps after $1 \times 10^{1} \mathrm{~s}, 1 \times 10^{2} \mathrm{~s}, 1 \times 10^{3} \mathrm{~s}, 1 \times 10^{4} \mathrm{~s}$ and $1 \times 10^{5} \mathrm{~s}$ 

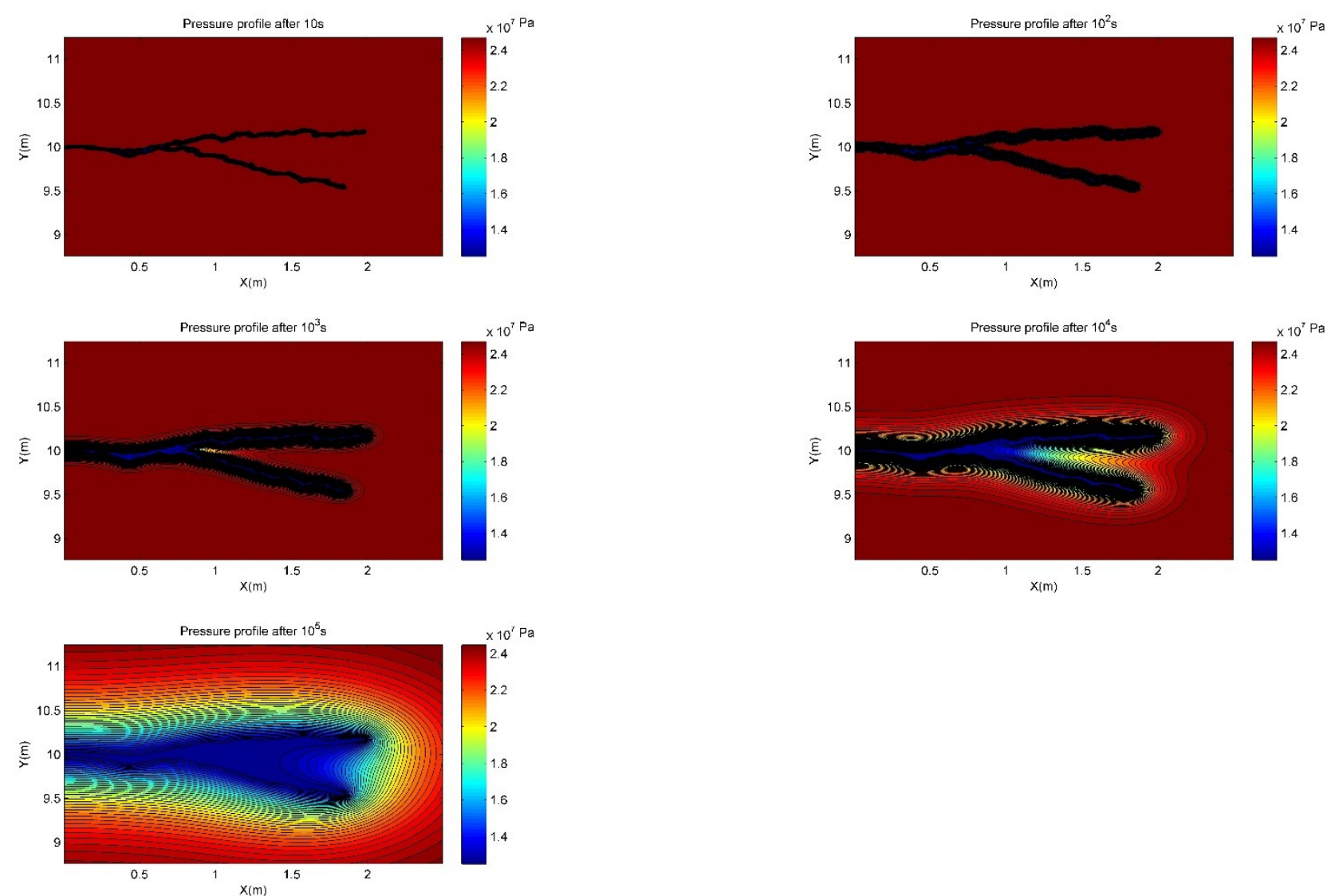

Figure D. 15 - Mono-branched fracture 11 pressure maps after $1 \times 10^{1} \mathrm{~s}, 1 \times 10^{2} \mathrm{~s}, 1 \times 10^{3} \mathrm{~s}, 1 \times 10^{4} \mathrm{~s}$ and $1 \times 10^{5} \mathrm{~s}$ 

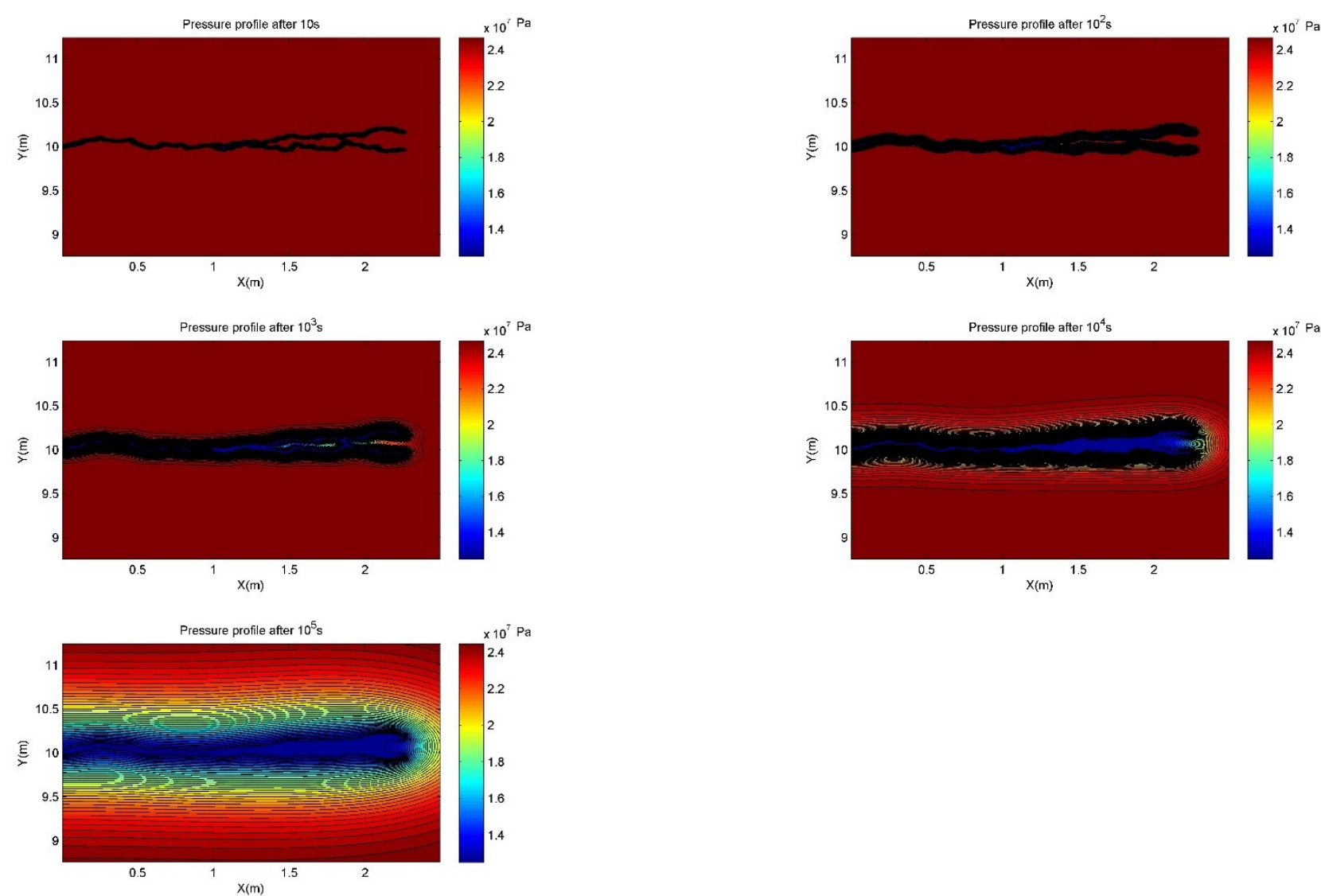

Figure D. 16 - Mono-branched fracture 12 pressure maps after $1 \times 10^{1} \mathrm{~s}, 1 \times 10^{2} \mathrm{~s}, 1 \times 10^{3} \mathrm{~s}, 1 \times 10^{4} \mathrm{~s}$ and $1 \times 10^{5} \mathrm{~s}$ 

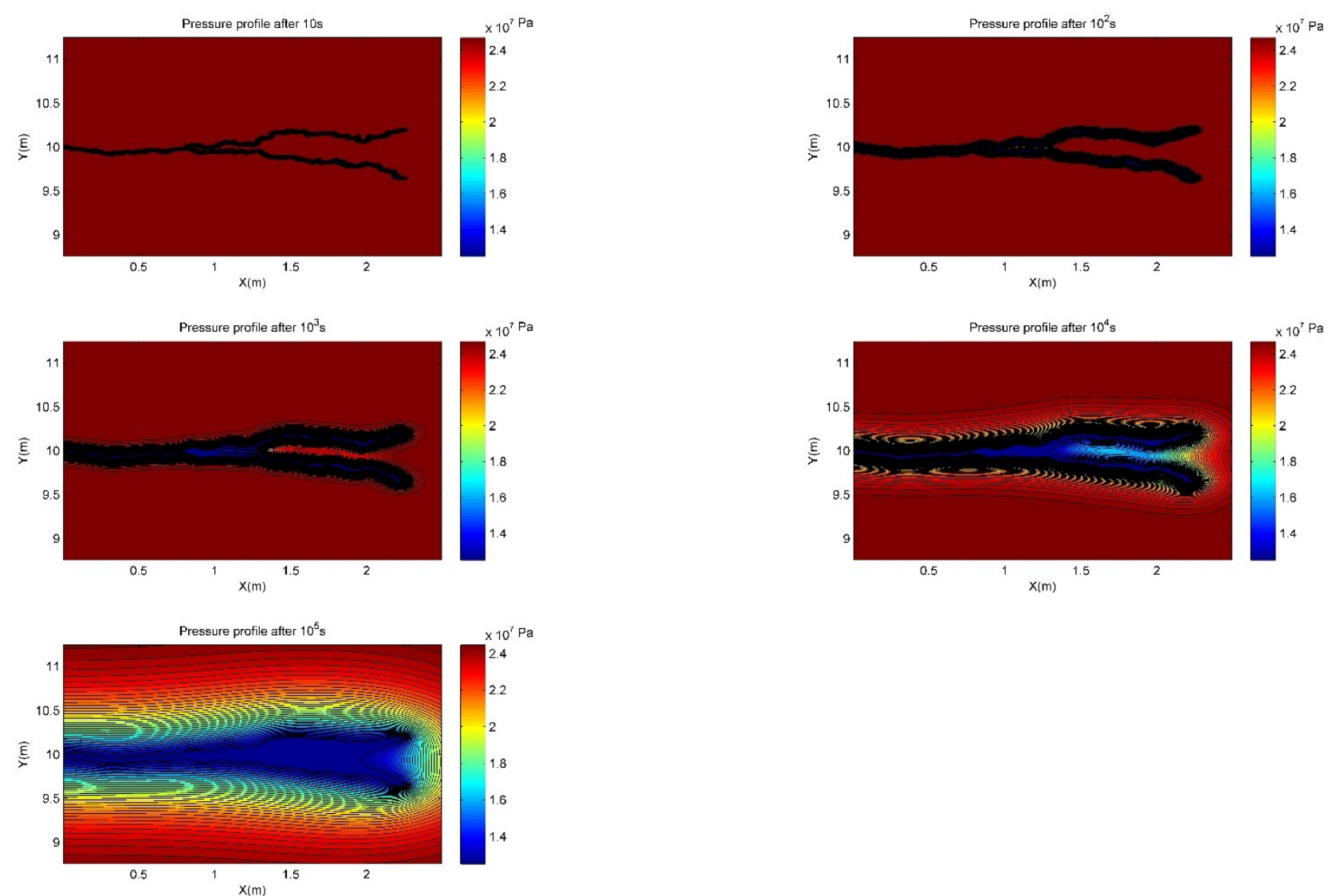

Figure D. 17 - Mono-branched fracture 13 pressure maps after $1 \times 10^{1} \mathrm{~s}, 1 \times 10^{2} \mathrm{~s}, 1 \times 10^{3} \mathrm{~s}, 1 \times 10^{4} \mathrm{~s}$ and $1 \times 10^{5} \mathrm{~s}$ 

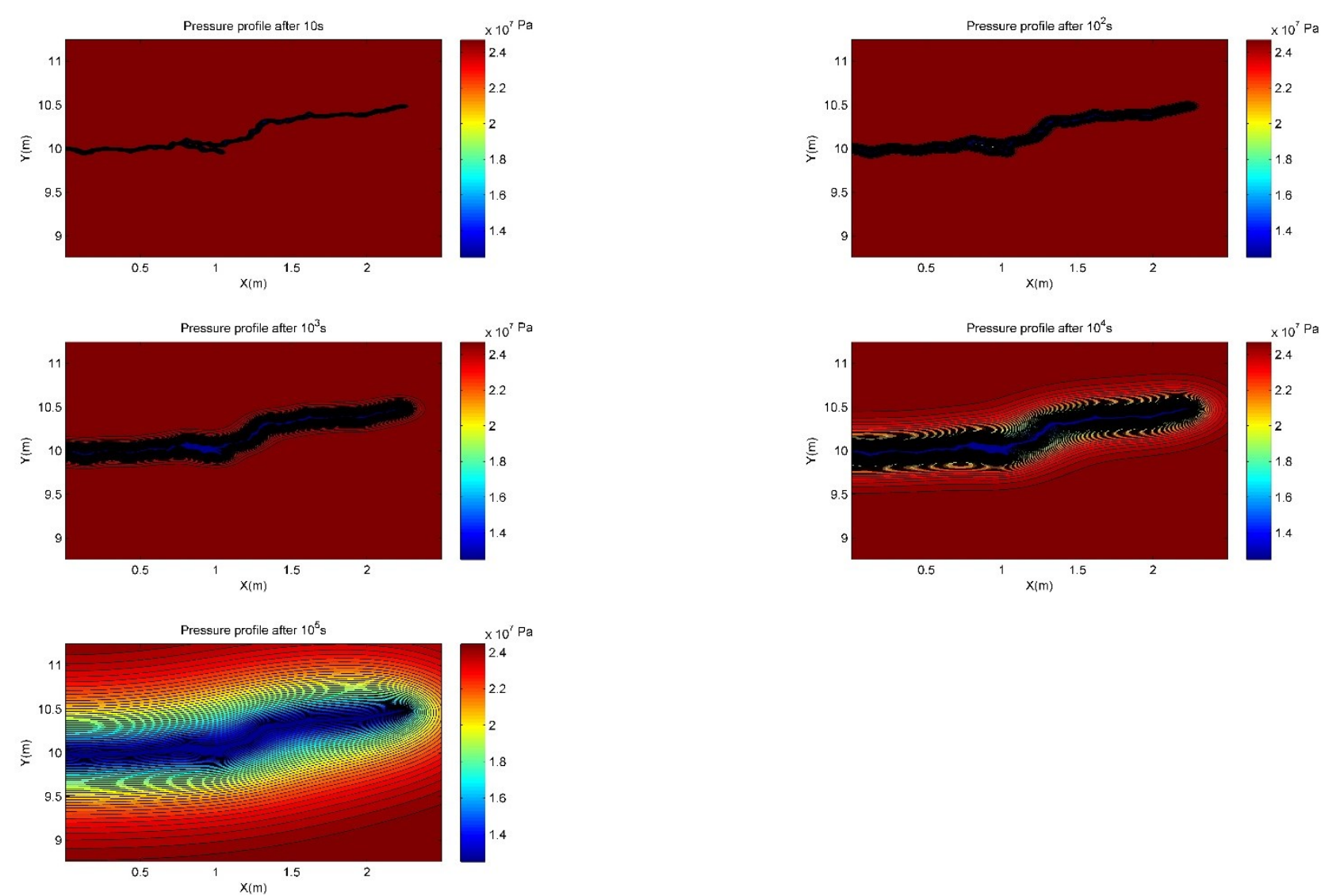

Figure D. 18 - Mono-branched fracture 14 pressure maps after $1 \times 10^{1} \mathrm{~s}, 1 \times 10^{2} \mathrm{~s}, 1 \times 10^{3} \mathrm{~s}, 1 \times 10^{4} \mathrm{~s}$ and $1 \times 10^{5} \mathrm{~s}$ 

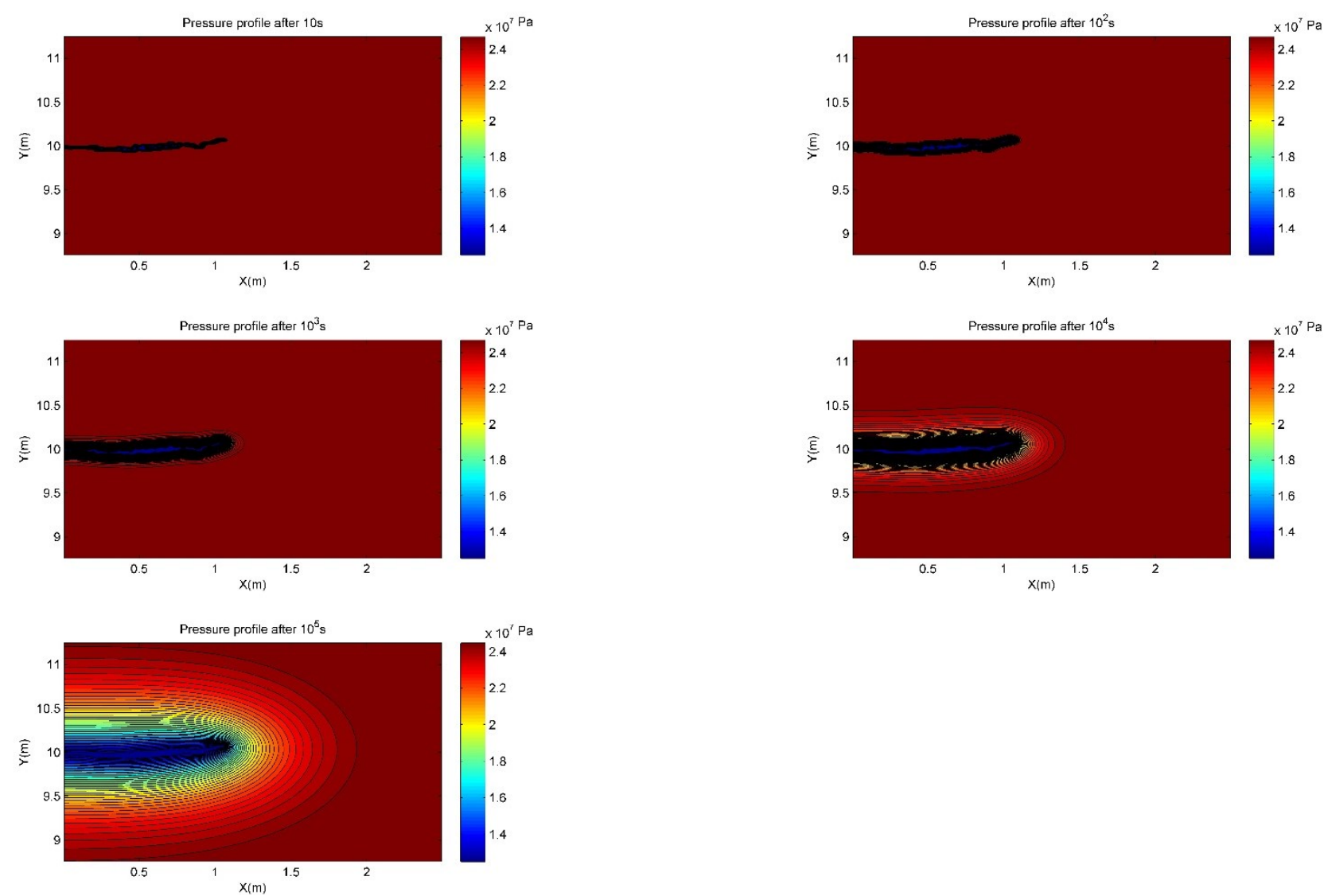

Figure D. 19 - Mono-branched fracture 15 pressure maps after $1 \times 10^{1} \mathrm{~s}, 1 \times 10^{2} \mathrm{~s}, 1 \times 10^{3} \mathrm{~s}, 1 \times 10^{4} \mathrm{~s}$ and $1 \times 10^{5} \mathrm{~s}$ 

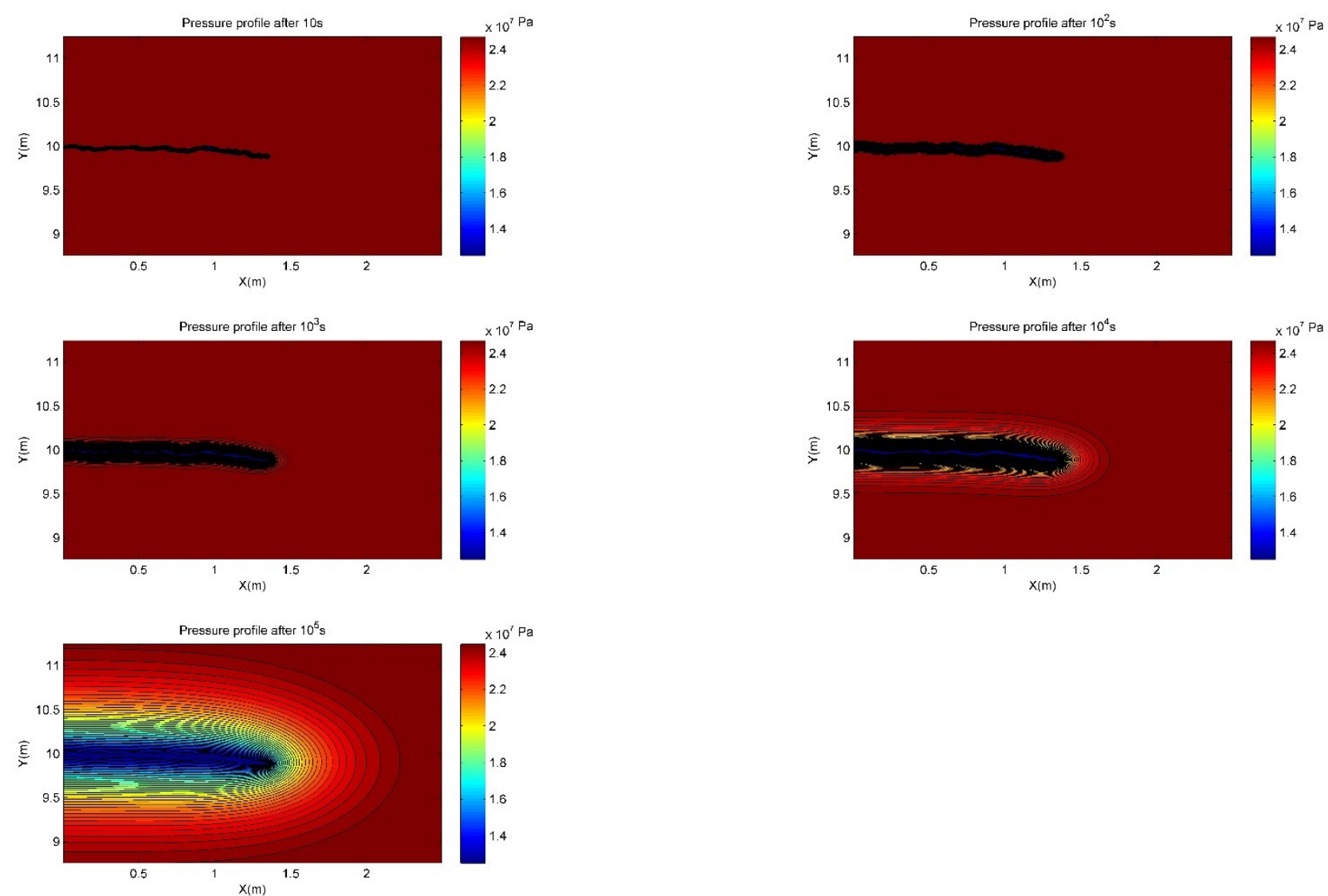

Figure D. 20 - Mono-branched fracture 16 pressure maps after $1 \times 10^{1} \mathrm{~s}, 1 \times 10^{2} \mathrm{~s}, 1 \times 10^{3} \mathrm{~s}, 1 \times 10^{4} \mathrm{~s}$ and $1 \times 10^{5} \mathrm{~s}$ 

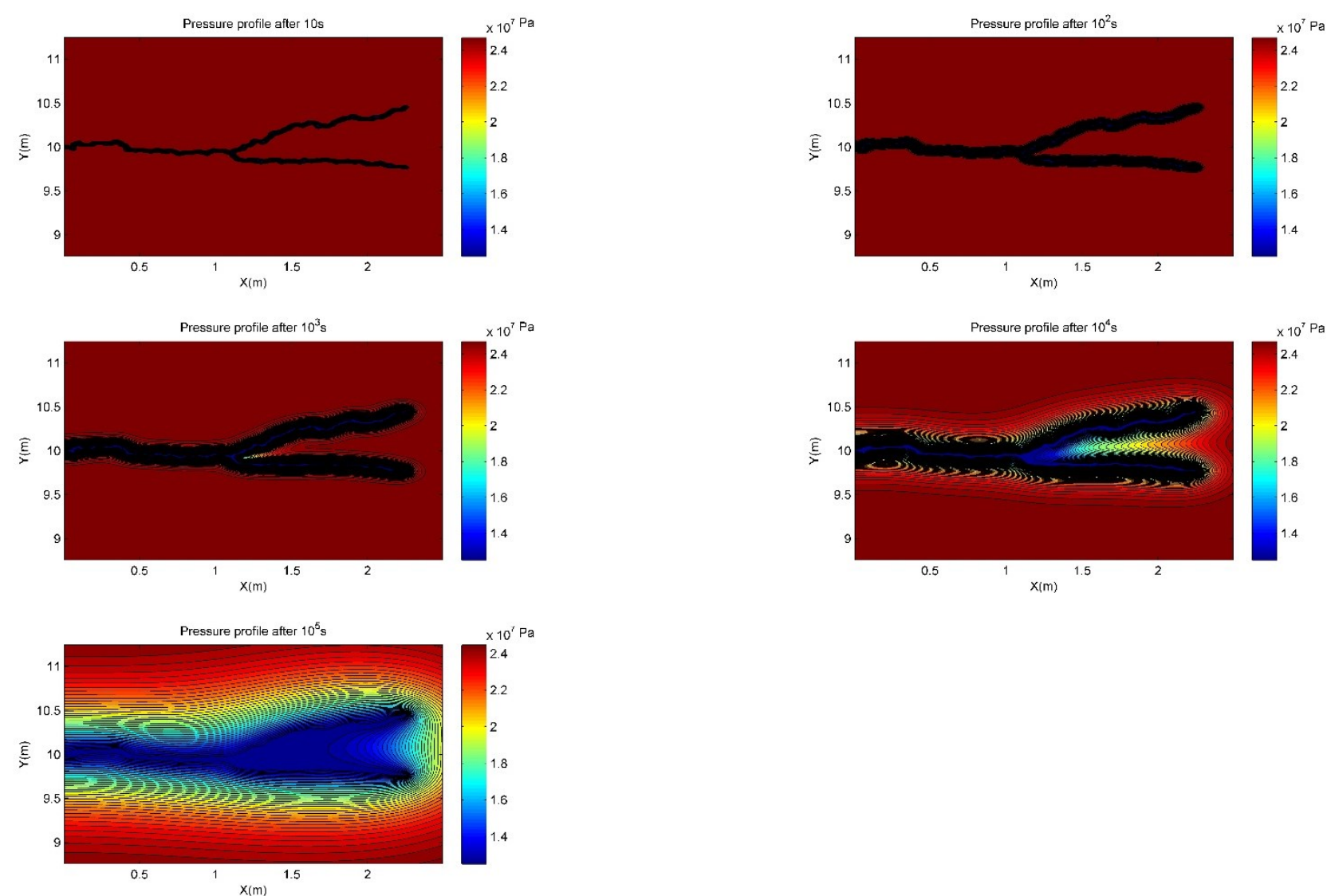

Figure D. 21 - Mono-branched fracture 17 pressure maps after $1 \times 10^{1} \mathrm{~s}, 1 \times 10^{2} \mathrm{~s}, 1 \times 10^{3} \mathrm{~s}, 1 \times 10^{4} \mathrm{~s}$ and $1 \times 10^{5} \mathrm{~s}$. 

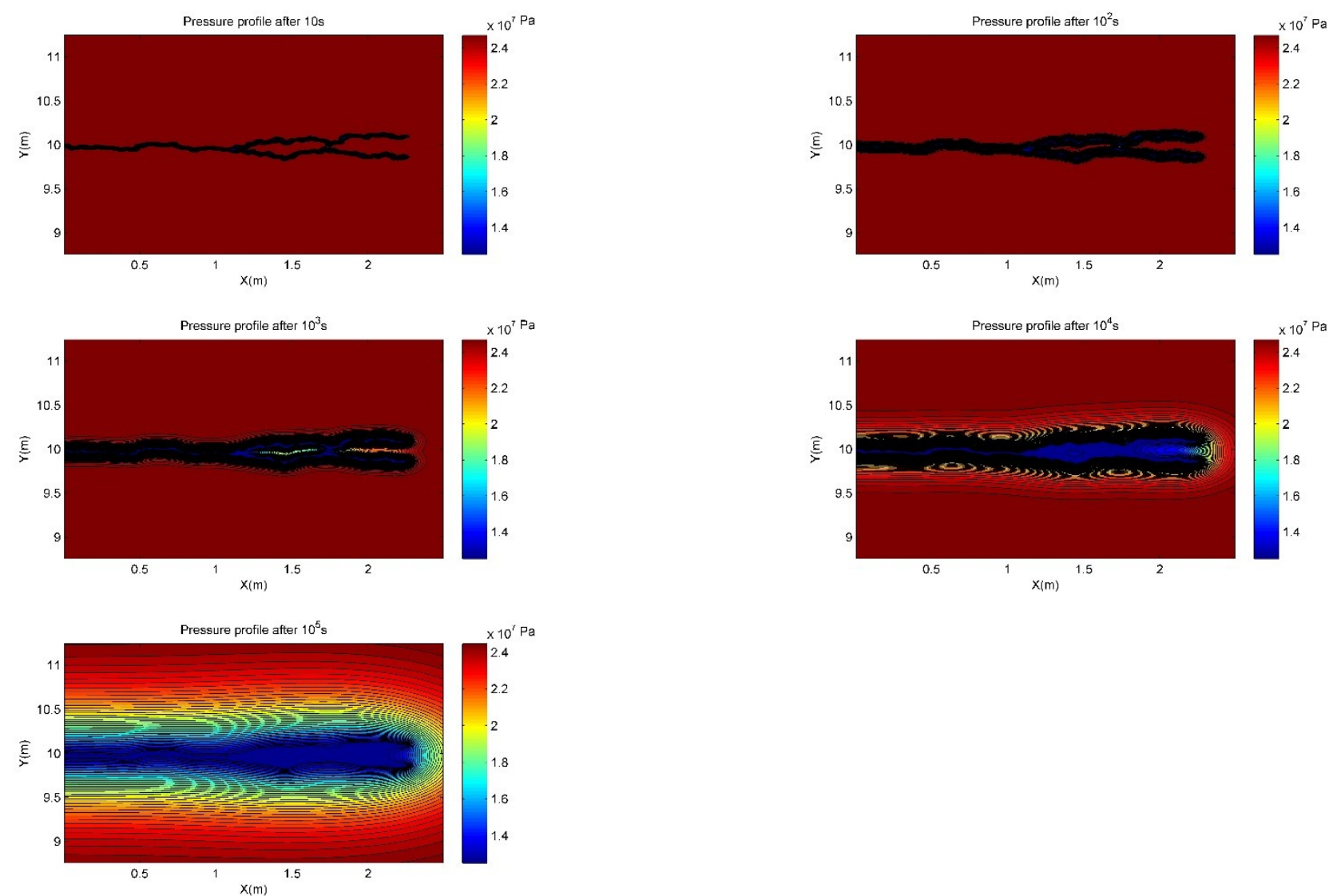

Figure D. 22 - Mono-branched fracture 18 pressure maps after $1 \times 10^{1} \mathrm{~s}, 1 \times 10^{2} \mathrm{~s}, 1 \times 10^{3} \mathrm{~s}, 1 \times 10^{4} \mathrm{~s}$ and $1 \times 10^{5} \mathrm{~s}$ 

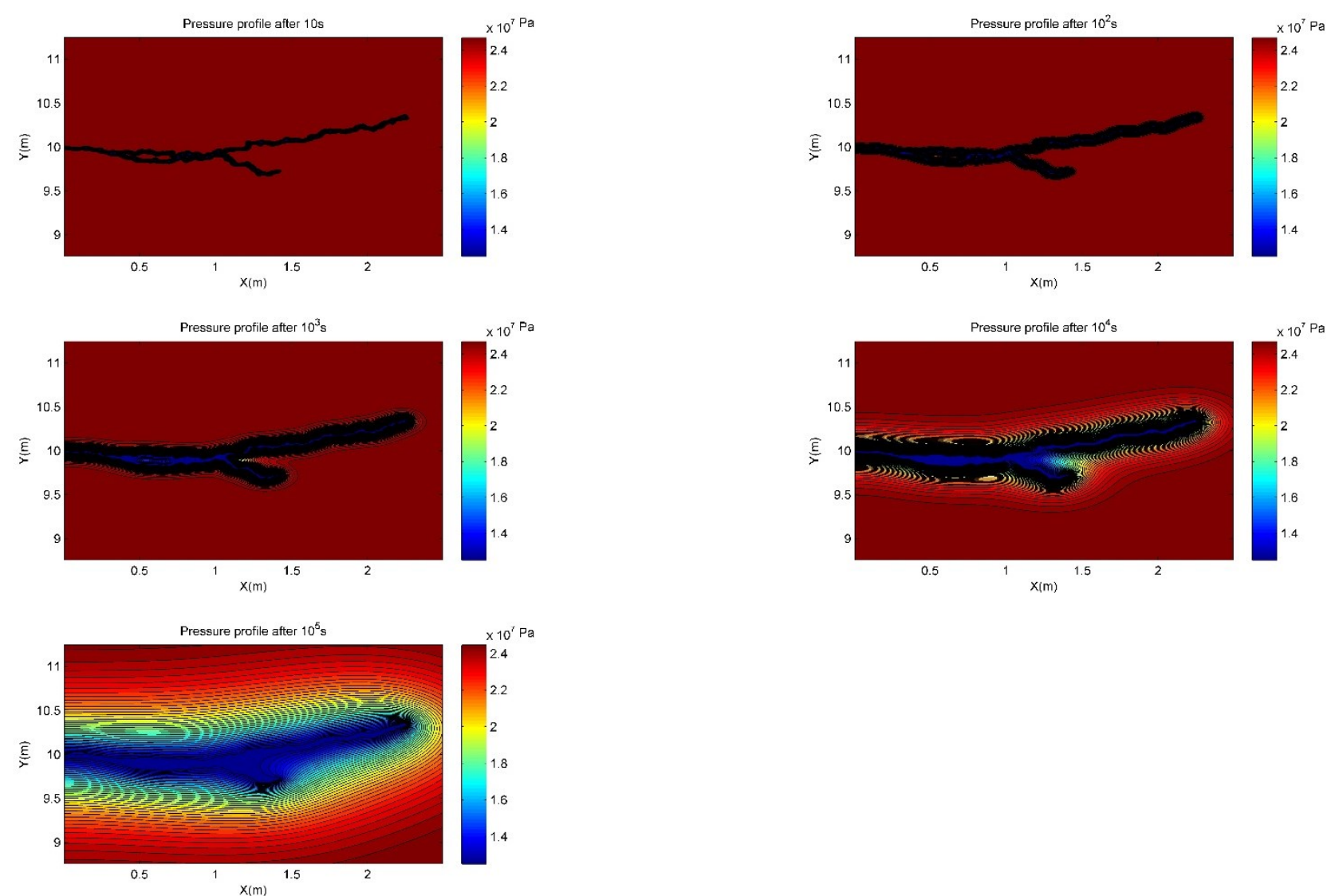

Figure D. 23 - Mono-branched fracture 19 pressure maps after $1 \times 10^{1} \mathrm{~s}, 1 \times 10^{2} \mathrm{~s}, 1 \times 10^{3} \mathrm{~s}, 1 \times 10^{4} \mathrm{~s}$ and $1 \times 10^{5} \mathrm{~s}$ 

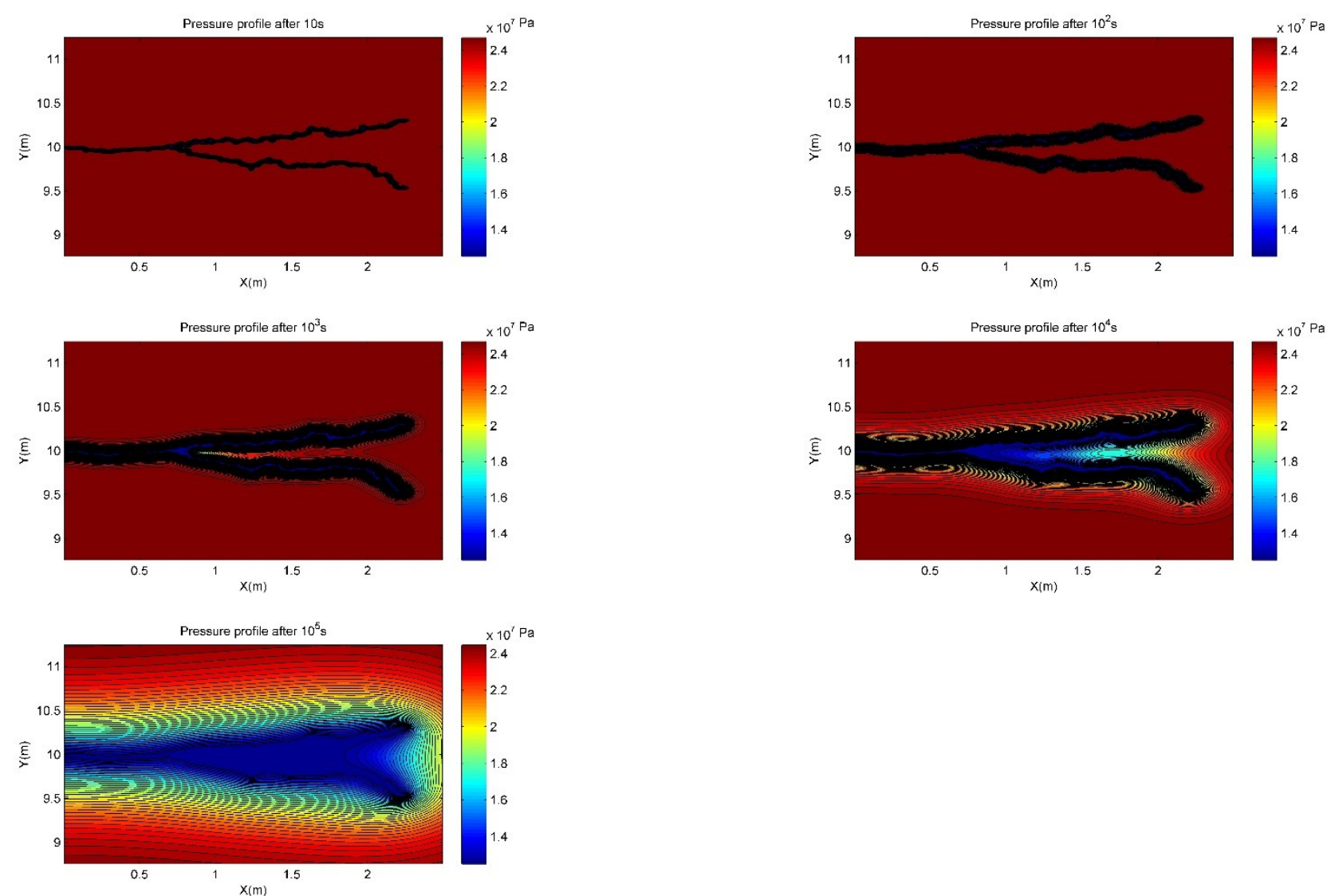

Figure D. 24 - Mono-branched fracture 20 pressure maps after $1 \times 10^{1} \mathrm{~s}, 1 \times 10^{2} \mathrm{~s}, 1 \times 10^{3} \mathrm{~s}, 1 \times 10^{4} \mathrm{~s}$ and $1 \times 10^{5} \mathrm{~s}$ 

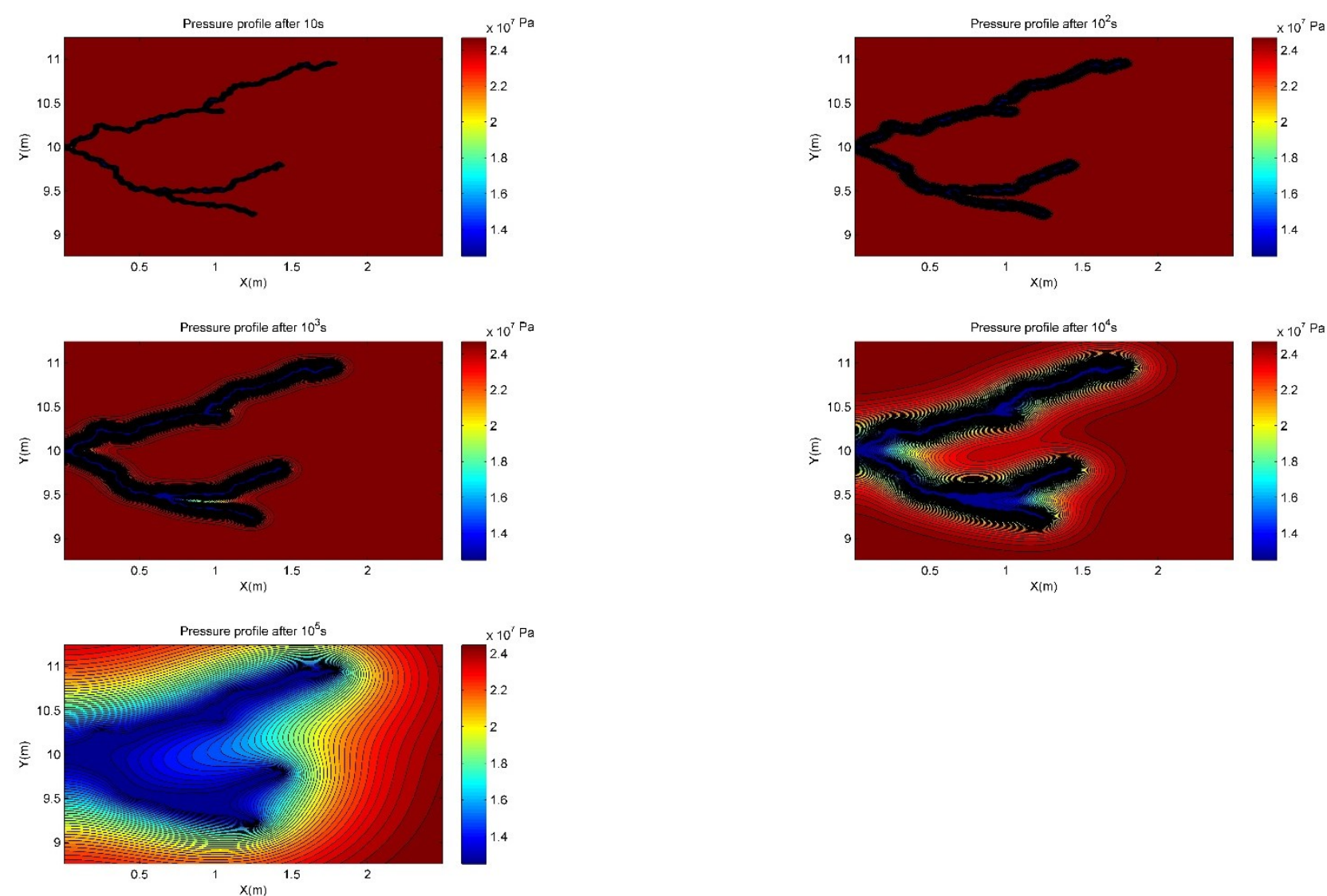

Figure D. 25 - Dual-branched fracture 1 pressure maps after $1 \times 10^{1} \mathrm{~s}, 1 \times 10^{2} \mathrm{~s}, 1 \times 10^{3} \mathrm{~s}, 1 \times 10^{4} \mathrm{~s}$ and $1 \times 10^{5} \mathrm{~s}$. 

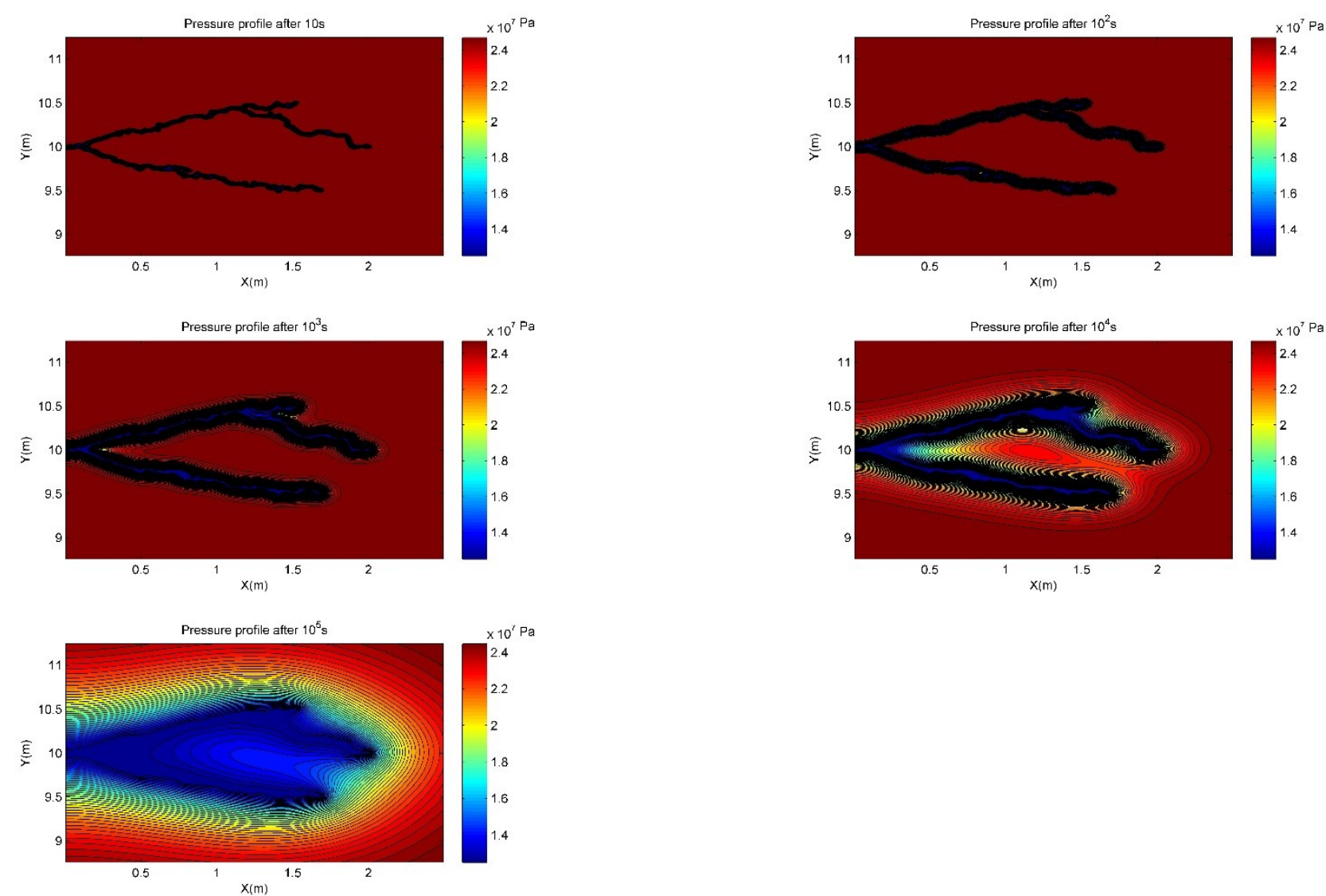

Figure D. 26 - Dual-branched fracture 2 pressure maps after $1 \times 10^{1} \mathrm{~s}, 1 \times 10^{2} \mathrm{~s}, 1 \times 10^{3} \mathrm{~s}, 1 \times 10^{4} \mathrm{~s}$ and $1 \times 10^{5} \mathrm{~s}$. 

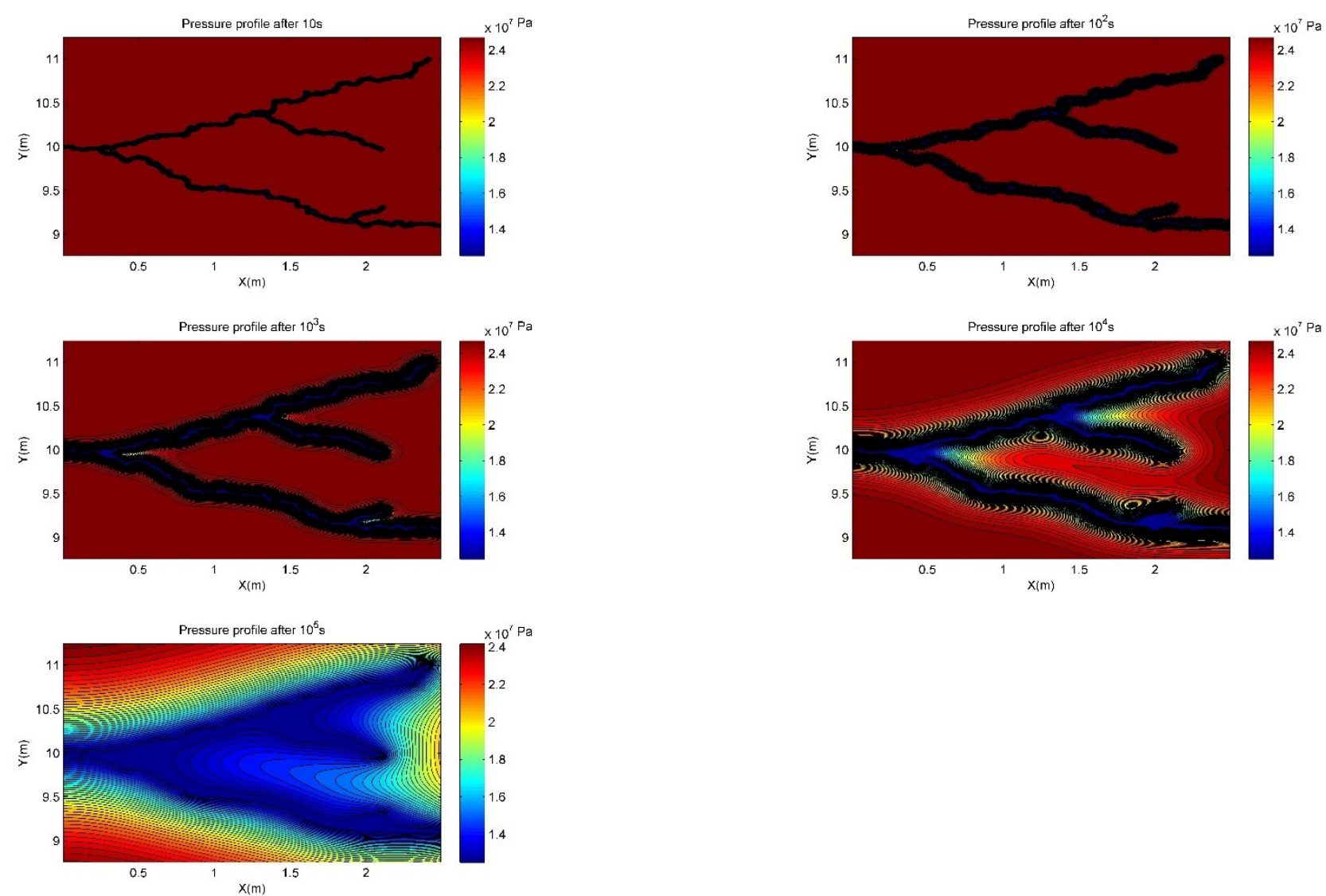

Figure D. 27 - Dual-branched fracture 3 pressure maps after $1 \times 10^{1} \mathrm{~s}, 1 \times 10^{2} \mathrm{~s}, 1 \times 10^{3} \mathrm{~s}, 1 \times 10^{4} \mathrm{~s}$ and $1 \times 10^{5} \mathrm{~s}$. 

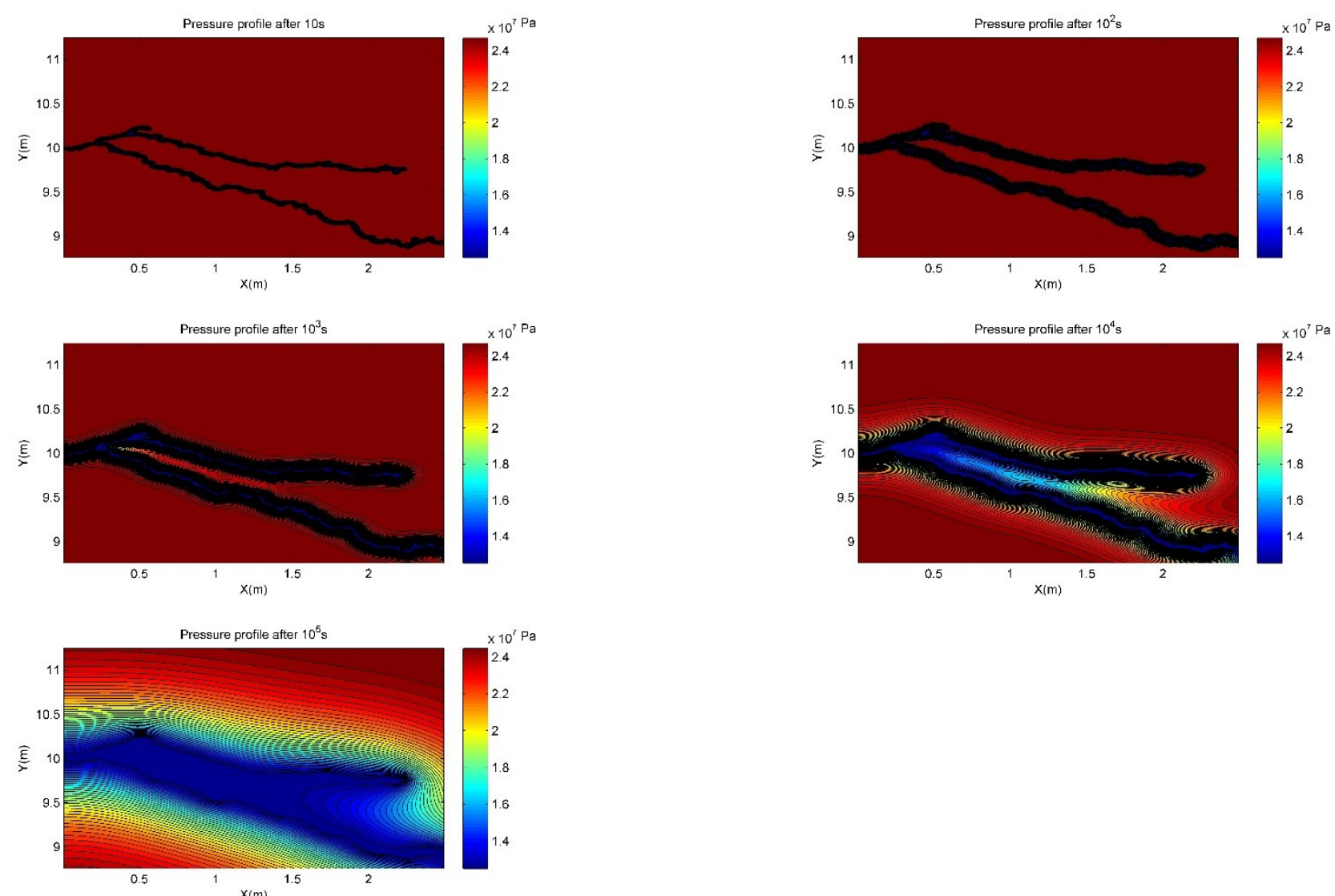

Figure D. 28 - Dual-branched fracture 4 pressure maps after $1 \times 10^{1} \mathrm{~s}, 1 \times 10^{2} \mathrm{~s}, 1 \times 10^{3} \mathrm{~s}, 1 \times 10^{4} \mathrm{~s}$ and $1 \times 10^{5} \mathrm{~s}$. 

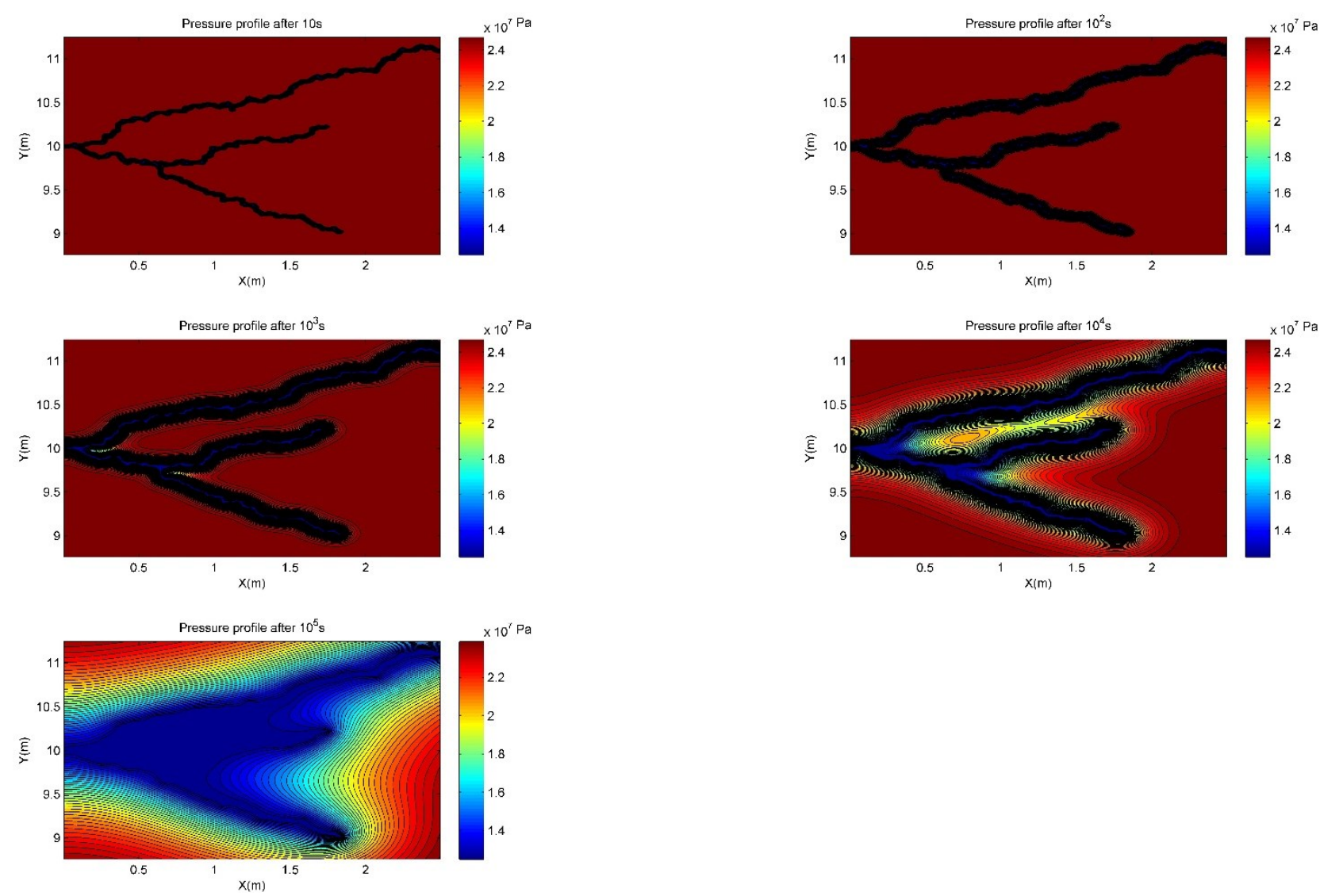

Figure D. 29 - Dual-branched fracture 5 pressure maps after $1 \times 10^{1} \mathrm{~s}, 1 \times 10^{2} \mathrm{~s}, 1 \times 10^{3} \mathrm{~s}, 1 \times 10^{4} \mathrm{~s}$ and $1 \times 10^{5} \mathrm{~s}$. 

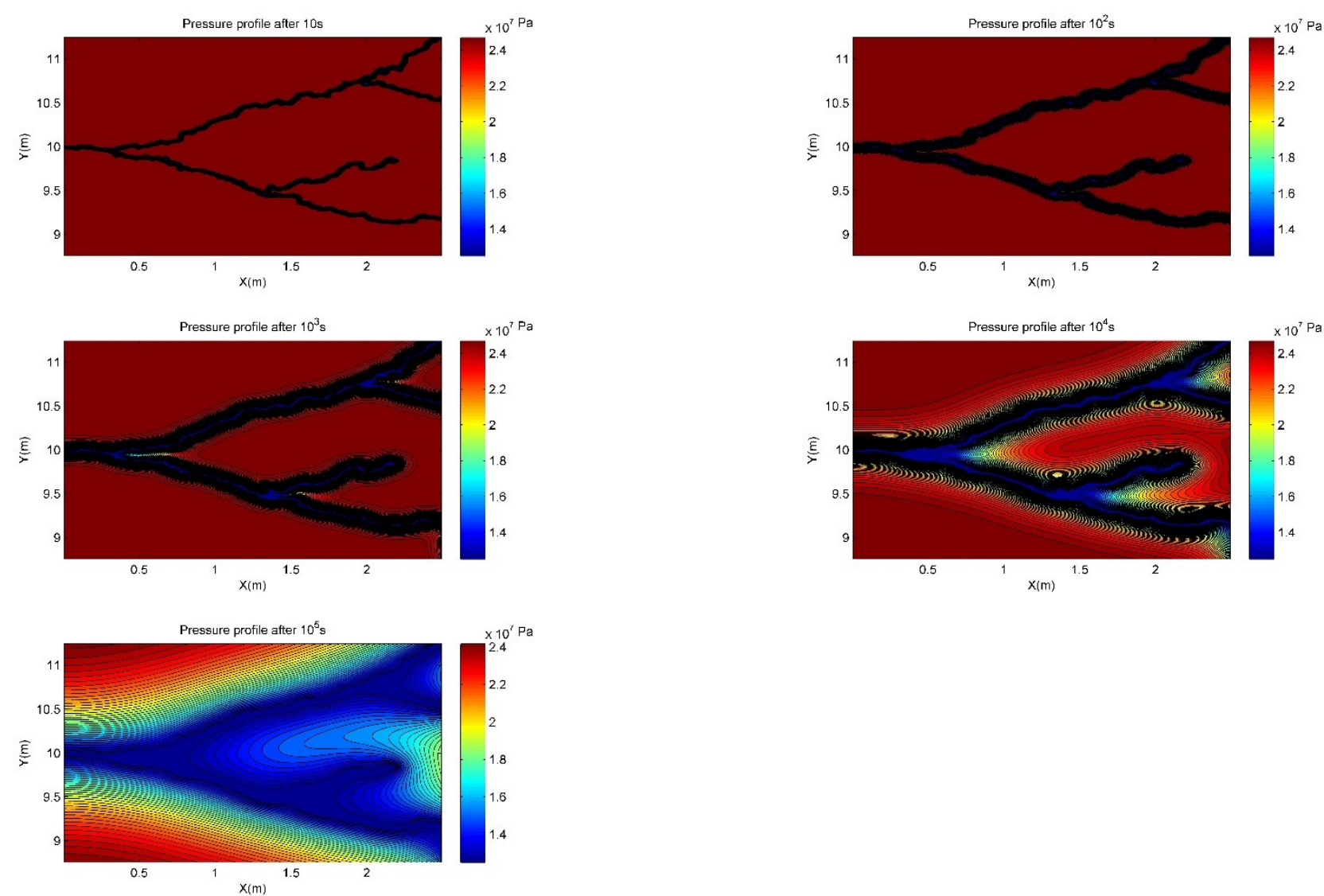

Figure D. 30 - Dual-branched fracture 6 pressure maps after $1 \times 10^{1} \mathrm{~s}, 1 \times 10^{2} \mathrm{~s}, 1 \times 10^{3} \mathrm{~s}, 1 \times 10^{4} \mathrm{~s}$ and $1 \times 10^{5} \mathrm{~s}$ 

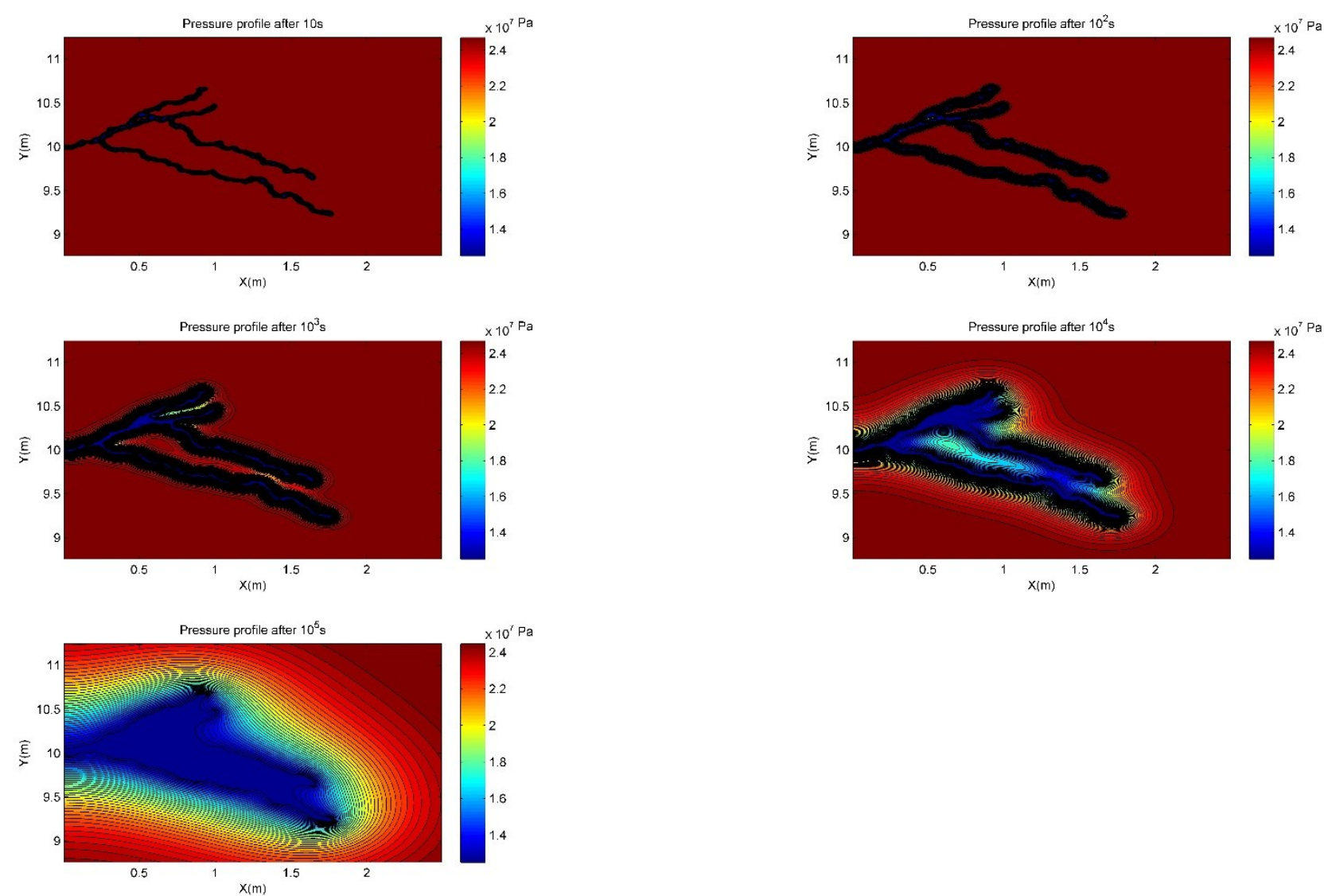

Figure D. 31 - Dual-branched fracture 7 pressure maps after $1 \times 10^{1} \mathrm{~s}, 1 \times 10^{2} \mathrm{~s}, 1 \times 10^{3} \mathrm{~s}, 1 \times 10^{4} \mathrm{~s}$ and $1 \times 10^{5} \mathrm{~s}$ 

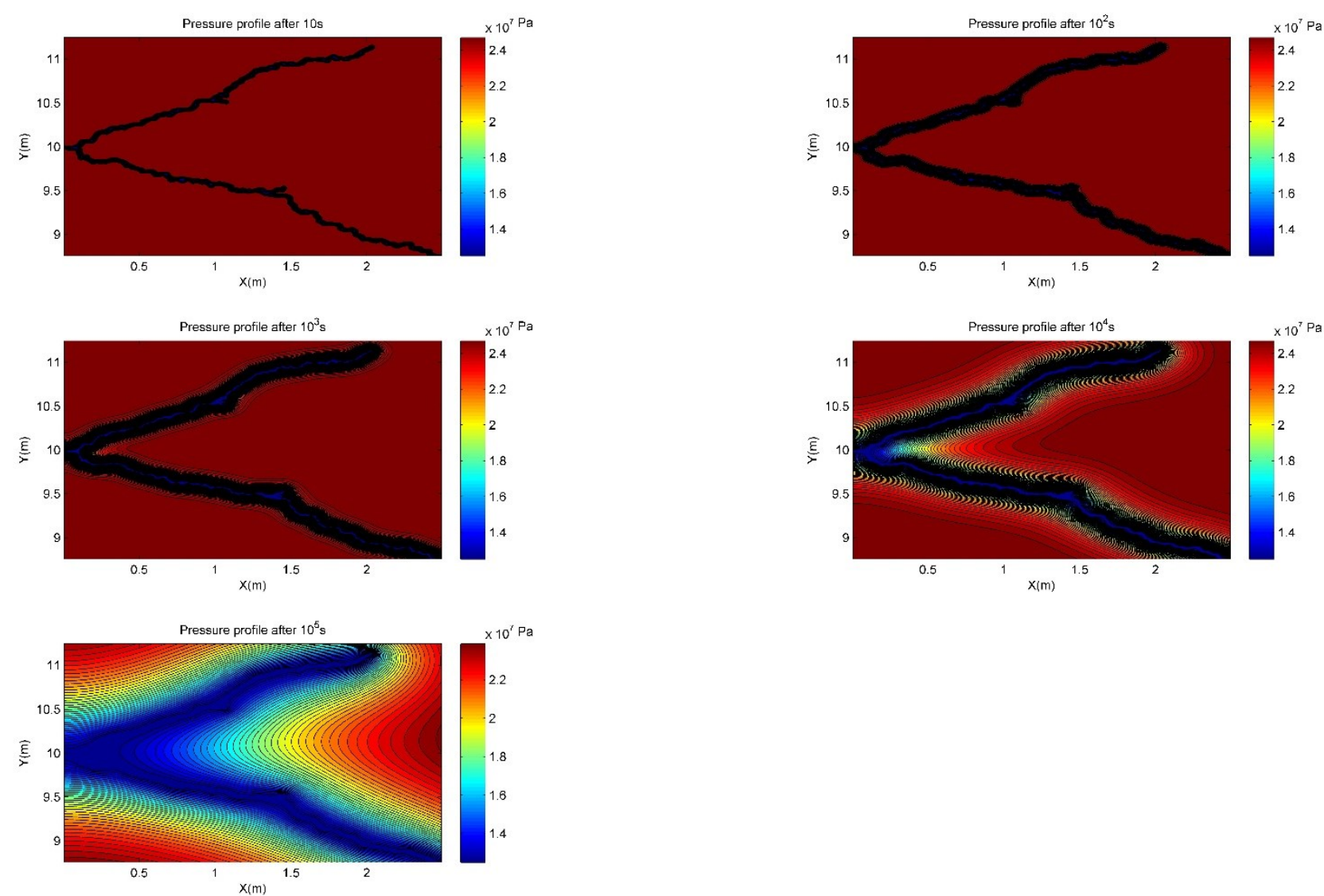

Figure D. 32 - Dual-branched fracture 8 pressure maps after $1 \times 10^{1} \mathrm{~s}, 1 \times 10^{2} \mathrm{~s}, 1 \times 10^{3} \mathrm{~s}, 1 \times 10^{4} \mathrm{~s}$ and $1 \times 10^{5} \mathrm{~s}$. 

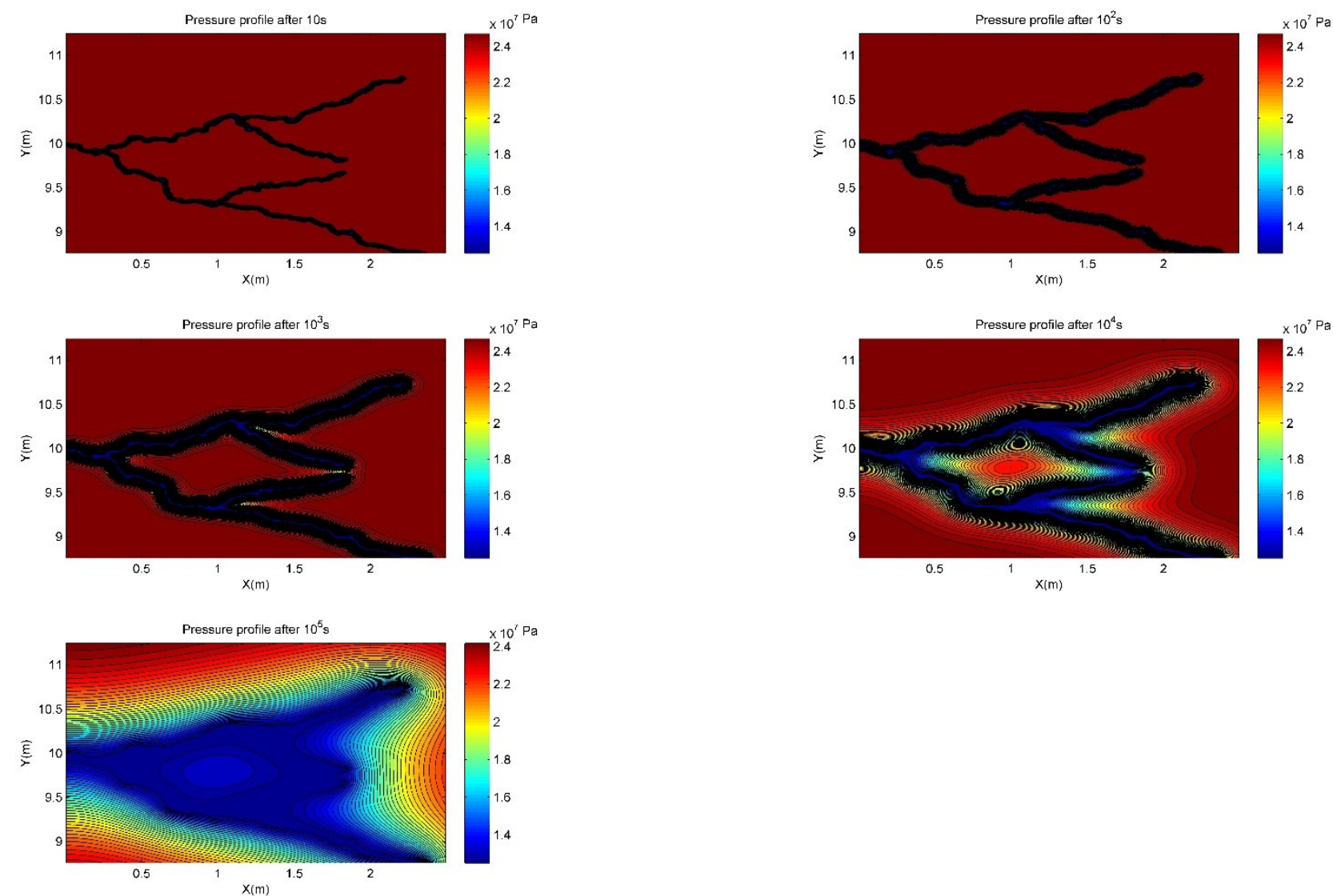

Figure D. 33 - Dual-branched fracture 9 pressure maps after $1 \times 10^{1} s, 1 \times 10^{2} s, 1 \times 10^{3} s, 1 \times 10^{4} s$ and $1 \times 10^{5} s$. 

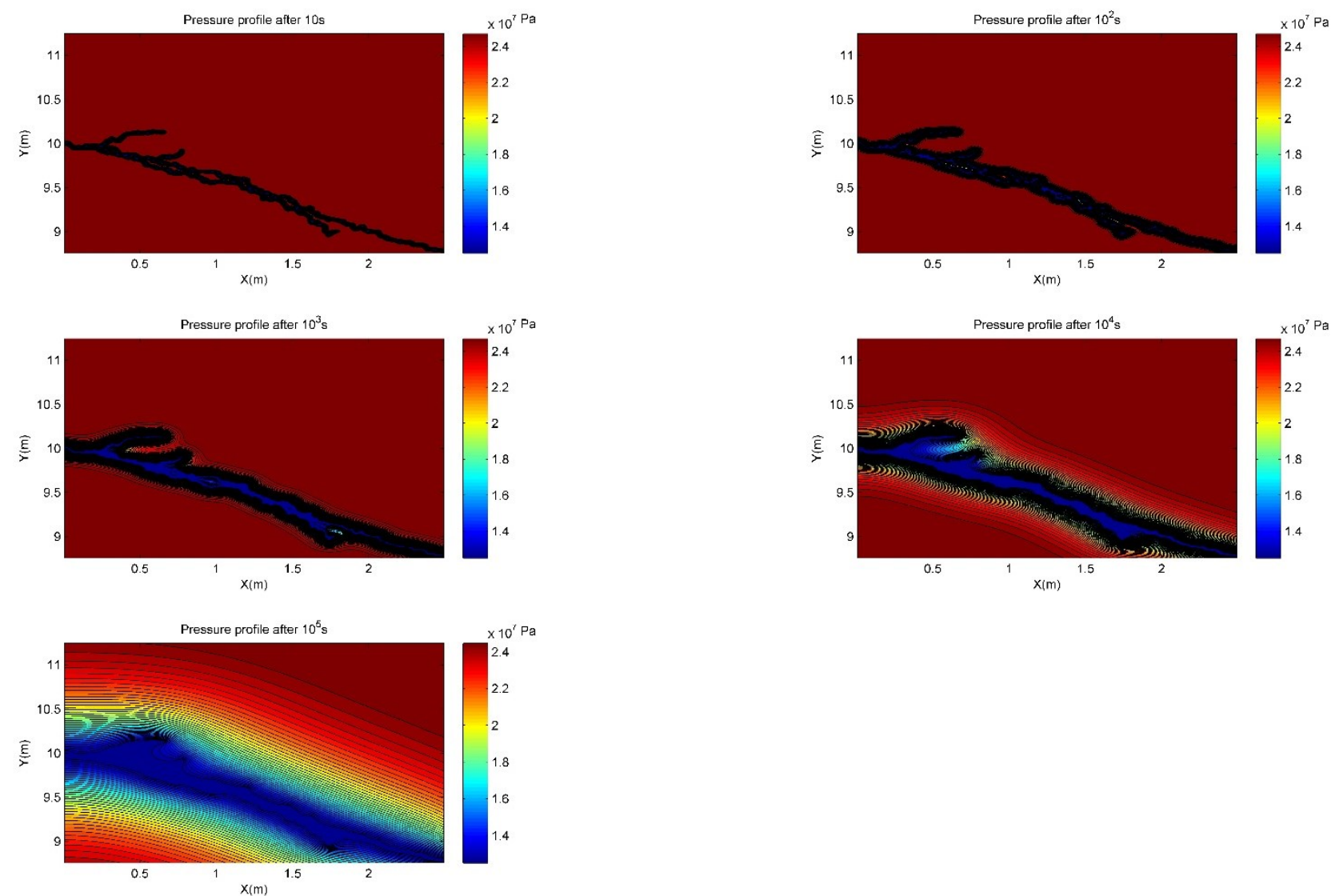

Figure D. 34 - Dual-branched fracture 10 pressure maps after $1 \times 10^{1} \mathrm{~s}, 1 \times 10^{2} \mathrm{~s}, 1 \times 10^{3} \mathrm{~s}, 1 \times 10^{4} \mathrm{~s}$ and $1 \times 10^{5} \mathrm{~s}$. 

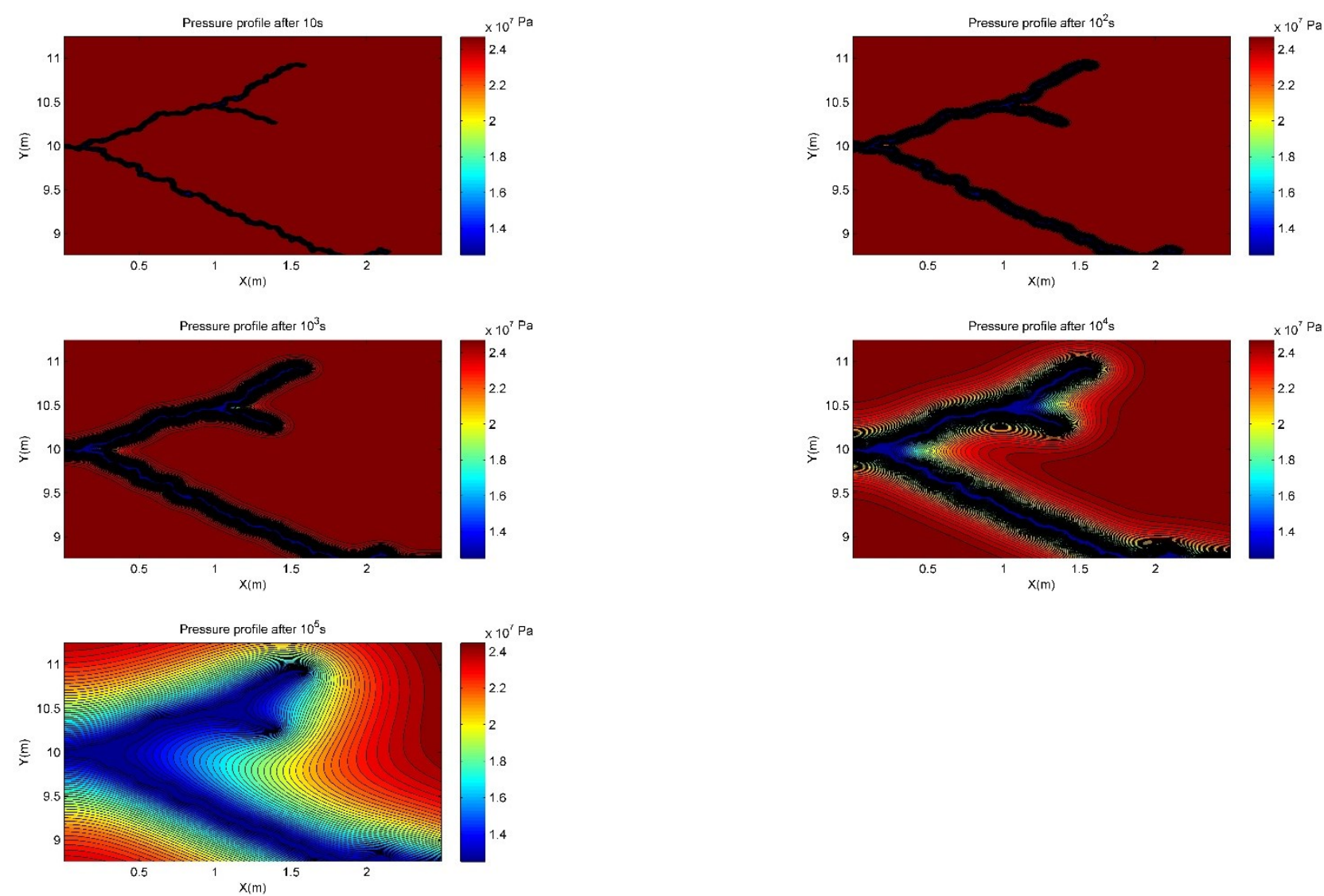

Figure D. 35 - Dual-branched fracture 11 pressure maps after $1 \times 10^{1} \mathrm{~s}, 1 \times 10^{2} \mathrm{~s}, 1 \times 10^{3} \mathrm{~s}, 1 \times 10^{4} \mathrm{~s}$ and $1 \times 10^{5} \mathrm{~s}$. 

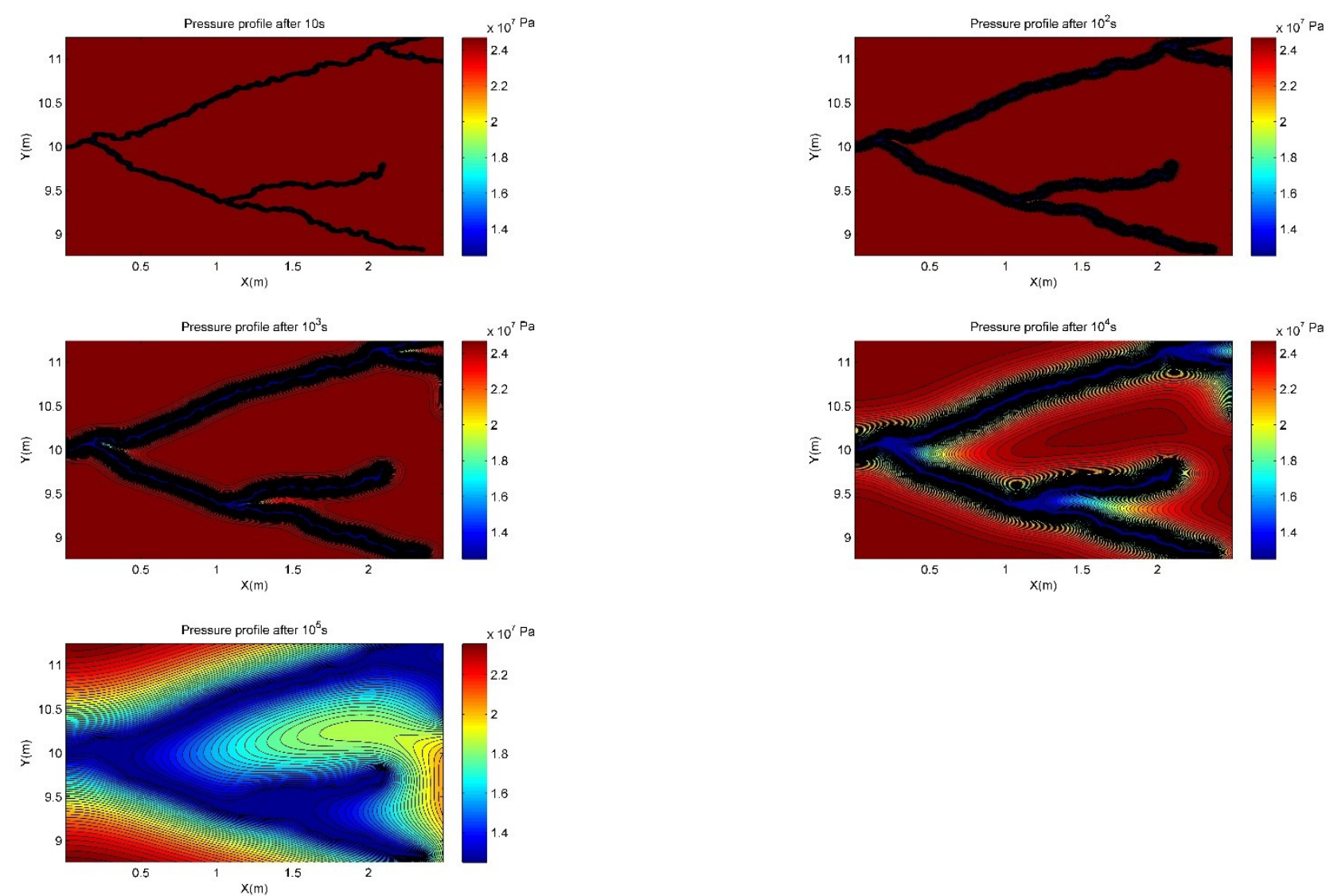

Figure D. 36 - Dual-branched fracture 12 pressure maps after $1 \times 10^{1} \mathrm{~s}, 1 \times 10^{2} \mathrm{~s}, 1 \times 10^{3} \mathrm{~s}, 1 \times 10^{4} \mathrm{~s}$ and $1 \times 10^{5} \mathrm{~s}$. 

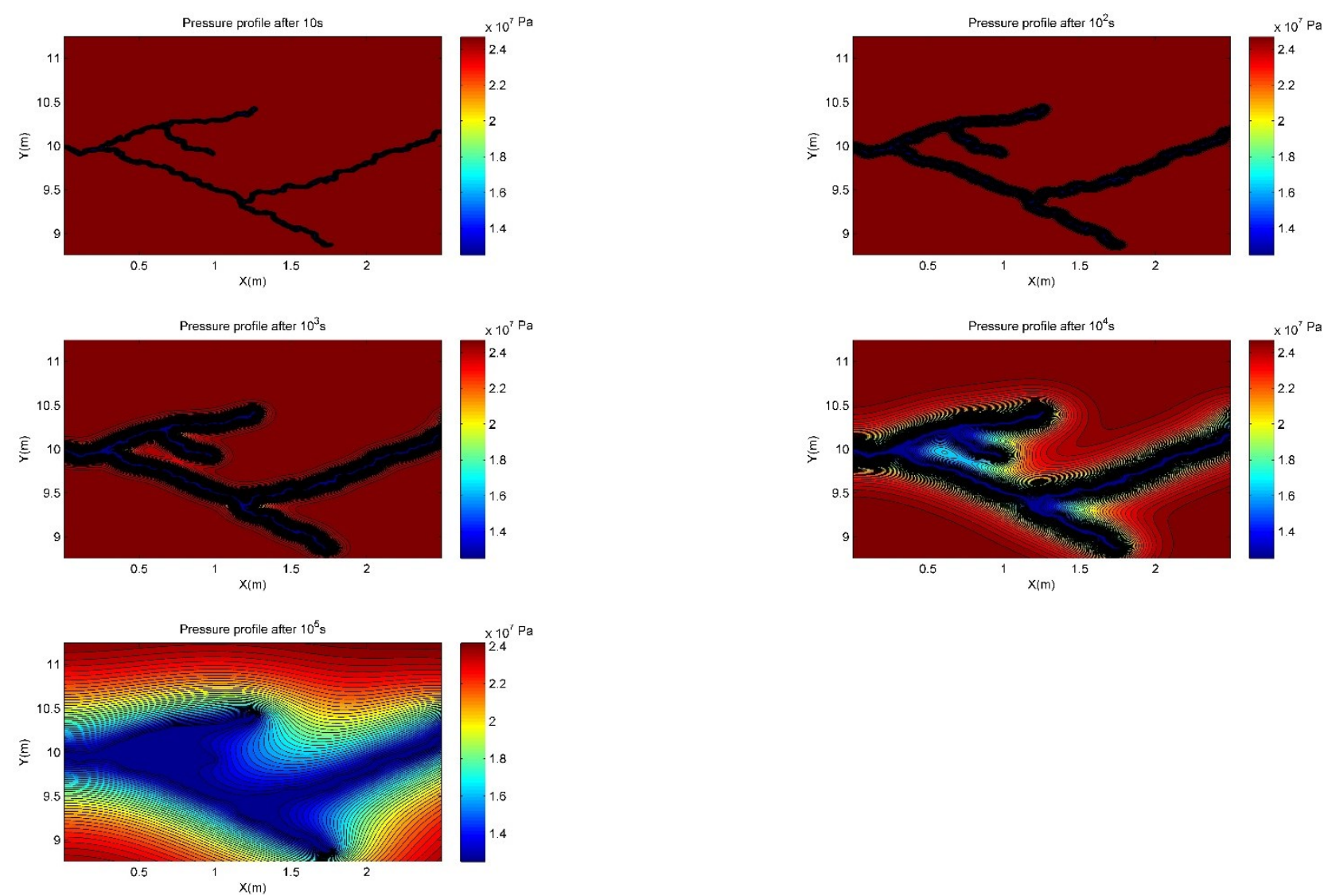

Figure D. 37 - Dual-branched fracture 13 pressure maps after $1 \times 10^{1} \mathrm{~s}, 1 \times 10^{2} \mathrm{~s}, 1 \times 10^{3} \mathrm{~s}, 1 \times 10^{4} \mathrm{~s}$ and $1 \times 10^{5} \mathrm{~s}$. 

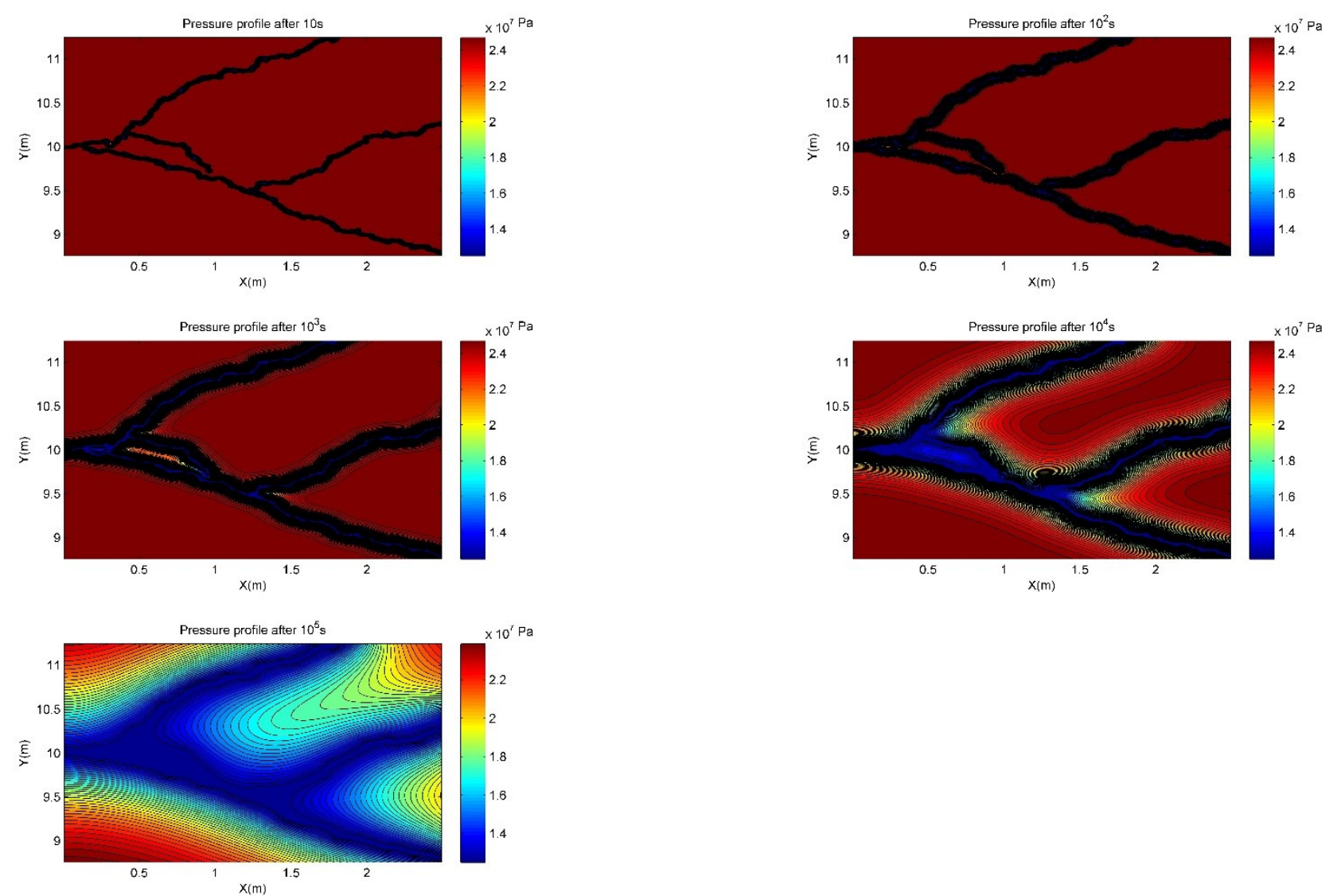

Figure D. 38 - Dual-branched fracture 14 pressure maps after $1 \times 10^{1} \mathrm{~s}, 1 \times 10^{2} \mathrm{~s}, 1 \times 10^{3} \mathrm{~s}, 1 \times 10^{4} \mathrm{~s}$ and $1 \times 10^{5} \mathrm{~s}$ 

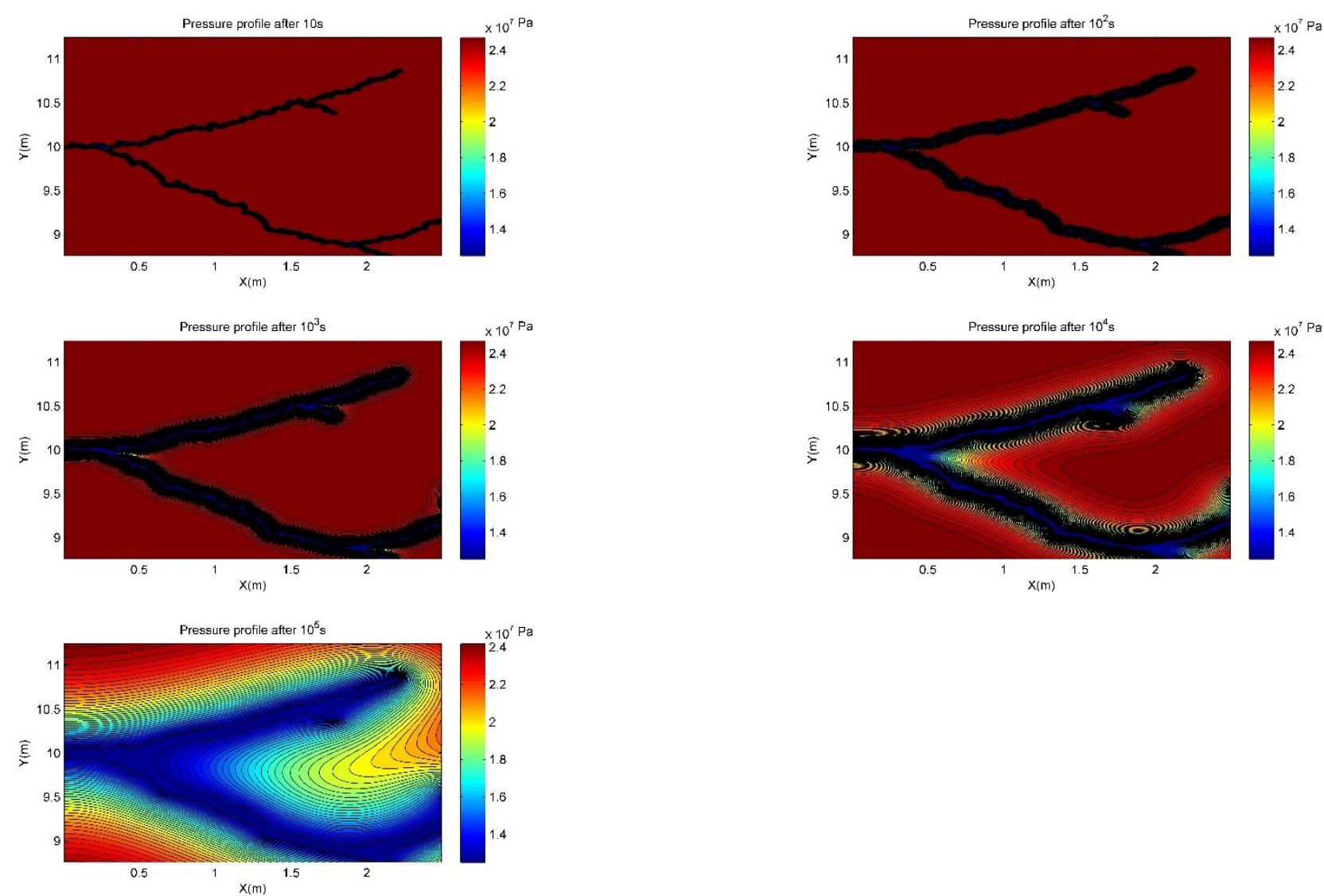

Figure D. 39 - Dual-branched fracture 15 pressure maps after $1 \times 10^{1} \mathrm{~s}, 1 \times 10^{2} \mathrm{~s}, 1 \times 10^{3} \mathrm{~s}, 1 \times 10^{4} \mathrm{~s}$ and $1 \times 10^{5} \mathrm{~s}$. 

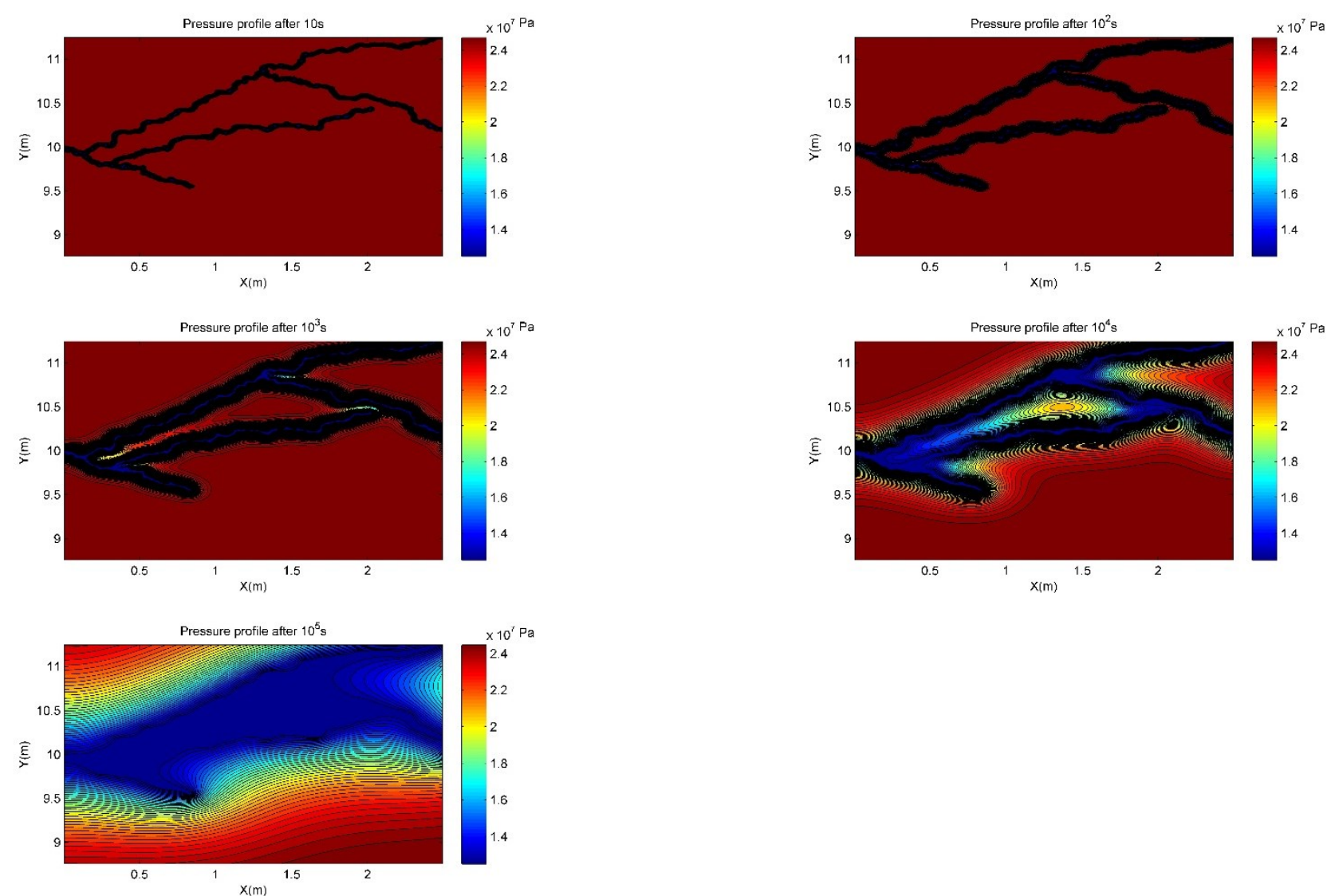

Figure D. 40 - Dual-branched fracture 16 pressure maps after $1 \times 10^{1} \mathrm{~s}, 1 \times 10^{2} \mathrm{~s}, 1 \times 10^{3} \mathrm{~s}, 1 \times 10^{4} \mathrm{~s}$ and $1 \times 10^{5} \mathrm{~s}$ 

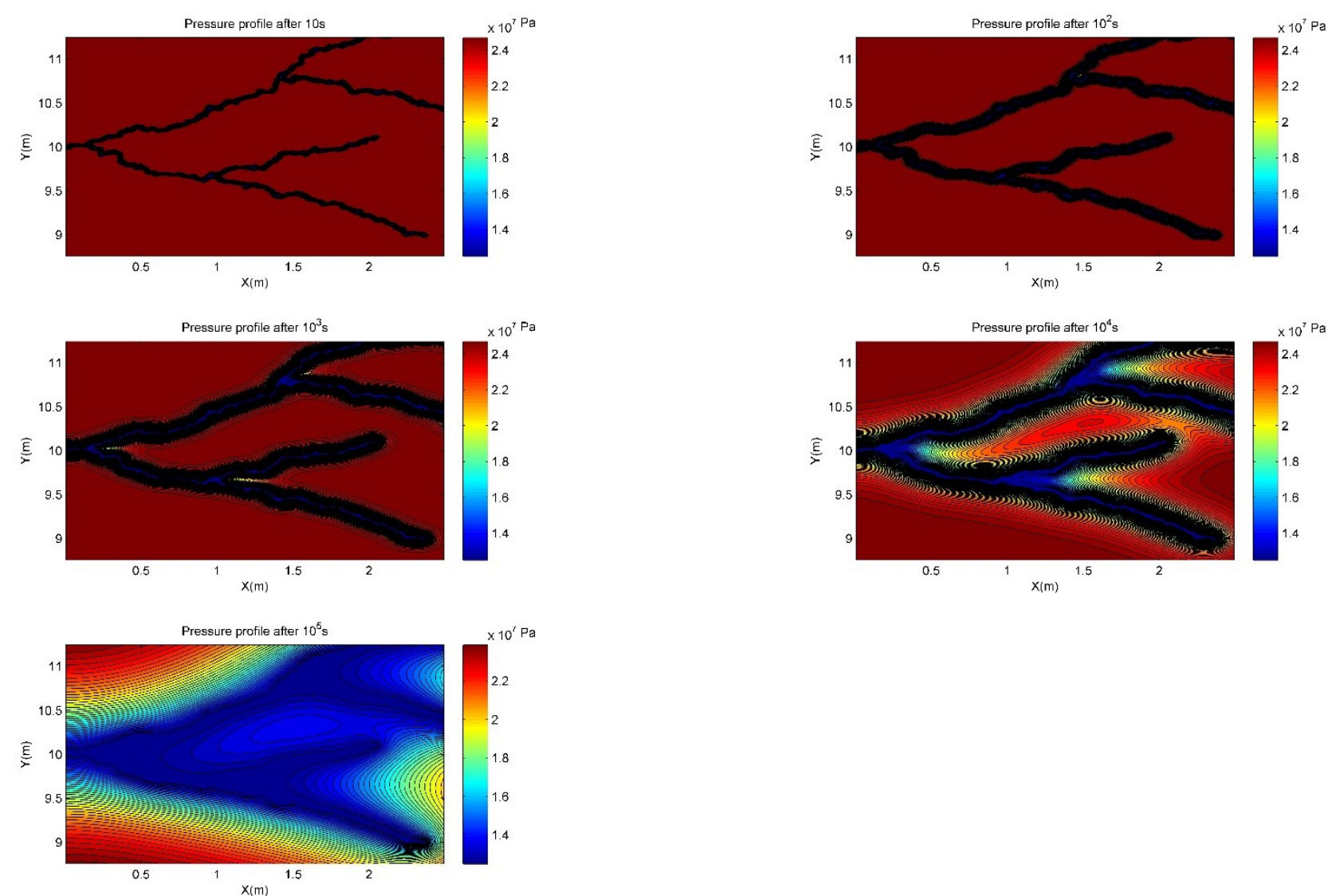

Figure D. 41 - Dual-branched fracture 17 pressure maps after $1 \times 10^{1} \mathrm{~s}, 1 \times 10^{2} \mathrm{~s}, 1 \times 10^{3} \mathrm{~s}, 1 \times 10^{4} \mathrm{~s}$ and $1 \times 10^{5} \mathrm{~s}$. 

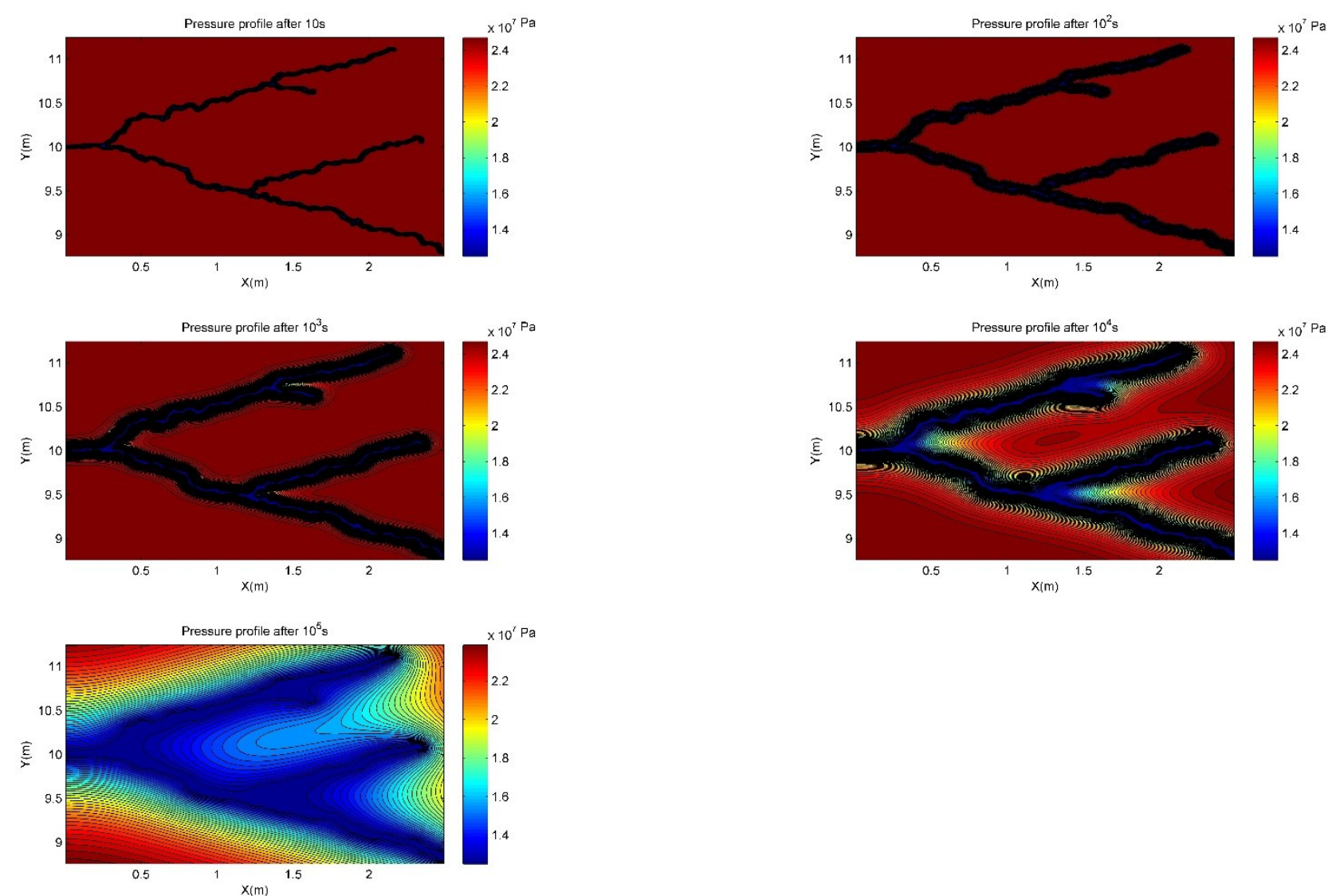

Figure D. 42 - Dual-branched fracture 18 pressure maps after $1 \times 10^{1} \mathrm{~s}, 1 \times 10^{2} \mathrm{~s}, 1 \times 10^{3} \mathrm{~s}, 1 \times 10^{4} \mathrm{~s}$ and $1 \times 10^{5} \mathrm{~s}$ 

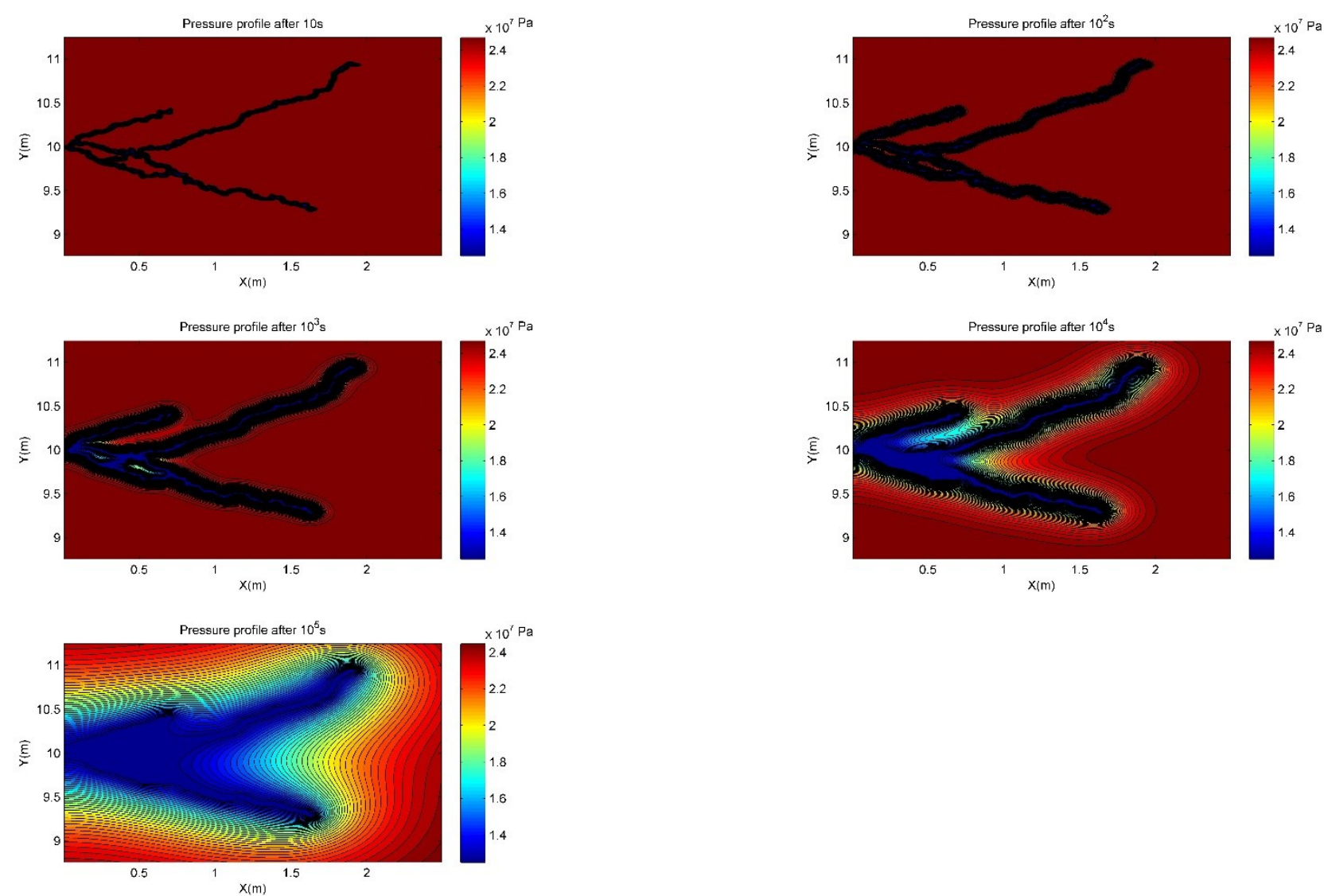

Figure D. 43 - Dual-branched fracture 19 pressure maps after $1 \times 10^{1} \mathrm{~s}, 1 \times 10^{2} \mathrm{~s}, 1 \times 10^{3} \mathrm{~s}, 1 \times 10^{4} \mathrm{~s}$ and $1 \times 10^{5} \mathrm{~s}$ 

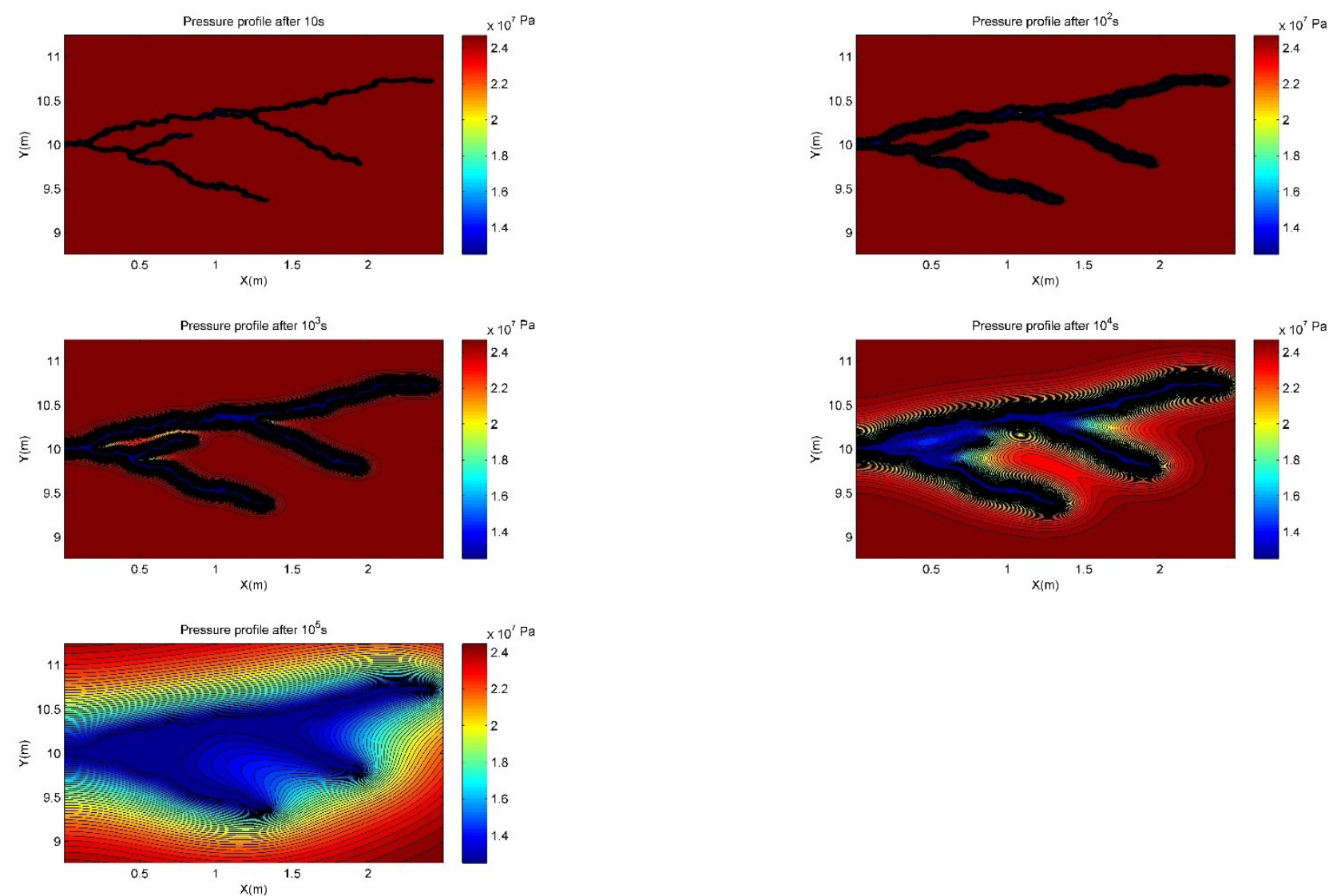

Figure D. 44 - Dual-branched fracture 20 pressure maps after $1 \times 10^{1} \mathrm{~s}, 1 \times 10^{2} \mathrm{~s}, 1 \times 10^{3} \mathrm{~s}, 1 \times 10^{4} \mathrm{~s}$ and $1 \times 10^{5} \mathrm{~s}$ 

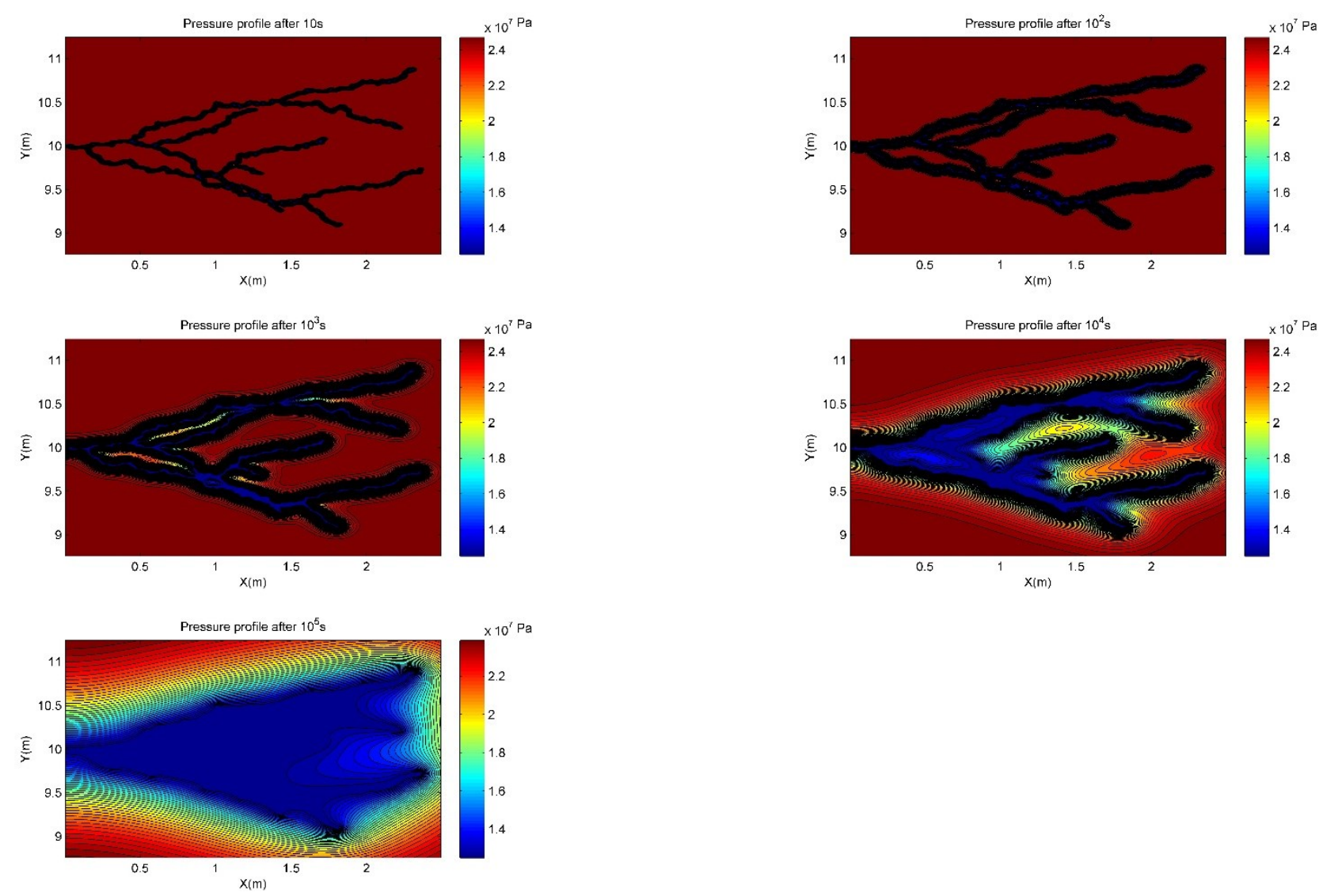

Figure D. 45 - Tri-branched fracture 1 pressure maps after $1 \times 10^{1} \mathrm{~s}, 1 \times 10^{2} \mathrm{~s}, 1 \times 10^{3} \mathrm{~s}, 1 \times 10^{4} \mathrm{~s}$ and $1 \times 10^{5} \mathrm{~s}$ 

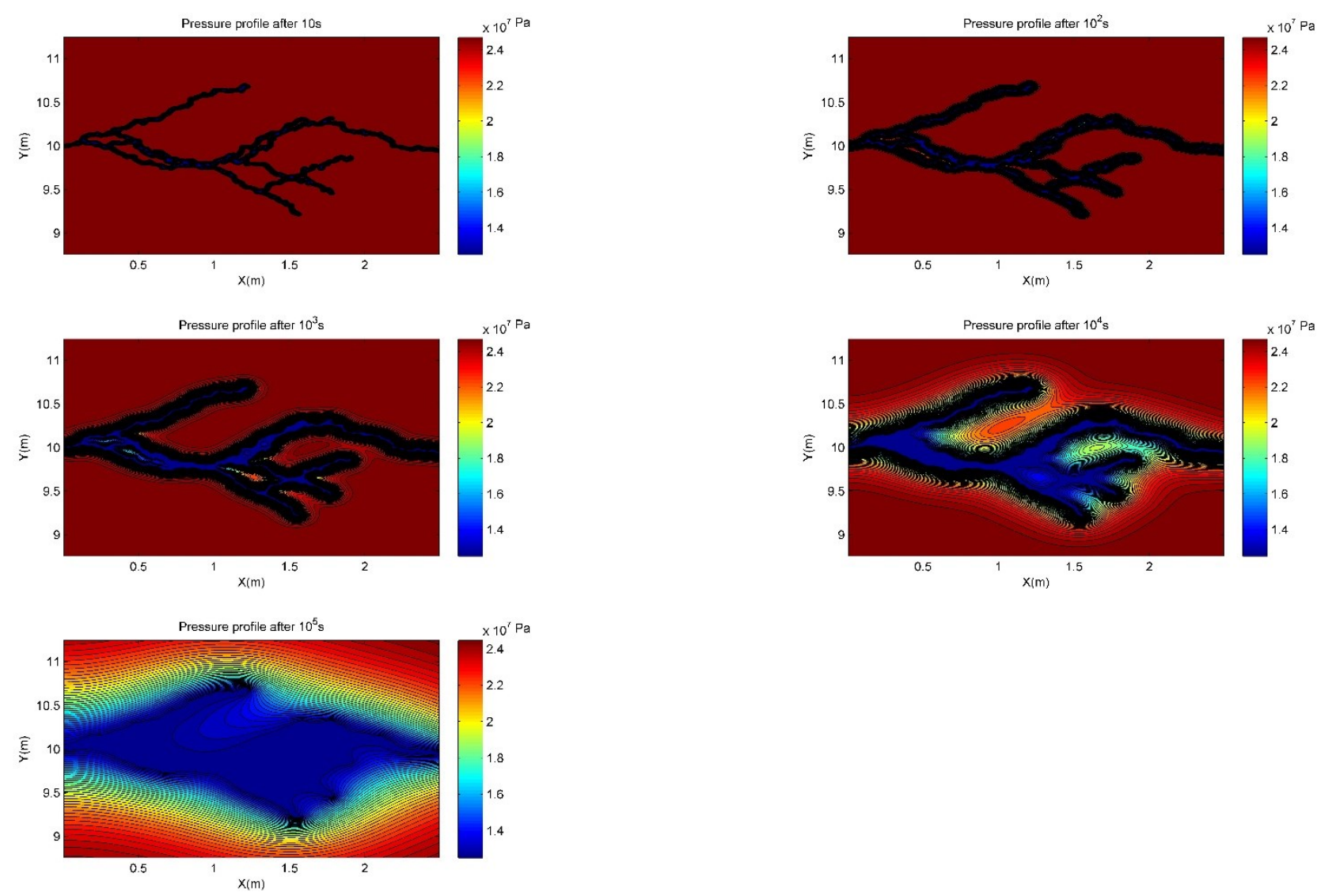

Figure D. 46 - Tri-branched fracture 2 pressure maps after $1 \times 10^{1} \mathrm{~s}, 1 \times 10^{2} \mathrm{~s}, 1 \times 10^{3} \mathrm{~s}, 1 \times 10^{4} \mathrm{~s}$ and $1 \times 10^{5} \mathrm{~s}$ 

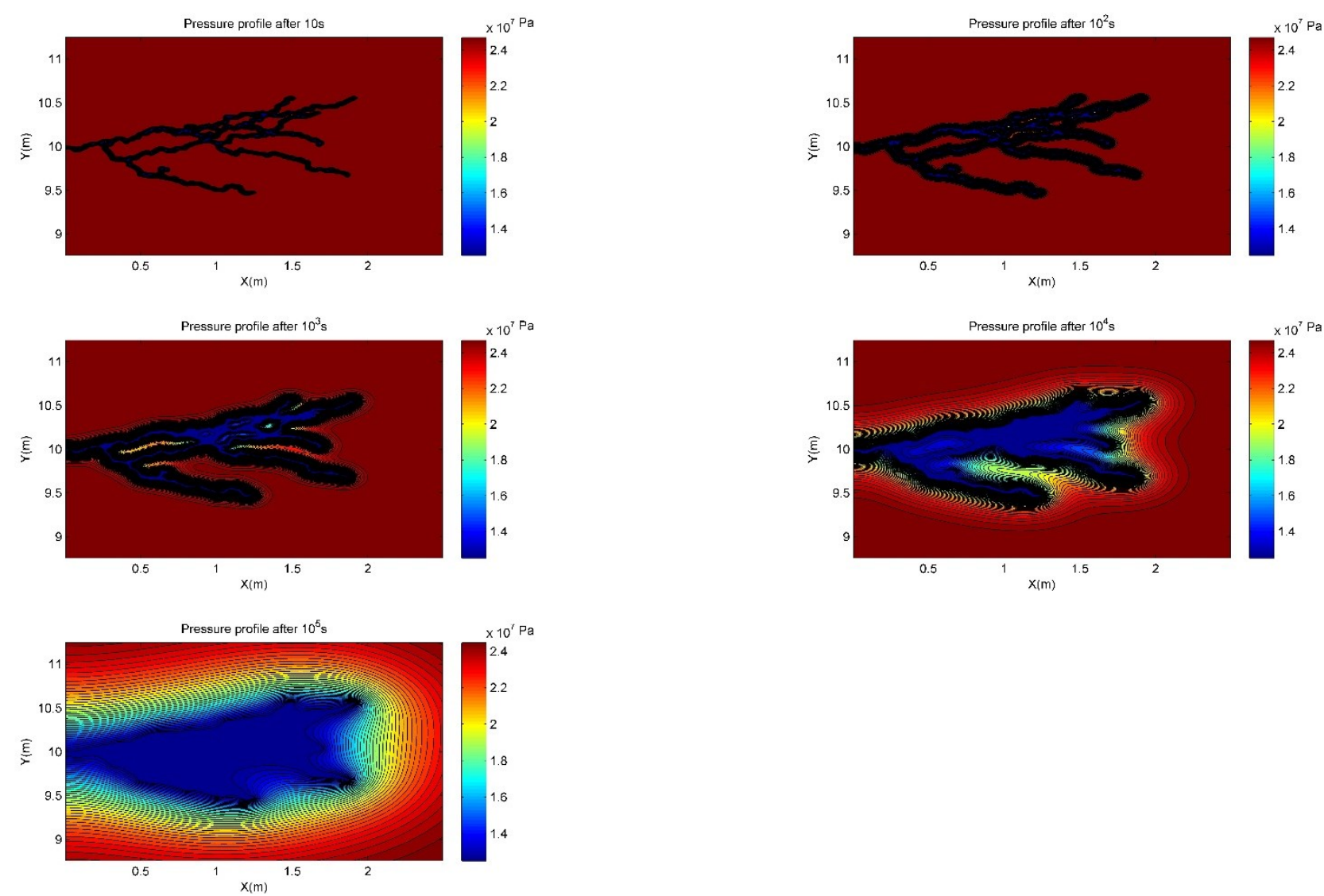

Figure D. 47 - Tri-branched fracture 3 pressure maps after $1 \times 10^{1} \mathrm{~s}, 1 \times 10^{2} \mathrm{~s}, 1 \times 10^{3} \mathrm{~s}, 1 \times 10^{4} \mathrm{~s}$ and $1 \times 10^{5} \mathrm{~s}$ 

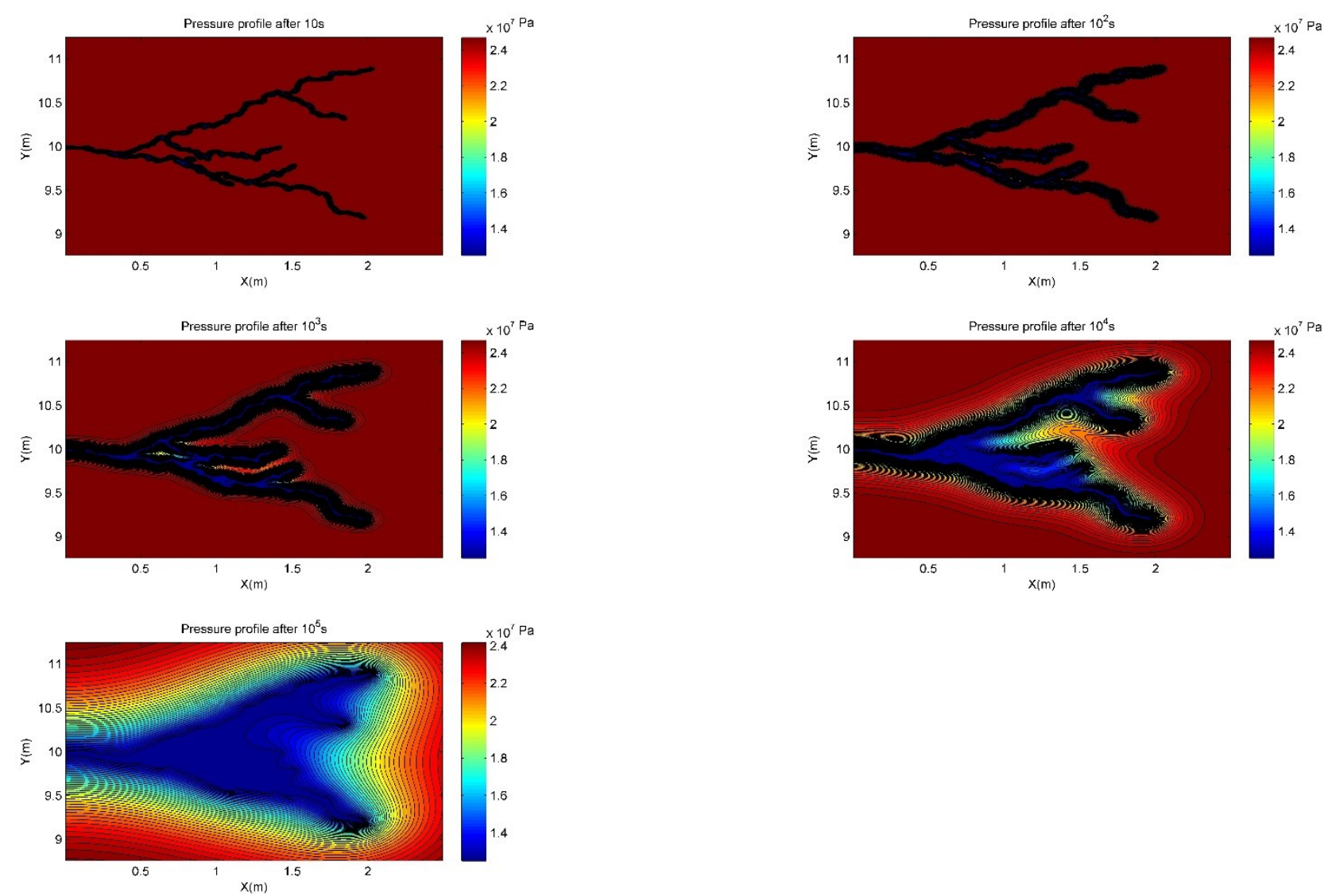

Figure D. 48 - Tri-branched fracture 4 pressure maps after $1 \times 10^{1} \mathrm{~s}, 1 \times 10^{2} \mathrm{~s}, 1 \times 10^{3} \mathrm{~s}, 1 \times 10^{4} \mathrm{~s}$ and $1 \times 10^{5} \mathrm{~s}$ 

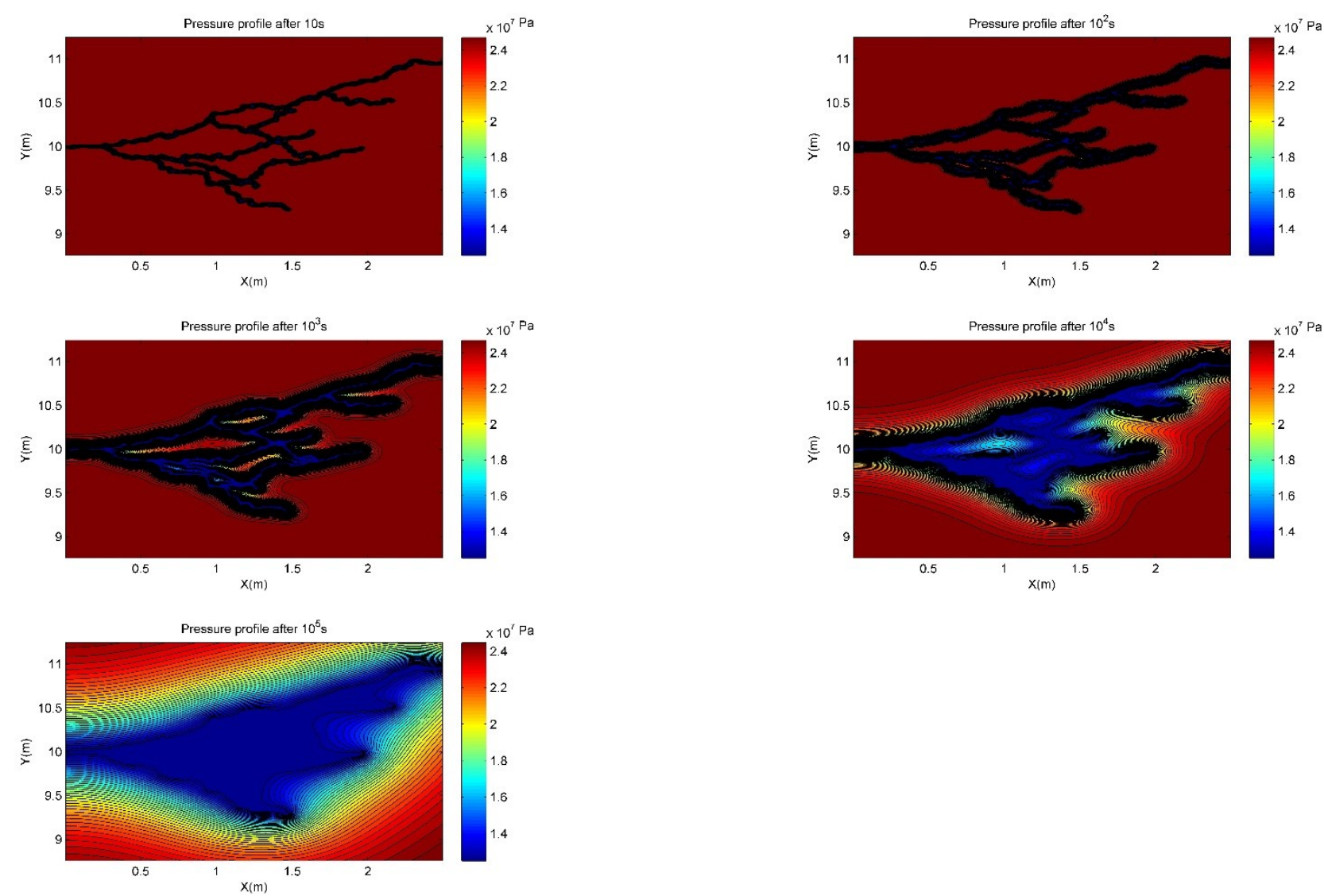

Figure D. 49 - Tri-branched fracture 5 pressure maps after $1 \times 10^{1} \mathrm{~s}, 1 \times 10^{2} \mathrm{~s}, 1 \times 10^{3} \mathrm{~s}, 1 \times 10^{4} \mathrm{~s}$ and $1 \times 10^{5} \mathrm{~s}$ 

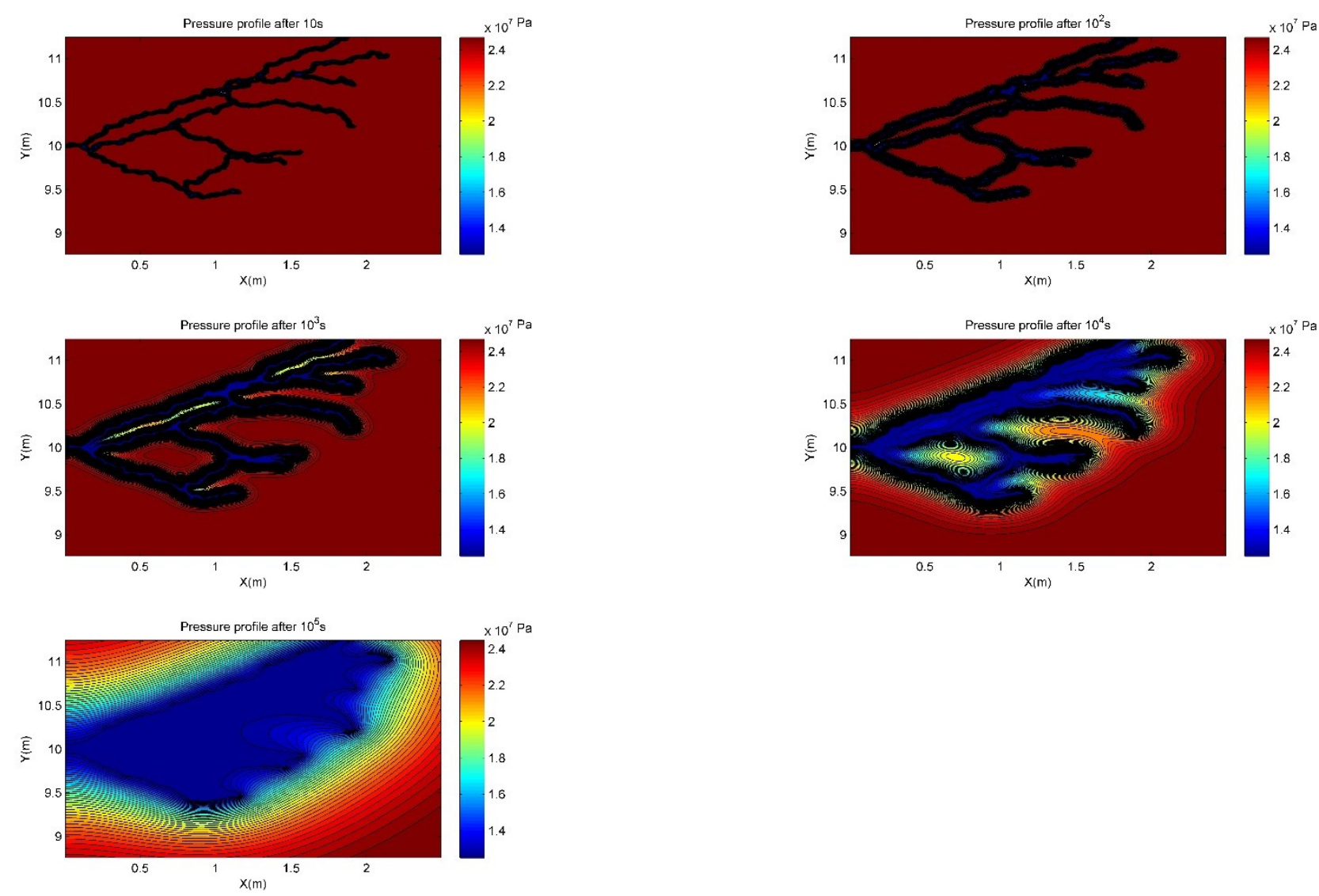

Figure D. 50 - Tri-branched fracture 6 pressure maps after $1 \times 10^{1} \mathrm{~s}, 1 \times 10^{2} \mathrm{~s}, 1 \times 10^{3} \mathrm{~s}, 1 \times 10^{4} \mathrm{~s}$ and $1 \times 10^{5} \mathrm{~s}$ 

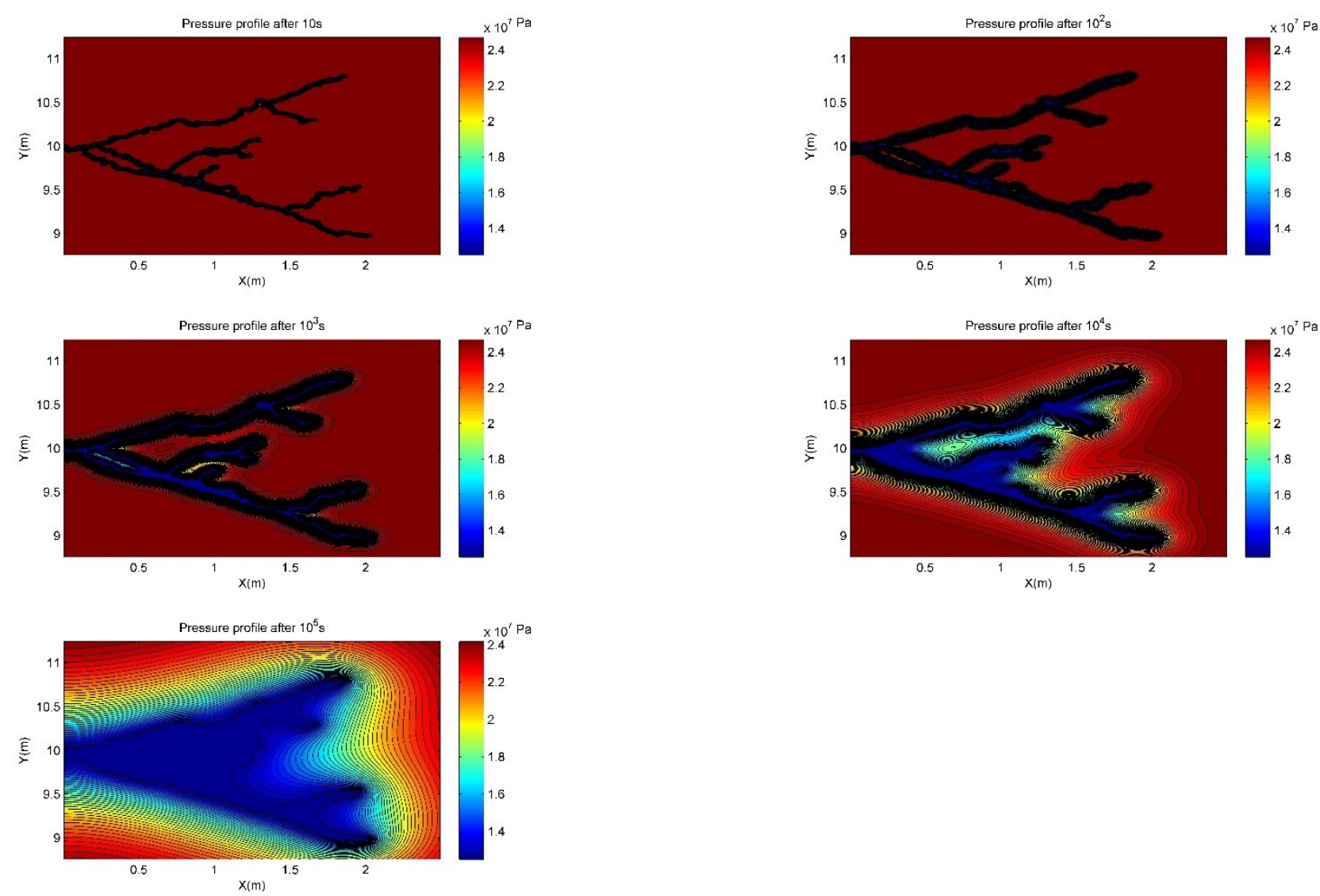

Figure D. 51 - Tri-branched fracture 7 pressure maps after $1 \times 10^{1} \mathrm{~s}, 1 \times 10^{2} \mathrm{~s}, 1 \times 10^{3} \mathrm{~s}, 1 \times 10^{4} \mathrm{~s}$ and $1 \times 10^{5} \mathrm{~s}$ 

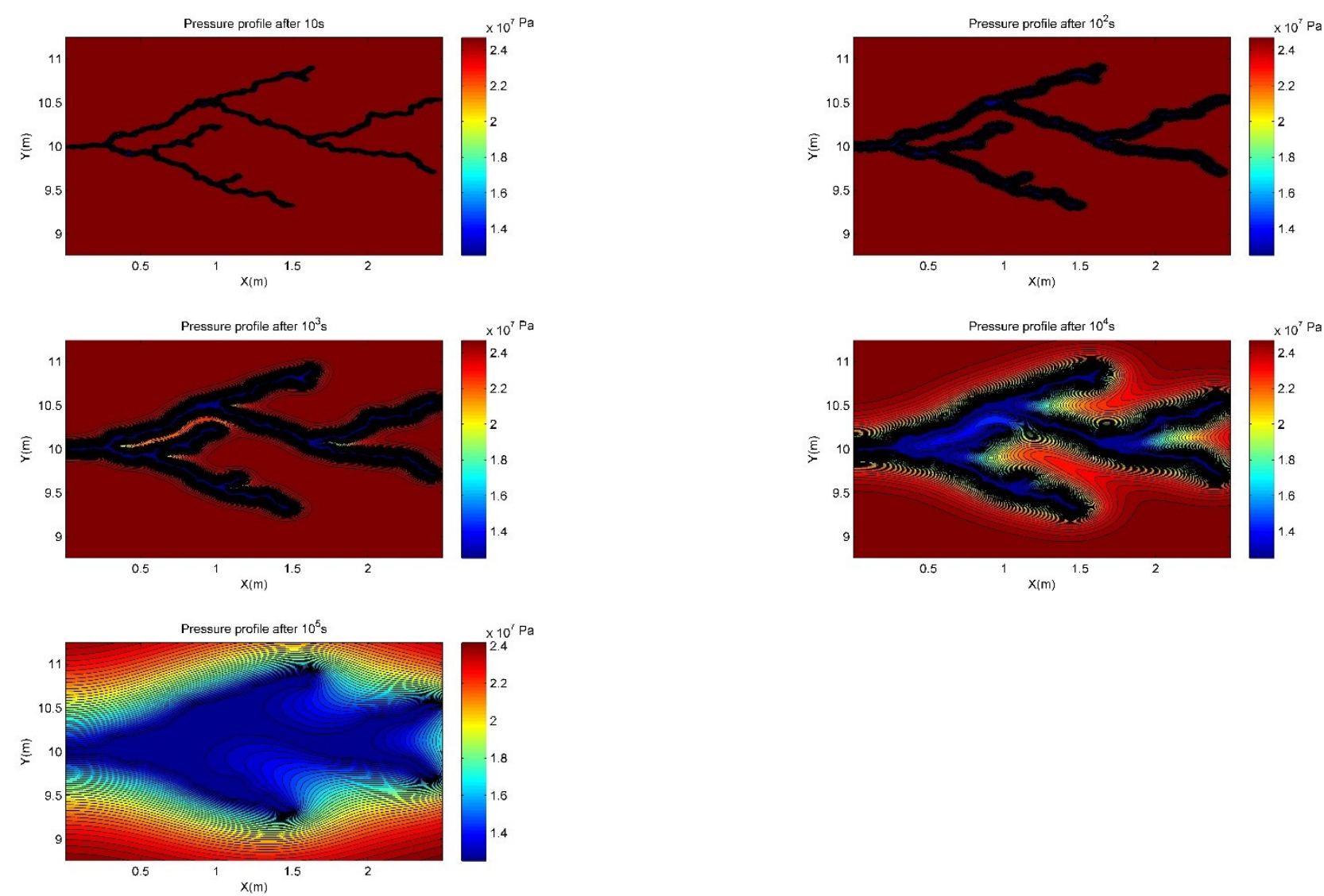

Figure D. 52 - Tri-branched fracture 8 pressure maps after $1 \times 10^{1} \mathrm{~s}, 1 \times 10^{2} \mathrm{~s}, 1 \times 10^{3} \mathrm{~s}, 1 \times 10^{4} \mathrm{~s}$ and $1 \times 10^{5} \mathrm{~s}$ 

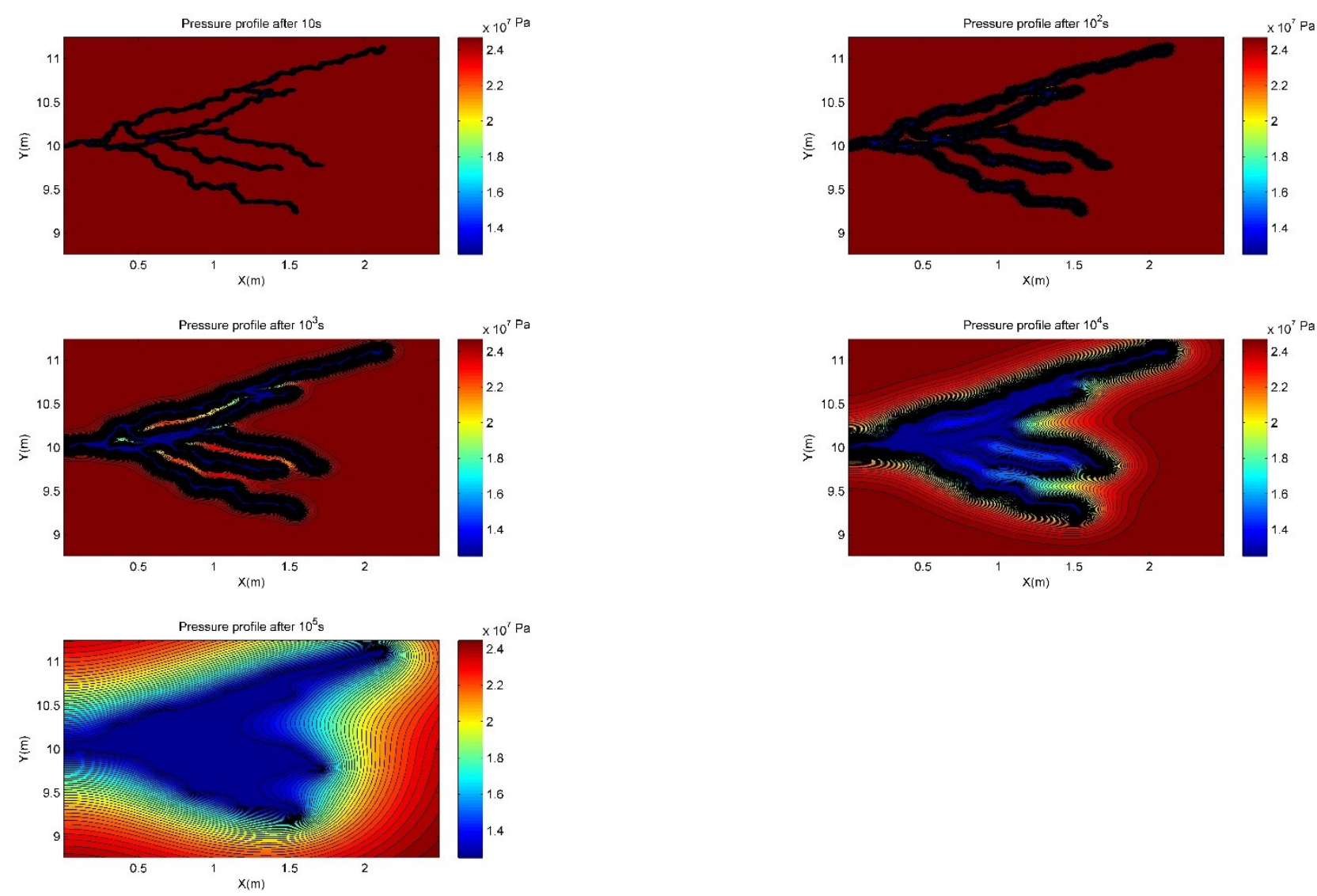

Figure D. 53 - Tri-branched fracture 9 pressure maps after $1 \times 10^{1} \mathrm{~s}, 1 \times 10^{2} \mathrm{~s}, 1 \times 10^{3} \mathrm{~s}, 1 \times 10^{4} \mathrm{~s}$ and $1 \times 10^{5} \mathrm{~s}$. 

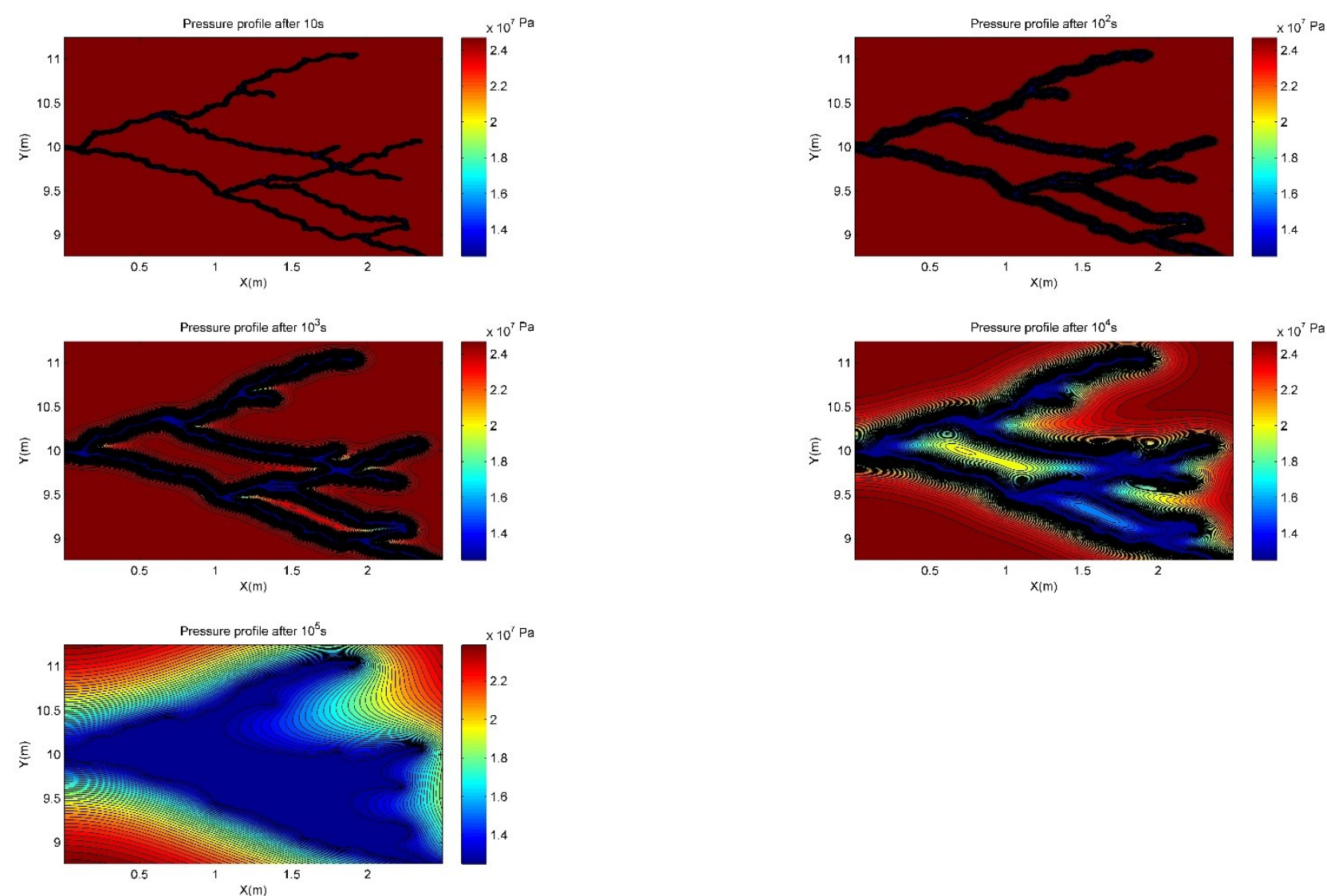

Figure D. 54 - Tri-branched fracture 10 pressure maps after $1 \times 10^{1} \mathrm{~s}, 1 \times 10^{2} \mathrm{~s}, 1 \times 10^{3} \mathrm{~s}, 1 \times 10^{4} \mathrm{~s}$ and $1 \times 10^{5} \mathrm{~s}$ 

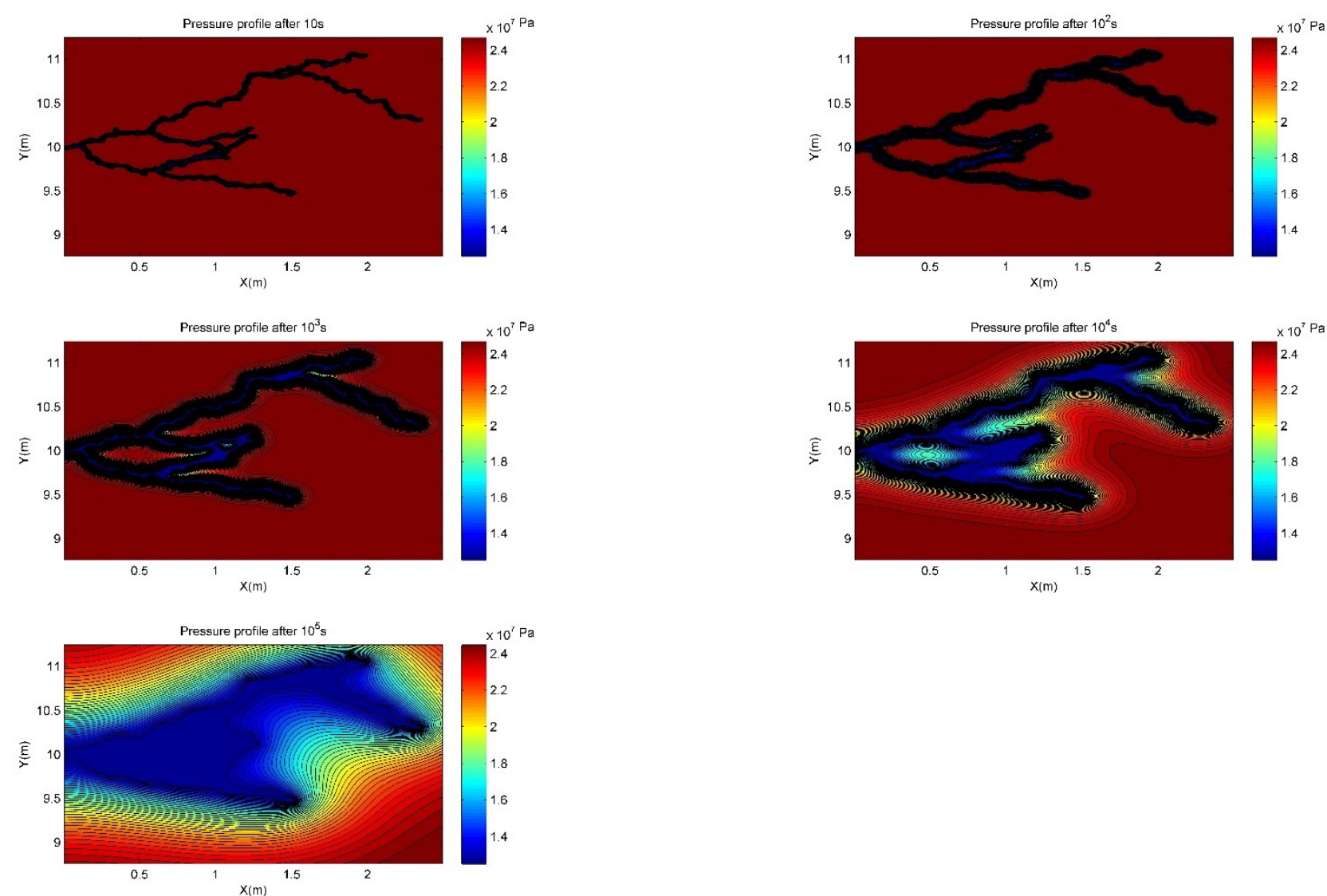

Figure D. 55 - Tri-branched fracture 11 pressure maps after $1 \times 10^{1} \mathrm{~s}, 1 \times 10^{2} \mathrm{~s}, 1 \times 10^{3} \mathrm{~s}, 1 \times 10^{4} \mathrm{~s}$ and $1 \times 10^{5} \mathrm{~s}$ 

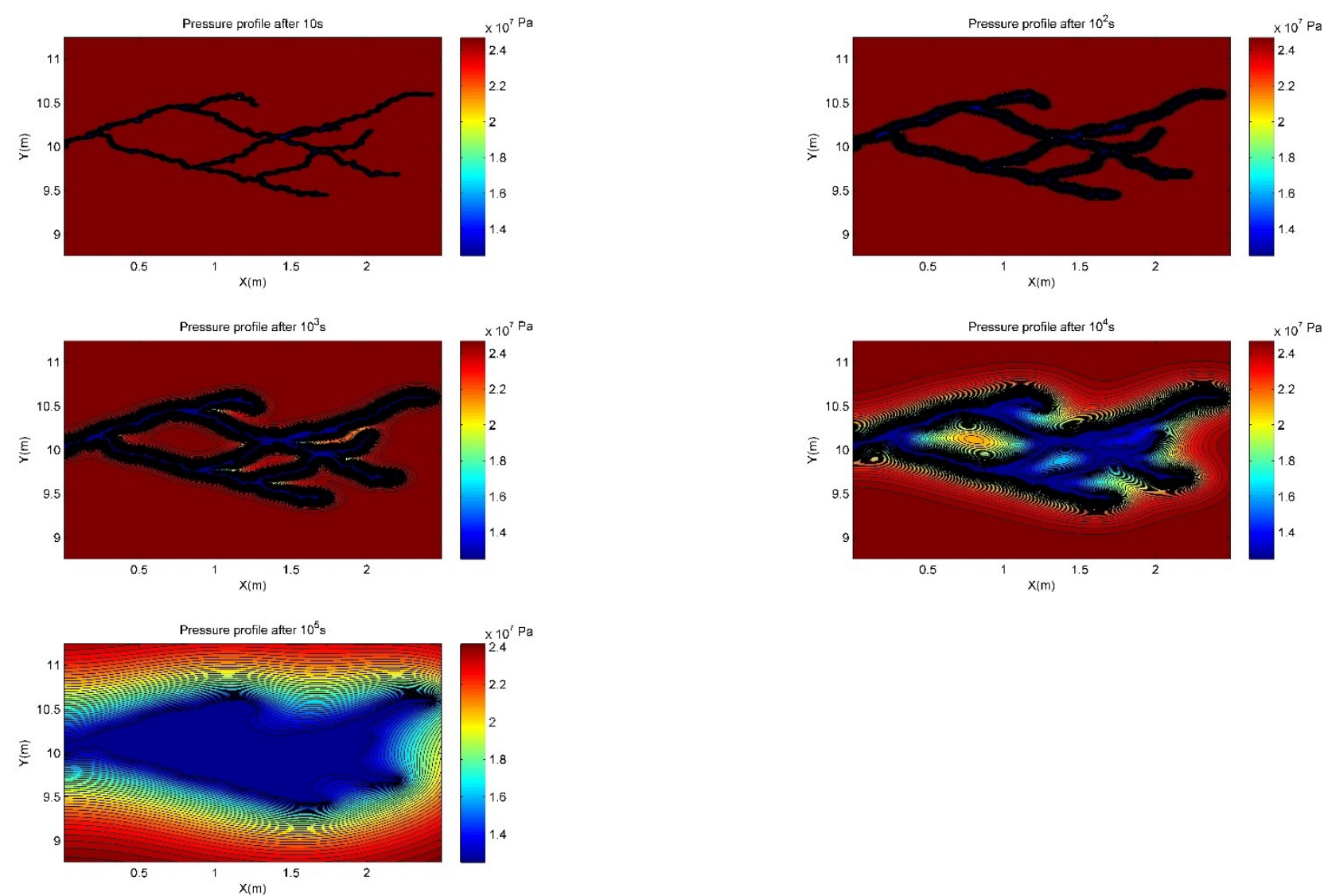

Figure D. 56 - Tri-branched fracture 12 pressure maps after $1 \times 10^{1} \mathrm{~s}, 1 \times 10^{2} \mathrm{~s}, 1 \times 10^{3} \mathrm{~s}, 1 \times 10^{4} \mathrm{~s}$ and $1 \times 10^{5} \mathrm{~s}$ 

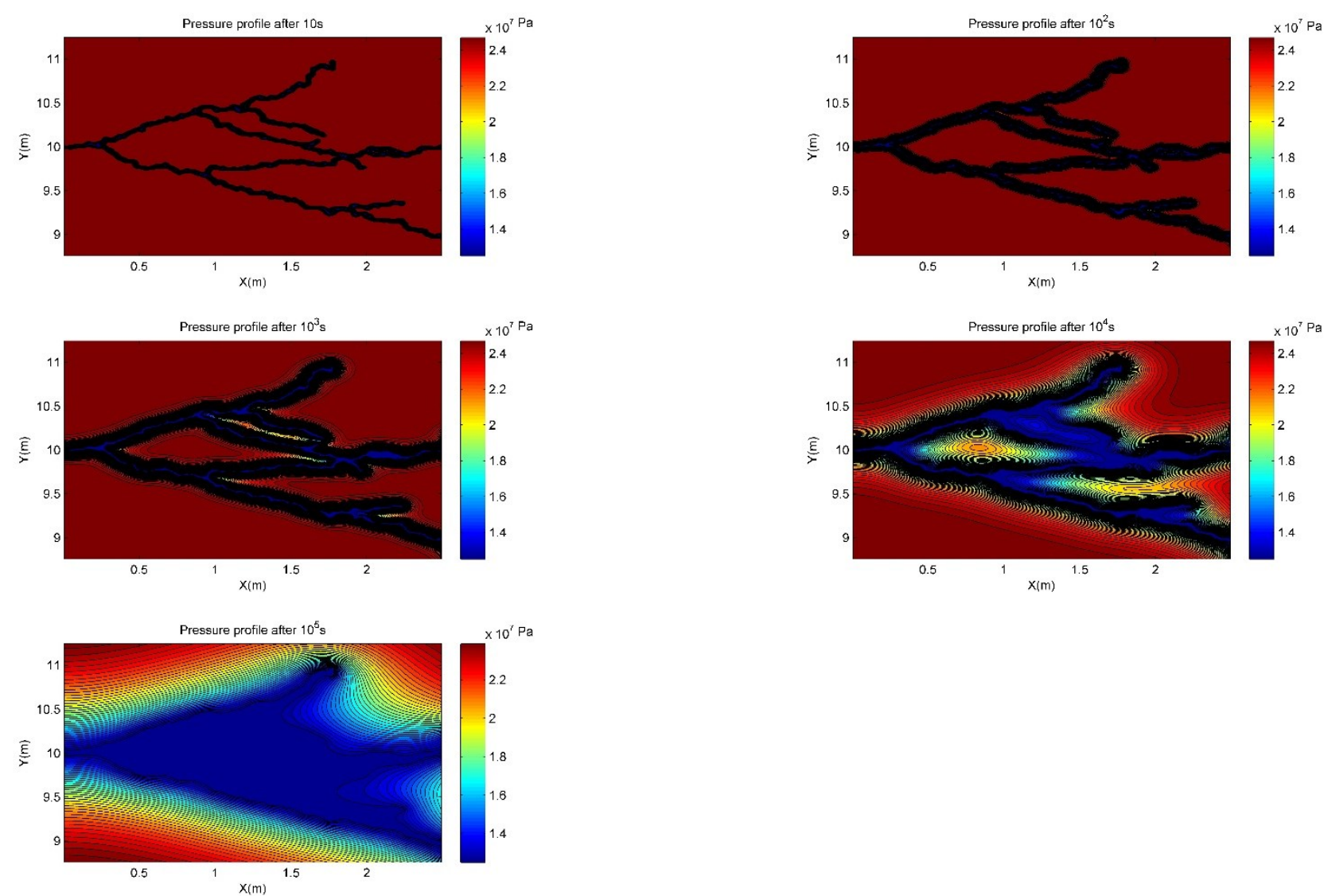

Figure D. 57 - Tri-branched fracture 13 pressure maps after $1 \times 10^{1} \mathrm{~s}, 1 \times 10^{2} \mathrm{~s}, 1 \times 10^{3} \mathrm{~s}, 1 \times 10^{4} \mathrm{~s}$ and $1 \times 10^{5} \mathrm{~s}$ 

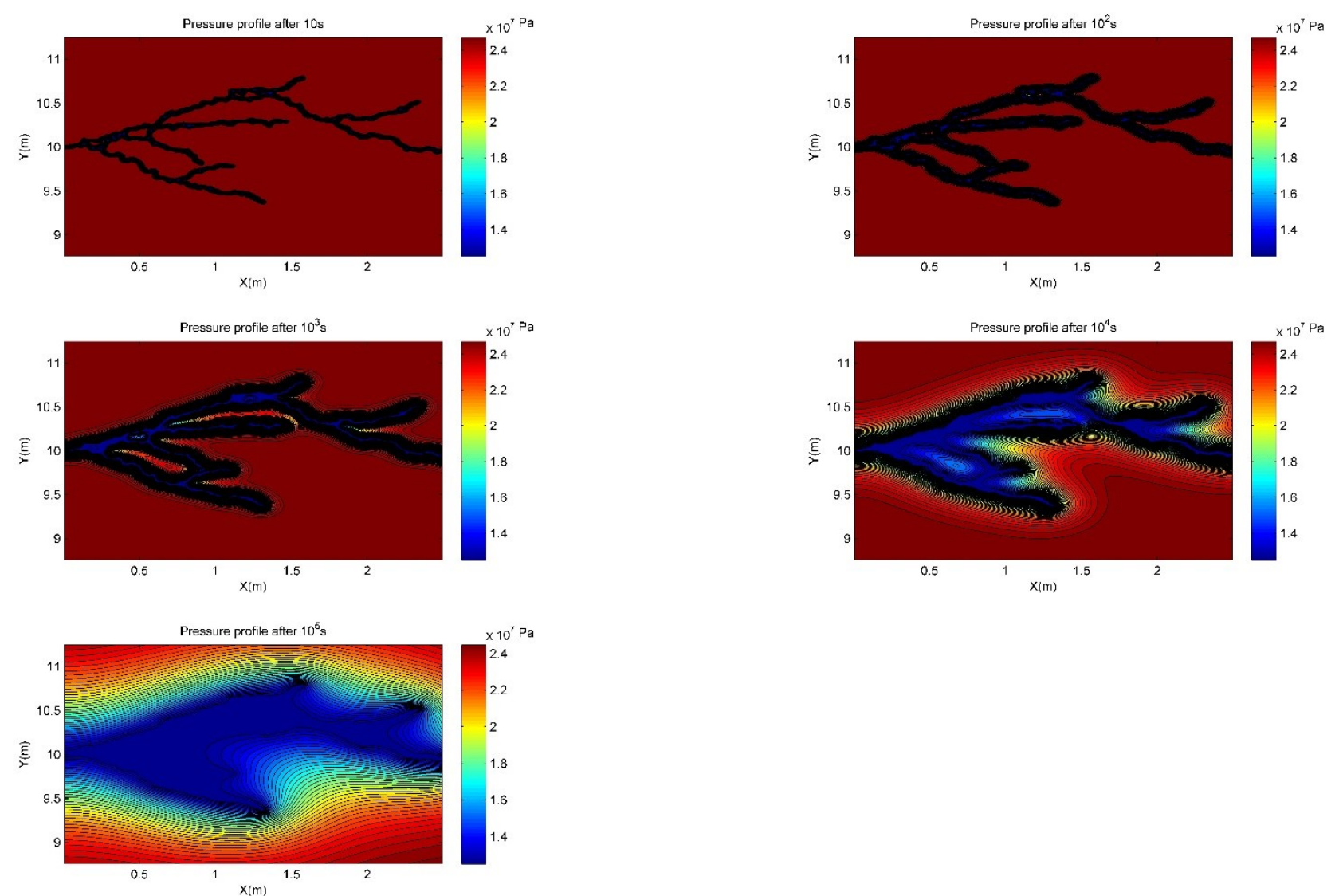

Figure D. 58 - Tri-branched fracture 14 pressure maps after $1 \times 10^{1} \mathrm{~s}, 1 \times 10^{2} \mathrm{~s}, 1 \times 10^{3} \mathrm{~s}, 1 \times 10^{4} \mathrm{~s}$ and $1 \times 10^{5} \mathrm{~s}$. 

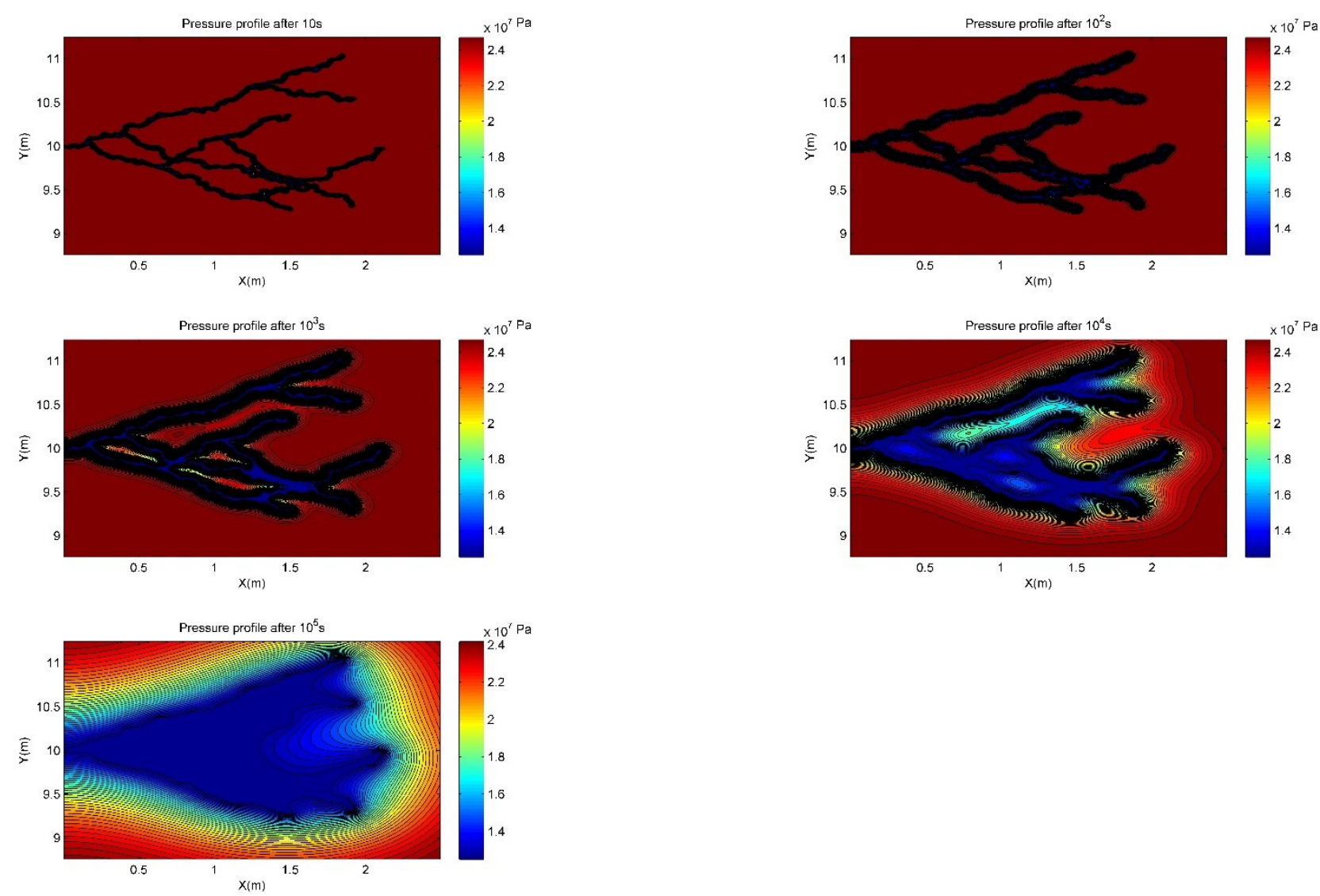

Figure D. 59 - Tri-branched fracture 15 pressure maps after $1 \times 10^{1} \mathrm{~s}, 1 \times 10^{2} \mathrm{~s}, 1 \times 10^{3} \mathrm{~s}, 1 \times 10^{4} \mathrm{~s}$ and $1 \times 10^{5} \mathrm{~s}$ 

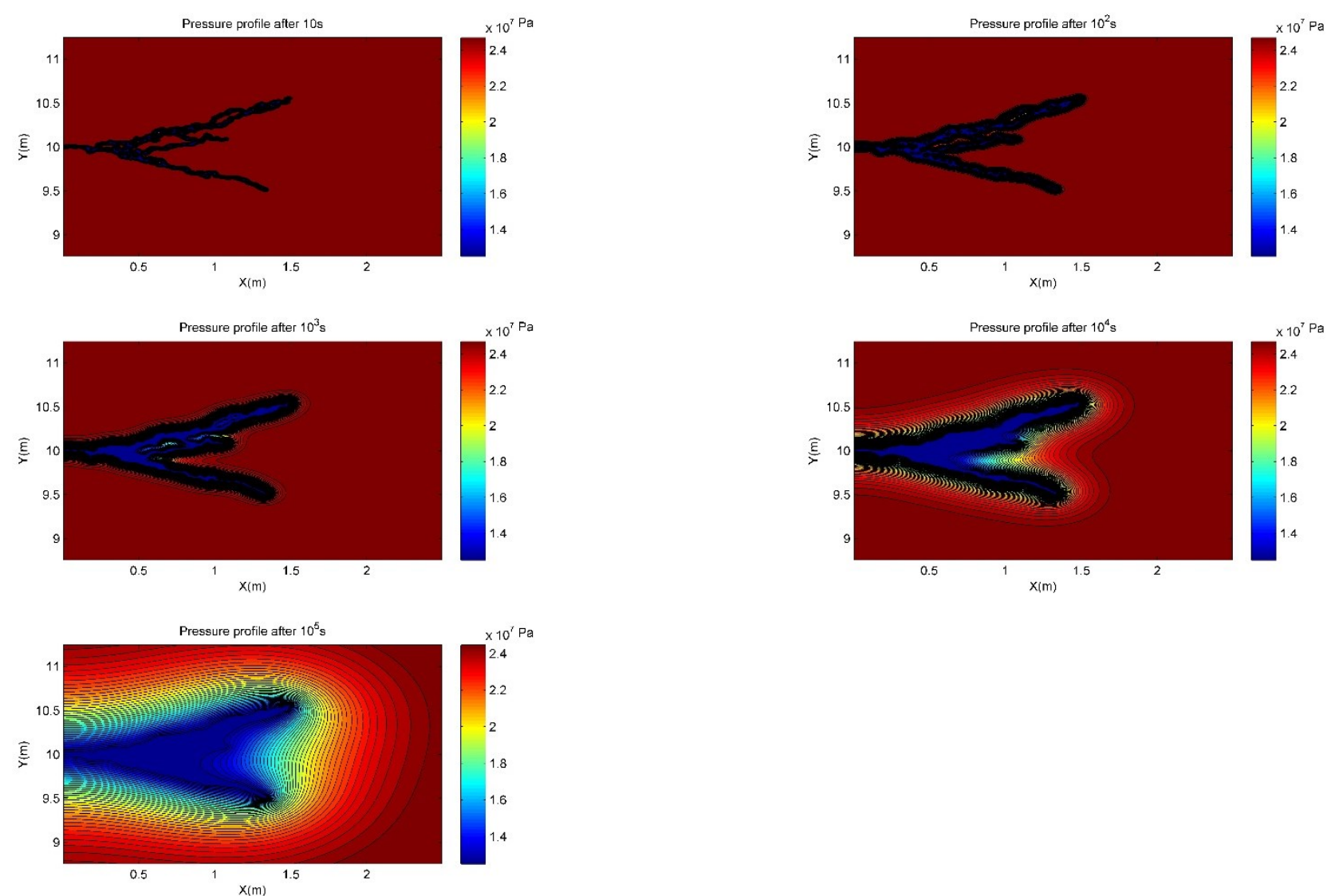

Figure D. 60 - Tri-branched fracture 16 pressure maps after $1 \times 10^{1} \mathrm{~s}, 1 \times 10^{2} \mathrm{~s}, 1 \times 10^{3} \mathrm{~s}, 1 \times 10^{4} \mathrm{~s}$ and $1 \times 10^{5} \mathrm{~s}$ 

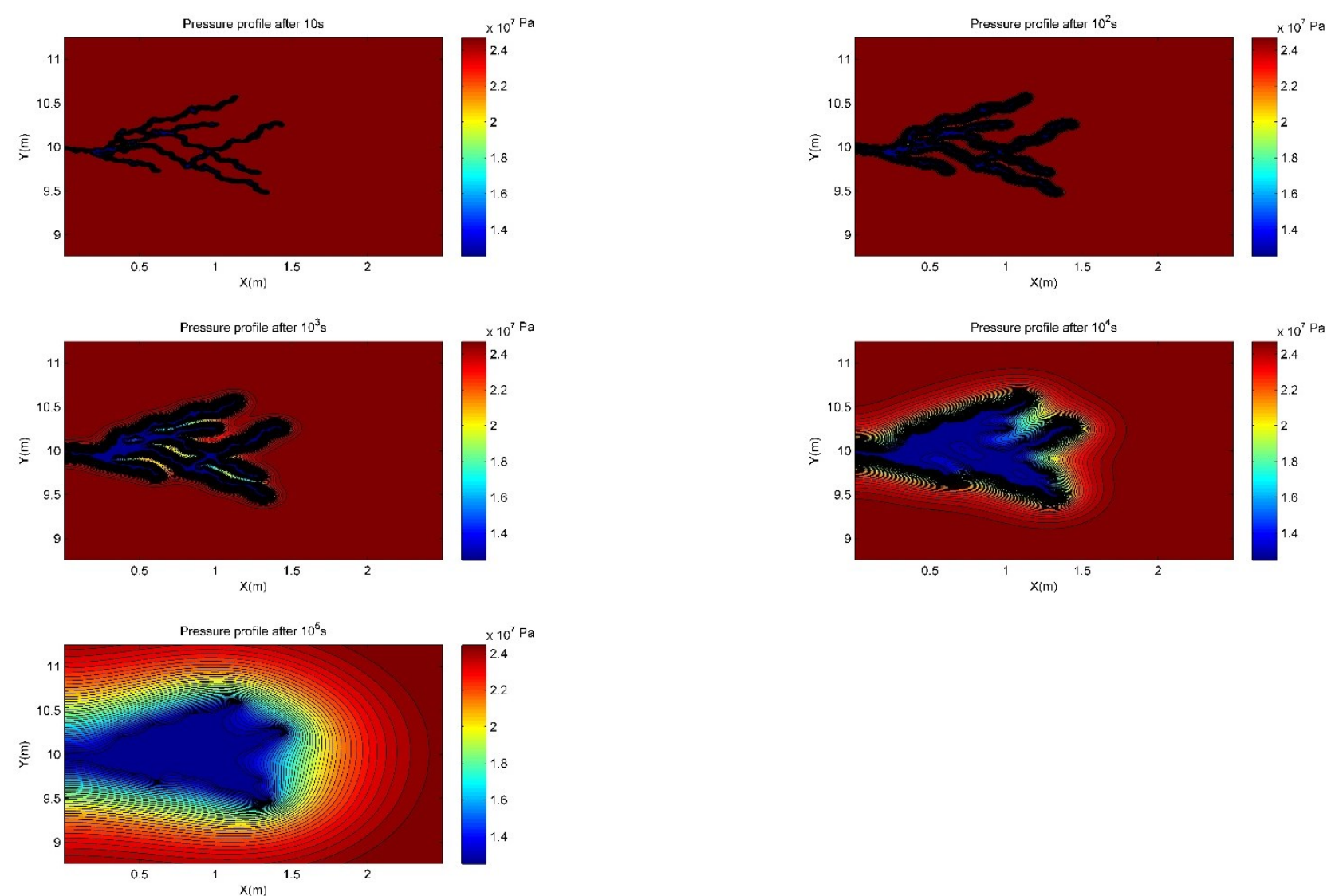

Figure D. 61 - Tri-branched fracture 17 pressure maps after $1 \times 10^{1} \mathrm{~s}, 1 \times 10^{2} \mathrm{~s}, 1 \times 10^{3} \mathrm{~s}, 1 \times 10^{4} \mathrm{~s}$ and $1 \times 10^{5} \mathrm{~s}$ 

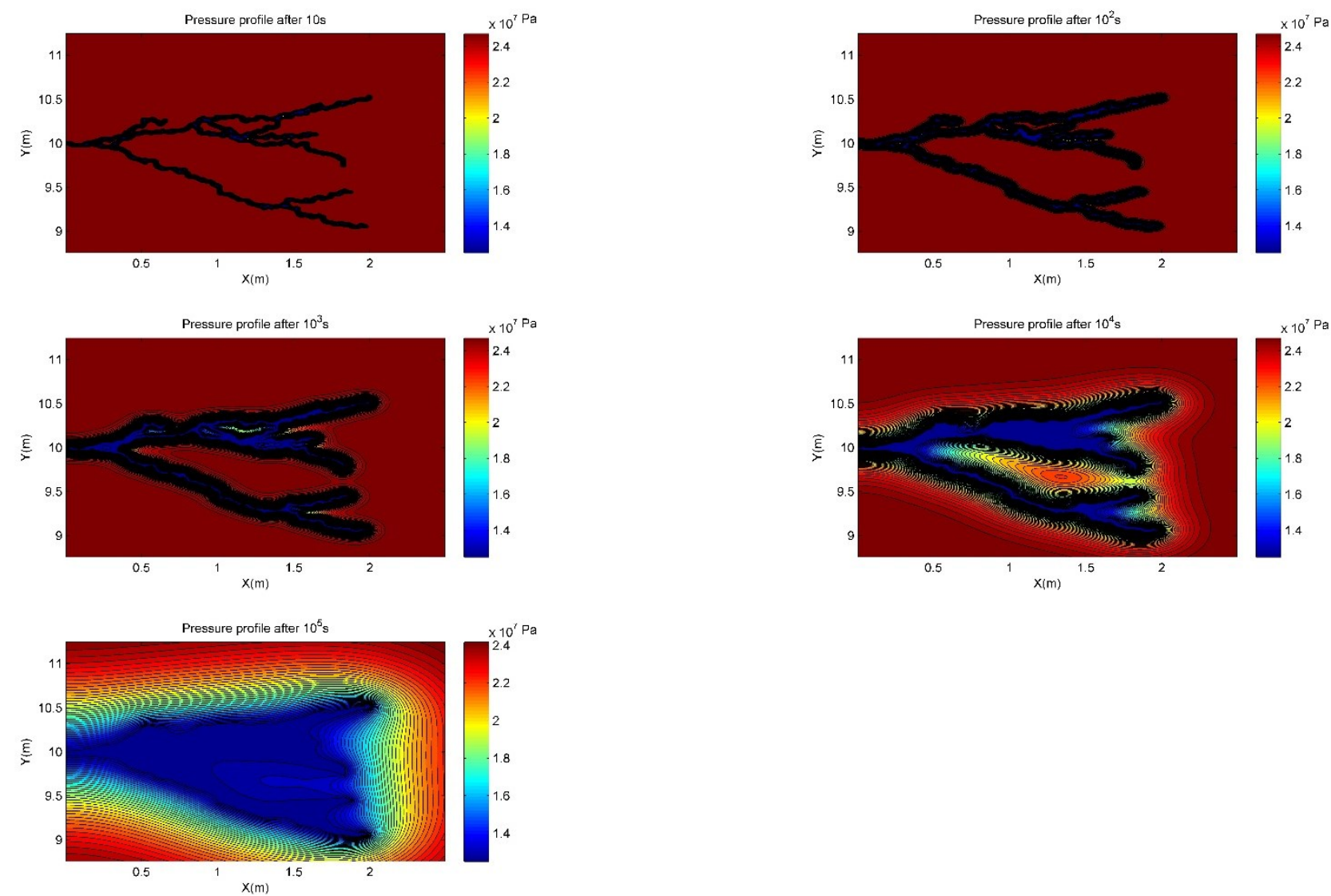

Figure D. 62 - Tri-branched fracture 18 pressure maps after $1 \times 10^{1} \mathrm{~s}, 1 \times 10^{2} \mathrm{~s}, 1 \times 10^{3} \mathrm{~s}, 1 \times 10^{4} \mathrm{~s}$ and $1 \times 10^{5} \mathrm{~s}$ 

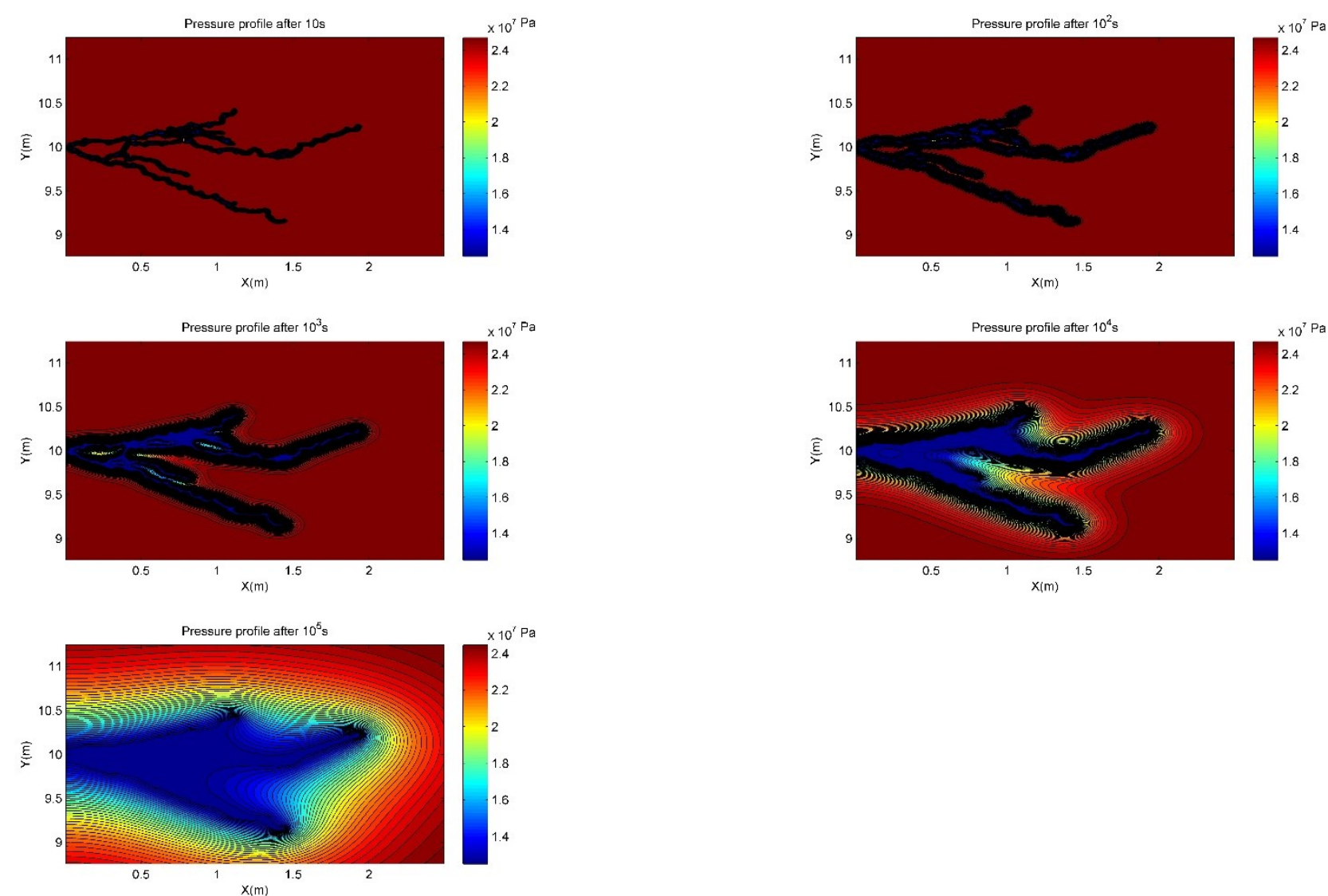

Figure D. 63 - Tri-branched fracture 19 pressure maps after $1 \times 10^{1} \mathrm{~s}, 1 \times 10^{2} \mathrm{~s}, 1 \times 10^{3} \mathrm{~s}, 1 \times 10^{4} \mathrm{~s}$ and $1 \times 10^{5} \mathrm{~s}$ 

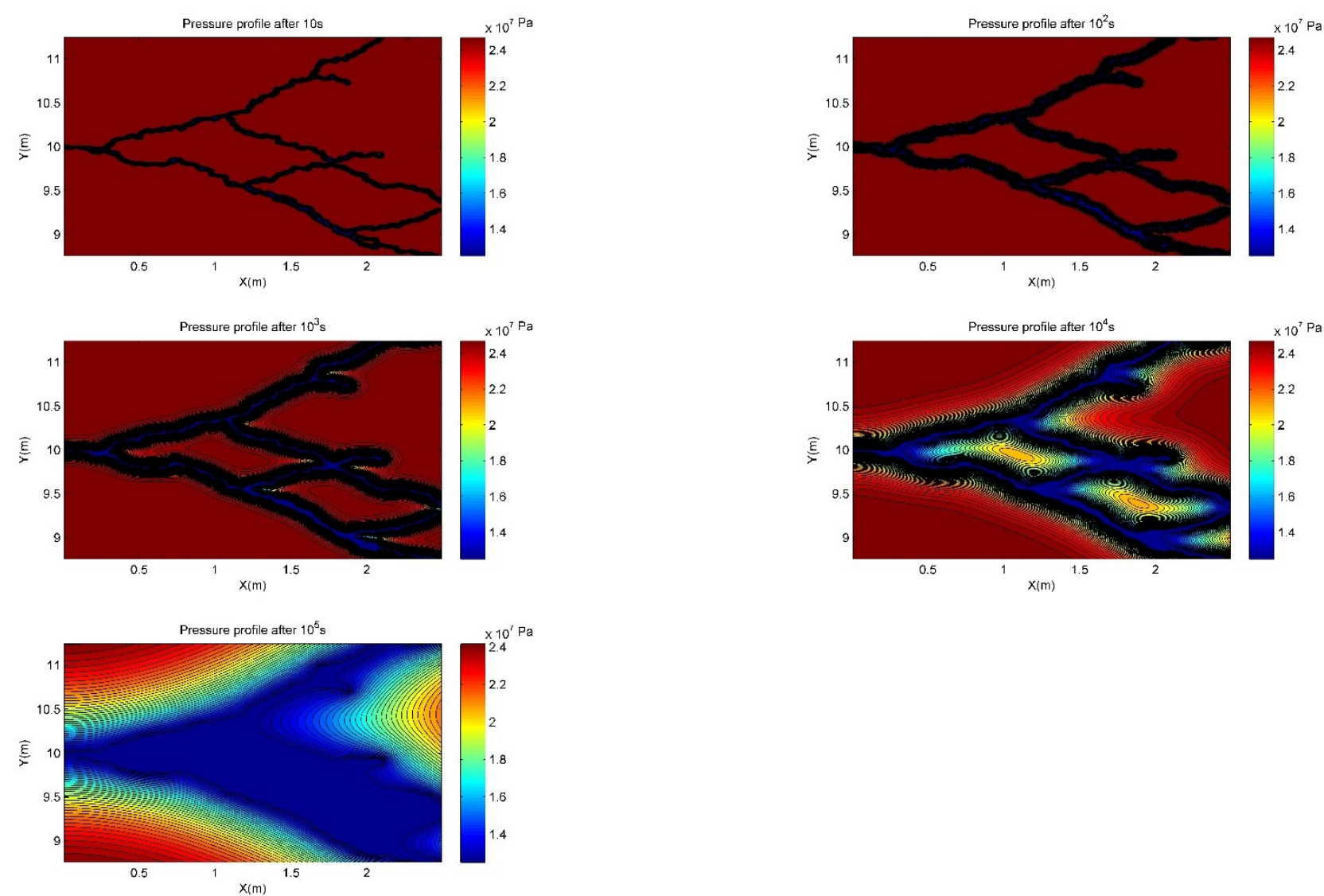

Figure D. 64 - Tri-branched fracture 20 pressure maps after $1 \times 10^{1} \mathrm{~s}, 1 \times 10^{2} \mathrm{~s}, 1 \times 10^{3} \mathrm{~s}, 1 \times 10^{4} \mathrm{~s}$ and $1 \times 10^{5} \mathrm{~s}$ 

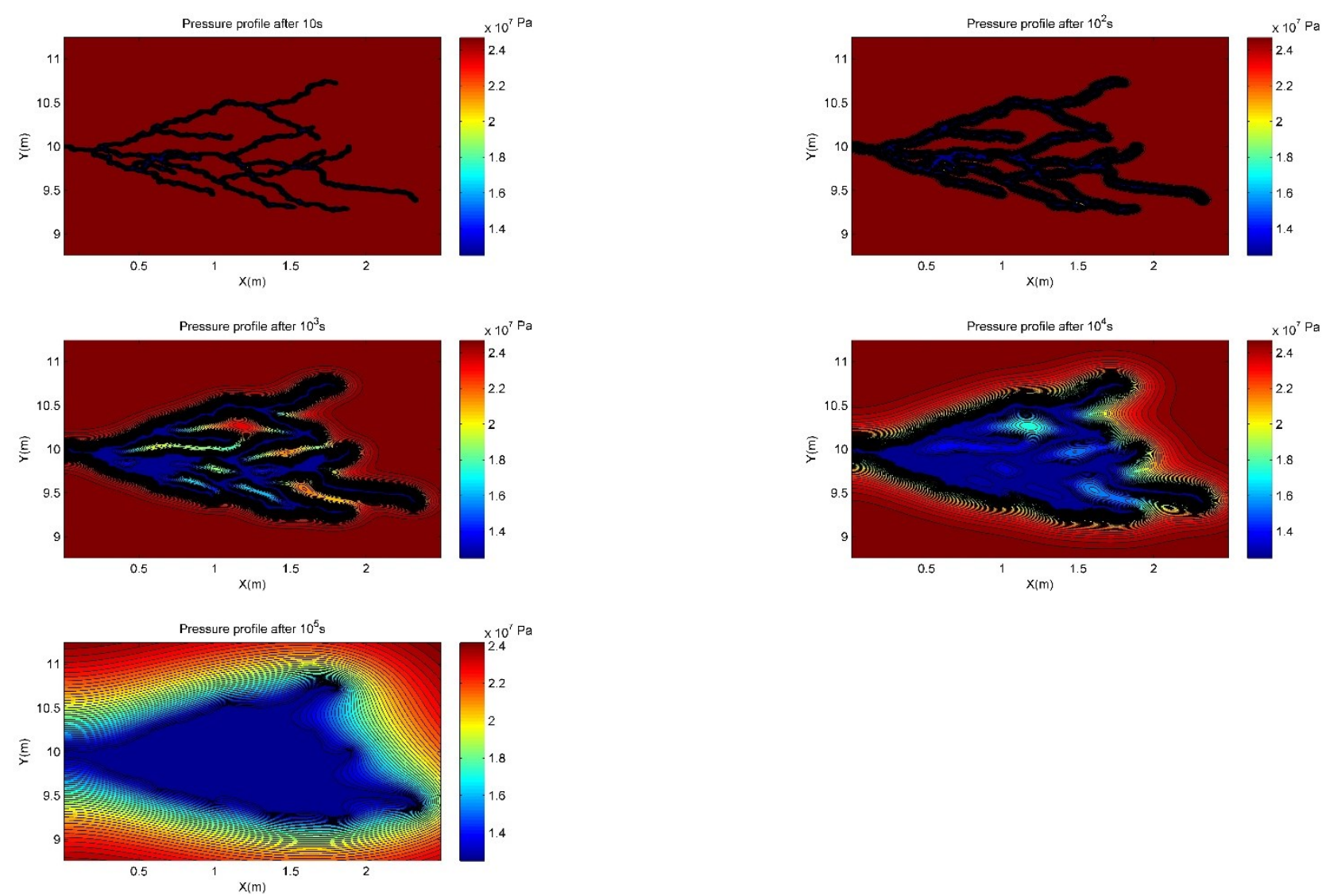

Figure D. 65 - Quad-branched fracture 1 pressure maps after $1 \times 10^{1} \mathrm{~s}, 1 \times 10^{2} \mathrm{~s}, 1 \times 10^{3} \mathrm{~s}, 1 \times 10^{4} \mathrm{~s}$ and $1 \times 10^{5} \mathrm{~s}$. 

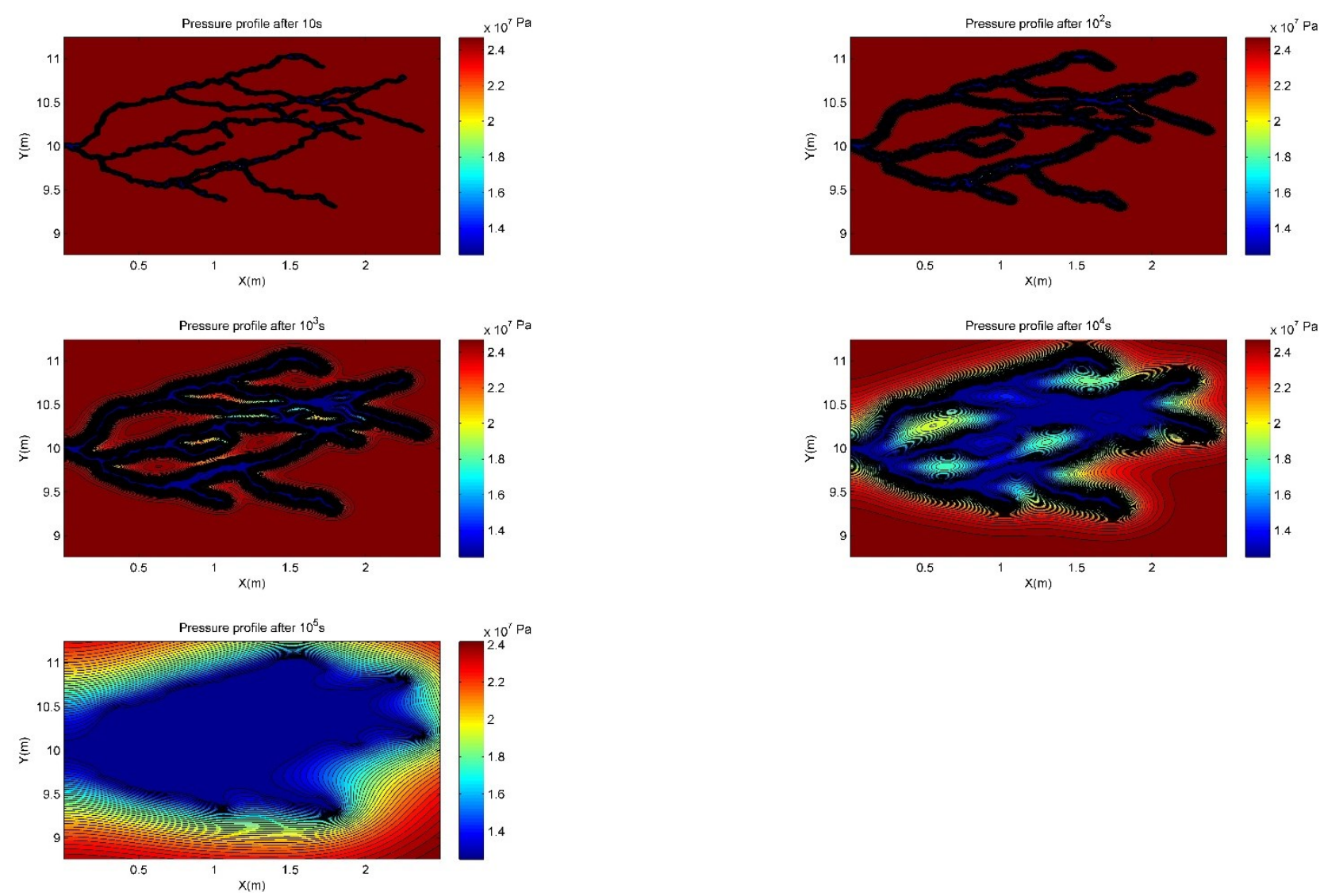

Figure D. 66 - Quad-branched fracture 2 pressure maps after $1 \times 10^{1} \mathrm{~s}, 1 \times 10^{2} \mathrm{~s}, 1 \times 10^{3} \mathrm{~s}, 1 \times 10^{4} \mathrm{~s}$ and $1 \times 10^{5} \mathrm{~s}$ 

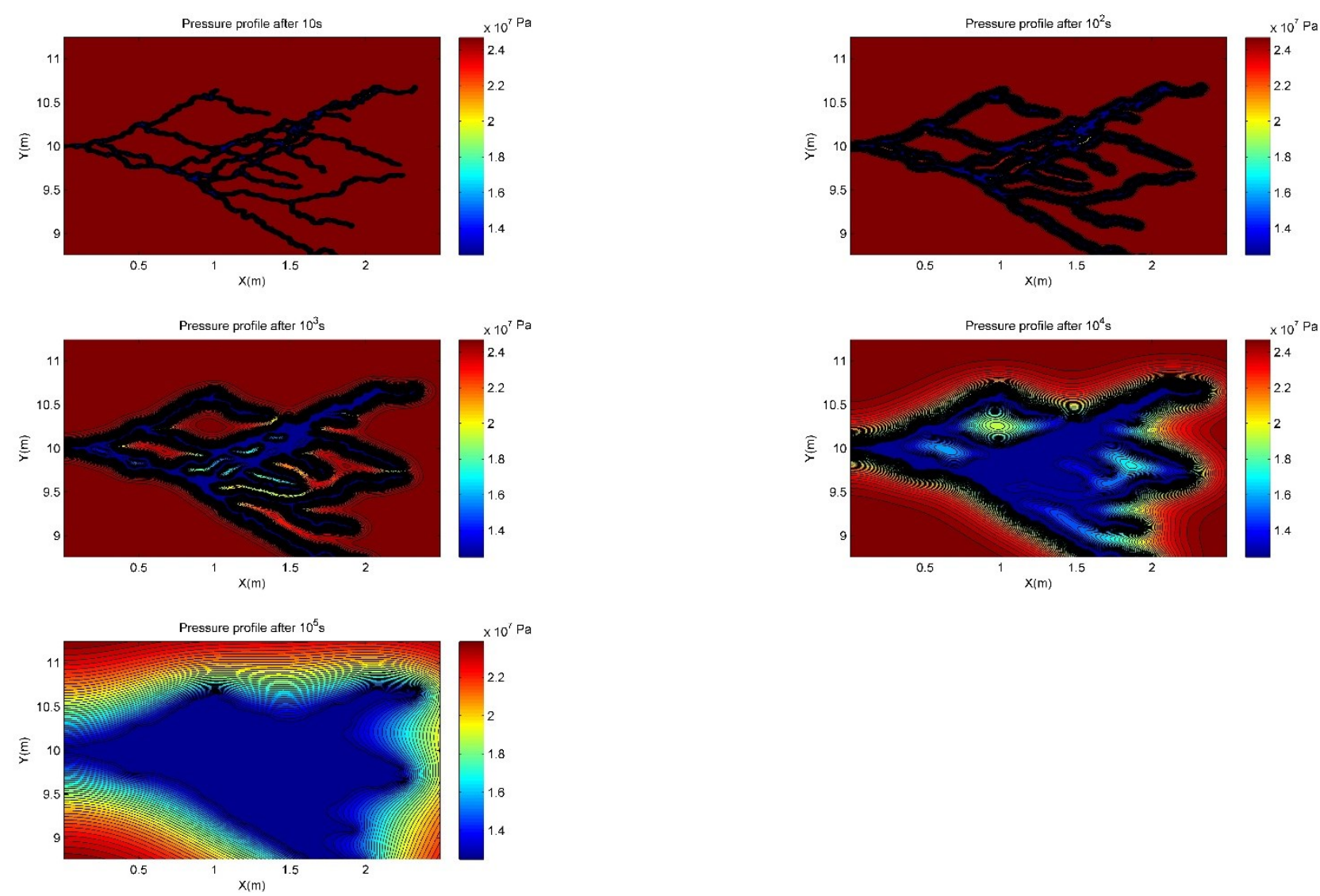

Figure D. 67 - Quad-branched fracture 3 pressure maps after $1 \times 10^{1} \mathrm{~s}, 1 \times 10^{2} \mathrm{~s}, 1 \times 10^{3} \mathrm{~s}, 1 \times 10^{4} \mathrm{~s}$ and $1 \times 10^{5} \mathrm{~s}$ 

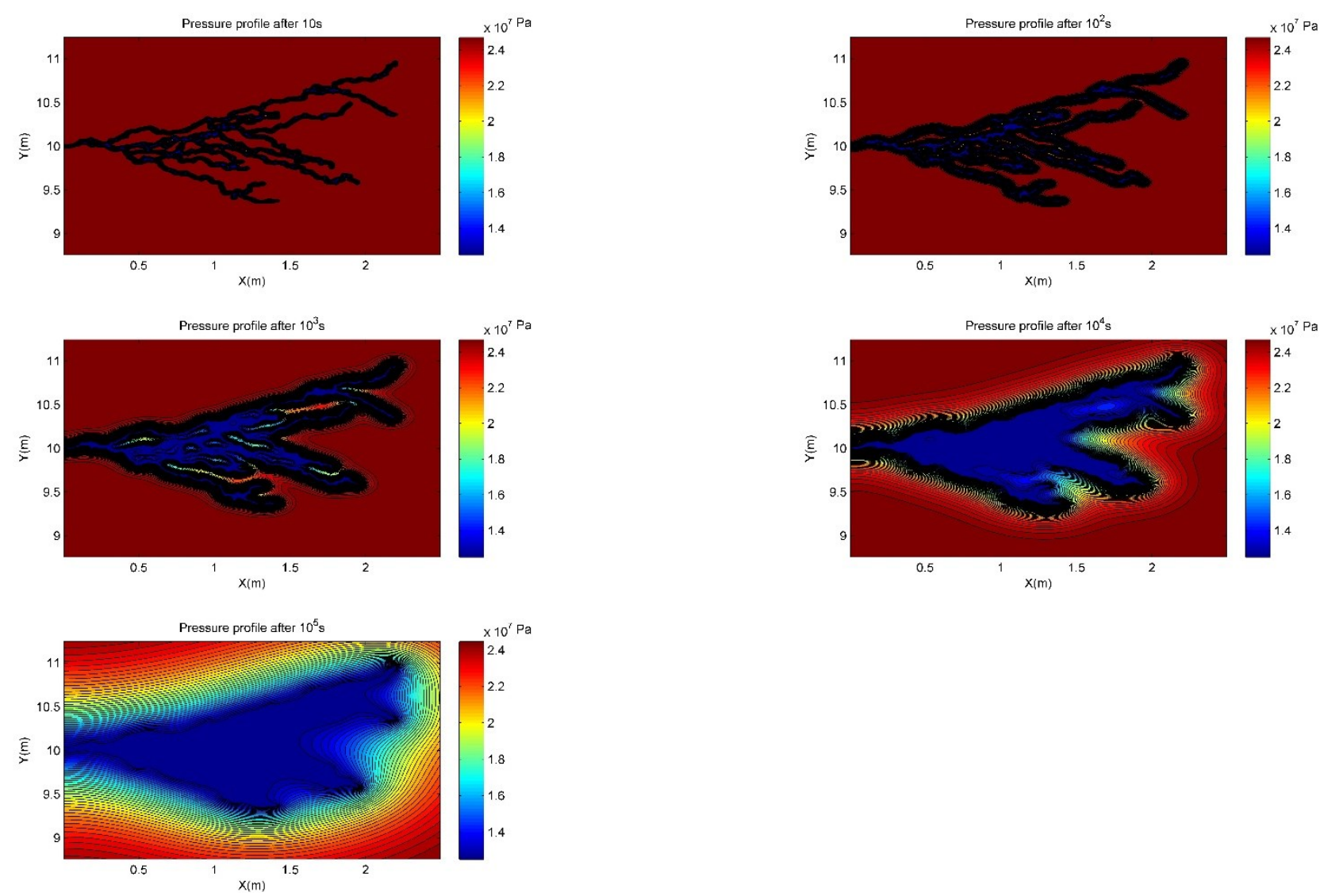

Figure D. 68 - Quad-branched fracture 4 pressure maps after $1 \times 10^{1} \mathrm{~s}, 1 \times 10^{2} \mathrm{~s}, 1 \times 10^{3} \mathrm{~s}, 1 \times 10^{4} \mathrm{~s}$ and $1 \times 10^{5} \mathrm{~s}$. 

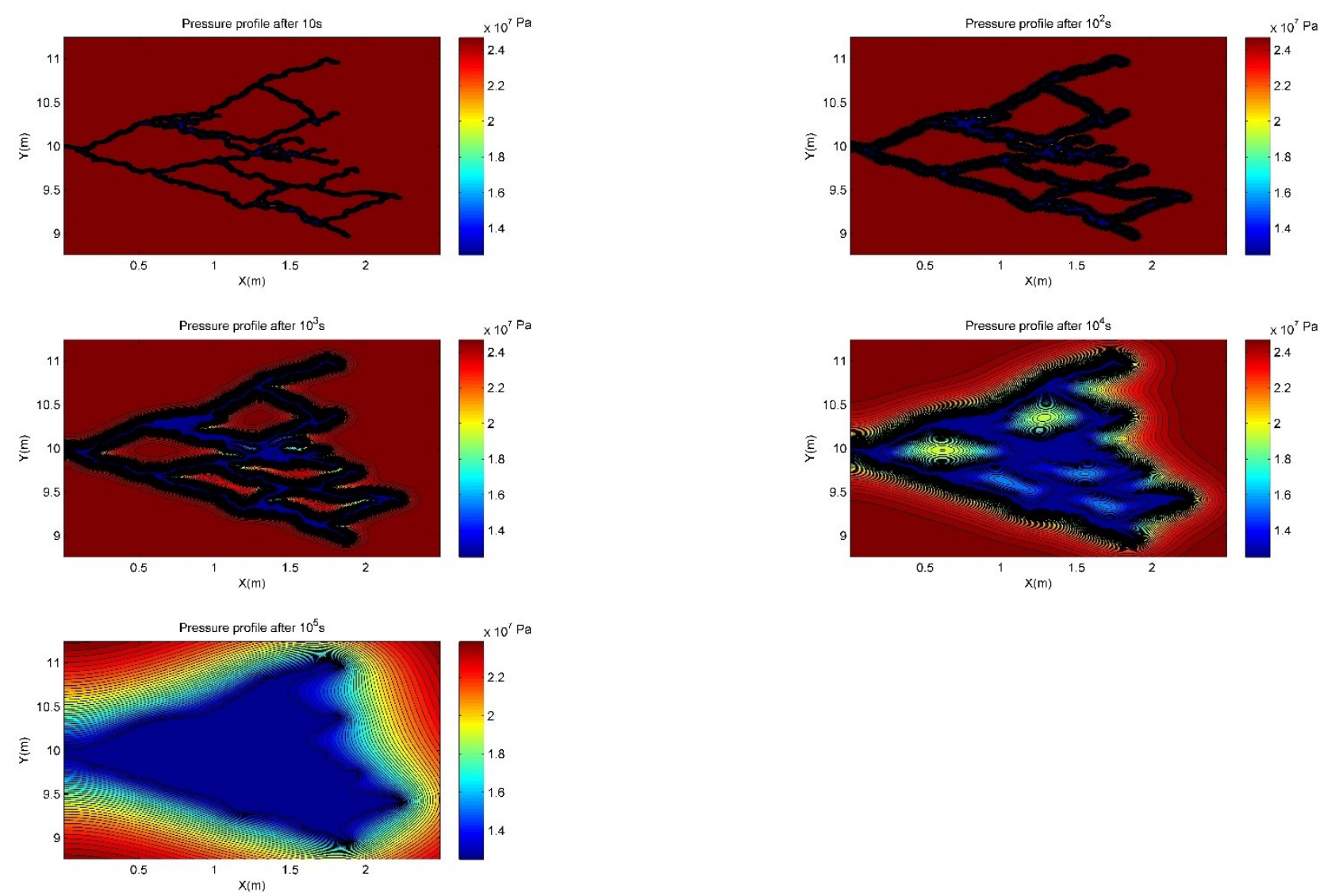

Figure D. 69 - Quad-branched fracture 5 pressure maps after $1 \times 10^{1} \mathrm{~s}, 1 \times 10^{2} \mathrm{~s}, 1 \times 10^{3} \mathrm{~s}, 1 \times 10^{4} \mathrm{~s}$ and $1 \times 10^{5} \mathrm{~s}$ 

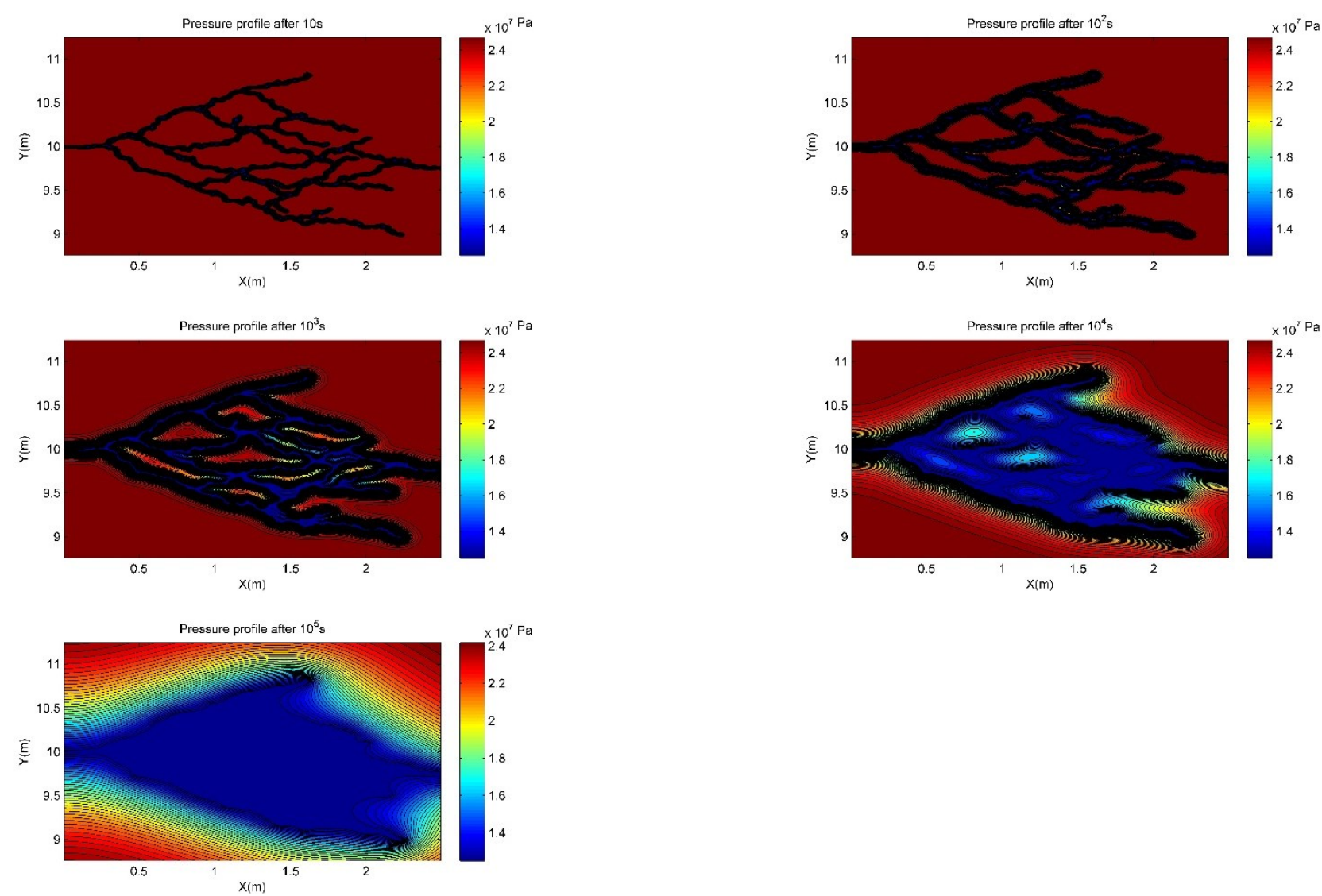

Figure D. 70 - Quad-branched fracture 6 pressure maps after $1 \times 10^{1} \mathrm{~s}, 1 \times 10^{2} \mathrm{~s}, 1 \times 10^{3} \mathrm{~s}, 1 \times 10^{4} \mathrm{~s}$ and $1 \times 10^{5} \mathrm{~s}$ 

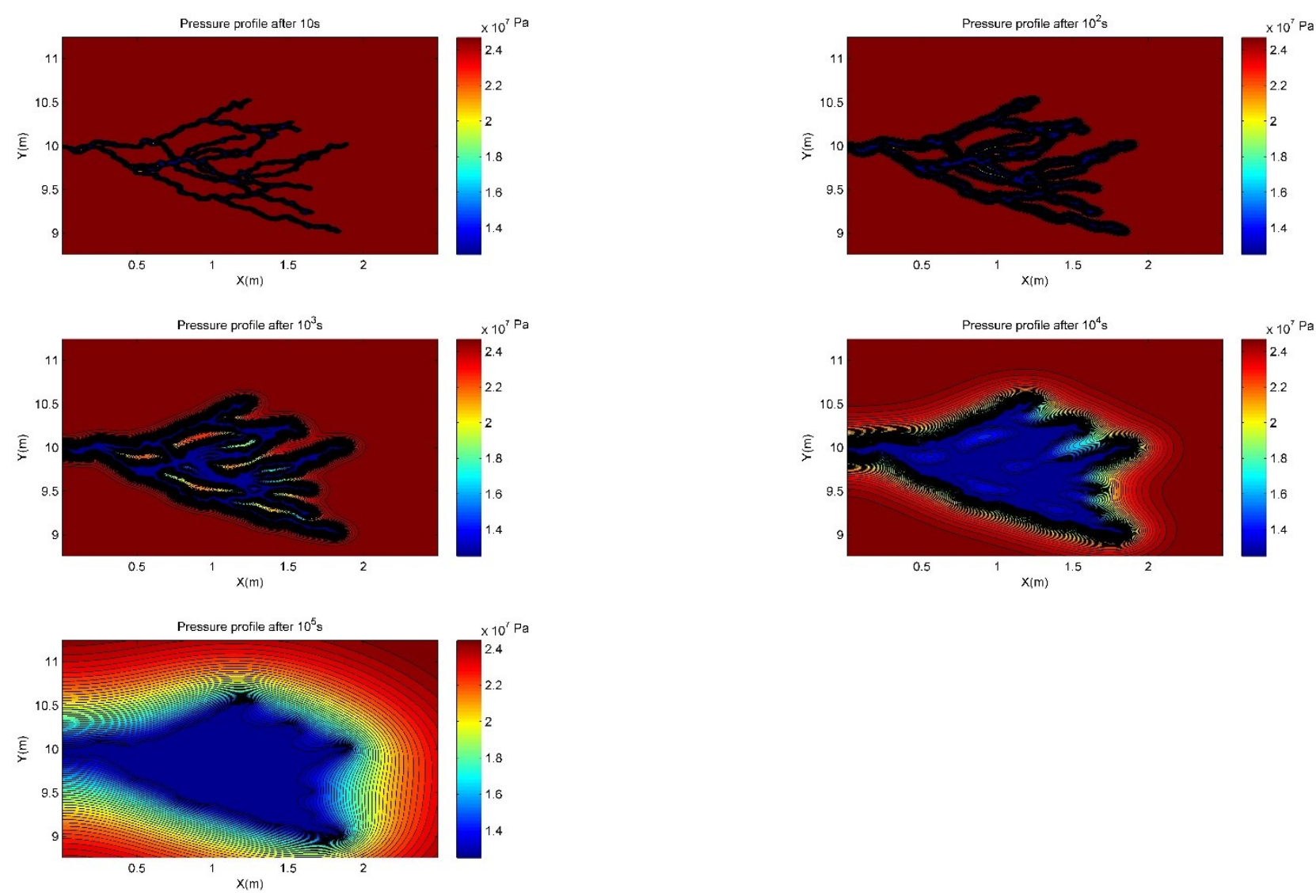

Figure D. 71 - Quad-branched fracture 7 pressure maps after $1 \times 10^{1} \mathrm{~s}, 1 \times 10^{2} \mathrm{~s}, 1 \times 10^{3} \mathrm{~s}, 1 \times 10^{4} \mathrm{~s}$ and $1 \times 10^{5} \mathrm{~s}$ 

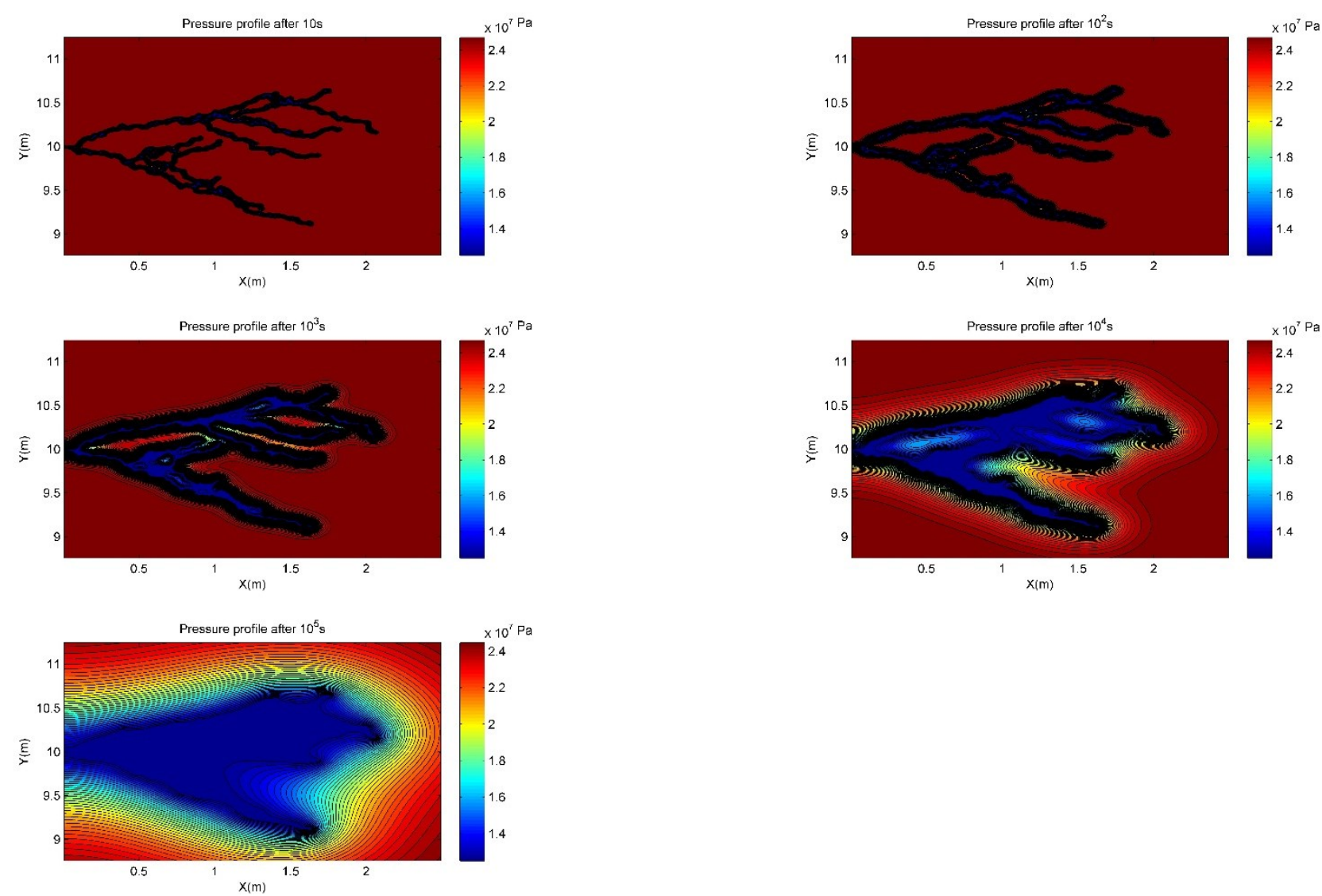

Figure D. 72 - Quad-branched fracture 8 pressure maps after $1 \times 10^{1} \mathrm{~s}, 1 \times 10^{2} \mathrm{~s}, 1 \times 10^{3} \mathrm{~s}, 1 \times 10^{4} \mathrm{~s}$ and $1 \times 10^{5} \mathrm{~s}$ 

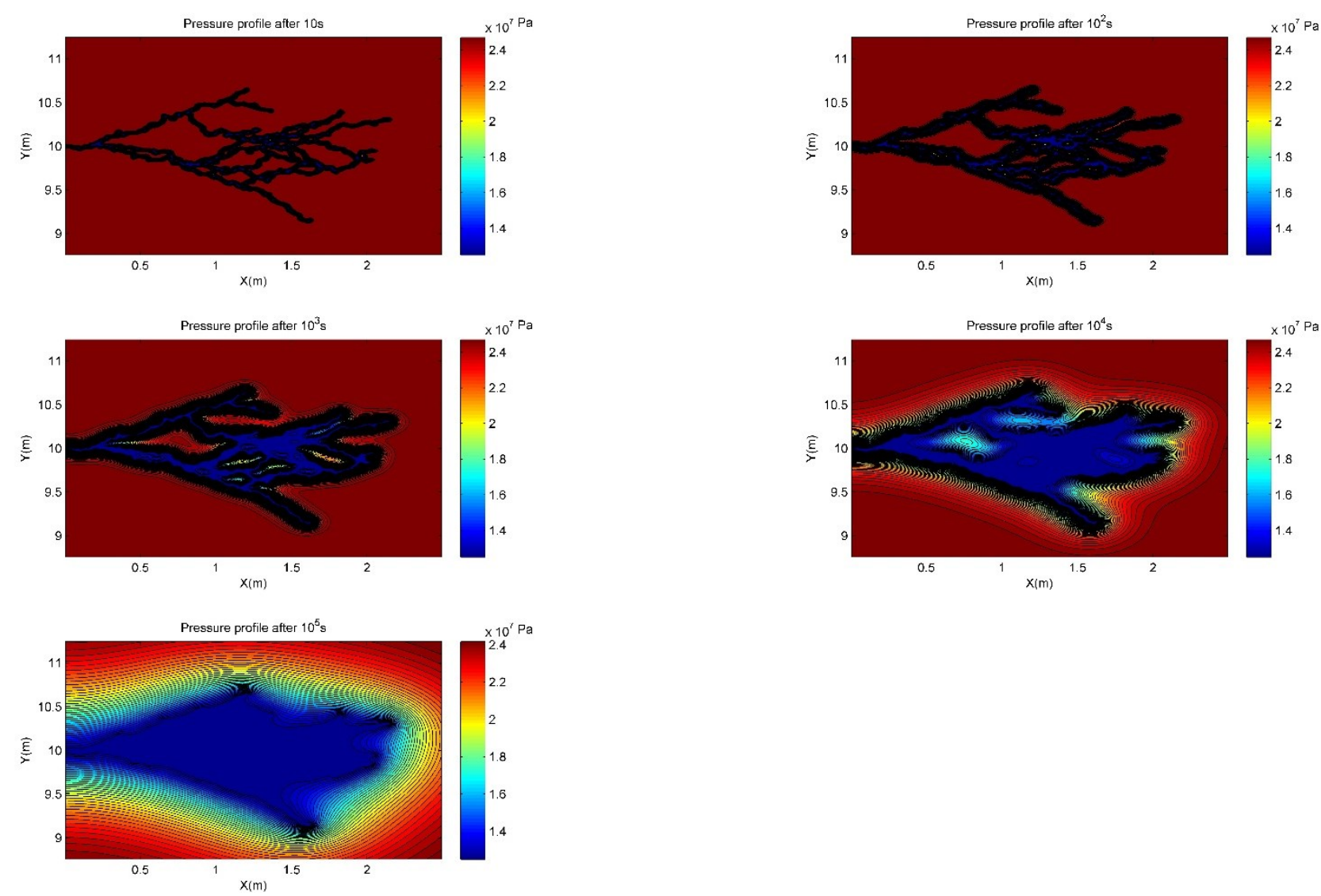

Figure D. 73 - Quad-branched fracture 9 pressure maps after $1 \times 10^{1} \mathrm{~s}, 1 \times 10^{2} \mathrm{~s}, 1 \times 10^{3} \mathrm{~s}, 1 \times 10^{4} \mathrm{~s}$ and $1 \times 10^{5} \mathrm{~s}$ 

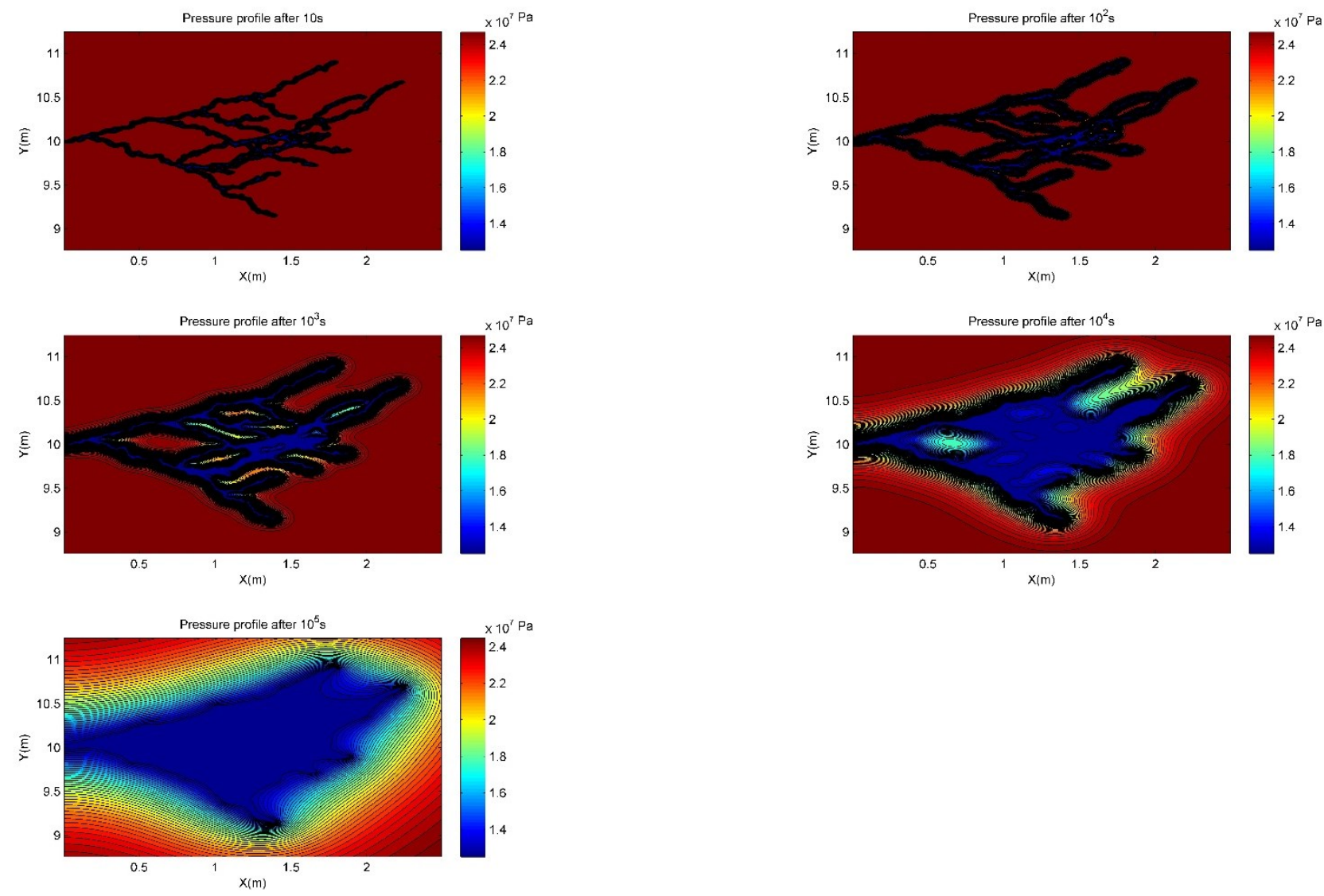

Figure D. 74 - Quad-branched fracture 10 pressure maps after $1 \times 10^{1} \mathrm{~s}, 1 \times 10^{2} \mathrm{~s}, 1 \times 10^{3} \mathrm{~s}, 1 \times 10^{4} \mathrm{~s}$ and $1 \times 10^{5} \mathrm{~s}$. 

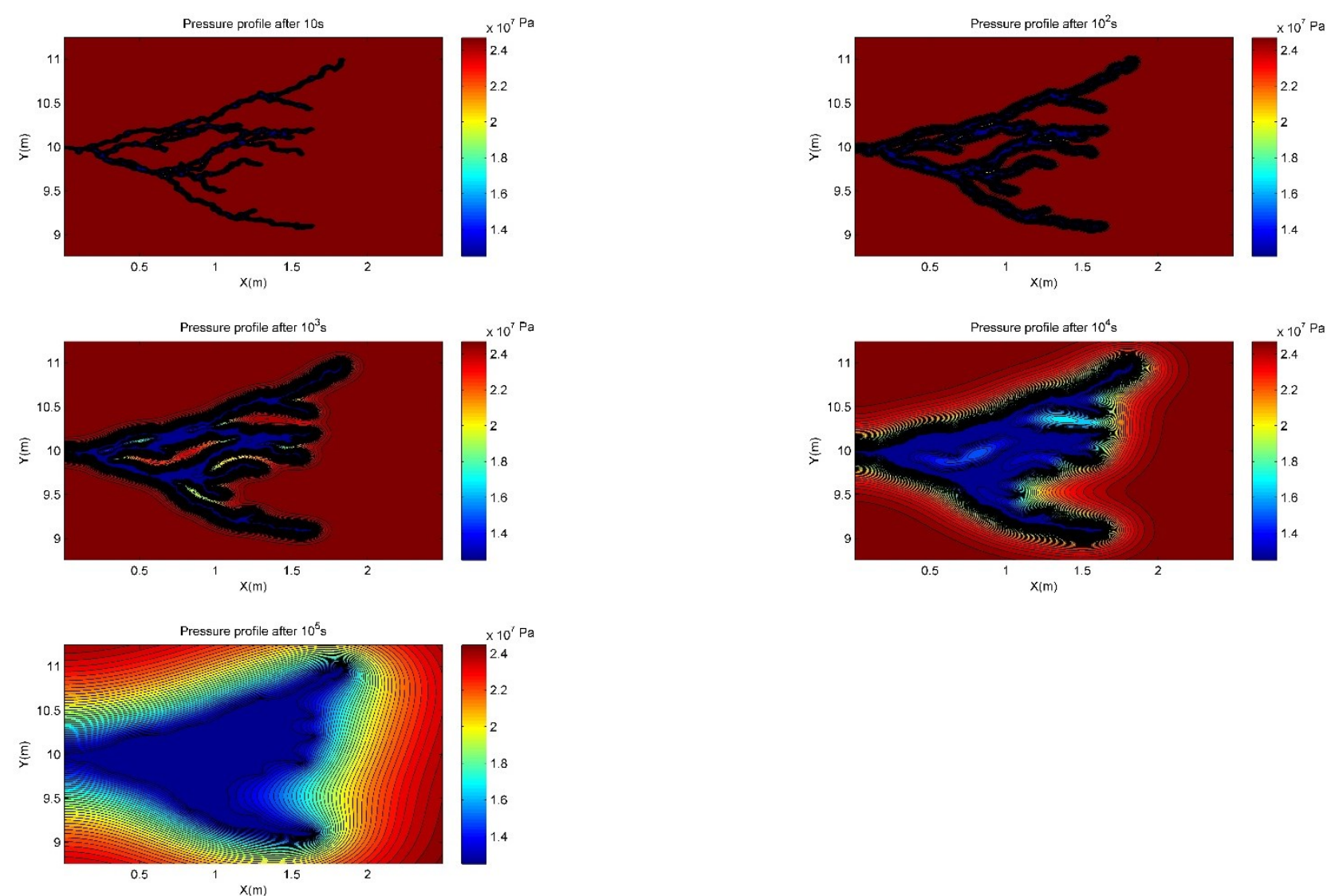

Figure D. 75 - Quad-branched fracture 11 pressure maps after $1 \times 10^{1} \mathrm{~s}, 1 \times 10^{2} \mathrm{~s}, 1 \times 10^{3} \mathrm{~s}, 1 \times 10^{4} \mathrm{~s}$ and $1 \times 10^{5} \mathrm{~s}$. 

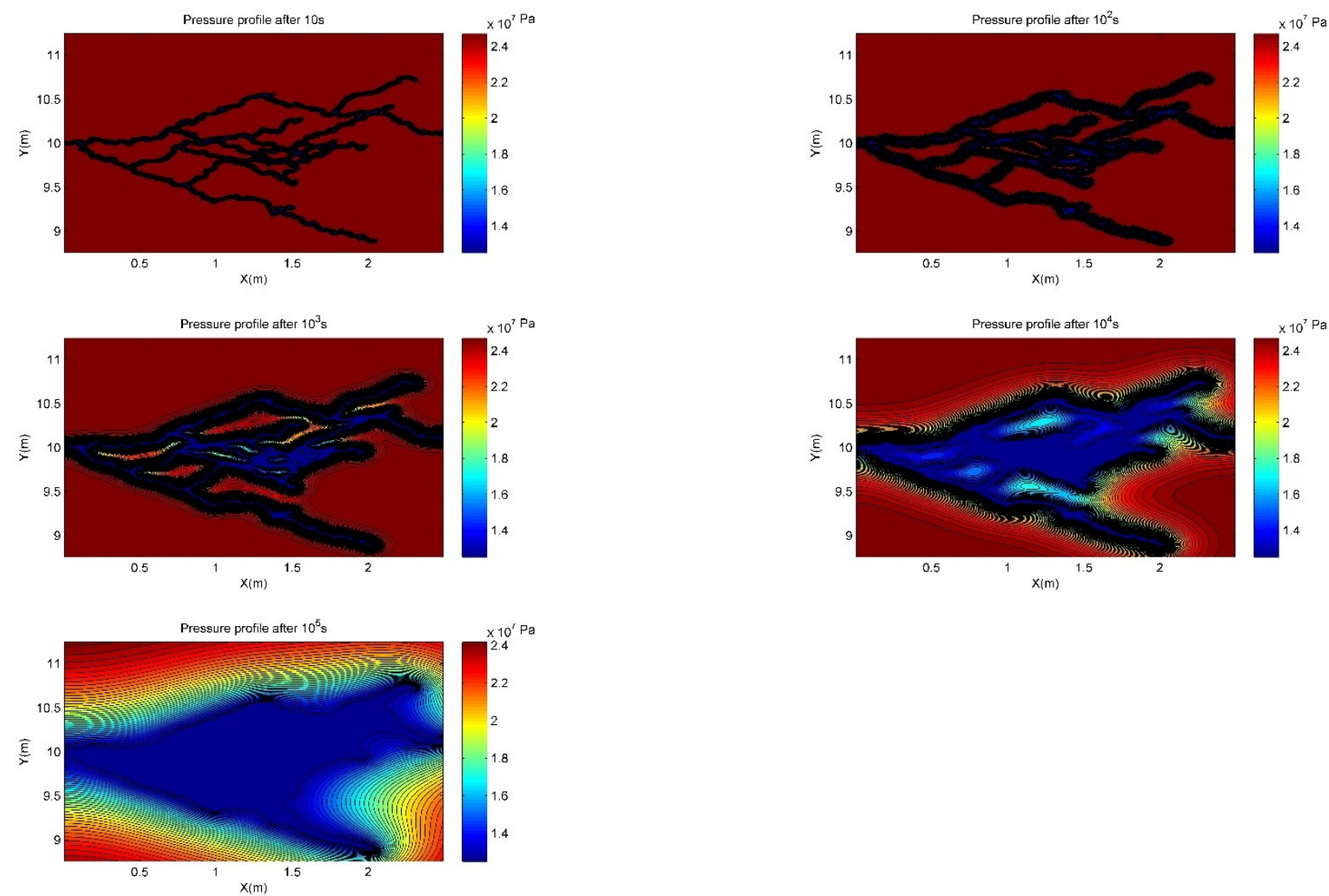

Figure D. 76 - Quad-branched fracture 12 pressure maps after $1 \times 10^{1} \mathrm{~s}, 1 \times 10^{2} \mathrm{~s}, 1 \times 10^{3} \mathrm{~s}, 1 \times 10^{4} \mathrm{~s}$ and $1 \times 10^{5} \mathrm{~s}$ 

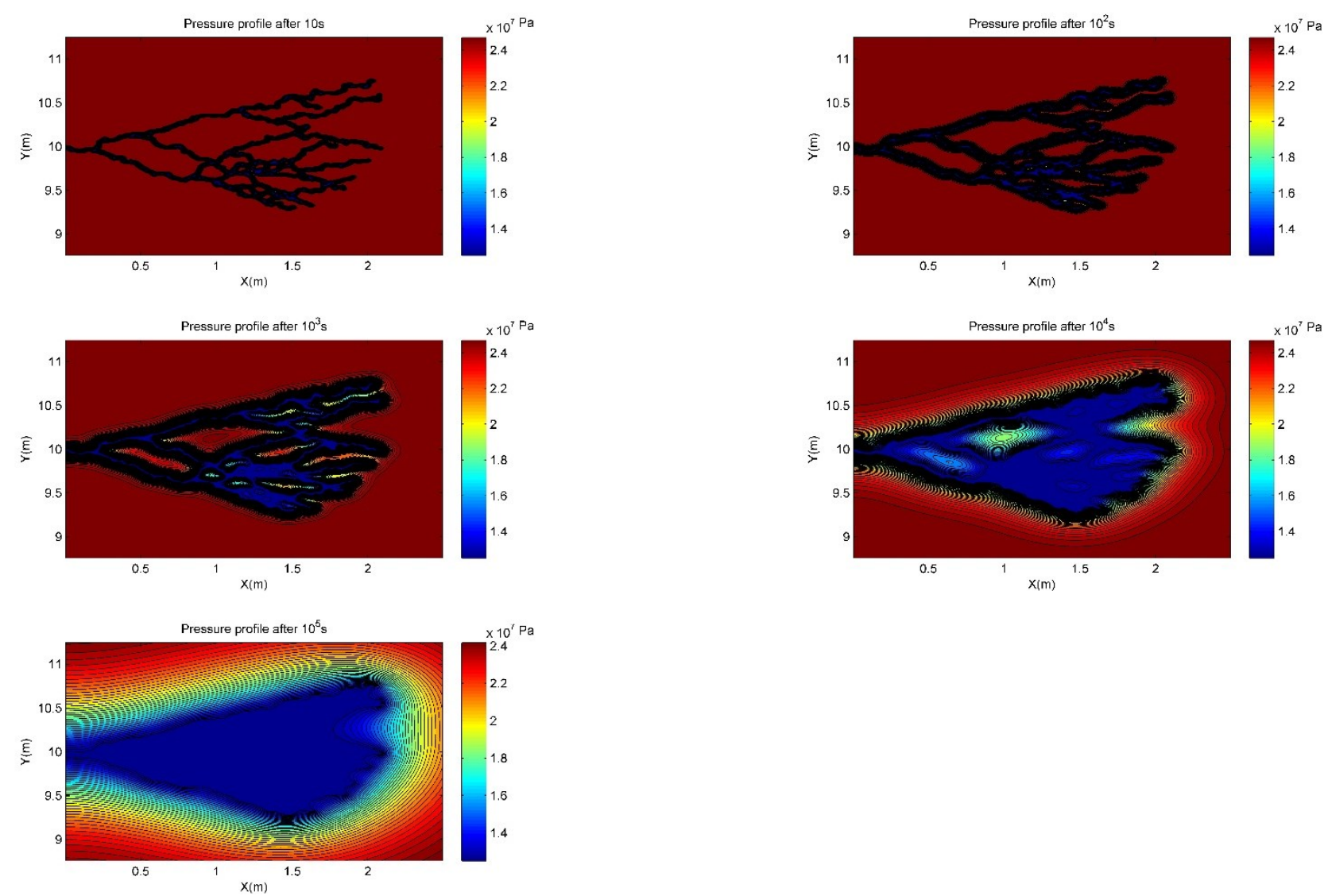

Figure D. 77 - Quad-branched fracture 13 pressure maps after $1 \times 10^{1} \mathrm{~s}, 1 \times 10^{2} \mathrm{~s}, 1 \times 10^{3} \mathrm{~s}, 1 \times 10^{4} \mathrm{~s}$ and $1 \times 10^{5} \mathrm{~s}$. 

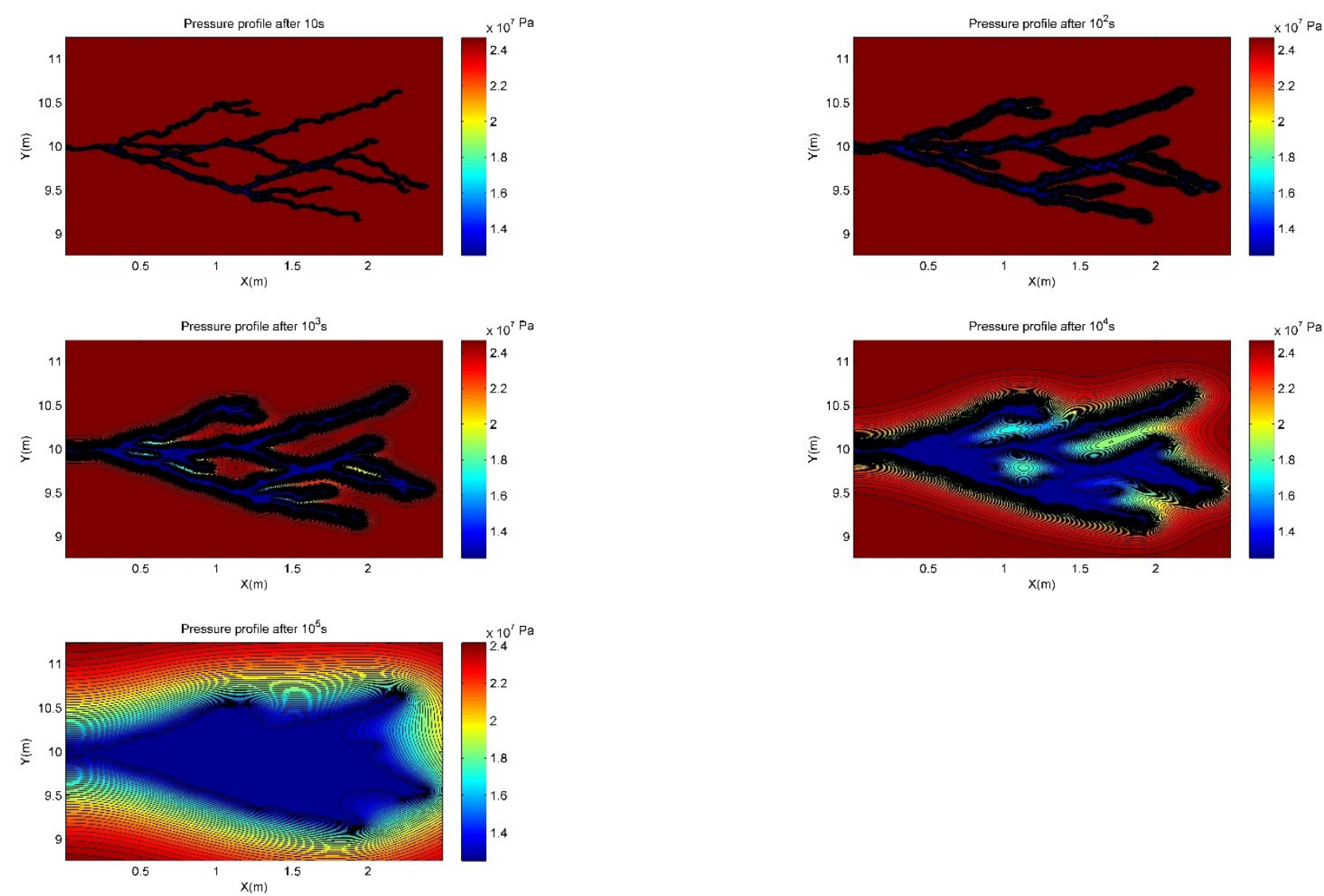

Figure D. 78 - Quad-branched fracture 14 pressure maps after $1 \times 10^{1} \mathrm{~s}, 1 \times 10^{2} \mathrm{~s}, 1 \times 10^{3} \mathrm{~s}, 1 \times 10^{4} \mathrm{~s}$ and $1 \times 10^{5} \mathrm{~s}$. 

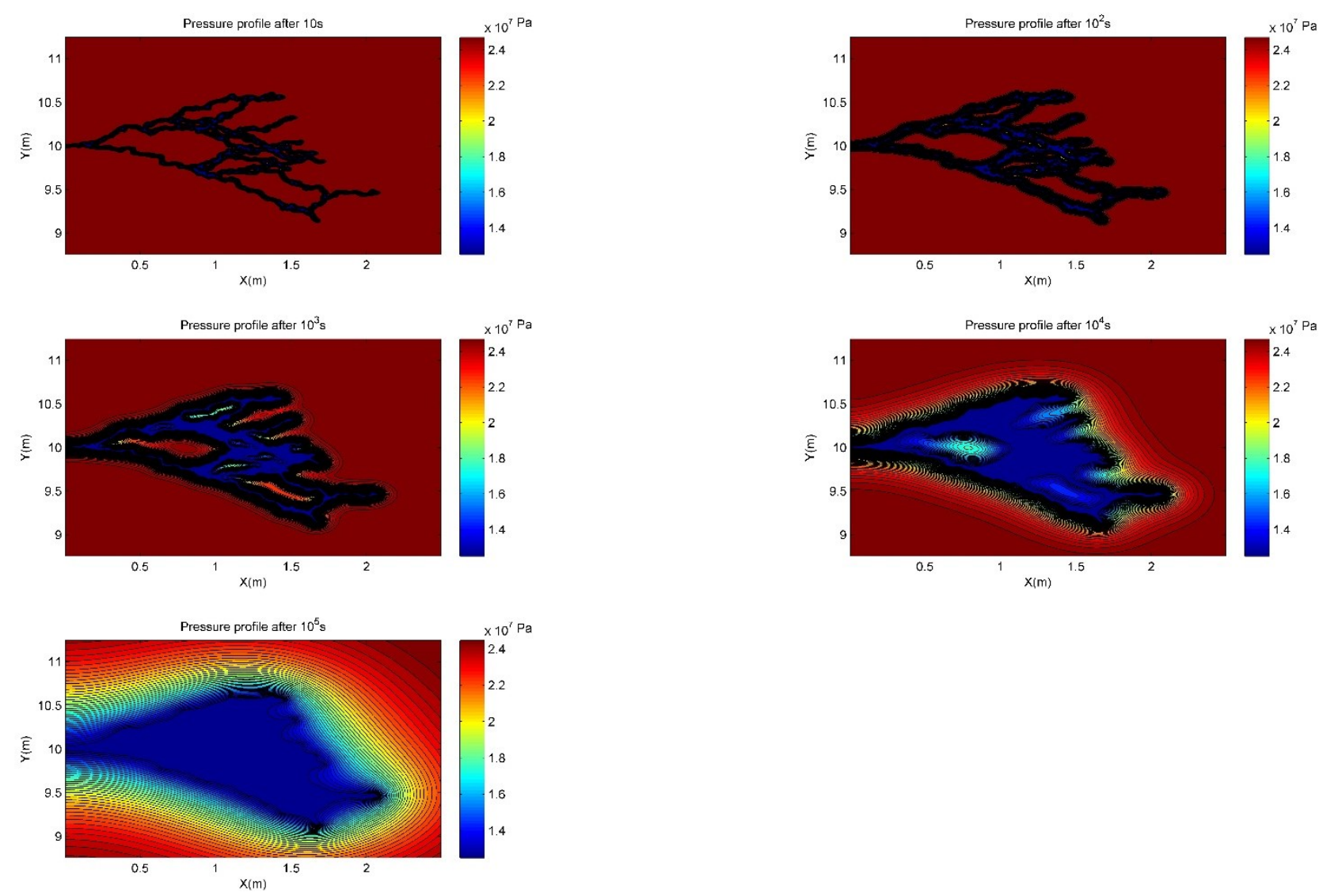

Figure D. 79 - Quad-branched fracture 15 pressure maps after $1 \times 10^{1} \mathrm{~s}, 1 \times 10^{2} \mathrm{~s}, 1 \times 10^{3} \mathrm{~s}, 1 \times 10^{4} \mathrm{~s}$ and $1 \times 10^{5} \mathrm{~s}$ 

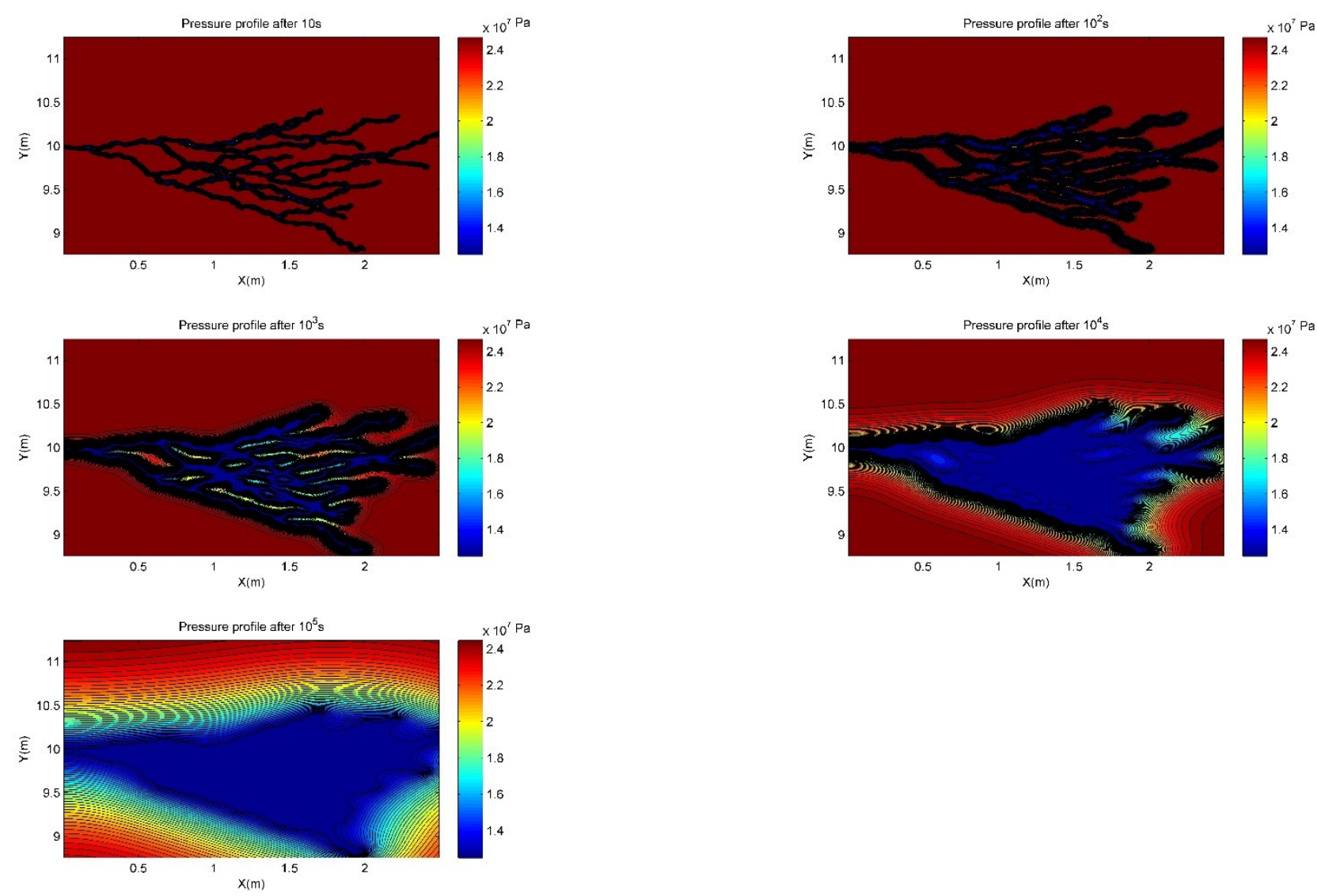

Figure D. 80 - Quad-branched fracture 16 pressure maps after $1 \times 10^{1} \mathrm{~s}, 1 \times 10^{2} \mathrm{~s}, 1 \times 10^{3} \mathrm{~s}, 1 \times 10^{4} \mathrm{~s}$ and $1 \times 10^{5} \mathrm{~s}$ 

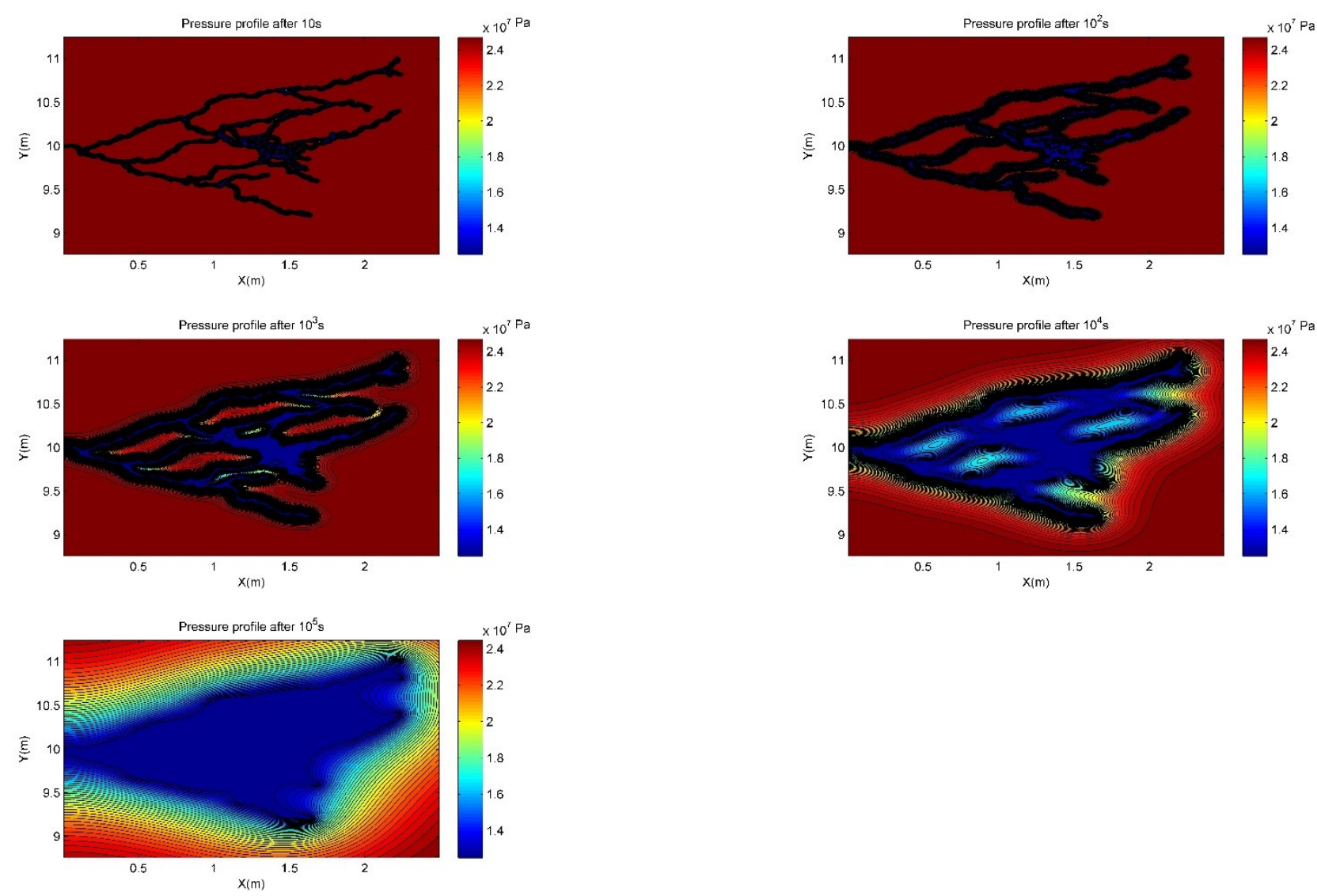

Figure D. 81 - Quad-branched fracture 17 pressure maps after $1 \times 10^{1} \mathrm{~s}, 1 \times 10^{2} \mathrm{~s}, 1 \times 10^{3} \mathrm{~s}, 1 \times 10^{4} \mathrm{~s}$ and $1 \times 10^{5} \mathrm{~s}$ 

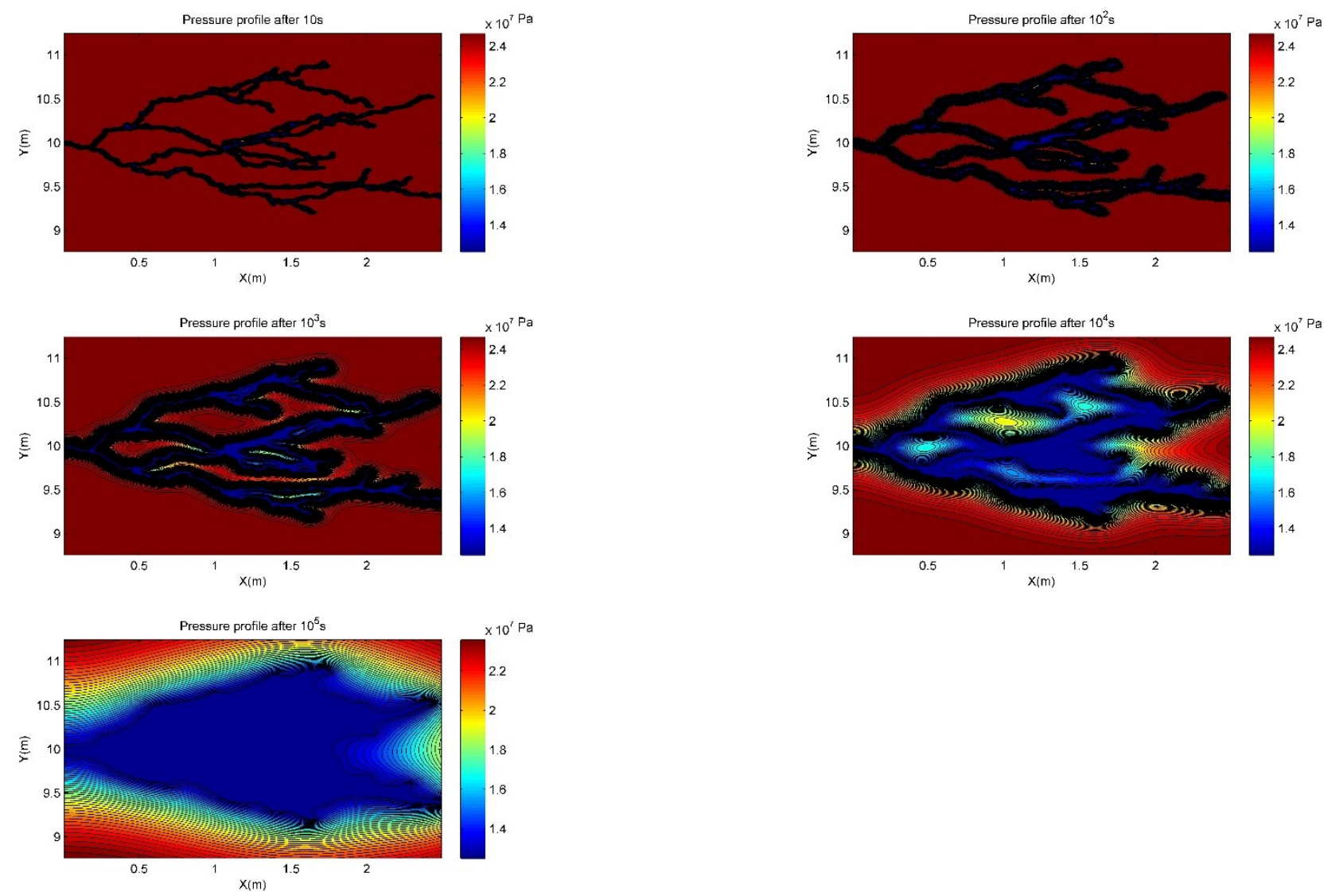

Figure D. 82 - Quad-branched fracture 18 pressure maps after $1 \times 10^{1} \mathrm{~s}, 1 \times 10^{2} \mathrm{~s}, 1 \times 10^{3} \mathrm{~s}, 1 \times 10^{4} \mathrm{~s}$ and $1 \times 10^{5} \mathrm{~s}$. 

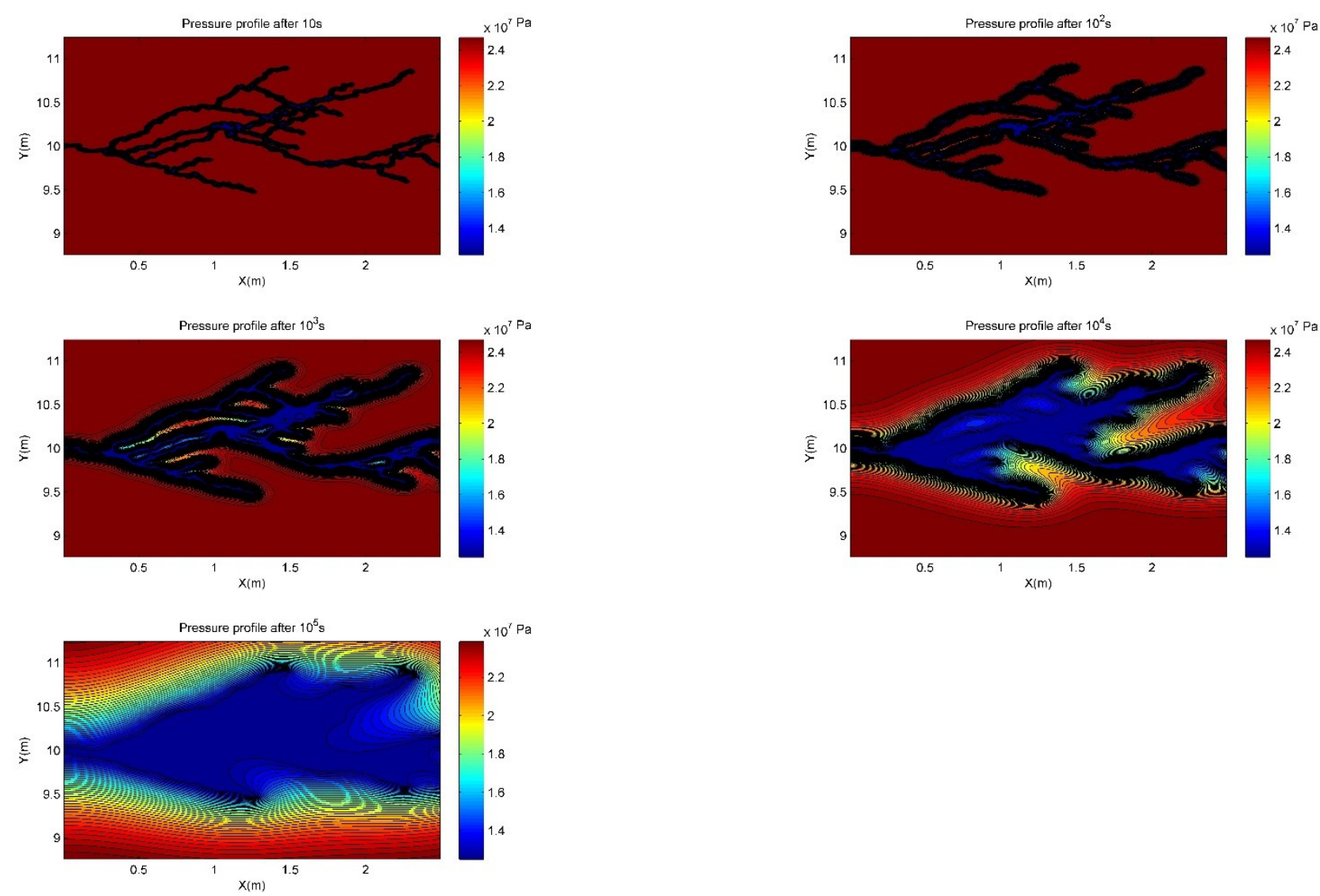

Figure D. 83 - Quad-branched fracture 19 pressure maps after $1 \times 10^{1} \mathrm{~s}, 1 \times 10^{2} \mathrm{~s}, 1 \times 10^{3} \mathrm{~s}, 1 \times 10^{4} \mathrm{~s}$ and $1 \times 10^{5} \mathrm{~s}$. 

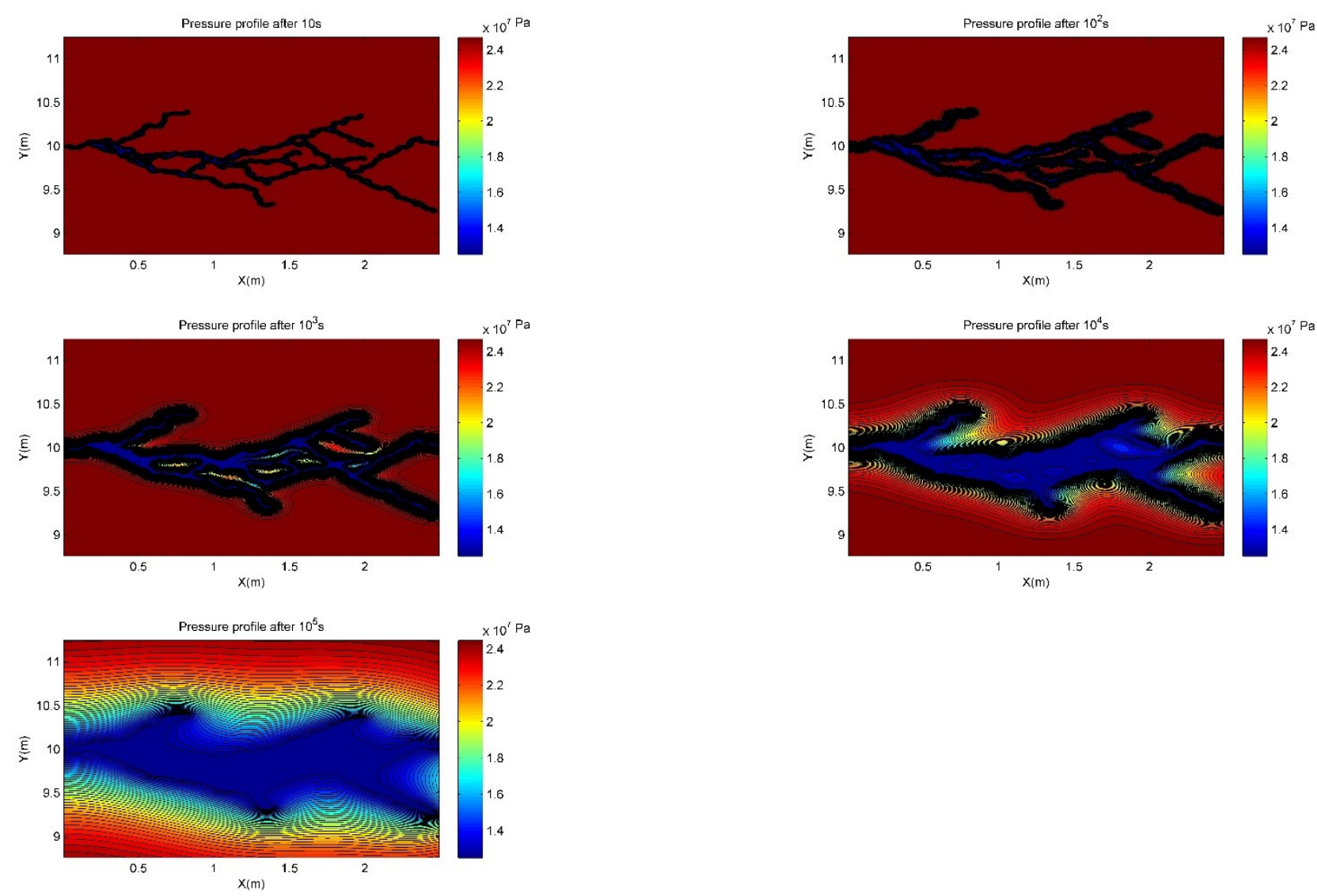

Figure D. 84 - Quad-branched fracture 20 pressure maps after $1 \times 10^{1} \mathrm{~s}, 1 \times 10^{2} \mathrm{~s}, 1 \times 10^{3} \mathrm{~s}, 1 \times 10^{4} \mathrm{~s}$ and $1 \times 10^{5} \mathrm{~s}$ 